UNITED STATES DEPARTMENT OF THE INTERIOR GEOLOGICAL SURVEY,

Spectrographic and Chemical Analyses of Geochemical Samples and Related Data from the Tanacross Quadrangle, Alaska By

Richard M. O'Leary, Steven K. McDanal, Christine M. McDouga1, Gordon W: Day, Gary C. Curtin, and Helen L. Foster Open-F1le Report 76-422 1976

This report is preliminary and has not been edited or reviewed for conformity with U.S. Geological Survey standards and nomenclature. 
Introduction- 1

Description of sample media- 2

Method of preparation- 5

Methods of analyses 7

Explanation of data- 11

References- 13

Illustrations

Figure 1. Map of Tanacross quadrangle showing sites at which samples of stream sediments, stream bank sod, aquatic mosses, and heavy mineral concentrates were collected

2. Map of the Tanacross quadrangle showing localities where analyzed rock samples were collected-_--.--

Table

1. Spectrographic anc chemical analyses and related data 


\section{INTRODUCTION}

Reconnaissance geochemical studies were made in the Tanacross quadrangle during the summer of 1974 to aid in determining the mineral resource potential of the quadrangle and to outline areas of anomalous metal content. For these studies, geochemical samples were collected at 607 sites at stream draining areas ranging from 5 to 25 square kilometers at an average sampling density of one site per 20 square kilometers (fig. 1). Samples were also collected at an additional 35 sites in swampy areas. The following sample media were collected at most sites: (1) Heavy mineral concentrates, (2) the detrital, minus 80-mesh stream sediment, (3) the oxide residue (oxalic-acid-leachable fraction) of stream sediment, (4) aquatic bryophytes (mosses), and (5) streambank sod (mixed organic and inorganic material) collected beneath the water level. In addition, 866 samples of rocks were collected at various localities within the quadrangle (fig. 2). 


\section{Description of sample media}

Stream sediments and heavy mineral concentrates were collected in active channels of streams. The material in samples of stream sediment ranges in size from fine sand and silt in areas of low relief to coarse sand in areas of high relief such as the Alaska Range in the southwest corner of the quadrangle and scattered high areas in the maturely dissected terrane north of the Tanana River. The minus-80-mesh stream sediment and the panned concentrates represent detrital material that has been mechanically introduced into a stream from bedrock and colluvium within a particular drainage basin. The minus-80-mesh sediment may reflect the presence of outcropping mineralized rock upstream. The heavy mineral concentrates are useful for determining the distribution of certain heavy metals and resistate minerals such as Au, Pt, cassiterite and scheelite. 
The secondary Fe and Mn oxides coating stream sediment particles (oxide residue), and aquatic bryophytes are considered scavenging agents that concentrate elements that have been leached from bedrock and colluvium and are migrating as ions in solution. The oxide residue of stream sediment contains secondary iron and manganese oxides, together with silica and alumina as major components along with the trace elements scavenged from solution. These components are extracted from the minus-80-mesh sediment using a weak oxalic-acid solution (Alminas and Mosier, 1976). A leachate residue is produced by this extraction process that is a derivative sample of the detrital stream sediment. In areas of low relief, where sulfides and other unstable ore minerals have been removed by chemical weathering, this oxide-rich derivative sample has the following advantages over the minus-80-mesh stream sediment: (1) the level of trace metal content is about an order of magnitude greater in the oxide residue than in the detrital sediment, and (2) there is a greater contrast of metal values in the oxide residue than in the detrital sediment. Weakly anomalous metal concentrations that might go unnoticed in the detrital stream sediment are enhanced in the oxide residue because the dilution effects of the detrital sediment are removed. 
Aquatic bryophytes were collected beneath the water level from streams and from shallow ponds in swampy areas. Generally either alliergon cordifolium (Hedw.) Kindb., calleirgon giganteuin (Schimp.) Kindb., or Fontinalis duriaei Schimp. were collected at each site. Identification was made by Hansford T. Shacklette of the U. S. Geological Survey who followed the nomenclature of Crum. Steere, and Anderson, (1973). The species were treated the same in this study. The bryophytes concentrate elements in solution primarily by absorption (Brooks, 1972). The bryophytes apparently also concentrate certain heavy minerals such as cassiterite (Curtin and others, 1976) by the entrapment of particulate matter.

Streambank sod was collected beneath the water level from streams and swampy areas in the terrane north of the Tanana River, but usually collected above the water level from streams in the Alaska Range. The streambank sod was not present at many of the sites in the Alaska Range, and therefore was not a useful sample medium in this terrane. The streambank sod consists of a mixture of inorganic material ranging in size from silt to fine sand, and dead roots and other pieces of decaying organic material. The el ement content of the sod comprises elements scavenged from solution primarily by the organic material and the element content of the detrital material.

Rocks were collected from outcrops at most sites. Several samples were collected at sites where the bedrock showed indications of alteration or mineralization. 


\section{Method of Preparation}

The geochemical samples were prepared for analyses by the following treatments:

The rock samples were crushed to -0.25 inches $(-6.35 \mathrm{~mm})$ using a chipmunk crusher. The crushed rock was split with a Jones splitter and ground to a -150 mesh using a verticle pulverizer with ceramic plates.

The stream-sediment samples were air dried and sieved through a 80 -mesh sieve. The -80 mesh fraction was saved for analyses.

An oxide residue (oxalic acid leachable fraction) was obtained by the procedure described by Alminas and Mosier (1976). Fifty mls of $1.5 \underline{N}$ oxalic acid was boiled in a beaker containing $5 \mathrm{~g}$ of -80 mesh stream-sediment. After 5 minutes the liquid was filtered while hot through a quantitative, fast filter paper. The filtrate was taken to dryness on a hot plate and placed in a muffle furnace at $450^{\circ} \mathrm{C}$ for four hours. The residue was then pulverized with a mortar and pestle to a fraction smaller than 80 mesh. This fraction was saved for analysis. 
Heavy mineral concentrates were sieved through a 40 mesh sieve and the -40 mesh fraction was saved. The fraction was further separated with bromoform into two fractions: a light mineral fraction having a specific gravity of 2.86 or less and a heavy mineral fraction having a specific gravity greater than 2.86 . The light mineral fraction was discarded. Magnetite and other magnetic minerals were removed from the heavy mineral fraction by use of a hand magnet and a Frantz Isodynamic magnetic separator set at 0.2 ampere. This magnetic fraction, containing primarily magnetite, ilmenite and hematite, was labeled $C-1$ and was for analysis. The remaining heavy fraction was again sent through the Frantz Isodynamic magnetic separator at setting of 0.6 amperes. The non-magnetic fraction at this higher setting containing primarily zircon, apatite and sulphides, was labeled C-3 and retained for anaiys is and mineralogy.

The streambank sod was air dried and sieved through a $2 \mathrm{~mm}$ stainless steel sieve to remove rock fragment and large pieces of litter. The minus $2 \mathrm{~mm}$ fraction was saved. This fraction was placed in an evaporation dish and roasted until the organic material in the sod was completely ashed. A split of this sample was saved for gold analysis.- Another split was sieved through an 80 mesh sieve and the -80 mesh saved. 
The bryophytes (mosses) were rinsed at the sample site and were dried at the field camp in a cloth bag. The moss was further prepared in the laboratory by hand massaging the dried material to remove remaining sand and silt. The samples were then pulverized in a Waring blender and ashed in a muffle furnace at a peak temperature of $500^{\circ} \mathrm{C}$.

\section{Methods of Analyses}

The rocks, stream sediments, and stream bank sod samples were analyzed by a 6-step, DC-arc, semiquantitative emission spectrographic method described by Grimes and Marranzino (1968) for the analyses of geologic material.

Ten $\mathrm{mg}$ of prepared sample was mixed with $20 \mathrm{mg}$ of pure graphite powder, packed into a 0.25 inch $(6.35 \mathrm{~mm})$ diameter preformed graphite electrode, and burned in a DC arc for 135 seconds using a $1.5-\mathrm{m}$ Wadsworth mounted-grating spectrograph. Spectra were recorded on $35-\mathrm{mm}$ SA-1 film in groups of 23 per film. Thirty elements were determined.

The observed spectra were compared visually to standard spectra using a 20x comparator. Results were reported as the approximate midpoints of geometric brackets whose boundaries are $1.2,0.83,0.56$, $0.38,0.26,0.18,0.12$, etc. These midpoints are $1,0.7,0.5 ; 0.3$, $0.2,0.15,0.1$, etc.

The precision of a reported value is approximately plus or minus one reporting value at 68 percent confidence and two reporting values at 95 percent confidence. Iron, magnesium, calcium, and titanium are reported in percent; all other elements are reported in ppm. Data qualifier codes, defined below, were used with some reported values. 
The approximate visual lower limits of determination for the 30 elements included in this report are, for those given in percentage, iron, 0.05; magnesium, 0.2; calcium, 0.05; and titanium, 0.002; for those reported in ppm, manganese, 10; silver, 0.5; arsenic, 200; gold, 10; boron, 10; barium, 20; beryllium, 1; bismuth, 10; cadmium, 20; cobalt, 5; chromium, 10; copper, 5; lanthanum, 20; molybdenum, 5; niobium, 20; nickel, 5; lead, 10; antimony, 100; scandium, 5; tin, 10; strontium, 100; tungsten, 50; vanadium, 10; yttrium, 10; zinc, 200; and zirconium, 10.

Heavy mineral concentrates and the oxide residues of stream sediment were analyzed by the same procedure as described above with the following exceptions. To eliminate the spectral interferences caused by high concentrations or iron, five mg of prepared sample was mixed with 20 $\mathrm{mg}$ of pure graphite powder and $5 \mathrm{mg}$ of pure Arkansas quartz. The spectra were compared to standards based on a $10 \mathrm{mg}$ sample weight, therefore, all values were doubled. Doubled values occurring between midpoints were rounded to the higher midpoint (e.g. $20 \mathrm{ppm} \times 2=40 \mathrm{ppm}$ but reported as $50 \mathrm{ppm})$.

The approximate visual lower limits of determination for the 30 elements are twice the original values given and the double Jower limits occurring between midpoints are rounded to the higher midpoint.

The bryophytes were analyzed by a DC-arc, semiquantitative emission spectrographic method developed by Mosier (1972) for the analysis of plant ash. 
Five $\mathrm{mg}$ of plant ash mixed with $10 \mathrm{mg}$ of buffer (graphite containing 10 percent calcium carbonate) was packed in a preformed graphite electrode (ASTM S-13), and burned in a DC-arc using a $1.5 \mathrm{~m}$ Wadsworth mountedgrating spectrograph. The split slit technique utilizing a Hartman diaphragm and step filter assemblage described by Mosier (1972) was replaced with an automatic filter positioner device developed by Curry and others (1975). Specta were recorded on 35-m SA-1 film in groups of 23. Twenty-nine elements were determined for each sample. Results were obtained by the previously described 6-step visual comparison method with approximately the same precision.

Iron, magnesium, and titanium are reported in percent; all other elements are reported in ppm.

The approximate visual lower limits of determination for the 35 elements included in this report are, for those reported in percentage, iron, 0.005; magnesium, 0.01; calcium, 0.05; and titanium, 0.001; for those reported in ppm, manganese, 10; silver, 0.1; arsenic, 200; gold, 2; boron, 5; barium, 20; beryllium, 0.5; bismuth, 1; cadmium, 1; cobalt, 5; chromium, 2; copper, 1; lanthanum, 20; molybdenum, 2; niobium, 20; nickel, 5; lead, 1; antimony, 20; tin, 5; scandium, 5; strontium, 100; vanadium, 5; tungsten, 50; yttrium, 5; zinc, 100; and zirconium, 10. 
Selected samples were analyzed for gold, copper, lead, and zinc by atomic absorption methods developed by Ward and others (1969). Arsenic was determined by a colorimetric method developed by Ward and others (1963). Mercury was determined by a mercury-vapor detector developed by Vaughn and McCarthy (1964).

At present, the lower limits of determination for these elements in ppm are gold, 0.05; copper, 5; lead, 5; zinc, 5; arsenic, 10; and mercury, 0.02 . 


\section{Explanation of Data}

The data listed in table 1 include analytical results for the 7 sample media. The table also contains data on the characteristics of four of the sample media (texture and percent ash) and of the streams from which the stream-related samples--stream sediment and stream-bank sod--were collected.

For all the sample sets the data are arranged so that column 1 contains the sample numbers shown on figures 1 and 2 . The latitude and longitude are shown in columns 2 and 3 . Columns in which the element headings (denoted in capital letters) are preceded by an $\mathrm{S}$ contain the emission spectrographic data. Six additional elements ( $\mathrm{Au}, \mathrm{Hg}, \mathrm{Cu}, \mathrm{Pb}, \mathrm{Zn}$, and $\mathrm{As}$ ) were determined in the minus-80-mesh stream sediment and in the ash of streambank sod. The prefixes for these elements refer to the analytical methods used as follows: $A A$, atomic absorption methods; INST, instrumental method (atomic absorption); and CM, colorimetric method. Element concentrations were measured in parts per million except for $\mathrm{Fe}, \mathrm{Mg}, \mathrm{Ca}$, and $\mathrm{Ti}$ which were measured in percent. The element content of the streambank sod and aquatic moss is reported on an ash-weight basis.

Data qualifier codes were used with some reported values. Definitions of the qualifier codes that follow the analytical data are: $\quad B=$ no data available, or sample not analyzed for this element; $N=$ not detected at the level of detection or at the value shown; $L=$ detected, but below the limit of determination or below value shown; and $G=$ greater than the value shown. 
In the minus-80-mesh stream sediment, oxide residue, and streambank sod data sets, the column immediately following the analytical data contains the $\mathrm{pH}$ data for the stream waters. The $\mathrm{pH}$ values were measured at the time the samples were collected and, therefore, reflect the $\mathrm{pH}$ of the waters at only one point in time during the field season.

Data on percent ash are given for the streambank sod and the aquatic mosses in the following column. These values represent sample weight after ignition.

The numbers in columns $4801-4804$ in the data sets for minus-80-mesh stream sediment. oxide residue, and streambank sod ash, express, characteristics of sample texture, and characteristics of the stream from which the samples were collected. Column 4801 contains data on the stream order at a scale of 1:63,360 in the following manner: First order streams are coded 1. These streams have no well-defined tributaries and drain areas generally ranging from 2-11 $\mathrm{km}^{2}$. Streams coded 3, 4. and 5 are the third, fourth, and fifth order streams which drain much larger areas than the first and second order streams.

The data in column 4802 denote stream width in meters or fractions thereof. Stream depth, in meters and fractions of meters, is shown in column 4803.

Column 4804 contains data on sediment texture as follows: 1 = stream sediment composed mainiy of silt and fine sand; 2 = stream sediment composed mainiy of sand; 3 = stream sediment composed mainly of coarse sand gravel. All the data in columns 4801-4804 were determined in the field. 


\section{REFERENCES CITED}

Alminas, H. V. and Mosier, E. L., 1976, Oxalic acid leaching of rock, soil and stream sediment samples as an anomaly-accentuation technique: U.S. Geol. Survey open file Rept. No. 76-275.

Curry, K. J., Cooley, E. F., and Dietrich, J. A., 1975, An automatic filter positioner device for emission spectroscopy: Applied Spectroscopy, v. 29, no. 3, p. 274-275, May/June 1975.

Grimes, D. J., and Marranzino, A. P., 1968, Direct-current arc and alternating-current spark emission spectrographic field methods for the semiquantitative analysis of geologic materials. U.S. Geol. Survey Circ. 591, 6 p.

Mosier, E. L., 1972, A method for semiquantitative spectrographic analysis of plant ash for use in biogeochemical and environmental studies: Applied Spectroscopy, v. 26. no. 6, p. 636-641, Nov/Dec., 1972.

Ward, F. H., Lakin, H. W., Canney, F. C., and others, 1963, Analytical methods used in geochemical exploration by the U.S. Geological Survey: U.S. Geol. Survey Buli. 1152, 100 p. Ward, F. N., Nakagawa, H. M., Harms, T. F., and Van Sickle, 6. H., 1969, Atomic-absorption methods of analysis useful in geochemical exploration: U.S. Geol. Survey Bull. 1289, 45 p.

Vaughn, W. W., and McCarthy, J. H., jr., 1964, An instrumental technique for the determination of submicrogram concentrations of mercury in soils, rocks, and gas, in Geological Survey research 1964: U.S. Geol. Survey Prof. Paper 501-D, p. D123-D127. 


\section{REFERENCES CITED}

Alminas, H. V., and Mosier, E. L., 1976, Oxalic acid leaching of rock, soil and stream sediment samples as an anomaly-accentuation technique: U.S. Geol. Survey open-file rept. no. 76-275.

Brooks, R. R., 1972, Geobotany and biogeochemistry in mineral exploration: New York, Harper and Row, $290 \mathrm{p}$.

Crum, Howard A., Steere, William C., and Anderson, Lewis E., 1973, A new list of mosses of North America north of Mexico: The Bryologist. v. 76 , no. 1, p. $85-130$.

Curry, K. J., Cooley, E. F., and Dietrich, J. A., 1975, An automatic filter positioner device for emission spectroscopy: Applied Spectroscopy, v. 29, no. 3, p. 274-275, May/June 197.5.

Curtin, G. C., Day, G. W., Marsh, S. P., O'Leary, R. M., and Tripp, R. B., 1976, Geochemical maps showing the distribution and abundance of $\mathrm{Sn}$ in the Tanacross quadrangle, Alaska: U.S. Geol. Survey, Misc. Field Studies, Map MF-767K, 1 sheet, scale 1:500,000.

Grimes, D. J., and Marranzino, A. P., 1968, Direct-current arc and alternating-current spark emission spectrographic field methods for the semiquantitative analysis of geologic materials: U.S. Geol. Survey Circ. $591,6 \mathrm{p}$.

Mosier, E. L., 1972, A method for semiquantitative spectrographic analysis of plant ash for use in biogeochemical and environmental studies: Appl ied Spectroscopy, v. 26, no. 6, p. 636-641, Nov./Dec. 1972.

Ward, F. N., Lakin, H. H., Canney, F. C., and others, 1963, Analytical methods used in geochemical exploration by the U.S. Geological Survey: U.S. Geol. Survey Bull. 1152, 100 p. 
Hard, F. N., Nakagawa, H. M., Harms, T. F., and Van Sickle, G. H., 1969, Atomic-absorption methods of analysis useful in geochemical exploration: U.S. Geol. Survey Bull. 1289, 45 p.

Vaughn, W. W., and McCarthy, J. H., Jr., 1964, An instrumental technique for the determination of submicrogram concentrations of mercury in soils, rocks, and gas, in Geological Survey research 1964: U.S. Geol. Survey Prof. Paper 501-D, D. D123-D127. 
Minus 80 mesh Stream Sediment:

Elements not looked for: S-Fe, S-Mg, S-Ca, S-Mn, S-B, S-La, S-Ea $S-N b, S-S C, S-V, S-Y, S-2 r$.

Elements looked for, but not detected: S-Ag, S-As, S-Au, S-Cd and S-Sb.

The following elements were not detected except in the samples listed:

$$
\begin{aligned}
& \text { S-Bi...... sample TX } 6435 \text { (15 ppm) } \\
& \text { S-Mo...... samples TX } 485(10 \mathrm{ppm}) \text { and } \\
& \text { TX } 3245(10 \mathrm{ppm}) \\
& \text { S-Sn...... samples } T X 317 \mathrm{~S}(15 \mathrm{ppm}) \text { and } \\
& \text { TX 639S (30 ppm) }
\end{aligned}
$$




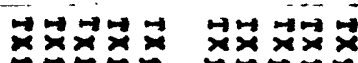
ம

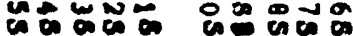

$\vec{x} \vec{x} \vec{x} \vec{x} \vec{x}$ $\bar{N} \bar{N} \backsim \bar{N}$ TNN a 4 क
부젖주점 至:

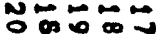

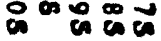

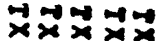

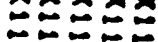

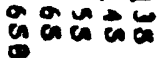

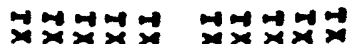
$-\infty=0$ $N=0$ 웅 co 0 \%

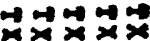

$4 x \times 2$

$5090 \%$

añ

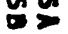

우요웅

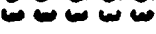
vacu un -

a. a o o

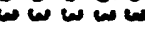
un ence

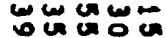

a o. 00 wa ט ט u u

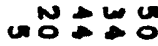

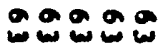
vacus on $a+\infty+\infty$

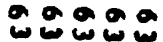
unu un w. Nu
09000

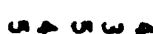
OSNG ט
2000요 $\rightarrow a=0$ ज这

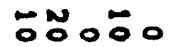

운운웅

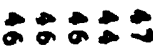
녕웅
-

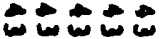
L: 우웅

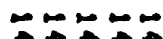
둔 ล W $\because \div \div \div \div$ ம م

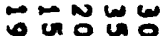

$\because \div \div \div$ ש م

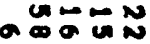
uñ $\because \div \div \div$ ש NNNDN WNa w)
ざさきた

N

- N.
픈

ט.N.N

든

- 이요

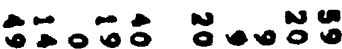

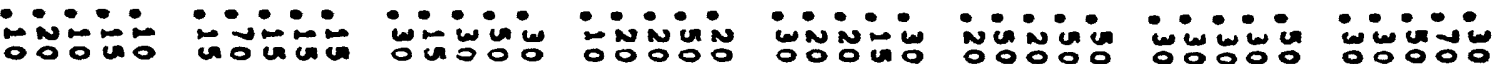

$\sim-\infty-\infty-N \infty-\infty$

$x=$

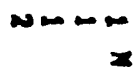
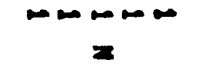

$-\infty \rightarrow \infty$

$x=2$

W w w w

w w w w

$\omega \infty \omega \infty$

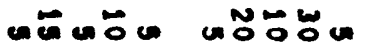

무유요

10

जํㅜㅇㅠ

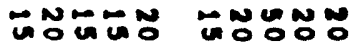

ڤํㅇ유유

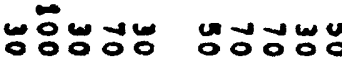
꿍르 ำ ㅇํㅇํํㅇำ 둥후웅 옹ํㅇㅇํㅇ 붕분 ㄴํㅇㅇํㅇํㅇ

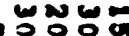
눙ㅇㅇㅇㅇㅇㅇ

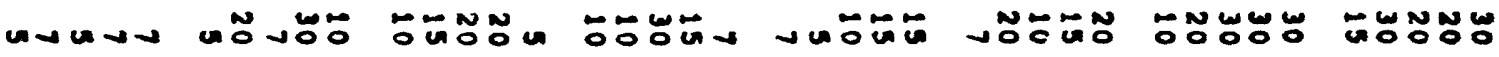

00000000000 000 000
0000000000 -
00000 - 000
0 $00 \% 0$

100
00000

0000
00000

00.00
00000 000000 $=00000000$
0000000000

00000

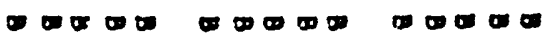

$000 \% 0$ 0002
00000 00000
00000 00000
5

ज茴囩 


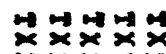

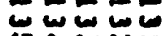

L - w N

o 0 \% $\rightarrow x-4 x$ $x \times x \times 2$ 以NN业 두요 $\rightarrow \vec{x}+\vec{x}+\vec{x}$

NNNNN

G is on

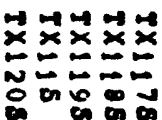

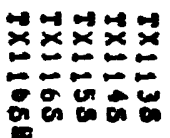

부ㅈㅜㅕ년

$\times \times \times x \times$

Б二5ㅇㅇㅇ

N的只电。
부ㅈㅓㅕ며제

xखx:

엉요웅

엫ㄷㅇ
00000

$0+00$

08000

- 00

00000

-

00000

00000

- 0080

00000

- 00

000

000

00000

000

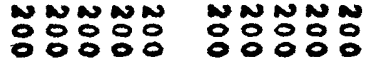

$z=z x=$

$z=z x$
ํํㅇํํㅇํำ

$2+2 \geq 2$
ํํㅇํํㅇํำ

ํํㅇํํㅇํำ

$z \geq z \geq z$

주지
ํํㅇํํㅇํำ

ลัดํํํำ

:ํํㅇํㅇํำ

I $\geq \geq z$

$z=2 \times 2$

2 $2 \geq 2=$ $\therefore 0: 0$

운윰응

$x \geq z=2$ $\div 0000$ 옹여요

e $0=2$
웅웅 온옹용 I 220 운웅웅 $x z z=2$
웅웅 은온옹

웅웅: zox $=2 x=$ $\div \div \div \div 0$ 운운오옹

$z=z$ $\div: \div \div 0$ 언하옹요 $x \geq x=2$
00000 0000 옹용요

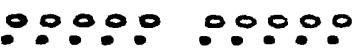
ioj:o inio: $=$

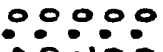

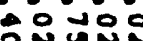

$\geq$
00000 00000 NNNN

$0 \% 00$ $\therefore 00 \div 0$ NNNND

$z=$

:0:0: 유:

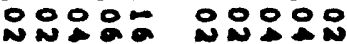

$=$

$=$

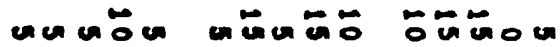
or ํํำ $\cos$ tot

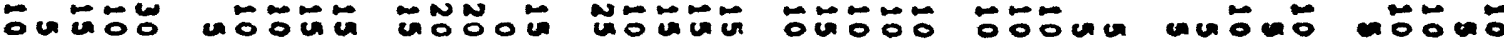
.

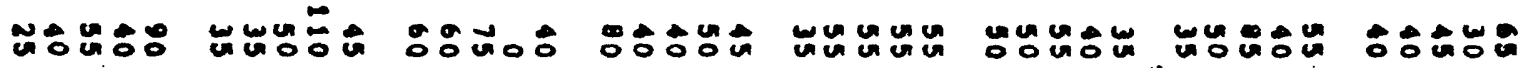

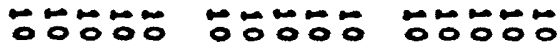

$z z=x$ $z \geq z \geq z$

$z \geq z \geq z$

unu uno a.:-

cucua unucia 00

a nu -

뚜융

ㅜㅜ웅

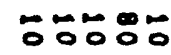
$z z z z z \quad z z z z z-z z z-z$

ㅜㅜ웅

$\div 0 \div 0 \%$

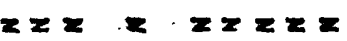

uncan unumu on coun wisi is:is:

$\infty$

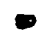

unuma uncana ovicio oivio. -

NMNMN
NNNルN

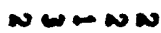

$\boldsymbol{N} \sim \boldsymbol{N} \sim$ 
- 2000웅 caco -

- ana

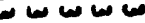

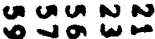
๒๐ is ogana NN운무웅 ーニ Sa

$\rightarrow N-N$ 유.

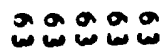
드

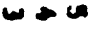
둥웅 o. 0000

200 un
- oro ט

is 0 is 80 ט

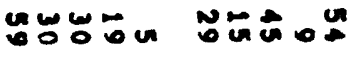

\section{$\therefore=5 \div=5$

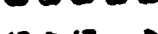 \\ - Naㄴa \\ - Nㅜㅇํ․ \\ $\sim-\infty$ $\because \leq+\infty$

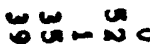 \\ $-\infty=\infty$ क ט. مN N $\therefore N=$} तே co a c a

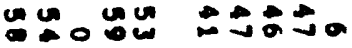

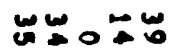

ロேー 4

(1)

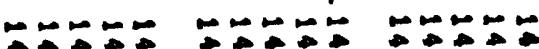

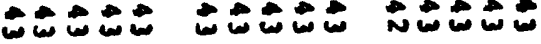

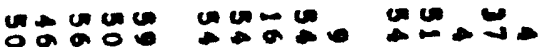
๑艹

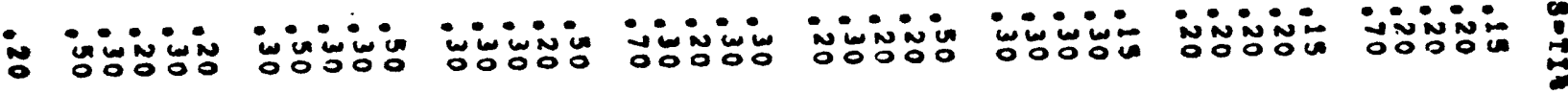

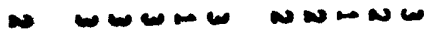

$=$ $z=$
$=$

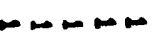

$z=$ $\sim \infty-\infty$
-

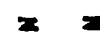

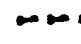

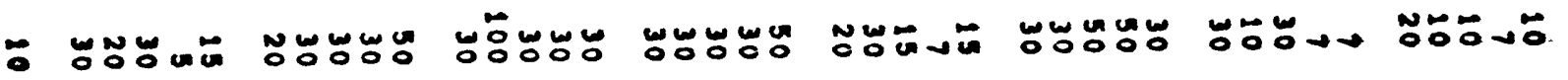

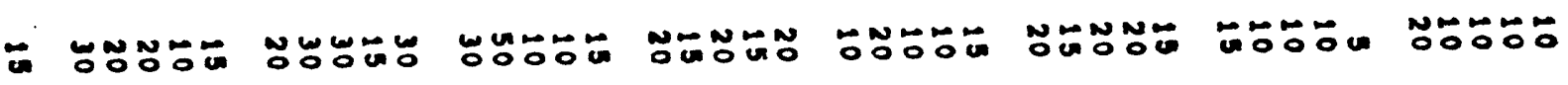

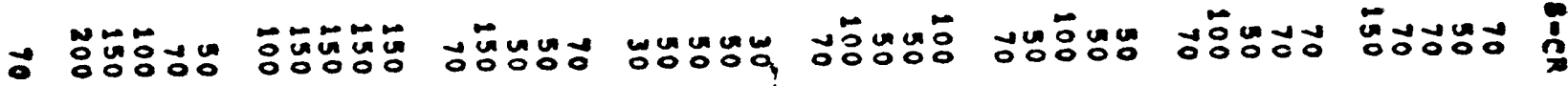

- 00000

- 0000

00000

- 1000

00000

0000000000

00000

100

.000 0000
00000

\$0000 1000
$-0005$
- 00000 - -000

00000 0000
0000000000 00000
00000

$-\infty$ $\begin{array}{rrr}00000 & 00000 & 00000 \\ 00000 & 00000 & 000\end{array}$

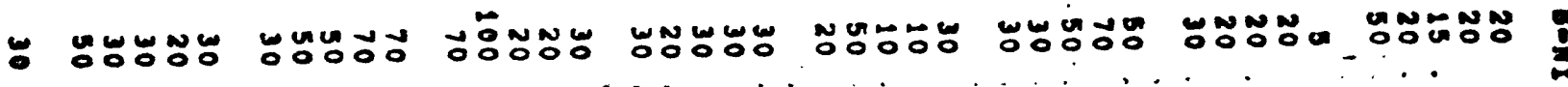


- 0000000000

- 000

- $20=$

00000

10000

00000

$0000 \%$

- 000000

00000

- 00

00000

0000

6

이요

$-2=0$

\section{- NNNNN}

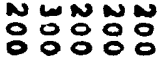

능영용용요

NN요용

NNㅡㅇㅇㅇㅇㅇㅇㅇ

Nㅜㅇㅇㅇㅇㅇㅇㅇㅇㅇ

NNNNN

a $z z z z z$

$z=z$

z. $z x z$

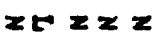

XEx $x \geq$

$z z z x$

NNNNN 응영ㅇㅇㅇ

$x \geq x=2$
영영운 웅운웅

웅유웅

z
은운운운

웁웅웅웅

$=\infty 2$ $z=2 z$

$z \geq z=2$
운운운웅

$z \geq z=$
운운운운

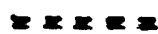

$\therefore 0: 0$

๑nùa

$x=2 x$

\section{- 00000}

00000

웅웅

00000
$0 \% 00:$

$-$

$$
z=
$$

00000

:00:-in

00000

$\div 0000$ NNADO

NN으요요

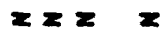

0000

눙웅

$z x$

00000

$00: 00$

Nㅇㅇㅇㅛ

$z=$

00000

$\therefore \circ$ in: WN《只品

$=$

$$
<2
$$

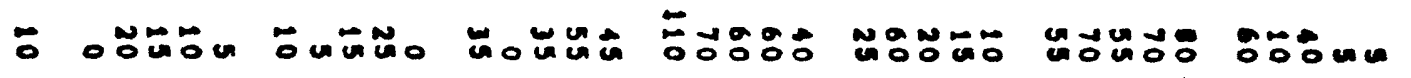

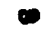

-

$\div \div 00$

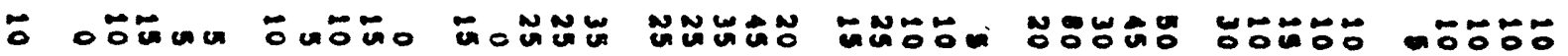
-

○岗ठี -

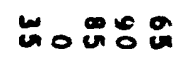
$\infty$
ロேーッ

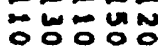
뜽ํํㅇํำ 8

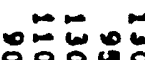

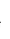

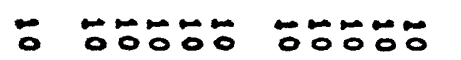

- $=7=\pi$

a unugun un ungas

$\therefore$ :00: $: 0^{\circ}:$
ธํํㅇํㅇ $x=5$

$\therefore 00$ $\infty$
유융응 $z=$

unoue. is in:
우융 $x=2 z$
ํํㅇํํㅇ $=2$
ธํํำ:

$n=2=$

50000 $z=z 2$

gagas anoo na: : $: 000$

$\because 0$ in:-io 6 00000

$$
+\infty
$$

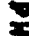

:

: $\infty$

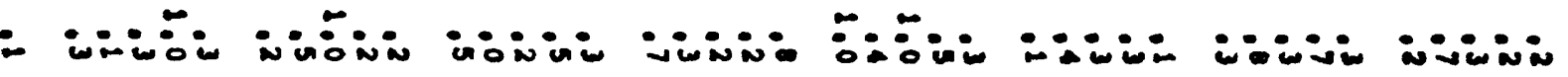




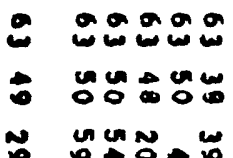

웅요

co- 0

$06=-6$

숭요 on

Nuñ

$N \in=0$

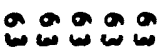

ט

NOA

문

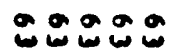

요

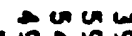

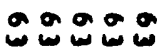

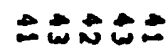

$\rightarrow \infty w$

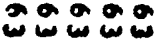

-

$\infty \omega$
운운

- uña

$\omega-\sigma=0$

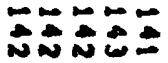

ดטu-

-

둥
프뇨

후웅

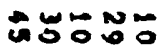

\section{NAE}

UN

- 0 a
きささされ

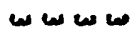

-

:
든

- யbua

U 0 웅

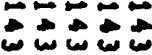

जus-

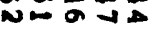

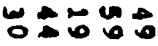

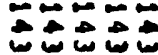

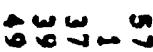

ش

w n en u

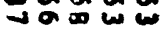

जูए๐

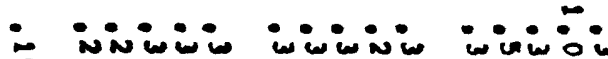

NON

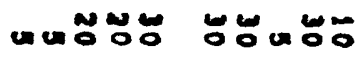
$\leftarrow$

뜽ํำ

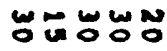

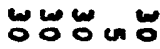

5

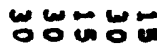

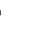

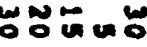

-

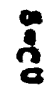

0000000000000010

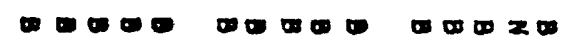

0000000000

00000

00000 - 00000000
0000

00000 - 000
0000000000

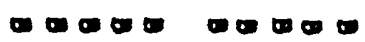

$000 \% 0$

$\infty 0 \infty$

00000 .

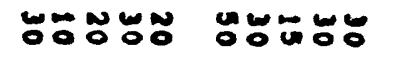

00000

0000

00000

00000

00000

0000

00000

0.00 
- $0000000000000 \% 0$

00000

00000

00000

0000000000

-

$-00$

00

000

$-\infty=0$

$-\infty=$

- E0000000

n nnnNa nnnNn

nNNNN

nNnNa

NNNNN

nNNNn

ํํㅇํํㅇํำ

$\geq x \geq 2 z$

NNNNN

$z \geq x \geq z$

$\geq z=\pi$

ดัดัด

$z \geq z=2$

\section{anisio}

$z=z=$

\section{운웅요}

$x=2$ i̊ㅜㅇ:-

$z z=0$
웅우우

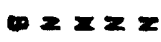

遥宫:

$z=2 z$
은운옹웅

$z z z z z$
品品宫:

$z=2 x z$

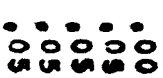

$z z=20$
- 00000
$\Rightarrow \quad 0000$ $=$
00000

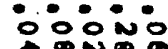
-
00000 웅우

$=$
00000

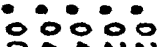
-

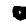

-0000

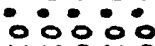
ㅇN옹용ㅇㅇ $x \geq$
- 이이 둥영영

응ㅇ

$\div 0000$ O TNNN

00000

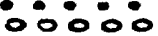

$z z=$

$\because \operatorname{arco}$ Ex

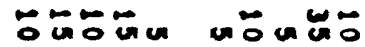
$0.0 \%$ $m$

Nㅜ요유

(a) ตับษั $-\because \cdots+$

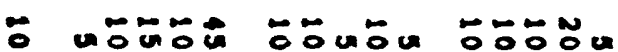

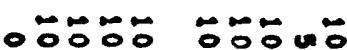
ேㅜ융

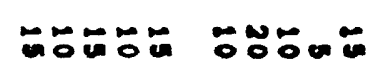
- 0

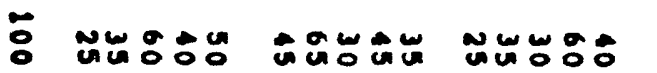
$\overrightarrow{0} \div \div$ -

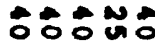

뭉후응

후눙 ต 600 c $\leftarrow$

$\because$ 무웅 후웅 م0000 ㅜㅜ융 $0000 \%$

$000 \%$

00000

후웅

z zezz $z z x z=$

$z z z z$

$y \geq x=$

$z 2 z=2$

$z z=z=$

$z z=z z=z z=$

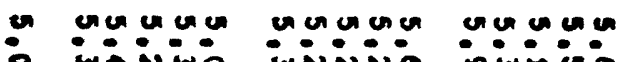
$\therefore \because$ en.

an. un.

arion

uncus noun. o O0. -10 $\infty \omega$

$\because \div 0$ 0000 :0i mะonn

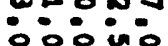

w$\div 0 \% 0:$

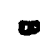

No00m o in in

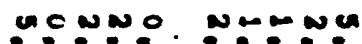
inoin ojio: : a - 
- 9000

- * w w

0000 w w

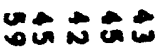

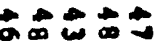

-aga

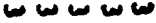

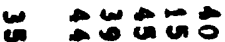

-

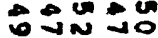

uño:

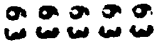

un $u n u$

10

$u=0 \%$
두요

u un un

कू⿻二丨

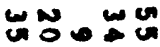

ing

c u $u=0$

\%

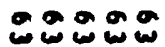

unun

$\rightarrow$ us un

טN

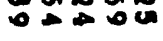

o. 000 co T)

$\therefore-00 \div$

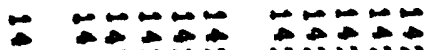

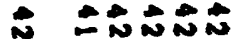

- Unuaro NANNAN ถั -0ㅎำ

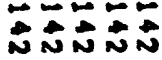
Nָำ ๑๐్ $\because \because 2 \div 2$ NNNNN 㫣 :

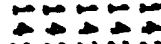
ลNNNA - 0 . 눙
NลN $N=\infty$ 舟 $\therefore$ 뜽응

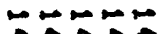
NANAN 뜬 -

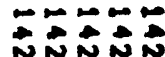

i $\rightarrow 04$ ubun

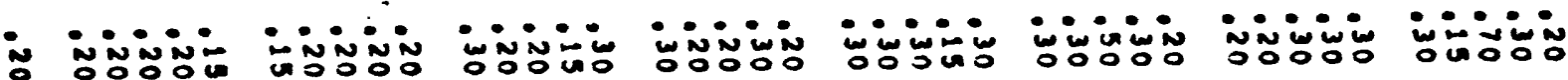

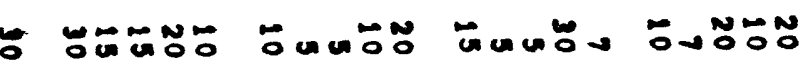
5

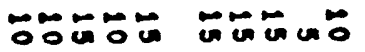

뚜우ำ

ำำ

2

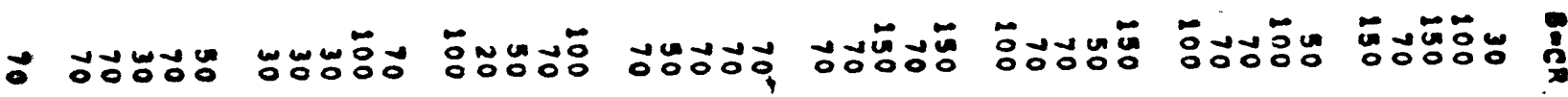

NN

$00 \rightarrow \mathbb{0}$,

$$
\text { - }
$$

- 00000

00000

00000

00000

10000
0000
00000

1000
00000 0000
00000 0000
00000 0000
00000 - 00
- 000000000000000

- 0000 0000
0000
00000 0000
00000

- 000
00000

- 00
00000

0000
00000

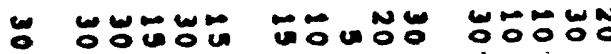
WNWNN
जU

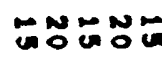

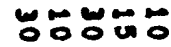
(5) 
$\therefore$

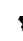

0000000000

- 0.0000

00000

0060

00000

0000

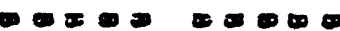

00000

0000

0000

000

00000

000

8

$\begin{array}{llllll}N & N N N N & N & N & N & N \\ 0 & 0 & 0 & 0 & 0 & 0\end{array}$

กับัก๊กัั

กลNกN

ถั:อั:

กNกNล

:ัะัะัะ

ลัดัดัดัดั

$z=z z$

$x \geq x \geq z$

ลัลํํํำ

กับกับั

$z x z z z$
: $: 0: 0: 0: 8$

a $z=\infty z 0$

:aini:

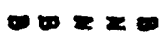

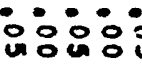

$z \infty \geq \infty=$

iòioio

inimio:

iniogio

$x=z z$

$z z=\infty z$

$z \geq 0 z$

웅웅

우웅웅

$z z z z$

$z=z=$

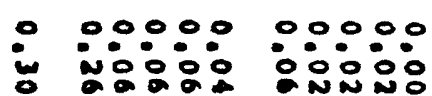

$=2$
00000

$\therefore 000$

$-$
00000

:00:0 OㅗN
00000

$\therefore 0000$

$\rightarrow$ $\because 0: 00$

$\therefore 00$

$=$
00000 $\because: 00:$ $1008 \%$

$00: 00$ : in:0: : Un:

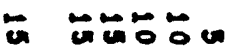

\begin{abstract}
แำ
\end{abstract}

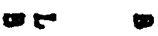

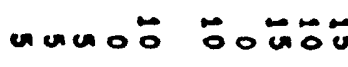

m $\therefore 0$ \%ับง
जับแลั0

$\div$

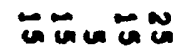

ํํㅇํำ

프은

$0 \div 050$

ơnuo:

두응

(

.

ธํํำ

ज范

?

ธேัธธธั

$$
\text { - } 100
$$

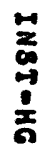

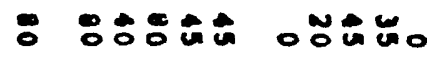

승ํㅇㅇํㅇ

U. 영응

훙ํㅇํำํํㅇ

응ํํำำ

ㅁํํㅇำแ

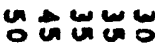

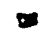

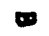

-

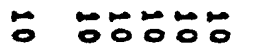

ேํㅇㅇ

ํํㅇํㅇ

훙후웅

ธ유유

50050

ัํㅇํำ

ธํํㅇํำ

$x+5=2 x$

CE $=50$

22202

$z \geq z x z$

$z \geq \geq z=$

$z z z z z$

$z=x \geq z$

$z z z z z$

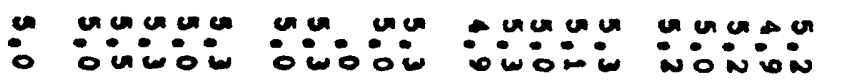
-

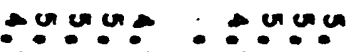
OWONO

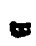

encu un inisis

un uno in:

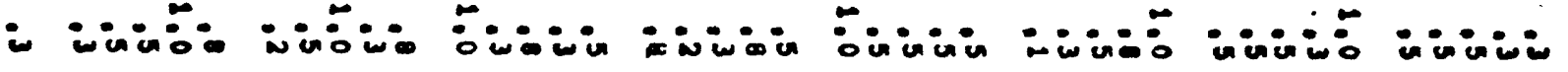


- igia

an - unuáa

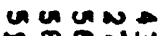
coun un

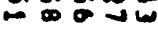

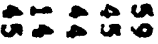

09.

ONNNN

Nỡ
운운

ט

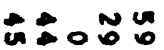

운웅

누영

$=-\infty$

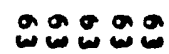

둥ำ

ตำำ

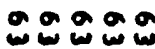

$\omega \bullet \operatorname{un}$

wousa año

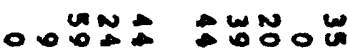

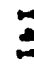

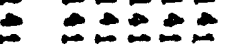

- $\omega$

- $00 \%$

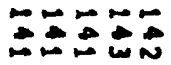
م

م

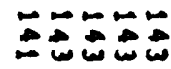
م

us 0

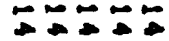

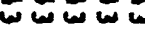
w 6

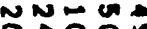

$\because 5 \div \div$ யーーのー

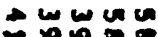

$0 \div 000$ $\because \div \div \div$ 吣

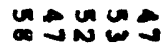
-

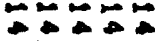
エMNEニ us a u ata: anda

Nun

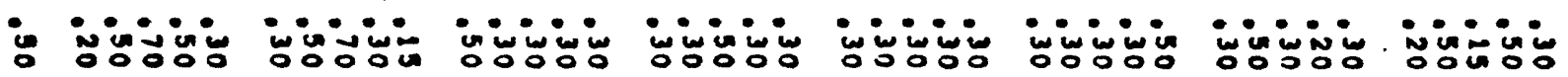$$
\text { 5 }
$$

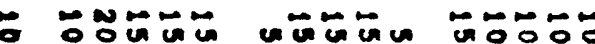

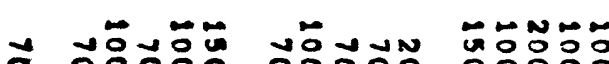

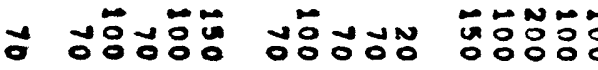

붕용훙뭉유 10000

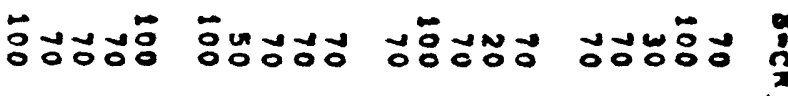

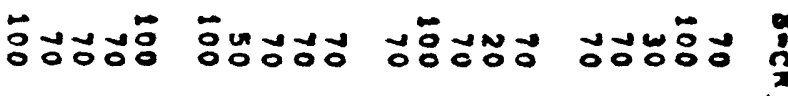

$=$

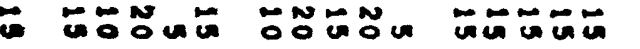
$-$

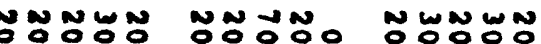
$z=2 z=2$

- $00 \% N N$

4
NONONO N NONON

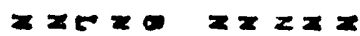

Nㅜㅇㅠ $z=202 \geq 2=25$
NONON

$z=2 z$
NONO

trex
00000 0000

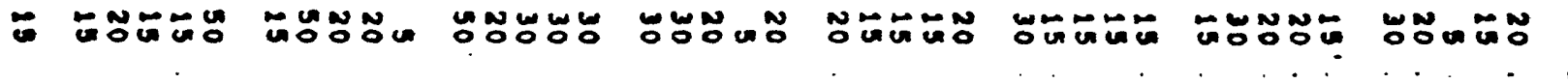

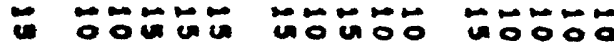

유유 CEC $=0$
ธேธธะ

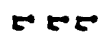

ัํํํำ 


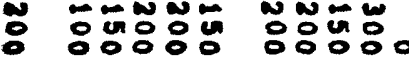
ํㅜㅇㅇㅇㅇㅇㅛ ㅇㅇㅇ융요

แัะั้

용 $-$

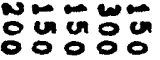

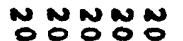

부융ㅇ

응융ㅇㅇㅇ

NONON

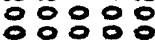

$z z=z$

z $z x z$

O

NNNNN

웅응융

$z z z z$
NNNN\% 응영융

$z \geq z=2$
บNำ

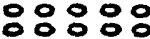

$z=z z$
00000

000
- 웅영연 웅운운

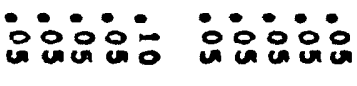

운운운웅은

z $\geq 2 x$

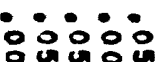

- $x \geq-5$
웅웅

$=x \geq z$
ํํㅇㅇㅇㅇ

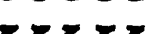

룰

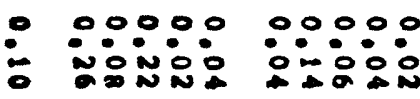
$z$

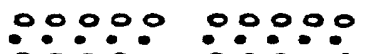

$0000 \%$ 여으
옹ㅇㅇㅇㅇㅇ

0050 :0?:?

웅ㅇㅇㅇ
00000

$10 \div 00$

$$
\pi
$$

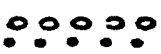

우에

둥은웅요

$z \geq 200$

- Nỡ

un uํํㅇ

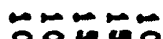

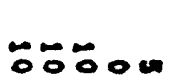

$\because$ ○

z $x z z z$ $z z=z$

ㅜㅇ우웅 Eete

후웅

ํㅜ웅웅

ํㅜㅇ후웅

$z z z z z$

$z \geq z=2$

$z \geq z=2$

- concu co

a cun un

ancon

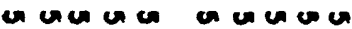

anco acouc

couman

- c c c

unuca ca ivivivi.
ㅜㅇ웅 $z=2 x=$ ด uncan. wivi. con on an

:i: :-

$y$

๑

$\omega \boldsymbol{N} \boldsymbol{N}$

$\omega \sim \omega \sim \boldsymbol{N}$

NN $N \cdots$

$\sim \infty \omega \sim$

$\omega \omega \boldsymbol{N \omega}$

Nผ $⿻ 上$

:

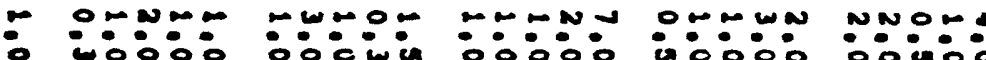

or On

NoONO

ON口:O inioir in: i:

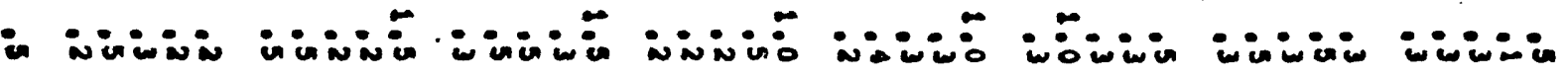
$\stackrel{8}{\mathbf{0}}$ 
- 우웅

a $\rightarrow u$ un

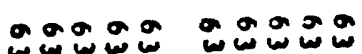
$\operatorname{lum}_{n \rightarrow \infty}$ u un un

wuN

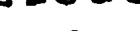
ேே두

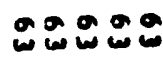

- o. o. 0 누웅

UN $\rightarrow \rightarrow 0$

웅요

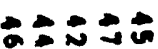

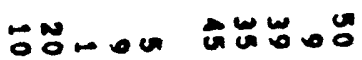

-0000 00000 wa

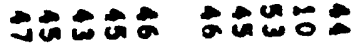
ט. ๑

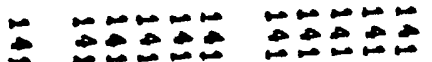

OEニ

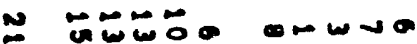

- $\infty-\infty$

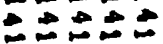
$\approx \approx 0$

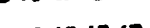

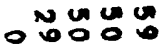

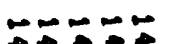

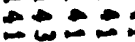

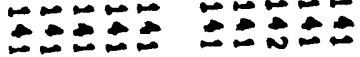
Dowo

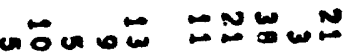
ำ

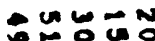

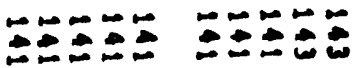

Nơỡ NOW

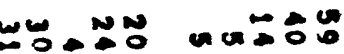

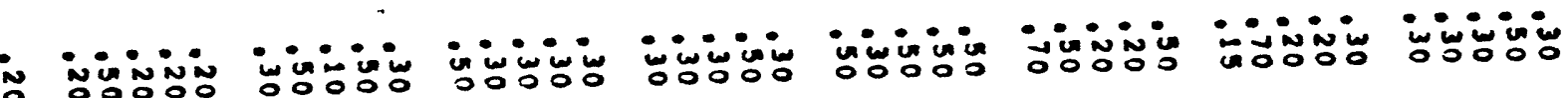

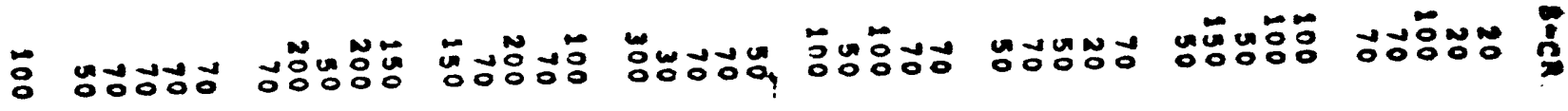

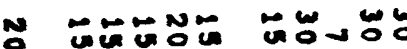
$\cos 0$

00000 00000 00000 00000

$\infty+\infty 0$
00000 0000

$$
-5
$$

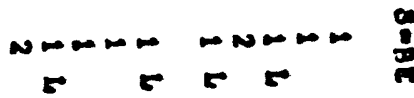

두응

두웅 c 

W

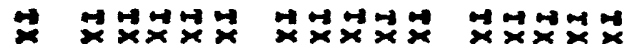

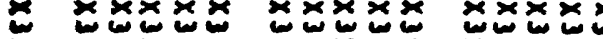

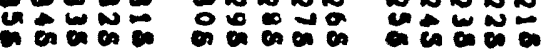

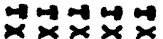

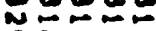
용 2x. $\vec{x} \dot{x} \vec{x} \dot{x}$

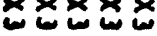

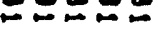

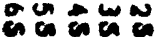

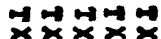
$x \times x \times$ $\sim \infty 0$ - 0 es 0

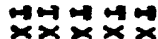

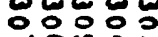

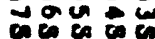
$x \times \times x$

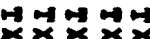

$x \times x \times$

잉ㅇㅇ $\omega=0 \leqslant 0$
- 000000000000000

- $0=00000$

- 0 0\%ㅇํㅇㅇํㅇ

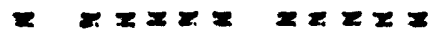

○ NONONO NNONNN
$-000$

NNㅡㅇํㅇำ

:ำ:ำ

rexz=

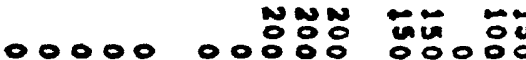
- 0.0

ถัดัดกัด

tezzx $x$
ลัดัดัลั

$z z \geq z z$
ลัลัลัดัลั

$2 x 2 \geq 2$

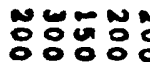

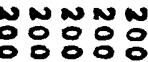

$=2 x \geq$
ํํํำ

I $x \geq z z$
영운웅운 운웅운운

- $x=2 z$

$z=0 z$
00000 웅ㅇㅇ웅 $z=z$
$-0: 00$

- 영용요
은운운운

$z z=z$
운운운운

$z \geq z \geq$
운웅웅은

$x \geq 0 x=$
웅ㅇㅇㅇㅇㅇ

$z \geq z=$
운운은웅웅

$x \geq x$
웅운웅:

m $x \geq z z$

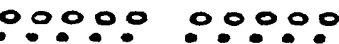

$=$
웅웅ㅇㅇ

$x$
웅ㅇㅇ웅
00000

웅ㅇㅇㅇ

$=2$
00000

ㅇㅇㅇㅇㅇㅇ

NN요요요

00000

웅이 웅웅

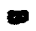

$z=2$

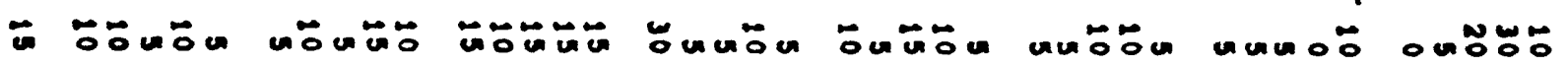

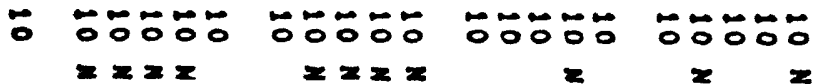

$\therefore$ : $:-$ un un

in:-in ung

unu un inio: andug untum tuncan ONin:

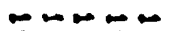

$0000 \% 0 \%$

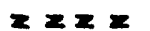

$z x \geq z z$

ㅜํ유

무융우 

$z z=z$

2

$\boldsymbol{N} \sim \boldsymbol{N} \sim$

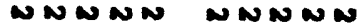

Nヘルー
$\boldsymbol{N} \sim \boldsymbol{N} \boldsymbol{N}$

UNM⿻上丨

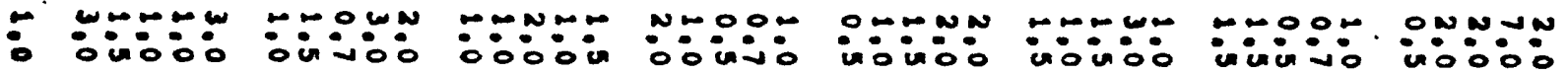

- 


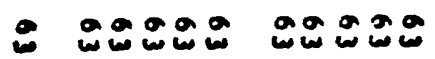

ט

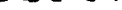

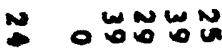

$\omega>\omega \omega$

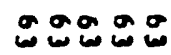
w Nu $\rightarrow$ N ingag anoag

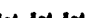

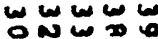
ํํㅇํำ
की

N orogo 0

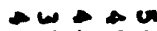

$\omega u \rightarrow N$

Gw.

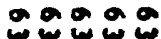

unu un

넌
0090

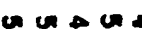

IN⿴N口

U a

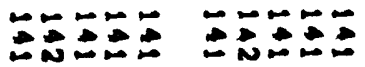
W 똥요웅

Nu

$\square-\infty$

$5=5=$

$\angle E D E=$

$\because \infty-\infty$

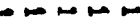
טั0 Nou (a) UN NO

uึ

$\because=5=$

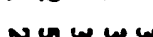

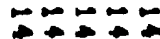

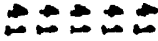
NNNN D 6000 $N \infty=\infty$ जिए人⿺ ज. 000

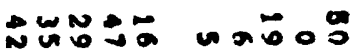

$\infty \ldots+\infty$ NNNNN

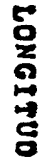

- inivir inivio ن : Uivi:

NiNN:

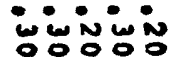

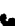

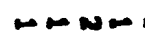
$\sim \sim \sim N$ NーM

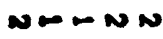
N $N \boldsymbol{N}$ NNNール

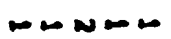
$\infty$

$$
z \quad 2
$$$$
\geq=
$$

$\div \div \div$ $\because \div 0 \%$

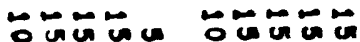

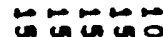

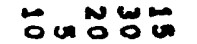
ロேッ゙ー 부웅

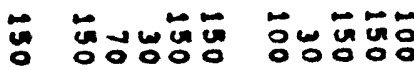

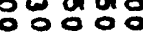

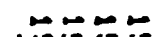
ㅂํำu 후융 둥 웅ํำ
능 om 0
ํํㅇํㅇㅇㅇ

$\rightarrow \ddot{0} \ddot{0}:$ 용요

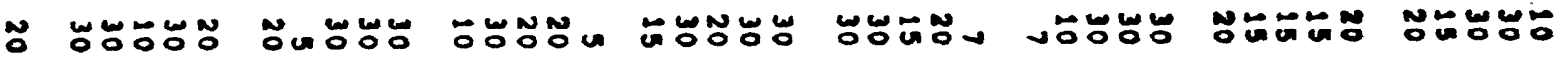

- 00000

- 00000

00000

00000

00000 -100000000000000
00000 0000
00000 $0000=$
00000 0000
00000 1000
- 00000

- 0010
00000

00000
00000 $\square 000$
00000 -
0000000000 $0=0000000$
00000 00000
00000 0000
5

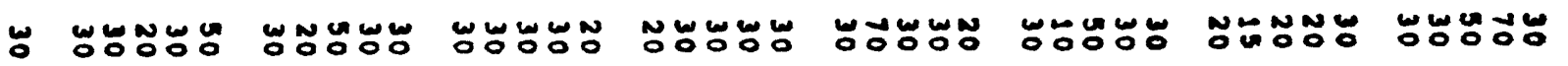
$0000 \%$ 
00000

- 000

00000

00000

00000

00000

$1 N N N N N N N N$

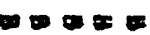

$=0+\infty$

$\infty \infty 00$

$-\infty<0$

$-00$

กักำกั

กับับ

พบกั

กับับ

ก บับ

ก บ บ $:: 8::$

$:: 0: 0$

$z z z z$

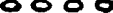

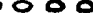

ละ:ะ

$\therefore: 80:$

วัะด:

$z z z z z z z z z z$

$z z z z z$

$z \geq 2 z 2$

$z x \geq z=$

$x \geq x \geq z$

2. $2 \geq 2$

$\therefore: 0: 0$

anuinún

$=225$ $\therefore 000$

iㅜㅇㅇㅛ

$\therefore 0: 0$

$x=\infty$

$z=2=$

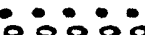

운요요

$\therefore \therefore:-$

8

$z z=2 z$ $z z a v$ $\therefore 0$.

$2 x-0=$

c $x \geq z$

$\therefore \therefore: 0$

$202=2$

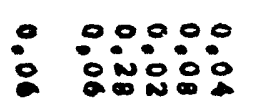

00000

00000

00000

00000

00000

8000

눙우

웅ㅇㅇㄴ

웅웅

$\because \therefore: \circ$

$z$

$=$

10

00?:0

웅ㅇㅇㅇ

00000

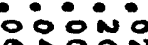

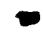

ㅜㅜㄷㅡ c

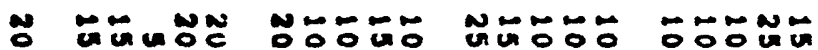

NNo

n̊ำ

$0 \div$

-

$\rightarrow$

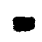

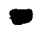

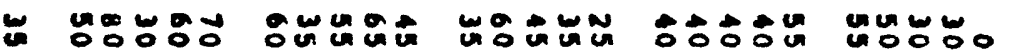

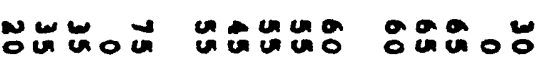

-

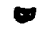

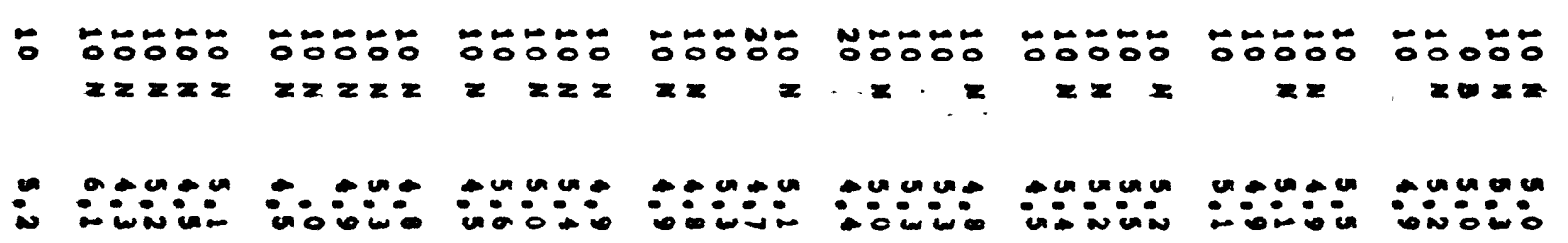
-onou batomo

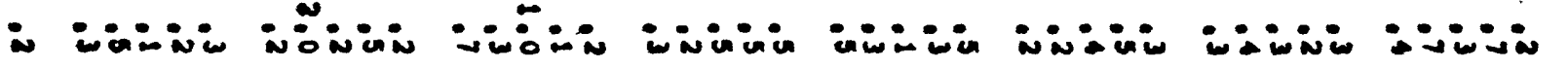


- 웅웅

年

- NNN N

a cown

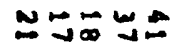

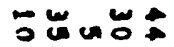

20000

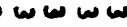

INU

N̋:0
00000

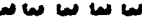

שั טu aso 000

O०

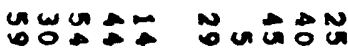

웅aㅇ

م

nNnUU

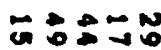

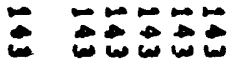 \\ - Mca \\ - MnNu} 㔚部 BW G

$-\infty=\infty$

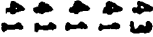

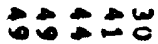
ט.

$\rightarrow \pm \infty+\infty$ $\rightarrow N N=N$ waำ

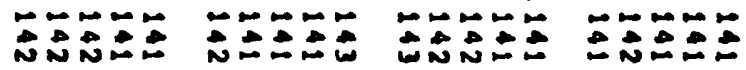
NNمUN Uمㅇㅇํำ

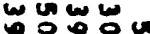

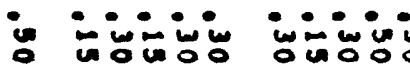

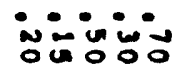
wo:
ن우웅 .

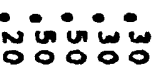
0000 م0

ivi:

$\omega-\infty-\infty$

$=2=$

$\infty-\infty \cdots$

$=$
-

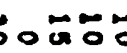
-

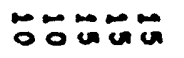

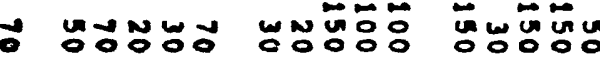

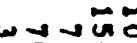
0000

ज. (

붕부융

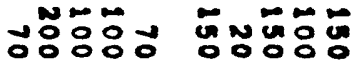
3

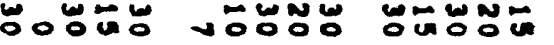

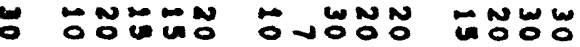
WOOOK -

000000000000000
00000000000000

000

00000 00000
10000
$+$ 


-00000
00000
0

은 웅웅융영 $x \geq 20$
운운운운운

웅웅운웅

r z $x$

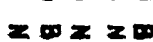

둥운운요

은영웅웅ㅇ

z $2 x=2$

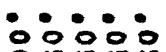

은운웅웅

웅ㅇㅇㅇㅇㅇ

ingu

$x \geq 2=$

exEz

$\because: 0: \div: \div$

=
이웅

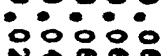
$=$

웅웅

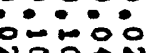

00000

00000

00000

$0: 00 \%$

웅ㅇㅇ

\section{- $=$}

:

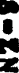

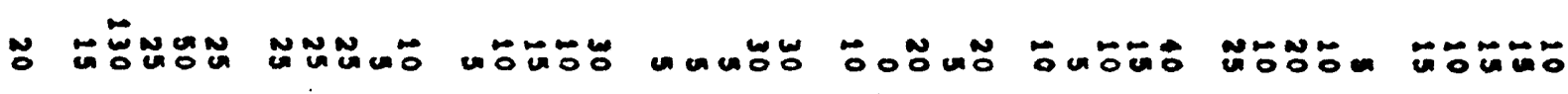
-

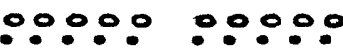

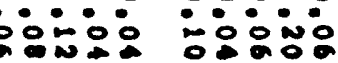




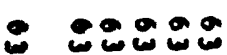

o. 0000

o.o. 00

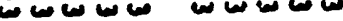

-

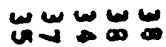
\pm D

0.900 Na Oజ N
20020

(1)

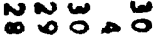

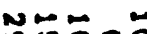

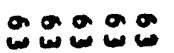

ט

-OOOO

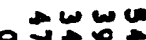

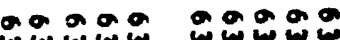

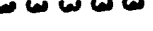
$\because \because N \& N$ w w w w ํㅠㅇㅠㅜ

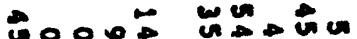

$\because \because \div \div \div$

-

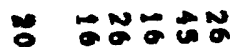

-

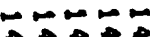
NANNN

ONDN

NNNNN $\underset{n+\infty}{* \rightarrow \infty}$ $\overrightarrow{N N B E E}$ NN w o N

ט

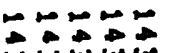

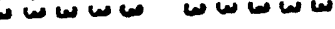

$\approx N$

INNAN

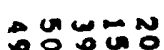

0 0. $\because ロ+\infty$

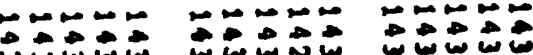

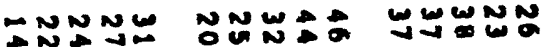

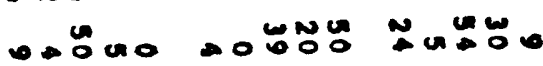

-

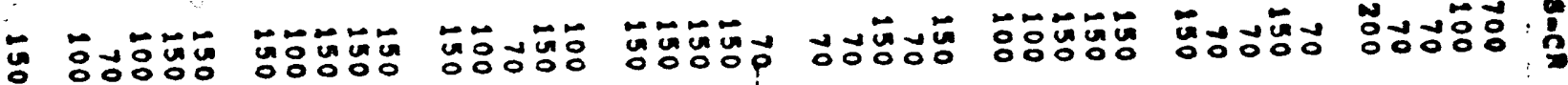

NDーN-

$\begin{array}{rrrrr}-\infty & -\infty & -\infty & -\infty & -\infty\end{array}$

-

Nam

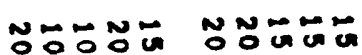

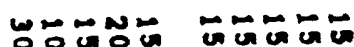

N⿴囗十

뚜융ำ

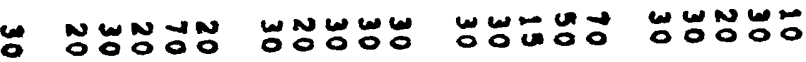

00000 $000<0$ 00000 00000 10000 - 000

00000 0000

00000 10000

00000 0.00

00000

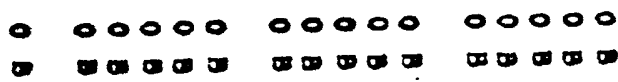

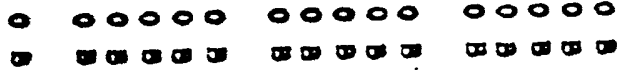

00000 00000

00000

- 00000000 $\begin{array}{lll}00000 & 00000 \\ 0000000000\end{array}$ wo:

3

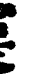

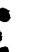

$\cdot \cdot \cdot$

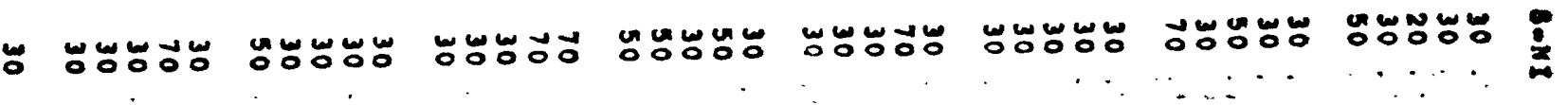

Oோ

$\div \div \div 0$ พํํㅇำ 
- 00000000000000000000

- $00=0$

영영영

$z \quad z \geq z z 2$
000

กับกับั

ะะะะ

$z z z z z$ 응융유: $x \geq z z$
NNNNN
$00 \times 10$

NNNNN 응융ㅇㅇㅇ $z \geq z \geq 2$
00000 - 100

Nㅇㅇ으응 $x \geq 25$
00000 I I

NNNNN 융융융

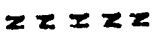

00000

1000

NNNNㅇ응 Oด: $x z z z=$
00000

- - to t

NNNNN 융융융
- 웅영우 웅웅 unuou

r $z=022$ $\div 0000$ 000 Un - $0 x \geq 2$
뭉ㅇㅇ웅 जuno

$z \geq 202$

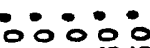
언의운

$x z z z z$
연운운

$z 2 z z 2$

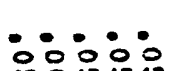

언은요

$z a z 22$

03000

- 0000 000:0 0000: 0000:

00000

00000

00000

- $z x z z$

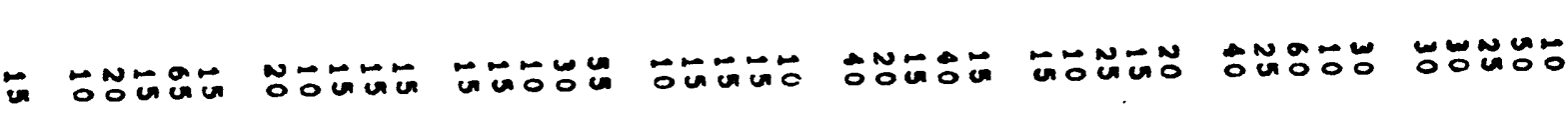

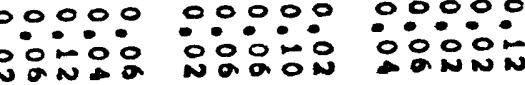
z

$z$

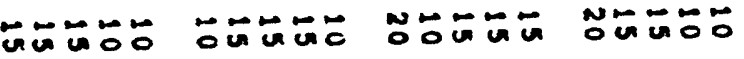

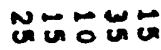

OOOFO

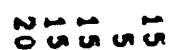

ํํㅇำ

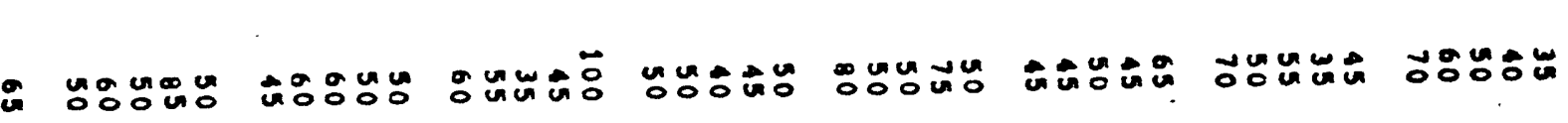

둥ㅎㅇ웅

우웅

뚜우

두웅우

뭉유유

०००0\%०

느융 유융

$z=z z z$

$z z z=$

$2 z=$

$z z z z$

$\geq z 2$

$z z 2 z=$

$z z z$

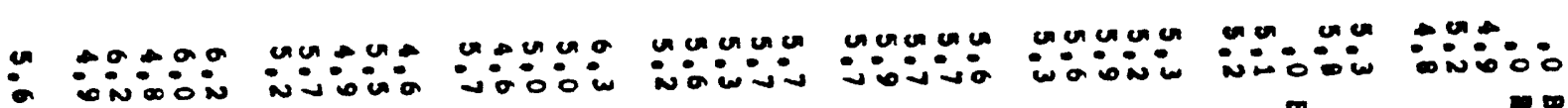

-

$\infty$

오

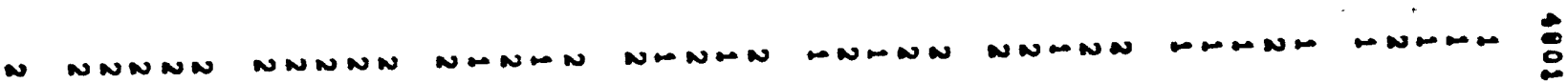

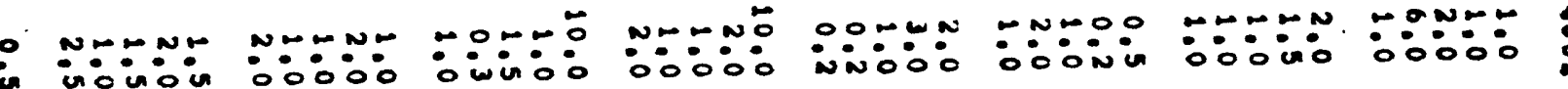

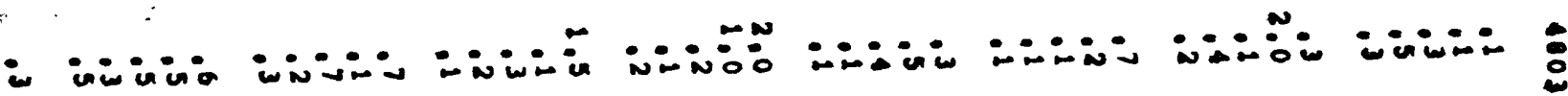

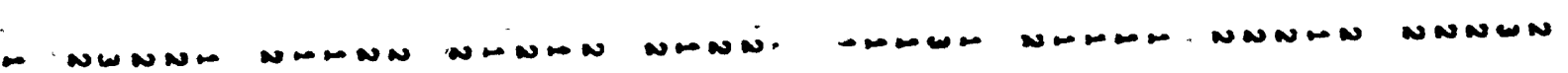




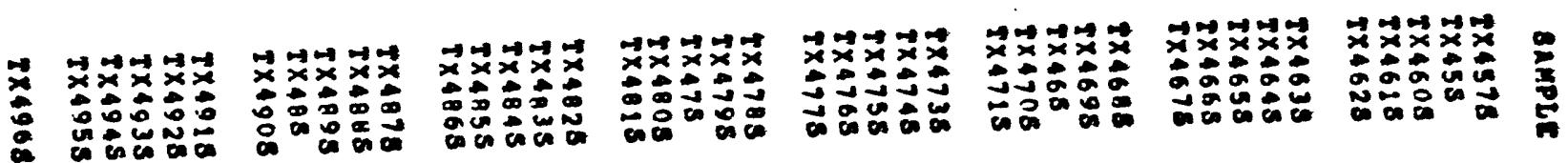

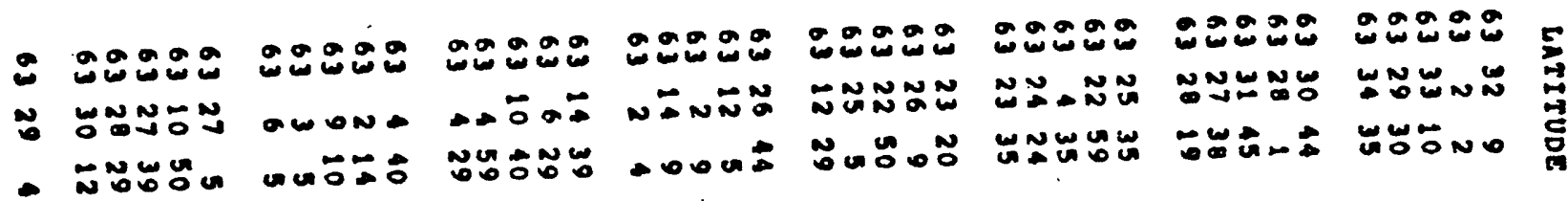

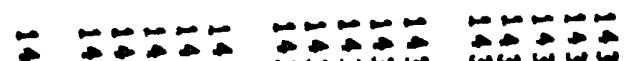

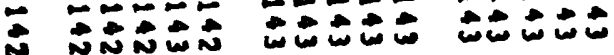

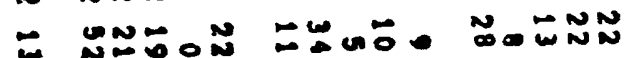

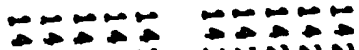

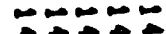

nand

$\pi$

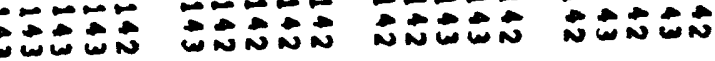

nNurN

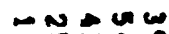

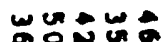

$\omega \rightarrow N-\omega$

シ ヘニた

axd

$-5$

ס

प w w

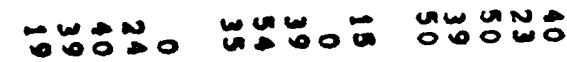

농유:

๒

N:D

ज๐ำ

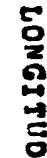

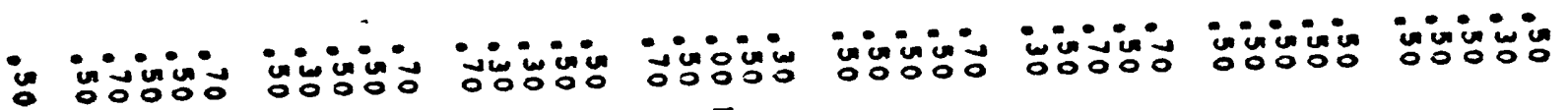

(1)

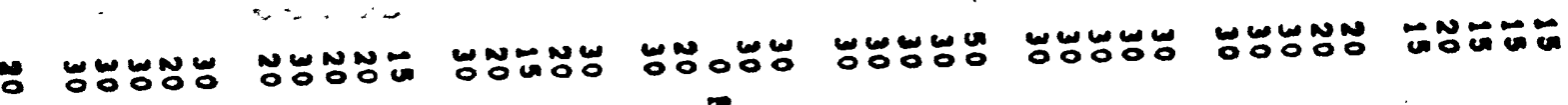

NNMNN $N$ N

(7)

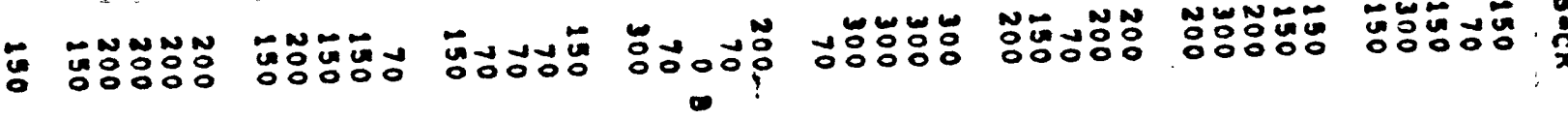

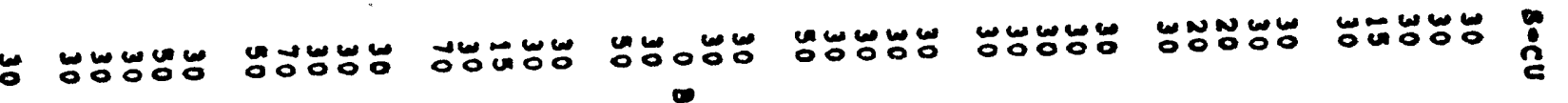

- 0000000000

-

$+\infty 00$

0000

0000000000

00000

100000000

0000

00000

00000

00000

00000

10000

0.000

5

- 0000000000

00000

00000

00000

0000

00000

00000

0000

00000

00000

2

- 0000

- 00

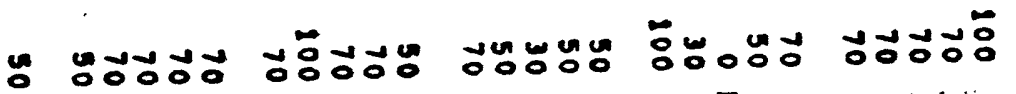

$\rightarrow \rightarrow \infty 0$

1000

ํํㅇํำ

ㅎํㅇํㅇํํㅇํํㅇำ

ํํㅇํㅇㅇํㅇ

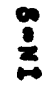

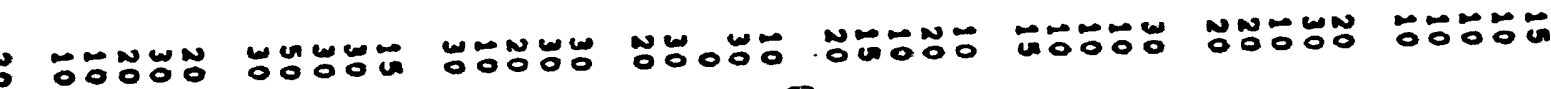
- 23 
- 00000000000000000000

\section{a}

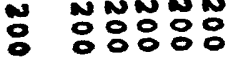

$\therefore=2$
1000

$-\infty$

NWNNN

ะัะดะ

$z$

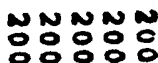

$x \times 5=$
ํㅕㅇ유유 $\geq \infty 22$
00000

0000
00000

000
00000 $-\infty=0$

NNNNN

OO:O: $: O: \%$ $z \mathbf{z}$

\section{NONONO}

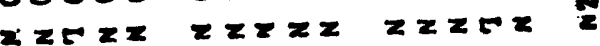

00000 0000 ㄷㅇㅇㅇㅇㅇㅇㅇㅇ

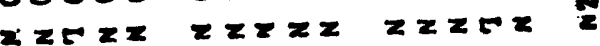

:
웅운운웅 웅ㅇㅇㅇㅇㅇㅇㅇㅇㅇ 붕ㅇㅇㅇㅇㅇ $x=0$

우웅웅웅 운운옹

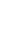
$z=w z=2 z z z$

$\div 0 \div 00$

- $\div \div 0$ $z \geq 20 x$

z $z z=2 z$

- 00:0

-

00000 -Ninio - NON응

00000

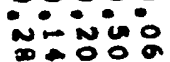

00000 00000

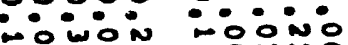
NOO WO $=$

OWNLN

누융

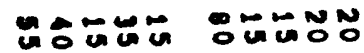

$0 \div \div \div$

$\because \div \div 0$

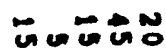

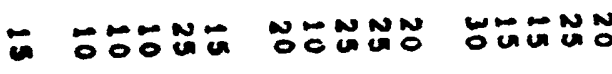

\begin{abstract}
O० G
\end{abstract}

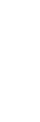

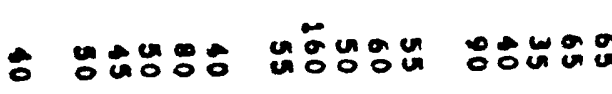

ํㅜㅇํํ -

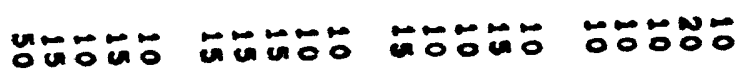

$\div \% \div \div \div$

- $0 \div 0 \%$

-

$\therefore 0: 0$ 후웅 $z z z z z$

ㅜㅜ웅

$\geq \geq x=$

두웅 c5

Nم:0\% $x=2$

\section{-} $z \geq z=$

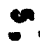
웅웅 $\therefore 0 \div 0 \div 0$ -0:0 1000 0000 00000

00000

OONOO

- 00000 ono 00

- 0000 - 00
10000 0000
00000 10000 $=m=2$

\%๐ั0
OONOO - 0
00000 $-000$
4000 i०

웅웅 10

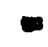

NONNE: 00000 10000

잉ㅇㅇ 웅ㅇㅇㅇ

00000 :000: - wor 100 - 10 ம00

100

웅웅

훈웅

-0ㅜ웅

뚱웅 $+\infty 00$

(10

ம 000

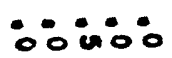

뭉웅 -$$
0.0000
$$

00000

ONOOO

$\infty=\infty+\infty$
- $\infty$
जOLN:

0

문둔

$-\infty=0$ 00000

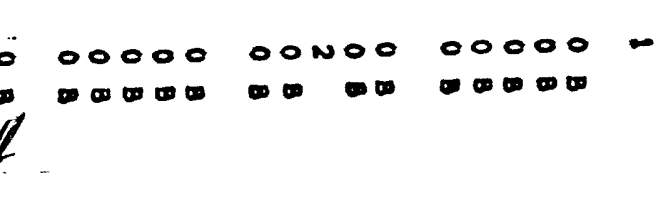

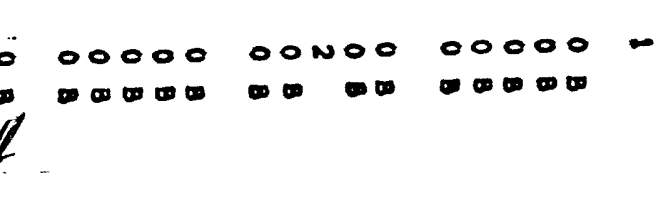

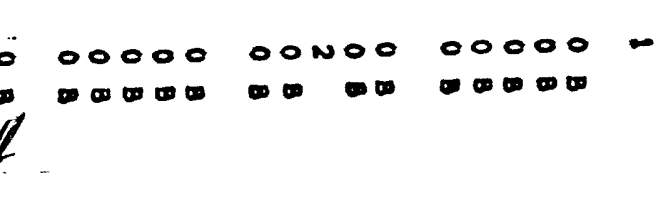

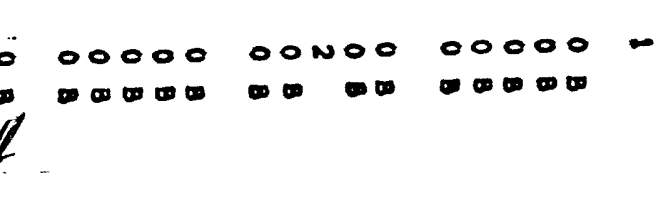

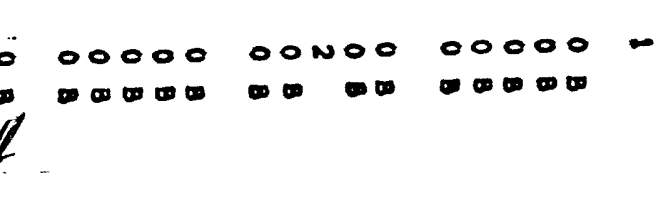

: 
- 운요

- $\approx 0 N N$

$\because \quad=\Delta N A$
문뭉

S

a
06

$\sim-N=N$

N

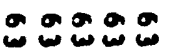

D

un

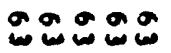

Nㅜ숭

UNG UN

ODG Un
0.

NNON-

Un $0=6$ a

NNNNN

10둥

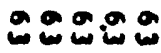

Now

कผ
-

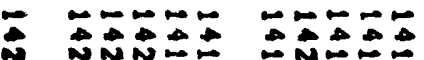

- no OA:

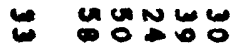

un un un

־0 unoo

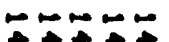

シลニลN

U. U⿺

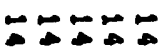

NANAि

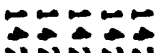

$\because \div \div \div$

แลNこE

Uूo
م

Nッニ5テ

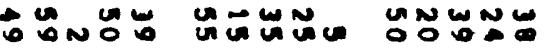

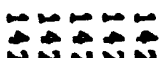

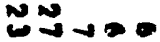

Gั0

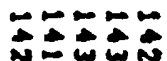

ำ

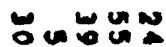

-

$N-\infty N$

NNNNN

NUNNN

NMNNN

WNNND

$\omega \boldsymbol{N}$

W

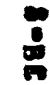

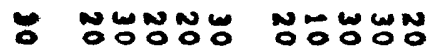

NONONO

NoN Non

WNOO\%O

WNONO

\%ONO

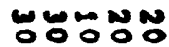

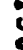

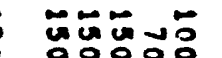

드응

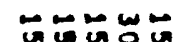

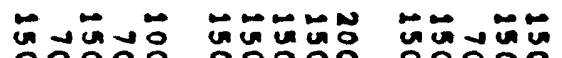

$\begin{array}{llll}N & N & N & 0 \\ 0 & 0 & 0 & 0\end{array}$

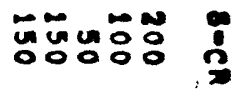

8

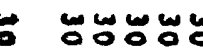

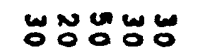

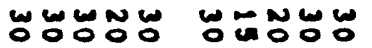

WN

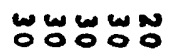

W\%:

W\%⿺辶一

2

- 00000

- 600

- 00000

- 1000

$\rightarrow$ t)

00000

- 0000

00000

0000

00000

00000

00000

00000

10000
< =0
00000

00000
00000

-
00000

ט
00000

0000
00000 0000
$00 \% 00$

- 0
0000000000 -1000 000 


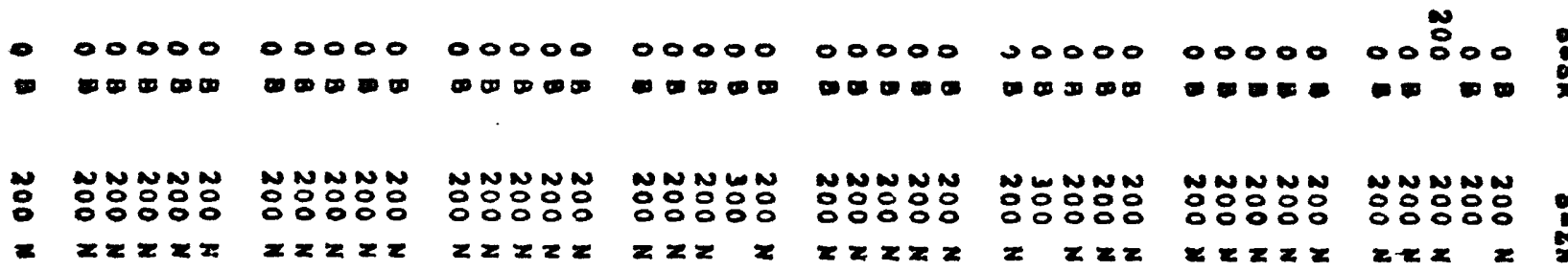

- 둔운웅

웅웅

온은옹

웅ㅇㅇㅇㅇㅇ

$z \geq z=2$
운운웅웅

무웅

$x \geq z \geq z$ $z=200$
읍웅운운운

잉ㅇㅇ

$x \geq z=$

$\therefore: \div:$

$z a z z z z z z z z z z=z$

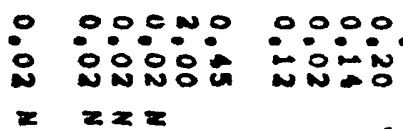

$N \quad \div \div \div \div$
00000 $\therefore \circ \div 0$ in ( $)$

\section{$\because 0: 00$} $z$
웅유

00000

0.0000
0.000

$\infty$

$\because 0: 0: \quad 00 \div 0:$ NㅗㅇN

$x$

à

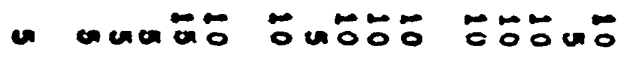
z

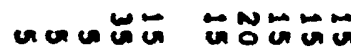
r

añ

NTNNN

NoNNG

-

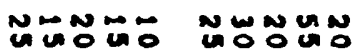

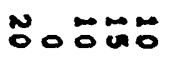

뭉ำ

EN N N

$\infty$

c.

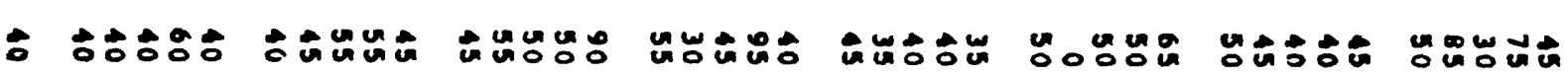
-

\section{$\because \approx 000 \%$ 유융}

$z z z z$ $x+2 x$

$\div 0 \div 0 \%$

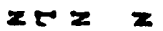

$\because 20: 00$

$\because \div \div \div \div$

$\rightarrow \infty$ - 00 ino - 0
두웅우 $z=x$
두웅

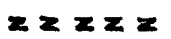

웅웅 z $\approx \geq$

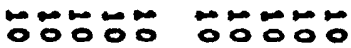
$z z z z z \quad x z z z z$

$\therefore \div 0$ -
후웅웅 00000
뭉:$\infty \infty$
뭉ㅇ 유유. 1000
웅

OnOON

$0<000$

$000 N 000000$

$\infty$

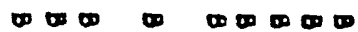

00000

OO-No

(I)

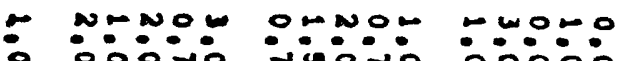

$\infty$
$000 m 0$ $\div 00 \div 0$ un $=\omega$
00000

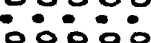
மய
01000 이요

$\infty \quad \infty$

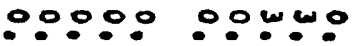
in óñó ம :

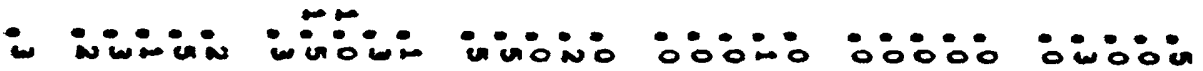

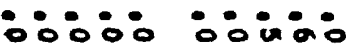

OOOOO OONNO $+\infty \infty+\infty, 0$ 


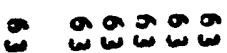

00050 ט

00000 nes cut

20000

NNئ日O

NNN N

NNNNN Na

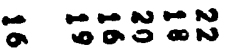

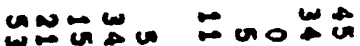

- wun

a o 000

운에의

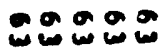

문

NNNNN

$\because \div \div \div$

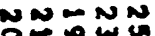

NNN N

10:

unva

- Nun $=\infty$

$\because 6: N N$

-

$\because \approx+\infty$

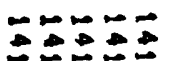

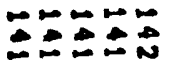

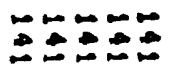

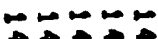

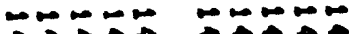

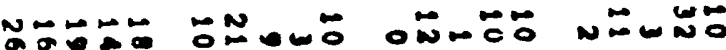

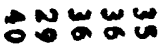

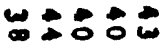

$\omega \geq \infty \mathbb{W}$

WNNOE

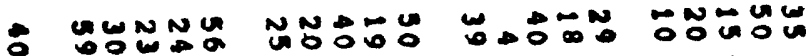

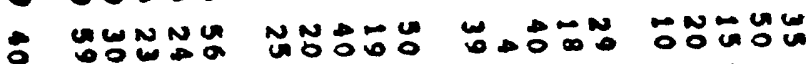

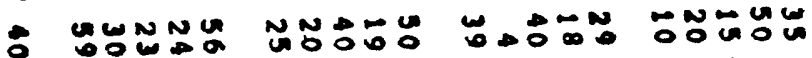

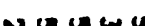

a

$\omega \rightarrow \infty$

טه

웅
2
흔

no 000

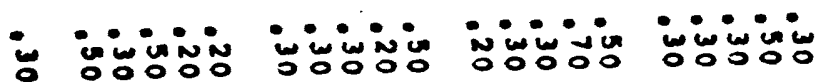

$\infty$

$\omega N \omega N \omega N \omega N \infty N$

$\boldsymbol{N} \boldsymbol{N} \boldsymbol{N} \boldsymbol{N}$

ஸ⿻
MN N N

$\omega \sim \boldsymbol{N} \boldsymbol{N}$

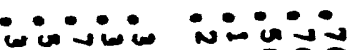
iñó ocona

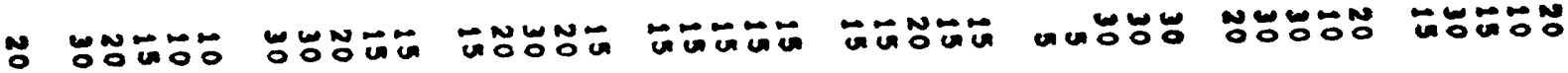

- nمr n

: 우웅 눙영ㅇㅇㅇ
tOFON 웅웅웅 مـ

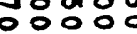

숭ㅇㅇ융 Nㅜㅇ융유

(n)

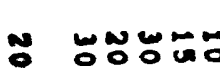

พัํํㅇํำ

NNWWN

NNO NOO

№요요

- 00000

- 0000

00000

00000

00000

$+\infty 000$

0000

0000

00000

$0+000$

00000

0000

00000

$-1000$

00000

- 000000000000000

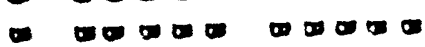

00000

00000

00000

00000

00000

00000

-

0000

00000

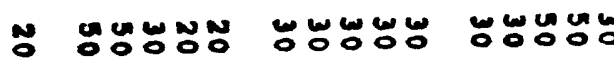

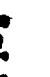


- 000

- $00=0$

00000

Ш

00000

- 00.0

00000

0000

0000

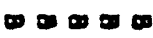

00000

$0+\infty 0$

00000

6000

00000

$-00$

Nㅡㅇ영유

NNNNN

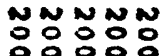

$x \geq x \geq z$

$x x z z x$

$z z z z z$

NNNNN

융ㅇㅇㅇㅇㅇ

$y \geq z x$

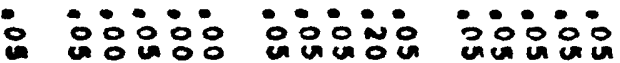

$x \quad 20=0$

$z \geq z=$
운운웅운웅

$\div 0 \div 0 \div$ 는요용 $z=\infty 2 x$ $z \geq x \geq z$ $\div 00 \%$ 운운은 - $x \geq z z$

웅ㅇㅇㅇ

$\div \div \div \div 0$ 운운용은

$z \geq z \geq z$

$x \geq z x=$

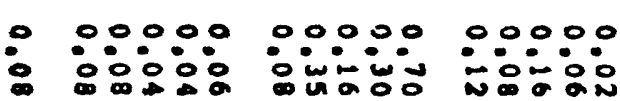

00000 웅요

$=$
00000

웅우

$x$
00000

웅:

NNANN

$z=2$

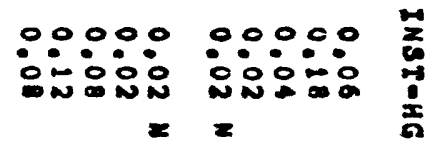

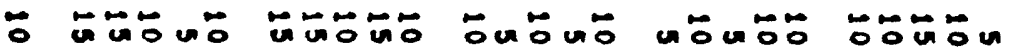
$\operatorname{MrON} 0$ $z \geq \infty$

ํํำ๐ ேํำ เ

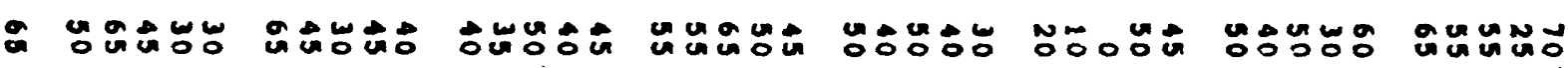
0

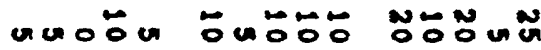
T5

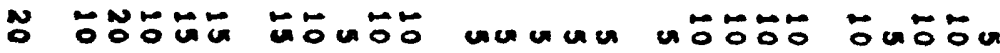
$\div \div \div \div 0$ ํํㅇํㅇㅇ

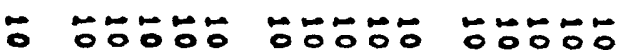

ํํㅇํㅇㅇํㅇ

ํํㅇํำ

ํํㅇํㅇำ

$z z z z$

$z \geq z \geq z$

$z \geq z \geq z$

$2 \geq 2 z$

$z=5 x$

an unum

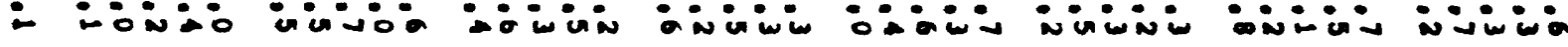
0

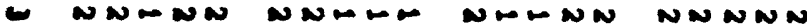

$\boldsymbol{N} \boldsymbol{N} \boldsymbol{N} \mathbf{N}$

$\boldsymbol{N} \boldsymbol{N} \boldsymbol{N} \boldsymbol{N}$

$\omega \sim \boldsymbol{N N}$

$N \boldsymbol{N N} \boldsymbol{N}$

o oning

00 On: ino: in iniso:

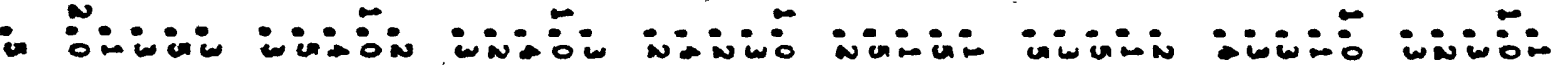




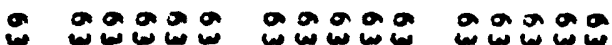

0.000

00000

운요

운웅요

or or o 0

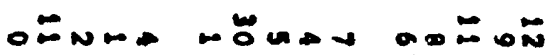

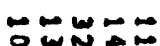

ேேニニー゙

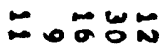

ن

뭉두

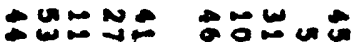

U๐

$\omega \sim N \infty$

טم

$N=N u \in$

u unN

UN: No

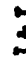

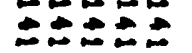

$\because \div-$

הח

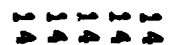

$\sim \infty \cdots+\infty$

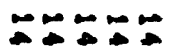

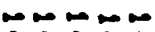

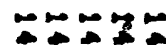

× w-a.

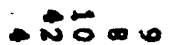

Uaニ

O००

ヒேேニ

A 0 w

ung

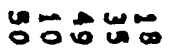

जڤ

พบำ

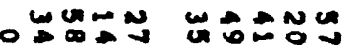

ニEAED $\triangle E ニ E N$

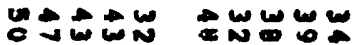

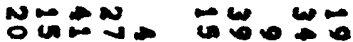

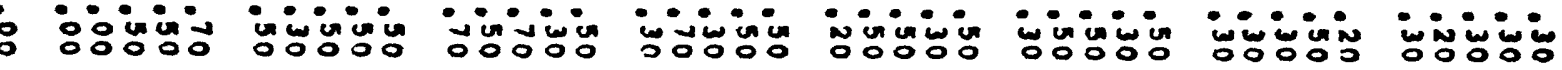
- 0

$00 \omega$

can w w w w

w

Non N

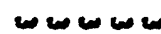

NDNM

$\boldsymbol{\omega}$

$\sim \sim \omega \sim$

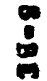

- 00 Nั\%

WNWW

NWw口

NNEWL

مN N

NNWOE

\%유ㅇㅠㅡㄴ

ํํำแ

- $\infty$

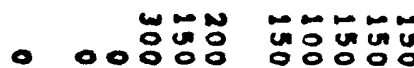

渵品

OUNOOO

- 0 $0 ㅇ ㅇ$

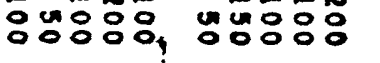

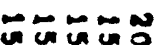

두은

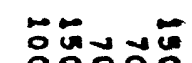

5
$\vdots$
$\vdots$
0
0

. 
- 000000000000000

00000000

WNNNN

$\therefore:: ㅇ$

nNnNN

웅ㅇㅇㅇㅇㅇㅇ

$z z=2$

$z \geq z \geq z$
:ำ:

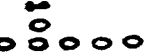

00000 $0=00$

0000

00000

-

NNNDN

응응응

NNNNN 응ㅇㅇㅇㅇㅇ

$z \geq z \geq z$
กํํํํำ

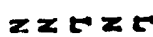

00000

00000

- $0=2$
- $\div \div \div 0.0 \div 0$

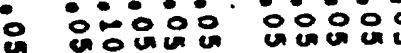

z $z z z$

$x \geq z=$

응

$\therefore \therefore 0$

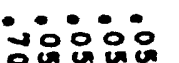

$\because 000$

ini:i:

$\because \because 0: 0$

$z z z z$

azoz

$z=2=$

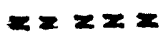

.

$000 \% 0$

zox $2 z$
- :00:0

0000000000

init :0000

00000

$\because 00 \div \div$

00000

ciois $-10 N$

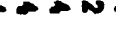

$$
\text { - : }
$$

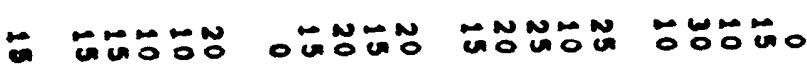
- $\div \div 00$

\section{0} $0 \div 0 \div 0$

00000

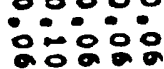
:000:

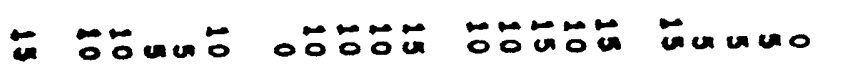
$\leftarrow$ -

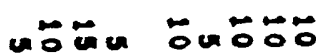
$\geq=$

NOOOOM

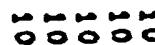

ㅜㅜำ

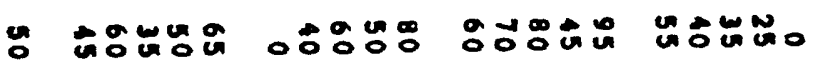
$\infty$ $\infty$

NO용

unuan

in $\rightarrow \rightarrow \infty$

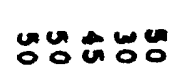
$+$

000

(2)

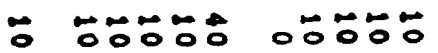

Nㅇ융 우융

ㅜㅜ웅

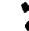

z ezz

$0 z z=2$

$z=2$

$z z z z=$

$z z z z z$

$00 \div 0 \div$

$\angle 000$

$\because$

\%ัธธ์ัด

3

$\because$ an:a: ab:u:

unana unu-u

natio

ionis

ancus

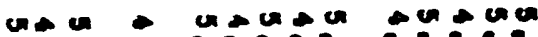

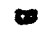
-

N NNNNE NNWMN

NNNMN CRNAR

N N W N N

NNNON

NーN N N

$\omega N \sim \infty \omega$

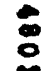

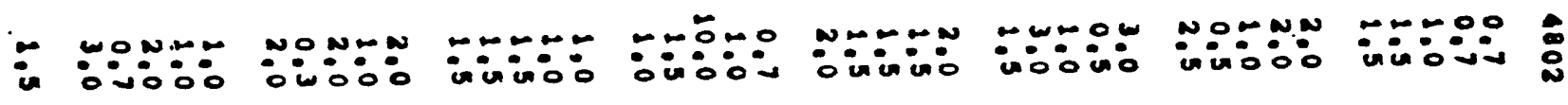

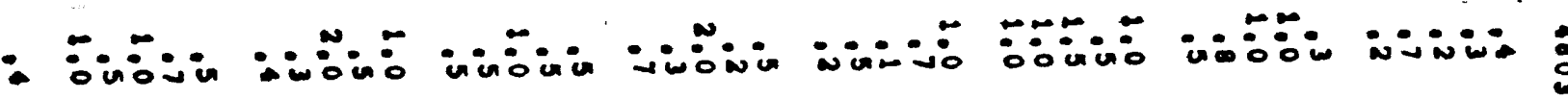

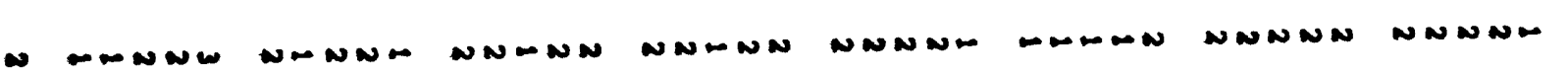




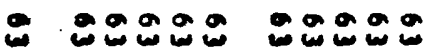

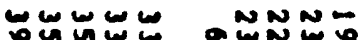

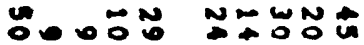

i. o. 00

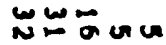

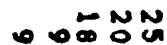

\%ogo

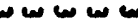
NDN 0 OON
운요

NNM WN: asoas एक $\infty \rightarrow-\infty$ - u⿺𠃊十
00000

tan 0

Nw onogo המס

-

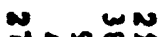

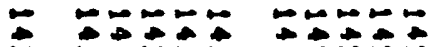

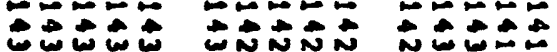
NWNONO Nő

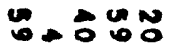

Uू⿱

- o

$\infty+\infty=\infty$

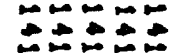

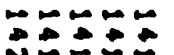

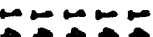

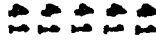

$\check{E x+\infty}$

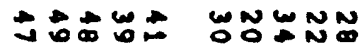
Dิ

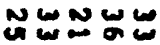

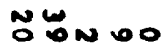

$\omega \sim N \vec{G}$

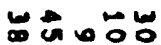

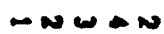

エNー

$\overrightarrow{0}=\mathbb{0}: \mathbb{0}$

ตั $=0 \%$ ○ै

$\vdots$

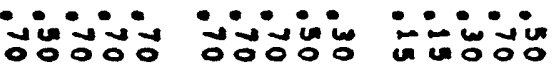

isivin NON

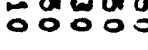

\section{ivivis} óo ơ⿺

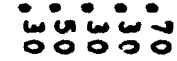

ज: 눙

w w w w

- WNNW

$\omega \omega \omega \infty$

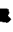

$=$

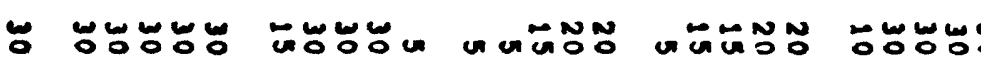
$x \geq$

GONON

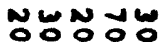

ㄴํำ

- ONnNin

WOOOLN NNOEN ㅜㅜ으웅

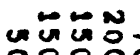

부요

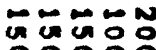

NWw

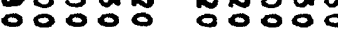

00080

$0000 \%$

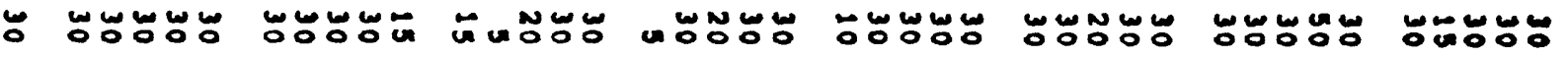

- $00000 \% 00000000000000$

- 0000
$-000$
00000 00000
00000

- 0000
00000

000 $\infty 0000$

00000
- 00000 N00000 00000

$-\infty=0$ $\infty=\infty \infty$
00000

- 000
00000 $0000=$
00000 0.000
00000 0000
00000 00000

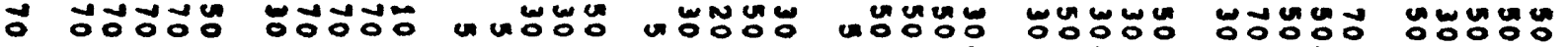

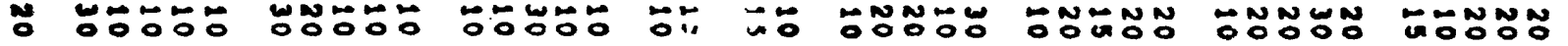


- 00000

- .000

: 용요

z $z z z z z$
¿0000

2600

NNNNN

응웅융

$z z 2 z z$
00000

1000

NNNNN

응ㅇㅇㅇㅇㅇㅇ

$z Z z 2 z$
00000

- 100

NONNN

융융유

$z z z z x$
00000

10000

00000

2000

00000

0000

00000

- 000

NNNNN

영ㅇㅇㅇㅇㅛ

Nㅜㅇㅇㅛ

$x \geq z z=$

c $2 x=2$
응응용응

$z z z z z$
NNNNN ㅇㅇㅇㅇㅇㅇ $z \geq z z$
운운운웅

웅

$z x z z z$

का 2022
우잉 운요는

$z=x \geq z$
둥웅웅웅

$z \geq 0 x$ $\because \div 0 \div 0$

돈요용

$y z z z$

뭉웅 은오옹요 $z \geq z 2 z$

00000

00000

응ㅇㅇ

00000

00000

00000

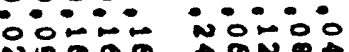
ㅇN요 봉영 $=$

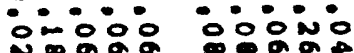

00000 i. ํํㅇำ $x=2$

z

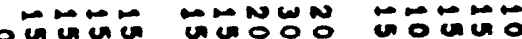
-

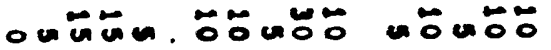
05

e
- Onumo 00000

$\omega$ $=5$

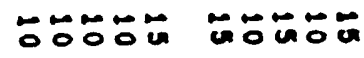

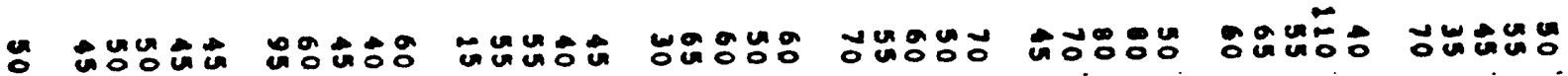

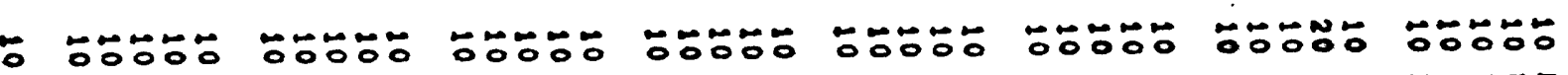
$z z z z$ $z z z z z$

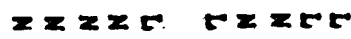

$z z z=z$

$z \geq x$

$z z=2 z$

ancun

- unao

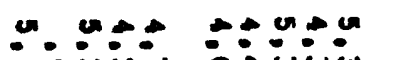

$-00-$ un

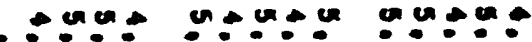

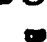
-

-

$\because \quad:=$

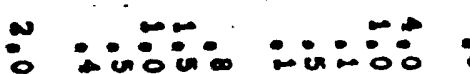

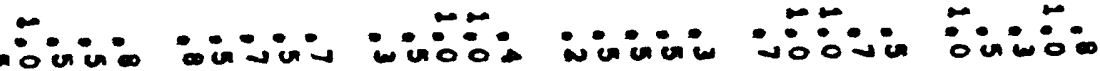

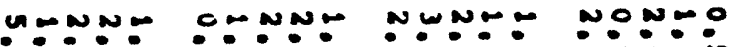
inovin 


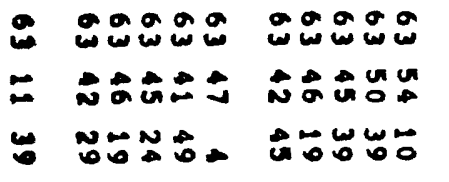

$\begin{array}{lll}0 & 0 \\ w & 0 & 0\end{array}$

$\rightarrow$ 눙

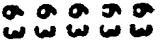

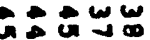

NuMN

N

ON=O
온운 $\rightarrow$ 넝하 م

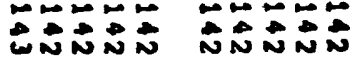

ט. ON⿴囗十

U⿴囗十丁:

W

$\because \operatorname{UNNN}$

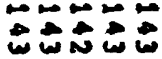

WNA

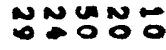

5
둔
2
름

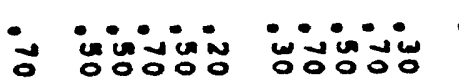

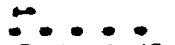

on wow

inisiv

نำ:

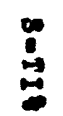

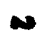

Wwom

Nw w w

w w w w

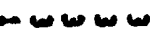

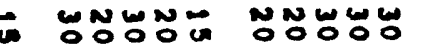

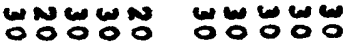

$4000 \%$

$\underset{8}{\mathbf{8}}$

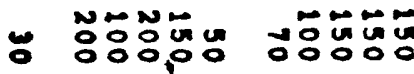

ज부눙

붕융용ㅇㅇㅇ

ज응ํํ융

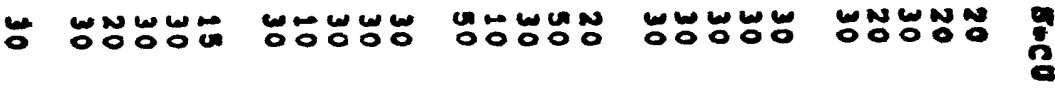

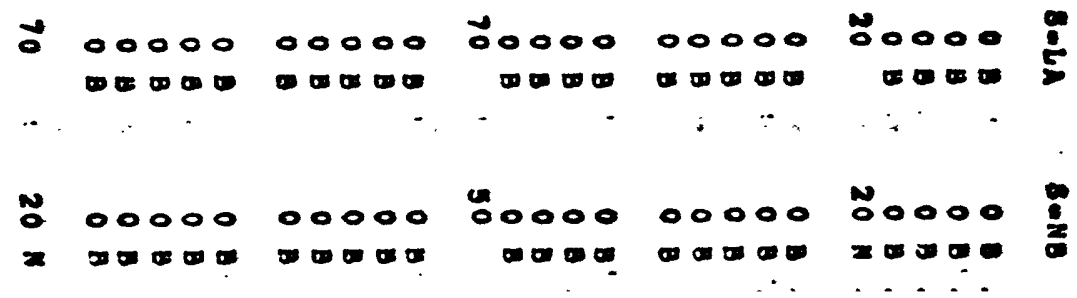

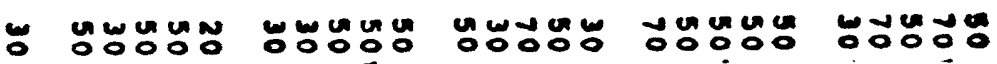




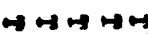
$\times x \times x \times$

00000

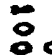

< 000

- 따미

000

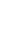

20000

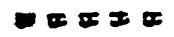

$\circ$

0000

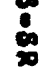

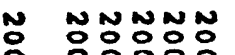

NNNNO

NNNNO

NNㅇㅇㅇㅇㅇ

NNNNN

잉

$z=z z$

$z x z=2$

$x \geq z=2$

$z \geq z=2$

:

운웅운

운운운웅웅

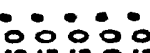

운운웅

$\because \therefore \circ:$

$z z z z$

$z x x \geq z$

$z z z=2$

$z=z z$

unuma

$z=2 z=$

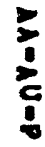

- OOOOO
용ㅇㅇ

00000

유N옹

$z$

:0:ㅇ

응으은

00000
$0 \div 000$
080

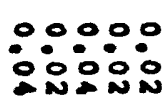

$z=$

$\sum_{0}^{\infty}$

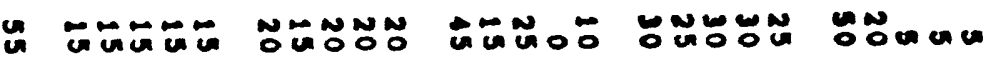

$$
\text { . }
$$$$
\text { . }
$$
-

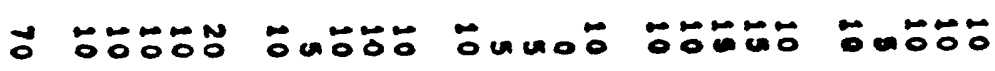

-

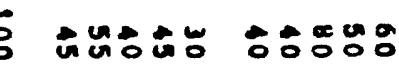

웅ㅎㅇㅇㅇㅇ

의영

कीजu:

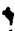

$\begin{aligned} \circ & 00000 \\ & =2 z z z\end{aligned}$

ัㅇㅇㅎㅜ

둥ㅎㅇ

$z=2 x=$

우웅우

ㅜㅜ웅

$z z z z$

$z x=2$

z $2 \geq z$

- anen:

un une

oncug un

$\because 0 . \div$

a 00

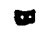

.

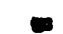

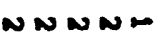

$\boldsymbol{N \omega} \boldsymbol{N}$

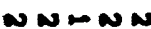

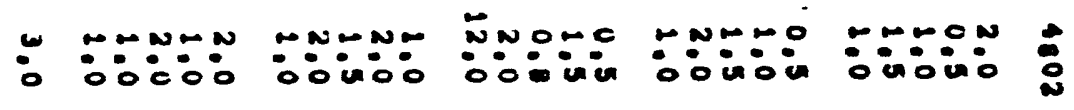

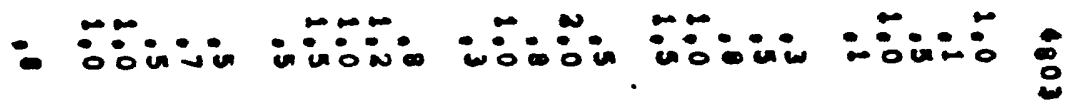

$\infty$

N

טח

$\omega \infty \sim$

$\infty \sim$

טח 
Nonmagnetic Heavy mineral concentrates:

Elements not looked for: S-Fe, S-HI , S-Ca, S-Mn, S-B, S-Ba, S-La, S-Nb, S-Sc, S-Y, S-Y, S-Zr.

Elements not analyzed: $\mathrm{AA}-\mathrm{Au}, \mathrm{Cu}, \mathrm{Pb}, \mathrm{Zn} ; \mathrm{INST}-\mathrm{Hg}$; and CM-As.

Elements looked for but not found: S-Au, S-Cd.

S-As was not detected except in samples TX9C3 (500 ppm) and TX160C3 (700 ppm). 
버ㅁㅕㅓ법

장응ㅇㅇㅇㅇㅇㅇㅇㅇ

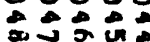

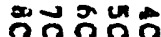

เथس

중영중영

กัธก์

- o 0

o. 000

مأس

$\omega \boldsymbol{\omega \omega}$

ט $\infty \omega$

$\rightarrow$ ○一 س

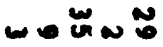

Noous

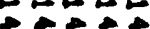

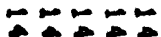

WWNA

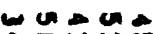

n: $\rightarrow+\infty$

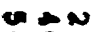

- 0 - 0

๑

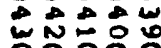

$\vec{x} \vec{x} \vec{x} \vec{x} \vec{x}$

ㄷㅇㅇㅇㅇㅇㅇㅇㅇ영

wै.

กรณ?

- o o o

ט

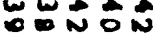

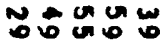

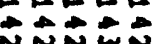

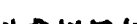

๒๐ッロ

$\vec{x} \vec{x} \vec{x} \vec{x} \vec{x} \dot{x}$

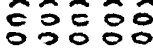

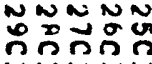

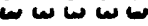

$\therefore \omega=0$

WUN 000

$\because \div \div \div$

แล் ลே

-

un

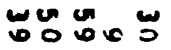

- 0000

$\vec{x} \vec{x} \vec{x} \vec{x} \vec{x}$

o

NN음

ลก๊ิ๊ิกำ

น

onoos

o

도웅

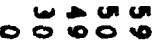

부저ㅁㅓㅓ젖

중응ㅇㅇㅇㅇㅇ

등ㅇㅇ

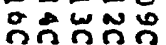

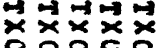

응응ㅇㅇㅇㅇㅇ

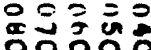

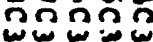

컺벚벚셩봉

ㅇㅇㅇ응웅응

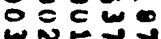

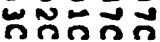

กรกล

a. a co

$00>00$

-0.00

مَ

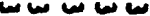

훙두

ס م

- م

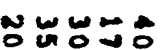

$\div 2=0$

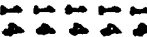

ดะNล์

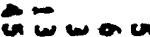

숭

Un

$\because \div \div \div$

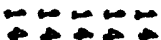

m a

שc

W

مِّ

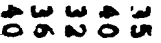

مَّ

N $\begin{aligned} & \text { N } \\ & 0\end{aligned}$

w영

$\div \div \div \div$

$\because \div \div \div$

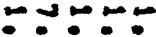

-50:

0000000000

$z z=z \geq z$

0000

$\therefore \circ: 00$

00000

$\div: \div$

$\div \div \div \div 0$

$\div-\div$

$\geq 2 \geq x$

NNNNN NNNNN

NNNON

$z \geq z z z$

$z \geq x x$

$\geq x \geq z$

$z \geq z=$

NNNNN

NNNNN

NNNNN

$N \infty N N N$

$\geq \geq 2 \geq 2$

$z z \geq z z$

$z \geq 2 z=$

$z \geq \geq \geq z$

NNONONO

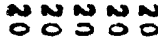

NONON

NONONONO

NNONON

ONO

NDNON

NONON

$z<2 z=$

$z z \geq z=$

$z z z z$

$z \geq 2 z$

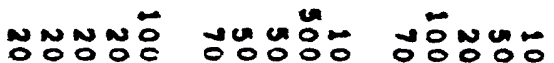

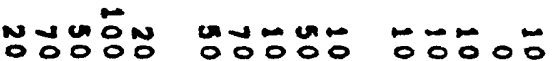

N N N $=$

Nㅡ유요

D

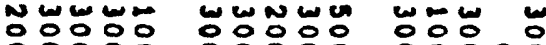

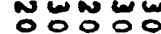$$
\text { - }
$$

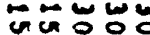

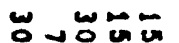
$\infty$

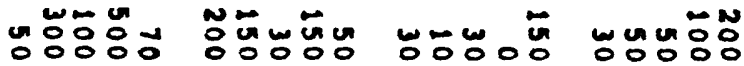

구웅

G요

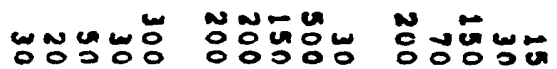
+

(1)

00000

व

$0<000$

00000

00000

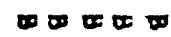

00000

0000

00000

10000

00000

- 10000

00000

-

00000

0:000\%

무유유

두ㅇㅠㅜㅇ

100000

후웅

$z z z z$

$z z \geq z$

$z x z z z$

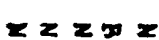

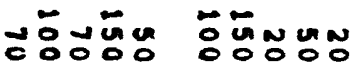

NONONO

NONONO

$x \geq z x=$

$z z=z z$

$z \geq z z$

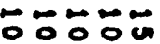

NNNNN

Nㅜㅇํํ융

NN유

$z \geq z x$

5

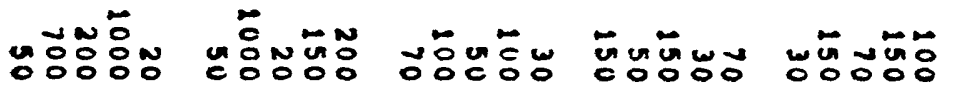

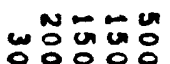

जேㅜํ요

uns

OOCट0 O0000

$=$

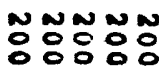

NNNNN

nNNNN

nNNNN

NNNNN

NNNNN

응ㅇㅇㅇ

$z z z x$

$z \geq 2 z 2$

응응

NNNNN

$\geq z \geq z$

$z z z z z$

NONOU⿴囗十ำ

טمก

NENONO $z=$

$z=$

กักัดกับ

응요

$z z z z$

NONN

$z z z=$

n以ー

옹ㅇㅇㅇ

둥응유웅 00000

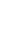

$z x z=$

$x z z z$

웅유웅

$z z z z$

응응등응

$z \geq x=$

E

00000

00000

00000

00000

ununa

unuma

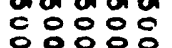

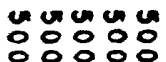

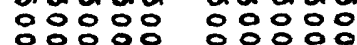

웅융융유

$z=$ 


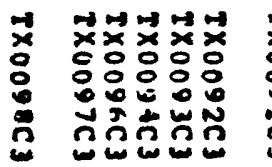

$\overrightarrow{ } \vec{x} \vec{x} \vec{x} \vec{x}$

중중중

웅융융유

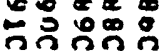

วะณอ

- 20000

웅.

蛅さむ

Nด的䓃

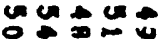

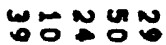

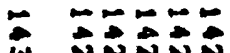

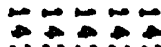

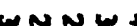

WN

ט

U $=\operatorname{lin}_{-\infty}$

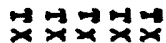

중징중잉

舟

นี้ก็กำ

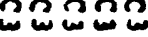

- 우웅

at ins

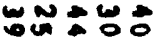

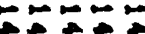
NลNล̃

un $u=N$

M UNN

O U U N

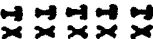

응ㅇㅇㅇㅇㅇㅇㅇㅇ

옹ㅁㅇㅇㅇ

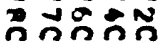

และ

우요

둥두

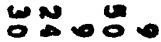

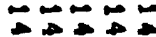

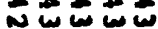
林さ

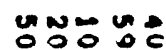

$\therefore$ แこลNล

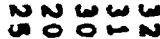
unNwN

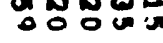

$x \times x \times$

คำ

0000

W

פم
퍼ㅁㅓㅓㅓㅓㅕㅓㅕ

응응응응

궁요

응응응응

엉영

วิกัก๊

종중중중

응ㅇㅇㅇㅇㅇㅇㅇ

ํำ行行

กรณ?

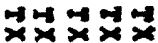

중충중

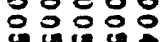

ผำำำ

ถุกณณ

$\frac{1}{3}$

00000

00000

- a 00

ס

ผั

तिए

- 4 -

-

ㅇํำ

론

$\because ே \div \div$

-

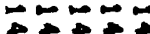

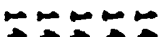
NNNNN

س

ס

ט.

슨

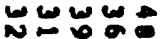

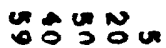

$\because \div \div \div \div$

$x \quad z=2 \pi$

:0:0:-

NNNNN

$2>2 z=$

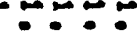

잉ㅇㅇ

$x x z=2$

$z \geq z=$

NNNNN

NNNNN

$z z z z$

$Z X Z Z$

№̛ㅁํㅇ

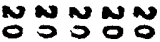

$z z x z$

$x \geq 2 x$

NONON

2 $2 x 2 \pi$

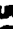

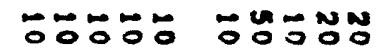

ํㅜㅇㅠㅜㅇ

مNN-

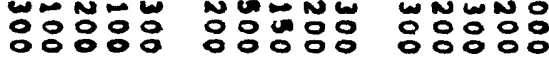

uㅜㄴ

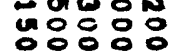

טم: $\therefore 0 \div 0: 00 \div 0$ $z x z=$

NNNNN

$Z=Z z$

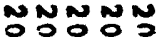

$x=2 \pi$

Nㅜㅇㅛ

NNO

$2 \geq 2=2$

NNNNN

$Z=\quad I=$

NNNNN

$x \geq z=$

웅웅

$z z 2 x z$

NNNNN

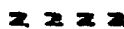

NNNONON

z2 $2 x$

NNNNN

OOOOO

CH,

웅유

$z z z z 2$

NNNMN

$z Z z Z z$

NNNNN

2000?

$z=2 x$

NONO

50000

$\infty$

00000

$z \geq z \geq z$

NNNNN

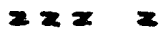

NบNN

$x \geq 2 z 2$

- Un⿺

OOOOO

- Now जo: 000 녕영ㅇㅇㅇㅇㅇㅇ 눙영ㅇㅇㅇㅇㅇㅇ

Nㅜㅇㅇㅛ

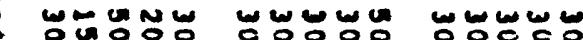

w0\%

뚜ำ

W6 $000 \%$

w⿻ Non (1)

6

0000000000 1000

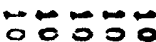

$z z z z$

OONON 00000

우유

$z z z z=$

NONNON

OO OOO

00000 0000

วேேேே

z $\geq 2 \geq 2$

NONONG

00000 1000 \%๐० $z \geq z \geq z$

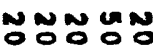

00000 0000

ㅜㅇㅇㅇ

$z z z z=$

NNONN OOOOO

00000 - 1000 ธேธัธะ $z=x \geq$

NoN 00000 - 00 С००० $x=z$

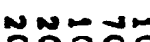

$$
x=
$$

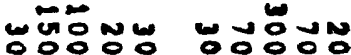

WONO $\leftarrow$ $z$

NNNNN 응응ㅇㅇㅇㅇㅇㅇ $x \geq z=$

NNNNN . NNNNN

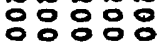
$x \geq z \geq z$

ㄷㅇㅇㅇㅇㅇㅇㅇㅇㅇㅠ

$z=2 z$

$x \geq x \geq 2$

$z z z z 2$

o

$=$

(a)

00000

00000

00000

$100000=000$

1000

ungun un

unu una

융유유

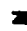

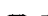

$z z=z$ unumuna

응영영

$z z z=z$

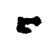

NNNNN :ํํำ $x=2 x$

NNNM NONONO
00000 - 000

unuma 영영ㅇㅇㅇ

웅영영영

- $z=x x z z$ $64: 7$

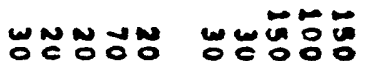
8
8
8
0 0
0
0
8

응용ㅇㅇㅇㅛ $x \geq z=$ Nㅜㅇํㅜㅇ 


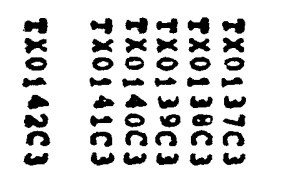

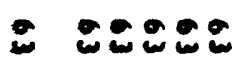
ธลัดัニ

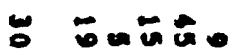

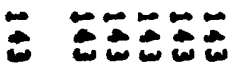

- *0心

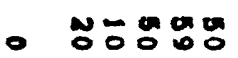

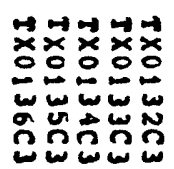

is ogo Ex=0u

$\therefore \therefore-5$

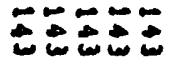

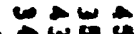

ดั

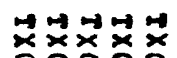

응ㅇㅇ응ㅇ응

늘 NN

ละละ

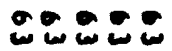

can una

○以

○०00

\section{こニさむ}

บลシัニ

N.0.

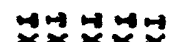

증항중중중

N正芯

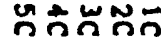

แล 20

-

ancun

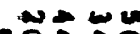

nos-

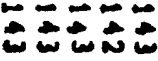

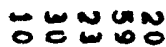

的管出

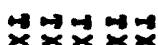

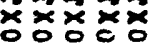

든둥

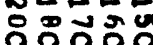

วกกล

훈두

cunuener

$\rightarrow \infty-\infty$

药。 un

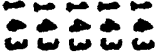

ONNN

पू⿱
버면버버

종중중중

ーேニニ

ลันัก๊กำ

س

둔둥

$u \rightarrow u \rightarrow c$

ㅇํㅇ춘

をニニニた

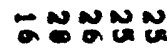

ตैe 0 -

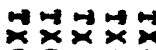

응ㅇㅇㅇㅇㅇ응

등ㅇㅇ응

กํํํำ

س

둔둥

수웅웅

ज०O००O

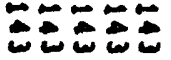

Nט

$0 \div \div 00$

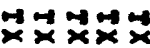

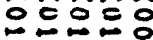

응ㅇㅇㅇ

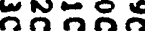

든

$\sum_{0}^{2}$

क्षव:

ลேேニシ

Un $N 0$

$\stackrel{5}{4}$

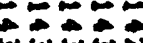

Ш

ニัㅜ

วิธี้ำ $-\infty-\infty$

- :00:0

$z \quad z=z z$

N NNNNN

- IETE

○ NNONO

$-5-\infty$

00000

$z=2$

$\leftarrow \div \div \div$

웅:

$z x \geq 2 z$

NNNNN

NNNNN

$$
\geq 2
$$

NOㅇㅇㅇㅇㅛ

$x \quad x \geq 2 z$

-

8

:

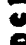

$\infty$

000\%

$z=z z z z$

6

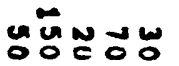

융ํํㅇํํㅇ $=$

NNNNN 등응ㅇㅇㅇㅇㅇㅇ NNㅠ용ㅇㅇ $z=2 z=$

$z z=z$

$\underset{0}{0}$

$=$

NNNNM

$$
=\mathbf{z}
$$

E

2

-

응ㅇㅇㅇㅇㅇㅇ

\section{๕ั}

unumu 응유:

unumu

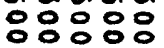
$z x=z$

Nㅜ유요

$z \geq z x z$

무웅우

แัดับับ Zxz= 구유

UNMNN

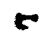

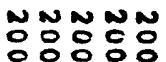

ZZZZZ.

MNN NN

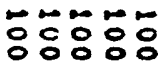

ZE =

O-NNN

6<smiles>[CH]=CC=S</smiles>
:00:0

$\geq \geq 2$

$\div 0 \div 0 \div$

$z \geq z \geq z$

$\div \div: 00$ 00000

NNNN

טN

$\sim \boldsymbol{N} \boldsymbol{N} \boldsymbol{N}$ r 5

$z=$

Nㅜㅇํํำ

NNNNN 0000

$z x \geq z 2$

NNNN

$\geq \geq 2 z$

두웅

10000

I $2 x$

OONOONO

ลับีักับั

Eับñ

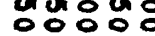

Nwww 응유:

แัดำกับ

$\div \div \div$ 10000

$z=2 x=$

$\div 0 \div 0=$ 00000

$z z z z z$

NNWNN $z \geq z \geq 2$

UN山N $z \geq z=$

Nㅜ융

$\geq z=2$

NNNN

$\geq 2 \geq=2$

NONONO

두유

$40 \omega n$ 명:용

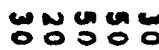

ฉ

umm nunnn 눙요

는응

تีWسN 응응영

닝ㅇㅇㅇ

00000 - 0

由ण

00000 00000 웅ㅎㅇ

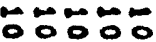
$z \geq 2 z$

$z=z x z$

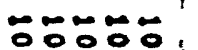
$z z z z z$ $z z z z$ (200 $z=z$

$\because N a n$

Nonn

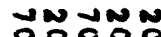

NNNNN

붕요

2

ง m n

wamon

N

$-$

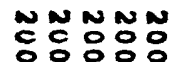

NONONO

$z z z z=$

$z z z z z$

NOSNONO

z z z z z

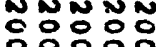

$z z z z$

NNNNNN

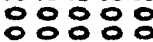

$z z z z z$

NNNN

งกำ

NWN

5

$=$

NNNNN OOOOO

$z z z z=$

NNNNG O000\% $z=z$

응응응웅

$\because \div \div \div$

웅둥ㅇㅇㅇ둥응

rxt

$z \geq z$

a w 


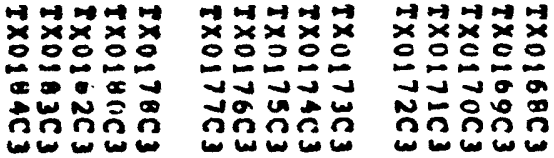

- 000 w

ڤニ

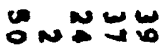

\#正

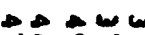

ज:

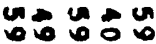

00000 w 6 w

G U U u

$\infty \rightarrow c$

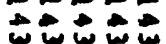

un on una

aक wa

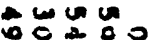

- 0000 o cus un

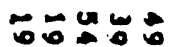

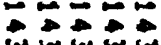
$u+m-\infty$ NONO BNoNu $\vec{x} \vec{x} \vec{x} \vec{x} \dot{x} \vec{x}$

응ㅇㅇㅇㅇㅇㅇㅇㅇ

5.5.0

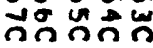

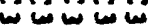

- $00 \%$

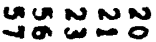
ט

$-2=0$ a a GーNOa №ñ

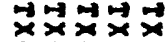

응ㅇㅇㅇ응응

aju uv

กิธี่ํํำ

-

แ

-

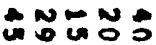

$\rightarrow \infty=2=$

un un

$0 \rightarrow N \leq \infty$

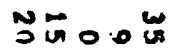

$\vec{x} \vec{x} \dot{x} \vec{x}: \vec{x}$

응ㅇㅇㅇㅇㅇㅇ

unuvu

กีก์ที่ัน

- 00

w

-ேேニ

คै

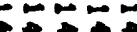

$\omega \leq=0$

0 ớn:

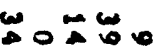

벚버ㅈㅓㅗ젖

ㅇㅇㅇㅇㅇㅇㅇ응

जún

กิธีี้์็

此的

- o. o.

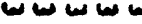

ㅜㅡ눙

w

$00=00$

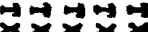

응ㅇㅇㅇㅇㅇㅇ

-

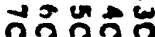

กรวละ

疍

0.00

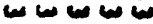

- a m a

- *6

$$
\underset{7}{7}
$$

$\div=\div$ ش

우의

ேேニேே

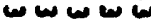

ânu un

ט。

숭무

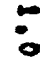 \\ : \\ $\omega$

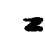 \\ $\overline{-}$}

○

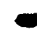

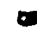

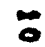

$=$

○

-

ะ ะกับัั -

ั กัดกํัดั

x zx=x

ะ กับับั้ก $z=2$

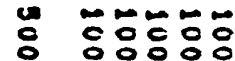
$z z z z=$

ำํำ

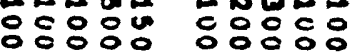
एक 50

- 00000 - . 0000

num un :ㅇ: $x=2 z$

$0: 00: 0$

$z z z z z$

$\omega \sim \boldsymbol{N} \sim$

$z=2 z=$

NNNNN

$Z Z Z Z X$

50500

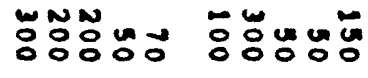

00000

NNNNN 융등융

ก NNNN

WNNNN

NNNUN

0000000000

uncun un

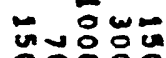

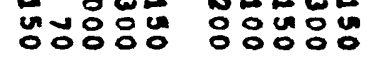

$0: 0: 0$

$z=$

NNNNN

ze

$z$

anu 03000 $z=2$

$\div 0 \div 0 \div$ $z=z=$ $\because \div \div \div \div 0$ $z=$

NNNWN NNNNN

$z=$

ธีดับํํำ

ํํㅇํํㅇํำ

$z 2 \geq 2 z$

$z 2 z x=$

$z \geq x \geq z$

แักับัด

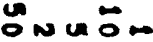

NNNUN

OODONO

ํํㅇํำ จำ

$2 \times 223$

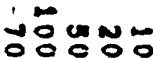

NGNN-

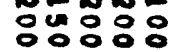

กับับั

แล-พn

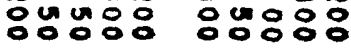<smiles>[CH]=O</smiles><smiles>[13CH3]</smiles><smiles>[131IH]</smiles>

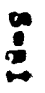

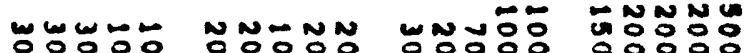

MnN

N

กN

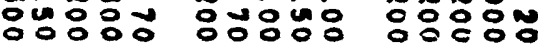

8

$=\div \div 5$

-

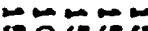

Uמ

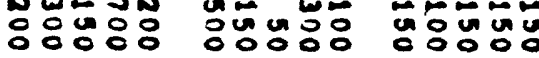

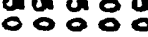

ㅇํㅇํㅇㅇำ

응ㅇㅇㅇ

50ㅜㅇㅇ

$\div \div \div 0$

$00 \div 0$

융ำ 05050

$z z z z z$

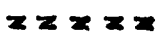

$z=x=$

$x \geq \geq=2$

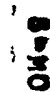

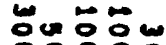

BNonuna

iñ⿻上丨

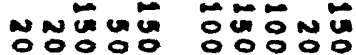

$00: 0 \%$

ㅇํㅇㅇㅇ

응응

:

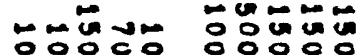

UNG

Nルே以

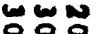
TE

nNNNN 응융응

nNNNN 뜽ํㅇ응

응융응

유ㅇㅠㅜ

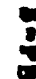

NNNnN nNoñn

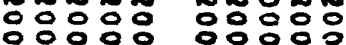

NNNNN 응융융

$z x \geq z x$

$\geq \geq \geq \geq z$

$m=2=$

$x \geq \geq x$

$z \geq z=2$

NNNNOO

NNNNN

NNONONO

ONONONO

ONONONONO

$2 z 2 x$

$2 x=2$

$z=x$

$z=2 x$

$x=2=$

N느무요

ヘ

두음

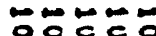

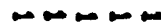

응동유

ㅇ:ㅇㅇㅠ

$z=2=$

$m=20$

$z=2 z$

$z z=2 x$

$x=2=$

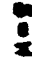

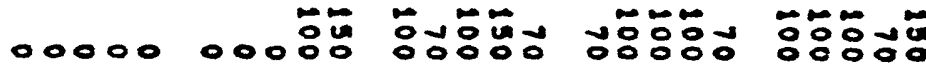

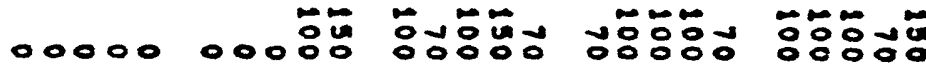

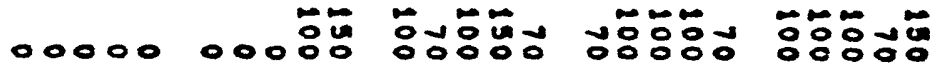

$-\infty 00$

- 0

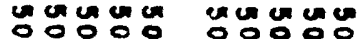

80908 


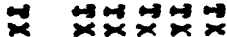

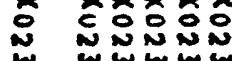

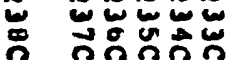

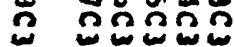

- 20000

- ovonara

- ovega

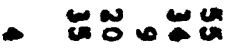

$\approx \div \div \div \approx$

-

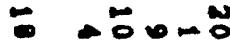

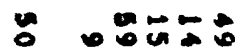

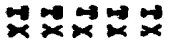

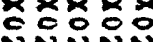

WWNNN

군

ลำ

a. 000 ט

$\operatorname{con}_{0} \in \mathbb{a}$

曲

菓ニニ

样

ヘニニニะ

o: $2: 5=$

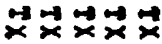

c co o

NNNNN

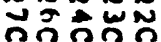

ละธละ

- 0 0 0

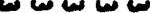

unging

טNN

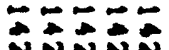

以ே

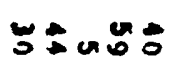

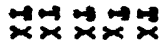

중송응

NNNNN

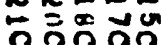

ลㅗㄴ

- aora

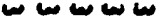

un: ún

$u+N u n$

GO융

モニミニニ

แ un

cas

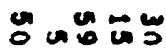

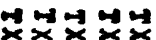

ox

NNNNN

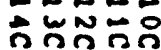

곤

0.000

$\omega \omega \boldsymbol{\omega} \omega$

:

ט.

ニニニニー

ニニニこた

U

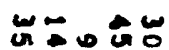

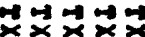

등ㅇㅇ응응

OCOOOC

䍒颉苗

กรวกิ

0000

ou unu

D

NDNE

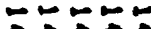
WNNAE OUGU. N⿴囗十

은?

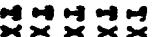

중증중

$505: 0$

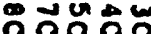

วกรถ

00000

تص

N口E

ษேேㅇ

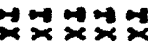

증중중

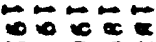

ํㅗㅇํำ

$-000$

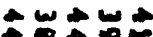

- cow.

$\rightarrow 0 \times 00$

ローーー

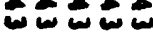

مي

뜬

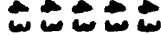

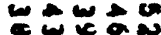

م

웅 $\because$ - $0: 0: 0$

$x \quad z=2 x$

N NNNNN

$z=z=z$

:

$x \quad z x y z=$

$\because$ 후웅

-

- Nํ유 -

ㅜㅜㄴ

GUNON

$\div \div 0 \%$

$x \geq z=2$

แ $N \cdots N$

$z x=2$

NNㅇํㅇ

ax $x=$

$0 \% 0 \% 0$

5

NNNN

OOOOO

$\div \div \div \div$

숭영영

NمNONO

NN N

$x=x \geq 2$

$z=2 x$

둥후웅

ㅜㅜ웅

TEE

:0:0:

$\infty+\infty$ 0000

$z \geq z \geq z$

무웅

z. $z z z z$

$N \omega N \infty N$

NNNNN

Z.ZZZ

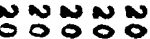

N $00 \%$

$z x \geq 2 z$

$z 2 z x=$

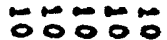

두유유

뭉응

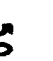

$\div$

3

ํํำ

NN OOOO

CON口

$\div \div \div$

두웅

$\because-\div \div$

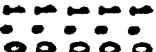

$z z z z z$

NNMNN

$z \geq z \geq z$

$z \geq 2 \geq 2$

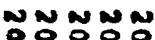

Nㅜㅇํํㅇ

zIzZz

$z z z z z$

ஜே๐

뚜웅

-anou

un

- 00000

- -

00000

000

00000

00000

0000

-

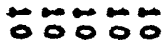

후웅

훙ㅇํㅇ

$z z z=z$

$z=2 z=$

$z \geq z z$

$z=z=z$

Nㅇㅇㅇㅇㅇㅇ

NNNNN

NOㅇOOㅇ ONONO

5

○ N

NONNN

NNNOOOO NNNWO

TO

$r=\boldsymbol{E}=$

$=2+52$

$z z z z$

$\sin 2 \pi$

E

NNNNN

CNNNQN

nNNNN

Cㅇㅇㅇㄷㅇㅇㅇㅇㅇ

동ㄷㅇㅇㅇㅇㅇ

융ㅇㅇㅇ

$z Z z Z z \quad z Z z Z z$

$z=2 z=2$

$z z z z z$

)

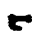

$\therefore \quad-\infty-\infty$

응ㄷㅇㅇㅇㅇㅇㅇㅇ

$z \quad z=2 z$

NNWNN

NONNO

NONOU $z=2 z$

$z \geq z=$

둔 응응양ㅇㅇ

등ㅇㅇㅇ둥ㅇㅇㅇ

둥

응영영영

$x \geq z x z$

$z x z z z$

$z \geq z=z$

00000

100

눙

$z \geq z=$

00000

10000

후웅

$z=x$

مَ

CEETE

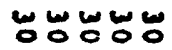

CErter

以NUNN

NWNN

- 2

- -5

NNNNN

$\because: 05:$

$x \geq z \geq z$

NNNNN

:ํํำ

$z \geq z z z$

№O№:

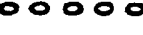

$z x z z z$

No응 용

NWNNG

뚱응요

$z=2=$

z $2 x$

뚜음

응영융

등ㅇㅇㅇ융융

동동융

$z \geq z=2$

$z z z z=$

$z \geq z$

동ㅇํㅇ

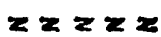




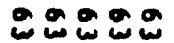

운운웅

운

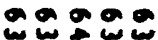

웅aㅇ영

운웅웅

טNN

"

놈요

-usun

ט두

×0:-

$=5000$

ט

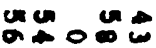

눈후웅

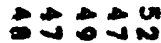

م

눙둥

- Nun

운연웅

우의

-

돔

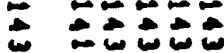

ڤேニニニ

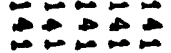

$=\infty-\infty=$

$=\infty \div \div \div$

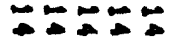
ט

w w

wen un u

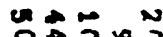

N000ज 0.050

- ua $a$ un

un $u$ u

טN

$\Delta-30 \%$

coco:

$\square \div \div \div=$

N5000

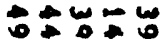

NTNWN

$\therefore 0=0$

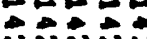

NNNN

두

두.

actos

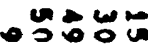

$5 \div \div$ 00300

- 222x

$\div \div \div$

$z x \geq z$

$\boldsymbol{N N \boldsymbol { N } N}$

CETEE

$2 \geq 222$

O ONONO

NNNNN $x \geq 2 x 2$

$\div$ 웅ㅇ 5

- WN$=222=$

50000 LEETE

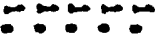
00000

무융ำ

$z=2 z z$

$z z z z$

ngnun

$\omega \boldsymbol{N} \boldsymbol{N} \boldsymbol{N}$

$22 z$

NNN NON

NONON

$=2222$

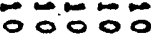

2 2 : 2

ํํㅇํำ

C5t

TEETE

00000

प5E5

Wח-ñ

Nㅜ응

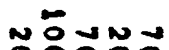

눈형 №ำ

$\because \because \div \div$

$x \geq z=2$

NNNNN

NNONOO

$2 x<2$

ó 0 < o OOOOOO

무웅

부웅

ㅜㅜ웅웅

뭉ํำ

누ㅇㅠㅜ웅 5 E

- 00000

- 000

- 두웅

z $z x z z$

- Nㅜ용
00000 100 후유 $z \geq z z=$

NONNNN
0000000000 10000 ㅜㅇㅇㅎㅇ $z \geq 2 z$

NNNum
0000000000 .0.00\% 둥웅 $x \geq z \geq z$

NOWNO
00000 훙후 $z z=x$

NNNNN

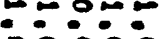
00000

지 $\mathbf{x}=$

NNONN

กักักัดั $z \geq 2 z$

유융 CEDER

ํํㅇํํ $=$ =

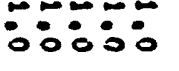

$z=z z$

NWN

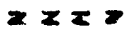

NMNNN O50\% $2 \geq 222$

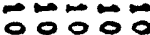
CEt5e

ํํㅇ응

$0000 \div$ ‥

00000 c 000 둥ㅎㅇ $x \geq 0=$

NN NN -

WNNONO

NNNNG

NNNNW TEE

ONNNN

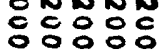
$r=r$

Ex $2 z$

NNWN OOOOO CE =

NNNNN 응영ㄷㅇㅇ $z z x z$ $z z z z$

NNNNN 응영융동응

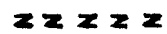
NNN NONO $z z=2$

\% ONNONO

NNNNN

NONUNO

NNNNN

NNNNN OOOOO

z

$z=$

$z=2$

응 응등흉융융

$z x z z z$

응둥듕ㅇㅇㅇ

동ㄷㅇㅇ응응

등용웅용응

$z z 2 z=$

$z z z z$

$z z z z z$

홍홍ㅎㅎ

$z z z z$

00000

00000

00000

00000

00000 ৫>-

- 000

00000 1000

00000

0000

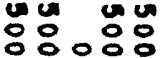

non 응ㅇㅇ

능ํํㅇํㅇ ㅇํㅇ응

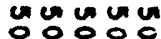
$\therefore: 0: 0$ $z z \geq z$

00000 000 ธัธธธ์ธ $x \geq x z$

NNN

NNNNN 20 5

NNNNN 응등ㅇㅇㅇㅇㅇㅇ $z x z 2 z$

象NNN 응응융 $x \geq z=2$

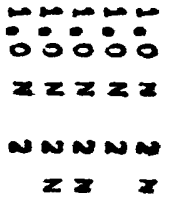

NNN N $z=2 z=$

두웅유 CE

ํㅜㅇ뭉ํㅇ =

ํㅜㅇㅠㅜ웅 -

00000 


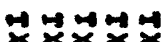
중중충 w w NNNN

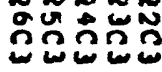
แ รกํํํำ

- 0000

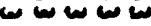
- a o o u a en $\omega N$ 0.

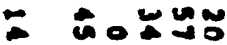
ニㅡ。․

×ニニー

×

and $-\infty$ - OÑ.:

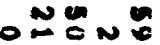
ㅇํำ:0

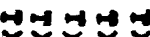
중중중응 U 敦和

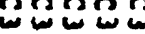

- a. a 0 $\omega \omega \omega \omega$ ט.5 ๑๐๐ั०

$5=5=$ coowa ใด้

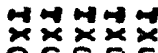
ww ー

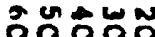
ล2

0.000 w w

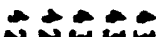

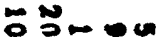

$\therefore$ シニシニニ

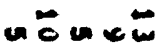
중중중종 w. กร์ एक

- arao w w w w

도누웅

ㅂ.

ํㅜ운

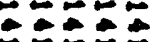

$\sim \boldsymbol{N}_{\boldsymbol{N}} \stackrel{\boldsymbol{N}}{\boldsymbol{N}}$

ำด

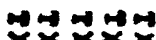

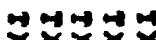

중중중

ww.

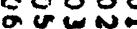

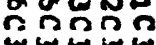

0000

แ山

忨ロニた

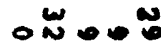

$\because \div \div=$

NN

$=\omega-\omega 0$

b. Su

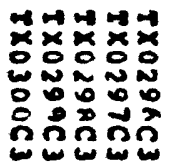

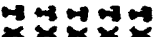

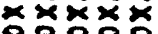

옹ㅇN요

우웅우

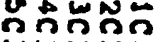

5

- 0000

w w w w

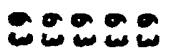

a unu

눙는

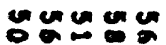

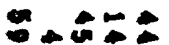

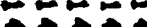

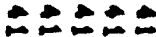

$-\infty-\infty$

$-2=50$

뚜요

แ์

$\leftarrow 0 \% 50$

$\because 5 \div \div 0$

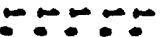

-

దோ-

$\leftarrow \infty-\infty$

$x=x z z$

00000

00000

00003

$0: 00 \circ$

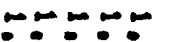

-

$z x z z z$

zx $x \geq z$

$z z z z z$

00000

:0:00

$\div 0 \div 0 \div$

$z \geq z 2 z$

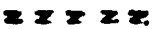

$z z x z z$

TNNOKN NNNNN

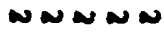

NNNMU

UNNNA

NNNNN

N N N N N

$\omega N N \omega N$

- $\angle z=2 x=$

$z$

$=$

$z=z z$

$z \geq z=\approx$

$z \geq 2 z$

NNNNN

N N N

NNNN

NNNNN

N ON

NONNN

$x=2 x$

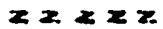

$2<22 z$

$x z z z z$

$z=z \geq z$

$z=2 z=$

๐ㅜㅇํํ

후웅

ㅜㅜ웅웅

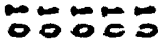

TEE

5

55

$\leftarrow 5$

$4 \div 0 \div 0$

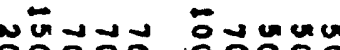

in 2 눙

ำำ

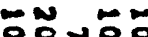

00000

00000

00000

00000

\% ํํㅇํำ

두유응

ㅇํㅇํํำำ

ํํำ

우웅유

TEEE TE

to

CERE

$=605$

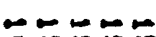

๒พัดธะ

:

:

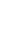

:

0000000000

00000

00000

00000

00000 - 00

வ

두웅

두웅

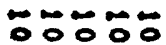

ㅜㅜ웅

$00000 \% 0000$

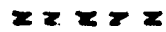

$x \times 2 x=$

$x \geq 2 \geq z$

$z z z z z$

\% NONONON NNONON

ONON N

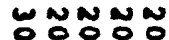

NONNO

NENONO

00000 -

๐ㅜ웅

00000

$z x \geq z z$

00000

- $\infty \omega \infty$

둥ㅎํㄴ?

$z=2 x=$

NNNNN

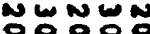

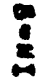

WNN

NNNUL

$$
=2
$$

WN WNN

WOONON

NNNM

$=$

5

$\mathbf{z}$

CE

ON

NONONNa

NNNNN

NNNNN

NNNㅇㅇㅇㅇㅛ

- $x z=$

$z x \geq x=$

$x=x x=$

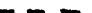

$z \geq 2 z=$

กัดํัดำ

$z \geq z 2 x$

$\div$

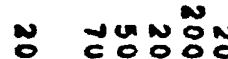

NNNNN

Nกตก

ต

8

nann O000 00000

$x=5$

-

ค

웅융유융

두유

ㅜㅜ유유

00000

$z E \geq z=$

$\geq x \geq z=$

$x z=z=$

형융영

$z x z=2$

도융응 용유 $x=2$ 응응응 $x \quad z=t$

NENNN ㄷㅇㅇㅇㅇㅇㅇㅠ $x \geq x=2$

NNNN O000\% - LE

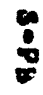

NNㅠㅇㅠ ㅇㅇㅇㅇㅇㅇ융 $z \geq z \geq z$

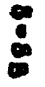

NANNN

NNWNN

: $=$

orom 등ㅇㅇㅇㅇㅇㅇ IEx 2 응응응 


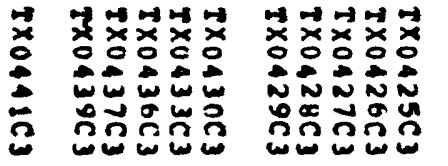

$\therefore \quad 0000$

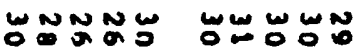

○

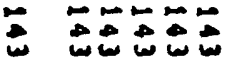

Na盛

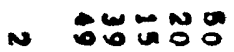

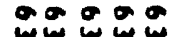

$\vec{y} \vec{x} \vec{x} \vec{x} \vec{x}$

웅ㅇㅇㅇㅇㅇㅇ

NNNN年

ผํํํำ

00000

w w w w

주에N

जि $00 \div$

००

$\because \div$ ש $N$

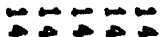
س

WNN

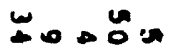

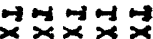

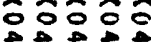

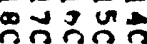

w w

00000 un w w w w

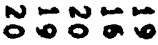

wat in

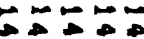

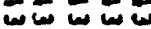
ON N un

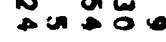

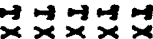

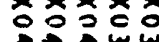

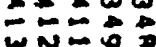

ผํำดั

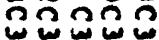

9.0.0. w w w w w

NNNDN

U⿺辶⿻日禸

$\leq \pm \leq=$

ט

$\sum_{\infty} \omega_{0}$

$\because \quad \div 000 \div$

뭉ํㅇํㅇ

x $2 x \geq 2$

$\div 0 \div 0 \div$

$z \geq z z$

NNan

NNWNN

NNNNN

$x \geq 2$

NNNON

NNONON

NNNONO

z z z z

$z<2 x=$

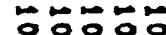

둥후웅

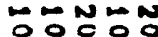

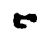

TE TEETE 웅융 영ํํㅇ

$-$

후웅

두ㅇㅠㅜ

n-

으유유 O응응

$0: 00: 0$

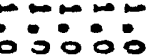

$x \geq \geq 2 z$

NNNNN

$Z 2 Z 2=$

MNNMN

$22 \geq 2$.

NNNNN

NNNNN

a $2 \geq 22$

$z \geq z 2 z$

जEOOOO

두융웅

$z=5$

ए

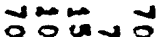

Б-5 옹ํํㅇ영

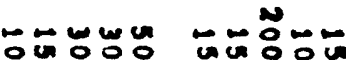

ํํㅇำㅇํㅇ

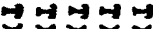

중중중

我

ปีํำ जิ

วละก:

0000

un un a un

- $\infty$ o

온

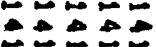

WNNNN

O- U. UN

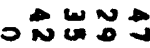

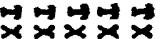

응중ㅇㅇㅇㅇㅇ

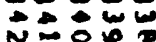

ํㅜㅇํำ

กรวลี

$x \times x+2$

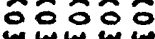

ww w

तีค์ ำ

กรก 20

00000

- o. 00

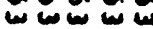

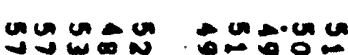

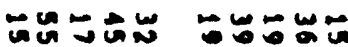

in

$\Xi E ニ E=$

WNNNN

N

ân $=0$

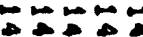

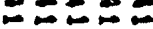

NN・゙ー

ஸேェ

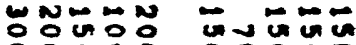

$2 x \geq 2 x$

:00:0

$z x z=2$

NNNNA

$z \geq \geq 2 z$

NONNN

$z \geq z x$

무융

$z$

$\div: 0: 0:$

$z z z z$

NNNNN

$z=2=2$

NNONONO

zXz2

뚱ㅎํ

DEE

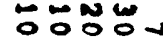
웅응

\section{ธ}

ํํㅇ융ํㅇ

=

c

5

5

뚜웅 $=0$

뭉ํㅜ웅 t..

00000 $\infty 000$

مே0\%

$z \geq z z=$

WNONO

00000000

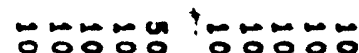

$z \geq x=$

$z \geq x=2$

Uோ๐ $\leftarrow$ 5

w $2=2=$

NNONON

NWNNW OOOOO Nㅜㅇํㅇำ

22222

$=2$

8

$=$

$x z z z z=z z$ $\begin{array}{lll}N & N & N \\ O & N & 0 \\ O & 0 & 0\end{array}$ $x x z z$

$\ddot{0}$

CONOOON

NONO NO

NNNNN

$x \geq z=2$

$z=z$

$z \geq z 2 x$

뭉둥용

뭉요

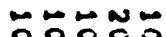

$z z z z=$ 00000 $z=z x=$

00000

$z x z z$

응ㅇㅇㅇ $z=2$

00000 வ10

00000 由ण 000

00000 띠

00000 1000

00000 -

00000 -

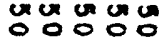
융ㅇㅇㅇ응

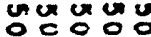

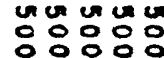
영영ㅇㅇㅇ

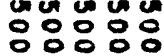
$x z=2$

vanum

등응융유

z $25 \mathrm{x} 2$

$z z z z=$

ununa

ษuñu

응영ㅇㅇㅇ

$x=2$

$\div 0 \div 0 \div$

zIzzz

NNGN

$x=2 x$

ํㅜㅇํㅠ

z $2 x z$

드융유

- 5

웅 등영영응 ॠ

00000

(1)

두ㅇㅠㅜ웅

$x=2 z$

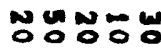

능용요

NNNNN 


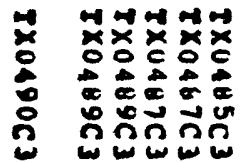

- 운후

- $\infty+\infty$

- 훙ㅎㅇ

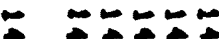

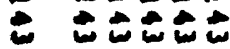

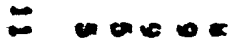

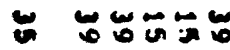

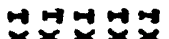

중충중

$+\infty+$

ผั

และ

둥. $0 \div$

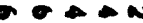

N

מש

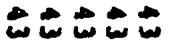
NNNND NNDA N
0000 -NNNN

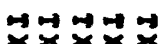
당중중응 車 กํํํำ w2.

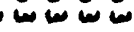

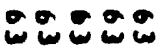
W טֶ:

-

$\pi$ แNANE $\rightarrow$ N مش ט

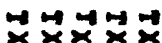

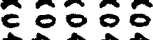
\%。웅 ทั่กั่กัก ละกับ

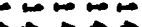
$\overrightarrow{N N}= \pm N$

N $ง \cup u$ cow

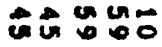

- $\div 5: 0$

$-\infty-\infty$ $\therefore 0000$

$z=2 z z$

$: 050: 0$

- $x z x=$

L NanNa

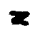

$\geq z$

NNNNN

NNNNN

$z x z$

○ Nㅜㅇㅠ

$z=2 x=2$

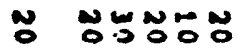

-

- 웅영부

NNNNN

$x \geq x$

ํㅡㅇํํㅇํㅇ
NNNN

ZXZZ

ธํํำำ $\div-\infty=$

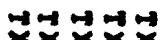
중중주웅

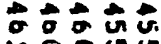

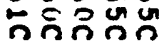
ฉล

00000 " w w

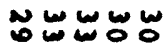
둥후웅

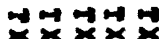

둥중중증웅

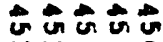
กำกัก

0.00 w w w w

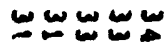
anu un NーN N wUUNa

-

무ㅇㅠㅜ

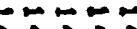

ANADA

-ONO

$\because \div 00$
는

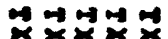

중으웅웅

눈영

ํํํํำ

-

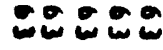

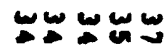

cun

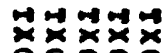

응ㅇㅇㅇㅇㅇ

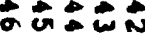

ก็กลักล

w

テーッー

ㅅN추

ONNN

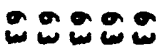
$\omega$ a n<smiles>[CH]C=[CH]</smiles>

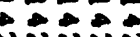
UNN טNNN תח مN

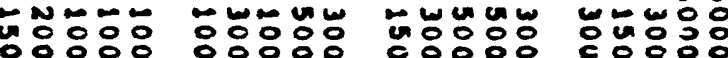

-

-

מח

-ேーー

- $-0-5$

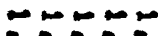

웅:0

잉우

$0: 00:$

$x=21$

$z \geq z=2$

$z \geq z 2 z$

$z z z z x$

zx $z=x$

NNNNN NNNNN

NNNNN

NN-NN

NNNNN

52222

$z z z=x$

$Z 2=2 E$

$z \geq 2 x$

$z \geq \geq z z$

NNNN

NONNO

NNNNN

N NONO

NONON

$22 \geq 2 z$

$2 \geq 2 \geq z$

$z z z Z z-2 z z z z$

$2222 z$

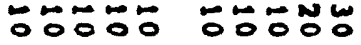

뭉ํํ유

뿌ㅇㅠㅜ

뚱부웅

5

5

0
0
$\vdots$
0
0
0
0
0

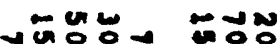

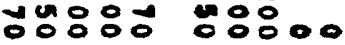

00000

- -

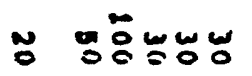

○

-

○

$z \quad z z z z$

b م

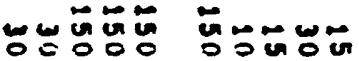

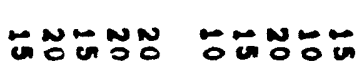

6

둥웅 5

núno

$=7$

00000 1000

0000000000

00000

00000 由 - 000000 1000 ㅜㅇㅇㅇㅠ 뚱후 둥후웅

$z 2 z=2$

Z $>\geq z$

$z z \geq z z$ ○ั0\%ั $z Z z Z z$

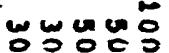
무융

WN NOE

뚱웅

S MNNN

N

RNNNN

NNNNN

$r=r$

TEE

응 듕ㅇㅇㅇㅇㅇ

NNONON

NNNNN

$z \quad z x z z$

$z \geq z z$

동융융응

Nㅡㅇ응용ㅇㅇ

$x \geq z z z$

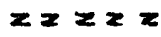

NNNNN

NNNNN

NNNNN COCOC

00000

NNNNN

z. $z 2 z$

$z z z z$

$z z z z z$

$x=2 x$

영등등융영

두웅

뜽둥ㅇㅇ

동영응웅영

홍ㅎ:웅

$z \geq z z z$

$z z \geq z z$

$z \geq z z z$

$z z z z z$

- 00000

00000

00000

00000

00000 10000

$\infty-00$

1000

由 =

a nunau

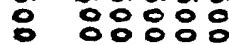

unun

unumu

unuma

응ㅇㅇㅇㅇㅇㅇ

응영융

웅영ㅇㅇㅇㅇㅇ

unuma

z $z=2 z=$ 


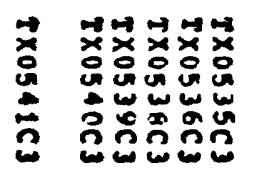

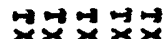

$\times \times \times \times x$

in unin

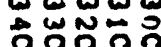

ह

- 0000

- or o 0

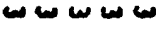

सू.

NNNNN

况ニニム

กํำ

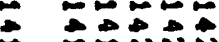

$\therefore \infty-\infty$ $\because A \div E=$

$=$ 6.0\%0 $\therefore=0$ WNW

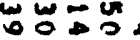

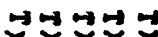
종중중훙 NNNNN 范 ถุํำ

anao ט NNENN ○以

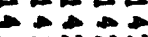
un Nolon

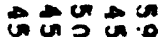

펴버법 중정중중 unum 造向 ถัก๊ร์

ono o o w w w $\because \approx N-N$ - 里 Nón

$\triangle \therefore \rightarrow=$ $\triangle N=$

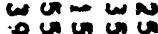

bun

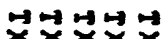
정중중

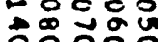
ลำลำ - 000 ט - NON N

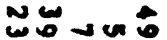

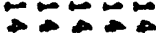
NNNA

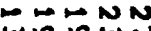

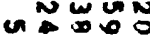

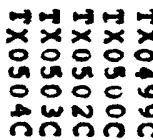

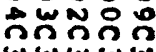

- o. 00 แผ w w NNNN ט

\section{$\vec{x} \vec{x} \dot{x} \vec{x} \dot{x} \vec{x}$ 융ㅇㅇㅇㅇำ}

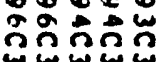

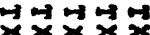
중ㅇㅇㅇㅇㅇㅇㅇㅇㅇㅇ 등ํㅇ

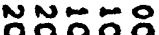
กำก

0000

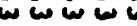
두웅 NNNNN סָ

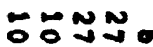

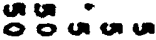

$=\infty+\infty$

مח مN

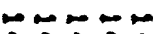
แล

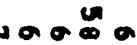
NNNNA แ - $-N N \div$ ט. N - ONNE

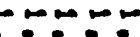
$\therefore 0000$ $z x \geq z$

00000

$z x \geq 2 z$

ロேー 00000

00000

$z x \geq 2 z$

$z z z z z$

NNNNN

NNNNN

NNNNN

$x \geq 2 \geq 2$

$22 \geq 22$

NNNNN NNOENn

N NONNN

NNONONO

03000

NNNNN

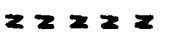

$\geq 2 \geq 2$

NNNNN

$=2=2$

NONONO

$z z 2 x z$

둥 눙ํํ

무ㅇㅠㅜㅇ

- 으유

두웅

CEC

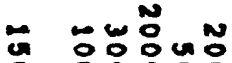

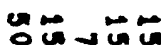
EE EE

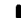

c

무우

$2 x 22=$

NWN ํํㅇㅇㅇㅇㅇㅇ

$0 N=0$ 영영응



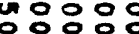

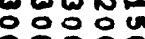
0000

$-\infty-\infty$

:0:0:

$\leftarrow \infty \div$

00000

$z x \geq z z$

$2 \pi 2 \pi$

UNNNN

NNNNN

$Z Z Z Z Z Z Z Z Z$

부웅

NNNNN

NONON

$z x \geq 2 x$

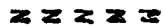

ONOOOO

5

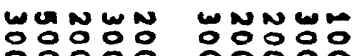

융ㅇㅇㅇㅇㅛ

둔

مー

$\sim-\infty \sim$

-

\%ำ re

ำง

- 00000

00000 - 0000 ㅜㅇ웅 $z z z=$ 10000 후웅 z $x \geq 2$

00000 由 두웅 $x \geq x=$

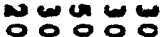

W\%

Nㅜㅇำ $x$

00000 00000 둥하 $z z z z$

W O

- MNㅜ웅 $z=$

Nㅠ용ㅇㅇㅇ $z=2 z$

NUNN z $z=2$

NONON $z=$

웅 $0800 \%$

$z \geq z=$

옹형유

$z z z=$

00000

00000 - 000

$F=00$ ununu 응ㅇㅇㅇ영

num un 응ㅇㅇㅇㅇㅇㅇ

$x=z$

$z z z=$
00000

Nํㅜㅇ

$=1$

NNNNNN

$z x=2$

NNNÖN

$x$

둥ㅇㅇㅇ ㄷㅇㅇㅇㅇㅇㅇㅇㅇ

$z \geq \geq z=$ 0000

00000 由ण口儿

00000 -

00000 00000

00000 - 000 0000

unun

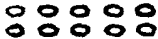

- ununu

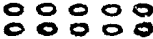

oñún 응응응 $x \geq x x$

NONO -

NNNNN 응융ㅇㅇ $x \geq z x=$ 훙

Nㅇㅇ융 ONO유 ON $z=z Z \quad z Z z=z$

둥응융융

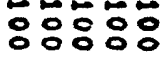
$z=z=$ $x=2 x$

vinunu unuma

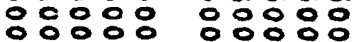
$x \geq \geq x$

$z x z z y z x z=$
00000
00000

00000 1000 $-\infty$ مே0\%ั $z \geq z z=$

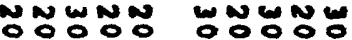<smiles>C[14CH]=[14CH]</smiles><smiles>[CH][CH]</smiles><smiles>[C+]1[C+]C=C1</smiles><smiles>[CH]1[CH]C=C1</smiles>
$n 5.5$ 


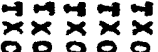

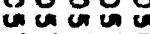

踏式

주ㅈㅓㅜ저ㅈㅓㅜ

응ㅇㅇㅇㅇ

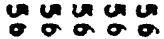

형영

วกรณก

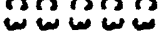

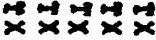

000응

जusun

กักักิิ

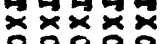

응ㅇㅇㅇㅇㅇㅇㅇㅇㅇ

unususu

wasus

และ

กล์กล

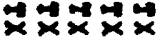

응응

ununa

जus

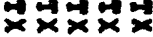

웅으운응

隹

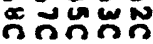

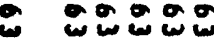

운 900

운운운

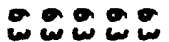

운둥요

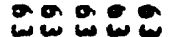

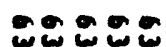

후웋

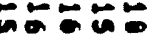

$\because \because 0$

NNNN

NNN N N

NNNNN

NNNN-

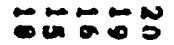

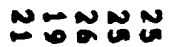

an

- uñ

- 0 -

$\therefore-0$.

은ㅇㅇ

aำ:

본

$\therefore \quad \angle E= \pm E$

$\div \div \div=$

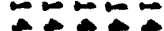

$\because ニ=\div$

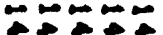

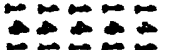

$\check{0}$

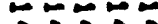
ש w

$N \rightarrow N$ N

wat

$\omega \neq \omega \omega N$

WNN

-

N

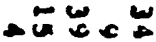

Bung

$n N$ an

$\omega \geq=\underline{c}$

ONMGN

ugang.

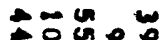

둥후

ด ธง ำ

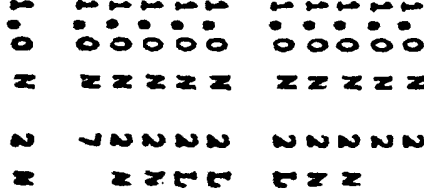

O NNNNNO

NNNNN

$x \quad 2 x \geq 2$

O 웅후웅 5

$5 \quad 5$

\%

c

ตํํㅇำ.

م-

ㅇㅇㅇㅇㅇ

$z=$

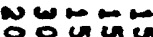

뭉둥 r $r$

- 00000

- 1000

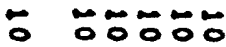

z $z z z z z$

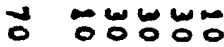
$x$

궁숭용 $F$

- NNNNN

응융유

$z \quad z Z z Z z$

CNNNN

동ㅇㅇㅇㅇㅇㅇ

$2 x 22 z$

○

NONNN

$z z z z$

응 응등융응

$x \quad z x z x$

- 00000

-

a muvuna

ㅇㅇㅇㅇㅇㅇ

$x \quad z z z z$

00000 $050 \div 0$

$z \geq z z z$

NNNNU

형등융웅

zZzZ

00000

caununu uñ
ேㅜㅇㅜㅜ웅

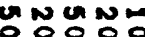

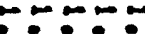

웅웅

מேー 0000

$z \geq z \geq z$

$z \geq z z z$

$\omega N \omega \omega N$

N

$z \geq z z Z$

$z z=z$

NNNN

NNNNN

00000

00000

ONNNN

$2 x 22 x$

$2222 x$

जํㅇ유유

N0유유

$\geq 2 z 2 z$

NONON

$z 2=2 x$

무융유

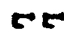

ㅜㅜ유요

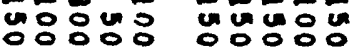

$\leftarrow N$

무유유

웅응

눙웅 0000

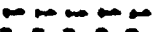
:0:0்

$z x \geq z=$

$5: 5:-$

:00\%்

$x \geq z \geq z$

NNNMN

Nㅜำ

$z \Sigma z=$

$z z z x$

NNNNN

OOOO

NNNNN

$z 2 z z=$

$z 2=22$

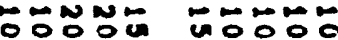

등융영영 영영영

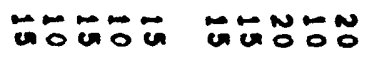

:

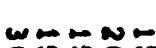

- w w

ேㅜํำ 5

0000000000

00000

00000

00000

00000

5

0000

1000

प1000

0000

1000

- 1000

05005

후웅

두웅

둥후웅

두유

$z \geq 2 \geq z$

$z z z z z$

$z z z z z$

$z=z z=$

$z z z z x$

후웅웅

$z \geq z z$

NONN

NNNNN

W

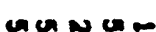

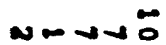

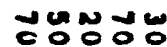

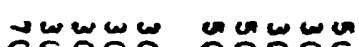

un

$\pi \rightarrow$ a com

NONNNN

응ㅇㅇㅇㅇㅠ

ㅇㅇㅇㅇㅇㅇㅇㅇ

NNNNN

$z z 2 z$

$z z z z$

응응등ㄷㅇㅇ

NNNNN

응용ㅇㅇㅇ

OOOOOOOO

$z \geq z z z$

$z z z z z$

$z \geq z z z$

NNNNN

응융ㅇㅇㅇ

$z z z z z$

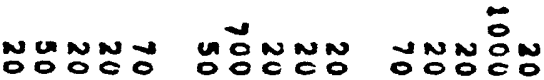

NNNNN

$z=2$

ํㅜㅇㅇㅛ

$$
2 z
$$

nx

둥융유 둥융융

루웅유

등융ㅇㅇㅇ

$z=$

ZZZZ

- 0000

00000

00000

00000

00000

00000

00000

由ण口卄0

- $=-\pi$

00 000

एष 0

-

unumur

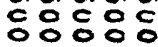

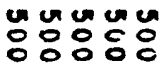

눙명영
씅ํㅕㅇํํำ

$z \geq z \geq 2$ 
or o. 0

00000

Du 0

ธับำล

Non-

4



$\rightarrow \infty+\infty$

$\because \infty$

- 눙요

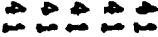
N $-\infty 0 \div 0$ พัOก:

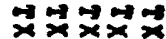
증중증 영ㅇㅇ응

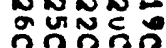
3ณ.

a 0 on o 0 w w

$+000$ 。

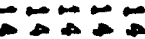

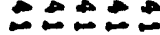
Nuس - NNEO
보벚버ㅈㅓㅜㅁㅕ 중중정 음영영 음 กิกีกักล์ ט

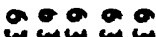
D$\omega \rightarrow U n N$ 콘

-

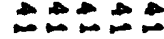
مै م $\vec{x}=\vec{x} \vec{x} \vec{x}$ 중정중

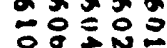

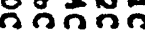

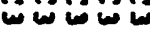

웅웅 - 0 0 â口

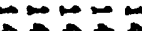
ニEニニ $-2000$ G

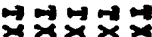
oㅇㅇㅇㅇㅇㅇ g운면 ㄷํ요은

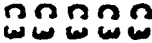

000 00 ロேニーニ

טa

$\leq N \leq 2=$

エேー

ニニシ心

$0 \rightarrow-5=$

Eேニニ

둥요

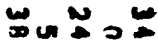

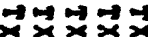
잉ㅇㅇㅇㅣ 는.

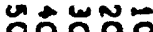
แேะष 프뭉

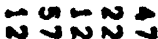

a.0.0.0

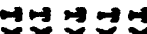
응ㅈㅇㅇ응 눈은 ¿ x๐ กรณ์?

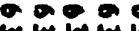

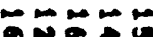

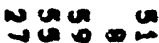

$\rightarrow-2=$ uㅢ:

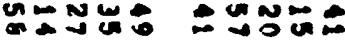

5

0 Nㅜㅇํㅇ $=2$

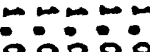

NNNNㅇㅇㅇ

XZX

NNW ํํㅇํํㅇ

NNㅜㅇ요용 응요

$z z 2 z$ $2=202$

동융융융 $x+2=$

00000 0000

ununu ㅇㅇㅇㅇㅇㅇ

$z z=2$ $z \geq z z=$

M $N N N$

$z z x z$

도융

영융둥ㅇㅇ

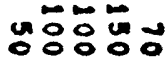

$\because 5: \div$

00000
$z z z z$

NNNNN

$\geq X \geq 2$

NNNNON

$42=2 z$

ํํㅇํำ

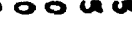

ㄸํำํำ r

Nㅕㅇ응녕융

-m心 $\therefore 0: 00$ $z z z z=$

NNNNN

$z \quad x=2$

ONOZONO

$z=2 z$

$22<22$

ㄷํำั

50505

to

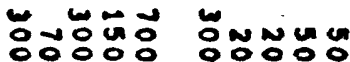
$=$

$\because 5 \div 0$

0000000000 - 00000000 $=2 \pi=$ $\because 0$ 웅

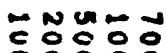

00000

ununca
ேேேேே

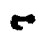

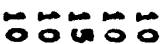

$=25$ Nํㅇํํ유

$=0$

$0+\infty$

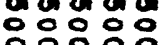

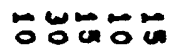
5

00000 0000 훙ㅎㅇ

$z \geq z z z$

후웅 $z x z=$

4

\%ำำ .

NNNNN 응ㅇㅇㅇㅇㅇㅇ $22 x 2 x$

둥 눙응 $\rightarrow \Omega$

눙응 0000

00000 00000 numun 응응ㅇㅇㅇ $z=z=$ $z=2$
00000 - 00 두웅 $z x z z x$

พே0\% $x$

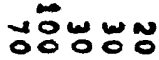

$\leftarrow$

NNNNN ㅇㅇㅇㅇㅇㅇ $z x z z z$ $\div$

눙응ํํㅇ

$0 \geq 2$

Gேㅇㅇㅇㅇㅇㅇㅠ $z=$

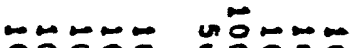
영융ㅇㅇㅇ융ㅇㅇㅇ융융 $x=x$

$x \geq z$

00000 1000

00000 0000

ununu ㅇㅇㅇㅇㅇㅇㅇㅇ

ununa 응응영응응 2

$=-\infty-\infty$ 웅응

$z z z=$

NNNNN

$z \geq z$

מ以山 $000 \%$

$x x \geq z=$

NNNNN $z Z z=$

NNNNN $z x=2$

NNNN $x 2 x 2 x$

$\circ 00 \% \circ$ $z z x z=$

NNNNN $z x 2 z=$

NONNN ZZI 2

Nㅜㅇㅠㅜ응 
Magnetic Heavy Mineral Concentrates:

Elements not looked for: SAFe, S-Mg, S-Ca, S-Mn, S-B, SABa, $S-L a, S-N b, S-S c, S-V, S-Y, S-Z r$.

Elements not analyzed: $\mathrm{AA}-\mathrm{Au}, \mathrm{Cu}, \mathrm{Pb}, \mathrm{Zn}$; INST -Hg; and CM-As.

Elements looked for but not found: SAg, S-As, S-Au, S-Bi, $s-c d, s-s b, s-2 n$.

Following elements were not detected except in the samples listed:

$$
\begin{aligned}
& \text { SHMo...... TX } 539 C 1(50 \mathrm{ppm}) \\
& \mathrm{S}-\mathrm{W} \cdot . \cdot \operatorname{TX} 543 C 1(100 \mathrm{ppm})
\end{aligned}
$$

058 



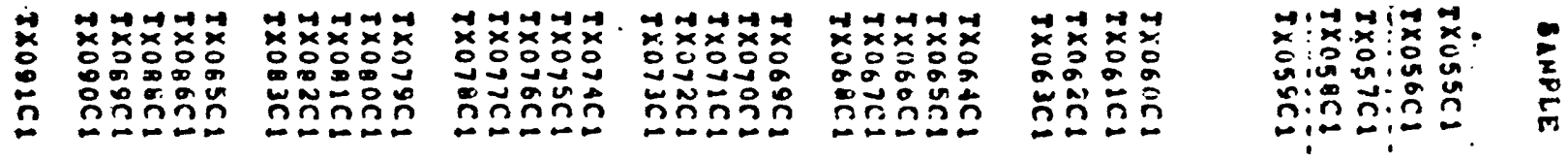

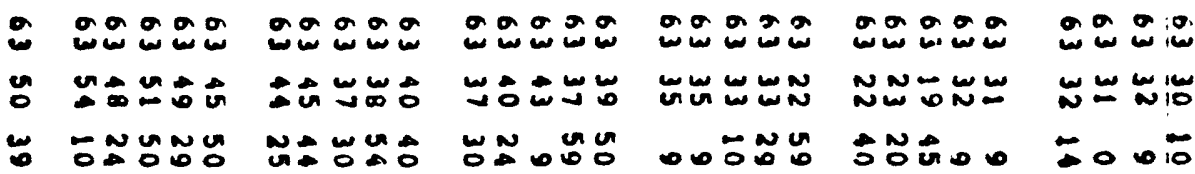

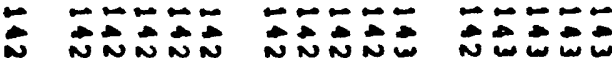

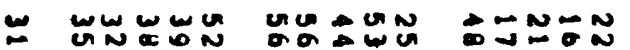
-

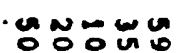

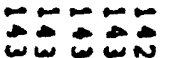
W
- Unu
モニะニニ

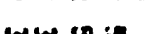

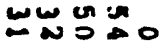

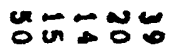
NAN 范

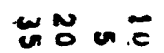

n

NNNNN NNNNN

$Z Z 2=2$

$Z \geq Z \geq Z$

NNNNN

NNNNN

NNNNN

$Z \geq Z 2 Z$

$z \geq z 2 z$

$N N \cdot N N$

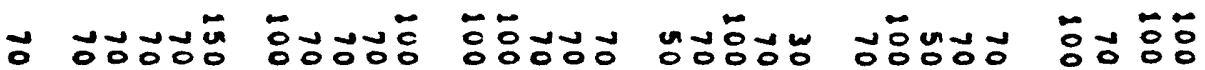

\begin{abstract}
10
\end{abstract}

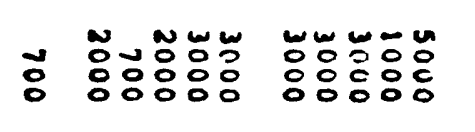

ungung an

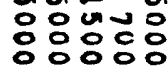

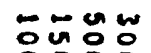

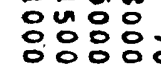

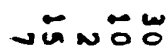

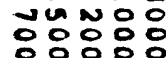

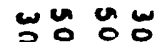

:융: $: 0$

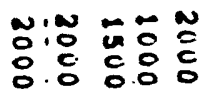

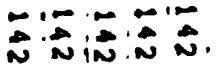

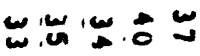

N:N:

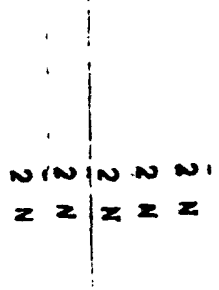

$\overrightarrow{0} \overrightarrow{0} \overrightarrow{0} \overrightarrow{0} \overrightarrow{0}$

:

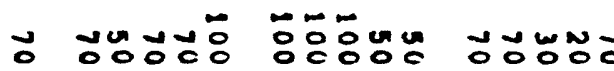

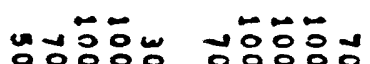

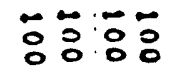

:ㄴㄷㅇㅇㅠ.

$\stackrel{n}{2}$

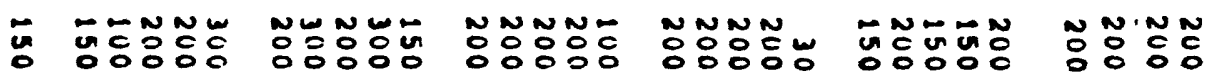

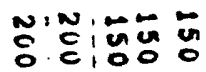

$\sum_{i}^{\infty}$

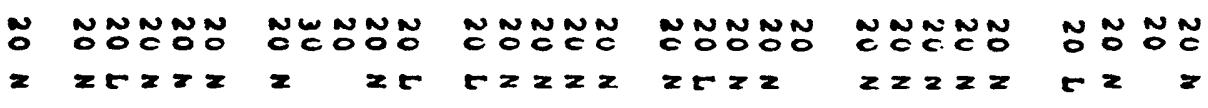

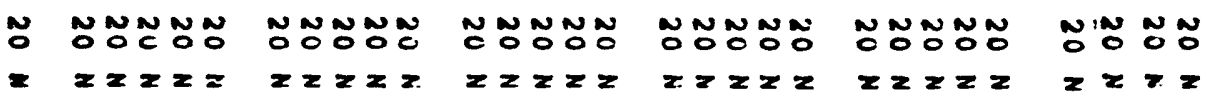

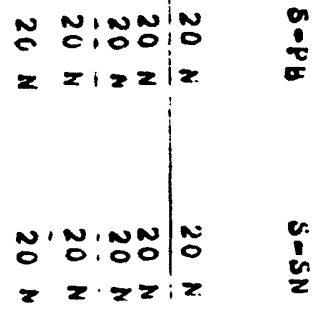

unumu ธัด: retez sununus un 동동영 $z$ $\tau$

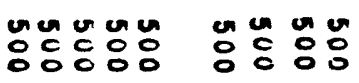

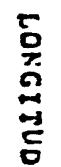

क्ष

$\frac{1}{3}$

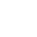


- orana

w w w w

a unu un

$\Rightarrow u \neq-6$

oroa 0 w w w un un u

Non

an on o م 6

or o o 0 ب

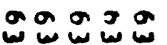

- a o a 0

00000

a a o o unu un u av v v

un un en

OU NO:

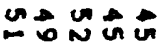

जि:-1

代出口此

No0:

$\rightarrow-1$ i 8

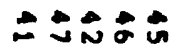

- 000

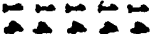

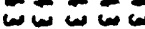
$\rightarrow G: N D$

$\rightarrow \infty+\infty$ w w ลงニั๐

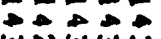
WN Noñ 해융

○พ $\square=\square$

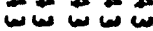

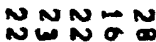
क

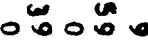

ேேロニニ むڤ屯心 NONNNND

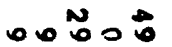

$\because \div \div \div$ むたた

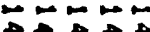
世屯出出 - NNニ ב

NAEN

O Oํำ

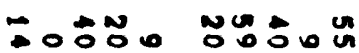

$\rightarrow \infty u$
$=2 \pi 2=$
NNNNN

$z=z$ $\sim N \backsim N N$ $z z x$
WNNNN $z \geq \geq \geq Z$
NNNNN CELZZ $\sim \sim \omega N \boldsymbol{N}$ $z z=2 z$
NNNNN NNNNN $z z z z z=z z z z$

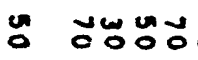

붕ำㄴำ ㅁํㅇํํำ ํํㅇํำ
붕영뭉무
붕ํํ우

둥ㅇㅇㅇㅠ 융융

응 후융

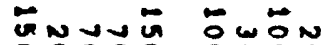
\% 용용요 O융요

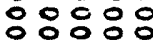

ํํำ 뭉ㅇㅇㅇㅇㅇ웅

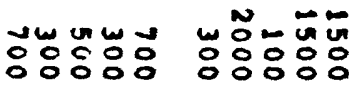

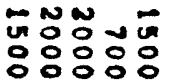

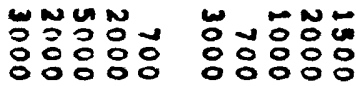

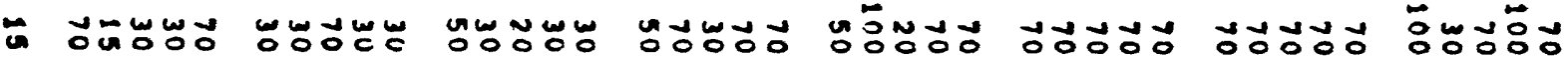

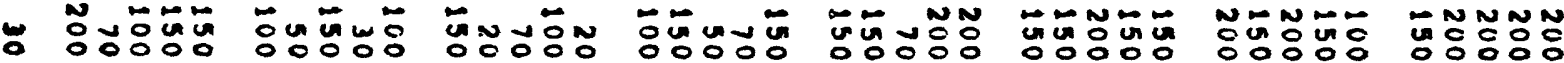

- NNNNE NNNNN

- trat
NNN ONN

$F \geq 2=$
NONOO

$r z 2 z=$
ONNNN

$z$
NNOÑN

2Et $=$
NONONO

$2 x 2 t 2$
Nㅡ요요

$z=$
○

$z x z z z$ $z \geq z=2$
NNN $0 N$

$z z z z$.
NNNNN

$z z=2$
NمNOOOON

$z \geq \quad z=$
NNNONO

$z z z z$
NONNN

$z z z z$
NON $z z z z$

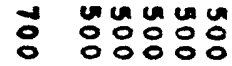

5

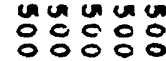

CEC unu uñ TEET

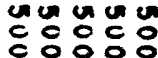
- 5 ununu ㅇㅇㅇㅇㅇㅇ

6 unumu unumu munum

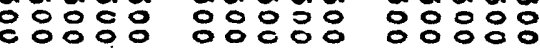

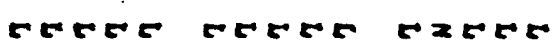




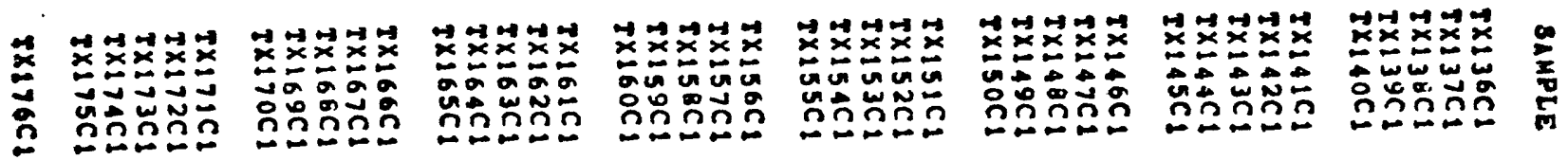

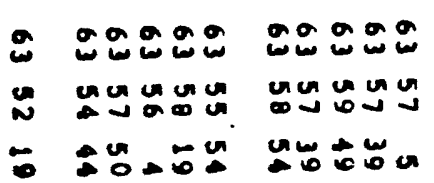

ซำ

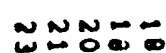

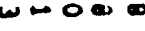

WONON

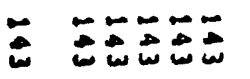

$\because-\infty-\infty$

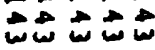

$\because \infty \div \div$

w⿻一⿻上丨

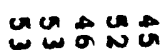

U⿺辶一

- wanu

\% a n.

$n \rightarrow N$

con un

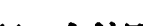

出口却政

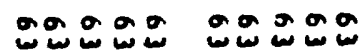

م-

שะ๐

$\because N \Rightarrow \overrightarrow{0}$ w. N oraga w

- 200

$=00=0$

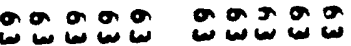
a $\rightarrow$ - n n GU I0: NONW

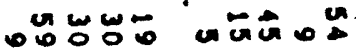

2

N $N \omega N \omega$

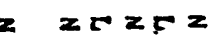

$C Z z=z$
$N \boldsymbol{N N N \boldsymbol { N }}$

$Z Z Z E Z$ $\because ニ \div \div$ Ш二屯ま $n=\cos _{\infty}^{n}$ O
ேேேこ

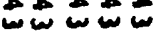

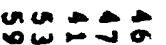

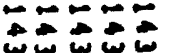

$\rightarrow$ un un u

जேवज०

$-\infty, 0$

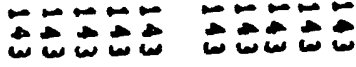

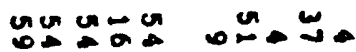

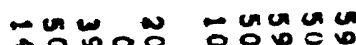

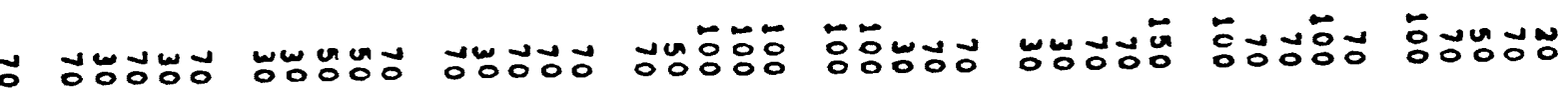

$\omega \sim \omega \sim N$

$\boldsymbol{N} \sim \boldsymbol{N} \boldsymbol{N}$

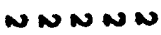

$\sim N N N$

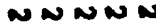

$T \geq \geq 2 \geq$

$2 \geq 2 \geq 2$

zदcz2

$z \quad z z z$

$z=\Gamma z z$ हो

ம

$n=-5$

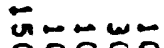

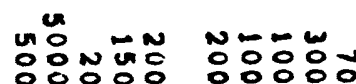

$\sim c \rightarrow N N$

.

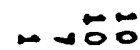

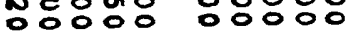
$z$

2

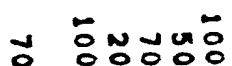

Wس

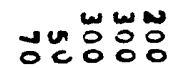

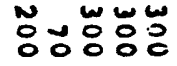

แัดั:ํํำ
둥명뭉 응ㅇㅇ
ถัดัวััดัะ

ทั่อัดัธุธั

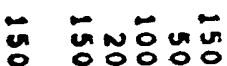
뭉ㅇㅇㅇㅇㅇㅇ

ํㅜㅇ동영

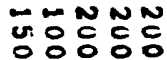

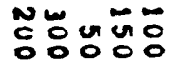

以ัธัดักั 능영융ㅇㅇ 등등 영ㄷํㅇํํㅇ
N ONNONO

$2 x+252$
NNONNON

CNETE
NลÕONO

z2 $\mathrm{E}$
พํํㅇํำ $z=1$
UO융유 NO융 $r=2=$
UMONONO

$z=2$

ํํㅇํํㅇำ 영:웅 ถุ 2

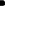


- agara

- cunuuva

- Gukñ

• oco 900 unu un un $\tan \sin 0$

$N=U \notin N$
20000

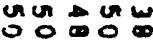
ư aso. 00 un $N \rightarrow N$ N
00090 un un w NDNOD

जि
언요

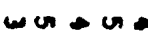

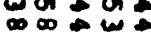

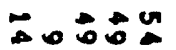

gos 000

on $\rightarrow$ un

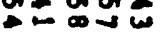

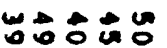

a o o 0 용

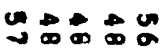

us n.

\section{N NENEA AEAEA \\ $\therefore \quad m-W=$ \\ \% 훙ำ \\ $\rightarrow$ i

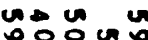

$\rightarrow \leftarrow \div=$ NNNUA

unu un:

$-\omega \omega=$

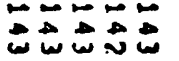

-

$\rightarrow \omega N-N$
N屯士

$\stackrel{\omega}{N}-\omega)$

$00 \div$

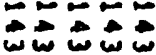

ט

مَّ

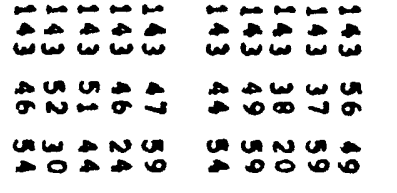

n. NNMNN NNNNN NNNNN NNNNN.

ZZZZE
$Z Z \geq Z \geq$ $z x=20$

$Z 2 \geq 25$

NNNNN

$z Z z Z$

NNNNN

NNNNN

NMNMN

$z \geq z \geq z$

$\geq \geq \geq Z \geq \geq \geq \pi=\pi$

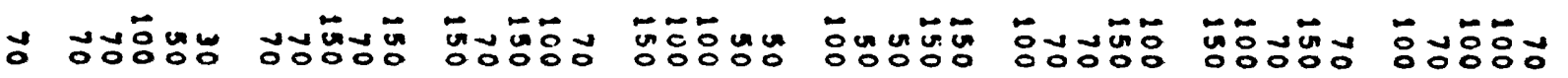

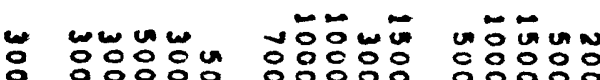

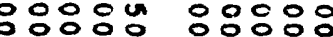

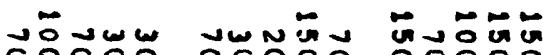

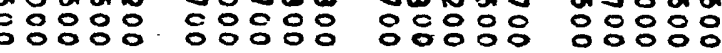

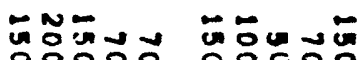

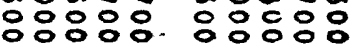

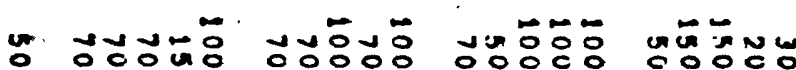

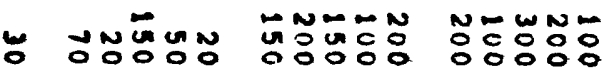

NNN

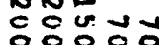

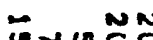

Gㄴํㅇํㅇ

NNWNN OOOOOO
COOOON OOOLOL
NNNN 응ㅇㅇㅇ

\section{NNN}

$z \quad z \geq 225$
NNNNN

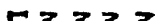

NNNNW

$z \geq z z$
ONNNO

zetre
NNNON

$z \geq t z=$
NNNON

$z \geq x \geq z$
NOO요

$2=52$
NNNNON

$2+22=$
0

\section{O NNㅠㅇㅛ \\ $z y z z z$ \\ NONNN \\ $z \geq z \geq z$ \\ CONON \\ $z x \geq z x$}

NONON

$z=2 z=$
NㅜㅇNㅇ

z Z Z I
Nㅜ용

$z=x z$
NNONNN

I $2 \geq x$
NNNNO

$z z=z$

\section{0
$\vdots$
2}

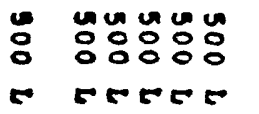

unuman

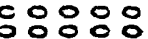

TETE unuma 등ㅇㅇㅇㅇㅇㅇ

TE

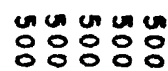
$\leftarrow$

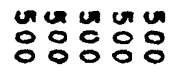

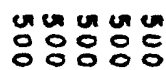

$\leftarrow$ CE 5

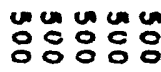

$-5$

unu un

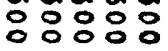

CETE 


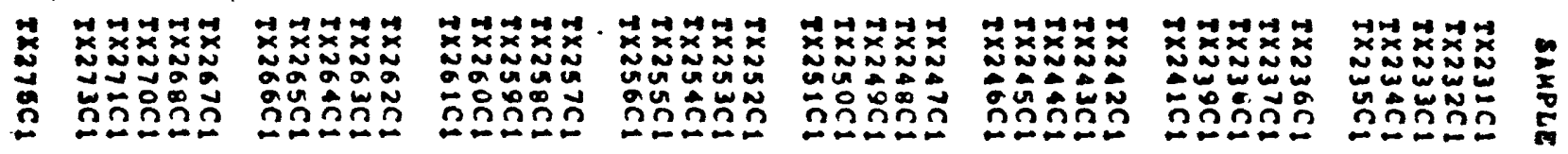

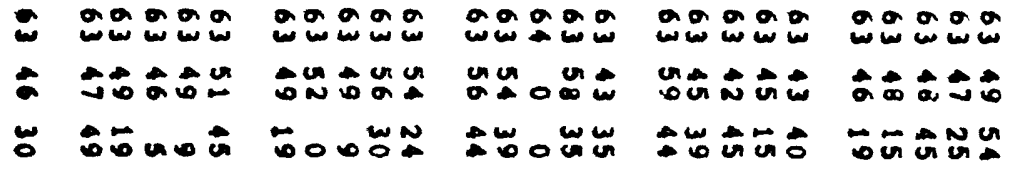

acoso

$u \in=u$ u

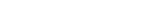

जữ

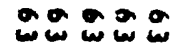

ununon

waw

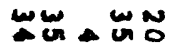

o. 0000

uncuen un 0 a 0 o

○出出告。

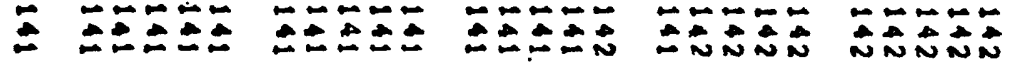 \\ or un unce

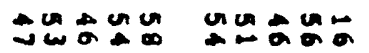 \\ unnaro

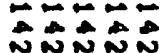 \\ $\Xi=\square=$ \\ $\because \div$ \\ $\infty \infty \propto \infty \omega$ \\ nNum \\ Non

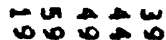 \\ ธำละ \\ NN $\overrightarrow{0}-\overrightarrow{0}$ \\ ०000 \\ DO० \\ Е๐。

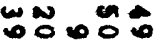 \\ 药藏。 \\ 0- Oैم \\ जि:50:}

NNNWN

N $⿻ 上 N \boldsymbol{N}$

NNNNN

งกแก

$z x \geq 2 z$

NNNNN

$z \quad z x z$

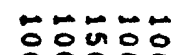

Dunun

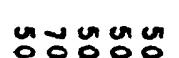

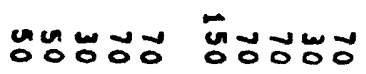

w.

옹ำ:

ธีธัธัธัช

؛̊

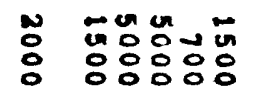

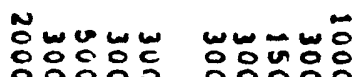

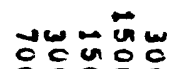

붕뭉융

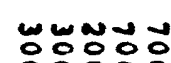

wow w

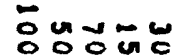

:ே

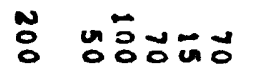

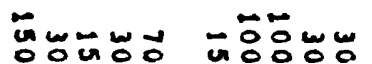

Noñ

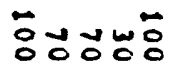

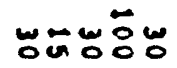

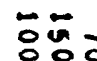

명ㅁㅇㅇㅇ

뭉ำ

$\ddot{2}$

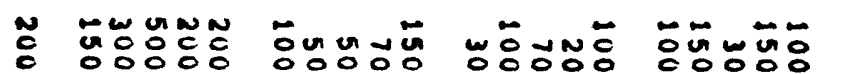

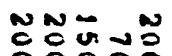

Oैพั้อัดธ

№́ñ Un

등융영

2
N DNONNN
5

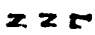
NNNNN
$22=2$

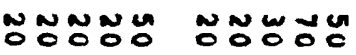
$z z z Z \quad z z$

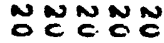
t 222

ONONONO

$r 2=2$
ENENO

$z 2 z 2$
กับONOE

- $z z z$

:

ONONONO

$z z z=x$
ONONONO

$z z z z z$
Nํㅇㅇㅇㅇㅇㅛ

$z=2 x$

\section{영 영영영:}

- TREF chunun 웅ㄷㅇㅇ융

ज下ET
농능응 $0000 \%$

nNTh ununu ㅇㅇㅇ웅융 $2=5$ ununuan un O०00: IETE
녕영영 rECEt ununu 융유 CECEt
농ํํㅇํㅇ응 응유유 Werte 
-

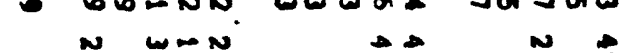
ज००००0 - wa जिए人 Nم ing a una una N⿴N ũu

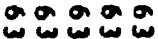
unuma ancos

$\vec{c} \div=$

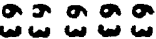

UN

or 9000

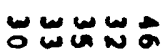
부요

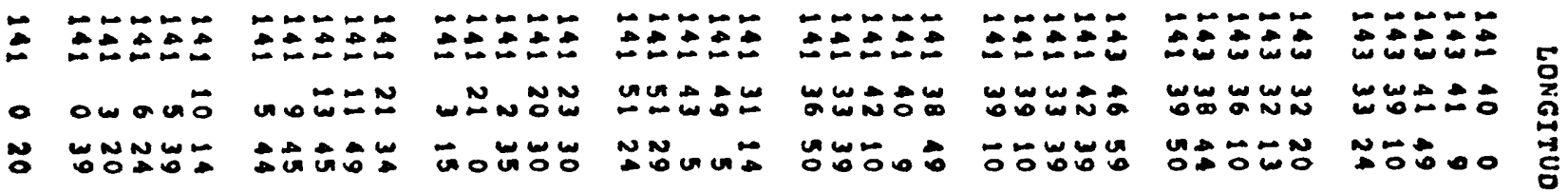

MT NNNNN NNNNN NNNNN NNNNN NNNNN NNNNN

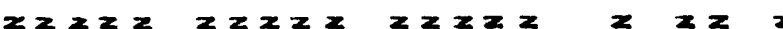
$\geq \geq \geq 2 \geq$
NNNNN :

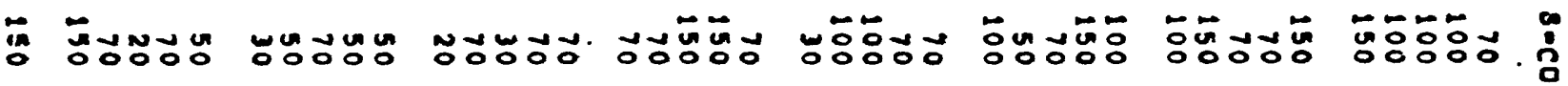

$\stackrel{8}{\circ}$

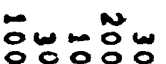
응ㅇㅇㅇㅇㅛ

जि

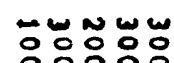

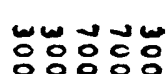
00000
ஜ 유음 r c
जิํํ융

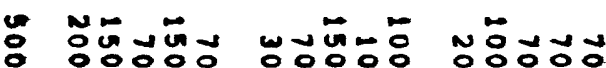
0000

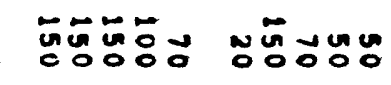

뭉ㅁㅇㅇㅇㅇㅇ

$$
\text { का }
$$

Nㅕㅇ융뭉용
능응요유
- $O O N O N N$ NONNNN NONNNN

$\geq 2 \geq 2$
22222
NONNO

$z \geq 2=$
NONNO

222
WNOZNO

$\geq z=$
NONNN

$\geq \geq \geq 2$
NONOO

$20=$ $\because$
O ONONON ONOOOOO OONONO NONOON NNNNN

$z z z z z$ $z z x z z z z z$
NONON $z \geq z \geq z$ NONONON ONOOOONO

$z z Z z=Z z Z z$

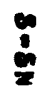

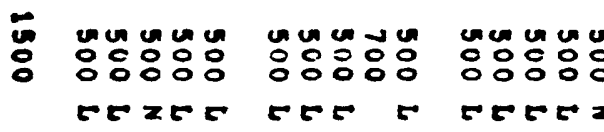

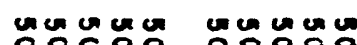

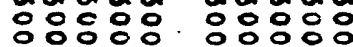
x $=5$

TEET unumu 잉ㅇㅇㅇㅇㅇ

tre 5
눙ㅇํㅇ용ㅇㅇ

ตํํㅇํㅇ 응ㅇㅇㅇㅇㅇㅇ CETCE 
0000000000000000

- 00000

- 00000

-
00000

00000

ம01000
00000

00000

- 10000
00000

00000

00000

ग0
00000 00000

00000 뚜
- In: on

uncua

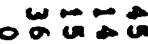
$\boldsymbol{\omega}$
00000 w w w w unuca ט ONMNON

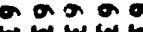
con

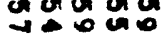
mong un 돈
- 000000000000000

000000

- 00000

-
00000

00000 $\infty x+\infty$
00000

00000

ण
00000

00000 00000

काषण
00000

00000

00000

कण

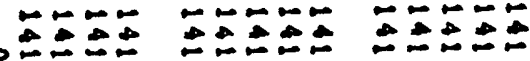

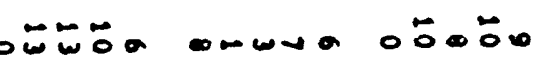

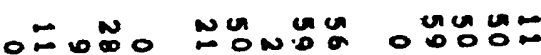
$\infty$
N NNNNN NNNNN NNNNN

$z \quad z Z z Z \quad Z Z Z Z Z \quad Z Z Z Z Z$
NNNNN

$z \geq z \geq 2$
NNNNN

$z \geq z \geq z$
NNNNN

$z \geq z \geq 2$
NNNNN

$z \geq x \geq z$ $\geq 2 x \geq x$
NNNNN

$\underset{8}{\infty}$

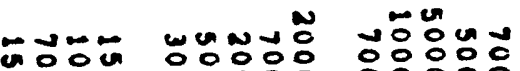

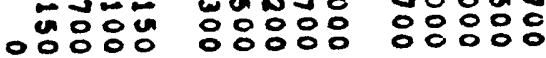
-
$\Omega$
- 000000000000000

- "10000
00000 00000
00000

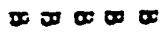

Nater

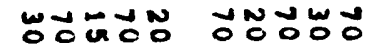

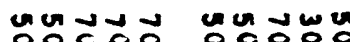

ט.

$\because 0 \div 0 \%$

wำำ

ํㅜ눙

붕ํํ유

드유유 00000
융며유

둥영영응

EัN O ONOOOOO ONNONCE

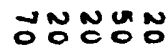

$z=$

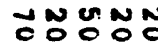

UNONON $z=$
ONONON

$z=z$
NNNONO

$z z z z$
NONON $2 z 22 z$
: NONONO

NONNN

$z z z z z$
NNNN N

$z \geq z \geq z$
NONONO

$z \geq 2 \geq 2$
NONNN $z z z z z$
NNONONO

$z \geq z 2 z$
NNONON

$z z z z$
№̛№ $z z z z z$

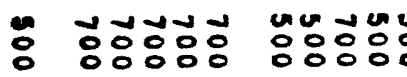

로유요

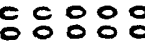

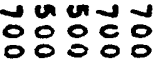

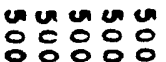
$z z$ un un un z 2 TE 2

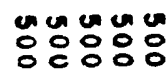
z z 2 C anuan

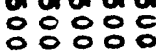
FEC $=2$ 


\begin{tabular}{|c|c|c|c|c|c|}
\hline 0 & 00000 & 00000 & 00000 & 00000 & 00000 \\
\hline$c$ & 00000 & 00000 & 00000 & 00000 & c 0 \\
\hline & $\begin{array}{l}0000 \\
0000\end{array}$ & $\begin{array}{l}0000 \\
00000\end{array}$ & $\begin{array}{l}00000 \\
00000\end{array}$ & $\begin{array}{l}00000 \\
00000\end{array}$ & 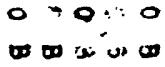 \\
\hline
\end{tabular}

\section{Na}

NNWNNN

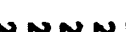

$z \geq 2 \geq 2$

NNNNN NNNNN

NNNNN

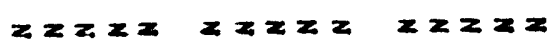

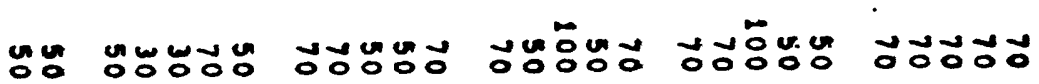
of 00000 0000

$$
\text { (1) }
$$

0000000

-

00000

00000
00000

00000

00000

$=000$

00000

0000

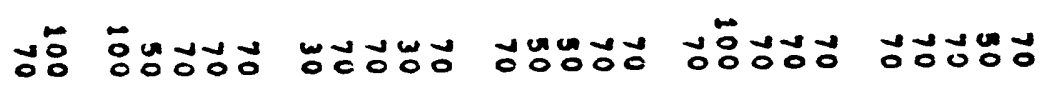

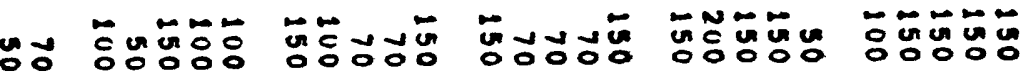

ONO UNOONON NONOOOO

NONON

Nợ:

ONONOO

$z=$

000

Oㅠ NOOO ONONON

NNONON

눙ํㅇ

NONOOO

$z x \quad x \geq z=$

$z z x z$

$z \geq \geq z$

$z \geq z=2$

$z \geq z=$ 

w 60

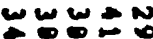
\%
운혀 WNNN مu
00000 as W世 두요 $\sim \sim N \sim N$ ה - wnu

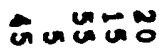

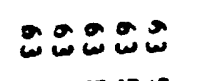

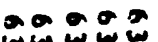
un un un

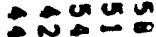

na

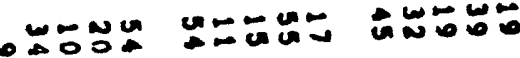

문두웅

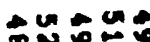

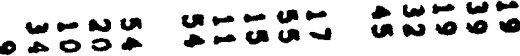

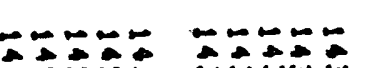
UNDEE $\triangle A B Z E$ ZN Nã

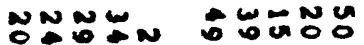

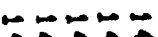

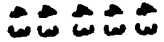

$=-\infty \div$ $N \underset{N}{N}$

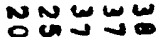
w u
$-0 \stackrel{\sim}{*} \mathbf{x}$

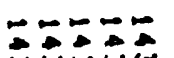

$\underset{\infty}{\infty}=$

$N N=n u$ WNNa ש ๘๐

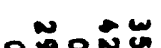

致热

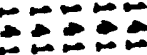
NNNNO

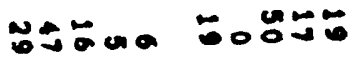

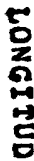
NUNNN NNNNN NNNNN NGNUN NNNNN N

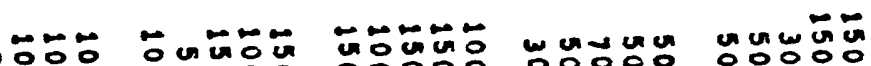

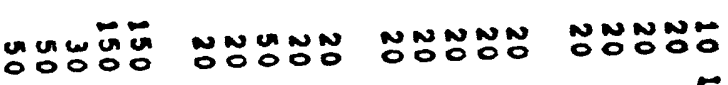

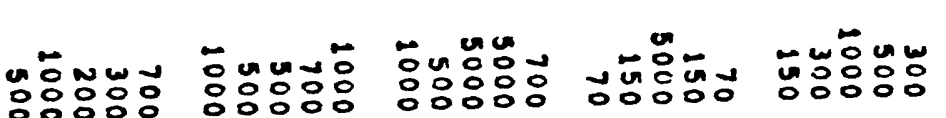
○
Uñ 용ㅇㅇ

० 0

$\therefore 0$ 응ㅇㅇㅇㅇㅛ o

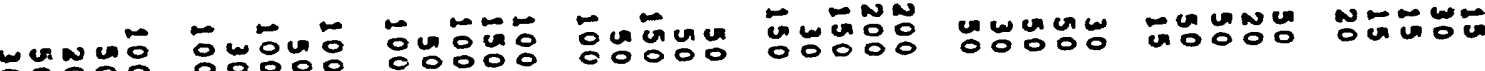

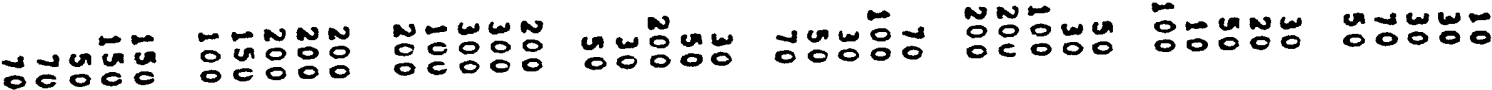

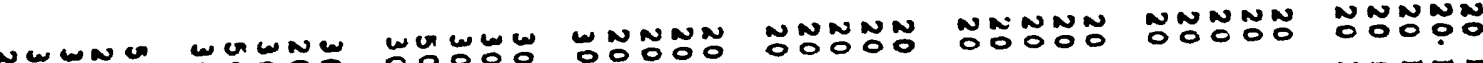

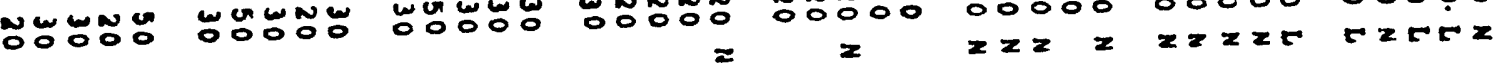

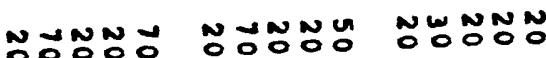
$z=z Z z=2 z=$

unumu unumu ununum

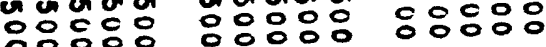
$z z z z z$
NNOOONO NONONO

NONONO NNNNN $x \geq x \geq z$

$y=2 z x$ $z z z z z 2 z z$

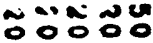

ununu c용요 $z z z z z \quad z z z z$ unumu 용ㅇㅇㅇㅛ

unumu

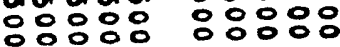
$z z z z y z z z z y z z z$ 


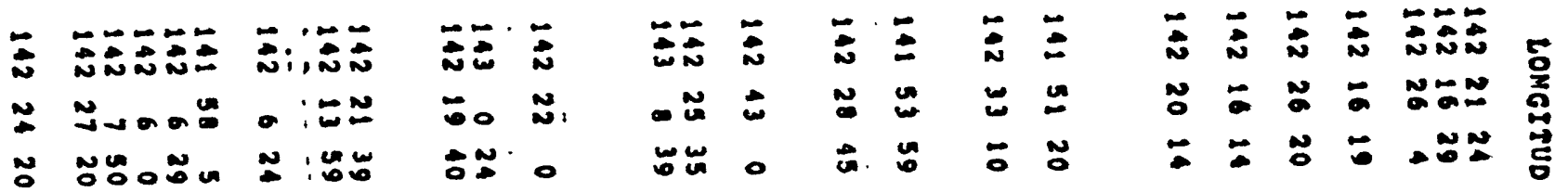

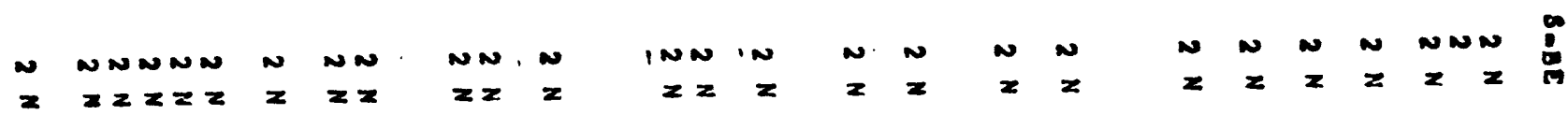

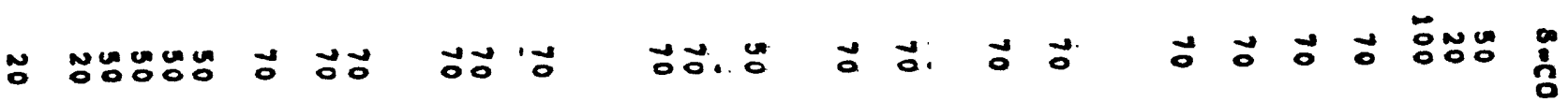

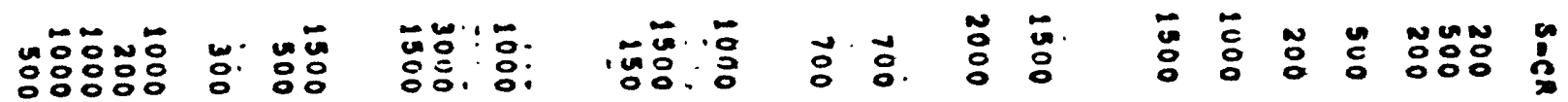

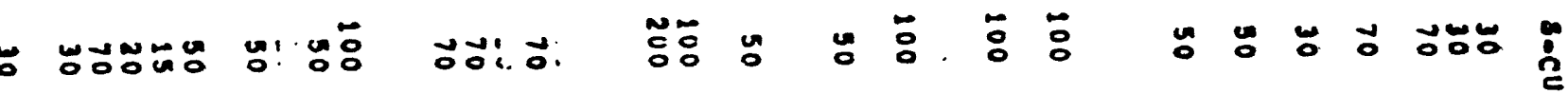

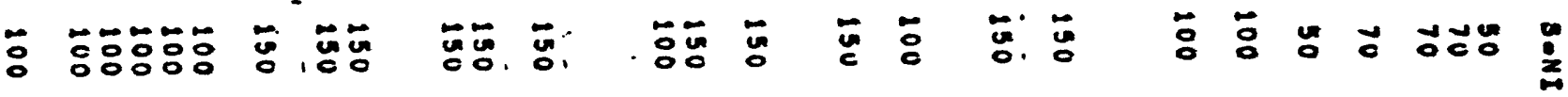

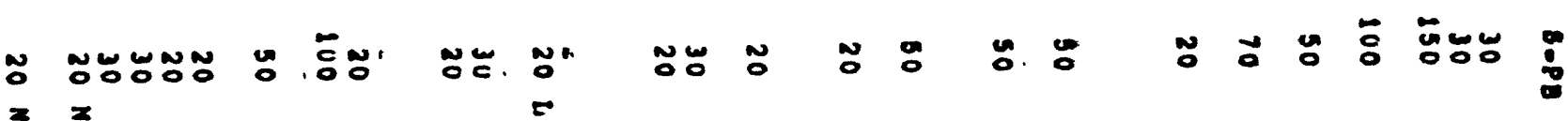

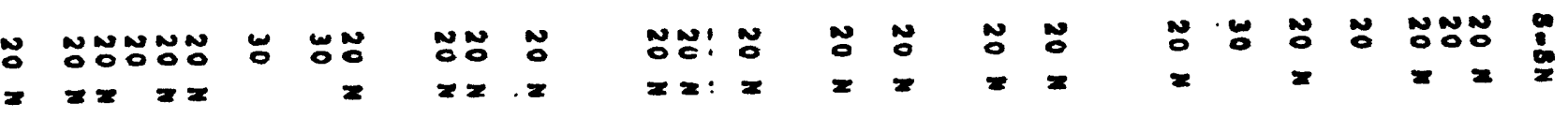

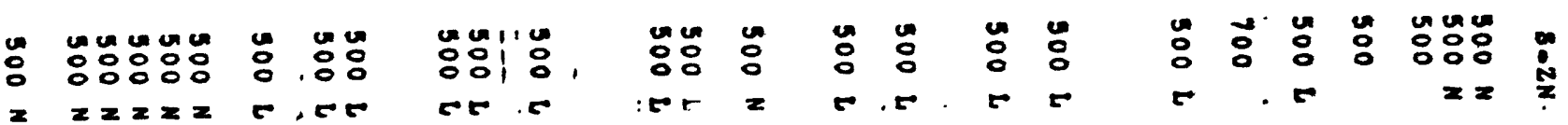
Bif 


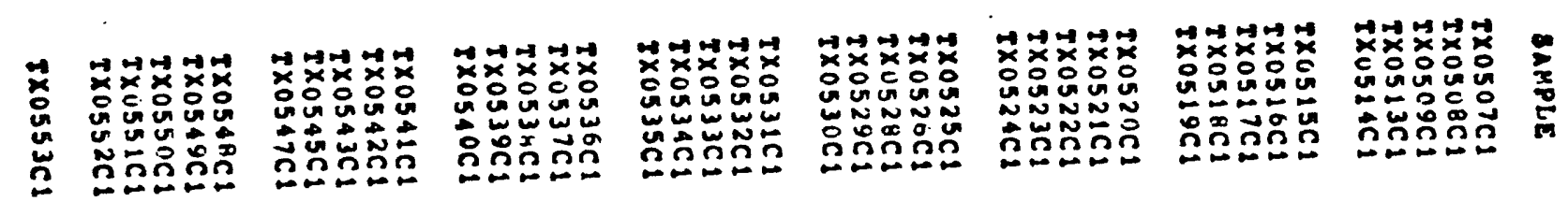

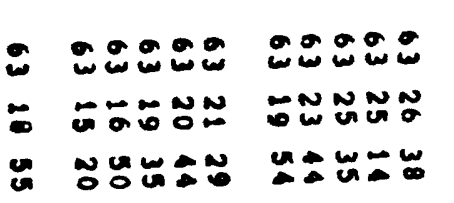

- or o

w

UN口 0

- 응

w 6

○NNNE

c $N \leftarrow \infty$ tna un

ถัก๊์
문

NNN-

-
6.

$N \leftarrow N=N$

ט

ㄴ 0
우우웅

-

uñu
운 운운 NNND

w $10 \%$

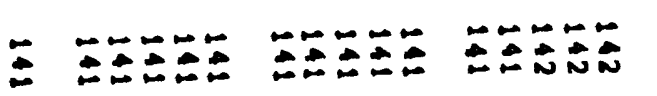

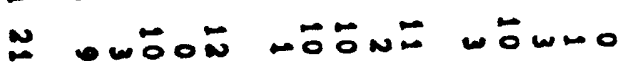

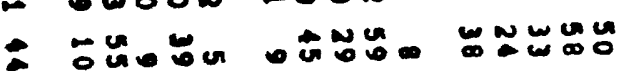

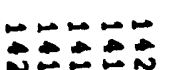

モニニニ

○蛅的

chus

$\operatorname{lno\infty }$

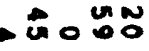

N"W:

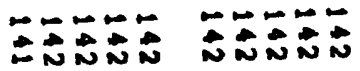

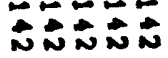

DOONA WLÑN 动ニ5

un:on

พ⿳八人口

ש

OSO

울
2
2
롱

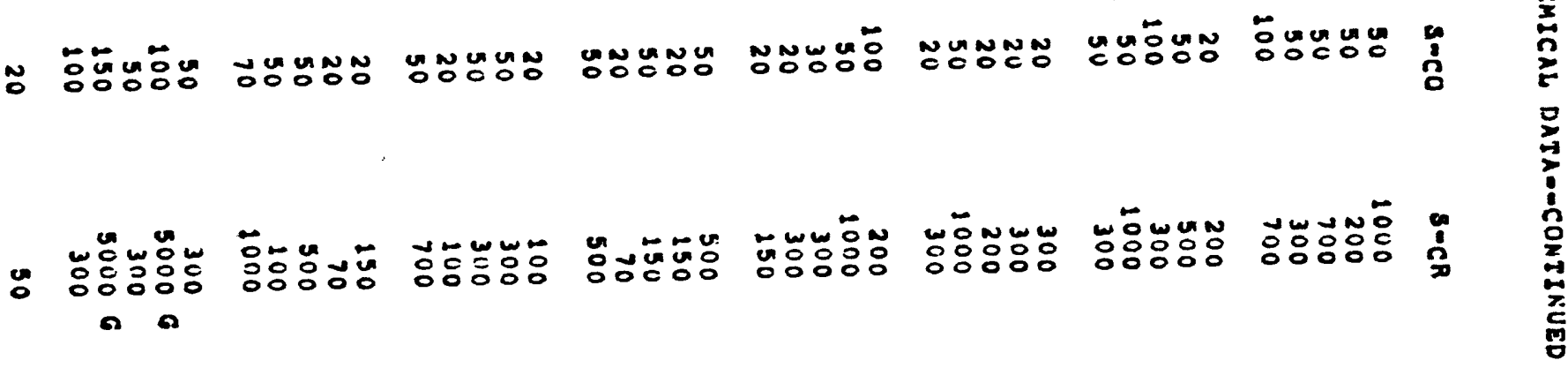

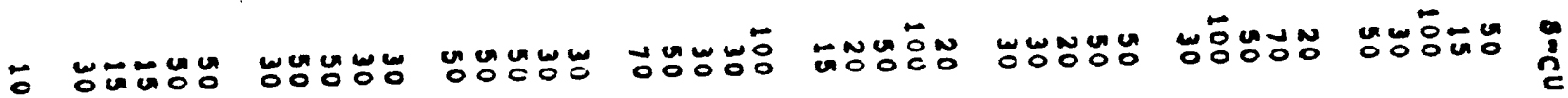

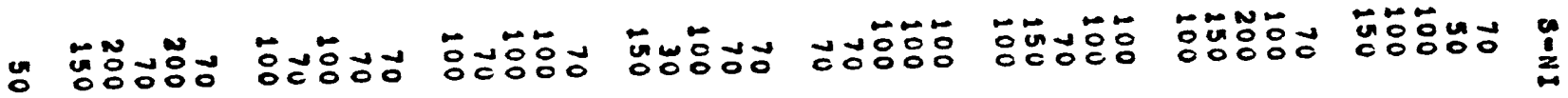

N $=25$
NNMN

$r 5$

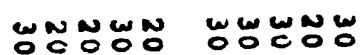

$z$

OOOONO

CE

N

$r=$

: 


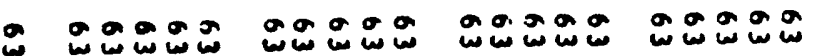

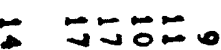

-

जேேேேே nasum

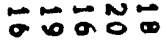

N

un $u$

N $N=$

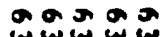

NNN

NNUN

UN
0

운운 NNN N

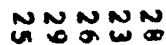
UニUNO

-

- w

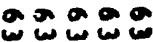
NNNND $\because=0$

$\because ே ニ ニ$

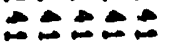

$\because ே \div \div$ 둥혀 जu 눙

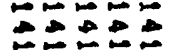

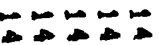
س $N \rightarrow N \omega w$ 눙ำด

م

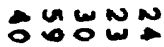

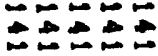
U山 UNN N U O
ேேニニミ

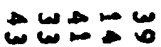
ט

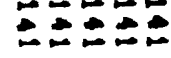
Nㅗㅇㅛ ON

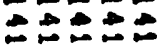

둥후

טั0 n NNNNN NNNNN NNNNN

I $\geq 2 \geq 2 x$

$z \geq x \geq 2$

$Z \geq 2 Z Z$

NNNNN

NNNNN

NNNNN

NNNNN NNNNN

$\stackrel{0}{0}$

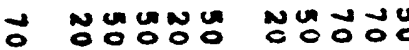

unuma

$\geq \geq \geq \geq 2$

ڤ

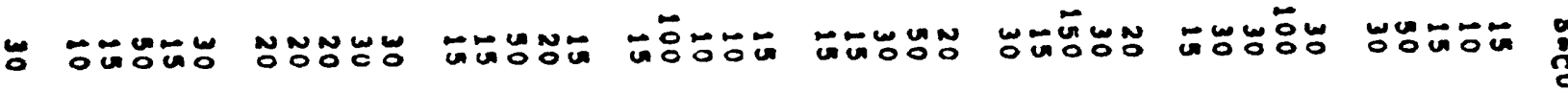
ㄷㅇㅇ유잉

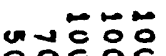

두융

ํㅜㅇำ 룽जㅇㅇㅛ 눙뭉ㅇㅇ w w ज़ํํ융

NONOOO ONONON

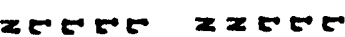

NONONON CEx $=5$

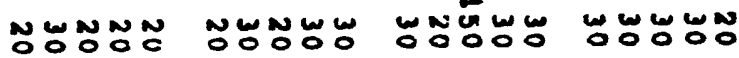
( 2
NNONON

$z=z=$
NONON

$z z x=$
NNNNN OOOOO $z \geq 2 z=$
WNONN

$z=$
NONOOONO ONONONO

$x=\geq \geq z=2$

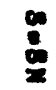




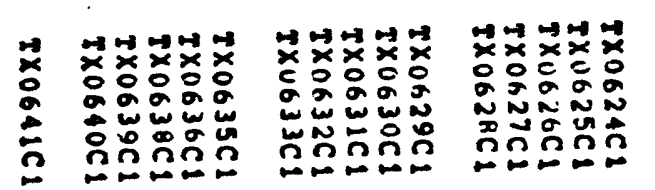

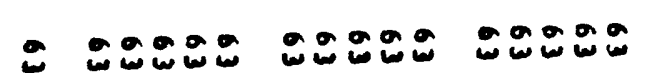

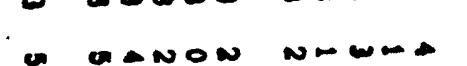

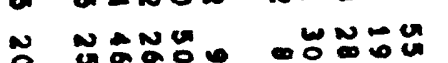

$-\infty \rightarrow \infty$

- 0000 w

10.0\%

a 000

- a 000 두

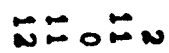
$\omega \rightarrow \infty \quad \sim$

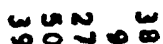

$\rightarrow 0-\infty$

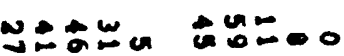

- 090

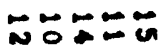
wN-
-

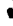

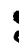

$\omega$

-

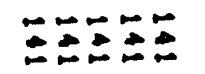

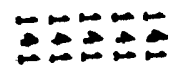

Fiar

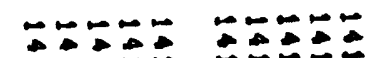

EE:

NWNN $\omega N \neq \omega N$

$\omega \omega-\omega$

an- 0

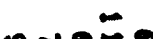

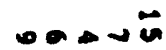

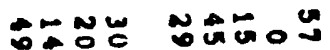

$n=\infty$

$-\infty \neq \omega$

N.

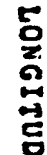

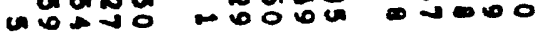

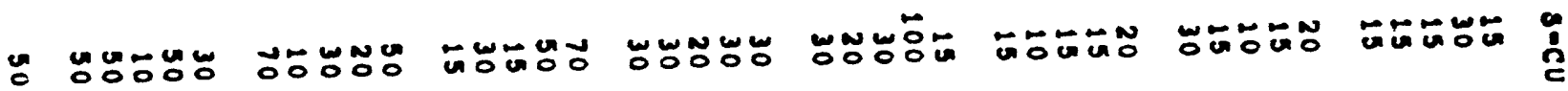

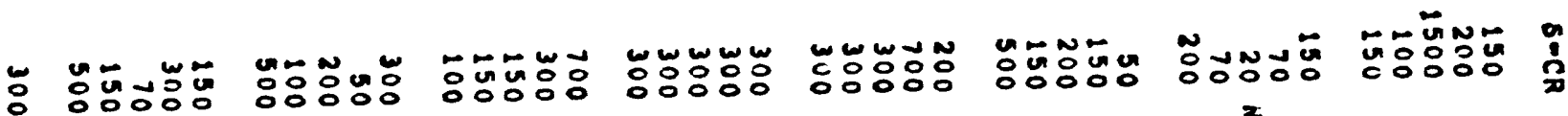

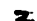

N N N $\boldsymbol{N} \boldsymbol{N}$

:

N $N N \boldsymbol{N N N} N \boldsymbol{N N N N}$

$z \geq \geq z$

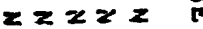

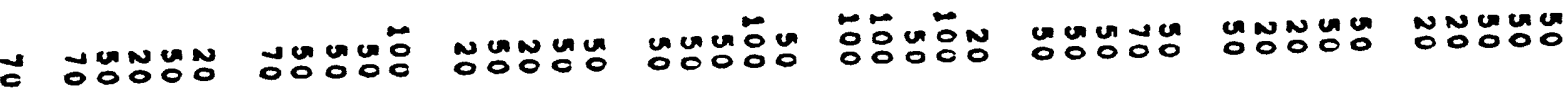


Oxide Residue:

Elements not analyzed: $A A \mathrm{Au}, \mathrm{Cu}, \mathrm{Pb}$, and $\mathrm{Zn}$; INST-Hg; and CM AS.

Elements looked for, but not found: S-Au, S-Sb. S-Cd was not detected except in samples TX 1235 (200 ppm) and TX 1245 (150 ppm). 


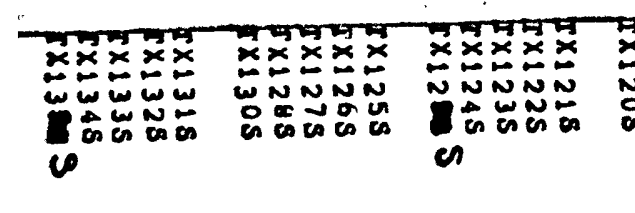

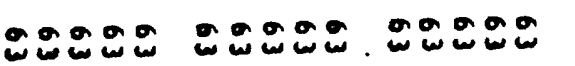
का $\omega \neq \operatorname{ung}$

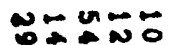
ungang cow nu un un ज̃

wagag a w un u a a w w. oc日oun $x \times x x x$

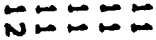

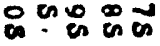

E

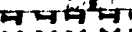
$x \times x \times x$

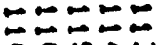

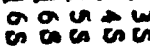
$\mathbf{\infty}$

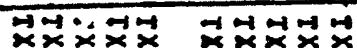
$0 \frac{0}{0} 00$ ㄷㅇㅇㅇㅇㅇ

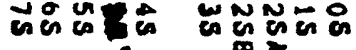
क
シேニேே

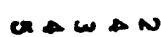

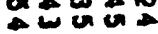
Now

OOONO

NONGNO

an

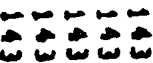

$-\infty \div=$

$\leftrightarrow \Delta \omega=\frac{0}{0}$

每 W

un

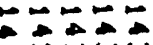

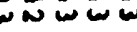

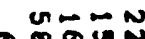

unn

जU:- $\begin{aligned} & n \\ & 0\end{aligned}$

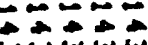
(2)

$N N N=N$

$-2=\ldots$

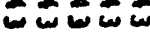
N NNNN ம - $0_{0}:$ $\because ே \div$

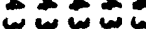
$\sim \sim \sim \sim N$

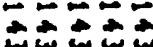

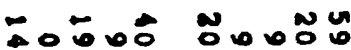

MNNNN NNNNN

- N N NN

NONNN

$N=0 N$ ลคก

$\Omega$

N N N W N

nNNND

NNNND $\therefore \circ 0: 0$ $\therefore$ in:

ำ.ำ.

0

0000.

NanN WMUMN :000: 00000 0000

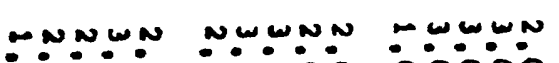
NNEN

NN

n N N N N N

W

w w w w w w w w

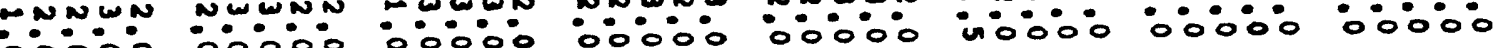
jumin

ivis

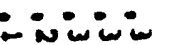

inisis

UNMND

inisin

$\ldots \ldots$

WONONO O००0。

Nㅜㅇํำ

Nمس 응영융유 영융융융

wU UnN 응ㅇㅇㅇㅇㅇㅇㅇㅇ

s

UnN Nun ㅇㅇㅇㅇㅇㅇㅇㅇㅇ

๑

a

NNG Un

응웅융응

응ㅇㅇㅇㅇㅇㅇ

a

$\Omega$

nแnแ

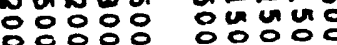

(2)

$\because \div:-$

잉우

in:000

:0:0:

$z \geq z \geq z$

00000

$z \geq z \quad z$

wuncun

영영응영

$z \geq z z=$

ununu

웅ㅇㅇㅇㅇㅛ

$z \geq z \geq z$

둔

영영영영영

5

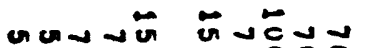
영융융 양융융 $z \geq z \geq z$

numun ㅇㅇㅇㅇㅇㅇㅇㅇㅇ $z \geq z \geq 2$

ununu 응ㅇㅇㅇㅇㅇㅇㅇㅇ $z z x z$ :0: $z \geq z z z$

unun unu

$z \geq z \geq z$

unumu 웅웅ㅇㅇㅇ

$z \geq 2 z 2$

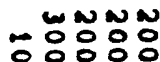

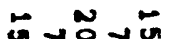

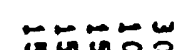

ח$-$

un

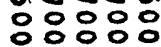

unn ơ 0 \% 잉ㅇㅇㅇㅇㅇ

$00 \%$ ?

? 00000

:

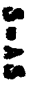
응영영융영영영 $z z z z \quad z z z z z$ $\div \div \div 0$ $z z z z z$

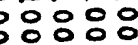

uña s. 웅영영 둥 응융ㅇㅇ

1 ㅁํำ 눙 120 응ㅇㅇㅇㅇㅇㅇ TE

- ONONO NONONON NNNN

UNNNN

N N N N

NNNNN

NNNNN

NNNNN C CE CEC TECTE

NON NNNNN NNNN N NONNN NNONN $2 x 2 z$ $z \geq z 2 z$ $z \geq z z 2$ $z \geq \geq z=$ $z z=z$ $z \geq z z=$

ㅜㅜㅇ N-N드는

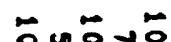
w 둥요웅 :

NWNNW NWNGN

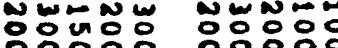
웅융요

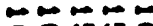

سN-

$\div W \% N$

풍궁융

:




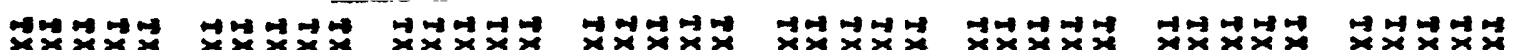
एक

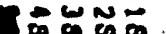
$\omega$

CMN MNERE

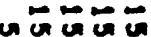

후으ํ

ษேษับ

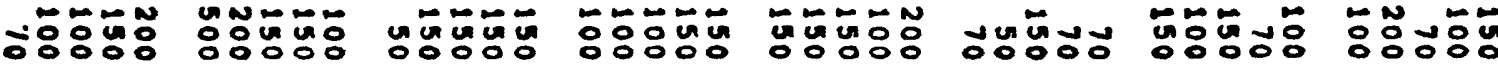

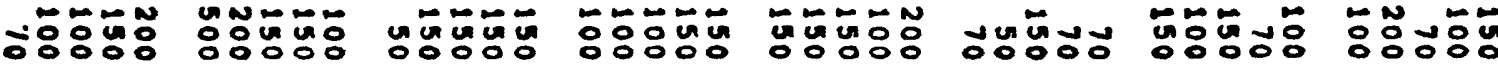

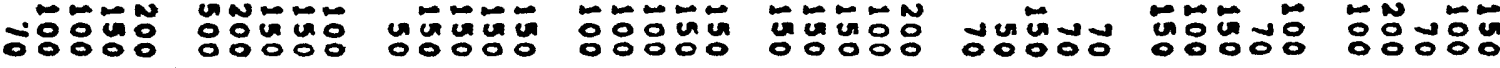

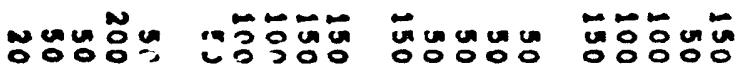

00000
00000
0

$z \geq z$

nugunga

$z 2 z$

Non 뭉ํㅇํ

무눙

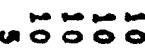

$z z z=$

nou un un

Nợug

$z \geq z=$

$z z z z$

กับข้อี

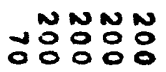

ผํํำ

$z z z$

gunuga

$z z x z z$

$-\vec{O} n N=$

Uㅇㅇㅇㅇ uñũñ

$\div 0 \div 00$

$z z z z$

onunu un

$z x z z$

n 읭ㅇㅇㅇㅇㅇ uñú o 000

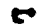

무ㅇㅠㅜ

$z z x$

uncuna

$z x z \geq z$

تே0-

जouño

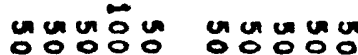
c

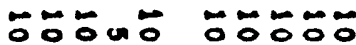

$z z x z z \quad z z z z$

UnU Uñ

z. $z \geq z z$

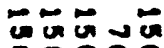

00000

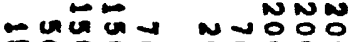

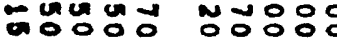
응ำ
등융유

uñ 눙ㅇㅇㅇㅇㅠ

부눙

ำํำ

뚠 . O0000

woño

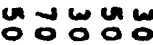

TNNN

NNWNE

MNNN
NN요

$2 z 222$

$\because$ NNNO

O.

웅ㅇㅇㅇ

NONNN

$z=2 z=$

\begin{abstract}
OOOOU
\end{abstract}
NONNN
2202
222

ONWN

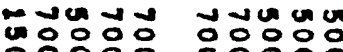

nunn
NONNN

NNONN

NNNNO

$z 2 z 2 z$

$z z z z=$

$z=2 z$

NNNN

NNNNN

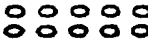

ㅇㅇㅇㅇㅇㅇㅇㅇ

NNNNN

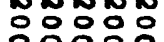
c

ununum

- ununa ㅇㅇㅇㅇㅇㅇ navuna ㅇㅇㅇㅇㅇㅇ
NONONO NNN ONO $z z x \quad z \quad z z z z$

NNNTN

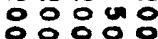
NNNNㅗ용ㅇㅇ

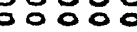

등응영융 $x \geq z \geq z$

an un

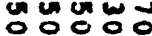
2

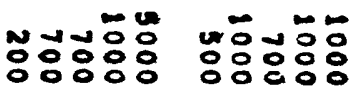

뭉

ㄴㅇㅇ영붕 잉융

a

जunvố ㅇㅇㅇㅇㅇㅇ응

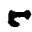

유융유 in $\because 00 \pi x$

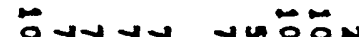

둥응영영

$z \geq z z=$

응응웅응

$x \geq 2 \times 2$

둥영응

$x \geq x \geq 2$

옹응웅웅

$2 x \geq 2=$

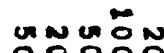

uñu

un a s

onuma 5

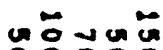
ㅇㅇㅇㅇㅇㅇㅇㅇ

뜽응중 ㅇㅇㅇㅇㅇㅇㅇㅇ

5

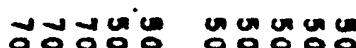
영영영 영융응

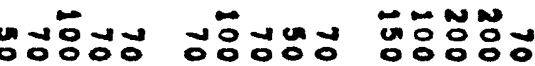

טח

$\sim \sim \sim \omega \bullet$

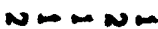

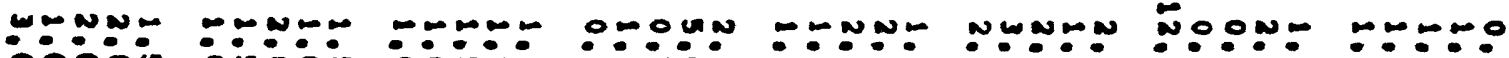

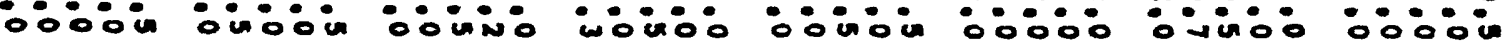

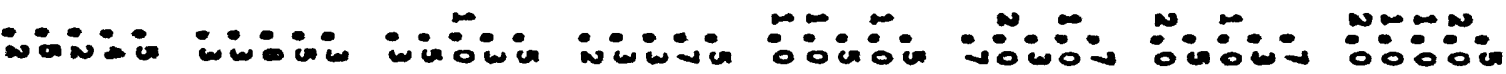




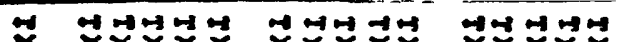

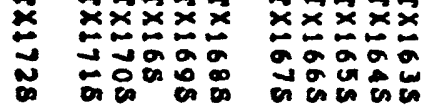
$x \times x x$ 웅후눈 웡웅.

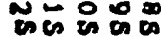

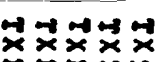
un un un

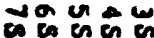

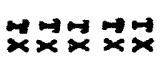

$u=\overline{0}$

on on on

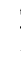

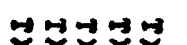

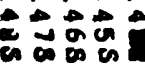
$\infty$

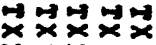

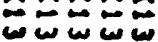
$0 \propto ⿻ 0$ os cis os on en

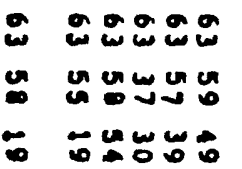

- 0000 w U日N ש o o o o o שே ANENO

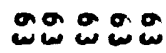

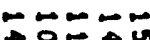

$\rightarrow 0400$

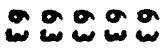

ज०

wo: -4

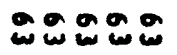

م $\infty$ a u un

c.

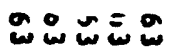

a

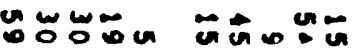

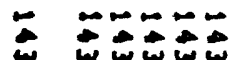

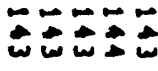

ט. No ต

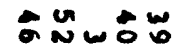

- NOONG

- NEONON

๑

NONONO

ดロan

Unn Un
-00000

$\cos 2 \pi x$

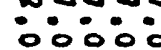

N

- ioió

NNWNN ०0:00

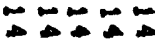

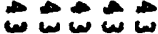
$u \operatorname{ur} \rightarrow n$ $0 \rightarrow n=0$

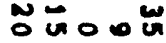

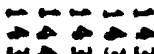
cou un 0 $\infty+\infty-\infty$ ш $\because$ ํㅜㅇ un w. जu०

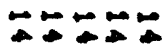

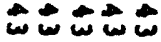
- unua $\because 0$ NO

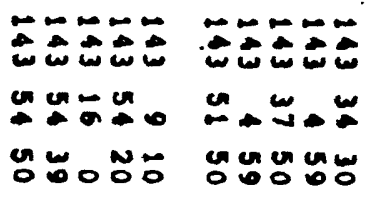

눌

ONONO NUNONO

NNNNN

anana

anana

$\Omega$

as

NNNNN OOO o

NNNNW NNNNN VNNNN :000: 0000 $\because \because 0 \%$

NNNNW :유:

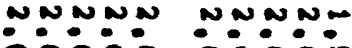
00000 0000

U m w

טw

-

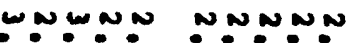
:00:0 :0:0:

00000 00000

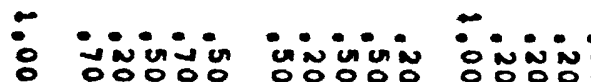

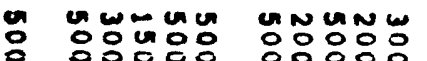

응 영융융유

ด

ค

$\because \div \div \div \div$

- 00000

$z \quad z z z z z$

a unumu

ㅇㅇㅇ영융

$z=2 z z$

: NNŬONON c

-

영 양융ㅇㅇㅇ

$\infty$

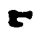

บ Nกั์

C 2

- uㅗㅇㅇㅠ

$\because 2 \div:$ 00000 $z 2 z 2 z$

unumu

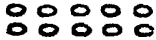

$2 \geq z=2$

UNNNN OOONO

- 2 N 응영융융유

ค

NNNNA

CE TE

TE
Unuñ 동ㅇㅇㅇㅇㅇㅇ

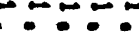
00000 $z \geq z 2 z$

nํํㄴ 응ㅇㅇㅇㅇㅇㅇㅇㅇ

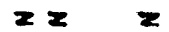

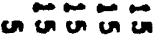
o o co

U w 귱ํㅇㅇㅇㅇㅇㅇㅇㅇㅇㅇ

wn w n

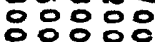

unuñ ㅇㅇㅇㅇㅇㅇㅇㅇㅇ ana

ตำ 영영응

a

$\div \div$ $z z z z z$

unvuna 응ㅇㅇㅇㅇㅇㅇ $z z=z$

Nㅜ용ㅇㅇ 잉녕ㅇㅇㅇ

$-0-\infty-$ oujo: xtryx

ununu ㅇㅇㅇㅇㅇㅇㅇㅇㅇ $222=$

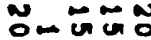
웅눙영

w w w w w w w

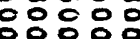

Nonu 웅ㅇㅇㅇ

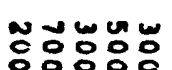

눙느

성ㅇㅇㅇㅇㅇㅇㅇㅇ

$\therefore \therefore:-$

$z z z z=$

Uunun

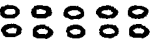

$z=z$

unuma

응웅ㅇㅇㅇㅇㅇ

$z z z z z$

numun

응웅ㅇㅇㅇ영

$z z z z$

ज药前。

NMN年

두웅

영웅응

NNNUN

nNNNO

TE $E$

CECE

NONNN

TNEN
TEN

NNNNN

NNMNN

CEE

N-ONN

NNNN

NONONO

NNONNN

Fyzzz

$z x z \geq z$

$z=2 z$

$z 2 z z$

沿䀡

พกำ

ㅇํㅇํํㅇำ
NONONON

$z \geq z x$

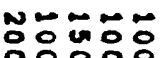

C

NONNN $z x \geq z=$

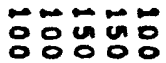

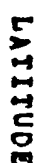


$4+44=$

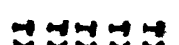

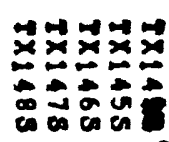

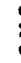

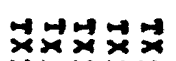

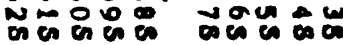

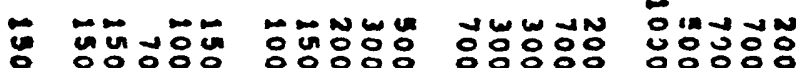

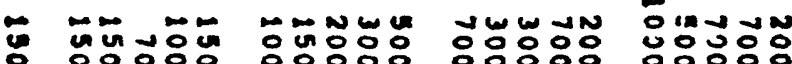

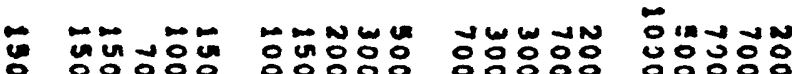

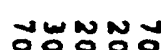

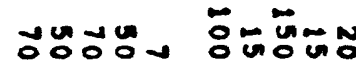

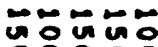

유:융

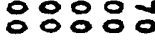

O0000

$\omega \omega \omega \omega$

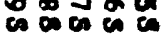

N NN NN N NNN

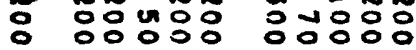
-

$\because$ ธธธธธธ $z=$

a ungungon

- Dzz

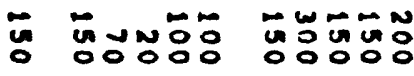

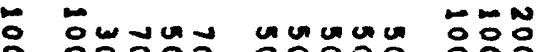

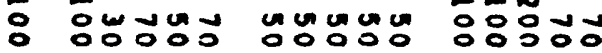

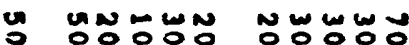

N NNNNN NNONONO

NONNONO

z $z z z z$

$z z z z$

$z \geq 2 z=$

N NWNNU

WWNN 응융유 $0: 08:$

NNNWN 융융유

NNNNN 융ㅇㅇㅇㅇㅇ r unungur $z x z z x$

unun wn ㅇㅇㅇㅇㅇㅇㅇㅇㅇ

NHNO 응ํํㅇㅇㅠ ธีธํํำ

Ū ㄴํㅇํㅇㅇㅇㅇำ

Ẽñ un un un un $x \geq 2 z 2$

Ungun un $z z z z z$

\section{unum}

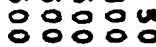

MnW

OOOOO

NNONONO NONONONO

NNㅇㅇ응

$z \quad 2 x=$

2222

NNONONO

$2 \geq 2 \geq 2$

NNNOOON

$\geq 2222$

NDNNN nNNWT

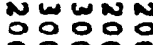

nNNNA 응유융 $+$

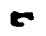

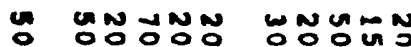

nNnNN

nNNNU 응응융

แONㅇㅇㅇㅇ

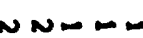
$\therefore:: ㅇ ㅛ$

unnu un

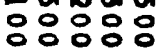

unumat 응융ㅇㅇㅇㅇㅠ $\frac{0.000}{10}$

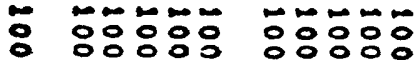
둥흉융 형융훙 뭉융융

$z z z z z z$

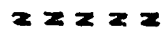
$z z \geq 2 z$ 22222 $z \geq 2 x x$

ํํㅇำ

$z \geq z \geq z$

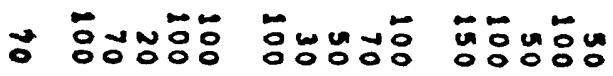
unvos: unauㅗㅇ ज.:

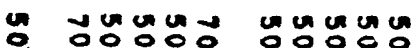
고융유유

드응

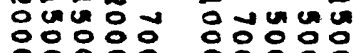

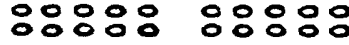

जטuี 영융ㅇㅇ

un 00000 5

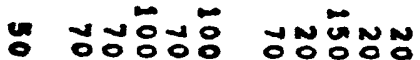
$z x z$

NON NON

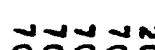

$\operatorname{mon}$

viting $z=x$ $=\approx$

* NNMNON NNNMN ONNNN NONNN NNNNN -

O-nNm $000 \div 0$ -

O0:0:0 :in:0:

$\omega \omega \omega N=$ ○:

$\ddot{0-\infty}$ 응영ㅇㅇㅇ $x \geq x \geq z$

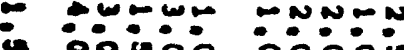

$\omega$

Nonow

UNO O०0:

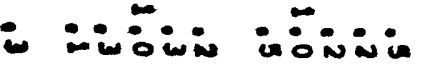

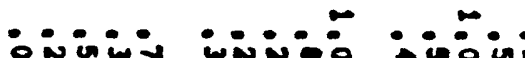
-

inis:

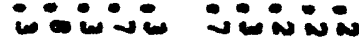




\section{- a웅aㅇ

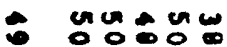

* Gun $-\infty$ asoso

$u \leqslant 0 u$

NOB ww宓

unum

등․․

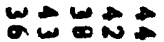

$\because \mathfrak{0}=0$
두요요

$\omega \neq+\omega=$

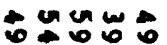

or o o o o

in $A$ is

○以on is on a

두웅

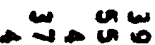

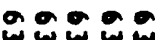
ununu $\rightarrow$ तो 0 $\because \div \div \div$ NลNล 눈 - $\therefore \rightarrow \div$ W $\rightarrow$ A

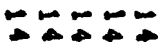
Nลิ uño. $\rightarrow$ a U.ONE $\because=-\infty$

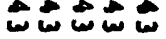
ס ש

W $W N u$ a

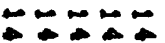

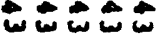
* ०0\%
둔 $a \rightarrow \infty \rightarrow$

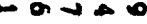
$\therefore \div$ ㅇํㅇ
ேㅜ둔

w

मि-

$-\infty=\infty$ 山ث onuma : wa n

- ONNNN ana

$\pi$ annam

- 00000

- núnan

- 0 :0\%

- invinisi

- núoúcó

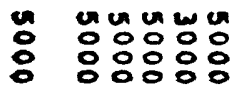

a aลa

$\because: 00: 0$

$z \quad z \geq z z z$

: 붕ㅁㅇㅇㅇㅇㅇㅇ

$z \quad z \geq z z=$

O N NONNN

$z=2 z$

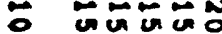

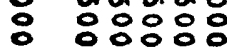

$\sim$ NUNN

- nunaner C 5

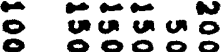

- vmun

- 8 ㅇㅇㅇ영
ENOUONON NENOOO

a

unn mu

$\therefore: 0.0$

NNmN-

$\because 0: \cdots$

N-N?N

runna $\therefore: 0$.

nan

$\therefore: 0$

Unu

:0:0.

andan

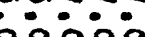

- canuna

:0.0

NNONONO

WNONON

a

unuma $\therefore 0: 00$

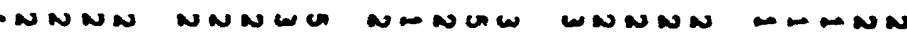

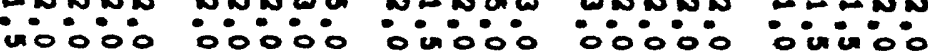

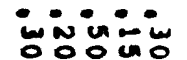

$\operatorname{ung} \cos$

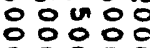

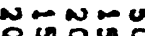

oñon

WNONONO

응ㅇㅇㅇㅇㅇㅇㅇㅇ

WO

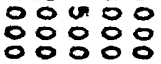

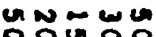

응영응

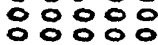

NMN

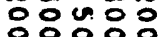
ㅇㅇㅇㅇㅇㅇㅇㅇㅇ 0

\section{$\div \div \div \div$ 00000}

$z \geq z z z$

$\div: \div \div \div 0$

$z \geq z z x$

unumu ㅇㅇㅇㅇㅇㅇㅇㅇ $z z \geq z z$

unununun 영영응

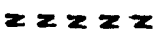

NNŨNN EE

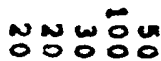
5

부

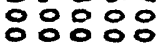

둔 ㅇㅇㅇㅇㅇㅇㅇㅇㅇ
누응ㅎㅇ웅

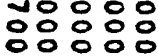

$z z z z$

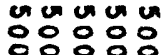
$z z \geq z=$

NNNNN OOOCO NOUNN NO

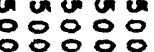

영융영융

$z x z z z$

NNNNN -

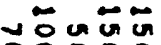

1050 뎡용요

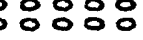
00000
뭉유 $z z z z$

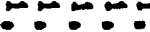
00000 $z z z z z$

unanu 응영영응 $z 2 z=2$

unumu 옹ㅇㅇㅇㅇㅇ $z \geq z z z$ Nㅡㅇ유유 NONONO te

둥ำ

분두웅

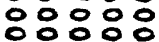

NNNNN TETER N CEXE

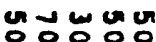

N N N N N 5 C NNNO゚N C T

Unum:
NNNNN 5

NNNNN EE $C$

wañ
NNNNN $\cot$

NN CEITE

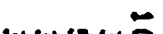
०0ํㅇㅇㅇ
NNNNN

हECE

-

NNNNN NWOKNW

CETE

wan un 00000 


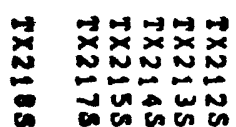

$\vec{x} \vec{x} \vec{x} \vec{x} \vec{x}$

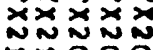
$=0$ 잉 $r$

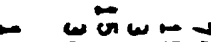

- 으음

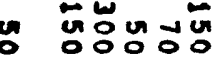

จุดับงที่ $\leftarrow$

두유:

$z \geq z$

\& gununu

$x \quad z z z z$

- HบnNN

ด ตับำำ

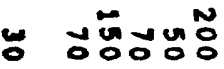

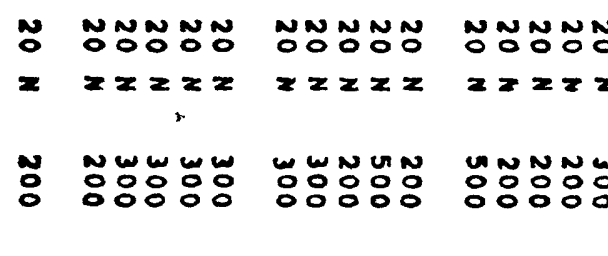

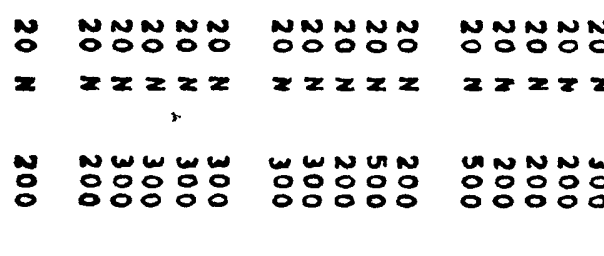

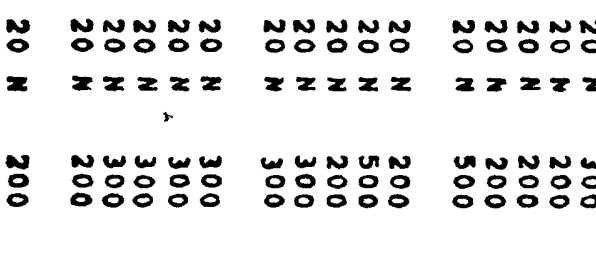

-

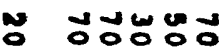

5

- uonua

- 눙영용ㅇㅇ

ununa

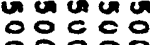
5

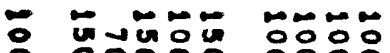

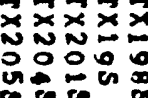

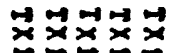
ஸे $\rightarrow$ U $\rightarrow$ W

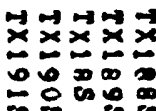

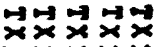
(

Den en un

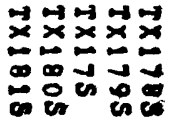

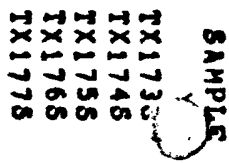

ज勿的落

牙的电 잉ㅇㅇ

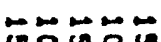

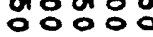

ธேே ㅇㅇㅇ영융

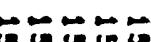

ỡo
뭉붕요

$$
5
$$

00000

$z \geq z z z$

Unu un un

tr z z z z

$\div-50$

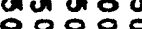
o 000 응

무우웅

$z \geq z z z$

ung:日uำ

$z z z x=$

जnota w

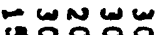
U 000

NOONON NNNNNN $222 x$

$x \geq 2 \geq 2$

NNNWN 융ㅇㅇㅇㅇㅇㅇㅠ

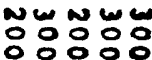

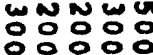

뭉ㅎㅇ

$z z z z z$

yoúc: ú

$z \geq \geq 25$

$\infty 0^{2}$ nusucu

NNNNN
웅

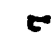

훙ํํ유

$z z x$

cuncúá

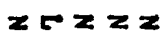

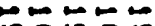

둥읭ㅇㅇㅇ
ேํํำ $z=z$

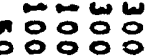

noño:

$x \geq 25$

:

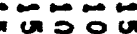
00000

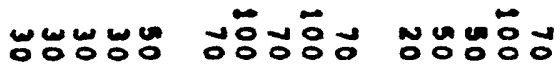

:

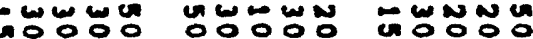

NNONON

NONON

$z z z z z z z z$

$z \geq 2 \geq 2$

NNNNN

WNNNN

-

Nw w un OOOOO Oㅇㅇㅇㅇㅇㅇ

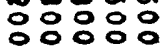

ununum

an w U 넝영ㅇㅇㅇㅇㅇㅇㅇㅇ영

00000

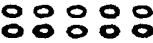

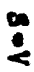

영영형융 형영융유

둥웅형형

웅형형

영융유

$z \geq z z z$

$2 z 2 z=$

$z \geq z z=$

$x \geq 2 z z$

$z x z=$

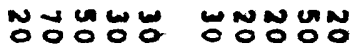

NUNMN

ํํㅇํํㅇำ

눙ㅇㅇㅇㅇㅇ

$=$

- $5=$

$x$

$=$

$=$

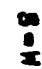

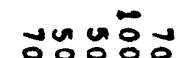

봉융중ㅁㅇㅇ

unumo

$\cos 2$

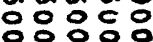

$+2$

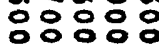

분 응영ㅇㅇㅇ

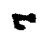

5

ํํํํํํㅇํㅇ

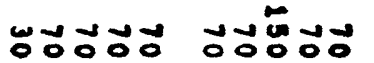

$\overrightarrow{0} \overrightarrow{0} \overrightarrow{0} \overrightarrow{0}$

무우웅

0

ำ ชั0ั0

w- -10 :00:0
- -0wnn w-0:u

- 00000 00ivio

- 0 o

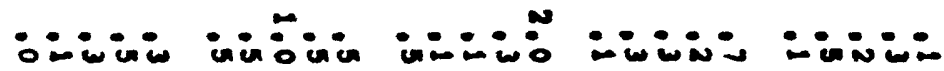
.

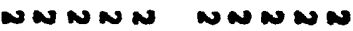




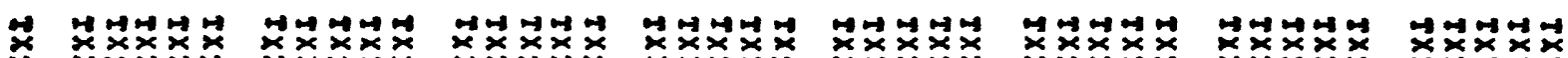

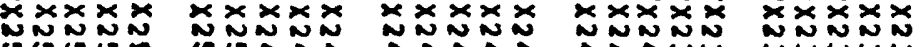

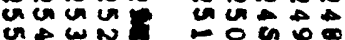

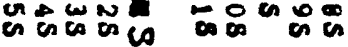

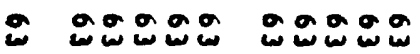

$\because A \Delta A+B \quad B+B$

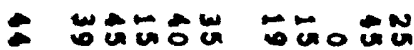

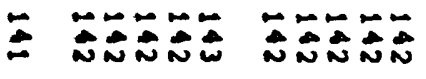

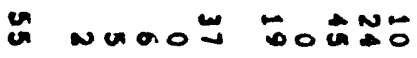

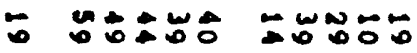

- NNNNO

$a$

OOONO

$\Omega$

a

$\sum \sum_{n=2}$

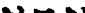

Nक⿻

م

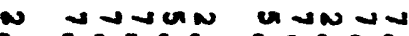

- 00000

$\because 00000$

$\therefore \cos (x)$

กอ

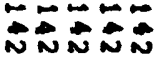

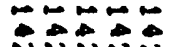

ind

-0oñ

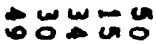

NONN
은 unuona เே부

ஸ.
문두요 unuaruon

a

w:D

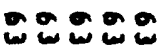
cucu un un No 웅우 a novio

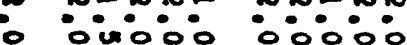

$\because 000$
ONONN

ด

numun

000 a 2

UNNNU :0:0:0

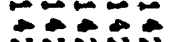
$N=-\infty$ in:

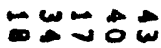

ow 0 :

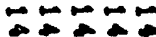
ลNANAN $\rightarrow+\infty$ in

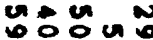

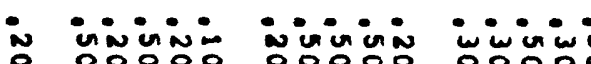

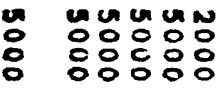

$\rightarrow \Omega$

$\because \because \because \div 0$

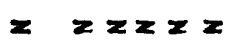

- ㅇㅇㅇㅇㅛ

$z y z z z$

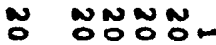

- 00000

$z \quad z=225$

O ETNN

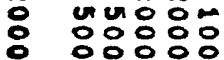

un un w un ㅇㅇㅇㅇㅇㅇㅇㅇㅇ

ค

a

uñ

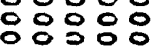

คด ด

$\div \div \div \div$

$z z \geq z$

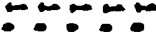

$\therefore 0000$

$z \geq z z z$

unuma

웅잉ㅇㅇㅇ

unumu

응ㅇㅇㅇㅇㅇㅇ

$z 2 z 2 z$

$z 2 z 2 z$

NNONON

응ㅇㅇㅇ

NONNON

$\geq 2 \quad 2$

$z z 2 z z$

此向

응응응영응

-

응ㅇㅇㅇㅇㅇㅇㅇ
证婇

wunna 응응융용

กด

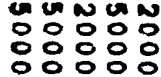

a

는

$z z z z$

ununu 응ㅇㅇㅇㅇㅇㅇ

$2 z 2 x=$

NNNNN

$z 2 z=2$

NNEN응ํํㅇํํ 응응ㅇㅇㅇㅇㅇㅇ ónog

$\because \div \div \div$ $z z z z$

unumer 응응응ㅇㅇㅇ $z x \geq z y$

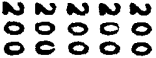
$z z z z$

CN-

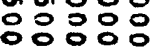

inivi

vinui o: Oó

awn wa 00003 ㅇㅇㅇㅇㅇㅇ응

a

$-\infty-$ $\therefore 000$ $z z z z=$

unumu 영응응응 $z x z x$ NONONN $z z x=$

분 ㅇㅇㅇㅇㅇㅇㅇㅇㅇ
NEN

?

$\Omega$

NONONO

an

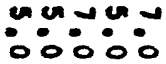

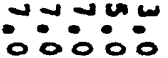

NNUN $\therefore \therefore \circ:$

UNNNN 잉ㅇㅇ

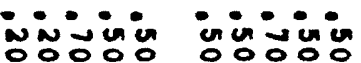

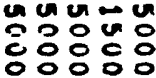

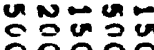
20

$a$

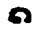

מEL $\div \div \div \div$ 00000 $z z=z z$

$z z z z z$

unúnún ㅇㅇㅇㅇㅇㅇㅇㅇㅇ응

unumur 응영ㅇㅇㅇㅇㅇ 닐 2

$z \geq z z$

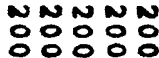
Nㅗㅇㅇㅇㅇㅇㅇㅇ $z z 2 z=$ $z z=z$

$\div \div \div \div$ 영ㅇㅇㅇㅇㅇㅇ

두웅 응영응응

ㅇㅇㅇㅇㅇㅇㅇㅇㅇ $\rightarrow$ qun $\operatorname{cn}$

$\omega \sim N \omega N$ NNNUN WN $N$ UN

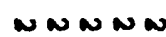
NN $N$ N N N $\boldsymbol{N} \boldsymbol{n}$ 6

ผ NMUN N $N$ a $N$ NNNGN NNNON $\sim \sim \sim \sim \sim$ N N N N 6

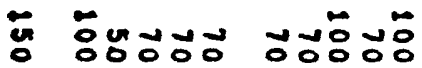

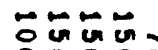

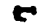
$z$ c tet $\leftarrow$

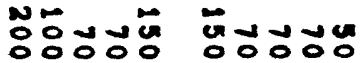
ถุ

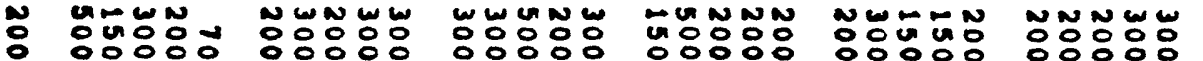

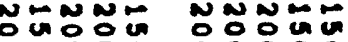

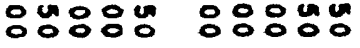


$\vec{x} \vec{x} \vec{x} \vec{x} \vec{x} \vec{x} \vec{x} \vec{x} \vec{x} \vec{x} \vec{x} \vec{x} \vec{x} \vec{x} \vec{x} \vec{x} \vec{x} \vec{x}$

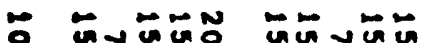

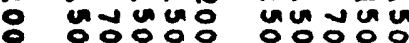

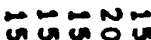
G0: 0000

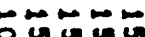

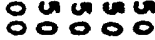

Gar ㅇํㅇㅇㅇㅇ

Nم

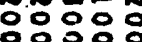
$n N N$
$\because O O O$
$O O$

문 N-

우유

영영

munu

- 승ㅎㅇ융

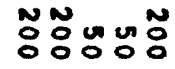

- Бே000

ேㅜ웅

두유유

$z z z z$

$z z x z$

$z z=$

$z \geq 2 x z$

a Munun

unu un un

unuuga

unugur

00000

z $z z z z z$

$z \geq z 2 z$

$z 2 z z z$

웅ㅁㅇㅇㅇㅇㅇ

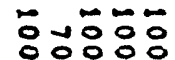

등후웅웅

ज둥ㅎㅇ

Бํํㅇㅇ

$z \quad x \geq z$

vonuun 00000

$z z z z z$

- Mํำ

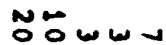

ज行

س

두응응

무유유

$z z z z$

unuma

○0000

00:

$z \geq 2 z 2$

분둥

जजㅜ웅

00000

ơํㅇํํ용

두융ำ

뚜웅

$z z z z$

ํํㅇํํㅇํㅇ

$z \geq z z$

붕ํํㅇㅇㅇㅇ

5

5

$\underset{0}{0}$

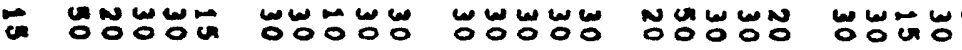

NWNNO

NONON ONO

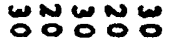

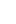

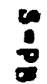

O

NONON

$z=2 z z$

\& $x \geq 2 \geq 2$

NNNNN

$x \geq x \geq 2$

OOOOOOOO

UUNun

:ํํㅇํํำ

NNNNN

西

NNNNN

$z \geq z z$

NNNUN

$z z z=$

NNN N N N

$z \geq z \geq 2$

NNNNN

ㅇํㅇำ

문

wผwนต

MUNNN

以ผ ㅇㅇㅇㅇㅇㅇ응

영영영

ㅇㅇㅇㅇㅇㅇㅇㅇㅇㅇㅇㅇ 눙용용용

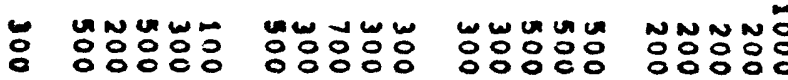

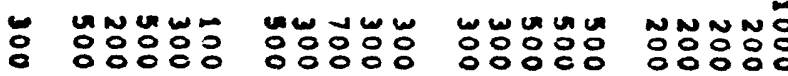

nNNN西

뭉

응요요요

UUn

영유유

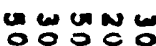

\%

on

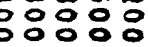

웅융후웅

ㅜㅜ웅ํㅇ

穴的

- $z \geq z z \geq$

$z \geq z 2=$

영ㅇㅇㅇ

$z z=z$

- 형ํㅇㅇ

붕

두웅

$$
z
$$

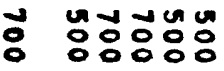

눙영영ㅇㅇ

더융ㅇㅇㅇㅇㅇ

5

$\because \div \div$

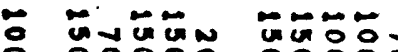

$\omega N-N N G$ $\div$

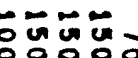

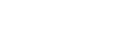

$\boldsymbol{N} \boldsymbol{N} \boldsymbol{N} N$
둥영융융

뚜웅

$z \geq z z z$

무을

응ㅇㅇㅇㅇㅠ

$x \geq 2 x \geq$

둥융융유

둥

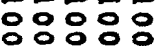

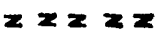

능용ㅁㅇ

$=2$

ำกับ

$=5$

$\infty$

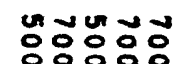

5

gun un un

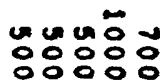

$z=$

두웅

Ev

$\because-\infty$

뚱융

ㄸํㅇํํㅇㅇํㅇ

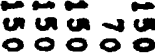

MNMU

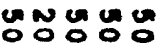

$z$

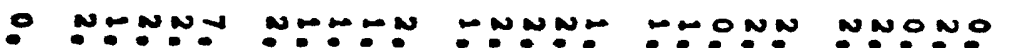

- 0.0 .0 .

0

$-\infty=0$

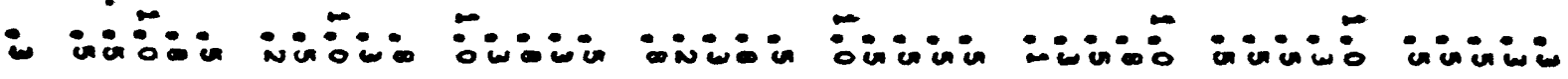

$\because \div \div 0$

$\cdots$

N N N

$\omega N \sim \sim N$

م

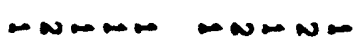

NNN-

$\boldsymbol{N} \boldsymbol{N} \boldsymbol{N}$

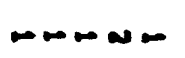


c. 20요웅

u canauca

\& núóno

- anoka

$\because \cdots$

$\therefore \quad \angle E= \pm \approx$

ம

* ONUNO

O ONONON ก

- nanun

- 0000:

N Namon

$\circ$ :00

nWNNT

$\therefore \therefore:$

v-nuna

i․:-

N NNN

०0000

NNNMN

이잉

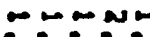

inivion

-nNNN

UIOOOOO

-nenu

iño:

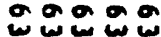
búugáa 엔도 충 네 พั0

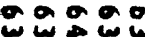

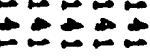
auㅛㅇ

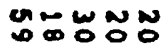

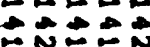
uuㅜㅇㅢ 웅잉
WNㅗㅇํㅇ

¿ No№n

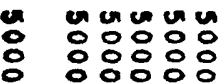

a nan

$\because \%: \div: \div 0$

$z z z z z$

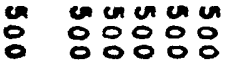

$z \quad z z z z$

O NNNNN

: 응영융

z $2222 z$

O WNNGE ○ ㅇㅇㅇㅇㅇㅇㅇㅇㅇ
Unn

옹응ㅇㅇㅇㅇㅇ

00500

กอ

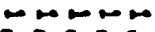

웅웅

$z z \geq z z$

unumu

ㅇㅇㅇㅇㅇㅇㅇㅇㅇ

$z z z z$

NNNEN

응ㅇㅇㅇ응

$z z 2 z$

ผ

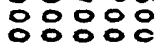

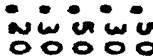

UnUNan

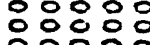

ด

a

$\because \div \div \div$

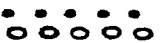

$z \geq z \geq z$

unuen un 응응ㅇㅇㅇㅇㅇㅇ

$z z z z$

NNNNN

응영융

$z 2 \geq 2 z$

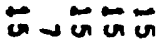
영영ㅇㅇㅇ

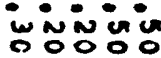

ํํㅇํํ응ํㅇ

o

แேะーะ 0000

$z z z z$

un un en n $z z z z z$

N N 잉ㅇㅇㅇ $z z=z$

任的向 ㅇㅇㅇㅇㅇㅇㅇㅇㅇ

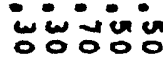

unum 영응용 응응응 ดกด

$\because \div \div \div$ 00000

$z z z z z$

Unu un un ZZZZZ

NNNNE 응응ㅇㅇㅇ

z2 2 2

unu

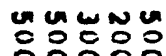
ㅇㅇㅇㅇㅇㅇㅇㅇ

an

$\div \div \div \div$ 웅우

$z z z z z$

a 영ㅇㅇㅇㅇㅠ I z z z

NNNNN 응ㅇㅇㅇㅇㅇㅇㅇㅇ $z z z=2$

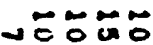
너응ㅇㅇㅇㅇㅇㅇㅇㅇㅇ

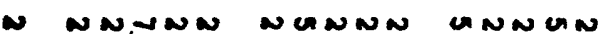
$\boldsymbol{r}$ CE $C$ TE N

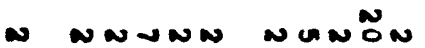
$\boldsymbol{F}=$ ET $T$

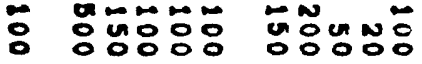
우눙 웅영 웅ํㅇㅇㅇㅇ Uñ

$\sim N N N N$

NNNNN

N N UNN

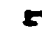

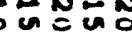
$\circ: \circ: \circ$

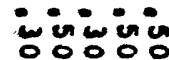

nunuma

wo unc 80808

คดคด

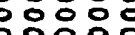

ana

$\underset{\vdots}{0}$

$\div \div \div$

$\because \infty \div \div$

$\therefore: 0: 0$

$2 \geq 2 \geq 2$

:

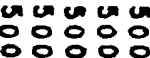

uncungun un $z \geq z z=$ $x \geq z \geq z$

NNNNN

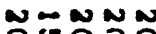
응응응 $z=2 z$

느ำ 응ㅇㅇㅇㅇㅇㅇ 
- m nor

מ

NTEN N

붕효 in

N-

ธேேேะே

nOOON

우ㅇㅝㅜ웅

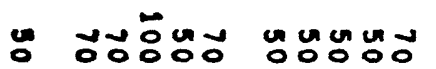
•

x

0

$=$

$\vdots$

เ

\section{-}

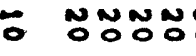

NNNNN

$z \quad z z z z z$

ร

: 2

ลัละัะัะั

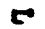

NE
NWWNN
NNNNN

$z \geq z \geq 2$

NONNN

$z z z z=$

NNUนNN ละดะ

unw :ஃะ:

ONNNNN
ONOOO
$z z z z$

․

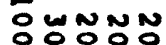
ะะะ:

$\omega$

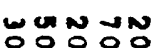
:ะะ:ะ

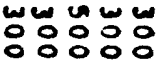

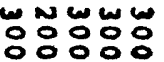

แัด ஃะஃะ

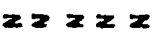

WNNNN c

象

$z z z z$

다다

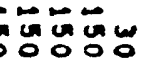

u unu

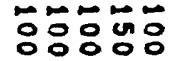

눙

एक्रा

ruñưncon

$$
=
$$

مே0ั0००

$z z z z z$

ㅜㅜ웅

$z z \quad z z$

unumuar

50000

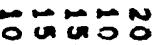

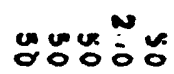
202

무유유 $x \geq 2=$

unungon

$z z z z z$ 뭉융둥융
5

$\stackrel{8}{8}$

$i$

$\sum_{\infty}^{\infty}$

$\vdots$

5

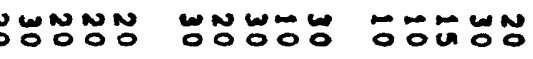

$:$

NNNNN NNNNN

NNNNN

NNNNN

$z=2 x$

$z 2 \geq 2$

$x \geq 2 z=$

:

นกำ

NWw un

cum nus

영융유

$x=$

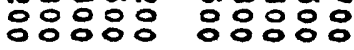

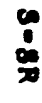

승영융유유

눙녕융융융 녕영용융

응 형융유응

مिए5

.0.

등ㅎㅇㅇㅇㅇ

둥융응

뭉응

مோே

$\circ 0000$

$z z z z$

$z z z z$

$z z z z z$ 00000 00000

ONONONO

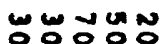

cos

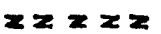

$252 \geq 2$

$z$

5

$r$

ํํㅇ뮤융

Nㅜㅇ으유

$z \quad 22 z=$

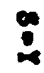

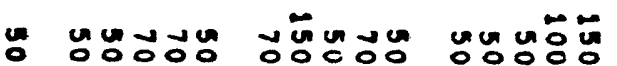

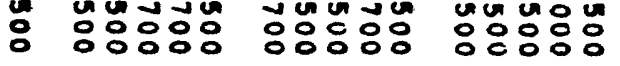

تีunun

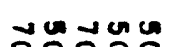

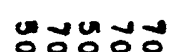
뜽융융융융

ununut 5 r

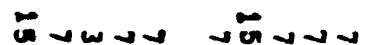

oे 무용 눙ㅇㅇㅇ

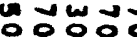

anc

พ두유 -

Nํํㅇํㅇ

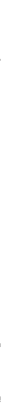

- - - wnen

$\omega \omega \sim \infty$

NNN $N$

- NNN *

- $-\infty \sim$

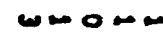

OONNO

TRENN

$\sim \omega N \boldsymbol{N}$

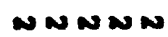
2

- $000 \%$ covino on:oi

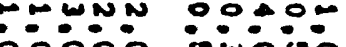

in:U⿺辶 anovio

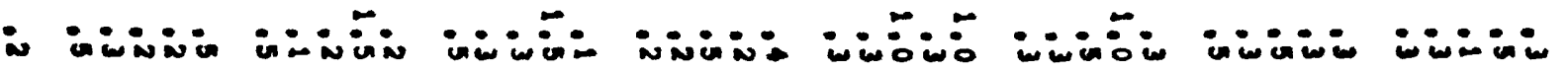


- $2000 \%$

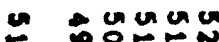

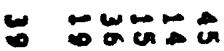

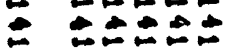

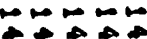

or on o o

un un un

ogono un un un

unn ia ouc

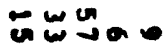

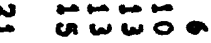

con on

$\leftleftarrows 0 \approx 00$

๓un uñ

- NNNONE กด

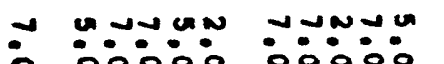

Nேー ónug

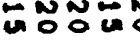
un $\rightarrow \rightarrow$ $\therefore=5=$

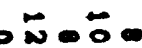

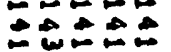
$\square-\infty \div$ ज穴凹 nuun No:NN $\omega \rightarrow=\overrightarrow{4}$

$\because 2 \div \div=$

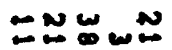

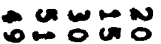

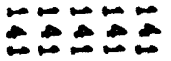

Nun

w

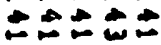

\begin{abstract}
anu
\end{abstract}
西

:00

每

OOOOO

NNMNN

-0:0

NNNNN

$\therefore 000$

NNNNN

०00\%0

w w

웅u․

س

○0。0

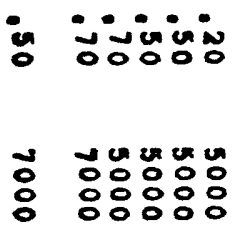

an

$z \quad z z z z z$

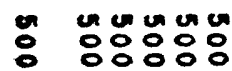

$z \quad z z z z z$

O NONNN

-

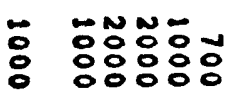

iniúa

แNंNi NON

vin in 잉 힝ํㅇ

unuce

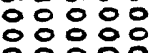

ก

acin the $\circ 000$

a $\Omega$

ง N N U w

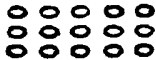

மோー $\div \div \div$ 00000 $z=z z$

uncíunu

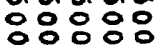
$z z=2 z$

NNNNN

C5 5

느응

융궁뭉응

궁융영응 $\therefore:: ㅇ$
NNNNN ceter

NONN

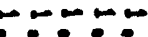
$000: 0$ $z z z z=$

ununu 응ㅇㅇㅇ응 $z \geq z z=$

ununu 영융유 $z 2 z 2 z$

nั0กnN

응응 r ret

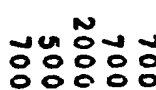

Oinivio ognos:

un UnN 응요 응ㅇㅇㅇ

$\Omega \Omega$

מே:0000000000

$z x z z z \quad z z z z$

ununu ㅇㅇㅇ융유 $z z z z$

Nㅜㅇ융응음

unuun un 응융ㅇㅇ $z \geq 2 z=$

$z z=$

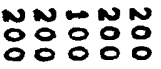

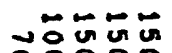
융ㅇㅇㅇㅇㅇ $z z=z$

动-

ตุํํㅇํํㅇㅇㅇㅇ ㅇㅇㅇㅇㅇㅇㅇㅇ inivis

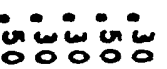

unun

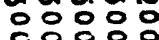

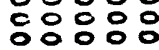

a 0 a

unum 영융유 응유: nan

$\because \div \div \div$ 00000

$\because-\infty-\infty$ :0000 $z z z z z$

$z \geq z z z$

ununu 용ㅇㅇㅇㅇㅇ

unumu $2 x \geq 2=$

웅응용ㅇㅇ $z z z z$

nNNNN nNNWN

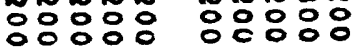
z $z \geq z z$ $z z 2 z$

NNOOOO

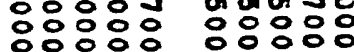

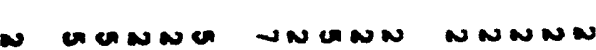
$\boldsymbol{N} \boldsymbol{N} \boldsymbol{N} \boldsymbol{N}$ กNกNล $\operatorname{sen} \cos$

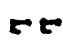

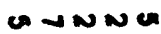

6

$$
\leftarrow
$$

5

c)

NONNN

N

NNN N N

NN N N

$\begin{array}{llll}N & N & N & N \\ 0 & 0 & 0 & 0\end{array}$

O OOOO

$z \geq E$

जผ

$\rightarrow \infty$ u e

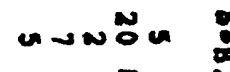

$z z z z z$

$z z z z z$

$z z z z$

- 훙

두웅

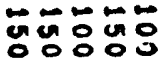

-0 -

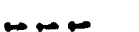

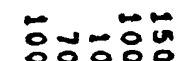
$z$

5ำ 5 훙휴웅융 


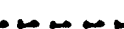

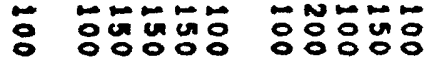

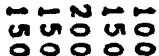

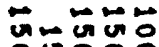

مثن

i

Eñ:

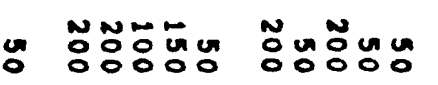

uㅜㅇ un

붕융유

O푸요 5

- Бேேேே0

ேㅜ웅

$z z x z=$

$x \geq 2 x \geq$

- vanun

$x \quad z z z z$

능영영융

- congug ununu $z z \geq z$

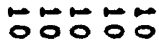
$z x \geq z$

unungur

$z \geq z \geq z$

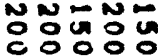

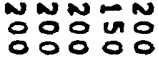

10050
$\quad 222 x$

$z \geq z z x$

뜸ํㅇㅇ

ㄷํㅇㅇㅇㅇ cunugur
두ㅇㅠㅜ웅

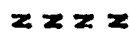

$z=5$

NNהT-

융ํํㅇํㅇ unuun un wo n 영영응 5

00500
$2 \geq 2 \quad 2$

ㄷํㅇำ uñ

$z z=$

둥영영 o óño

ํㅜㅇ용ํำ

이잉

जั0

잉ㅇㅇ

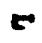

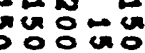

뚱응 능

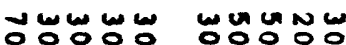

समी

mando

$\begin{array}{ll}\text { ज0유 } & \text { 00505 } \\ & =2 x=2\end{array}$

ucucuna

tr $x \geq 2$

unuma

$z \geq z z$

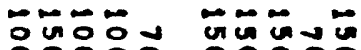

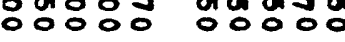

- unumo

MNNNN

N

mNMn

NON N

WNEN

$\div 0$

훙수

문ำ 0000\%

ONN

WNNNO

\% NNONON

NNNNN

NNNNN

NNNNN

NONNN

WONON

N

$z z z z z$

$z z \geq z z$

$x \geq 2 x$

$z \geq z z$

$z z z z$

$z=2 z$

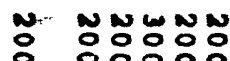

nNNNN

nun UnN

NNNNN

NNNNN

NNWN 000

$z=$

- 뜽융융

unnún

응응응

$z$

NON

ํํㅇำำ

Oㅇㅇㅇㅇㅇ

융유용

munnN

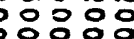

MN

응잉ㅇㅇㅇ응

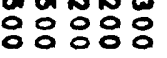

س

웅영영ㅇㅇㅇ

م

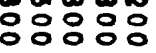

ununu

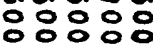

:

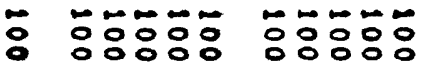

흥웅응응

둥훙ㅎㅇ웅

등옹ㅎㅇㅇㅇㅠ

응용융웅

두유웅

م

$z z=2$

$22 \geq 2 z$

$x \geq 222$

$x \geq x \geq z$

$z=2 z=$

$z=z$

$x z z x$

응ㅇㅇㅇㅇㅇㅇ

$z z z z$

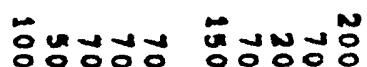

눙웅 ज्ञ

5

5

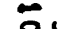

용 녕융융

긍영영영영

붕ㅎํㅇํㅇ

ㅇㅇㅇㅇㅇㅇㅇㅇ

-

-

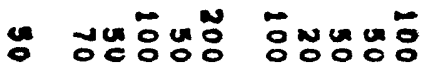

ก ติ

Noñon

$=$

$N$ NMNDN

MNNNN

NNNNN

- NNNN

NOMNN

$\boldsymbol{N} \boldsymbol{N} \boldsymbol{N}$

- $\boldsymbol{N} \boldsymbol{N} \boldsymbol{N}$

N-OOO

-

$\because-N=$

:00:0

:0ن்

$00-N N$

$\because 0$

-

inio: inisio

ONNNE ज०0:0

0
0
0
0
0
0
0
0

:

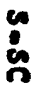

0
0
2
1

:

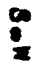

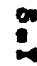

-

ununu un 웅영영

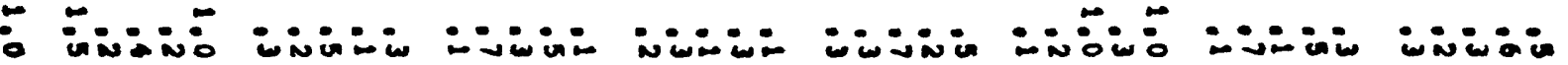


- o. 0 . 0

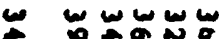

○N
- o o o o

ถ

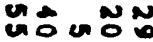

- 으의

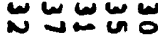
مuำ
우의. w w w م WA WN

\section{웅요

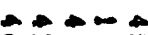

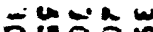 \\ - iu \\ un}

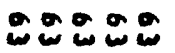
on un un

Nunn
문둔 ant

ס

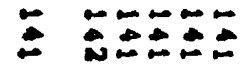

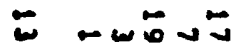

능ㅇㅇㅇㅇㅇ

N NONNNN

ด

$\because \infty-\infty$

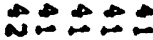

แ

$\leftarrow \infty-\infty$

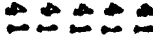

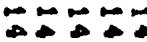
$=-$

an un u

ous ou

$-\infty N \tilde{\infty}$

N. No

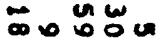

NNNNN a

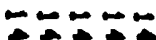

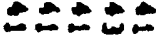
WNN ㅎํㄴํำ $=-\infty-\infty$

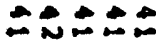
Nun

un

$\because-\infty$

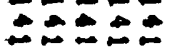

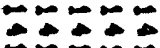
NNNN NNNNN 00000

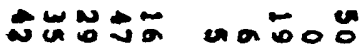

- unuma

NEN NO $\infty$

UNONO

ana

NNNNN :00:0

unuma

NNN

N w w w

wwa

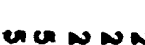
促

a

UNNN

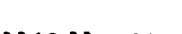

NINONO<smiles>CC1CCCC1</smiles>

UNNTN

-

unumana :0:\%: 웅웅

ำ

:0:-

unnum :0:00

- 00000 00000 :00:0

$\operatorname{lng} \cos (\log$

unumon

NNNNN

NanNN

unuma :0000 :0000 0000: 00:0:0

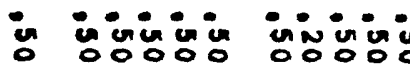

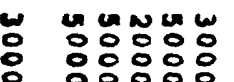

- $a$

nunum

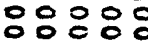

DQA

Ğต

웅ㅇㅇㅇㅇㅇㅇ

a

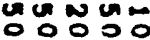

응ㅇㅇㅇㅇㅇ

a

$\div \quad 0 \div 0 \div: 0$

무유:

$z=z z z z$

$z \geq z \geq z$

두웅

$z z z z$

-

$0000:$

$z z z z z$

n anum

응융융

ununu

응ㅇㅇㅇㅇㅇㅇ

$z \quad z z \geq z z$

z 2222

GNNNN

O NNNONO

r TETE

CE unumu 응웅ㅇㅇㅇㅇㅇㅇ $x z 2 x=$

unuma 응영ㅇㅇㅇㅇㅇㅇ $z \geq z z=$

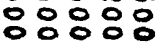

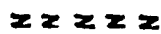

NONNN ete

NNNNN TETEC

NNNEN terte

wNNun

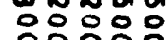

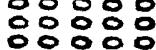

능 2 응 응응홍ㅇㅇㅇㅡ

4 둥

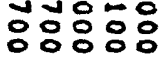

O

응 영영영영

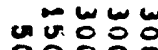
ㅇㅇㅇㅇㅇㅇㅇㅇㅇ

$\sim$

- NNNNN

z $z z=2 z$

ต 후웅
NNNNN

$z \geq z z z$

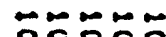

응웅ㅇㅇㅇ응 nenuso

NONONO $z \geq 2 \geq 2$

non 눙영영
NWNNN

$z=2 z 2$

는 anuö
U U U N N N r

NONON $x \geq 2=$

Jumuna

너엉

$\because \cdots$

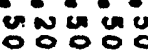

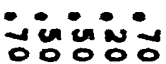

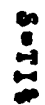

Nỡ 응응영영영

uww

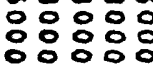

○

unuan 옹ㅇㅇㅇㅇㅇㅇㅇㅇㅇㅇㅇ $\infty a$

$\infty-\infty$ 00000 $z z z z=$

$\leftarrow \infty-\infty$ 00000

$-\infty-\infty$ ㄷㅇㅇ웅 $z x z z z \quad z z x z z$

unumu ㅇㅇㅇㅇㅇㅇ응

unum 영영ㅇㅇㅇ응

unuun 응영ㅇㅇㅇ응 $z z z z x$

$z z z z z$

$z \geq z z z$

NON Non

NNNNN 55

CEETE

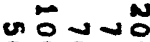

$\because \div \div \div$

등ㅇㅇㅇㅇㅇㅇ 응응영융 00000

Nㅜㅇ 응융유. 응융응 


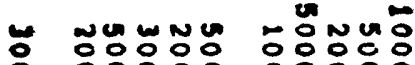

w w 응ㅇㅇㅇㅇㅇ 영융융

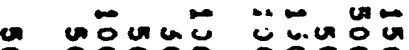

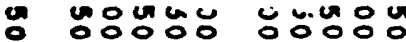

z $x=$

$x=$

$x$

- 05005

z zzzzz

- granugu

z $z z z z z$

- 훙ㅇㅇㅇㅇㅠ

ำ ㅇํㅇํํㅇ 뭉ํㅇ

두우

$z z z$

ununun un

$z z z z$

M 능유유

$z$

ज㱒可

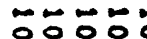
$z \geq z=$

$5 \div 5 \div \%$

0000

1n

$z \geq z$

00000

ungungun

$z z z=$

$z \geq z z$

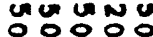

$z x z z=$

NON-

응 눙영

웅ํํㅇ

00000

س

เีลีำ

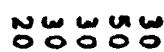

NŨ N -

$\because$ -

NNMUN

MNON

N U N N N

NUNNN

누웅웅

00000

$z \quad z x=$

gonuca

$z \geq z 2=$

oog:

$z x z z$

융명융융

두융유

둥휴융요

$\stackrel{2}{2}$

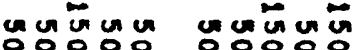

$z z \quad z$

$z=$

5

$\div 5 \%$

$\div 0 \div 0 \div$

$\frac{0}{3}$

unung

$z \geq z z=$

?a

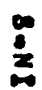

눙츄융

ํํㅇํํㅇํㅇ

:훙ㅇㅇㅇㅠ

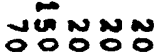

NONOOO

Nㅜ융

NNONON

NNNNN

$2 x=$

$z z z=2 z 2 z=$

$2 x \geq 2 z$

$z x \geq 2 z$

용 용ㅇㅇㅇ

nNnNa

NNNUU

Nan

UN

nNn

nNNNA

(⿻)

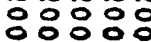

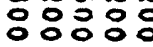

5

$z=$

- unuun

vnu un

unuas

da un un 융ㅇㅇㅇㅇㅇ

$=x$

untu unuma ㅇㅇㅇ영영 영영영

un unu un $=$

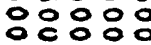

unuma

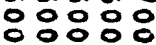

응 훙영융

능웅영웅

응융융유

응융융유

훙영뭉응

훙융융

응웅융유

뜨웅유

$x \geq z x$

$z x z z z$

$z=2 z$

$z \geq z 2 z$

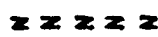

$z \geq z z$

$z x z z=$

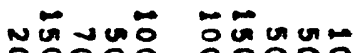

WNun

ำกี

กำ

z

uncon

(2)

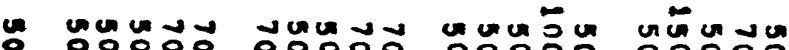

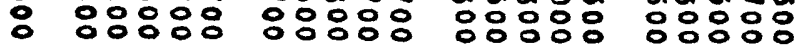

-

5

$\cos u \cos$

응ㅇㅇㅇㅇㅇ

$-$

ununu

ㅇㅇㅇㅇㅇㅇ

tre

navuc

잉ㅇㅇㅇㅇㅇ

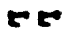

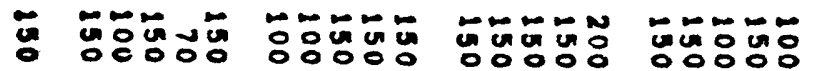

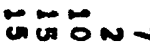

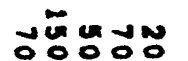

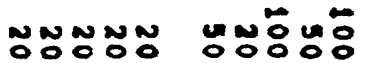

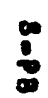

3
2
3
2
0
0
0

:

:

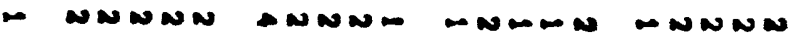

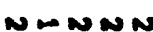

$\sim \boldsymbol{N} \sim \boldsymbol{N} N$

$\boldsymbol{N \infty N \infty}$

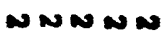

울

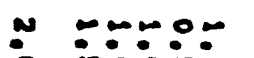

O-0 $\boldsymbol{M} \boldsymbol{N} \boldsymbol{N}$ (2)

nOMUN TnNON

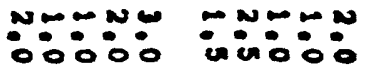

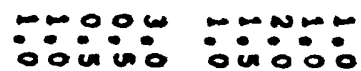

incio

00070

is

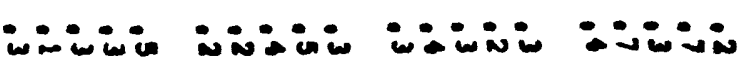




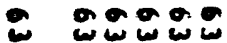

Oaga

- 0000

N

Nㅜㄴ

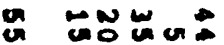

oun:

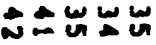

눙 $0: 0$

$-20 \div \div$

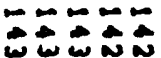

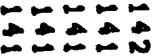
م

¿ NoñڤN

- uñNo जus: $\div \div \div=0$ Uñ जט:

- NOOOOOOO NONONO

NONONON

NONONO

NNNNN a

$\Omega \Omega$

ana

ansa

a

andun

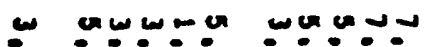

$\ln \pi n n$ ०००:0

:0:0\%

$\therefore 000$.

uUnun :0:0\%

unuma io:0:0

unana :000:

N N ०0 i००

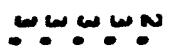

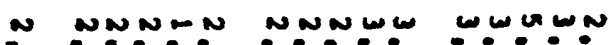

un un

w w w



N ०0:00

- O0ं0

O००:0

웅우

00 00000

00000

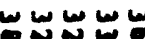

- i iño:

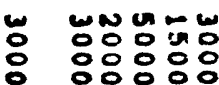

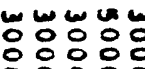

๑

ค

잉ㅇㅇㅇㅇㅇ

응ㅇㅇㅇㅇㅇㅇ

$\circ$

$z z z z z$

u vonung

영융영

z $z=2 z z$

- 뜽응

$\div \div \div \div$

00000

-mb

:000\%

zzzzz

$z \geq z=2$

ununung ㅇㅇㅇㅇㅇㅇ

$z x z z y$

붕ํํㅇํㅇ 응응

눙무요

UUNNO

5

a w두

U w.

55

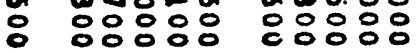

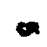

WNNNN netrug

$$
=r
$$

- NONONO

NNNNN

$z z z z z z$

$x \geq z 2 z$

- 둥 뚱루

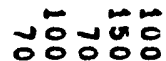

ํㅜ UnN NONONO $z z z=2$

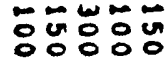

คด

$z z 2 x .2$

NNNN

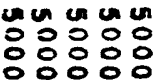

ana

$\therefore: 0$

$z \geq z \geq z$

unumu 영영

$z z z z z$

NWNGN -

-NMNN

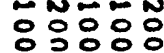

00000 ớñú

unn

응응요

융ㅇㅇㅇ

๑

m$\therefore:-0$

$z \geq z \geq z$

ununu

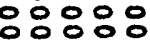

$z \geq 2 z z$

NNN요 CEL

MTN ㄷㅇㅇㅇㅇㅇㅇㅇ 응요 inis vuñon

wnw 응응ㅇㅇㅇㅇㅇㅇ

a

-mー 웅웅 $z z z$

unumu ㅇㅇㅇㅇㅇㅇ $z z z x$

NNNMN ter

以N N 응ㅇㅇㅇㅇㅇㅇㅇ 00000

जU 둥ㅇㅇㅇ응
NNN NON $2 z 2 z x$

Nunua 잉요
ن넌

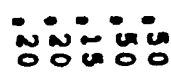

unnun

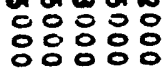
a

$=-5-$ 웅ㅇㅇ $z z z z z$

unumu

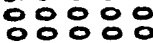
$z \geq z z z$

(

NONNN CTEEE

- una nó 응ㅇㅇㅇㅇㅇㅇ a 0

$\because \div \div \div 0$ $z \geq z z=$

ununún 응ㅇㅇㅇㅇㅇㅇ $z \geq z 2 z$

NNNEO 응융융 영ㅇㅇㅇㅇㅇㅇ

붕요용 CEE

NNㅜㅇ

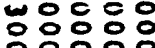
응응융
-

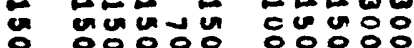

N

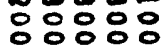

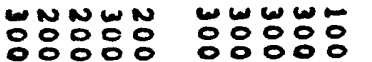

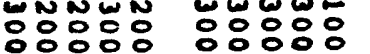

unation

NNNNN $z z z=z$

ONNN

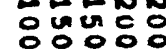

UNNON

NNNNN OOOOO $z x z z z$ 


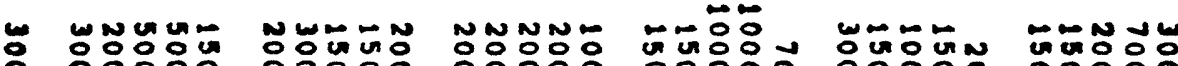

w些管出

-NNNG

능: $: 0$ :

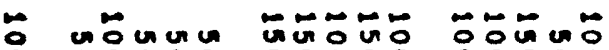
०००00

$z=$ z.

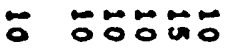

무융

$z z z z$

$z \geq x \geq z$

* vonunu

ununua

z $z z z z z$

$x \geq \geq E^{+} \geq$

으유요

¿OMEN जo: un

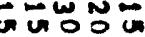
잉ㅇㅇㅇ

ஸே0ั0ั0 z 2 ㅈ. $2 x$ CFI $2=$ $z z z z z$

\section{r-un-}

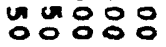
vunun gooú
No

응ํuuu $=$

N-

๐ே0ல0 $z=z$

Nㅜ융유 $z \geq x$

unung $z z z z z$

\section{둔}

웅요의 ㅇํㅇํㅇ

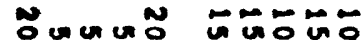
웡ㅇㅇㅇ

$x$

후유 $z \geq z x$

gunung $z z z z$

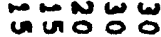

ேㅜ웅

$z \geq 2 \geq z$

unum o 0000

$z \geq z \geq z$

ดํํำด

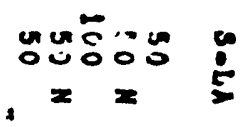

뚜ㅇㅜㅜ

$z \geq z=$

ununu

$\geq \geq \geq=$

วัดำธีอ
융ํㅇ $z=5$

ํํำ wow

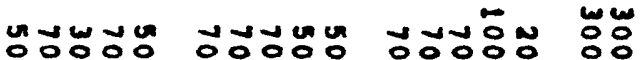

NOO융
OOOOO

NNONON

WN

NONON

N NNNONO

NONONN

WONON

$z=$

$z \quad z=2$

$z \geq z \geq z=z z$

NNNNU

NNANN

응영융

응ㅇㅇㅇㅇㅛ

nNMUN

$z x$

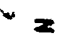

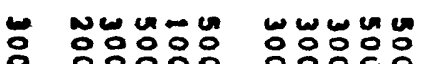

unơn 응영융

ㅇㅇㅇㅇㅇㅇㅇㅇ

nun $\cos$

nUUMN 웅ㅇㅇㅇㅇㅇ

UNON NO

OOOONO $z=2$

$x z=$

NNNNN 응ㅇㅇㅇㅇㅠ

nNMUn

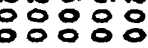

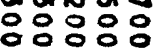

nun un ㅇㅇㅇㅇㅇㅇ
응영웅웅

NNNNN

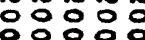

훙ㅎㅇ

눙ํํ융

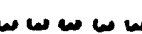

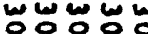

ேㅜㅇㅠ 6

Nuํำ 2

NNNNG 응ㅇㅇㅇㅇㅇㅇ

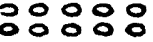

unNun

unuu

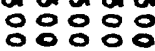

una

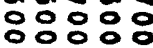
$<$

응 ㅎㅇㅇㅇㅇㅇ 영영영 형영영

영융융

융웅요

웅융ㅇㅇㅇㅠ

둥ㅇㅇㅇㅇㅇ

$z z z z z z z z z$

$2 \geq 22 x$

$z=2 x$

z 2 Z Z z

$z z=2$

$z \geq x$ z

무두

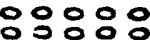

$x \geq 2 x=$

\& NONONO

MUnan

너우요

UUn $\cos$

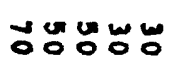

ANNNO

$=$

I

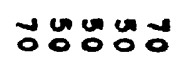

พํㅇㅇㅇㅇㅛ

$=$

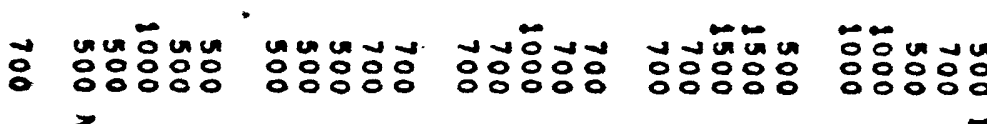

눈혀

영영영

건붕

영영융

unu un

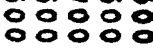

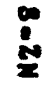

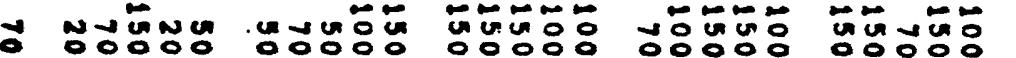
$=$

N NNNN

NNNNN

NNNN

$N N-N$

مמ NMN

WMNN

NNAN

NNNWM

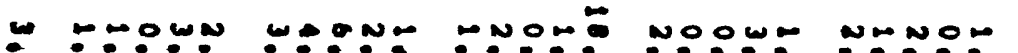

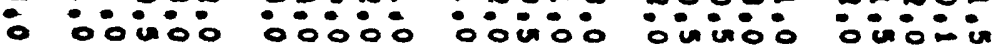

n-ON

NON

$-\infty=-\infty$

$\therefore 0 \div 0 \circ$

:

-

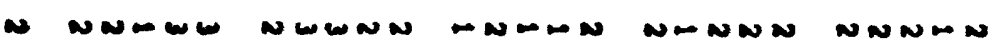

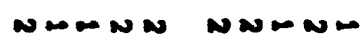

$\omega \sim \sim \sim \sim$

0
$\frac{1}{0}$
0
0

$\div$

$\underset{:}{0}$

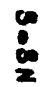

$:$

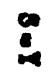

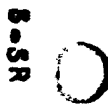


- 20090

운

0000

-

- oñoú

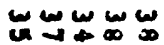

-

w w w w

م
우의 טم: ON N⿴囗大 Na

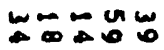

요웅 ט Nơ जजि०० o. on 0 o w 世\% o 10 aso. 00

-

H. 00
우의요

N๐O

was cu

\section{$\because=2 \div-\infty$

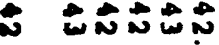 \\ - पूãañ \\ - W050.

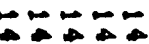 NลNANÁ ONGNA $W_{N \perp N}^{N} \mathbb{N}$}

ดENOG

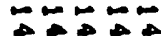
NNABA UN แ แNN $\because \infty-\infty$

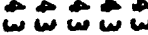
NO ถัต์ $\underset{\omega}{\infty}= \pm$

Nㅡ는

•
ேㅡ눈

-NNNG

0
0

a.

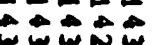

ON $N_{N} \rightarrow 0$

wN

$1000 \%$ $\because-\infty$ W ע. Nun
O늉ำ

a

a

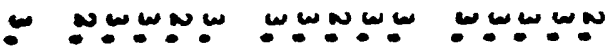

$\therefore$ :000:000:0 :00:-

N NWח

- jo: in :0000 0000U w un un 잉:ט:
NNWNM

WNNNN 웅웅
กับ ๑ an w w w w w

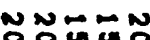
OOำ

ONONON

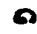

WUNNW

wunw ०0:00 WN

NWשn NNWNG

:००0:

00000

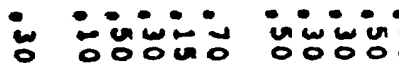

: 승영명영

$\boldsymbol{\Omega}$

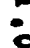

.

ร

\section{:}

영영명 $z$

$n$ MNUNAN re

O NONNN $\geq 2 \geq 2$

응 두웅웅 ㅇㅇㅇㅇㅇㅇ NNN $z \geq z z=$ NNNNN $z z z=z$ N ๕ேํํํํํ

U은 응ㅇㅇㅇㅇㅇㅇ

분 $=$

영형형뮹융

(2)
-

GNNNN

NNNNN

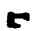

NNNNN $z z z z z$

NONON $z=2 z=$

겅겅ㄷㅇㅇㅇㅇ ถัวิธัดี

No

ㅇㅇㅇ뭉융
눙형영

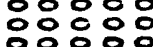
응응융용 o

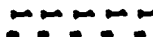
00000

$z \geq z z z$

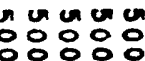

$z z z z$

겅붕용

מே

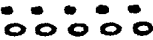

$z 2 z 2 z$

-m心 웅ㅇㅇ $z z z z z$

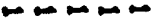
웅우웅 $z z \geq z 2$

unuun

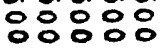
$z z z z z$

ununu ㅇㅇㅇㅇㅇㅇㅇㅇㅇ $z z z z$

cununa ㅇㅇㅇ영영

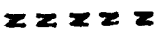
눙후응 붕응 뜽ㅇㅇㅇㅇㅇㅇ 눙영웅 00000

둥추웅 응영ㅇㅇㅇㅇㅇㅇ

구옹훙ㅁㅇㅇ 융융응

그눙요 응응응응

NNN N Nun ann EE $C$

NNNNN $x z 2 z=$

NONON $z=2 z$

NON NN $z=z z=$

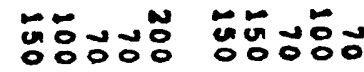

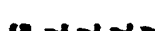
00000 


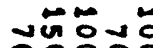

붕ㅎㅇㅇㅇㅇㅇㅠ

ENNNN

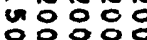

ำง

G유유

MNNN 00000

00000

응융융

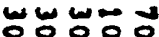

N waw 융영ㅇㅇㅇ영

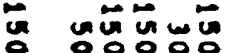

Nํำ

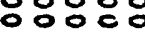
5

ปี่ํํำ

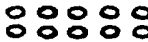

$\infty$

ํํㅇํํㅇ

z $z$

$\omega \sim \omega N N N$

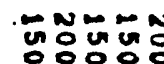

NNNNN

$N-N-N$

-

- ioisin o0jo: oivio

N-m-

$\div \div 0 \div 0$

OOn wN

$0 \div \div$

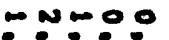

:

$\because-\div \div 0 \quad 000=$

:00:0 :00:0

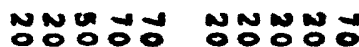

$=$

$z=2 z$

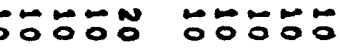
$z=2$

$z \geq z x$

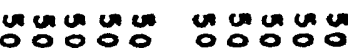

$z \geq z z$

$z=2 x=$

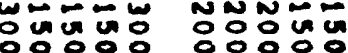

는ํㅇ

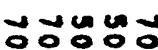

$$
25=2005
$$

ONONO ONOOOOO

ONN O O

NNN NON

$z \geq 2 z=$

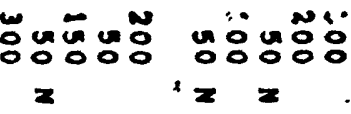

:

$\infty$
$\vdots$
$\mathbf{0}$
$z$

- ivivi iñiv 


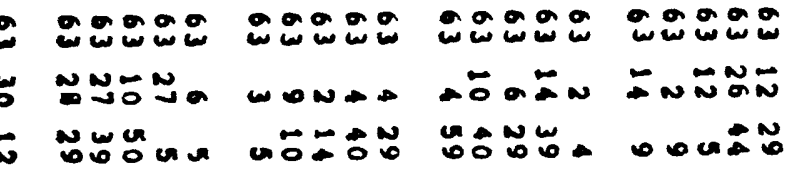

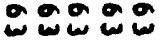
NNNNN שN

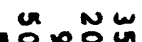
둔둥 $\sim N N N$

ס - voú
- o. ono

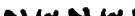
A-

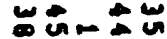

운웅

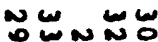
W0

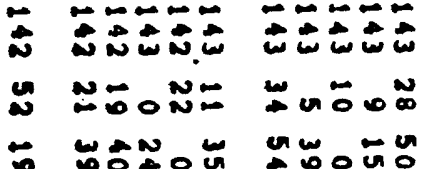

O ONONON a

w wnun

- :00:0

- MNMUN NNNNW

$\because 0000$ 0000

\& UNoñ

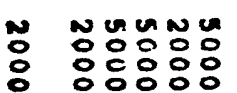

unun

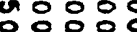

응융ㅇㅇ

s

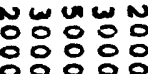

?

$\leftarrow \div \div \div$

$\because \div \div \div$

- 00000

z $z x \geq z z$ 00000

$z z z z z$

응영영영

$\because N$ NNN

unuuna 00000

$z \geq z \geq z$

$\div \div \div \div$

$z z z z z$

unuun

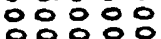
$z z z z z$

눙무웅

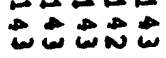
NUNND Б0 जun $\triangle A D A N$ N ưou $\square \div \infty \div \div$

ดڤ

WNֻ00

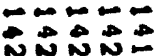

ט

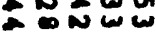

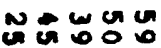

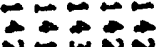
سִ 웅무

NENONO a a $a$

NNNUO

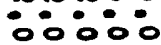

-ONNN

ก

NNNNN ?

NOOOOONON

Nㅡㅇㅠㅡㄴ

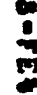

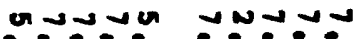
:00:0

०0:0\%

unuun

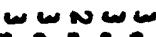
웅웅 $\frac{1}{2}$

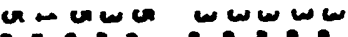

wn w w

oncunar :00:0

UWN WW oño:

00000

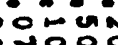
$0 \rightarrow 00$

an unu

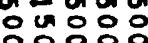
영ㅇㅇㅇㅇㅇㅇ

○

OOOOOS 응ㅇㅇㅇㅇㅇㅇㅇ

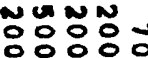

$z \quad z z z z$ 응영영응

Nㅡ융 ㅇㅇㅇㅇㅇㅇㅇㅇ

a

$\because \div \div \div$

$\div$

$\because \div \div \div$ 웅웅

$\leftarrow \infty-\infty$ 잉ㅇㅇ $z z z z z z z z z$

$z z z z z$ 00000 $z z z z$

ununa 응영영

ununu un 응응ㅇㅇㅇㅇㅇ

ununu 영융응 $z z \geq z z$

$z \geq z \geq 2$

$x \geq z \geq z$ 잉영영영 $z=z z$

붕영붕무 ஸूㅇํㅇㅇㅇ

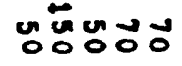

룽ㅎㅇㅇㅇㅇ

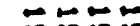

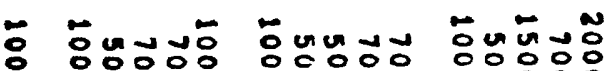

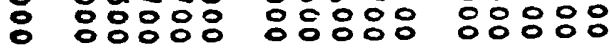

눙요융

후웡ㅇㅇㅇ

ำง 응응ㅇㅇㅇㅇㅇㅇ

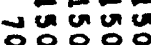
응ㅇㅇㅇㅇㅇ

\section{의 눙 ㅇㅇㅇ영ㅇㅇㅇ}

$=$

NกบกN

NNNMN

te

$$
\text { CE }
$$

C

N NNNNN

NNNNN

NNNNN

5

TE

5

NNNNN

NNNNN

NONONO

z $z=2 z z$

$z \geq z z z$

$2 \geq 2 z 2$

$z 2 x z z$

$2 \geq 2 z 2$

- 뭉무웅

웅붕영

후웅

N-N

UNON

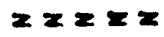

ตับ

웅융
NNONONO

$z z \geq z$

둥 ㅇํㅇㅇㅇㅇ
ㅇํㅇํํㅇํㅇ 응응ㅇㅇㅇㅇㅇㅇㅇㅇ
잉잉 $z z z z$

unuma 웅웅ㅇㅇㅇ $z z=2$

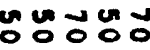

NNNNN $z z=2 x$

둥ㄷㅇㅇㅇㅇ

:

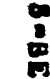

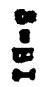
:

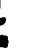

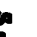

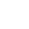




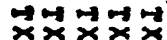

$x \times x \times$

00 :0

en w en e

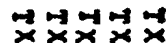

$\times \times \times \times$

$\infty \quad \infty 10$

0
0 co $\overrightarrow{x \rightarrow-1}+\vec{x} \vec{x} \vec{x}$

$2 \times x \times$

Did

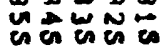

$\vec{x} \vec{x} \dot{x} \vec{x} \forall \vec{x} \vec{x}$

존

$\infty \leq ⿻$

ำ $\vec{x} \vec{x}+\vec{x} \times \mathbf{x}$ $x \times x \geq 1$ $20 \leq 5$ en es en

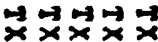

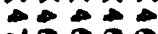
तूaे 응 ơ co

$\vec{x}+\vec{x} \underset{x}{x} \vec{x}$ $x \times x \times$ 중ㅎㅇ out wen

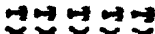
$x \times x \times x$ aua

- Naำ

UnM 응응응응응

NNENT 영영영

datumand 웅응 능

○ ㄸํㅇํํㅇ융

z

- 후후

z $z \geq z z$

\& onyuno

$z \quad z z \geq z z$

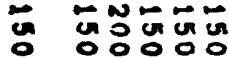

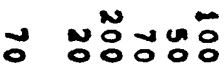

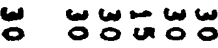

- OOOOO

$z=2 z 2 x$

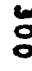

능 융용ㅇㅇㅇㅇㅇ $\leftarrow$

두웅 $z \geq z$

unuun 00000

$z z z z z$

WNNNN

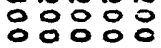

जNNON
NNNONO

$z=z z$

NNUNN

응응ㅇㅇㅇㅇㅇㅇ nํㅇㅇํำ

ज용ㅁㅇㅇㅇㅇ

$z=$

뭉웅우 $z \geq z z$

unuun 00000

z $z \geq z$

눙후웅 0 웅융

무워ำ

NNN N
Uก๊
잉응응

ำํํำ $r$

부웅 $=$

\section{ununua} 00000

$z x z z z$

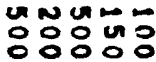

웅웅웅

NNONO

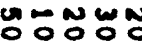

NNNNN

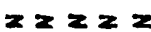

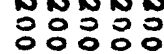

NNNONO

$z z z z$

WNWN 응융ㅇㅇㅇ
NNNNN

$x \geq 2 z$

UNUNN 융융응

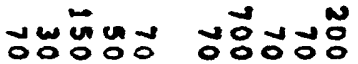

numun

$z z z$

둥ㅎㅇ

$z x \geq z z$

unumu

O5000

$z z z z z$

뭉응충

uno

won

$x=2$

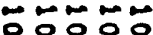

$z x z x$

unuuna

00000

$z z z z z$

ำํำ

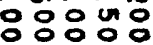

N 눙ㅇㅇㅇㅇㅇ

둥융ㅇㅇㅇ

둥 ơ 000

$z 2 x$

nongoño $z=2 x$

뭉유유

$z \geq 2 x=$

웅ํํ

unumu

엉응

ununa

00000

$z x \geq z z$

븡응융용

붕무웅

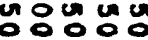

5

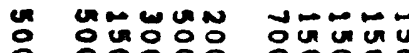

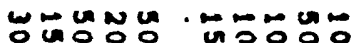

ñouuำ

NNNN

NNNNN

NNNNN

$z 2 z 2 z$

$z=2 z 2$

$z z z z z$

NNNNM

nuuna

ㅇㅇㅇㅇㅇㅇ

웅ㅇㅇㅇ응

응융융유

Non

5

:

녕영영

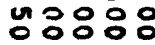

응융ㅇㅇㅇ응

ununu

namu 영붕영영ㅇㅇㅇㅇㅇㅇ

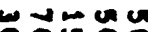

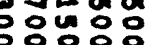

- 우웅ㅇㅇㅇ

두웅

두음

م

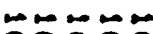

8

무음

-

0 응ㅇㅇㅇ

00000

$z=z z=$

응영ㅇㅇㅇ

응ㅇㅇㅇㅇㅇㅇ

$z x z x$

z2 $2 \geq z$

$z x \geq z$

$x \geq z-2$

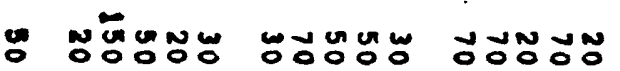

UN UNก

NONONO

Nan

OoOdo

$z=2$

- $=$

MNOLON

- $x=$

OOOONO

$z=2$

:

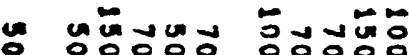

궁ํำ

s- 0 un

unumu

8

ununa 4añ

ำำ

acosian 응응응융응 $z=2 z$

$x=2$ $\leftarrow$

웅융ㅇㅇ

$\leftarrow$

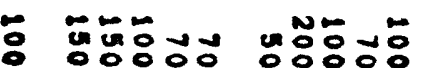

№:

UN $2 \div 0$

$\ddot{0} \rightarrow \ddot{0}+2$

o

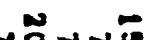

ㅜㅜ

으응

2

N Nom

NNN WN

NNNNN

WNN

NNND

$N \in \operatorname{Man}$

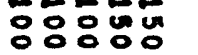

0ㅇㅇㅇ

a

-

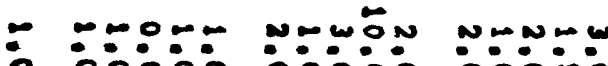

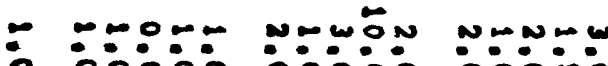

uncow

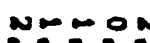

w山w几

NWNNN

coico:

$\div 00$

:0ं:i

$-0-0 \%$

OOO OON-O

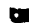

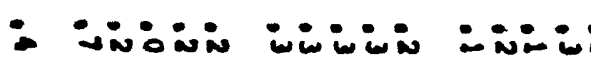
-

- - ONN NNNNN NNNNG 
6

- or on o w w w

$-\infty 0 N$

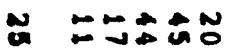

$\therefore \quad \therefore<\infty$

$\because$ woot

-

O NNONONONO

Nㅡ융ํㅇ

Nㅡㅇㅇㅇㅇㅇㅇ

Nํㅇํํํํำ

NNNNN

anasa

on a

a 200

- 0

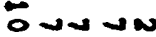

UNNWW

00000

rand and

Uun wow

00000

00000

שcusun an

00000

wnum

$\therefore 0: 00$

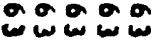

NONNNN

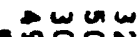

arog

مू์ N

ธักำ.

$: 00000$

unnun

ununon

WMUNM WLNWN

WNWW $\therefore 00 \%$

$0 \cdot 0 \cdot 0.0$

$\therefore 0=0$

Ww $000^{\circ}$

0000

$\omega \omega N \omega \omega$ $\because 0: 0: 0$

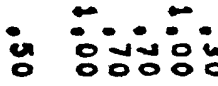

8

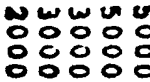

$: 0: 0:: 0$

$z \quad z x z z z$

영 영영명영

z $z \geq z z z$

$\overrightarrow{0}$

둥 뎡융명융

녕유융유.

ํํㅇํํㅇ응

응응융유

ํㅜㅇ용

응융영융

$\ddot{0}$

งN $N \omega *$

Nun MN

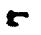

O ONONONONO

NNNNN

$z \quad z z 2 z$

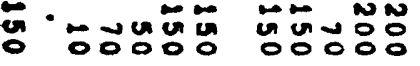
ㅁํㅇํํㅇํำ
UNNNN

$F$

NNNNA

$z 2 \geq 2 z$

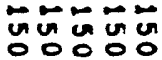

c $N$ on $N$

$\leftarrow$

บNNMN

$\geq z \geq z=$

的的 isin 00000

WNGN (1) 응융응

a 0

ค

$-\infty, \infty$ 00000

$z z \geq z z$

$\because \div \div=$ 00000

$z \geq 2 z=$

unumu

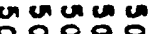
:ะ: $\geq 2 \geq \geq 2$  $z z z z z$

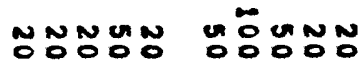
ODOOO

तேㅜㄷㅇ ㄷㅇㅇㅇㅇㅇ

\section{대웅ㅇㅇㅇ}

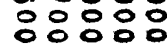

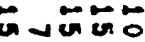
응응용

$\div: \div$ 00000

$z z z z z$

unu un un 융ㅇㅇㅇㅇㅇ $z \geq z z z$

WNNNN

능융ํํㅇ

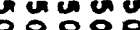
웅용유: $z z z z z$

$x=2$

둥 후 응영영응

2

NNNNN

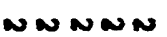
tr 5 TE

50

NNNNN

NNNNN

NONNN

$2=222$

22222

$z \geq 222$

NNNNN $x z z=2$

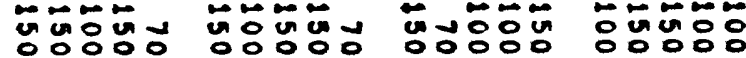


- 긍중ㅇㅇ

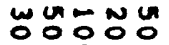

जnw- U

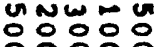

$=0 \sim 20$ जoㄴㅇㅇㅢ

ேோッா

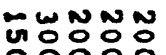

후는

ธํํㅇํㅇ ○००000

0000

onuun

ununo

붕ํำ

nugura

numun $=$

$$
z=
$$
zetzzz

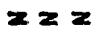

5

- ㅜㅜㅇㅠ Z2 Z2Z

유융

두융유

$z Z 2 z$

$z z z z z$

- yunún

ununu

x $\geq 2 \geq z z$

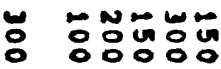

$z z z z$

요욤요

unumu

00000

z $z \geq z$

กักแับัธ

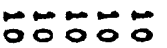

$z z z z z$

무유웅

$z z z z z$

unugung

unungu

$z z z z z$

$z z z z$

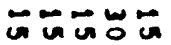

的药落它 ớc:

Nㅜ융

$z=z z$

두웅

$z x z z z$

vounu

$z z z z z$

non ungún

$z \geq z \geq z$

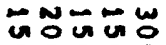

要动机

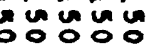

:

웅

$=$

8

nNNNG MUNGM.

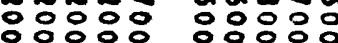

ㅇㅇㅇㅇㅇㅇㅇㅇㅇㅇㅇ

50unu ㅇㅇㅇㅇㅇ

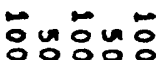

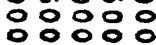
w영뭉웅

눙ํㅇㅇㅇㅇㅇ

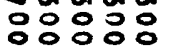

nonuman ㅇㅇㅇㅇㅇㅇ

nenum 영응ㅇㅇㅇ

un enc

응융유

둥응영웅

응웅융유

두웅웅 옹영ㅇㅇㅇ

영ㅇㅇㅇ

$2 x \geq 2 x$

$z \geq 2 \geq 2$

$x \geq 2 x=$

$z x \geq z z$

등옹응융응

$x \geq 2 \geq x$

훙홍형

$6222 x$

부에

ปูก

Nㅜ응

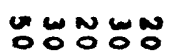

NNNNN

$=$

$z=$

$z=z z$

:

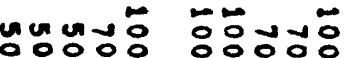
응ㅇㅇㅇㅇㅇㅇ $<x \geq$

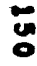

$\infty$

-NNMN

NNON

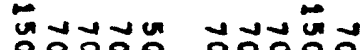

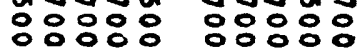

unu $u$ ㅇㅇㅇㅇㅇㅇㅇㅇ

노응

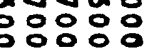

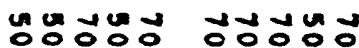

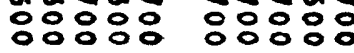

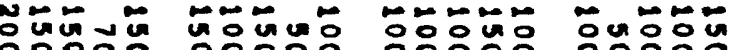

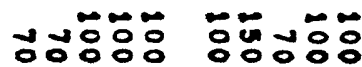

NNWN

N-NNM

MNNNN

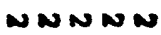

$\boldsymbol{N \sim \sim \sim \sim}$

$N \sim \sim \sim N$

:

-

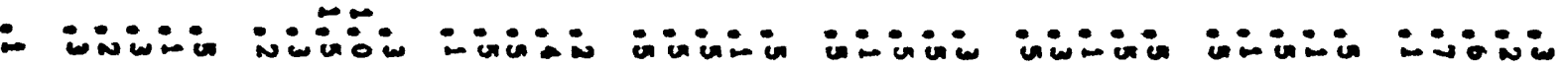


- 00500

00000

00000

00000

- 000 ON

NNNNN

UNN

nuo

N

- unuod

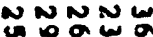

$000 \div 0$

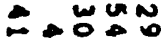

00000

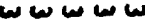

NNNNN

जि: ano. 0 ט ธேธே

UnNu - U⿺辶0

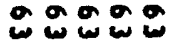

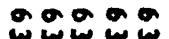

س

NNNNW

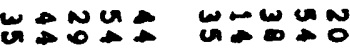

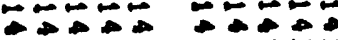

$\div \div \div$

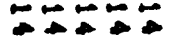

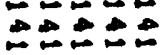

$\div \div=0$

퐁

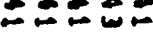

$\omega N \mathbb{N}$

중두

Nㅜㅇㅜ

○О

-

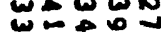

응 जú

vunum

0 :

-

م

Nㅜ융

W.

NONNN

an

NNNNO

ดคด

ana

w w N

w w w w

W $\omega \omega N$

- :0000

$\therefore 0: 00$

$\therefore 0: 0$

$\therefore 0: 0.0$

wunu

$\therefore: 00$

NNNNO

NNNNN

ดอด

nar a

NNONON

NNNNN

nasa

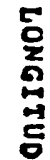

-

WWNNW

w w

W

ט w w w w

ww w w

네

MNUUN

:000 :000:

$\therefore 000$

$\therefore 000$

WNWN

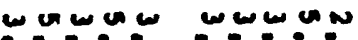
0000 00000

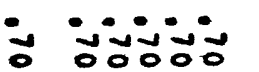

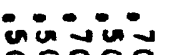

uninis

ن

univica

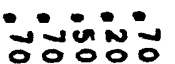

봉ํํㅇํㅇ

inin in 00000 कृष्ठ

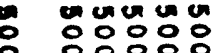
응응융

anMna

ununum

wnuw 응융응

ㅇㅇㅇ응ㅇㅇㅇ

옹ㅇㅇㅇㅇㅇㅇ

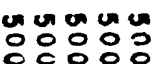

unnna

00000

ค

o

న

a

$\circ: 00: 0$

a na an

ก

a

wnus

잉ㅇㅇㅇㅇㅇㅇㅇㅇ

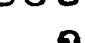

unun

응웅운두

ㅇㅇㅇ등영

กดด

$\div$ -

-

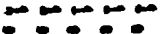

ம

$-\infty-\infty$

DrOH

웅웅

잉ํ

W以-

웅유

מRLR

$z z z z z$

$z z z z z$

웅우웅

$z \geq z 2 z$

$z z z z z$

anumun

un unn

anuma

ununu

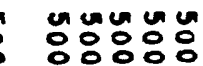

ununuan

unuunu

융ㅇㅇㅇㅠ

ㅇ:ㅇ:

응요요

$\therefore: 0: 8$

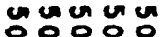

융유:

$z z \geq z$

$z z z z$

$z z z z z$

$\because 0 N-N$

งen ua

융즁ํํ

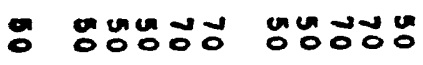

붕ํํㅇㅁㅇ 뚱유유

성ํํㅇ 5

- NNNEN

Nüuㅜ

ज药药

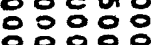

유:

$\circ: 0: 0$

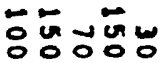

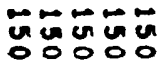
$\therefore: 0: 0$

oㅇㅇㅇ응

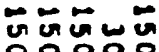
ㅇㅇㅇㅇㅇㅇㅇㅇㅇ

á⿴囗十⺝木

NNON 웅ㅇㅇㅇㅇㅇ

ㅇㅇㅇ응

$\frac{1}{2}$

:

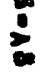

n nNNNN Nando

SUñon andun

Un Una

anunas

NNNN

ธே⿻上丨。

$\boldsymbol{c}$

○ ONONON

NNNNN

ONQNN

NNONO

NNNNN

N N N

NNNN

NNNNN

- zxzz2

$z 2 z 2 z$

$z=2 z z$

$z x \geq z=$

$z \geq 2 z z$

$z z z=$

$z z z z z$

$z \geq z \geq 2$

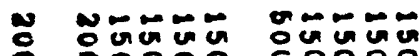

$=-N=$

$\because \div \div$

두두

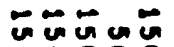

$\because-\infty$

-

oㅇㅇㅇㅇㅇㅇ

융ㅇㅇ융ㅇㅇㅇㅇㅇㅇ

00000

00000

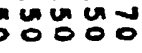

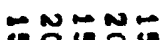

- WN-N

$\sim \infty-\infty$ 


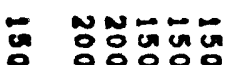

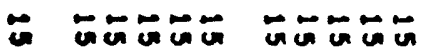

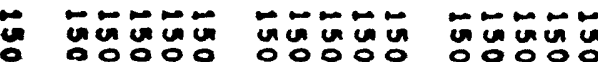

o.óo Gúñ:

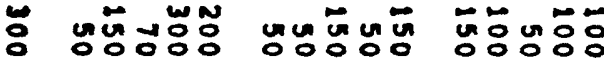
z

800

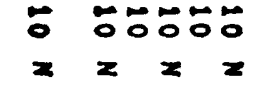
두웅

무유요

두응 z $x \geq$

- ánón

$z=2 x \geq z$

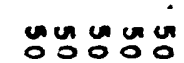
$z z \geq z z$ munn un $z x \geq z z$

NNN N N $\because N-N N$ 00000 o0ㅇㅇㅇㅇㅇ

Gencis o00
W. WN N N ะัะะั

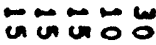

س 영영영

مَ

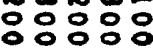
Ma ow

$0 \% N N N$ ㅇㅇㅇㅇㅇㅇ

U⿻一𠃋十

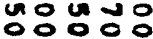

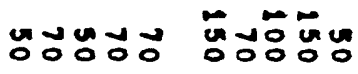
ैㅠㅇํํำ

웅

nis
WW UN 00000 00000

\section{- NONNN}

NNNNN

NNN N

NNONON

z $z \geq 2 z 2$

$2 \geq 2 z 2$

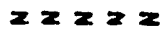

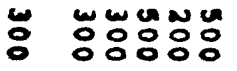

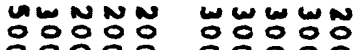

$z x \geq z z$

NONNN

$z 2 z 2 z$

NNNOั

$z 2 z 2 z$

NWNN

NNNNN OOOOOO

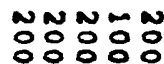

붕uํㅇㅇㅇㅇ $z z=$

\%

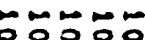

$z z z z$

unugung

$z \geq z z z$

$\begin{array}{llll}W & W & N & N \\ O & 0 & 0 & 0\end{array}$

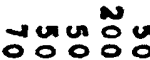

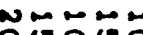
nouro $z z$

cựú $z z z z$

N. ㅇㅇㅇㅇㅇㅇ

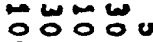
ตํํㅇㅇㅇㅇ 영융영 응ㅇㅇㅇㅇㅇㅇ

NNUNA

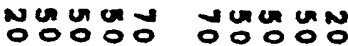

NNN NON

NNNNN

$z \geq z 2$

$\stackrel{0}{0}$ aํํㅇㅇㅛ

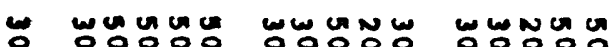

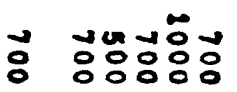

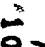

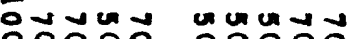
○0000

NGNUN 웅ㅇㅇㅇㅇㅇ

:

: 응영유유

둥융웅

ㅇㅇㅇㅇㅇㅇㅇㅇ

ungun

unumu

$\ln \pi 0^{\circ}$

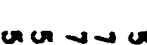

and

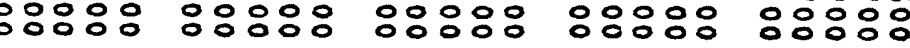

웅융유유

뭉웅융요

뭉유융

영영용

ํํ융유

후웅웅

$z \geq 2 z=$

$z \geq 2 z=$

trzx

zZz 25

$z 2 z x z$

$\div$ 융ํํ융

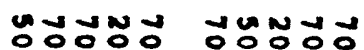

OONO

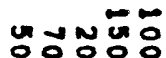

GNO

$z$

$=$

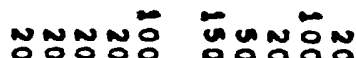
$x=2$

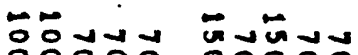
응응영융

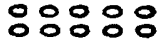

ㅊํㅇำㄴำ

거에융ㅇㅇㅇ

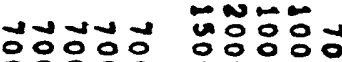

: 둥ํํ응

\section{뚱후웅}

느눙유

$\because$

구유요

눙우

4

UNO

붕ㅊㅇㅇํㅇ

$\omega \boldsymbol{N} \sim \boldsymbol{N}$

N NーM

$\omega \sim \infty \omega$

$\boldsymbol{N N} \boldsymbol{N} \boldsymbol{N}$

$\boldsymbol{N} \boldsymbol{N} \boldsymbol{N}$

$N N N \backsim N$

$\because \div N-\infty N=-\infty$

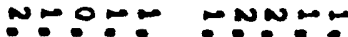

$\omega-\infty \omega$

$-\infty-\infty$

$\because 0: \div$ incis: 00000 0000: 010000 invivir 0 Ni:0

- 


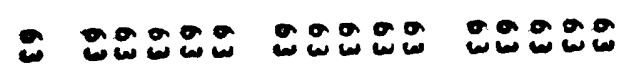

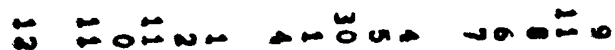

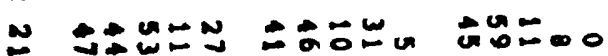

s.t.

$-\quad 2=0$

$\rightarrow \quad \omega \infty$

a on $\cos$

$\sim-\infty$

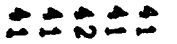

$+\infty+\infty$ N๐o $\infty \rightarrow \infty=0$

мate

ம 100

$\rightarrow \infty+\infty$ $\rightarrow+\infty \pm$ - $\omega-\infty$
운 운요 두운

W. or or on

-

ज政

W
- 0 a 0

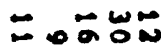

$N \rightarrow N$ un

$N \rightarrow$ a e u
웅요

$\therefore \approx$

a c a

-
- ․․웅

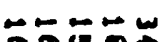
Unー $\rightarrow-\infty N$

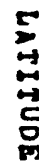

$-\infty=\infty=$

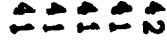

ע

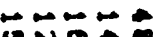

$=\infty+\infty$

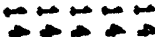

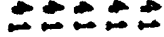

- $\rightarrow-10$

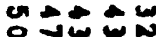

는

$N \leftarrow+$

w un

NDOUN o

ดัONกN NONONONO
NONNN

an a
NONONO

a

$\Omega$

NNNNN anas

w w w w

- concon connum

- 0000: 0:000

:००:०

W :0:0:

andand :0:0:

W

N

w w w $\div 0 \div 00$

W W W 000

waven :00\%:

$\omega m \omega n$

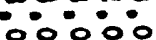

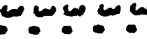

NONGN

NONONO

ana 0

DOSAR

NNNN:

ด00?

ranne

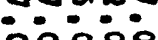

ancus a

:00\%:

我

- :-

- viniv uninis

c

\section{$:$}

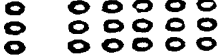

a $\Omega$

$\because \div \div \div$

- 0000

$z \quad z \geq z \geq z$

injus

anuna 응ㅇㅇㅇㅇㅇㅇ 응영융융

?

$\rightarrow$

$\because: \div \div$

$z \geq z z 2$

unum $z \geq z \geq z$

MUUNon 00000 $z \quad z \geq \geq z \geq$

- anuma

영영영

- 영ํㅇ

unnu

ㅇㅇㅇㅇㅇㅇ

ㅇㅇㅇㅇㅇㅇ

a n

$\because \div \div \div$

00000

$z \geq z z$

dununa ㅇㅇㅇ영

$z \geq z z$

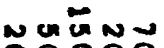
00000

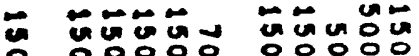

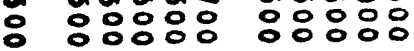

n aninan aunan EE

○ Nㅠㅇㅠ NONONO

z $z z z z z$

- 윙ㅇㅇㅇㅇㅇ

누웅

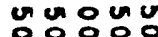
00000 영웅융

Wㅜㅜㅇ

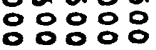

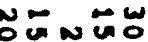

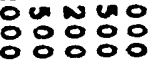

Uñ ㅇㅇㅇㅇㅇㅇㅇㅇㅇ

불ㅊㄹ

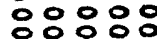

NNNNN

UNNNA E

ET

NNNNN $z \geq z \geq z$

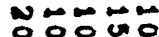
웅영영

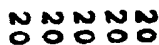
$z=2 z=$

जn $\rightarrow$ Un ज्ञ numun

NNNNN $z z x z z$

uㅗ뭉 잉유
$N \boldsymbol{N N N N}$

$$
C
$$

NNNNN

$z=2 z=$

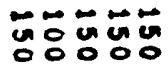
웅: $\because M N=$ 웅ㅇㅇㅇㅇㅇㅇㅇㅇ 웅ㅇㅇㅇㅇㅇㅇ

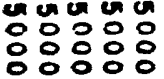

ค $\Omega \Omega$

$0 \%$ $z z z z x$

unumu 응: 0 : $z \geq z z z$

INNDU OOOO

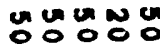

$\div \div \div \div$ $z z z z$

ununa ㅇㅇㅇㅇㅇㅇ응 $z z 2 z z$

-N N N

GOO ㅇㅇㅇㅇㅇㅇㅇㅇ

NNNNN

5

NNONN $z=2 z=$

NNNNN $z \geq z z=$

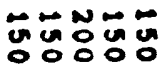

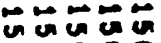

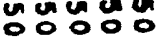

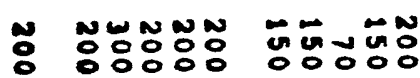

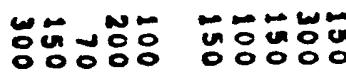
Oजㅛ용

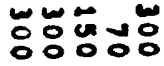

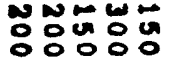




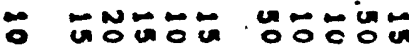

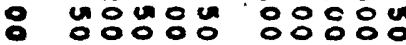

กีํํ응

กำกับ ดัดัด

a cusaro $z \geq x$

ตัตง

unuño

unumo

- ㅜㅇㅇㅠ

$z=$

$z$

$z \quad z z z z z$

무웅

$z z \quad z$

두ㅇㅠㅜ

- cunum

unusu

$x \quad z z z z$

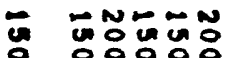
$z z=z z$

두웅

$z z z z$

núuún

unu un un

$z z 2 z$

$z z=2 z$

ज比药 óo

ज征 잉응

- 중ํㅇ

Nann

naw a

w늠 $z=$

$\%$

- NNNNN

NNNNN

NNNNN

z $2 x 22 x$

$z=2 z$

$z x \geq z x$

N WNNNN

NNNNN 응ㅇㅇㅇ응

ํํㅇ

NNNUN ㅇㅇㅇㅇㅇㅇㅇㅇ

눙두

n $z z 2 x=$

UNNNN

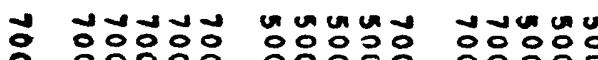

ununo

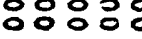

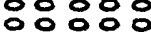
영ㅇㅇㅇㅇ

누웡 ㅇㅇㅇㅇㅇㅇ잉

두웅

$z z \geq z z$

unuug

z z z z

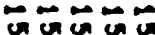

वी

ํํำ

nom un

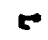

두웅

$z \geq z x z$

uanum

$z z z z z$

NEN N

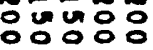

UWN-N

unur

00000

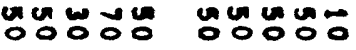

NNNNN NONONO NONONON

zx zxz

$z x \geq 2 x$

NNNNN

$z=2 z=$

NNNNNN

$z=2 x z$

N

WNNNN

N

OOㅇㅇㅇ

잉ㅇㅇㅇㅇㅇㅇ

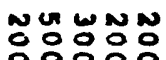
잉잉

충ㅇㅇㅇㅇㅇ

ununa

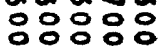

구눈ํํำ 응영융

등ㅎㅇ우응

둥ㅎㅇ웅

두응

응 형영융 형영영융

웅형융

홍ㅎㅇ영

$z z z z$

$z z z z$

$z x z=x$

호융유

$\cos 2 \pi$

NNUNN

ins

$=2 E$

GงunE

$z=2 z$

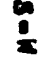

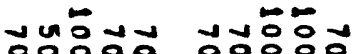

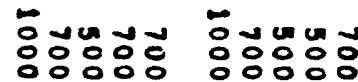

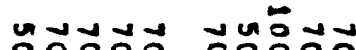

$\div 5000$

89800

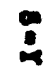

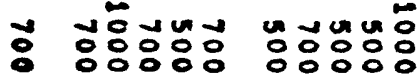

응영ㅇㅇㅇㅇㅇ

영ㅇํㅇ용

응ㅇㅇㅇㅇㅇ

둥주유

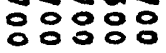

:

-

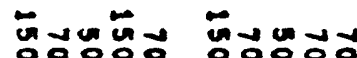

후우두

두응

-

N N N O

영영명 녕영응

:

a NNNNN WNOMn

NNWN

- N

UNM⿻上丨

MNNMN

MกNNM

$\boldsymbol{N N} \boldsymbol{N} \boldsymbol{N}$

:

- NONE -NONH

N-N- -

-

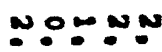

$-\infty-00$

$\therefore \div 0$.

$00 \div 0 \div$

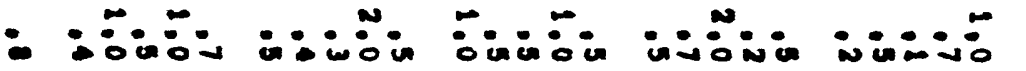

$\because \because \div:-$ $\because \div$

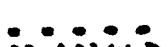<smiles>[13CH3]</smiles> 


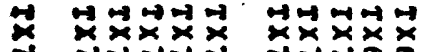
$\sin _{i=0} \vec{n}=\vec{~}$

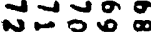

osen in en

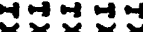
즘중 con on

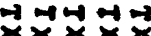
중중주

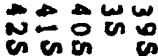

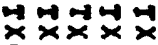
증즘즈 waw cis en en हn

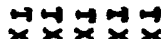
즘중

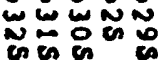

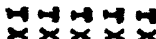

$2 \times x \times x$ NNNNN ân

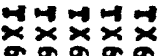

नू०

No⿺ in i

$\cos 00$

\&

ה

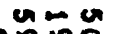

$\propto$

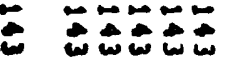

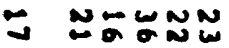

O

O NNONON NNONON NOEONO

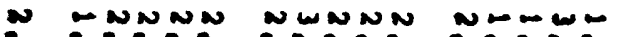

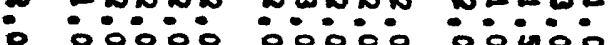

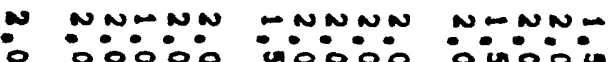
unO00\% is o : o 0

- añ

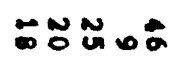

$\sin 000$

NWONN

an 0 \%
운요요

-

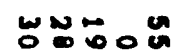
- $\rightarrow 00$

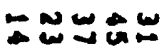

- 0 wis

w-

ש

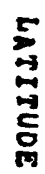

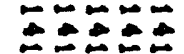

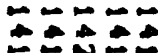

م

ט

ज़ण

$\rightarrow W=\omega N$

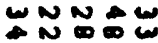

N 00 م

NU⿺𠃊一兀

๑๐ั0\%

두융

Non
ONONN

ana a

NONNN

anaso

NNNDN Oด

ona 0

NNNNN OOOC

NNNNN $\Omega$

s $\Omega$

$\sin 1000$ ununa $\log 2 \pi n$ ०0:00

MnUn $\therefore: \therefore 0$

UUnum 0:000

W W N w

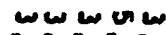

W

WUMn ט

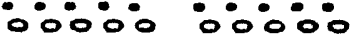
00000 00000

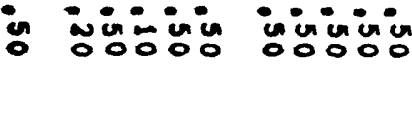
영영융ㅇㅇㅇㅇㅇㅇㅇㅇㅇㅇㅇ

nNGMN 옹ㅇㅇㅇㅇㅇㅇ ㅇㅇㅇ응

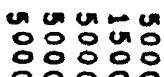

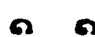

$\because \div 0 \div$ 잉잉

०0i:

$z \geq z \geq z$

$\because \div \div$ 00000

$z \geq \geq z z$

$z \geq 2 \geq z$

:

$z \quad z z z z z$

: 충ㅎㅇㅇㅠ -

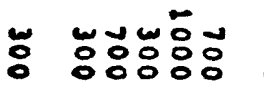

in un un un

$z x \geq z=$

능ㅇㅇㅇㅇㅇㅇㅇㅇㅇ

$z z z z$

$m+\infty$

०0000

$z \geq z z z$

00000

$z z \geq z z$

ununu

응ㅇㅇㅇㅇㅇㅇㅇㅇㅇㅇ

a คan

옹응응응

(0.08

aी० 0

$-\infty-\infty$ $\div 0000$

$z x z=z$

unung 응융융여

unumu

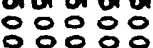
$z \geq z z=$

$z z x z z$

unumo 영영유 $x \geq x z=$

둥유에

뭉무

U UñON

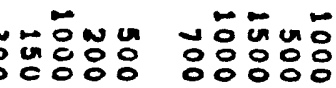

내용

붕ㅎํㅇํㅇ

บ N 응ㅇㅇㅇㅇㅇㅇ

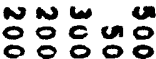
ㅇㅇㅇㅇㅇㅇㅇㅇㅇ
约落

inis

wunnu

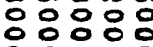

(

ตุำ

응융융유 웅응ㅇㅇㅇ

a

- 웅웅

$z z z z z$

$\because 0000$

$z z z z z$

un unu 영ㅇㅇㅇㅇㅇㅇ $x \geq z \geq x$

un un un

웅응응

$z z \geq z$

ano:

UNO

6

부운

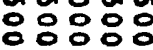

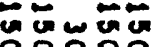
ㅇㅇㅇㅇㅇㅇㅇㅇ $\boldsymbol{c}$

บ NON N

$z \geq z \geq z$

- 둥후웅

NNNNN

NaUnN

UNNNN

GTMNon

NNNNN

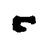

NNNNU Ct

NNNNN

Nㅜㅇㅛ

NNNNN

NNNNN

z z z z

$z \geq z z z$

NONONON

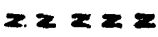

NNNNN

$z z=x$

붕붕웅

-

$-5=0$

드눙ㅇㅇㅇ

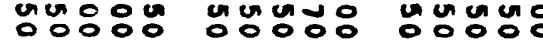

NNNNN

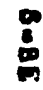

NON N

$z z=z$

두웅

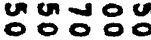


ษேㅜㅜㄴ

o ganougo

- 5

- :5u50

$z \quad z z z z$

- uñóñ

z $\quad z \geq 2 z$

: ํㅡㅁㅠ:

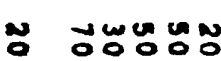

- NOMNONO numun 00000

ㄷํㅇํำ $z x \geq z$

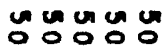
$z \geq z \geq z$

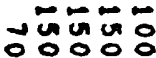

NNNON $z$

NNNNON

- NNONON

NNNN NO

$z \quad z z z z z$

$z 2 z 2 z$

용ㅇㅇㅇㅛ

- unmún

\& $80 \%$ 영

$-$

re

ค n n N N

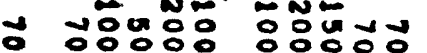

N NNNDN NNNNN

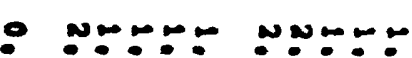

$\infty$

$\div \quad: 0: 0$

-

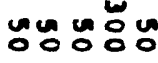

- 5

후웅

uñ ñú

$z=z z z$ 이잉

트유유

NNNEN

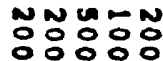
c

Nan응영영 $x$ z

붕ํํㅇํำ

두웅 -

둥ํํ

$z z z z z$

ununuñ

$z z z z$

붕ํํㅇํㅇ

$z \geq z z$

NNNE

Nㅜ음

잉의

녕영녕

U든

붕영영

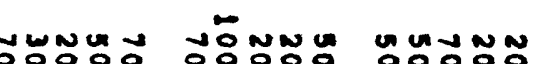
5

ONONON

NONON

NNONON

NONONO

$z \geq 2 \geq 2$

$2222 x$

$z \geq z=2$

NNNNNN

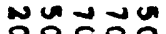

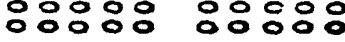

WONONO 응ㅇㅇㅇㅇㅠ

NONON $0000 \%$

NNNNN OOOOO

NNNNN OOOO ONONON $z=2 x=$

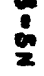

anu

und

눙으융ㅁㅇㅇ 응응용

응융ㅇㅇㅇ 응영ㅇㅇㅇ

ans 영휴영유

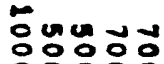

NNNNN 응융요
등응영융웅

$z=x z$

응형ㅇㅇㅇㅇㅠ

$z 2 z 2 z$

동웅융유

$z=z=$

ேㅜㅇㅎㅇ웅

00000

ONOOOOO OONONO

NONO

$z=z z=$

ดNNNO

$5=$

눙

눙엉ำ 00000

ㅁํㅇ형

누웅

งunus

눙후

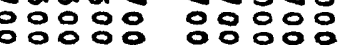

ㄸำ

영영드융 영영융융

흥응웅융응

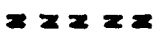

:

№유:

$z=2$

=

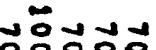
응영유

능ㅇㅇ

$\because \mathfrak{O} \div \because$

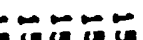

nーMN 1000 0000

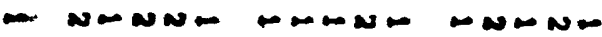

UNッー

00

N NNO

$\omega \sim \omega N \sim$

$\omega \sim \sim \sim \sim$

$\cos 20$

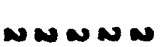

है

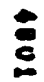

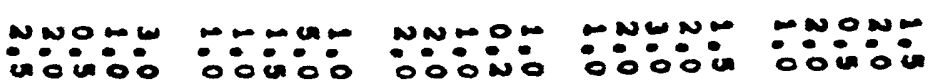


운 9090\%

arog a o

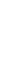

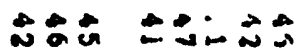

in $\begin{array}{ll}0 \\ 0\end{array}$

जुN

พั0

훈aㅎ

$\therefore \rightarrow \overrightarrow{0}:-$

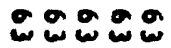

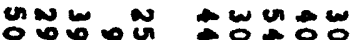

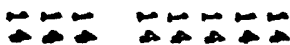

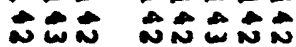

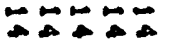

$\omega \infty$

مू

NลNNAN

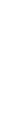

Nล⿱士心N心

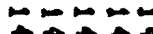
שָ

ㅎำ

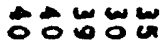

$\because \omega u \neq u$

NNA

U $\rightarrow$ Un

N

UNUNa

NONNON

NONONO

NONOON

○

กแก nกnกn

NNNNN 00000 $0: 000$

NNMNN

NNN WN 잉iㅇ 유:

WN

W

$\therefore \therefore$ $\therefore \circ:$

$\because 0000$

0000

:0\%:0

iañ

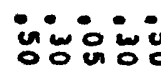

inivio

\%

ini:-

ivivinis

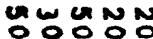

응ํำ

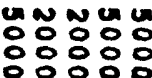

Nㅜㅇํํ능

๑ $\Omega$ ००० :००:०

$\therefore: 0$

$z x z$ 두웅

מ-R $x \geq x \geq z$

0000 $z z z z z$

unu unamu

용응

$z \geq z$ 응유:

$z z \geq z$

unumu

응영웅웅

$z z \geq z$

ํํㅇํํำ 융ํํㅇํํ -

$\div-5$ 잉

\section{Evn} ㅇㅇㅇㅇㅇㅇㅇ

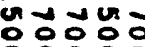

ㄸํㅇㅂํㅇํㅇ

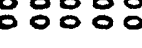

ตNN NGWN

ce

$$
5
$$

แ $\boldsymbol{N} \boldsymbol{N \omega}$ cote

Nㅜㅇํํ

$\because N-N$

$x z=$ $z=z z$

NNNNN $z=z z=$

مै

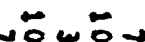
ஃะัะว

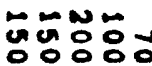

$:=0: 0$
000 in:
$x=2 x=$

un unnu

웅ㅇㅇㅇㅇㅇㅇ

$x \geq 2 x=$

$a n$ ํㅜㅇ $z \geq z \geq z$

encunun 웅ㅇㅇㅇㅇㅇ

$z z=2$

ธัละวะ

ลังัว

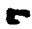

어웅ㅇ

훙충ㅎㅇ응 영융응

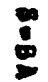

ง $\boldsymbol{N \infty \boldsymbol { N }}$

TEE

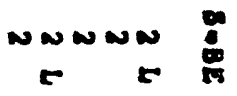

ONON-O

NNNONO

6

$z z=$

$z z z z=$

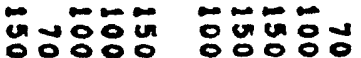




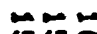
ํํㅇ

두웅 ํํㅇํํㅇํㅇ

ज征穴

范- Nم

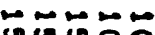

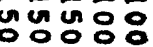

$\ddot{2}$

on

a a의요

- 5

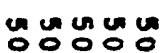

뚱웡ํํㅇ

nugunga

$+$

55

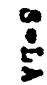

뚜웅

$z z=$

can

$z 2 z$

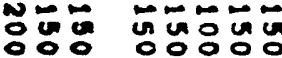

00000

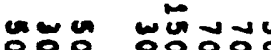

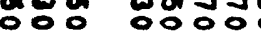

ํํㅇ

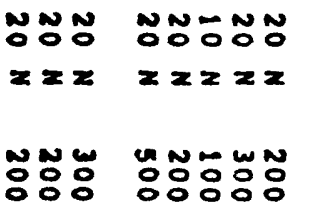

롱ㅇㅇㅇㅇㅇ 융응용ㅇㅇㅇ

형융 뭉뭉

$c=2$

$z \geq x \geq=$

ํㅜㅇ

훙ㅎㅇ

$z z z z=$

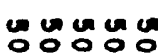

$z z x z$

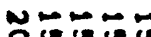

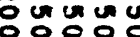

뭉 以ㅜㅇ

$x \geq 272$

붕후웅

$x \geq z$

붕ํํㅇㅇㅇ

$z x x z$

जưño

$z z \geq z=$

뚠두

ت00

뭉영영:

?

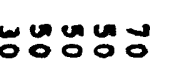

40

ํํㅇㅁํㅇํํㅇ

UีUn

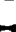

NNOOOONO NOEOOOO

:

NNNNN

O

NNNON

00000

NON N

$z z z z x$

$z z z z z$

mann

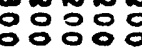

응ㅇㅇㅇㅇㅇㅇㅇㅇ

5

WNN

응융ㅇㅇㅇㅇㅇ

ํㅜㅇㅎㅁํㅇ

un a d a

옹영웅용요

$\div$

홍훙훙융

ㅜㅇㅇ뭉

Бேே0ே0

$z 2 \geq 2=$

$z z=z$

$z \geq z=2$

:

뚱ํㅇ

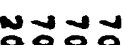

\section{政}

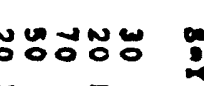
-

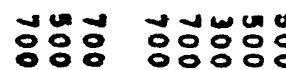

เัตัลัวัดั

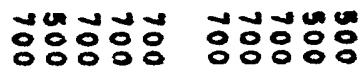

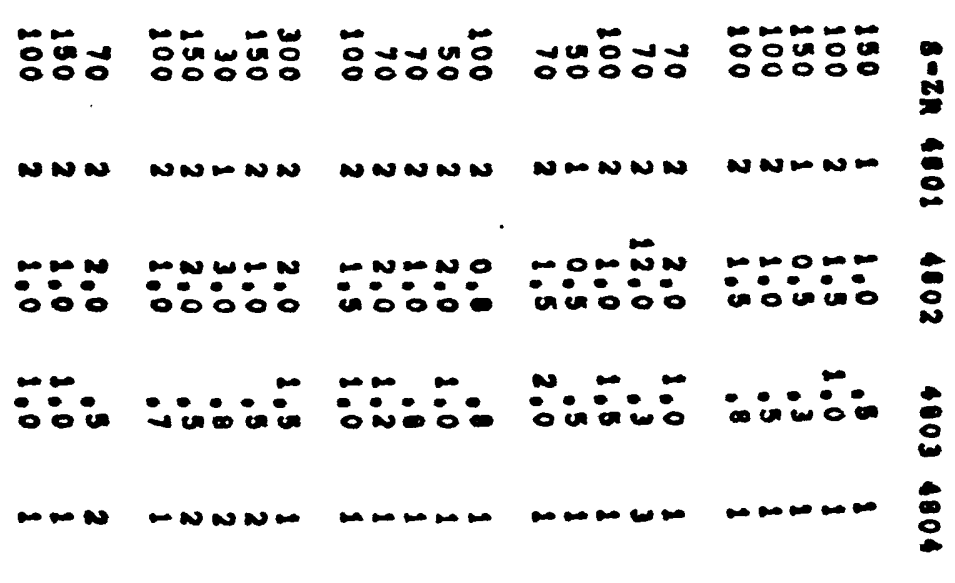


Ash of Moss:

S-CA was not looked for. Elements not analyzed: $A A \mathrm{Au}, \mathrm{Cu}, \mathrm{Pb}$ and $\mathrm{Zn}$; INST-Hg; and CM AS.

Elements looked for but not found: S-Bi, S-Nb, S-Sb.

S-As was not detected except in sample TX 606VC (500 ppm). 


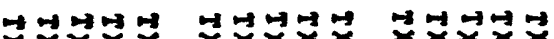
xхxхxх $x \times x x x$ 

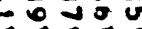

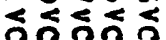

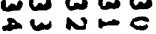

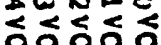

$x x \times x>$ NNNNN กัธ่์

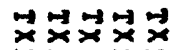
NNNNN AWN=3

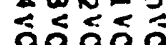

-1
$x$ $\therefore=5=$

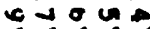
ถุํำ

$\rightarrow \underset{x \rightarrow 4}{x \rightarrow 4}$ $\therefore=\approx 二 0$ แบ눈 웅웅영 $x \times x=$ ถุํำ

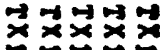

00000

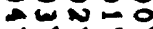

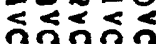

aoa onoo

b a

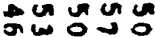

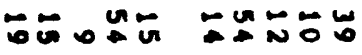

- a o a ט coñ unusun aro a o o uncon en $\rightarrow 0 \rightarrow \infty$ $n \neq u \in \tilde{c}$
00000 uncus un $\omega \rightarrow \infty \in$ NOMNO
웅요요 w van

-
00000 (4) जि जी 류유
00000

하영

능요

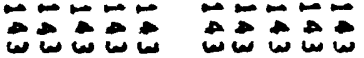
$\ddot{\infty}=\infty$ Numun 00 000 $\rightarrow \div 2=$ ش ONN $\approx \frac{0}{0}$ ต $\rightarrow \infty \rightarrow \infty$ CNUNO Nㅡㅇำ $\rightarrow=-$ ONNN年 - 0 แ

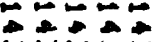

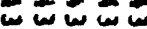

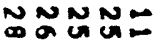
๑ దี้

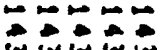
م NNAN $0 \div \div 0 \%$
투ㄴㅡㅜ

NニN心 ०ै
0
2
2
ê

$\div$

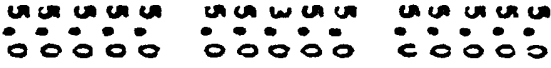

Da $a$ an $\operatorname{lng}_{0}^{0} \ln 0$ -0000 มด ununa c000: מลกล unumu 00000

na 20

unumu 00000 20000

wan un 00000 DSa

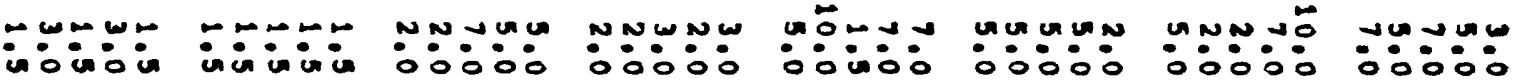

iniviv ininin inion

inin

○jini

넌 in

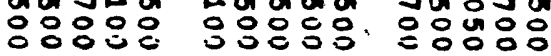
ㅇㅇㅇㅇㅇㅇㅇㅇㅇ . 000000

ninin: 영영응

winis 응ㅇㅇㅇㅇㅇ

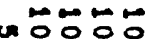
둥ㄴ

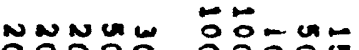

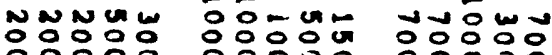

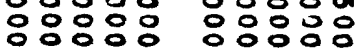
○

ก

คด

$\Omega$

누을 응웅응 응응ㅇㅇㅇㅇㅠ 응ㅇㅇㅇㅇㅇㅇ

둥

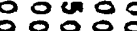
응ㅇㅇㅇㅇㅇ

คa

uㅜ웅웅 ㄷㅇㅇ영 옹융용 ? 20

00000 inis inivi bisivi Niwin in is:

inis

inivis

is $=0 ;$

NMNNM NNNNN NANNN

NNNNN

NNNNN

$N N N N N$

NNNNN

NNNNA

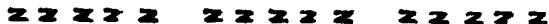

$z Z \geq Z Z$

$x \geq 2 \geq 2$

$z=2 z 2$

$z \geq z \geq 2$

$z 2 x \geq 2$

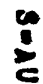

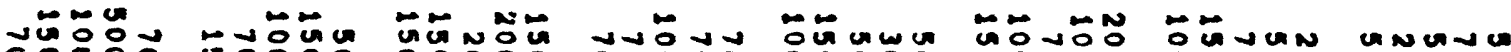
Oㅇํㅇㅇㅛ

:

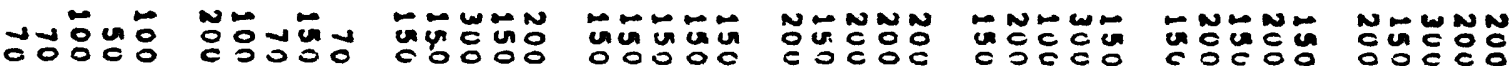

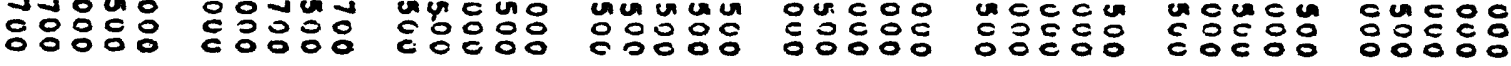




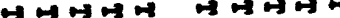

$x \times x \times x \times x \times$

屾

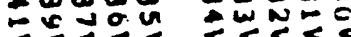

$\vec{x} \vec{x} \vec{x} \vec{x} \vec{x}$

NลÑNN

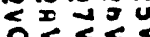

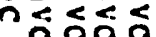

$\vec{x} \vec{x} \vec{x} \vec{x} \vec{x}$

NNNNN

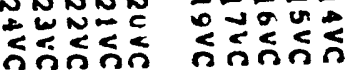

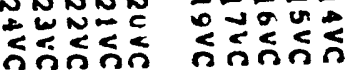

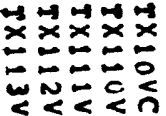

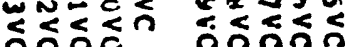

$\begin{array}{rl}-3 \\ x & x \rightarrow 4\end{array}$

$\div 0 \div 0 \frac{0}{0}$

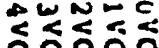

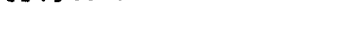

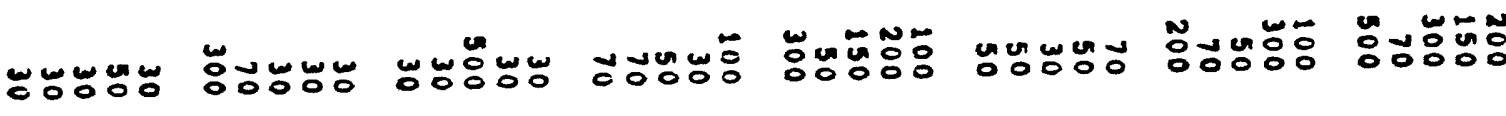

$\stackrel{8}{8}$

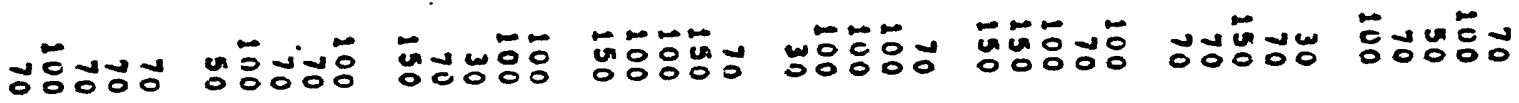

ְ.

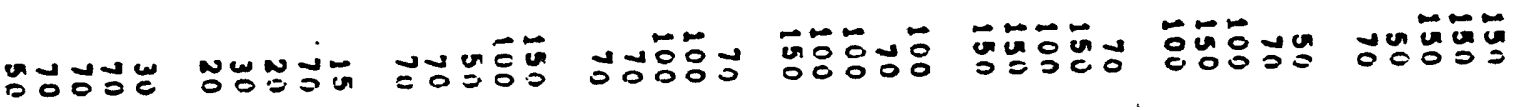

$\stackrel{n}{2}$

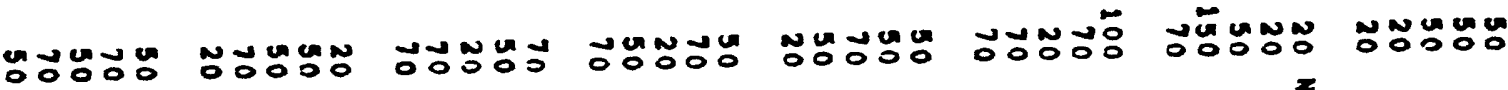

5 z

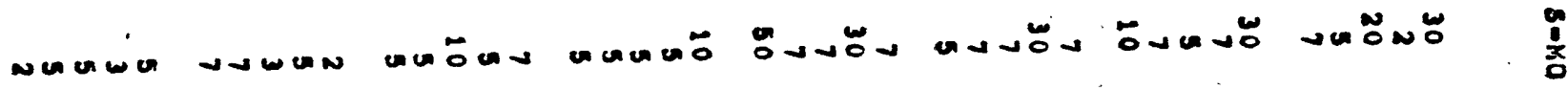

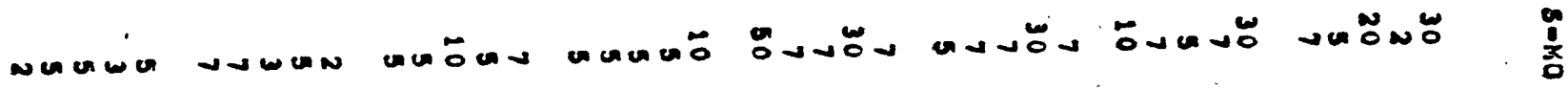

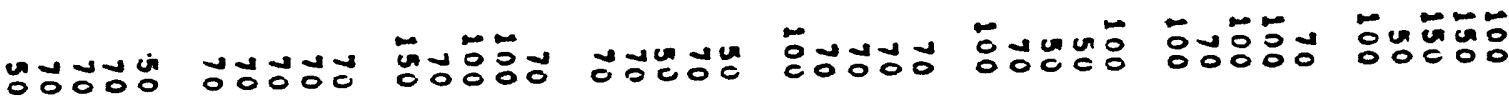

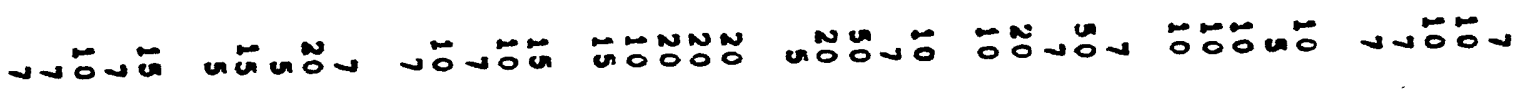

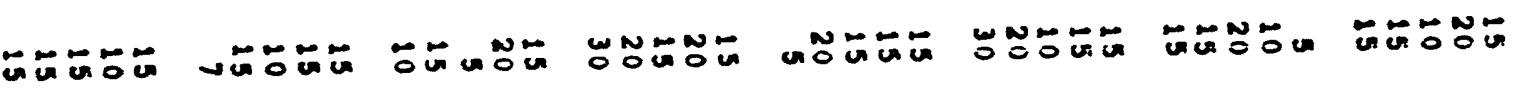

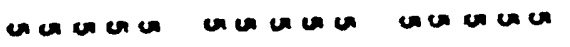

$=2$

는

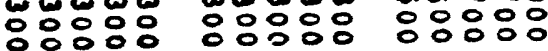

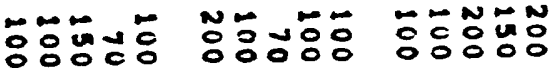

on unu

$z \geq z \geq z$

unum an

$x=\geq$

un un u

unumu

coñún

$z=2 z$

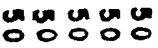

$z \geq z 2 z$

on 000

unumu

w

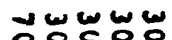

was

num

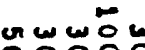

동ํㅇ응

unแas

$z 2 z 2$

$x \geq z 2 x$

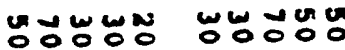

N

웅융유:

an n w w

ㅇㅇㅇ응응

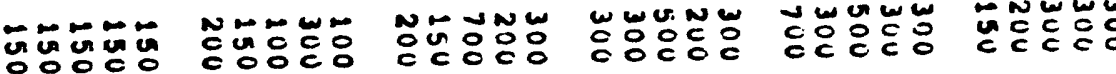

coco

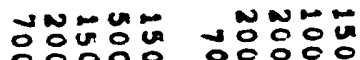

c⿻ำ

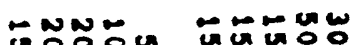

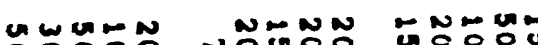
ㅇํㅇํํㅇ ํํㅇㅇㅡ

00000

:

$-\omega \sim \boldsymbol{N}$

w

N๐。亩

문유 
- $0900 \%$

ט u un a v

- 0 DNN

$\sim N N \mathbb{N}$ $a$
6
6 un on u u 足:

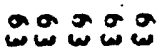

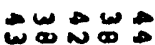

N口药 in a on w: $\rightarrow \leq=$ $\omega-N \omega u$ $00100 \%$ aro or on

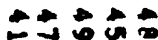
w u

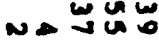

- o. on or un un a

$-\infty=0$.

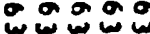
u un un a vis - जus. 0ே0
운웅웅

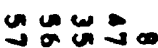

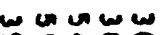

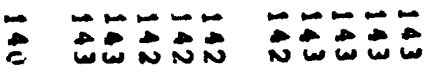

モニモニロ

듬

-

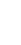

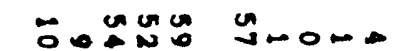

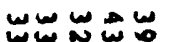

- un

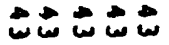

$\because ே \div$ W

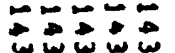

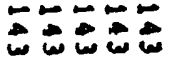

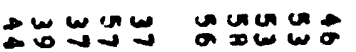

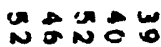

un a co

$\omega \mathfrak{0}=0 \div$

故范造

० w un

$\rightarrow N$

N
- año:

num un un :00:0

$\infty$

นas

ununu cicio

un un unca

ununu -0000 cio:0 nas a ac

$\Omega$

กดด

un unu un :00:0

ค $\Omega$

w wunu unumu :00:0 :00:0 ก0ก0

-
nNMUN $\therefore 0: 0$
W

:000:
WOOOO

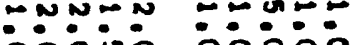
O0:0in: 00000

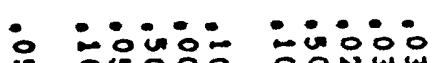

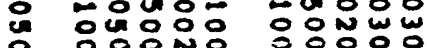
UIUNN 80000

눙

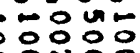

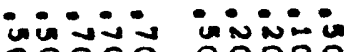

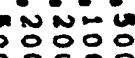
응응응

눈

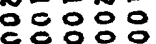

ज陋宁

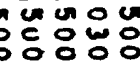

\section{d}

후유.

웅ㅇㅇㅇ 응ㅇㅇㅇㅇㅇㅇ anas

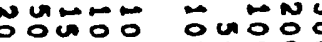
응융유 영ㅇㅇㅇㅇㅇ 영영영

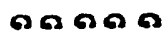

- 00000 00000 0000000000

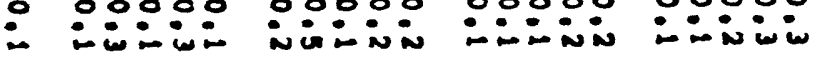
5

טNNNN N NNNN

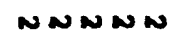

N $N \boldsymbol{N N}$

$z=2 z 2$

$z=z z=$ $z=2 z=$ $\omega \sim \sim N N$
$N \sim \sim N \Omega$

$z \geq 2 \geq 2$
טNN N

$z z z z z$
NNNMN

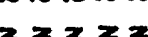

2

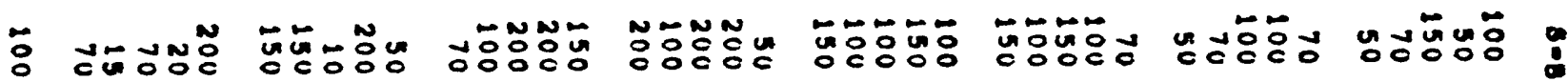

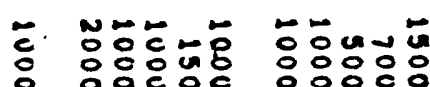

ํํํำ 웅응ㅇㅇㅇ
거융ㅇㅇㅇㅇㅇ 응융융융

궁중중귱유 $z=z 2 \quad z=2=$ 


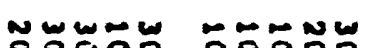

응웅유

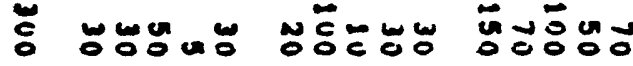
$\mathbf{z}$

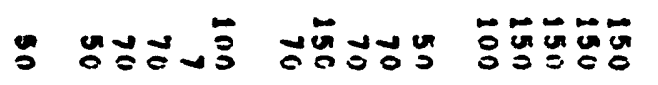

N NONON NOOOOOOO

$z=z z=z=z$

$\mathbf{x}$

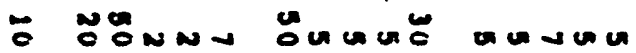
$z \geq$

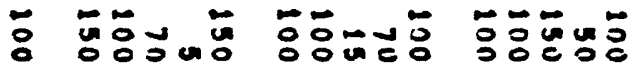

-

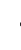

4

2

م

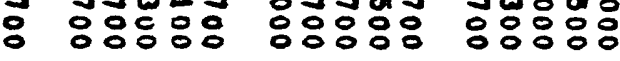
2

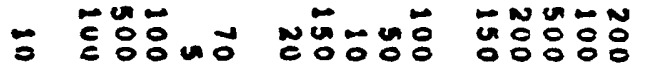

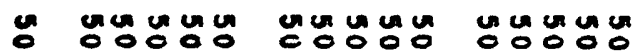

$z=2 z 2 \quad z z 2 z 2 \quad 2 z 2 z 2$

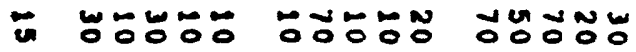
$=$

$$
x=
$$

N

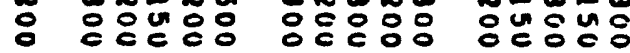

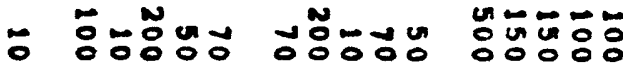

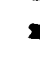

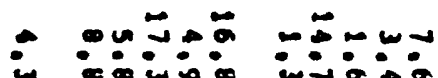

a o. $00 \div$

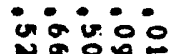

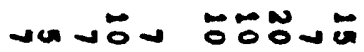
$z$

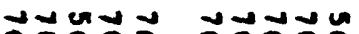

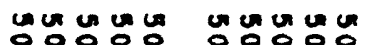

$z z z z Z z z z z$ $z$

NNHN NNNMN

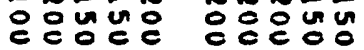

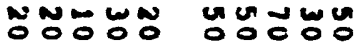

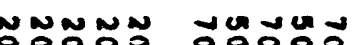
$z=2$

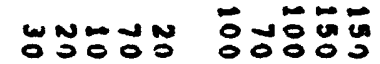
눙웡융

웅ํำ 므요요<smiles>C=CCO</smiles>

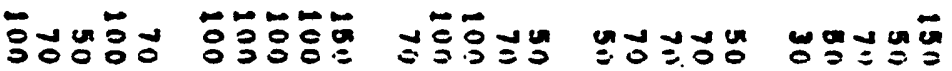

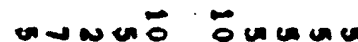

son on so

an $\operatorname{ang} \cos$

co

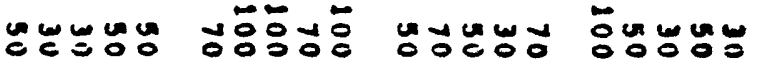

$\overrightarrow{0} \overrightarrow{0} 0 \overrightarrow{0}$

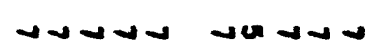

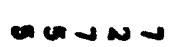

ง

unumar anouna $z 2 z \quad 2 x$

unuma Cr $2 \geq 2$

unouna $z x \geq z$

cosen a co

$x \geq 2 x=$

wu n w w w

w

눙요

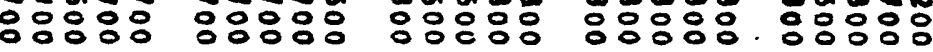

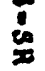

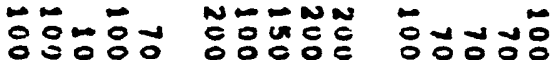
تُك 둥요 잉ㅇㅇㅇ yonguno

$z 2 z=2$

wo no:

Nேー cuñ van unos $2 z 2 z=$

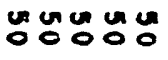

$z \geq z 2 z$

뚱ㅁํ:

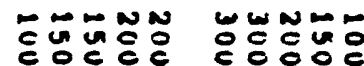

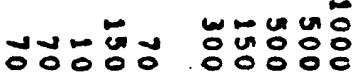

눙뭉

웅웅영붕융

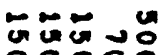

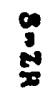

두ㅇㅠㅜ

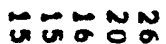

บNละO

$\dot{0} \sim \approx=$ :

$\underset{0=-\infty}{* \infty}$

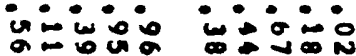
$\div \div=$

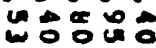


- 둔운운

00000 ம

w w w w w

acoso

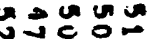

$\because$ oUñN

$\therefore \quad \square=5 \div \div$

$\because \div \div \div$ שN

$\Rightarrow \div \div$ NลNNN

๐NN

ำล

NN

든

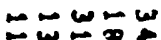

ผ N

N. $n=0$

แผ

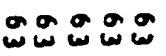

$u=m u n$

wa
‥ 운

a $\infty \ln _{0} \rightarrow \infty$

NUNND

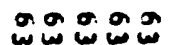
טan:

uャ-

군문 : NOU

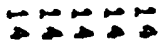

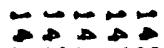

$\because \div=0$ NNNNN

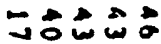
GNa טルー owo = سู - ถู ט 0 N טص山

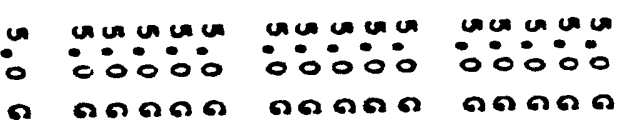

un unu 둥웅 ununu -0000 20620 unumu :000: cosar unuma 00000 ค月ล0

ununu c000\% ด्व

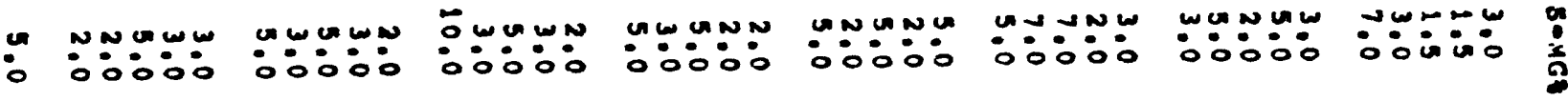

- initio 영 영용영 unini

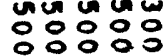

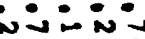
응ㅇㅇㅇㅇㅇㅇ i湿法

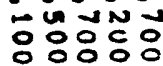

- ivini vinunivion

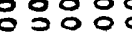

uninu 잉ㅇㅇㅇㅇㅇㅇ inisio

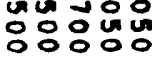

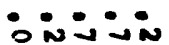
봉응용ㅇㅇ

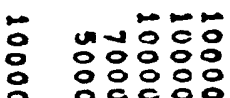

○
두응유

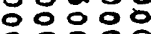
영ㅇㅇㅇ영

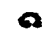

응훙응응 잉응ㅇㅇㅇㅇㅇ $\circ 0$ 영응

a D

\section{동ํㅇㅇㅠ}

ㅇㅇㅇㅇㅇㅇ응

00000

a 0

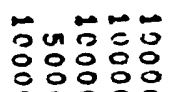

ana
긍ㅇㅇㅇㅇㅇㅇㅇㅇㅇ

응ㅇㅇㅇㅇㅇㅇ

a $a$

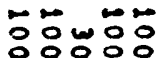
옹ㅇㅇㅇㅇㅇㅇ 영영영

a

무우웅 응응응ㅇㅇㅇ응 a $a$

- 00000

00000

00000 00000 0:00: 0:?:0

0000000000

in 000

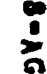

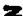

N NNNNN

$z=2 z 2 z$
$N N N N N$

MNMNN

NONNN

NNNNN

$2 \geq 2 \geq 2$

$z \quad z=2$

$Z Z Z Z Z$

NNNNN

NNNNN

NNNNN

$z \geq 2 \geq 2$

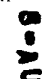

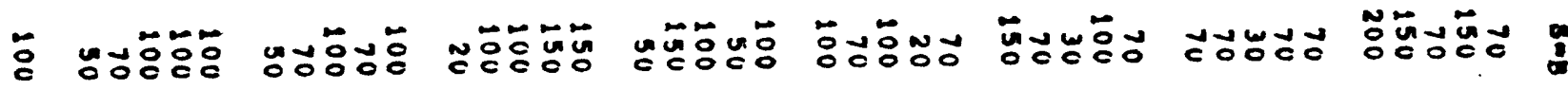

NENNE ZNNNNON NUNONN

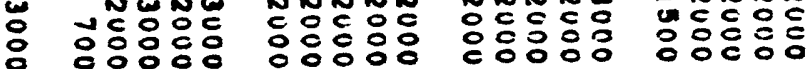

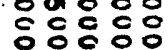

NNNNN

NN更U 등응유 등용듕유

는두웅 응등융

$\approx$

WNANA WNGRE

מw - Now

UN $N$ WN

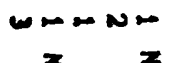

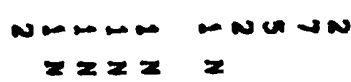

:

u wouknow

UNNNN

UNOAN UNNEN

NNNUU

NUNNN

NNNNN

NAN

: 


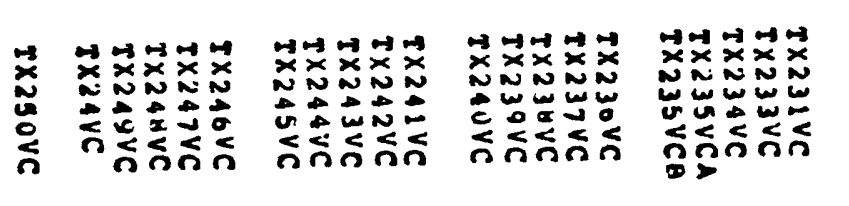

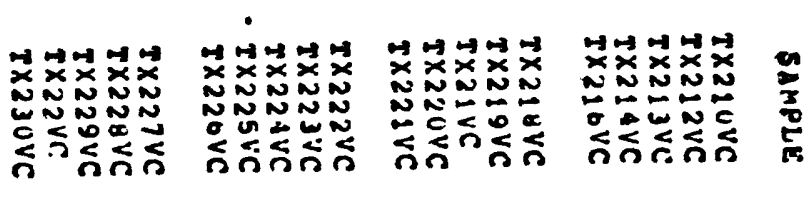

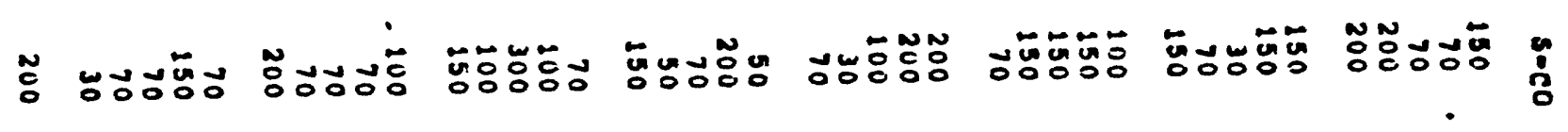

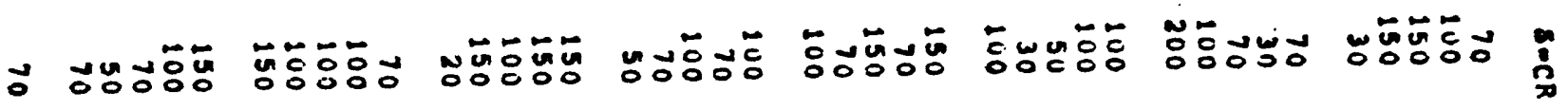

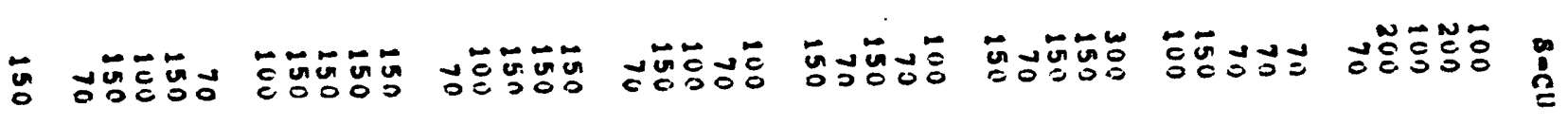

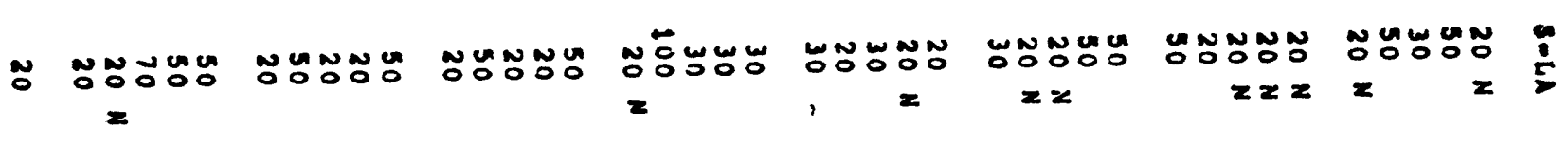

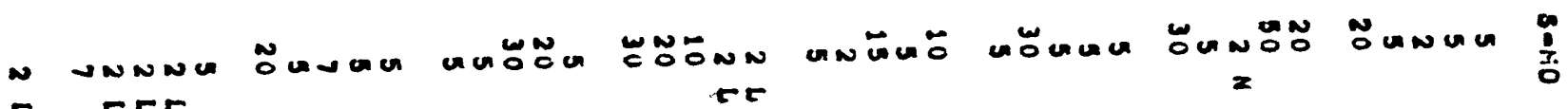

$r$ Trer tor

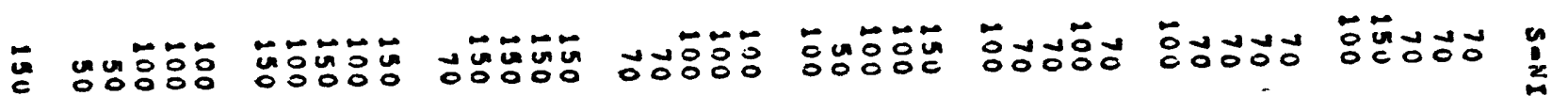

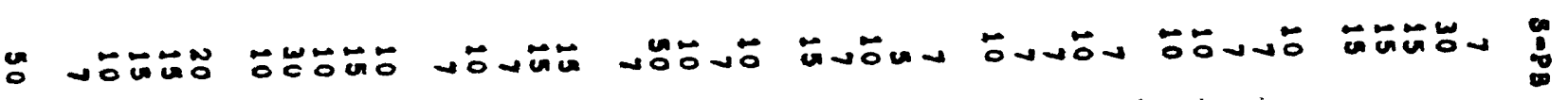

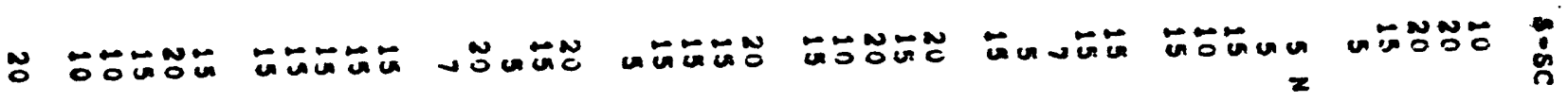

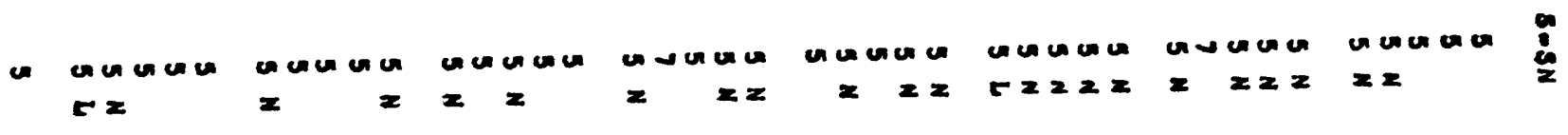

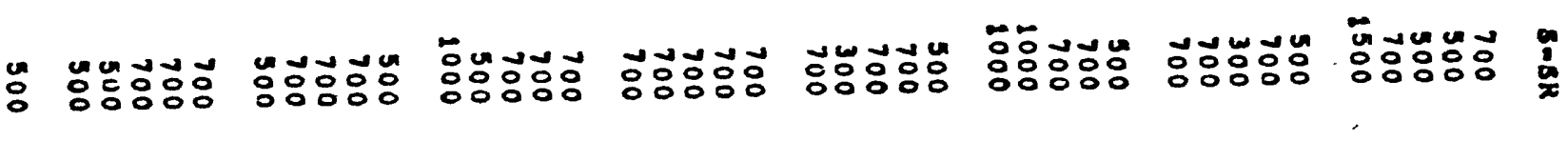

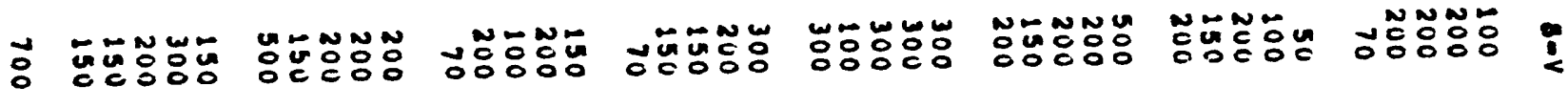

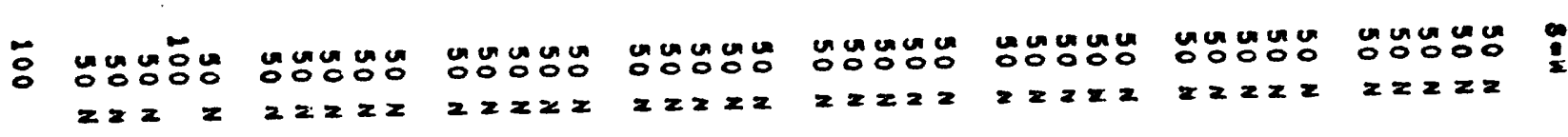

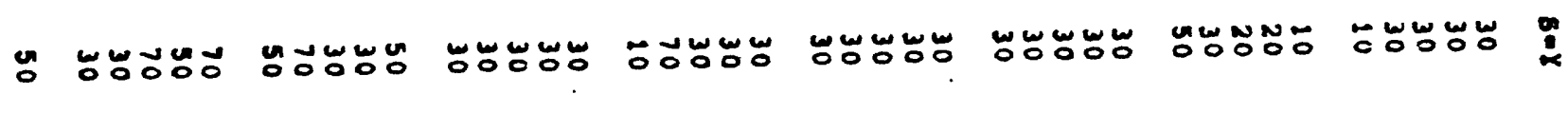

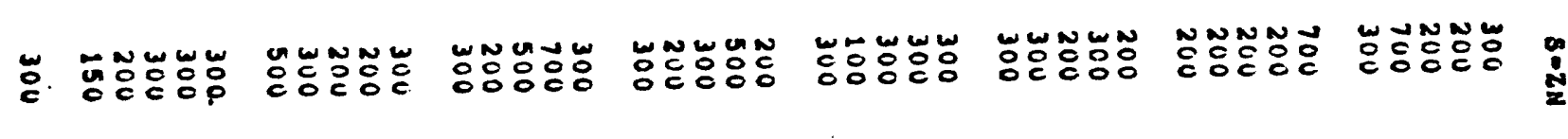

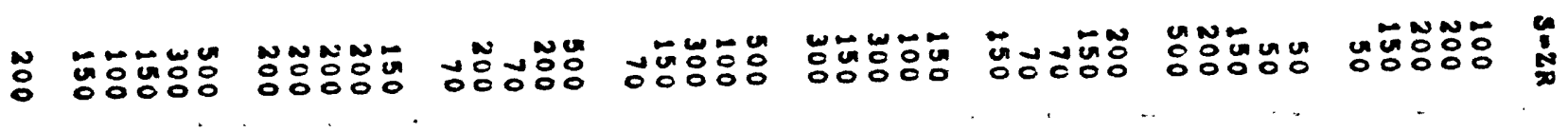

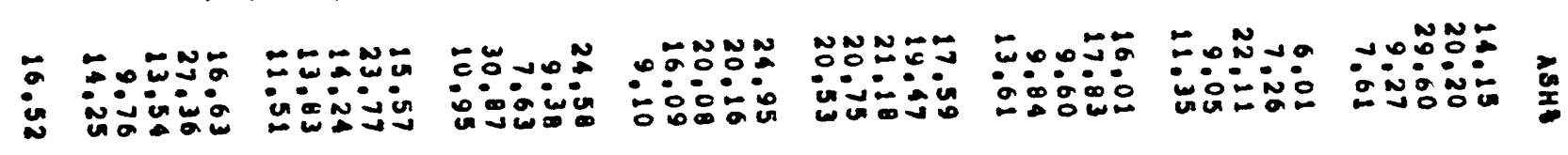




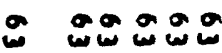

N $\rightarrow N N N$

- unu.

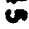

-

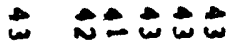

ேேேேே

مै

जUNㄴ영
N

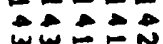

$\rightarrow$ un

NNNO
ع

जu u以s $\ddot{\square}=\square=$ unuan U nu o o U⿺辶一 ago o - unua

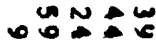

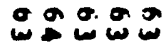

No $0: 0$

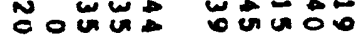

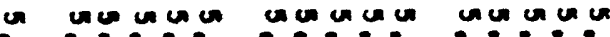
i:ioj ioici ieioio nanar ar 0 anaga un un un iㅇํㅇㅇ กล un un un 00000 $\operatorname{san} 6$ un un un c000\% andas unuma $\therefore \circ: \circ 0^{\circ}$

a) a

unugua o:00: anana

- jo:

Univin

in -10

넌

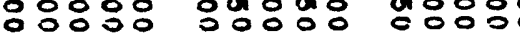

응응

$000 \%$

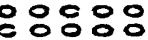

$\circ 000$

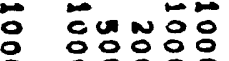
응ㅇㅇㅇㅇㅇㅇㅇㅇㅇ

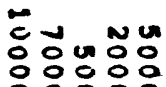

ค $0 \Omega \Omega$

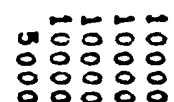

ดロ a
둥요융유 융ㅇㅇㅇㅇㅇ

Da
$5 \div$

웅은 응응ㅇㅇㅇ 응영응

$\Omega \Omega$ $\div 5000$

웅ㅇㅇㅇㅇㅇㅇ 응응응응

ดคดล0

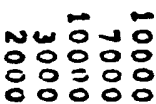

a $\Omega$

0 두웅

ㅇㅇㅇㅇㅇㅇ

ㅇㅇㅇㅇㅇㅇㅇㅇ

ค 200

- OOCOO OOOON

00000

00000

00000

00000

00000

융 and NunNi iNi心i ini:i

ERE

$x$

2

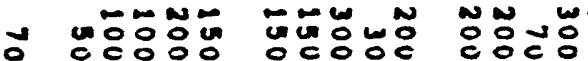

NWN-

ㄴำำ

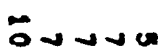

-

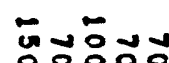
:

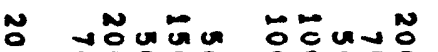

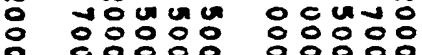

W 등ㅇㅇㅇㅇㅠ

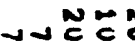
궝유융용

NNN N

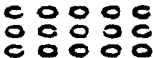

NNWNW :등등 웅ㅇㅇㅇㅇㅇ WẼ
- $n-n-n$

$-2$

ט $\rightarrow-$

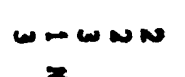

$\cos a \cos$

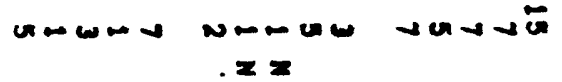<smiles>[CH]=[Co]</smiles> 


\section{)

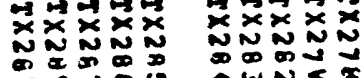

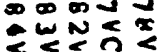

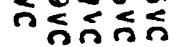 \\ $\overrightarrow{ } \boldsymbol{x} \dot{x} \boldsymbol{x} \dot{x} \boldsymbol{x} \dot{x}$ \\ NNN

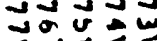

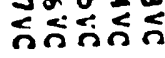

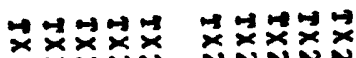

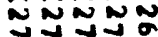

NN=C

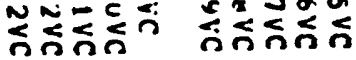

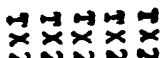

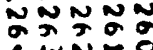

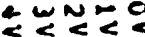

ธ์ธ์ธ์ธ์

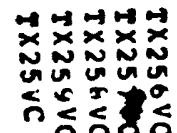

UNUNUN

U⿴囗十心

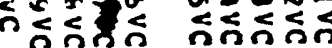

घ :<smiles>O[Hg]</smiles>

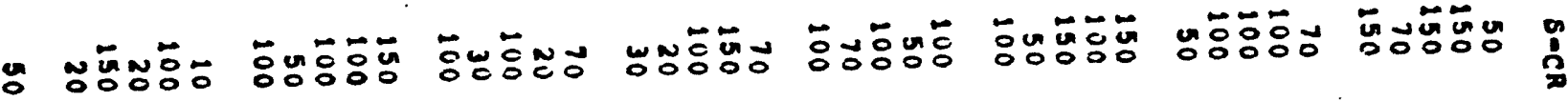

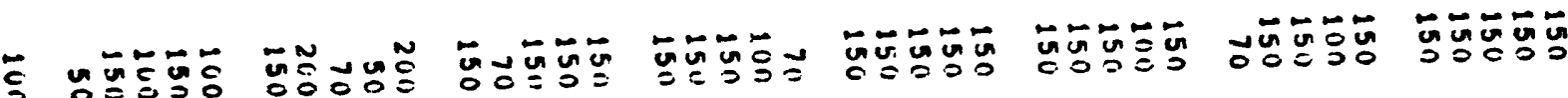

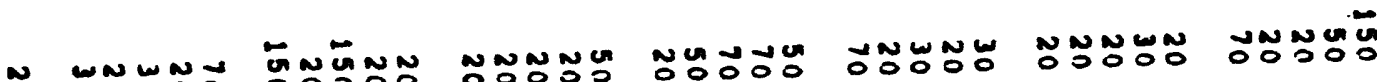

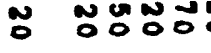

$z=2$

a anco $z$

a
MัOกNล
$=$ $z=$

บับ ก 2. 2.
$=$

UNNON

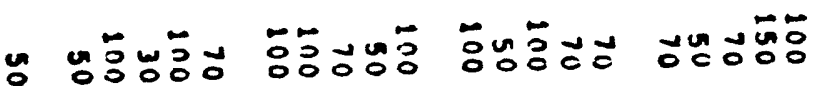

$\sim \sim N N \boldsymbol{N}$

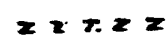

ง $⿻ \cup N$ $z \quad z=2$
ง nune nanum

$\stackrel{8}{5}$<smiles>[13CH3]</smiles>

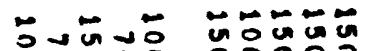

Cᄃ

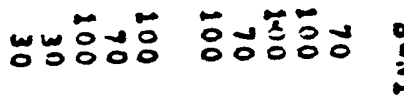

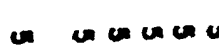

$z z z z$

: unumio

고능 응응응응응
藏客 0000

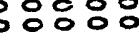

Tேั0

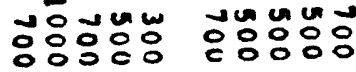
응ㅇㅇㅇㅇㅇㅇ 뜽뭉융뮹

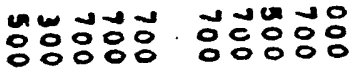

$\vec{\circ}+40 \div$

\section{,}

- nn n n n n n

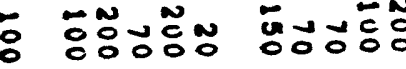

ununu

- 00000

$z$ z. 2222

․ํㅇํำชัด

$-N N W N$

응 웅유유

ungungun

$z \geq z 2 z$

שัด

แัด์ทัธ์ 욷유융
ํํㅇ융ํํㅇํํ

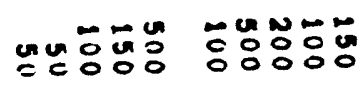
NNNㅡ유유 등응등융

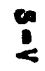

unumu núuer

$\begin{array}{llll}0 & 0 & 0 & 0 \\ 0 & 0 & 0 & 0 \\ 0 & 0 & 0\end{array}$ $z \geq z 2$

$z \geq z=2$

$z 2 z 2$

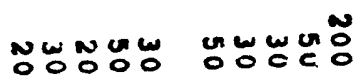

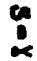

$$
z
$$

w

WWWNW WWONON

un un un

تم w w

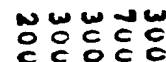

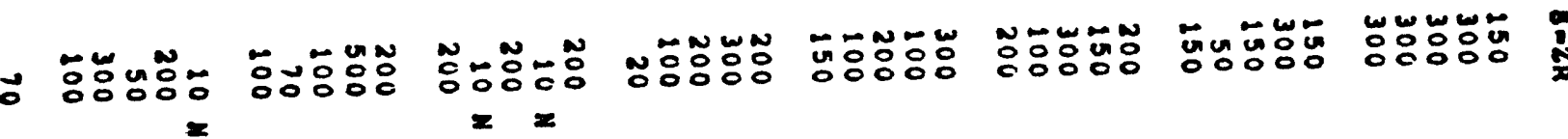

No $=0$

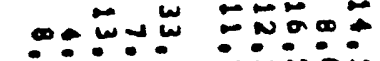

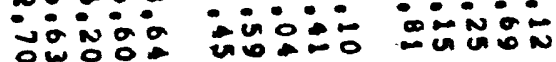

$\because \cdots \div-$

ニN

$=\approx 000 \div \quad 0200 \div$ 
- 20ำ

a on

운웅 un un

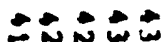

00000

00000 운웅

\% ww w w $\rightarrow \rightarrow \rightarrow$

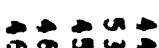

שั0 $\rightarrow 0 \pm \mathbb{N}$

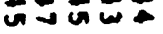

un un un un

$N N \rightarrow N G$

nu․․
00000

ฯ ํำ

- oc úo
ज象

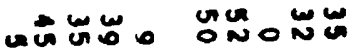

- 0000 w w w w un unga ว u.

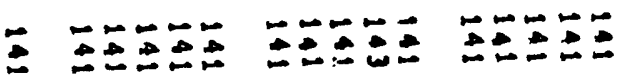

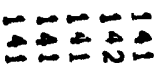

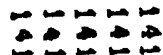

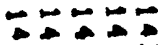

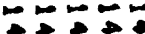

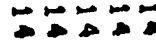
$-\infty N N \quad n N N U$

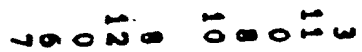
ou un nu unn

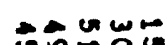
NOW $n \rightarrow \rightarrow \infty$ Nజ\% Oun $0 \div$ WW 每 var 뭉
- nucumu unumun

- 00000 :00:0

a ar a a rat

a numan ununu wankn

unu un un $\therefore \therefore 00$

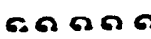

ungun un $\therefore 0000$

unumu un un c:00: un un un

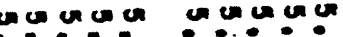

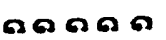
0000 0000 anana

ดกのด onana

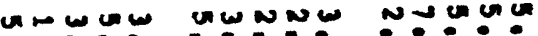
:0000 00000 00000 00000

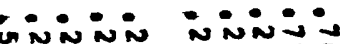

iำin

:

\section{$\infty$} $\frac{3}{2}$

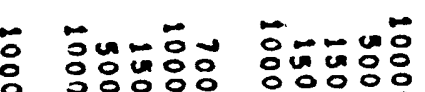

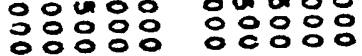

$\Omega$

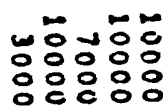

$\rightarrow 0$
응둥요 응융유 영영웅 a $\Omega$
응유융요

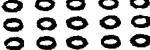

a $a$
둥요융

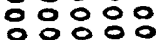
웅ㅇㅇㅇ ๑ $0 \Omega$

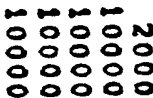
00000

웅운 ㅇㅇㅇㅇㅇㅇㅇㅇㅇ 웅영옹웅 $\Omega \Omega$

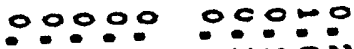

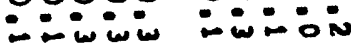
$z$ coc:0 $=$

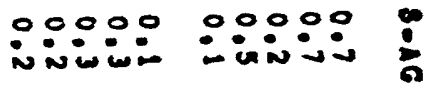

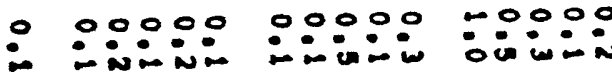
$x$
- nunna nnnan nnnnn $z=2 \geq 2=$ $z=2 \geq z$
MNNNN NNNNM

NNNNN

NNNNN NNNNN

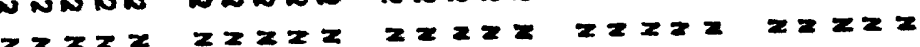

$\div$

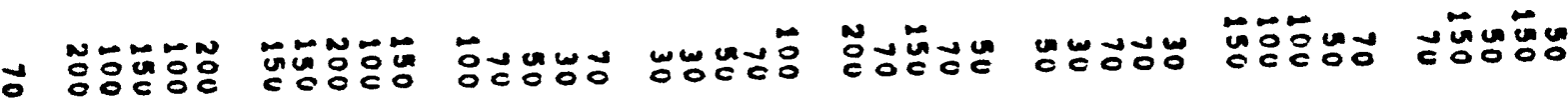

-

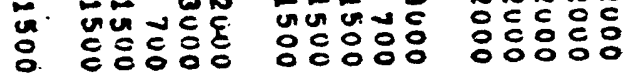

NNEたO

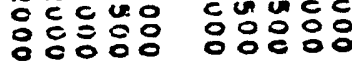

NEพ

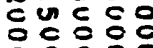
c응요

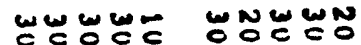

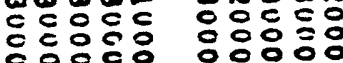




\begin{tabular}{|c|c|c|c|c|c|c|}
\hline 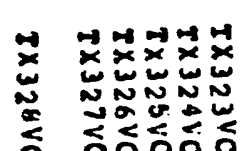 & 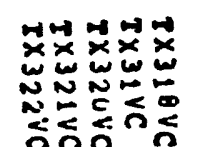 & 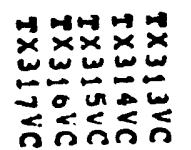 & 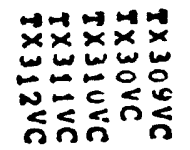 & 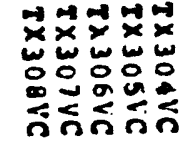 & 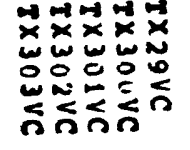 & 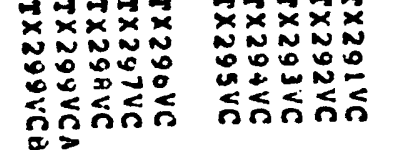 \\
\hline
\end{tabular}

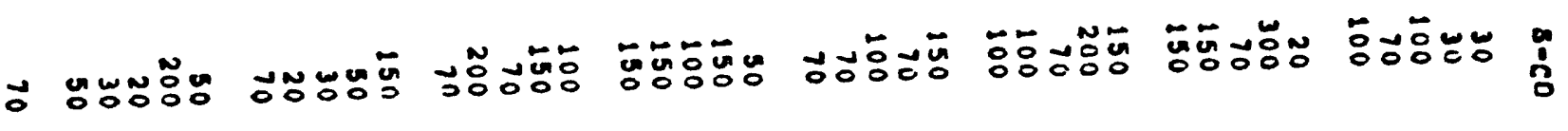

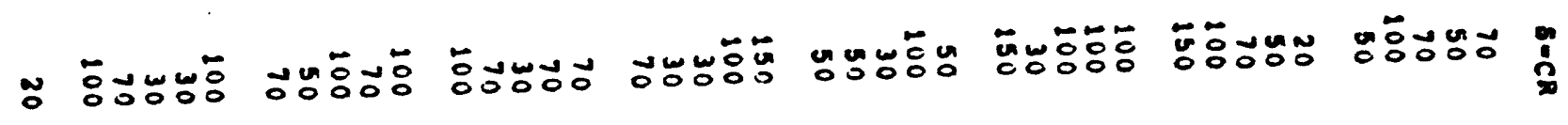

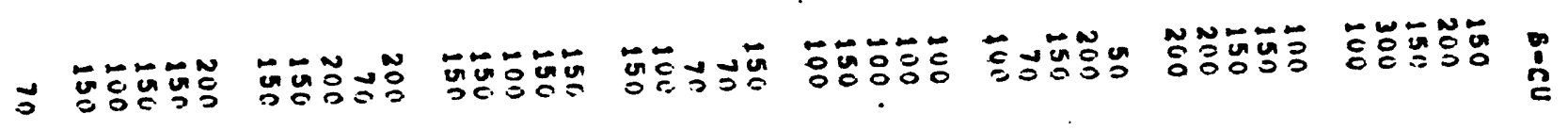

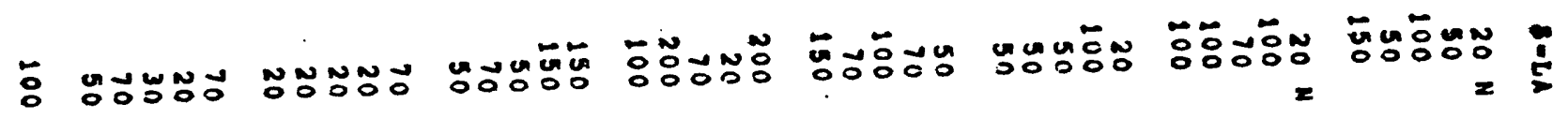

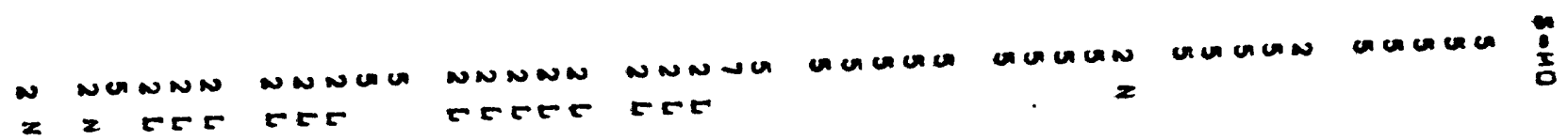

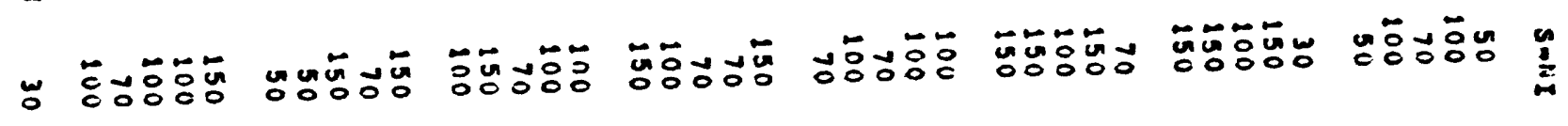

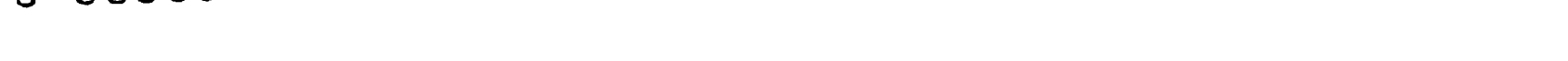

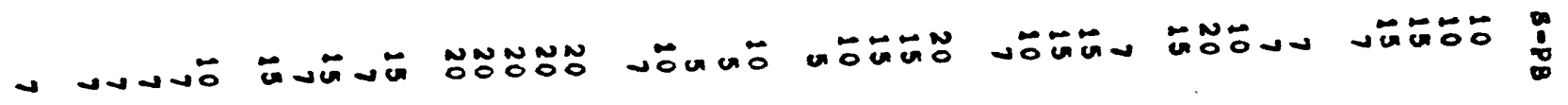

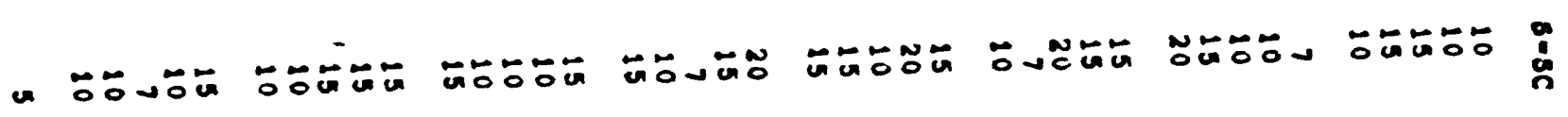

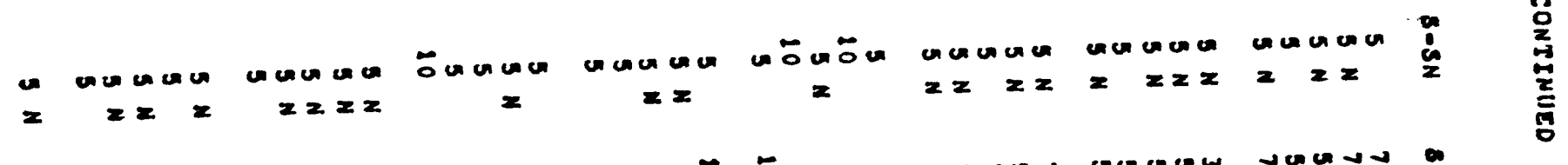

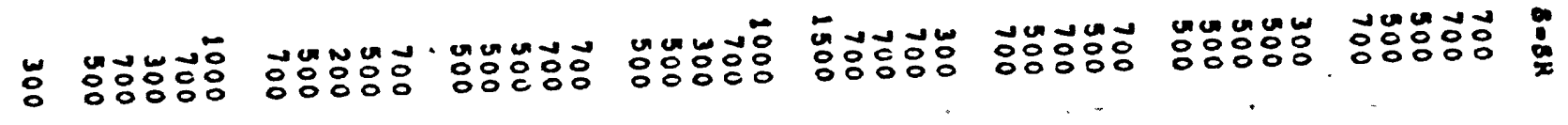

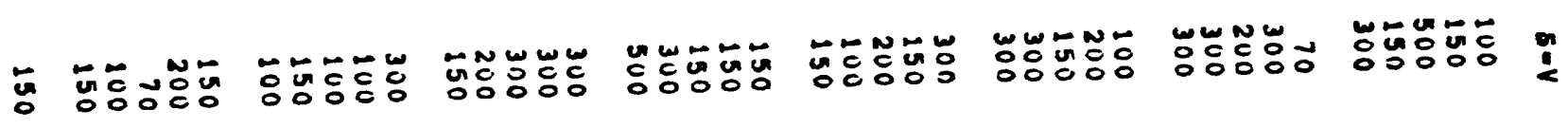
ك

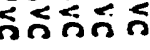

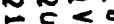
w w w西。 ㅇㅇㅇ잉 岩造贶 $0 \times$ x . 


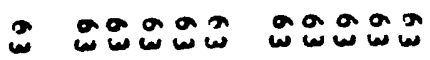

م ט

N NONNN wN?uU $n \rightarrow 60 \%$ agano orogas 政 $-N \& N$

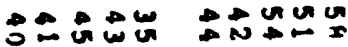

w ing

ununu

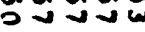

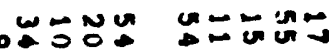

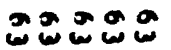

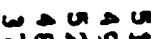

תח

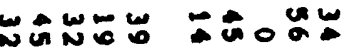

를 un un unu un

a $\operatorname{sana}$

מ - ununu :000: กล⿻上丨

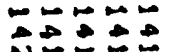

(ח)

$-\infty 000$

$0=0 \pi$

UN

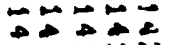
$\omega N \underset{\omega}{\omega}$

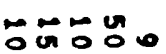

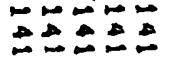

W م告范 $\because \div \div$

NNWNN

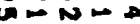

$N=\pi$

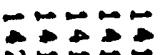

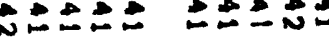

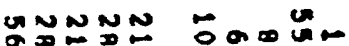

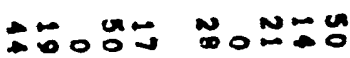

un un un un ununa ¿0000 00000

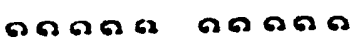

nuunco c000\% Snano an un un -0000 2050 muduca unanco :0000 c0000 nDas Don a $\frac{5}{3}$

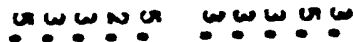
:00:0 00:00

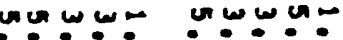

nNuUn

WNW

:0000 $0: 000$

i isin 영 -

- iiㄴ

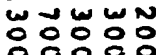

는in

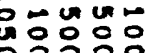

un un 농용응응

证沙 잉ㅇㅇㅇㅇㅇㅇ

UNㅜㅅㅜ

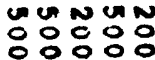

ㄱ.

수눈 NONONN

is

눈두 ㅇㅇㅇㅇㅇㅇ응

-
우어스융유

న

a
둥후웅 응응유융

20
둥ㅇㅇ응 응응ㅇㅇㅇ영

$a \Omega$
둥ㄷำ 잉ㅇㅇㅇ응 옹영응영

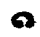

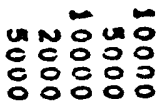
붕우웅응 응영웅ㄴㅇㅇㅇㅇ 응영ㅇㅇㅇㅇㅇ

\section{ㄷㅇㅇㅇㅇㅇㅇㅇㅇㅇㅠ} ㅇㅇㅇㅇㅇㅇㅇㅇㅇ

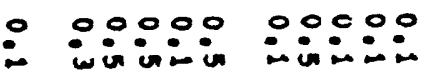
222

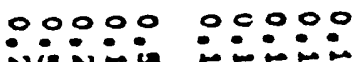

00000 $\because 0: 0 ?$ 泫法 CET 운웅 $r$
N NNNNN

$z=z Z z$
NNNNN $z=2 \geq 2$
NNNNN $Z Z=2 Z$
$\boldsymbol{N N \boldsymbol { N } N}$

NNNMN

NNNNN

NNNNN

NNNNN $z=2 z$

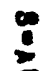

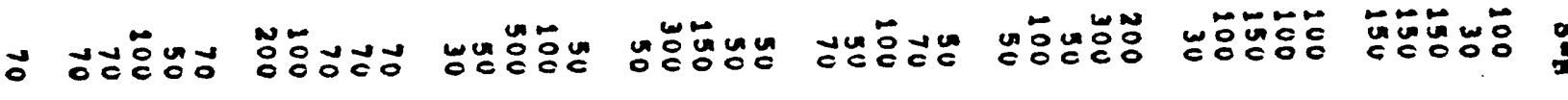

- N N N N

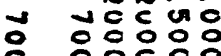

N

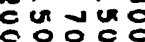

옹웅응응 응영융ㅇㅇㅇ

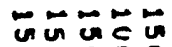
uñó ㄷㅇㅇㅇㅇㅇㅇㅠ

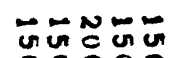

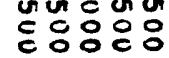

Nㅜㄴ Uñ⿺辶一兀 응ㅇㅇㅇㅇㅇㅇ
ง ق

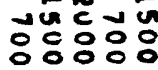
붕두웡ㅇㅇㅇ

מטm

(1)

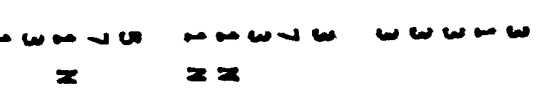

M

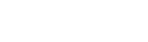

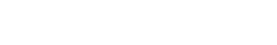




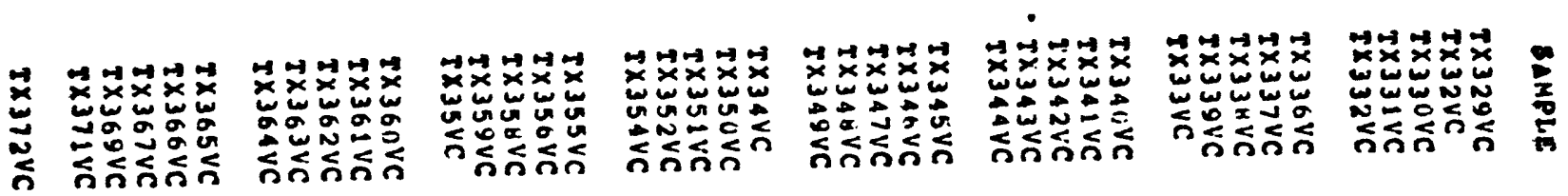

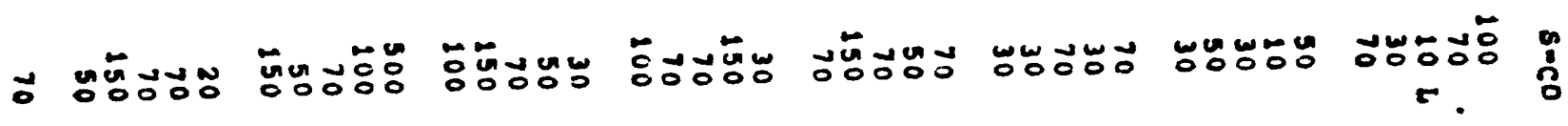

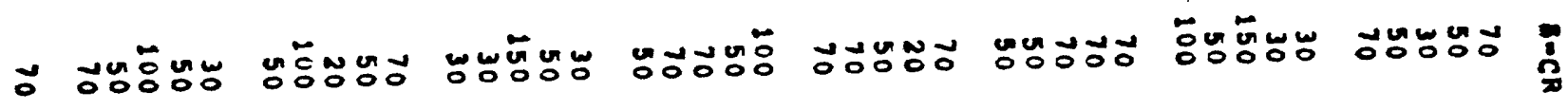

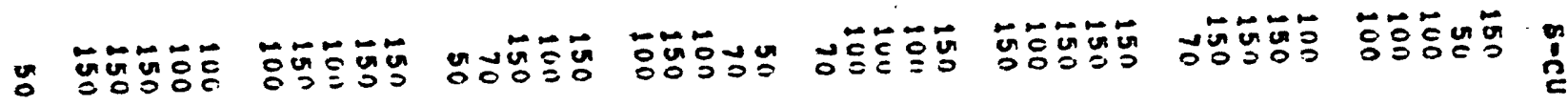

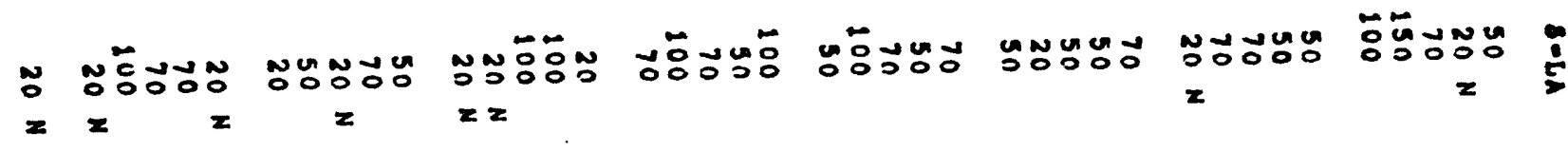

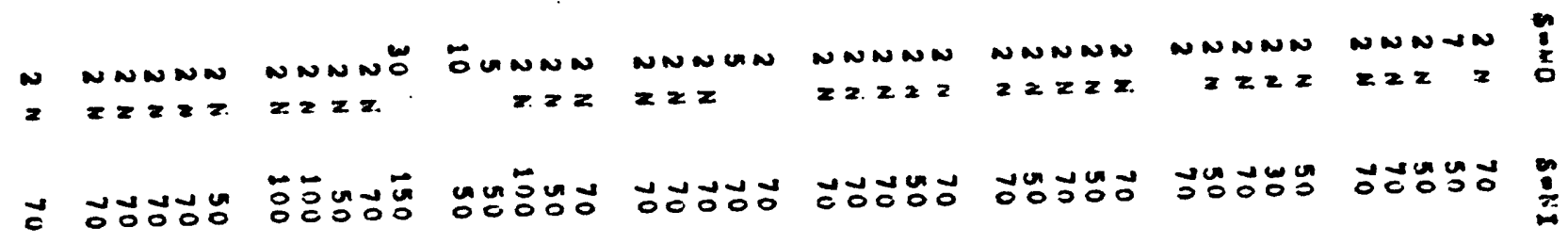

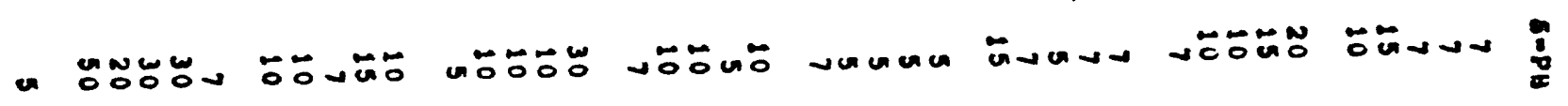

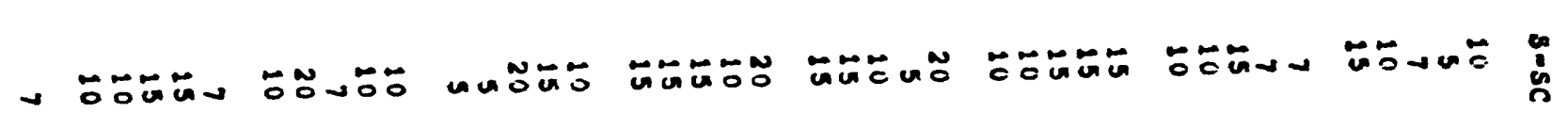

ta

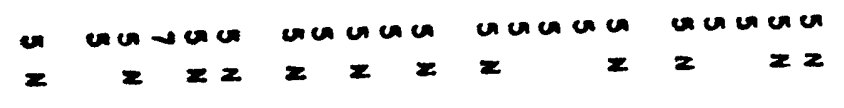

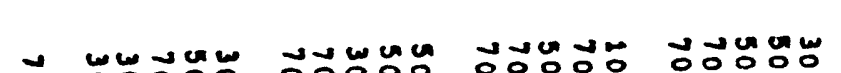

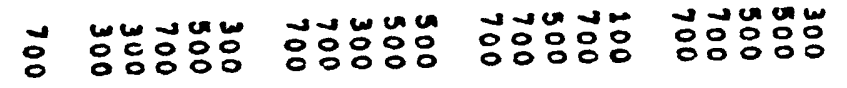

THNTR nNRNA

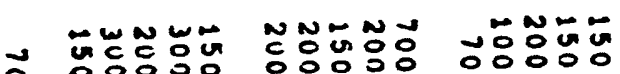

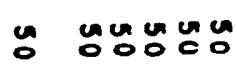

$z \quad z=2 z 2$

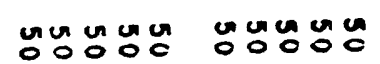

$z z z 2 z$ zzzz

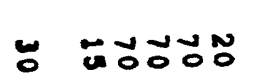

WU

مพ⿴囗十) (1)

:.

WẼ

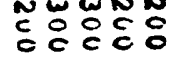

$C$ : unumu

$z z z z z$

ณํํำ

영융융 counconen

$$
z=
$$

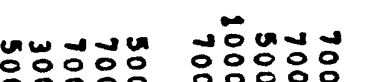
응웅융응
NNN品:

ENONCN

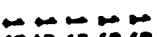

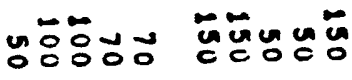

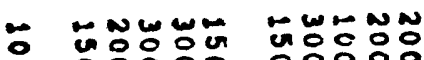

: 0 : :

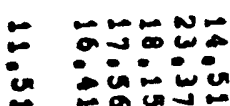

NN经业

$\because 0$

$0 \infty \infty \ln ^{\infty}$
थบำต 00000

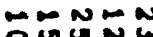
OG जU: - $=\infty$

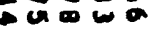

NWO:ON:

ununung $z \geq 2 z z$

뭉뭉ㄷㅇ

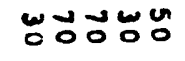

NWw 웅ㅇㅇㅇㅇ

CE: wnum uncouco: $z \geq z=2$

Nบำ

WWNWE WWNONO

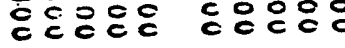

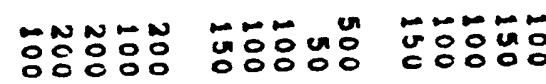

든ㄷำ $00 \div$ w UnN

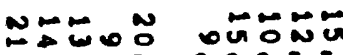
0 iñ
? 


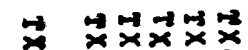 \&NSNN

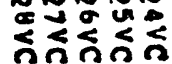

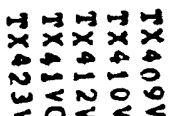

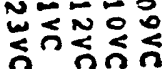

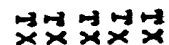

영ㅇㅇㅇ

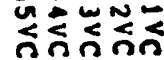

쿠ㅈㅓㅜ서ㅈㅓㅜ

*w.

c

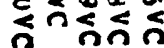

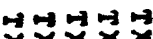

$x \times x \times$

\%

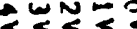

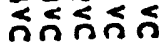

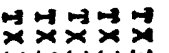

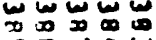

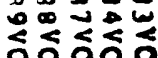

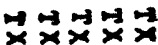

w

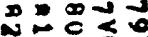
के $\vec{\sigma} \vec{\omega} \frac{1}{\omega}$

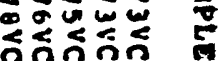

- a 000

*

م

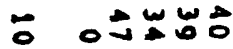

$\operatorname{lng} 00$

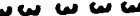
NoNNN un us

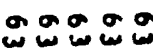

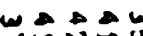

$w \neq N=0$
에요

orogo

누 ח

ơn

w $n \in \infty$

ongono

운운

00000

م

N

(1)

象

- 0 DN Nat-

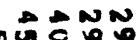

unus:

$\because N 0 \underset{0}{N}$

론

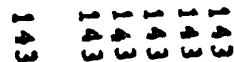

$N \rightarrow N N N$

* o- ono

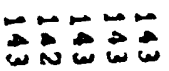
מ nunu $00-\infty$

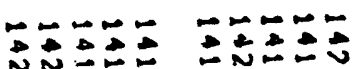
$\because \rightarrow \because E=\mathbb{N} N N$

UnU a

:

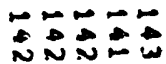
N w U U

$\sim N \omega-N$

○० 0 U.
キニシささ un was

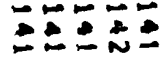
$\operatorname{lom}_{0 \rightarrow \infty} \rightarrow$

Nㅜㅇํำ

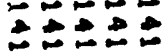

ט טN U UNN
- :0000 - $\Omega 0$ untun ununum 웅ㅇ an a unu un un :000:

a $\operatorname{son}$ unuug $\therefore \circ 000$

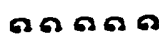

unumu c:00\% nana unu un un $\therefore \therefore: 0$

ดीด

unu un :0:-

nasa a vints

- n-m O:0:0 00:00 wnun wơnแ

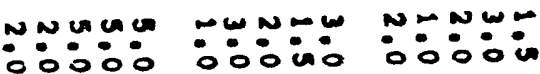

$\frac{1}{2}$

2

$\stackrel{0}{:}$
- 영영응

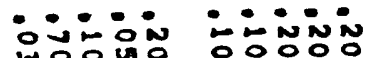
영응응영 ㅇㅇㅇ융융

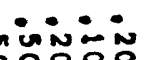

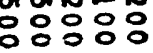

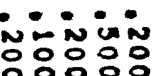

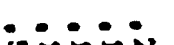

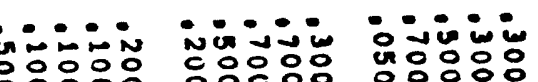

-

응 응융ㅇㅇㅇㅇㅇㅇㅇㅇㅇ

둥숭용

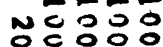
ㅇㅇㅇㅇㅇㅇㅇ

a

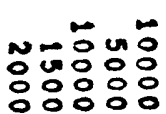

ก ค

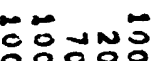
응응으 作응응

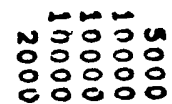

คด

\section{웅두웅 ㅇㅇㅇㅇㅇㅇㅇㅇ} 영영응

ด $\Omega$

ㅜㅜ눙

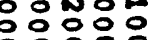

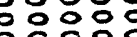

o
- 0000000000

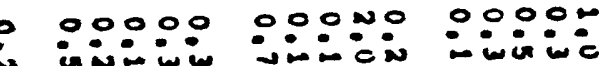

a $=$ o:0: :0:00 00000

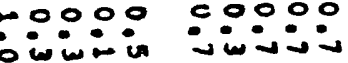
00000

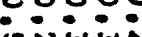
0000000000 inisin in:in 2
N $N N N N N \quad N N N N N$ NNNNN
N $N \omega N$ $z=2 x$

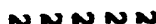

NNNNN NNNNN NNNNN

2

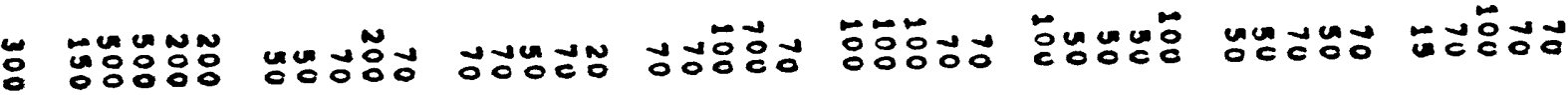

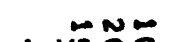

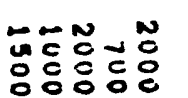



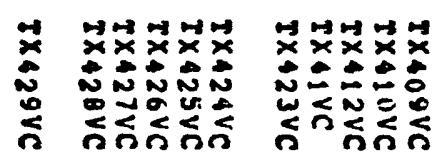

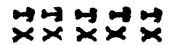

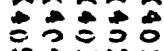

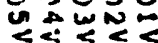

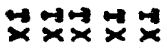

후웡

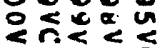

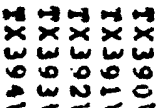

$\vec{x} \vec{x} \vec{x} \mathbf{x} \vec{x}$

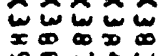

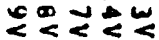

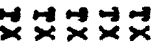

证

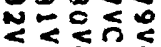

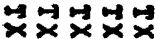

w西要

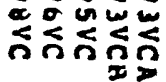

章

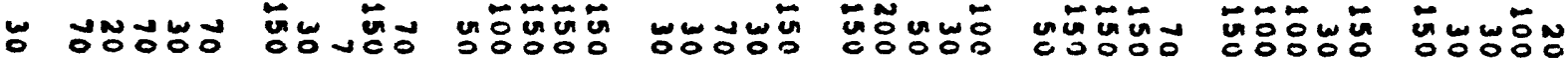

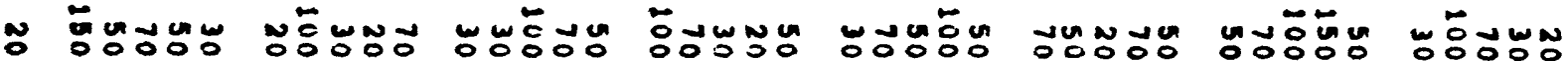

:

¿

:

:

undonand

WNO2N

Mn

WTNNA

OOO융 NOEONON

2

7.

$z=25$

$\begin{array}{ccc}N & \text { NONNNN } \\ z & =2.22 & 2\end{array}$

N N UN

NNNNN NNNNG

NONNNN

INNUNA NNNNN

$=22$

$\geq \quad 2=2.22$

$z=22$

2

$\angle z 2: 22$

:

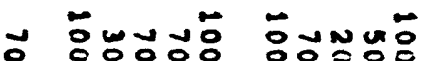

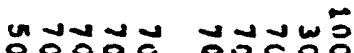

ேํำ

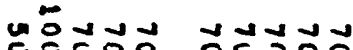

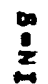

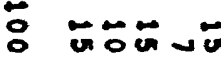

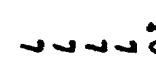

Uñ 20

जu:0\%

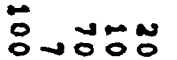

जuேㅡㅇ

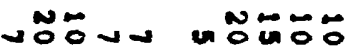

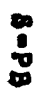

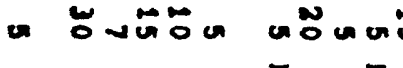

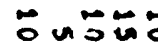

$\div 0 \div 0$

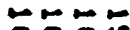

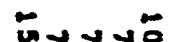

ㄷำ

$\cos \pi$

:

a ancian

a o u un

uname

Onumu

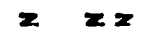

$z=z z$

$z \geq x \quad x$

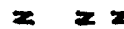

$2=$

$\rightarrow \quad 27 \rightarrow 0$

an wat

anenting

$\ln 40$

$\ddot{0}$

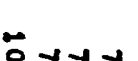

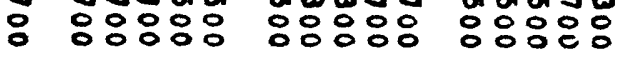

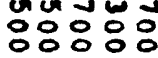

영ㅇㅇㅇㅇㅇ

영영응융

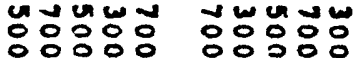

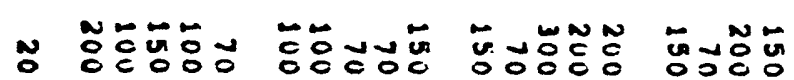

ununua

angugan

usungug

00000

z $z 2 z z z$

$z 2 z=2$

22222

\%

NWNOT $=$

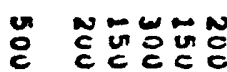

뚜ํํํㄹ

Oก:

n NNMN E它它它:

wำำ

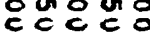

unumu 00000

WNDW w둔

co잉

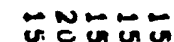

Uํํㅇํำ
ONWNOO

$\because N N N$

ज응융

unumen

uncunug un

únún

ugungun

$22 z 2 z$

zzzzz

$z \geq z 2 z$

$z z z z$

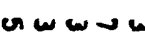

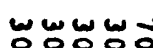



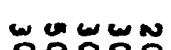

NUWNM NNNNM

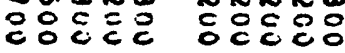

www

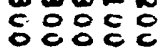

CUONON

EUㅇㅇㅇㅇㅇ

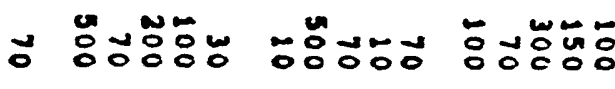
$\geq$

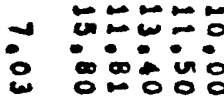

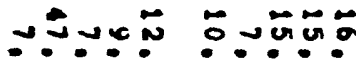

Non in
- - .

두워
ํํㅇำ

으ㅇㅠㅜㅇㅛ

0

ํํำำ

co

ํํㅇㄷํ유

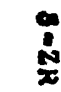

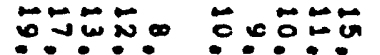

-

ำะัะะ

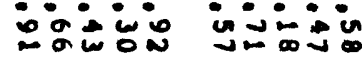

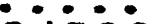

동년

- Mㄴ.

$\because \div 0$ $\because N: \div$ 


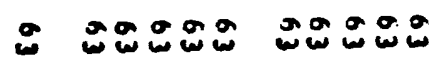
NNNNN NNNNND

N OGNAW

- Eugno

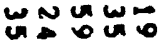

oging م $w \vec{u} \ldots \omega$

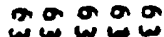
ט WN OW WOO

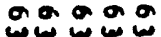

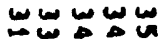
un uñ

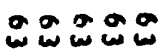
w w $0 n$

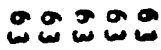
WNNWN O

w w w
웅ㅇㅇㅇ

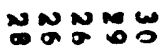
舟
点

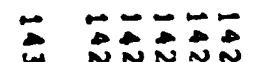 \\ - NNNNN \\ C NNDUN \\ - Eũ a}

$\vec{A} \div \vec{*}$ wu Uू
$\overrightarrow{A \rightarrow H}$ แล स $\$ N \underset{N}{*}$ Nu心
NE⿱E口心E W

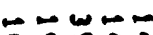

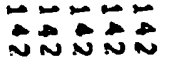
-NONO OूO $\because N 0 N$ $\because \div \div$ NลNNลN $N$ NNN $\triangle-N \rightarrow-$ NNNNE

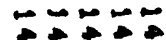
$\therefore \rightarrow \vec{\omega}=0$ Nu w ono

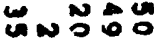

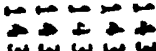
-

(1) $060 \% 0$
- acunun

- 0000 un un un -0:0: คดลล ununu $\therefore: 0: 0$ ดลกลล ununu :00:0 ด $\Omega$ unu un un $\therefore 0.0$ Dกลa

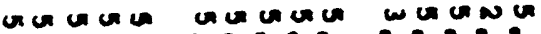
icio :000\% 00000 $\operatorname{20\Omega ONO}$

60 $\frac{a}{2}$

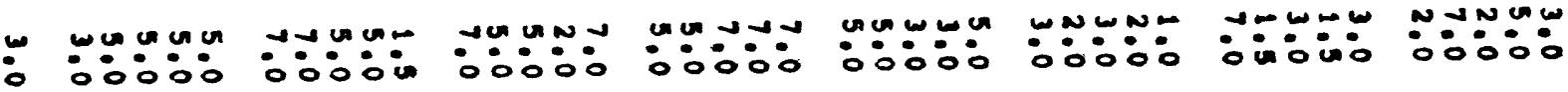

\section{a ninis inisin univis:}

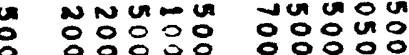
잉ㅇㅇㅇㅇㅇㅇ

$\therefore$

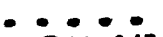

-

in univis

No: in

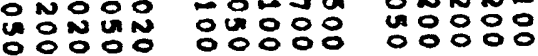

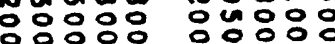

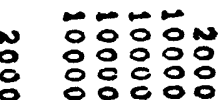
nuㅜㅇ

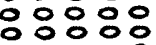
ค 9

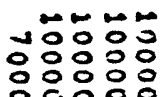

200
웅듕ㅇㅇ 응ㅇㅇㅇㅇㅇㅇㅇㅇ

a $\Omega$
혀유유 읃ㅇㅇㅇㅇㅇㅇ

a 동ㅇㅇㅇㅇㅇㅇㅇ ㅇㅇㅇㅇㅇㅇㅇ

ऽa 0
융무 0000

$\Omega$

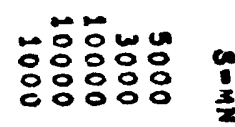

a
- 00:0: 0:0:0

in inisin

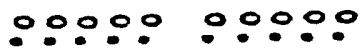
noini inini 5
00000 i.

0000 in $\rightarrow$ in OOC:O :00:0

N NNNNN NNNNN

$z \quad 2 Z Z Z Z Z 2 Z 2$
NNNNN $\geq 22 \geq 2$
MNNNN

NNNNM

NNNNN

UNNNN

NSNNN

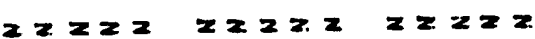

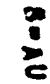

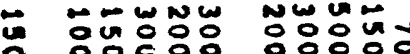

N.

두웅

wै

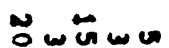

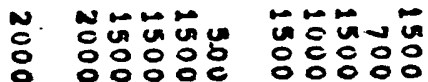

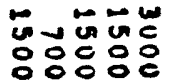
N

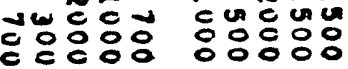

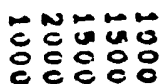

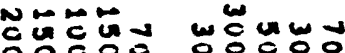

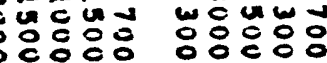

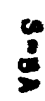

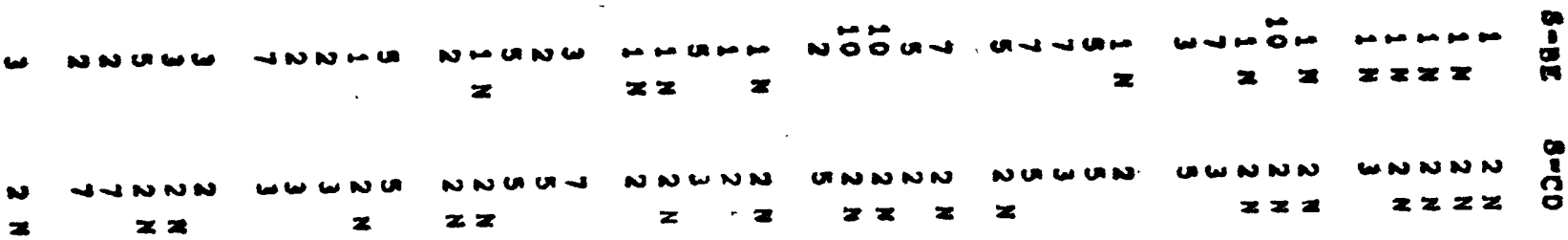




\section{댁

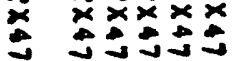 \\ Ints

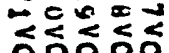

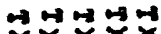

$\times x \times x$

ogasio

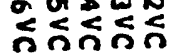

$\vec{x} \dot{x} \times \mathbf{x} \times \mathbf{x}$

蛅出出的

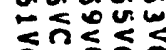

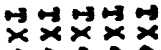

蛅动:

눙웅

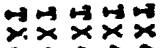

DE:

兹它

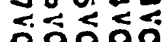

$\ddot{x} \stackrel{4}{x} \underset{x}{x} \vec{x}$

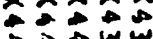

is

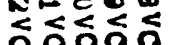

$\bar{x} \bar{x} \bar{x}$

10:0

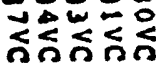

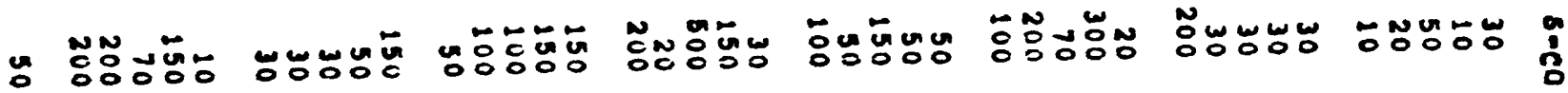

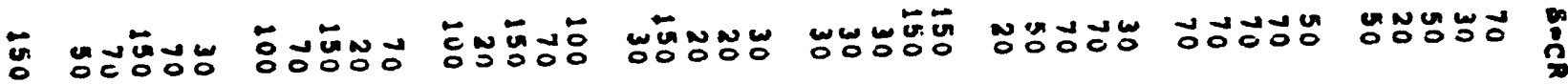

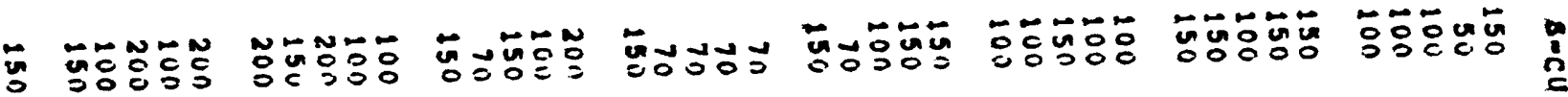

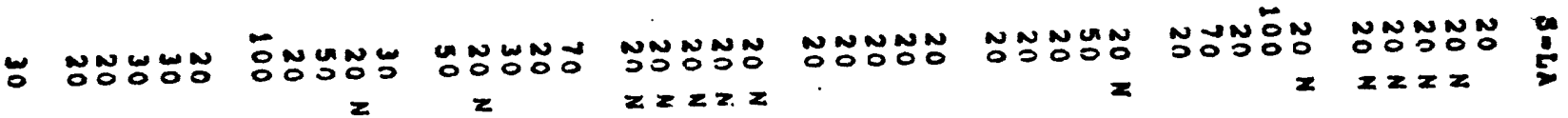

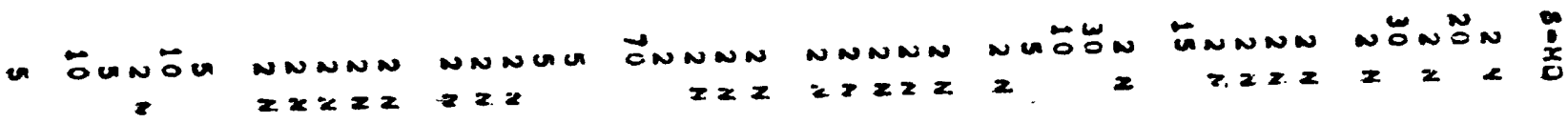

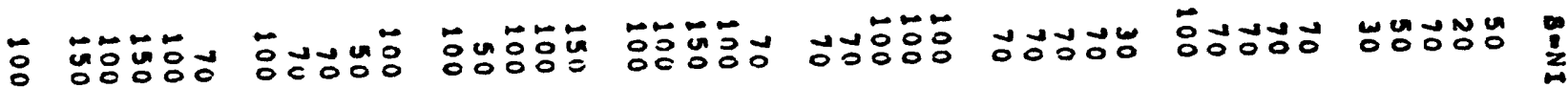

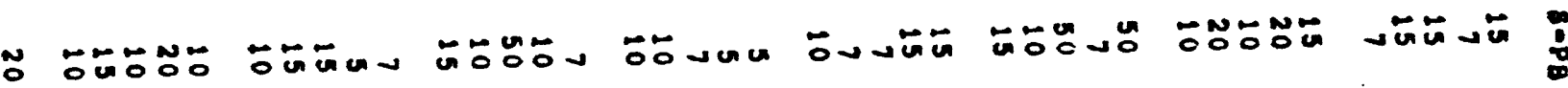

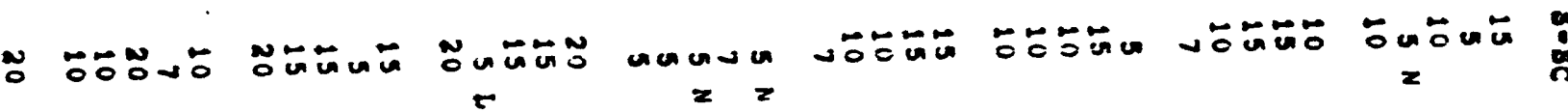

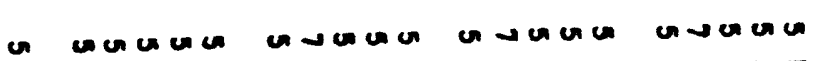

$=$

$z \geq z$

$2=$

$z z 2$

öunum

ounuso

שั0ข

$z=$

coneres

$2 z z z=$

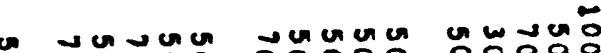

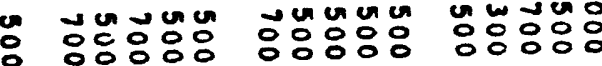

wữ un

गั

$\vec{\circ} \vec{\circ} \vec{\circ} \overrightarrow{0} \overrightarrow{0}$

무무요

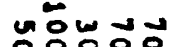

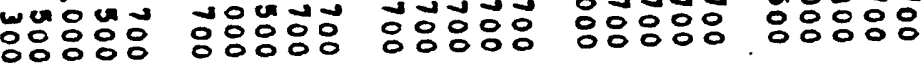

$\frac{1}{x}$

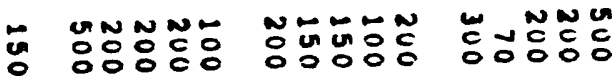

$\sim \omega$

WTWN

- Unw

NWNL

등휴융

00000

동동ㅇㅇㅇ영

능영영

잉ㅇㅇㅇㅇㅇ

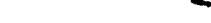

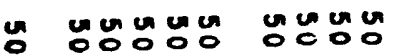

unugun

unumenos

uncuunan

gununu

ununu

on un ung

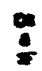

$z=2 Z z Z Z Z Z Z$

$z=2 z 2$

$z=222$

$x \geq \geq 2=$

$z z z z=$

$z z z z z$

$z \geq 2 z 2$

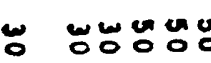

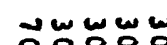

ungna

monWO

แัดํํํㅇํㅇ

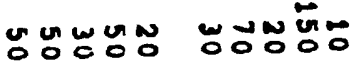

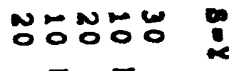

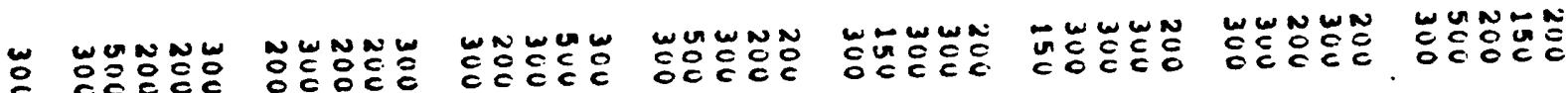
w.

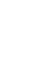

$z 5$

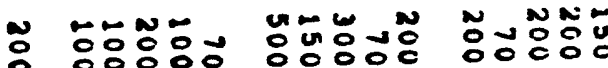

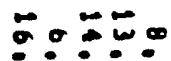

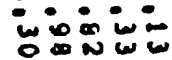

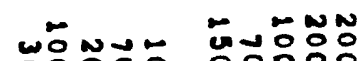

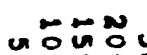

명ㅁํㅇ응

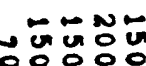

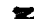

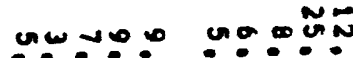

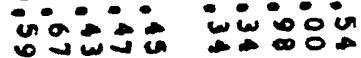

뚜

$\because \div \div \div=$ $\because n=0$ $\rightarrow N N=\infty$

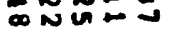

G $\because \therefore O N=$ 궁융 
- agago agooa

a

טNO

$\Rightarrow \quad \omega n-\infty$

-
N⿴囗十心

웅

UNU

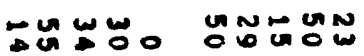

운우

$N \backsim-\infty N$

NU⿻人口

un

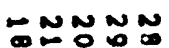

$-\omega u$
م:

N

Jaمn

ט

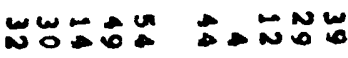

- 0000

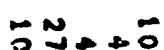
un $n$ : 点

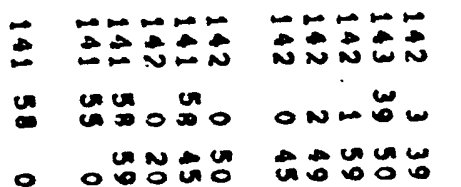

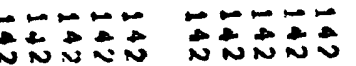
WN UูU

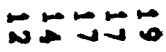

$u \rightarrow w n$

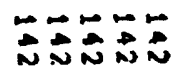
WN OW $\rightarrow$ D wun

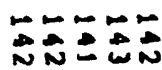

ou a a

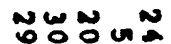

$\therefore=$

ONO

NENND

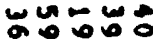

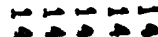
WNCEE ○N No⿱⺊⿱日一)
- cavenca vanuma

- :0000 0000\%

a nanaa ana unu un un :0: nanan uncunco :00:0 nanas un unu :00:0

nana unuma $\therefore \circ \therefore \circ$

ดのกのด wunn

$\therefore 0000$

ana

unuma io:00 00600

a wunar wunum

nRnNn nathm

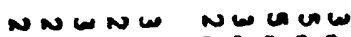

wwonch nuven

:00: 0:0i0: in:00:

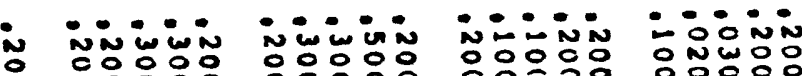
응응영 영영영

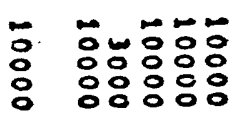

○

$\Omega \Omega$

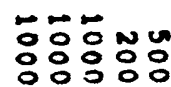

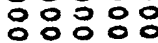

ana
뭉ㅇㅇㅇㅇㅇㅇ

응ㅇㅇㅇㅇㅇㅇㅇ

กดดล
웅웅유유

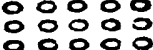

sana

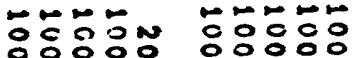
융융ㅇㅇㅇㅇㅇㅇㅇㅇㅇㅇㅇㅇㅇㅇㅇ onas NONONO UniñN

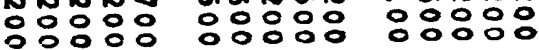
00000

- 00000

$\because \quad 00000$

00000 WNiNi
$\boldsymbol{N} \sim \boldsymbol{N} \sim$ $z 2 \geq 2 z$
NNNNN

$2 \geq 22 Z$
NNNNN

$z \geq z \geq z$
NNNNN $z z=2$

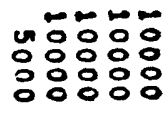
ㅇㅇㅇㅇㅇㅇㅇㅇㅇ

$0 \Omega$
NNNNN

NNWNN

융유융유 웅ㅇㅇㅇㅇ

a

NNNNN

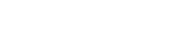

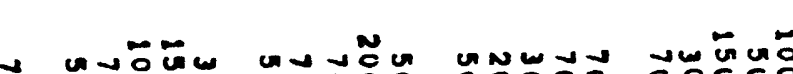

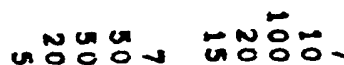
z. $z \geq z$ $z \geq z z=$ $z \geq \geq z$ o웅응 당 C잉이 C.8\%잉

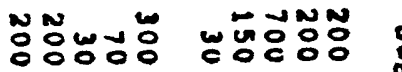

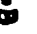

- ẼUNON NEN

NNTEN

WN

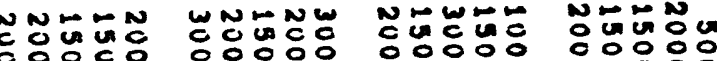

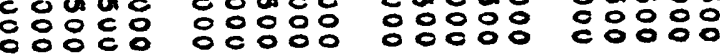




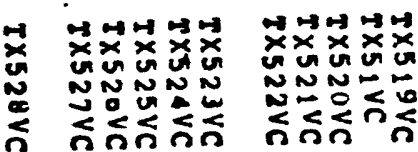

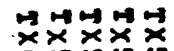
ज़ưu -

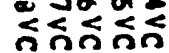

$\vec{x}+\vec{x}+\vec{x} \times \vec{x}$

जunu

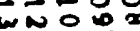

$\leqslant \leq \leqslant \leq 2$

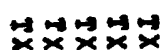

unugu

영요은

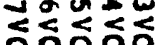

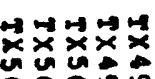

जी

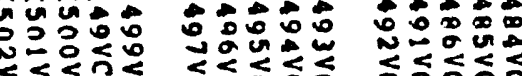

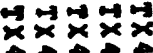

$x \times x \times$

$0: 000$

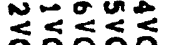

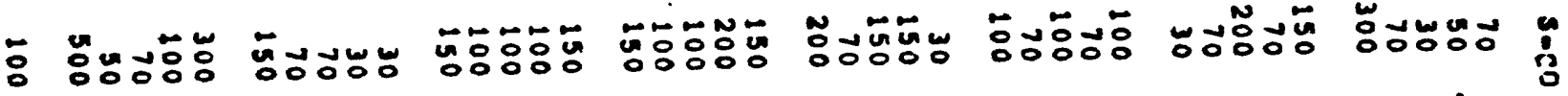

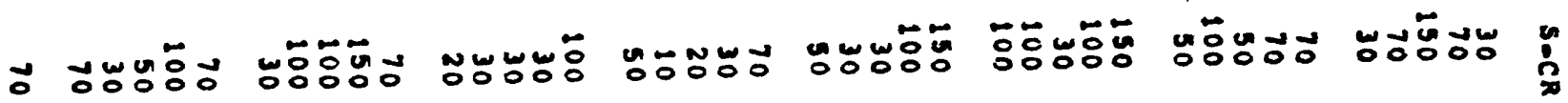

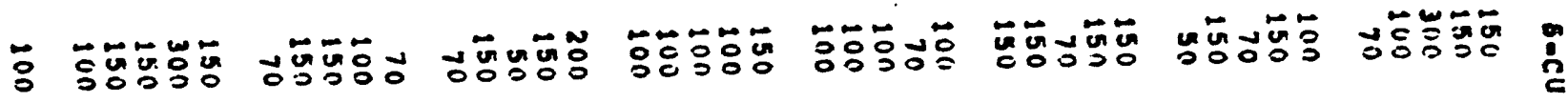

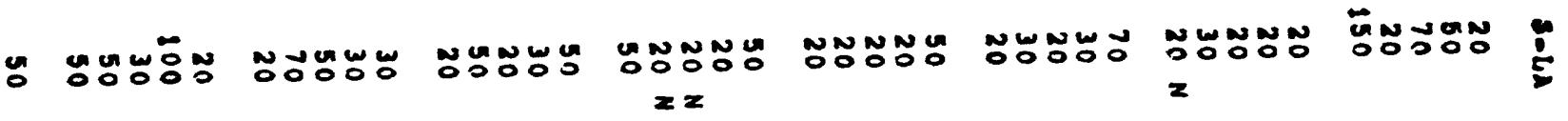

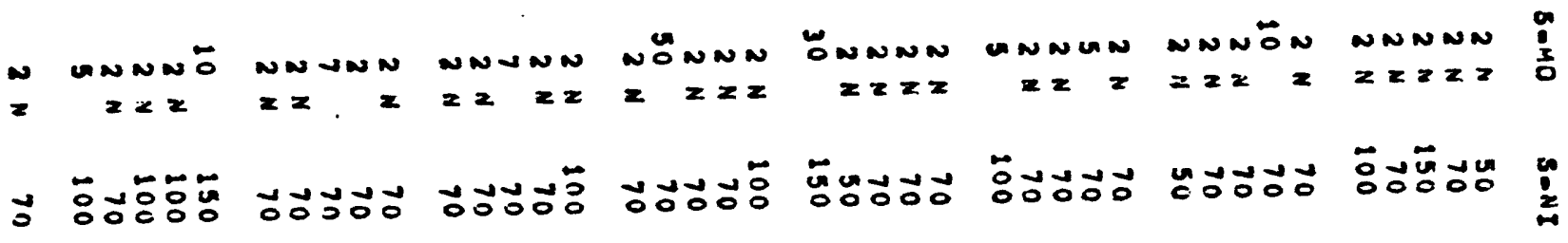

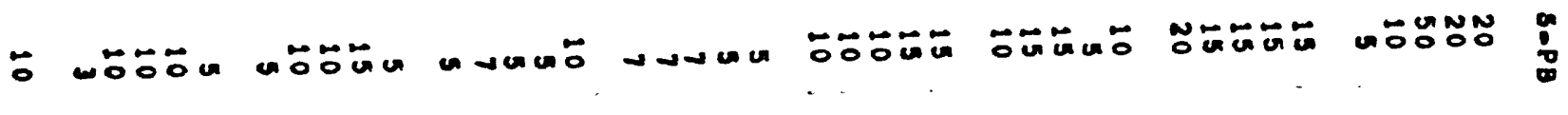

0

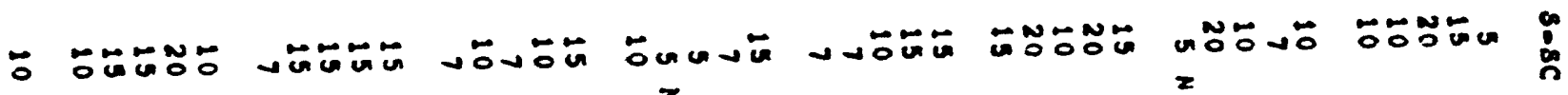

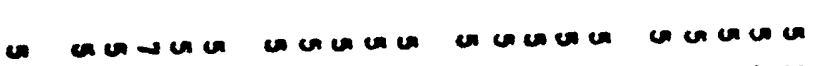

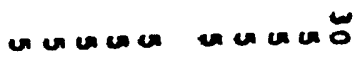

uncouco

cousun

in

$z \quad z 2=2$

$z=2 x \quad 2 x \geq 2 z$

$z=2$

$z x$

$2 \quad z \geq 2$ ?

a.

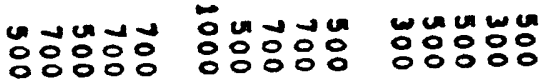

0
0
0

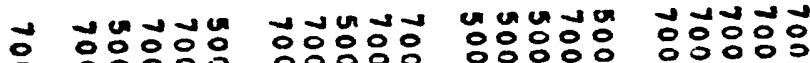

뭉주융용

0000

(5)

-

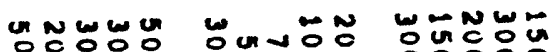

NaOSONO

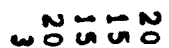

NNN-

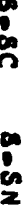

응 등영영 웅등응

$\therefore::$

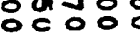

OOOOOO

ㅇํㅇㅇㅠ:

Oํำ

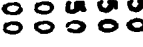

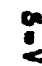

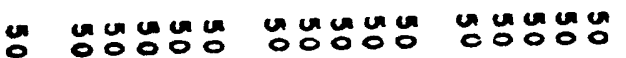

$z=2222$

$z z=2 z$

$z \geq z z z$

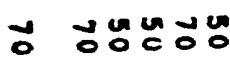

N WNN

ㄷ.․ 융유유

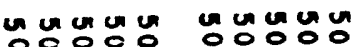

wa

$z 2 z 2$

ज䓠范口

o0000

$z z \geq z z$

땡명유

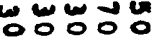

WNW

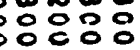

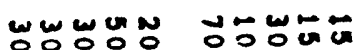
욷ㅇㅇㅇ융 영영 unculan 이잉

$z z z \geq z$

Nun 영ํํㅇ응

NN W

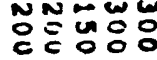

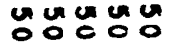

$<2 z 2 z$

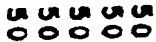

$222 z 2$

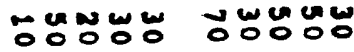

TWNN WONNN GWOOOOOOOOO

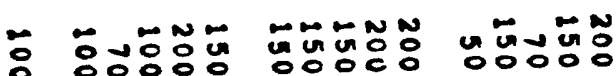
告,

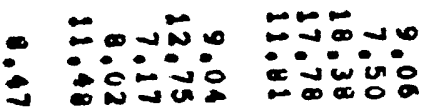
こロニ䓃 :un:o
등Nㅡㅇ융 영영눙

$=$

=un

تU⿴囗十 ผ 명응뭉

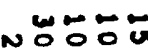
녕유음 뜽융융유유 :

๑NNG: Oin $\vec{\sigma} \sigma \vec{\sigma}$ in:-

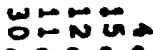
in: U: $: 0 \%$ 
- rorao

i w

N

- OQENE

$-7-5$

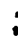

$\omega$

- $\rightarrow 0$ 융

900 90

ago o o

- 0000

NNN NN

NNNNA

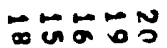

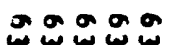

웅aㅇ

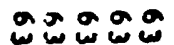

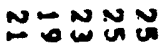

NNN

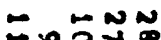

ט

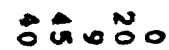

UNO

$N u=\infty-$

שח

$\because-\because N O N$

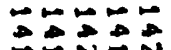

$\rightarrow \rightarrow$

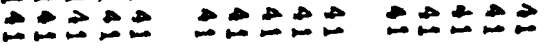

ס

-O 0 in

בָּ

$20=\infty 0$

Now0

Nッ大்

WNOW

ט
은

NNNWN

0

Uै=0 000
돔

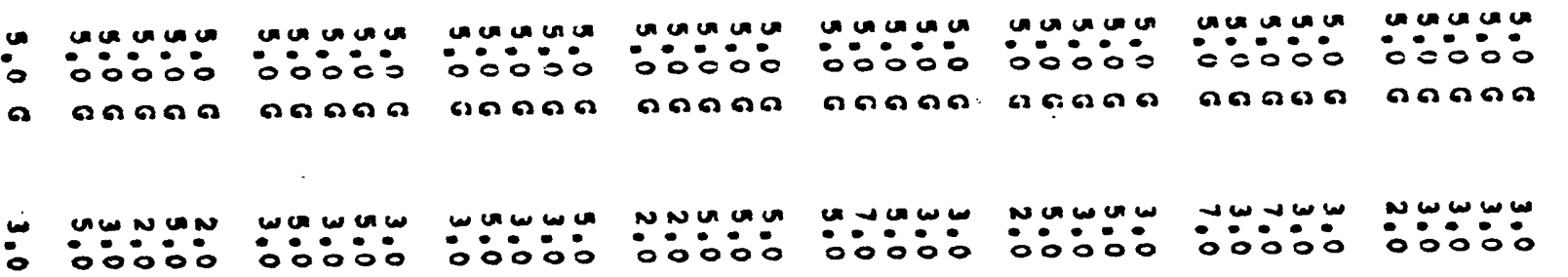

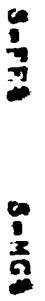

i innin nimin

- inivis

U一inis

군요

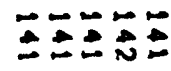

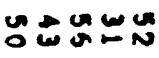

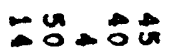

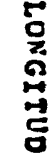

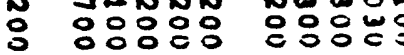

응응ㅇㅇㅇ

응용ㅇㅇ

응ㅇㅇㅇㅇㅇㅇ

in in in

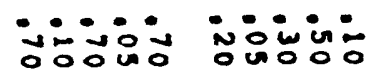

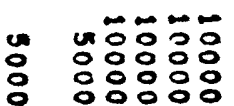

ana
퉁ㅇㅇㅇㅇㅇ 응응융요 응응영영

a

눙유유

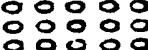

SaAa

넝ㅇㅇㅇㅇㅇㅇ

영ㅇㅇㅇ영

anas

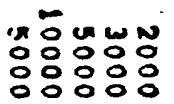

o
동숭ㅇㅇㅇㅇㅇ

응ㅇㅇㅇ영

a $\Omega$

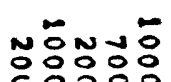

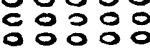

$\circ \quad 0$

둥후웅

영ㅇㅇㅇ응 응ㅇㅇㅇ

กลดดล
- 00000

z

N. NNNNN

2

$Z Z Z Z 2$
00000

inivi-
00000 Nivi co000 in: U i 2

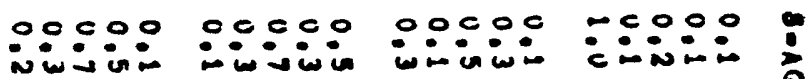

$z$

NNNNN NNNNN NNNNN

NNNNN

$\geq x \geq 2 z$

$z 2 z 2 z$

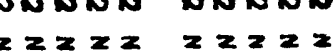

$2222 z$

$z=2 x=$

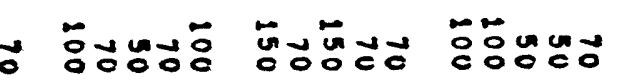
웅잉

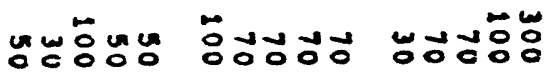

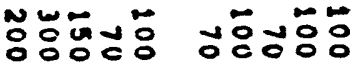

CNNEN

CON NW O000 


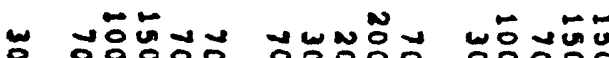

uñ

مैํำ

a

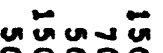

눙등ํํㅇ

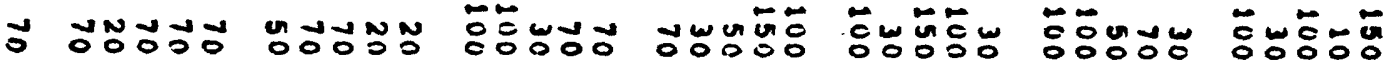

뭉뮤융

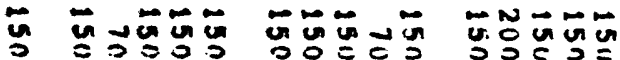

붕ํำ

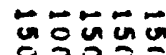

范詑:

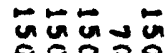

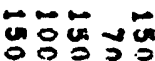

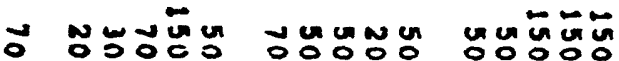
2

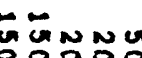
2

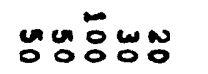

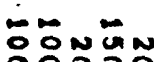

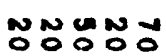

z

$z$

MNGNN

x

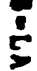

N NNNNN

NNNGN NNNNN

NNNUN NUONN

แกง

$\operatorname{LONNN}$

$2 \geq 2$

N Nun

$z z$

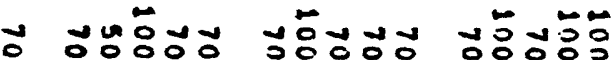

숭융

다에

$\div 2 \div$

$\div \div \div$

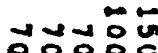
0000 00000 웅유

$\div \quad \operatorname{sen} 0$

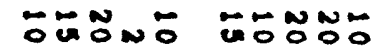

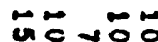

$\rightarrow \div 0$

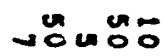

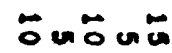

$\operatorname{sac} a n$

$\because \div \div \div \div$

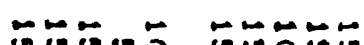

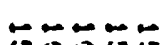

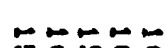

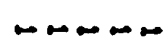

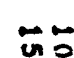

$\underset{20}{2}$

뚱ํํ

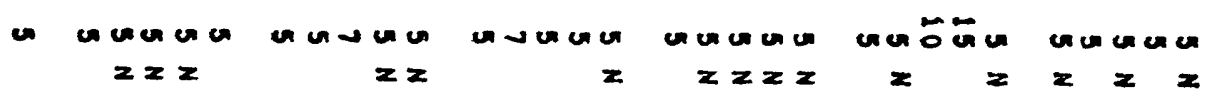

numa

unumo

$z \quad z x$

$z x \geq z z$

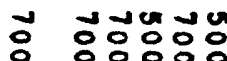

ํㅜㄴำ

un un

and

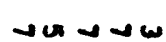

\section{0}

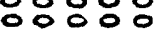

응영

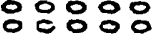

ㅇㅇㅇㅇㅇㅇㅇㅇㅇ

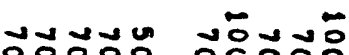
둥ㅇㅇㅇㅇㅇㅇㅇㅇㅇ

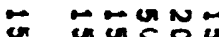

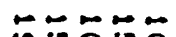

nN-NN

- WNNW

nNN응응융ㄷㅇㅇㅇㅇ 응응응응 㟧

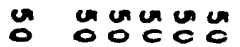

z. $22 \geq 2 z$

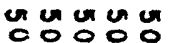

जưñon

unuman

$z \geq z z$

$z 2 z 2 z$

$z z z z z$

unungus un

$x z \geq z x$

vinum

$z z z z=$

unum

$z z z z z$

ungur un

$z \geq z z \geq$

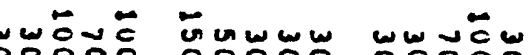

ํํำ

w w n w

(a)

ON $N=0$

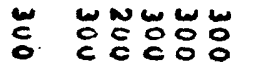

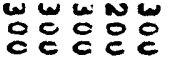

Nww

Nun

N山w

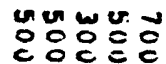

wㅜ

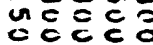

WWNWN 동응유

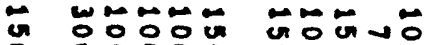

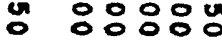

$\because \quad 00 \div \div$

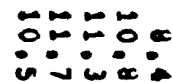
ivi

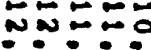
$\therefore-1$. ON NO
Nㅜㅇㅠ -O웅
Nㅠㅇㅠ in inio jo:

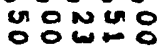


- 운웅

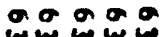
운 $-400$

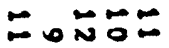
$\vec{u}=\overrightarrow{0}$ 운 -

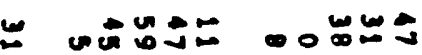

ט. 운요 $\overrightarrow{0}=0$ un un w on NNun

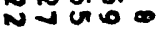

- 200 oraga -

(2)
운 000 $=N-N N$ na $\operatorname{co}$
오웅우 תN ט

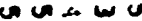
uno

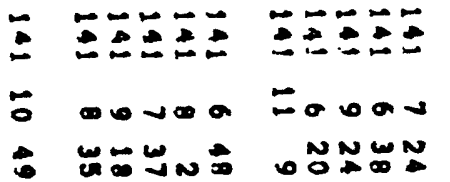

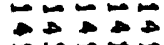
UNज:

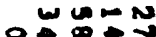

$\because \div \div \div=0$ 문둥

$\omega \neq N$ N $\square=\square$

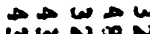

$\rightarrow N \rightarrow$

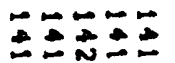
م

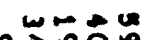
$0=500$ $\because ே ே ே \div$ مَ

WNN UN O DO

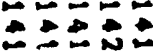

से

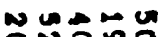

-

a nas unu un -000\%

anasa un un un 00000

ดลดดด unuun -00\%:

nanoa un un un ○0000

กลดกล unuuva

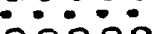
ดกดด wunua a councus

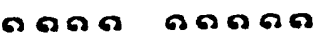

-

in

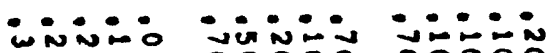

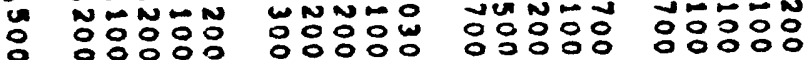

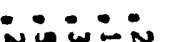
웅요

U士

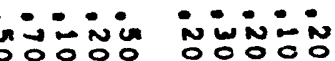

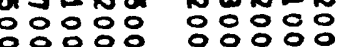

ivivin

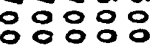

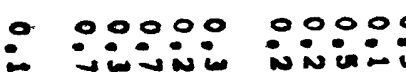

눙ํㅇㅇㅇㅇ

응ㅇㅇㅇㅇㅇㅇ

o
뭉웅뭉 응ㅇㅇㅇㅇㅇㅇ

a
ㄸํㅇํํ유요 응응응

o

$\Omega$
Nㅜㅇㅇㅇㅇㅇㅇㅇ 응응ㅇㅇㅇㅇㅇ

ana
NNNㅠ응 영ㅇㅇㅇㅇㅇㅇ

2

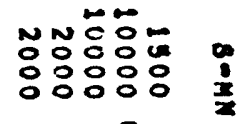

a

\section{อ}

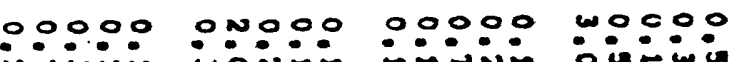

$000000000 n$ Nimi-

1000 won:-

M UNNNN NNNNN NNNNN NNNN $z=2 Z Z Z Z Z Z Z Z Z 2 Z Z Z$
NNNNN $\geq \geq z=$
NONNN

$z=2 z$
NNNNN

$z=2 x=$
NNNNN

$z 2 z \geq 2$ c

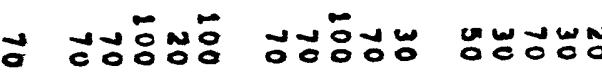

둔둔 등응ㅇㅇㅇㅇㅇㅇ

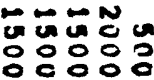

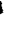

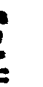




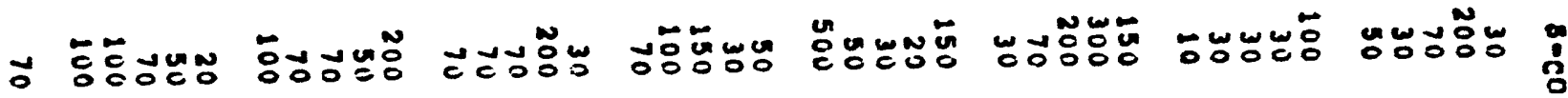

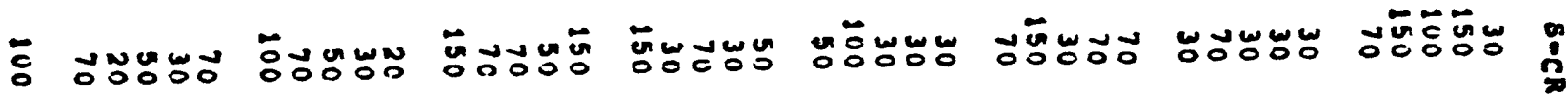

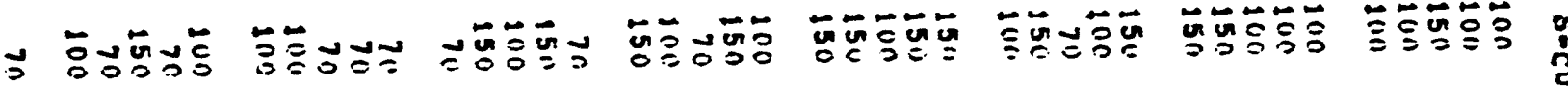

O ONOOОNO $z=$

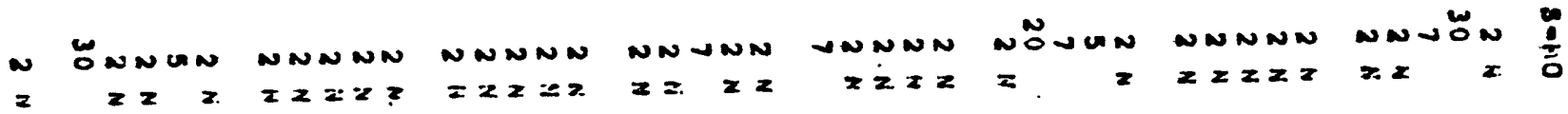

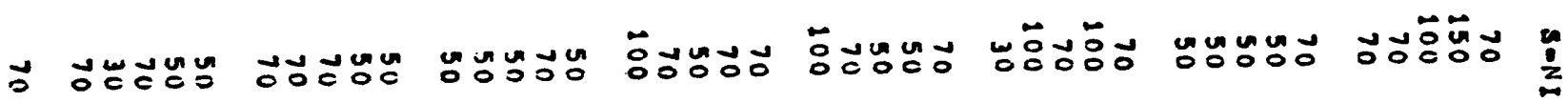

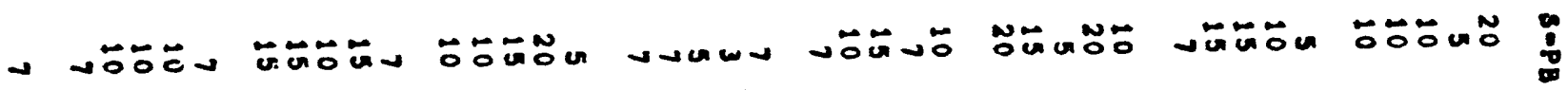

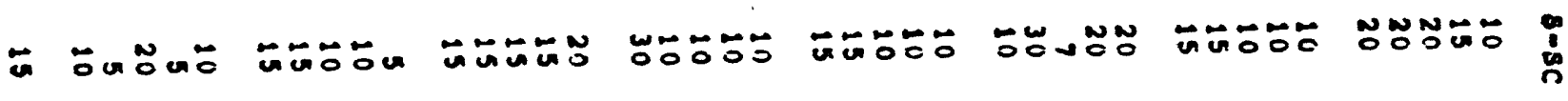

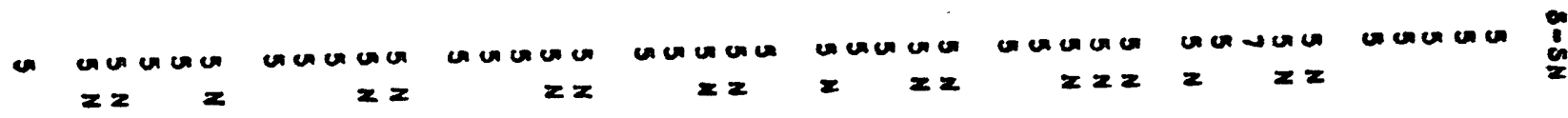

$\overrightarrow{:}$ :

(

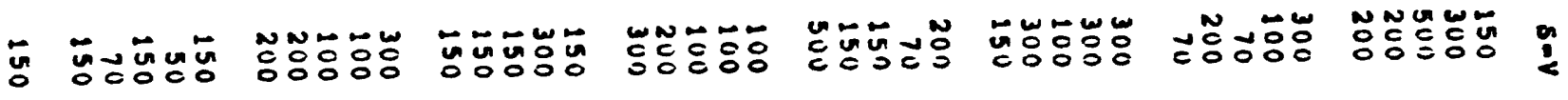

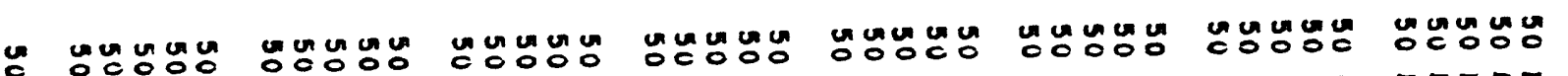

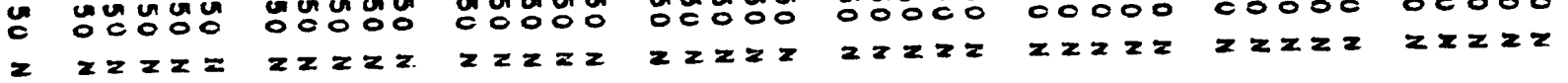

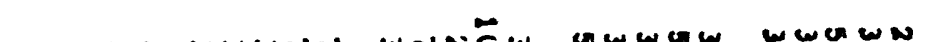

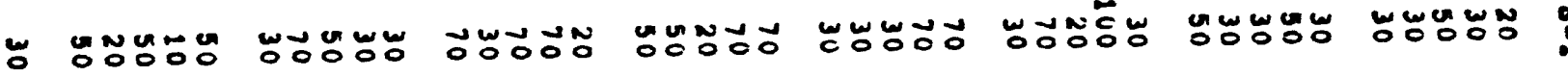

E

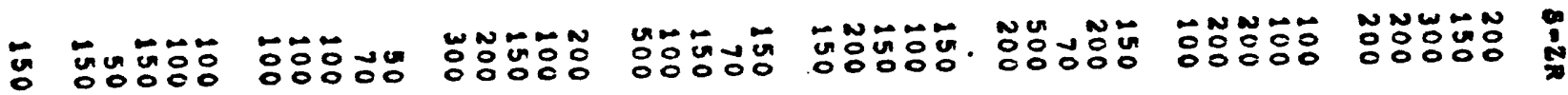

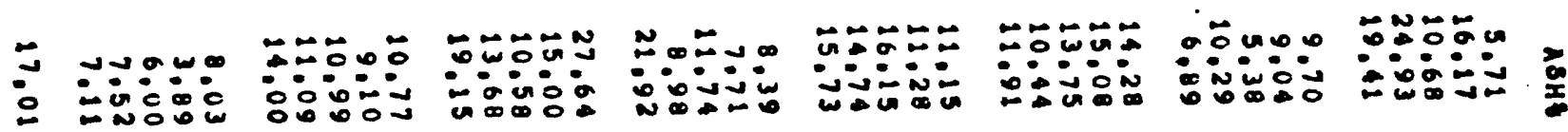


- 운요

w

- $\because 0$ N

$\because \div \div \div$

-

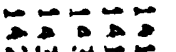

$\because \div \div \div$

טN

ص م

药 Nم:

$\pm \overrightarrow{0} \vec{x}=$

WேN

a nu una unumu

- 00000 00000

a a sal anasa

un un un :0:0\%

vunun -00:0

nanas unumuan un ico:i

ununa c0:00

no un un

की 020
운

ニNー。

$\omega N \rightarrow-n$

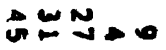

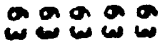

$N-\infty-\infty$

$\because N=0 \div$

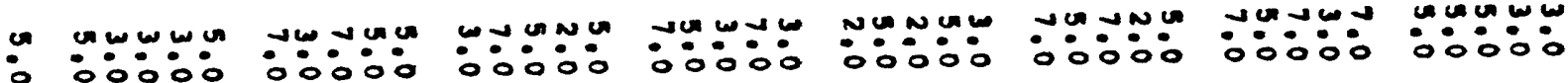

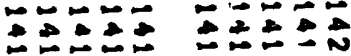

Nw

Nㅜㅇㅎㅇ

G- Nơ

0
2
2
0

i misin ivino jisio

- iDio

ininin ninin

- in

نNini

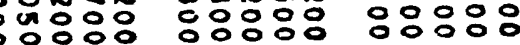

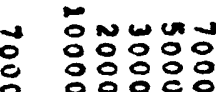

?

등ㅇㅇㅇㅇㅇ

응ㅇㅇㅇㅇㅇㅇ

a

드융ㅇㅇ

응ㅇㅇㅇ응

a

눙유융요

응ㅇㅇㅇㅇㅇ

ดロ 0

응휴

응ㅇㅇㅇㅇㅇ

두웅우

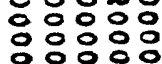

ดคด $\Omega$

ana

웅응유유

웅ㅇㅇㅇㅇㅇㅇ

ด 9

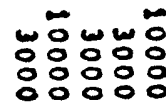

a

- 00000 00000 0000000000

00000

ONODO

00000

00000

$\because 0: 0: 0$

$z$

2

ivini

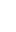

NNNNN

NNNNN

NNNNN

NNNNN

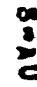

$z=2$

$z \geq z \geq z$

$\geq \geq \geq \geq \geq$

$z \geq \geq z$

$2 \geq 222$

$2222=$

$Z=2=$

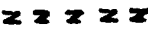

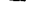

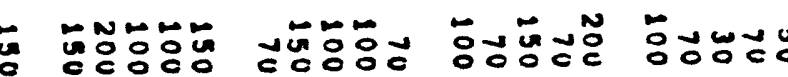

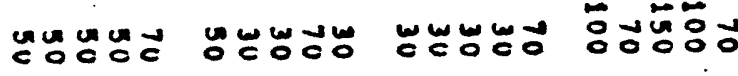

0

:

$\because N \sim \mathbb{O}$

\section{-}

N N N

$N N N=$

NNNND

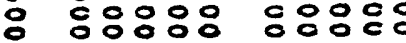
O응ㅇㅇㅇ 웅용ㅇㅇㅇํㅇ 웅웅ㅇㅇㅇ응 응응ㅇㅇㅇㅇㅇㅇ

COCEU ENUUE

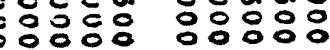




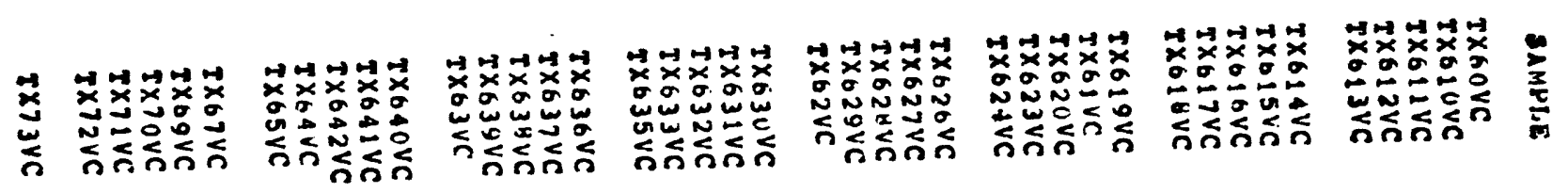

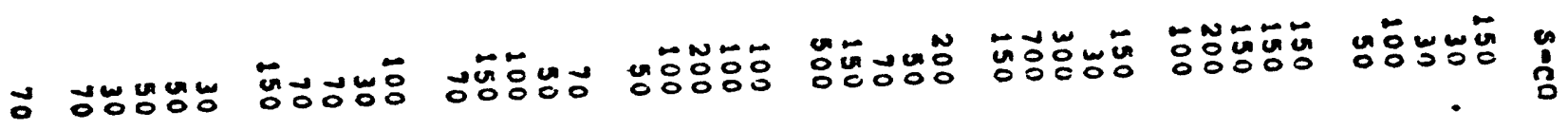

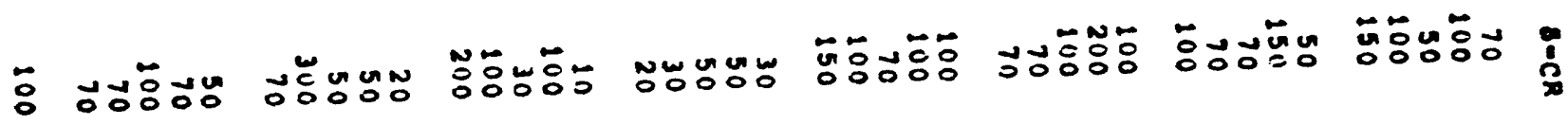

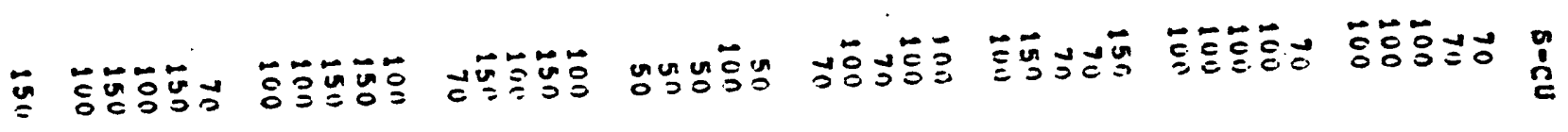

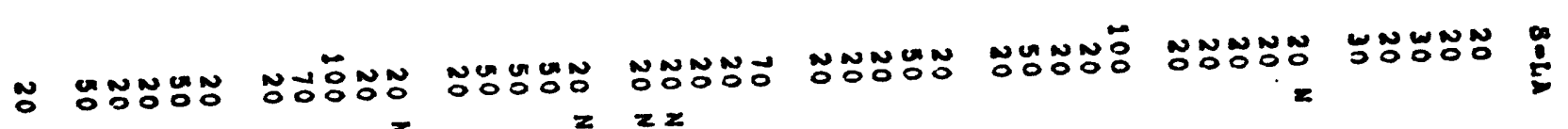

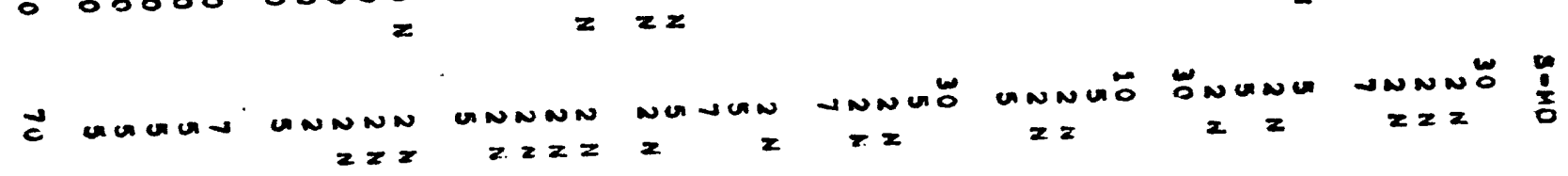

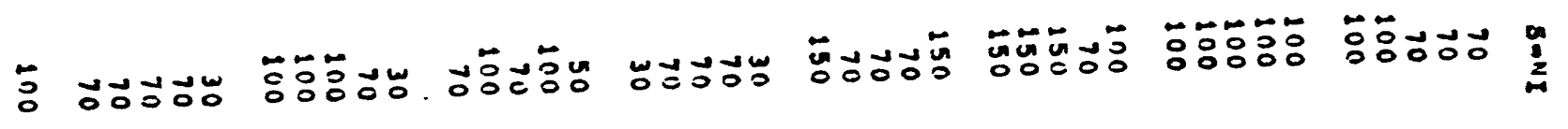

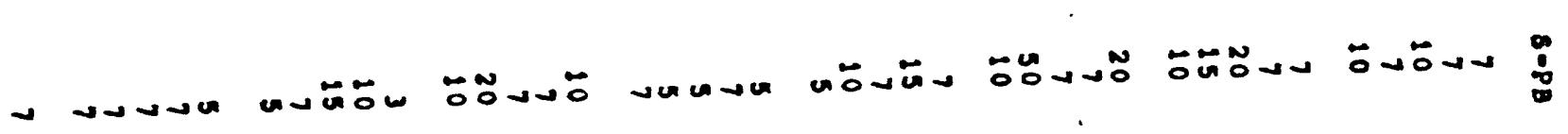

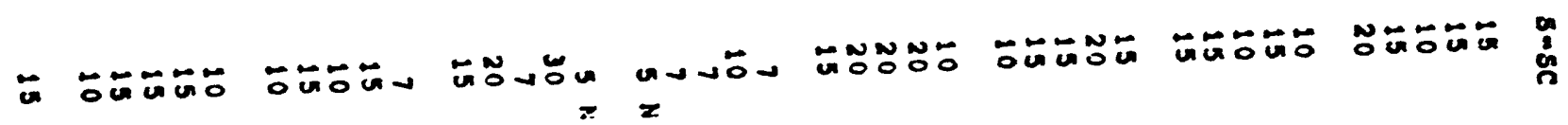

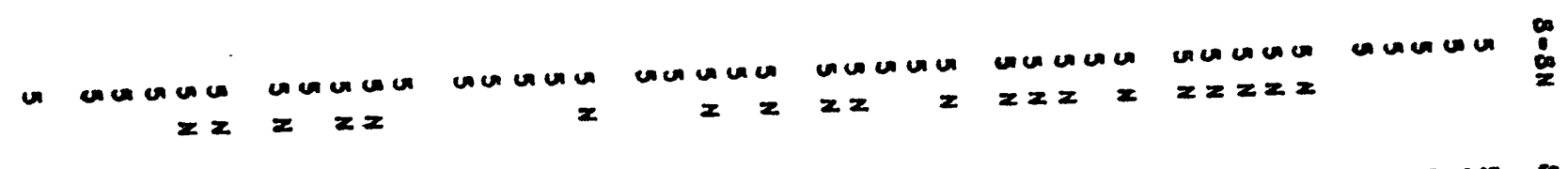

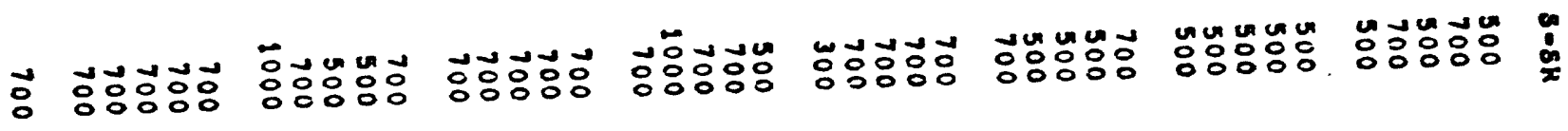

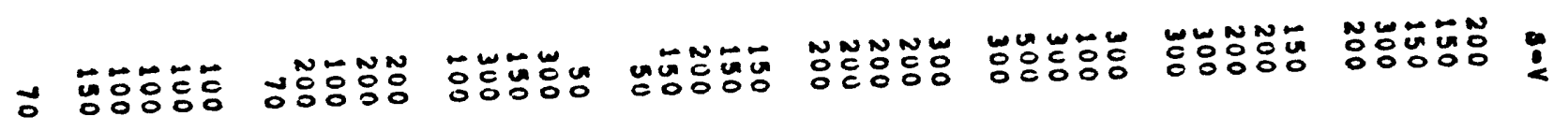

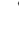

\& ํํㅇํํㅇํㅇ

Un uño: uno:

ununu

ununung

unu uno

uningun

z $z z z z \quad z z z z$

$z z z z z z z z z$

$z=z$

coloco

vinunu

능ํํㅇํㅇㅇㅠ:

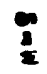

ㄸํㅇำ

$\ddot{2}$

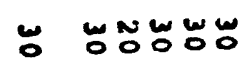

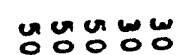

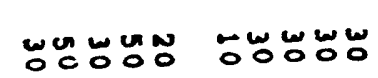

๘๐ัน์

तैWWWD

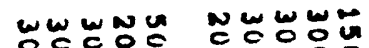

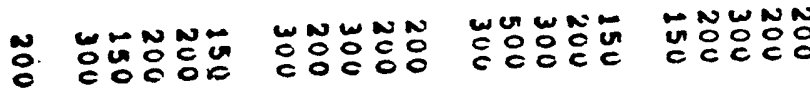

두일

000

돋읃

过品

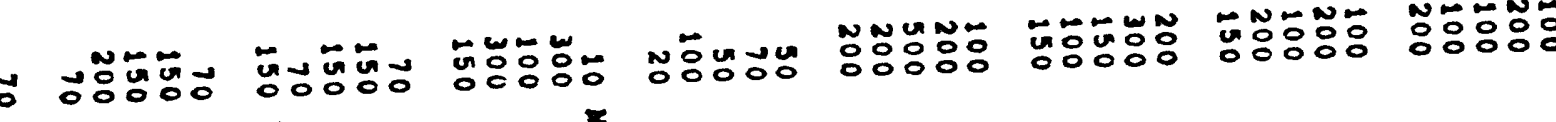

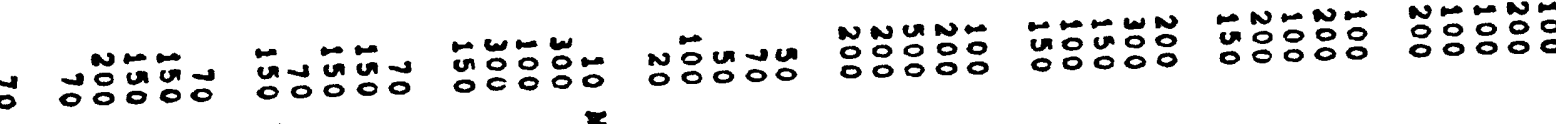

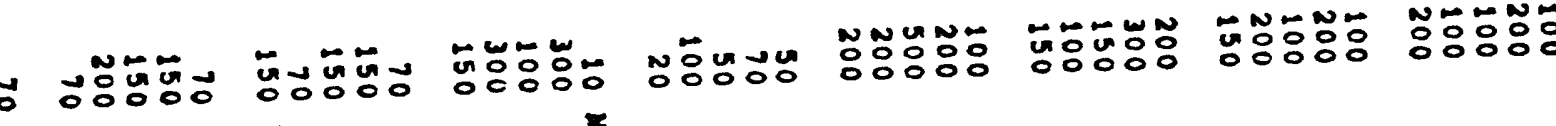

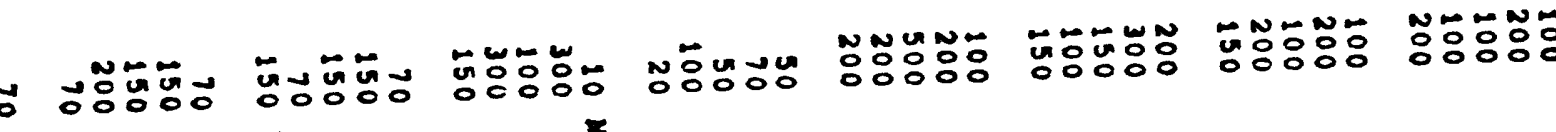

$\therefore$

$\cos 2 \pi$

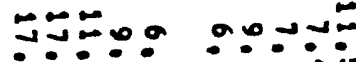

N:

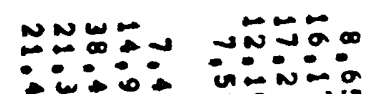

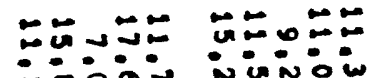

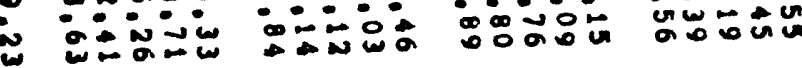

in: $=0 \div$

iw o요

Niñi

WNNNW 
00000 00000



웅aㅇ

웅aㅇ

운운

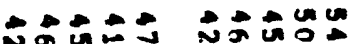

뚱ำ

ט.

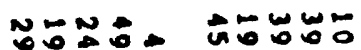

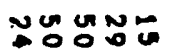

$N=w u$

w N U

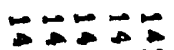

$\because \underset{N}{*}$

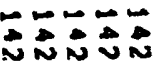

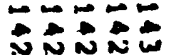

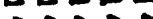

W- WN

뿌ำ

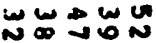

$U \cup=U N$

-

जNO

"

UNNNN

N

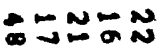

후 $u \neq=$

-

जำ

unumar unumu $\therefore \therefore: 0$ जU:

ดลกด

ดคกล

un unva

$\therefore 0 \div 0$

ununa unuman

$\therefore 0.0$

0.0

a กล

ดลa

ดอด 9

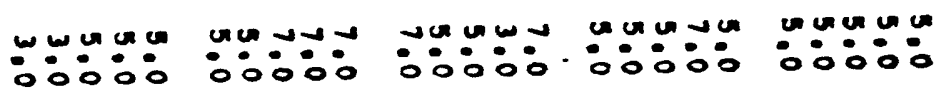

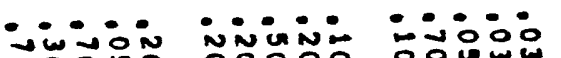

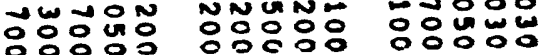

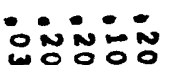

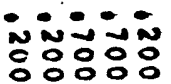

《드유 무ㅇㅠㅠ

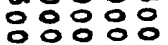

ㅇㅇㅇㅇㅇㅇㅇㅇ

응ㅇㅇㅇㅇㅇㅇㅇ

a a

ano

누우웅

둥후 영영융영ㅇㅇㅇㅇㅇㅇㅇㅇㅇ

누웡

a

$\Omega$

0000000000 O50: 00:00 0000?

NNNNN

NWNNN

NNNNN

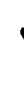

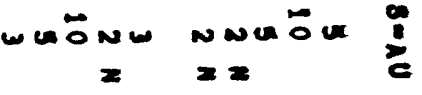

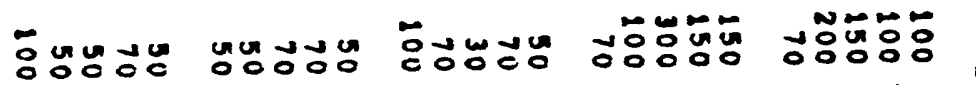

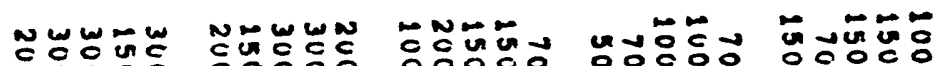

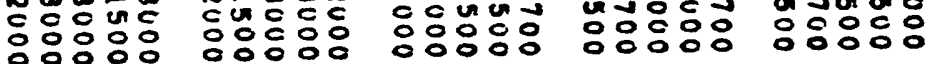

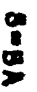

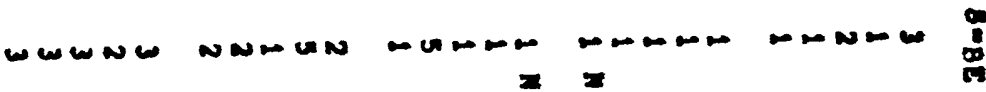

N U U UNN 


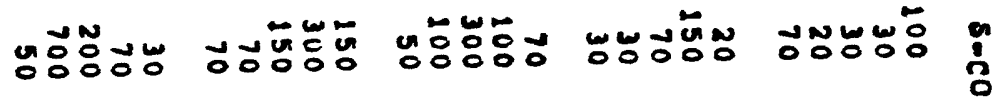

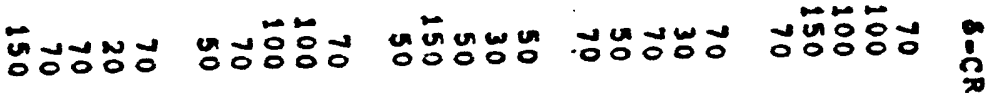

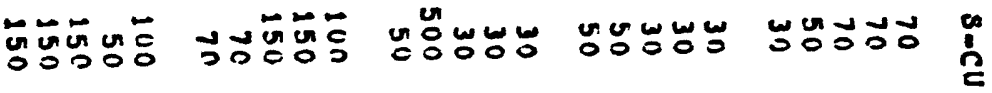

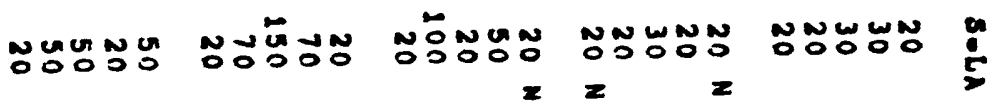

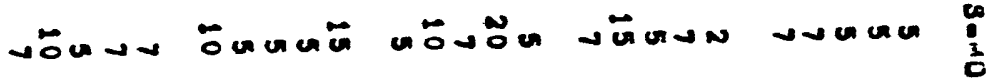

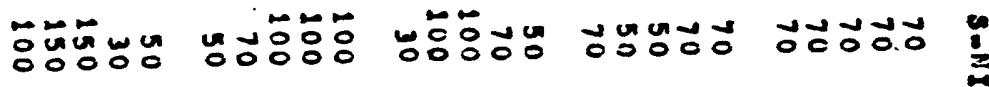
rusun

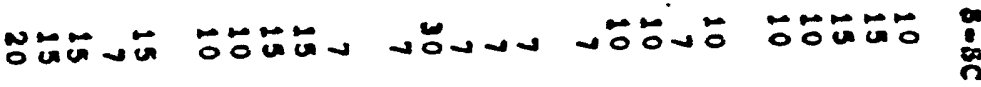

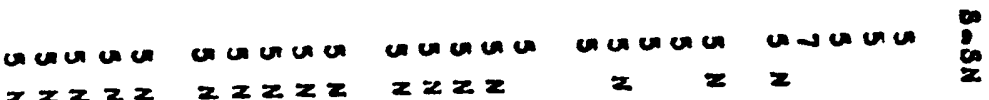

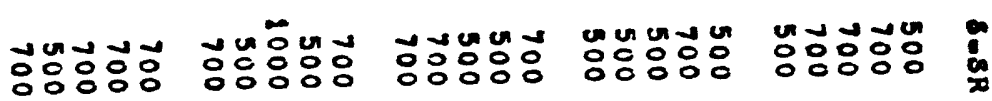

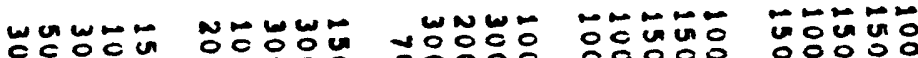

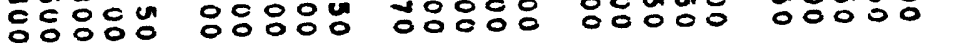

\begin{tabular}{|c|c|c|c|c|}
\hline $\begin{array}{l}\text { ingug unu } \\
\text { onoㅇㅇㅇ } \\
z z 2 z x\end{array}$ & 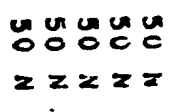 & 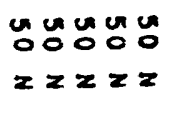 & 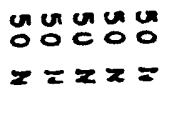 & 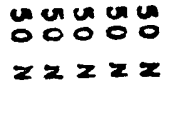 \\
\hline Wuñơ & 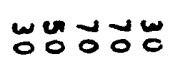 & 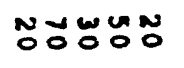 & NDWW & 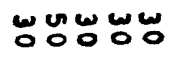 \\
\hline $\begin{array}{l}N U n \\
\circ \\
0\end{array}$ & 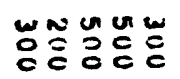 & WWNيّة & 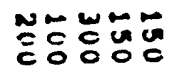 & 象产品 \\
\hline
\end{tabular}

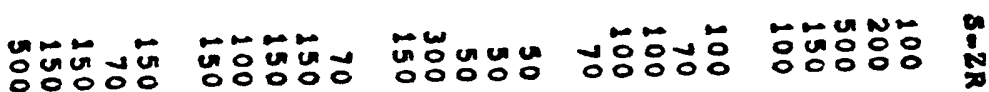

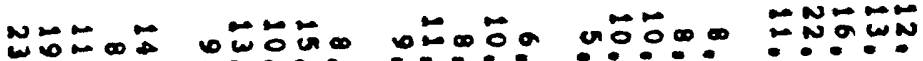
W 
Ash of Streambank sod:

Element not looked for: INST-Hg. The following elements were looked for but not detected: S-Au, S-Bi, S-Cd, and S-W.

S-As was not detected except in sample TX 634P (200 ppm). 


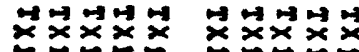

-

*

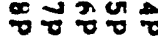
$\omega \omega \omega N$

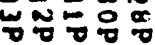

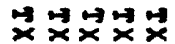
$x \times x x$ nNNNN

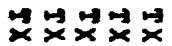

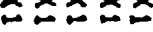
NNNN=

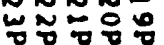

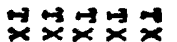
$\approx \sim 0 \div$

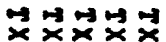

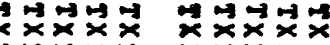

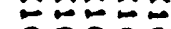

WN=ㅇㅇㅇ응ㅇㅇㅇㅇㅇ

乐宁出

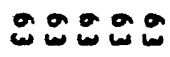
añ $\therefore$ un

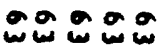
anu un L 0 - 0 on UNO

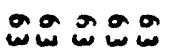
un unu

cin

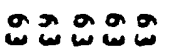
unueru W

운 un un un

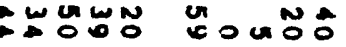

00090 ט שـ जि

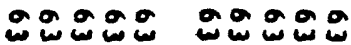

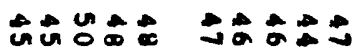

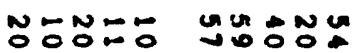

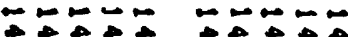

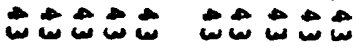
$-\infty \ldots$

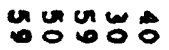
$\omega \rightarrow N=A$ W⿴N WO $-\infty-\infty=$ ش

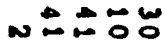
แN유 $\underset{\infty}{\infty}+\infty$ WNーニ Nữ O O Nw w w on
GNNNA

No
WDE

NNNNE

con un a

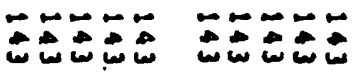

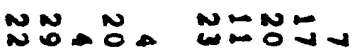

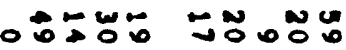
$+$

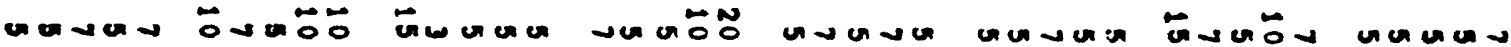

Oidoin

DOOOOO OOOOOOO OOOOOO

wо̆

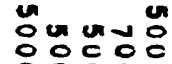
00000

ด

$\Omega$

uñ uñ co: 0

$a n$

둥ํㅇ 응영영응
둥ํ응 등응응

○

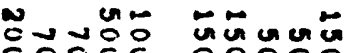

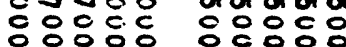

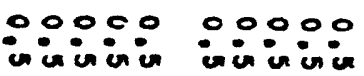

$z z z z z z z z z$
00000 ininivin $z \geq z=2$
00000 inivivis $z z z z z$
우웅 uviui $z \geq z z z$
00000 uninin

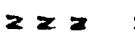

00000 in in in in

$z 2 z 2$
00000 in in in

$z x z z x$ $\vdots$

8
ООㅇㅇㅠ Nㅠㅇㅠ r

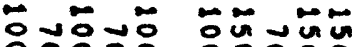
응영융흉영영영응
ํㅜㅇ서융유 응응응융유 동ㅎㅇㅇㅇㅇㅇㅇㅇ
뭉요융융
OONOO

te 5
00000

5

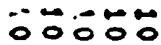
t 5 $\frac{0}{0}$
둥후

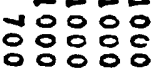

엉뎡응 응융융 


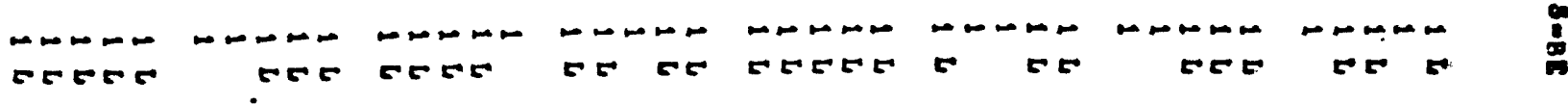

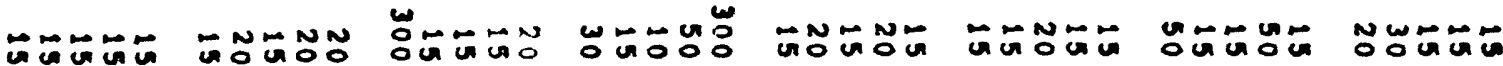

$m=N=-\infty$

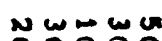

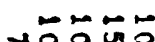

in

NNO

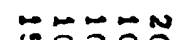

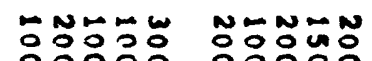

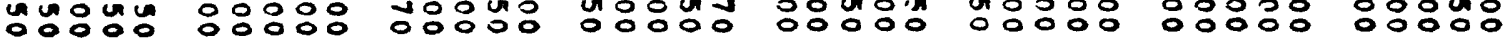

:

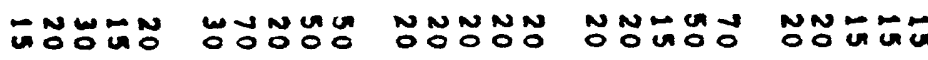

ENOEN

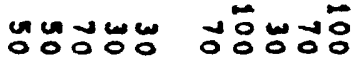

Noñ

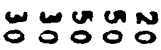

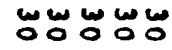

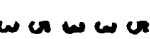
०0000

ำ ONONOO

$n \in u \in u$
$z z z 2 z$

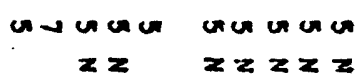

ununơ

unumu

$z \geq z x$

$z \geq z z z$

un unsu

$z \geq z=$

uncuno

$z \geq z \geq z$

unun un

$z \geq 2 z$

NONONO NONONO NNONON ONOOONONO NNNNNN

LE CETECE

ZETE
NONONN

Cot
NNOONO

C5E

$25 \geq 5=$
กํํㅇㅇㅇㅇ

Cate

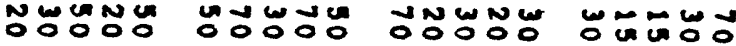

NNNGE NOEO
OOONON
NOW

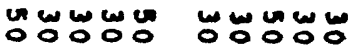

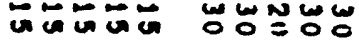

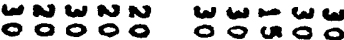

UENONO N

NNETN
NG NGN
Nㅡㅇㅠㅡㅇ NGNONO
ேㅜㄷㅎㅇ

$2222=$

ேㅜㅇํํ

무융웅

ํㅜㅇ두웅

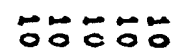

$z 2 z 2 z$

I 22

z 2 2 ?

ㅜㅜ웅

z $\geq 2=$

두웅ㅇ

$z x z z$

드융유

$z z z z$

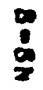

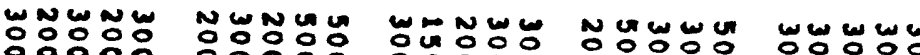

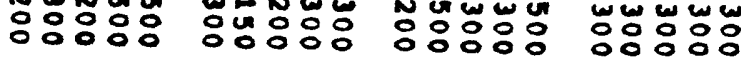
w⿻上丨

N $00: 0: 0$

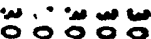

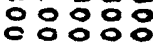

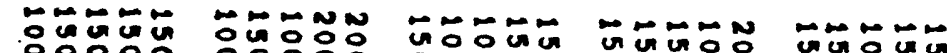

드음

N-NO 웅영영응

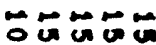

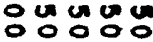


$\vec{x} \vec{x} \vec{x} \vec{x} \vec{x} \quad \vec{x} \vec{x} \vec{x} \vec{x} \vec{x}$

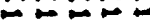

$\omega \omega \omega$

$\because \rightarrow \lim _{0}^{\infty}$

WNWN

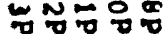

$\vec{x} \boldsymbol{x} \vec{x} \vec{x} \vec{x}$

NNNNN

a

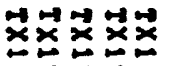

NNNN茫

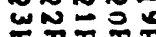

$\vec{x} \vec{x} \vec{x} \dot{x} \vec{x}$

$Z=\square=Z$

$\infty=50$

벚부버ㅈㅓㅜ

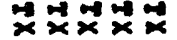

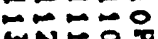

Nㅜㅇㅇㅠ
500-5 50 둥

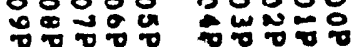

مس

Nㅡㅇ영용ㅇㅇ

$z \geq x z=$

Nㅠㅇㅛ

융융응

UNN NNN N

NNNNL

$z z z z$

$25 \times 2$

NNNNN

응ㅇㅇㅇㅇㅇㅇㅇㅇ

NNNNN

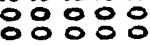

NNNNN

ㅇㅇㅇㅇㅇㅇㅇㅇ

$z z z z z$

$z z z z$

$\geq \geq 25$

NNNNN

웅ㅇㅇㅇ

$25 \geq 2 z$

NWNNE UNU U

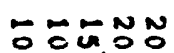

NNNN

WWN

LNNNN

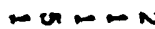

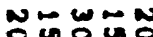

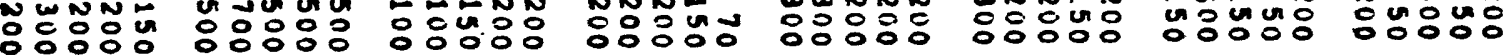

$\therefore: 0: 0: 0: 0$ uninu

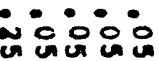

i웅용

운운웅요

$\because: 0: 0$

icio:

z. $z \geq \quad z \geq z \geq \infty$

$z z z z z$

$z z=2 z$

$z \geq z$

$z \geq z z z$

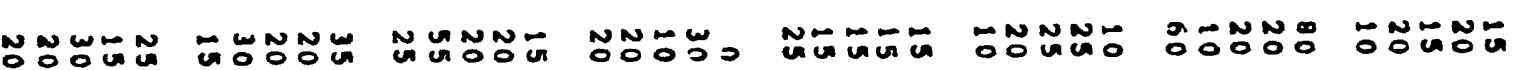
-

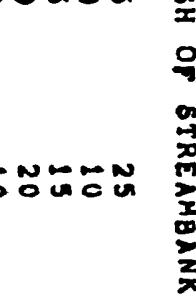

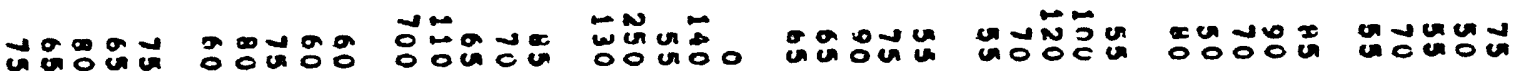
$\infty$

$\frac{2}{3}$

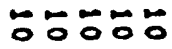

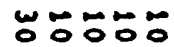

훙유

유유

두유

두융ㅇ 융웅

$z \geq z \geq 2$

ze $\quad 2 x$

z $\geq z$

$z \geq$

$z$

Cret<x

$z \geq z E z=5 z$

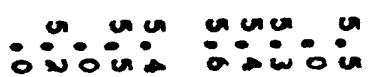

unumu

un unca

(a)

un un unuara caunum

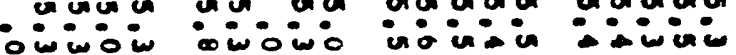
cr

$\infty$

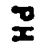

$\infty$ -

$\boldsymbol{c}$

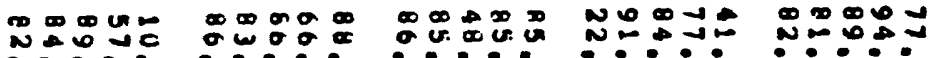

ㄱó

퓰

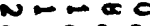
iñ

$\rightarrow \infty \infty 2$

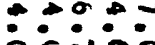

至

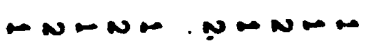

$\sim \sim \sim N$

$\sim \boldsymbol{N} \sim \boldsymbol{N}$

WNMN

NNNNN

NNWNA NNNND

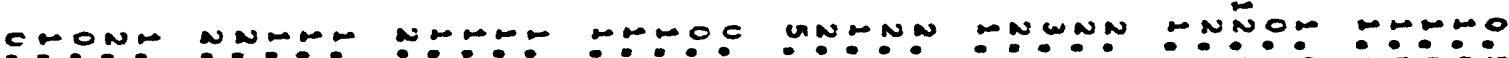

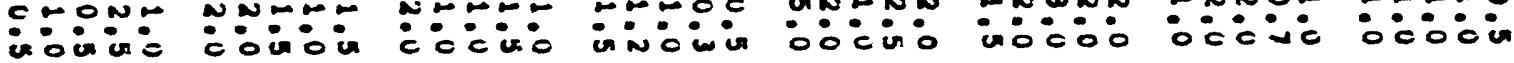

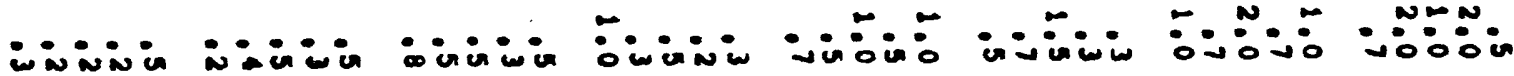


a 00000

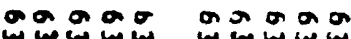

anoa 0

oraso

a s. 00 ט $w \rightarrow$ wa

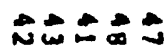

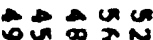
anciun in WNatco

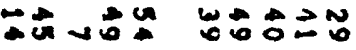

WONA

w

- 4 a w a ơ un un

$=$ N匹0

$\overrightarrow{0}-\overrightarrow{0} \ddot{0}$

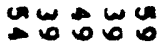

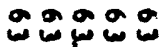

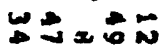

แั

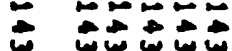 \\ 0 - م \\ N}

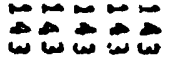

טש

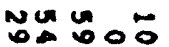

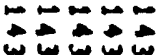

and

un

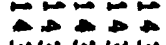

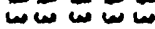

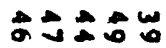
ำ

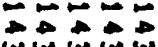
W un ㅎํำ

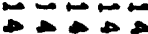
ט U⿴囗十⺝木 un 0 : $\because \square \square$

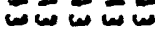
No

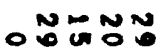

ro

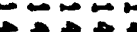
Nこむ山ー is us ס ט $0000 \%$ C

o onsones

crenona vando

and an

ancen

$\operatorname{lon} 2$

$\ln x \rightarrow$

$\ln a n \rightarrow$

- Namnn

NNNMN

WN-

DEN N N

$\div-\infty-\infty$

N-NNN

NNNNN

NNNNN :000\% $\therefore: 0 \circ$. 章

$\rightarrow$ MnEMN

$n=m-2$

-

$\because E N=N$

$\because-\div-n$

$\div \div \div \div$

눈

잉우

뚜에 응ㅇㅇㅇㅇㅇㅇㅇㅇ

뚱용 웅용응

무웡ㅇㅇ

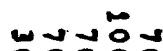
등응ㅇㅇㅇㅇㅇㅇ

L 응영영
$\Omega$

\section{c0000 inisivis}

$z x \geq z$

- 00000

- iviuivi

$z z z z z$ 00000 inivinis incion

$z \geq z x z$ $z x \geq z z$

\section{$000=0$ univio $z=$}

00000
inivivi
$z 2 z=2$

00000 unisin $z z=2$

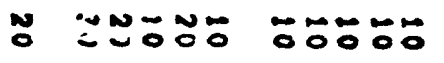
5
뭉영 te
유유 무유

rom
ேㅜ웅 5
ேㅜ웅 


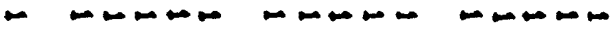

$\infty+\infty$

Mー

ח

$-\infty-\infty$

M M M

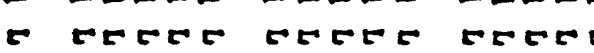

एECE CERE

Cक्ट

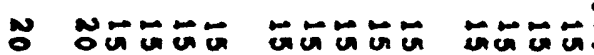

$\because \approx \div \div=$

Gúva

जேะே・

in $=\pi$

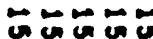

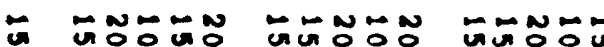

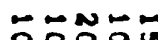

$-\pi 0^{2}-n$

Hama

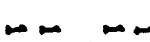

ara

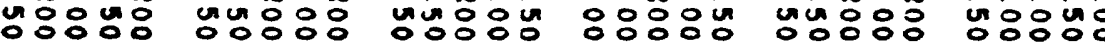

:

눙영융영

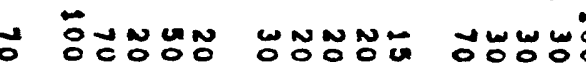

NonNon

TNONN

ט

\section{$r$}

NONOE NONONON

ฉ̊

ڤ ڤ艹

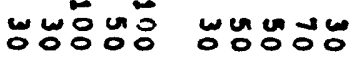

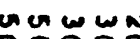

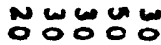

$\sum_{2}^{\infty}$

O ounan

unuma

unumas

uncuun un

unuma

as anu

unuoun

unano

$=2 x z$

$z z z z z z z z$

$x \geq x \geq 2$

$z \geq 2$

$z=2$

$z z z z$

$z \geq \geq 2 \geq$

:

O OCENOE ONOEO ON

NONNO

NNNONO

NNNNN

NONNN

NNNNN

CECE

$c r$

5 5

NNNNN

zete

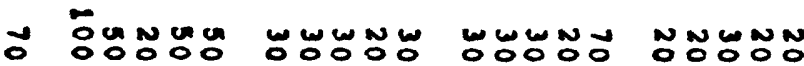

nNand

WNNWE-
NOONONO

U: NOW

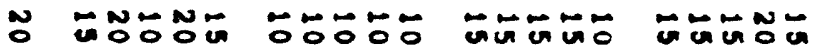

NG NOE

UOMON

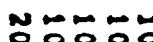

Gே 뜽

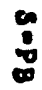

C OOOOㅇ NONOTO NONONO NONONN

NONNN

NanNa

$\because N=N$

NGN NON

:

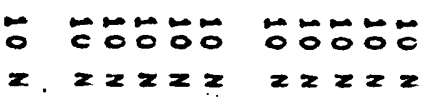

뚜ㅇㅠㅜ

z $212 z$

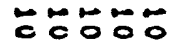

$z 2 z 2$

뚱후웅

$z z z z$

둥두웅

$2 \geq 222$

드웅웅

zzzz

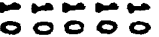

$z z z=2$
:

WسNN WNNN

NONONO

WNN Now

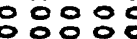

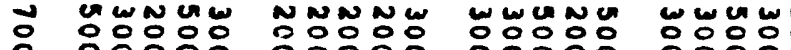



* WENLE KNONON

- NnNNN ㅇㅇㅇㅇㅇㅇㅇㅇㅇ z 20

NNNNN 응ㅇㅇㅇㅇㅇㅇ

$z z z z$

NNNNN

응ㅇㅇㅇㅇㅇㅇ

zzz
nNNNN

응영영

$z z z z z$
OODOO 응응ㅇㅇㅇㅇㅇ

$z z z z z$
NNNNNN

$z z x z=$
Nㅡ융유

$z z z z z$
NNNNN 응ㅇㅇㅇㅇㅛ $z z z z=$
-

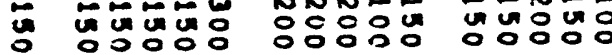

NUMN

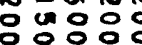

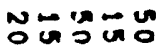
ㅇํㅇํํㅇํㅇ
UUNONO UNOOOOO UEOOOOO

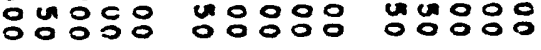

ㅇㅇㅇ웅요웅

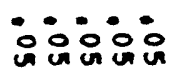

- $x \geq 5 z$ $z x z z$
무웅웅 웅ㅇㅇㅇㅇㅜ 옹운운으

웅ㅇㅇ

$z z z z \infty \quad z z z z z$ $\div 0 \div 0$

웅웅

잉우 $z z \quad z z$

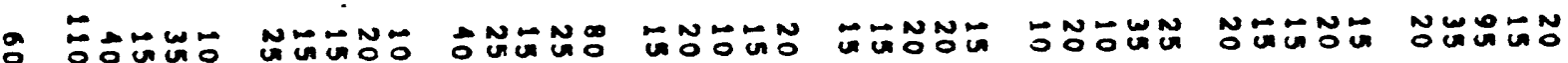
W

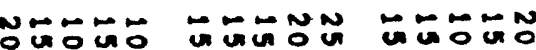

$\operatorname{COONO}$ -NGWN

NONON

In

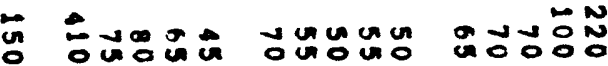

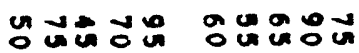

뭉요 누요

inisum

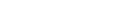

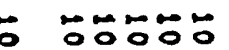

$20=$

ஸே0ั0\%

NOOC

Nㅜㅇㅠ

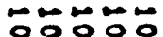

Ex $2 \geq$

$z$

$=20^{\circ}$

$z z=$

Б๐ํㅇํ

$z \geq z \geq z$

०ேㅜㅇㅠ

:....

0.

-

c. un

unaun

un in us an का

$+\infty$

$\infty$

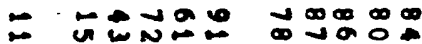

- acoil

$\infty \infty \infty N \infty$

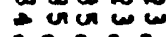
a: :0:

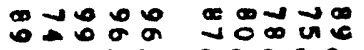
io: 0 isiois $\because$ unu unuuge ung no

$\infty$

푼

$\infty$

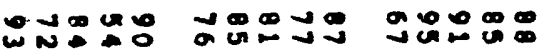
n:-0 $c$
2
2

:

- $0000 N$ NNNNR NNNOO

NDNNN NNNNA

NNNNN NNRNN NNNNN

3

- 000

$\infty 0$

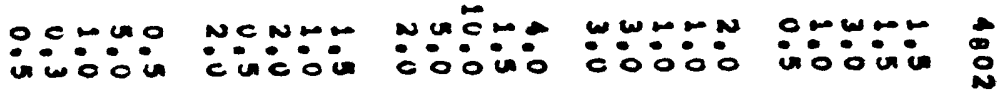

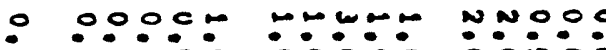
:00: :0:0: :0i:0

$\infty$ 000

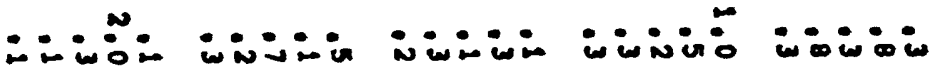
$\stackrel{0}{0}$ - 000

$\infty$ 
o 09090

a saunu

- ONwOW

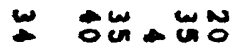

준에

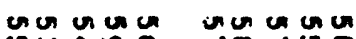

N n
문 ununu जी परत्र

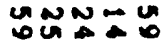

or an a d w un 0 u $\rightarrow-N$ N

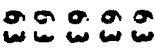
טun $\because$ UNa
\% unuau N icas unviun na 的会出

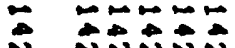 \\ anNan

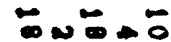

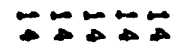 NANAN

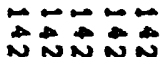

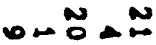 $\because \underset{\infty}{\infty}=$

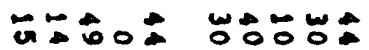 \\ NANA

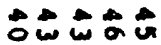

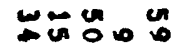 \\ जu} ơon un $\rightarrow \infty$ सेNAN धล:

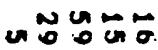

$\leftarrow+\infty$

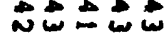
caㄷㄴ w:

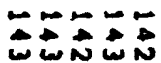
Nم大 Oํㅠ는

$$
5
$$

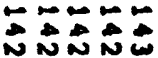

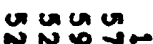
○OONN

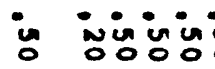

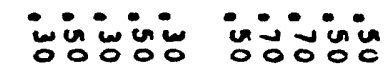

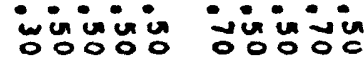

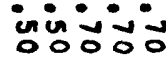
บับ

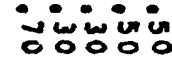

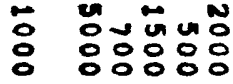
a

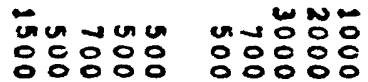

- uvini

$z \quad z z z z$
이잉 in in ini

$z z z z$
00000 un un un

$z z \geq z z$
UnNNㅜㅇ 응ㅇㅇㅇㅇㅇㅇ융 $\Omega$

กำกับ 융유

a
ํํㅇํํํํํำ

ํํㅇํํำ ㅇㅇㅇㅇㅇㅇㅇㅇ an
NUNN ¿:00: inivin $z z z z$ oc:00

$z \geq z z$
00000 univivi

$z z 2 z z$
00000 incuivin $z \geq z z=$
0 \%000 acinis $z=$
2

두융 유융 $+$ t-
ละํำ TE

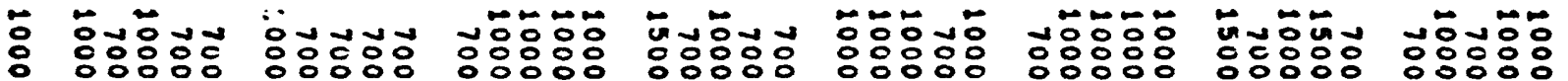




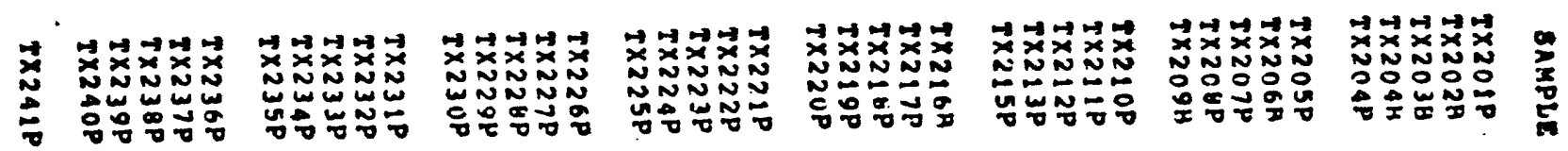

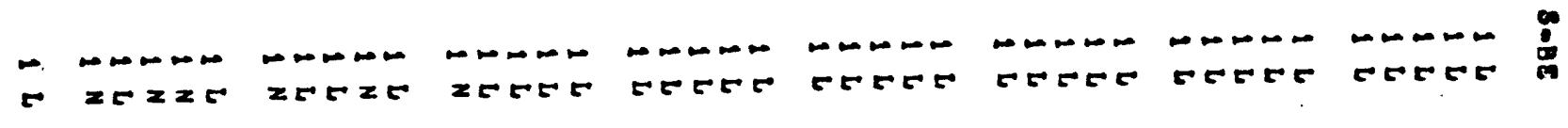

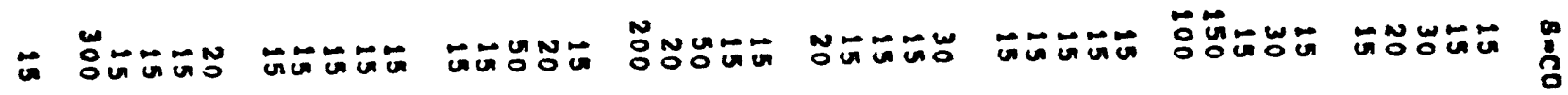

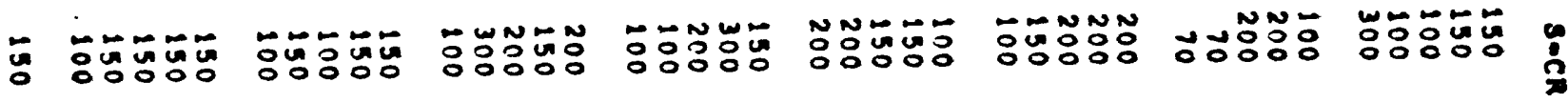

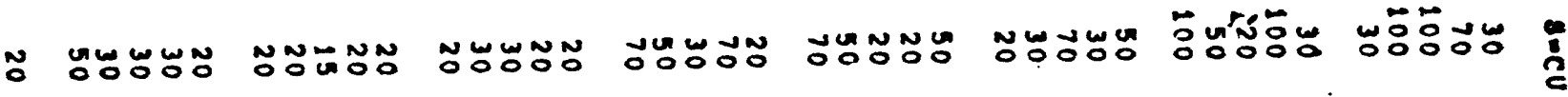

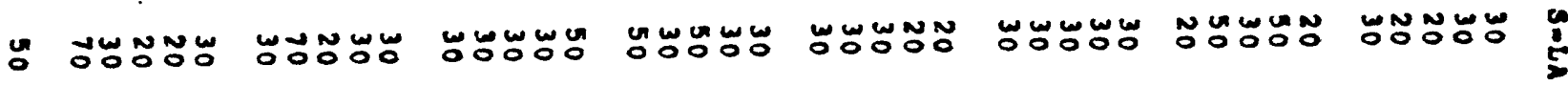

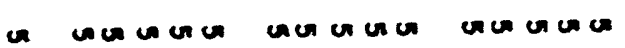

Öunum unumun

$\ln \rightarrow \infty u$

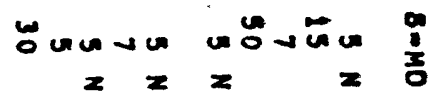

$z z z z z z z z=2 z z$

$z \geq z z$

$z z z z z z$

NONONOO NONONO

NNNNN

NOOOON NONONN

NOOOOO

IEx $=2 x \geq z$

C世: $=2$

NONON

NNNNN

CE $2 x=5$

$x \geq z=2$

$12 x \geq 2$

$x=25$

$2022=$

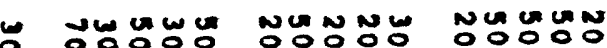

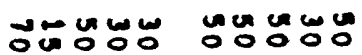

wongun

तै

:

$\underset{x}{2}$

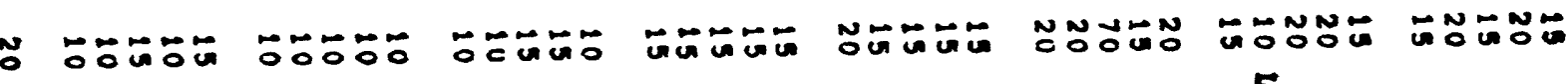

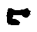

5

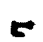

3

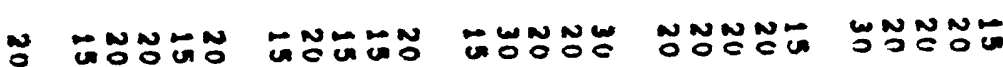

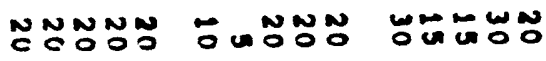

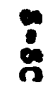

- $\overrightarrow{0} \ddot{0} \ddot{0}$

ㅜㅜ웅

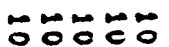

๐ேேேேே0

бํํ두

бே0ே0

$\div \div \div \div 0$

둥후웅

$z \quad z z=2$

$z z z z$

$z \geq 2 z=2 z 2 z=$

$2 z \geq 2 x$

$z=2 \geq 2$

$z \geq z 2 x$

$z=z z \quad \stackrel{0}{x}$

ڤ 응

cos

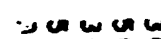

융유 영영융

$\frac{1}{20}$

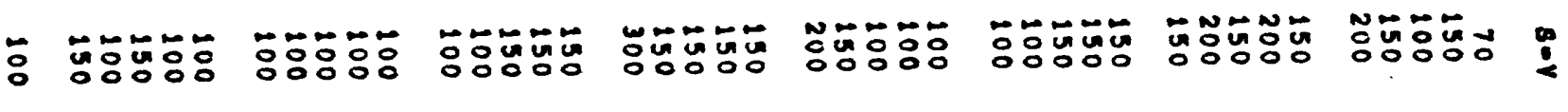




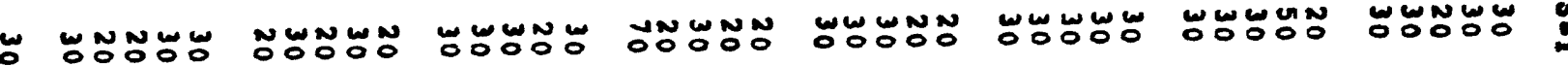

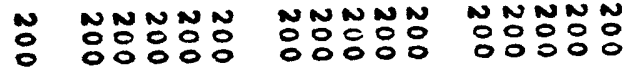
$z z Z z Z z Z z=z Z z Z$
$\operatorname{Ln} \cos 0$

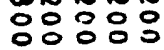

$z \geq z z$
NNNNM 응ㅇㅇㅇㅇㅇㅇ

$2+\mathrm{CH}^{2}$ 응ㅇㅇㅇㅇㅇㅇ z zzz
NNNNN
NNNNN

응옹융용

$z z=$
NWEN 융영영 $z \quad z z$

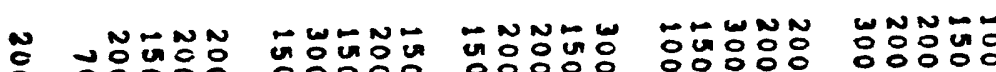

- ㄱㅇㅇ융ㅇㅇㅇ

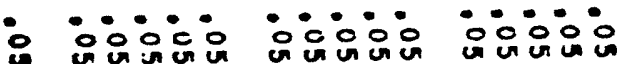

$z \quad z z z z$

$z=2 \pi$

$z \geq z \geq z$

웅운운

w $2 \geq 25$

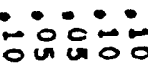

둥웅웅

ए5

$t=25=$

영유

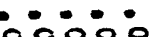

MnNu

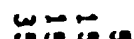

- W NOONO

W־EN

NONONO

I Unu un

una un

a

.

$\infty \geq 2$

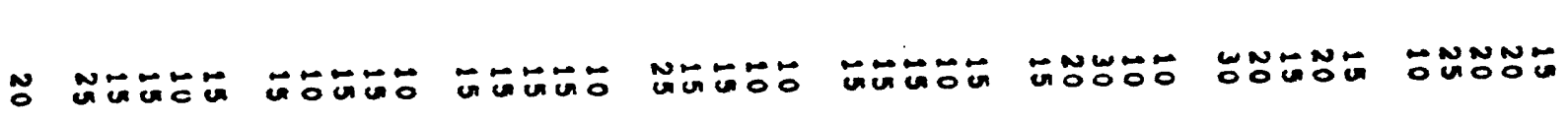

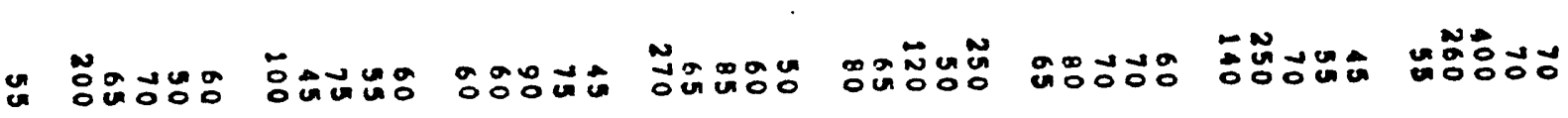
(2)

5

a $\quad n \rightarrow u \rightarrow$ u un unum c.

\section{$\omega \quad \omega \infty \infty \infty$ \\ - $\div$ -}

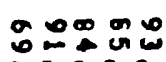
wo a ci

$\infty \infty \infty \infty$ - $0 N$

n小ąo $-1000$ - 05004 iói.

00000 N6\% ioin:

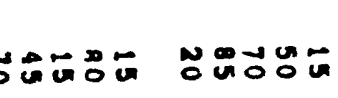

ט $\infty$

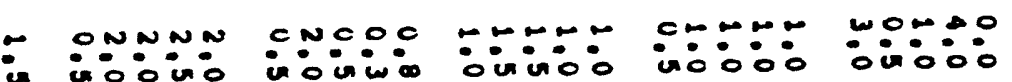

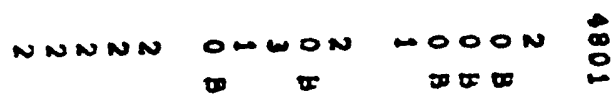

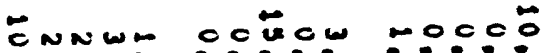

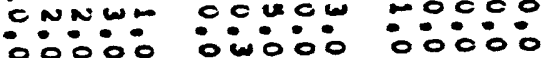
- 1000

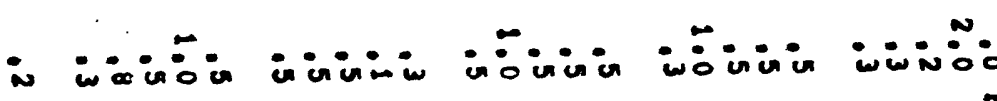

$\because \ldots$ inisio ×
in:O0:

ONNON NOOON - 


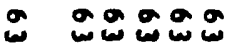

*

$\therefore 0^{n}{ }^{n}$

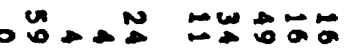

웅 90

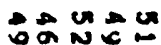

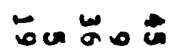

कोत

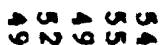
un uñ is:a पnd

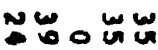

00000 טw w w was 得えだ

जिए

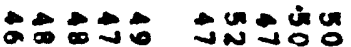

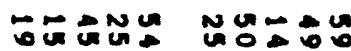

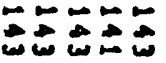

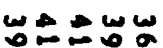

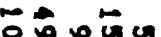

ニニニミ゙

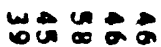
कu un

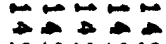

unca un un on $\infty \infty \cup-w$

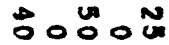

三走政 an un cu van

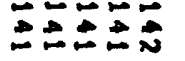

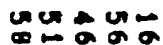

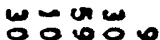

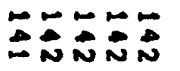
uñua $000=0$

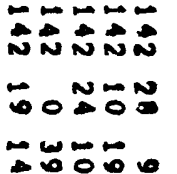

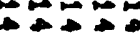
NลNAN

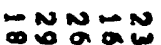
Wำด

\section{$r$}

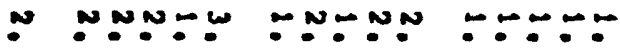

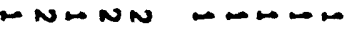

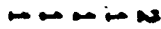

N-N

TnNNN :

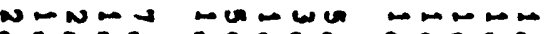

$\because \div \div \div$

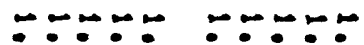

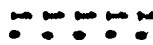

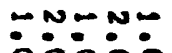

$\sin 2$

\section{$\ldots$}

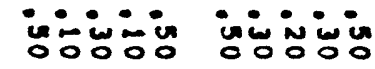

$\operatorname{ingining}$

$\therefore \therefore$

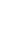

$\therefore$

in....

inivini

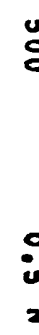

뜡ㅁㅇㅇㅇㅇㅇㅇㅠ

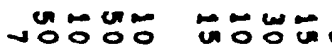

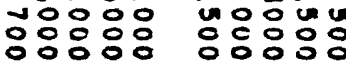
a

00000 in un in in:

0000000000 incis iv $z=2 z$ inirinis 00000 inivinis incinisin $z z z z=$ 00000

00000 inivivic in in in ivive $z=z x z=z \geq z$
:

$z \geq z \geq 2$

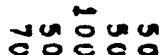

푸음 융융ㄷㅇㅇㅇㅇ

กัธธัด r

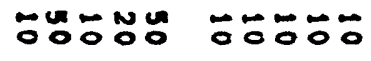

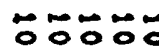

ธัดัธัธธ์ $\leftarrow$

ํํํํํำ

ேㅜㅇㅇㅡ

ำธะ์ - 
$\infty \quad \infty-\infty$

-n m

$\infty x^{2}$

ローRー

-

-

$\infty$

- M

$r$ CERE

$z=2$

CEE CEL E

CEDEC

$\operatorname{CE} 2=$

EEEZE HETZE

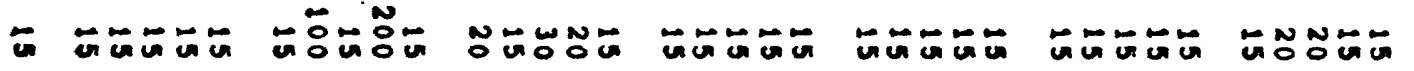

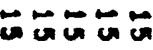

ó

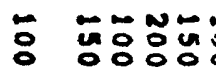

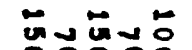

똥ํํำ

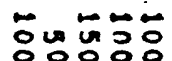

부웅

뜨융

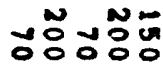

ํㅜㅇㅠำ

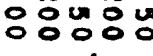

:

O

\section{$r$} =OLNO

NN NONO

¿́

O NONONO

"TWNN

Uñ

OกNำ

سم سم سم

N.

แำ

แั

$z$

z

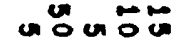

uncuono

unueron

unumen

unuou

$z \geq \geq z z$

$z z z z z$

$z z z z z$

$z 2 z \pi z$

on un un

uncan

$z z z z z$ Í

$x \geq E x z$

N ONONO

N N N N N

NNONN

NNNNN

NNONONO

NNNNN

NNNNN

$x=225 z$

t 2522

$5 \geq 250$

CEL 2

TEEE
NNNNN

CEXEE

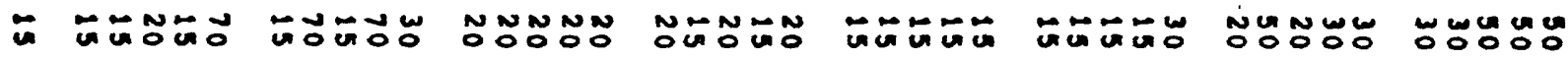

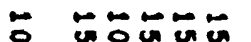

un 5

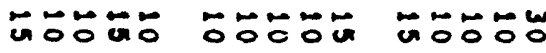

$\because \approx N+\infty$

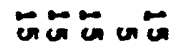

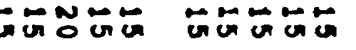

뜨ㅇㅠㅜ tr
CNOOOOO

뚱눙

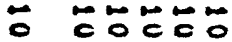

$z \quad z z=2 z$

O

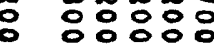

แั่ง 응ㅇㅇㅇㅇㅇㅇ

응ㅇㅇㅇㅇㅇ

OOO

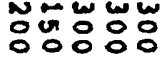

두ㅇㅠㅜ웅 $z z 2 z=$
두ㅇㅠㅜㅇㅠ $z z z z$

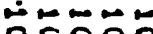
$z z z z z$
NNWEN ㅇㅇㅇ융유

NWW 응 영ㅇㅇㅇ

బ 응영응융

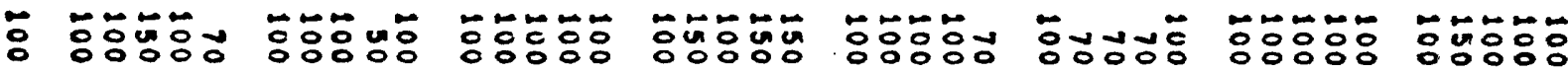


WNW

NWUEN MUNNN

NONON

טم

WNWNW

N. Nㅠ용ㅇㅇ

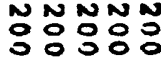

NNNNNN

NNㅛ용요

NNNNN

Oㅇㅇㅇㅇㅇㅇ

NNNNN

ㅇㅇㅇㅇㅇㅇㅇㅇㅇ

NNNNN

ㅇㅇㅇㅇㅇㅇㅇㅇ

$z=z z z \quad z z z z$

$z \geq x \geq x$

t5 Z比z

$z z \geq z x$

NNNNN

잉잉ㅇㅇㅇㅇㅇㅇ

$z z z \geq z$

RTnNm

- unoOO \%uñú

\section{RTNNN}

ה

ENONN

ज何药

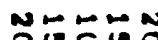

훙 ज응

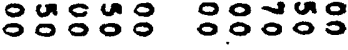

은든ㅇㅇㅇ

웅ㅎㅇ웅

웅웅

웅 0000

은둔웅

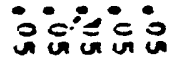

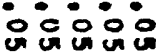

\section{:}

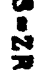

$x \quad z 2 z 20$

$x \geq 2$

$z Z z Z Z \quad z 2 z=1$

$z z z z z$

$z z z z z$

$z z z<2$

$z \geq z z z$

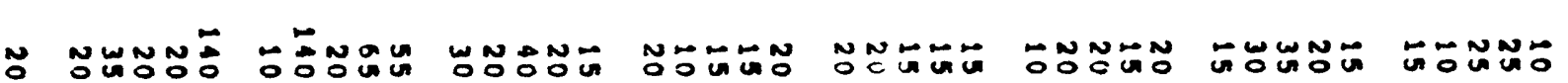

NMNG

$N \rightarrow \infty N \rightarrow$

$\operatorname{cin} \in \mathbb{R}$

N

im

NNNM

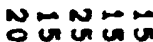

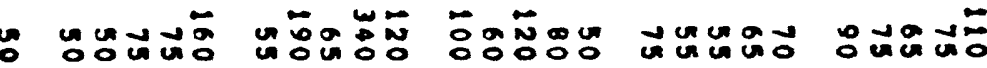

atuoun un

aㄴㅇㅛ

g:ogon

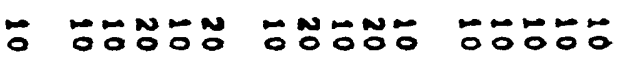

22

$\geq$

tre 22

unuma

- Disin

$\infty$

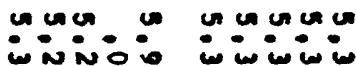

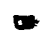

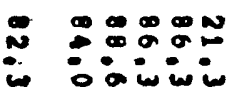

טN@N

U. 0 C 0

구용 ㄱan: :ำ $\therefore 0: 00$

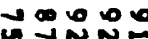

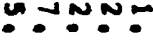

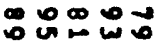

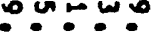

$\therefore 00$ $\div 000$

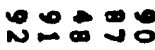
i0:0\%

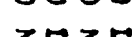

ununcer un unuma ow ow:

uncara io: in
NNNNN

OBD?N

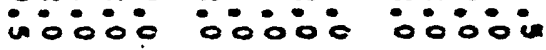

$\dot{2}$

-0.

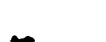

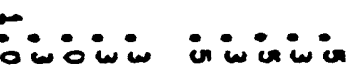

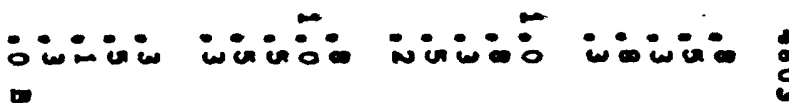




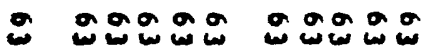

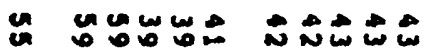 \\ - 0 เ

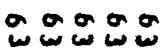

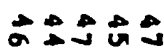

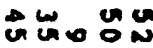

agoog

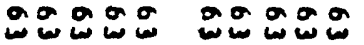

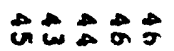

w w
Bun

Nugna
주요

unuan

iv

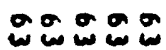

UNUNN

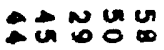

\%

N N N

- $0 \omega:=$

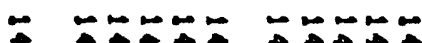

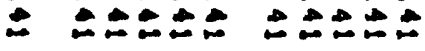

० D o ow

ט๐̆

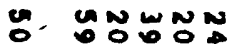

w: $\underset{\infty}{\infty} \infty+\infty$

$\sim N \ldots N$

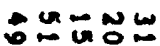

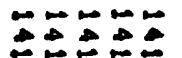

NNun:

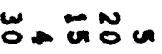

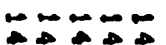

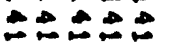

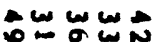

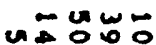

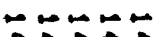

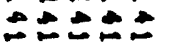

س

Nִ

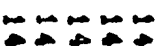

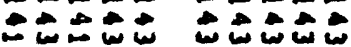

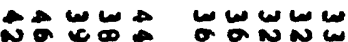

WUUO\&N ODON

O OOOOO OOOOOO

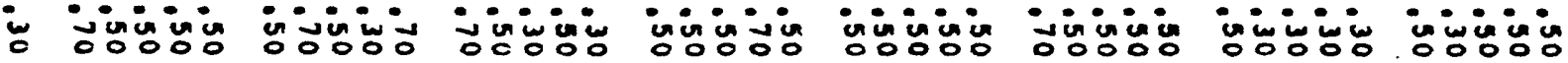

00000 inivivin

$z 2 z=2$

00000 invinis

$z z z 2$

0000000000 uvinin in in un

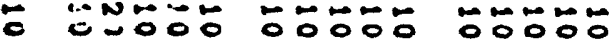
$+$ 5

ஸே0\%ั OOONO 5

$\div$ $r$

$50 \div 00$
$0 \%$

مै⿴囗十유

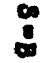




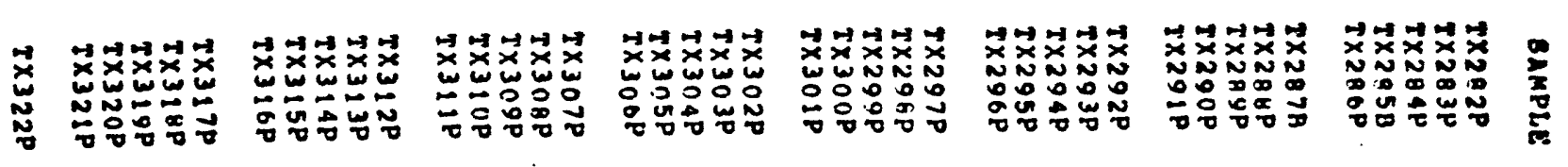

-

is

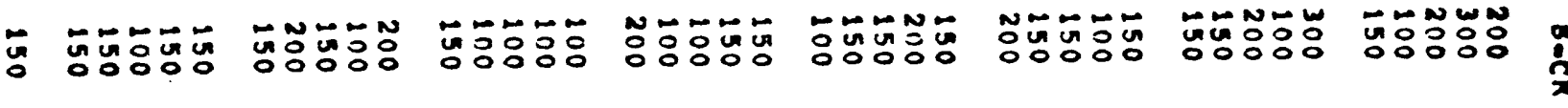

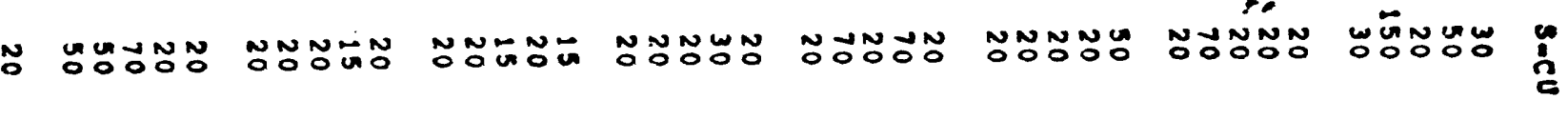

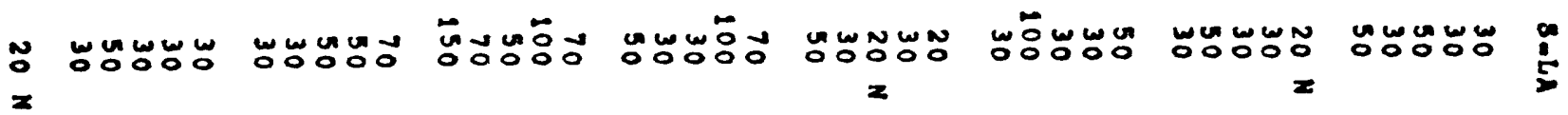

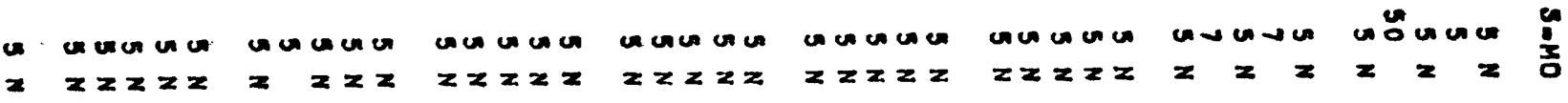

ล NONOONO NONOOON

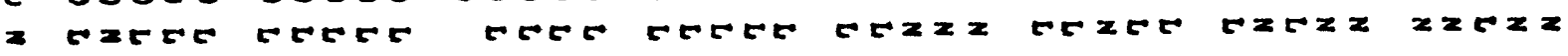

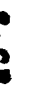

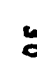

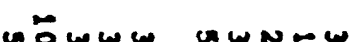

Wัดกลก

WNOกั

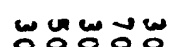

NENก

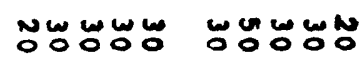

$i_{i=1}^{\infty}$

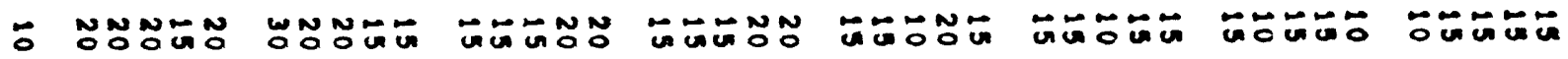

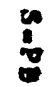

i

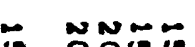

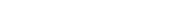

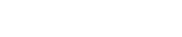

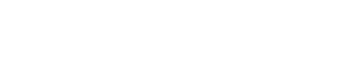

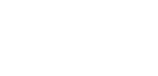

WOANO

:

우ㅇㅠㅜㅇ

Бே0ั0ั0

ேㅠㅇㅠ

무유

ธே0ั0லั

ேㅜㅇㅠ

\%๐ேே

$\because \because \because \div$

$z=z z z$

$z \geq z z$

$z z=2$

$z \geq z \geq z$

$z=2 x$

$z=z z$

$z z z z$

$\begin{array}{ll}00000 & 0 \\ z z z z z\end{array}$

:

19.

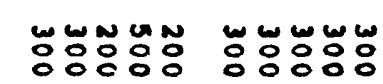

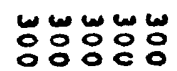

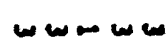

แ้นแัะ

กับแัะแั

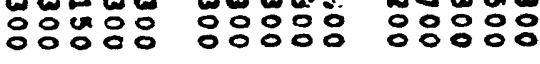

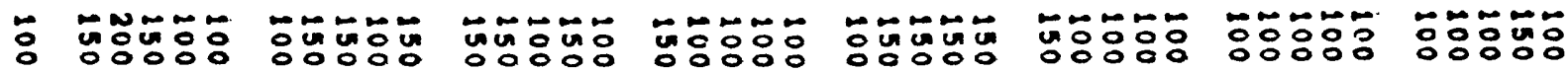




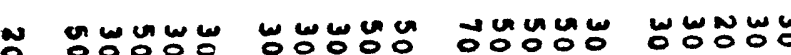

O OOOOOOO

NOOOON

$z$ r $x z z$

$z z z z z$
OOOOOON OSOㅇㅇㅇ

IIII
กับกับัก OOOOOO

$z z=z 2$
nNNNN 응ㅇㅇㅇㅇㅇㅇ

$z x z z=$
nNnNN 00800 $z \geq z=$

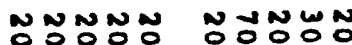

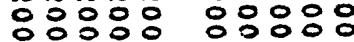

$z z z z$

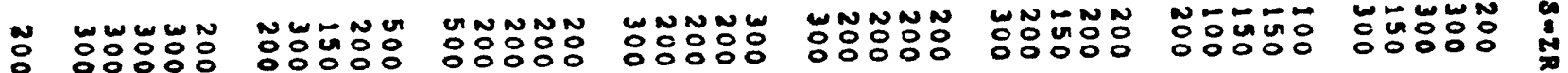

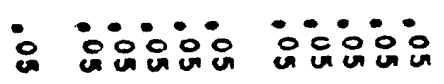

$z z z F z \quad z z z z z$

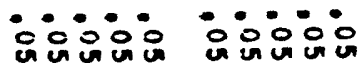
$z x z z z$
운응유

$z z z z z$

\section{운은융유}

$=2202$
원웡ㅇㅇ

$2 \geq 2 \geq 0$

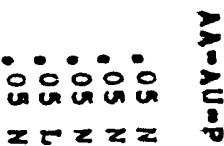

N ONOOOOO OOOUEO

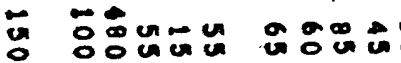

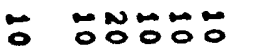
$z \quad x$

$\operatorname{aroun} a$

i. $0.0 \%$
뭉ㅎㅇ $z \quad z=$

c. unum $\therefore$ :

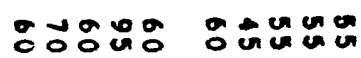

a

a o o uno

\section{c $\infty \infty: 0 \overrightarrow{0}$ \\ - wis:-}

ஸீல் $\because \div \div$
무유 무유

$z=$

cuuna unumu
후웅웅

con ancu

nivin
둥후

unucun a wivi
뚱웅

unuue aw:
O־ONO

2

ca cucu

-ino:

$\infty \infty \infty \infty \div$ $\omega \infty+\infty$

$0000 \infty$

$\because \operatorname{lnn}$

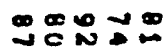

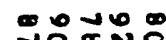

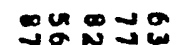

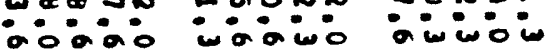
0 in:

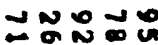
-

N NNMNN

NNNM

$\sim N \infty N$

$N \sim N \sim 0$ $\infty$

$\mathbf{8}$

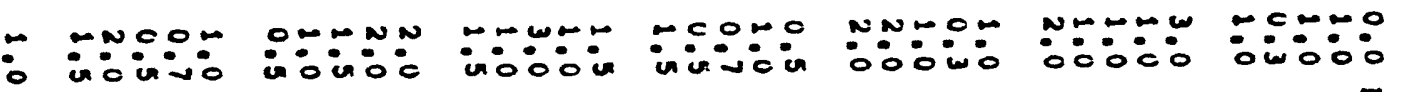

$\because 0 \div 0 \div \quad$ :

$\infty$

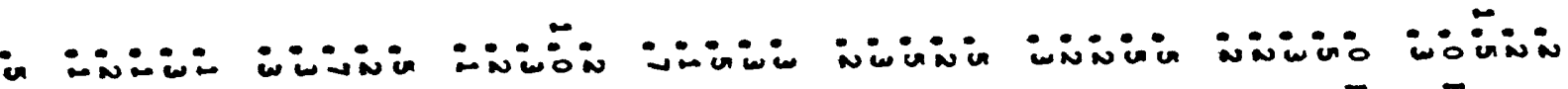
: 
- 0000

م

orogo

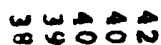

운웅

운둔

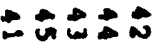

unum un

운

$u \in u \rightarrow n$

BUUNB

พ⿻上丨

10

$\neg \rightarrow M D N$

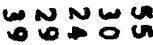

0 OD:

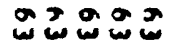

운

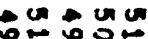

ununua

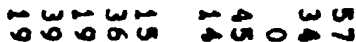

a

un u v u

NNW

\section{モュミミニ}

\section{シニニニ゙}

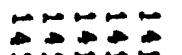

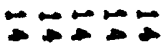

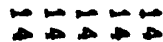

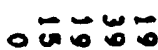
NWN م WNNNN

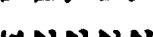

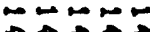

$\rightarrow-\infty$ ーREA

$\underset{n \rightarrow \infty}{\infty}$

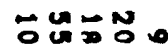

范范

जั0ั0

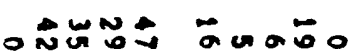

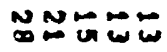

뭉

U๐

Non

$+00 \leqslant 0$

uno no no

$r$

02000

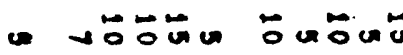

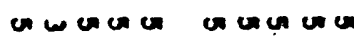

ton un un

$\cos 0 \cos$

ancono

onoun

NNNNN NNNNN

NNNNN

DNNN

N-NN-

NNNNN

00000

:00:0

0000000

00000

00000

$\therefore 0: 00$

00000

NWWNN NNNNN NMNNN NNNNN

$0.00: 00000$

NNNNN

NNNNN

NNNNN

NNNNN Oㅇㅇㅇㅇ O0:00

잉웅 웅유

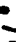

in:

a)

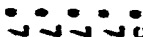

injo ivon

incivingin

iningino:

inivis: แำำํำ

inviginging

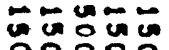 융유}

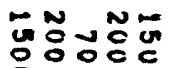

ज叶范范

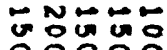

แลํㅇㅁำ ㅇํㅇㅇㅛ

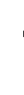

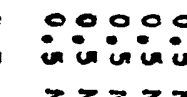

inivin

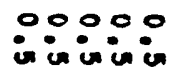

íco: in:o0 ininio $z \geq z z$

z2zzz

$z \geq z z$

$z z z z z$

$z \geq z \geq$

00000 iniviv $z \geq z \geq z$

00000 irinivi $z z z z$

눙 Nㅜ응

ธั0ำ

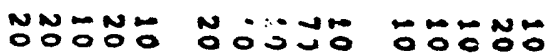

느ำำํํํํํํำ

ํํำำ

두응 
$\sim \sim \sim \cdots$

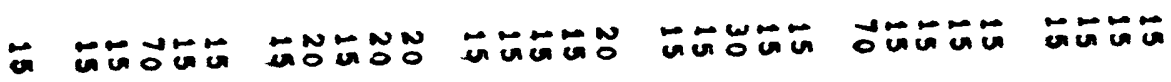

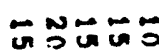

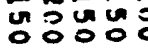

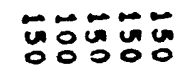

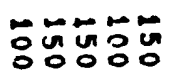

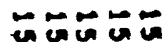

$-$

.

nato

Nㅠㅁ

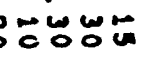

NOO

:

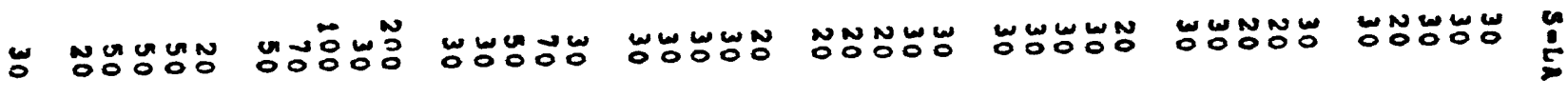

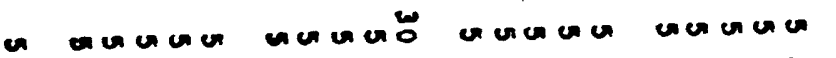

unumo

unuuer

oncung

unenen

$z=z z z z z z z$

$x z z z z$

$z z z z z$

7. $z 22 z$

$z \geq z z x$

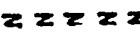

$z \geq z \geq z$

N NENONO NOEONONO

NONONO

NNNNN

NNNNN

NNWN

NNNNN

NONON

ㄴ.

$\infty$

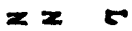

$z$

$z z z \quad z \quad x z z z$

$z z=2$

crezz=z

$z \geq 200$

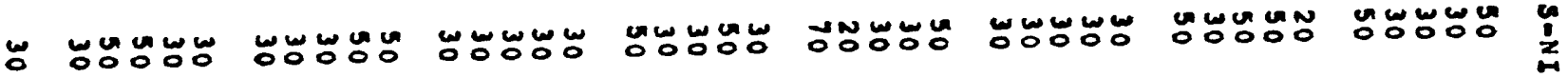

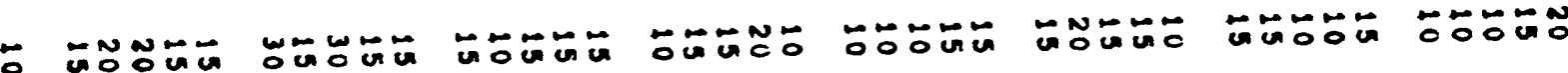

O OّO

\begin{tabular}{|c|c|c|c|c|c|c|c|}
\hline $\begin{array}{l}\text { 두웅 } \\
z=2 z=1\end{array}$ & 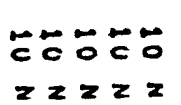 & $\begin{array}{l}\ddot{C} \ddot{0} \ddot{0} \ddot{0} \\
z z=z z\end{array}$ & $\begin{array}{l}\text { 두응 } \\
z z z z z\end{array}$ & 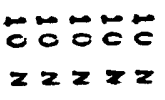 & 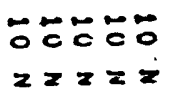 & $\begin{array}{l}0 \approx 000 \\
2 z=2 z\end{array}$ & $\begin{array}{l}\ddot{0} \ddot{0} \ddot{0} 000 \\
z=2 z=\end{array}$ \\
\hline س & 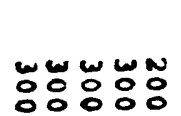 & 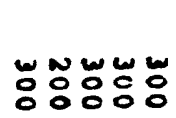 & 응영영영 & $\begin{array}{l}\omega \\
\circ \\
\circ\end{array}$ & 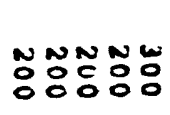 & 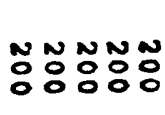 & $\begin{array}{llll}N & N & N & N \\
0 & 0 & 0 & 0 \\
0 & 0 & 0 & 0\end{array}$ \\
\hline
\end{tabular}

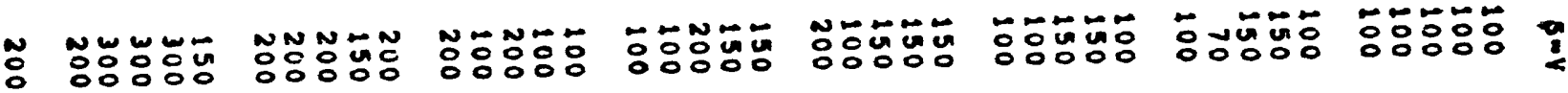




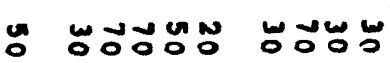

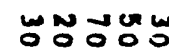

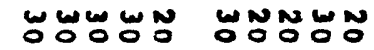

Un

ORNONON

Nㅗ융ㅁㅇㅠ

N NNNNN NNNNN

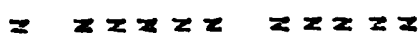

영융영ㅇㅇㅇㅇㅇ

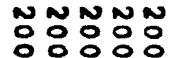

$z z=z$

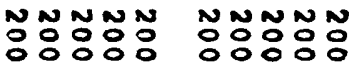

$z z z z Z F=2$
NNNNN

응영영
$25 \times 2=$
nNNNN

응융유
tZLEZ
NNNNN

응융융

$5=25$

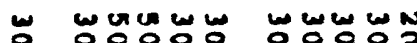

웅 웅융융 영융융

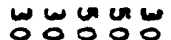
응응응ㅇㅇㅇㅇㅇㅇ www w 응용요 w ㅇㅇㅇㅇㅇㅇ응 ww ww $\therefore \circ: ㅇ$

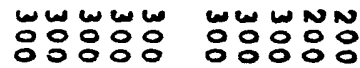

$x \quad z=2 x \geq z z x$

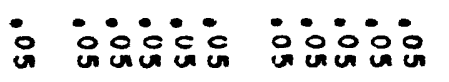

兑品俩:

i̊요용

in जi:

$z \geq z \geq z$

$z z z z z z z z z$

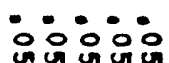

iिcióñ

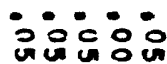

$z \geq x<z$

Zw $2 \geq 2$
,

م

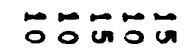

NonTR

NONON

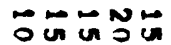

MTNNo NOONNO

\section{ํㅜㄹ}

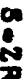

:

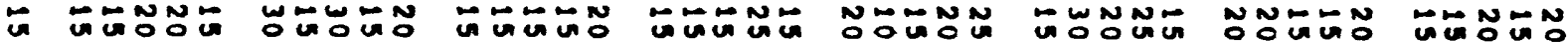

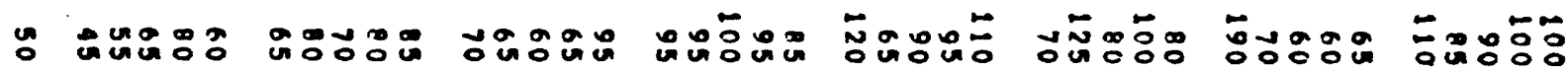

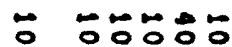

ํํㅇํㅇำ

우융

뭉두

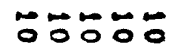

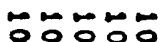

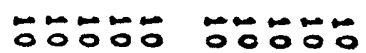

$x \geq$

$z=$

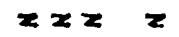

$z=$

z $z z z$

z 2

$z z$

$\because \quad-\infty i \infty$

ind a

$u=-u$ un

uvau- usuuv

unu un

unumerumuna

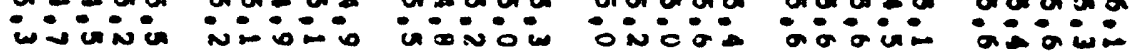

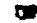

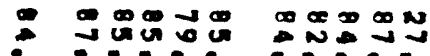

$\stackrel{\infty}{\infty} \underset{\infty}{\infty}=$

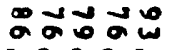

$\infty \infty \infty \infty$ in: ion:o ia:

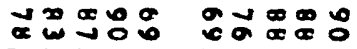
andio á:0
- $-N-O N N N N N 0$ $+$

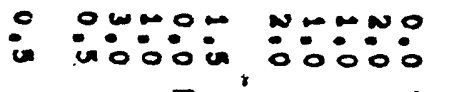
- $\boldsymbol{1}$ $\omega \sim \sim \sim \omega$

$\boldsymbol{N} \sim \boldsymbol{N}$

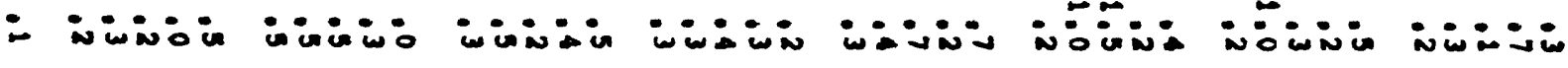
- 
- 20ำ 운운

00000

-

ํํㅇำ

- Nㅜㅇ휴융
م

눙
20003 ט

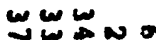

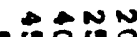

ongan - 00

NNNUE

Nㅜㅇํำ oriago

음aa

טw

ט

-

1900

كس

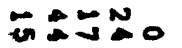

NUNN

NNN

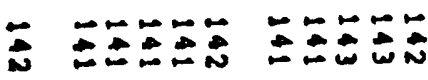

- 혜후

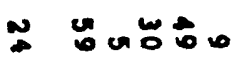
N $=$ 눙융

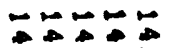
Nㅗㄴ N

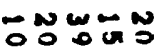

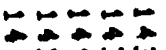
م $\omega x+\infty u$

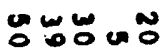

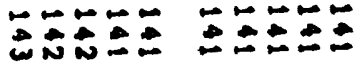

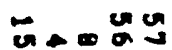
nun

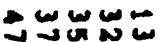

cro

on:

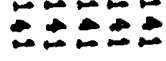

$\vec{\omega}_{0 \rightarrow \infty} \rightarrow$

Now

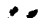

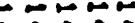

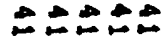

Woñ

caño

둥

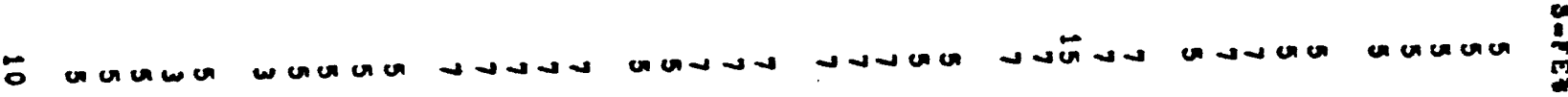

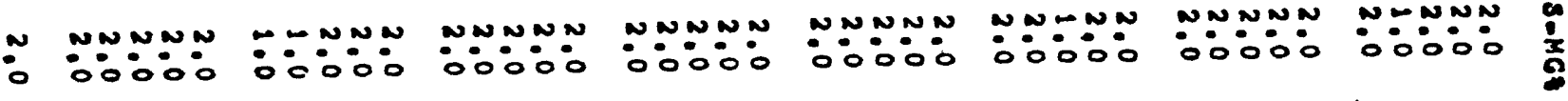

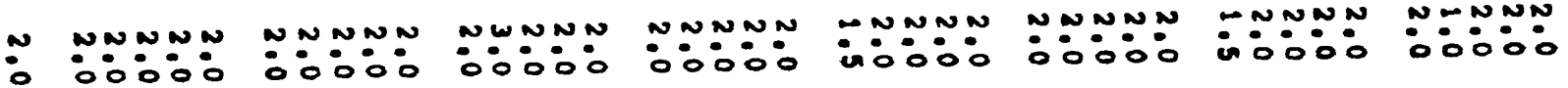

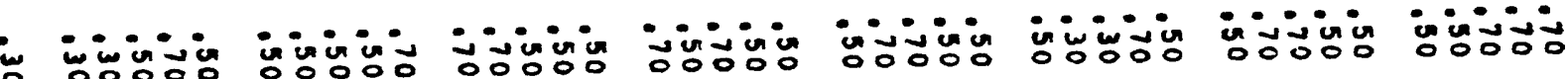

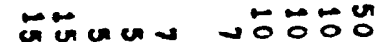

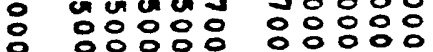

?

-

$z \quad z z z z=$

00000

00000

눙눙응

응ㅇㅇㅇㅇㅇㅇㅇㅇㅇ

ㅇ등ㅇㅇㅇㅇ

ㄷㅇㅇㅇㅇㅇㅇㅇㅇ

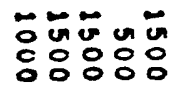

뜽ำ

ㅇㅇㅇㅇㅇㅇ응

0 
$\infty-\infty$

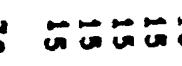

$\cos 20$

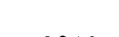

$\lim _{0 \rightarrow \infty} \rightarrow \infty$

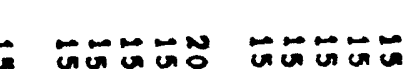

두우은

\section{a}

\section{NEVG}

8

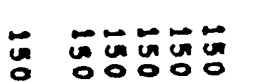

ज゙ज응

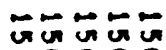

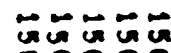

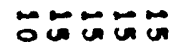
O००००

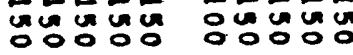

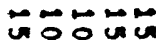

\section{$\sim \approx \div \div$}

ज요요요

?

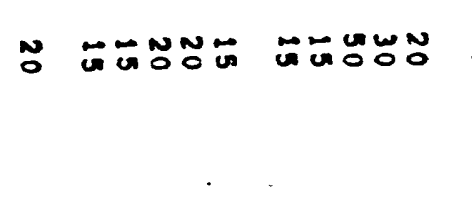

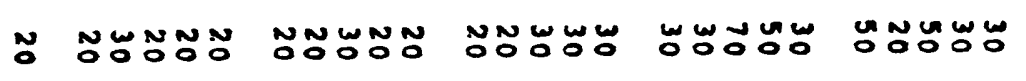
$x$ MWNONON NONOONO WENONO

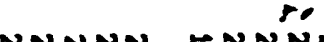

NONONO FNONOE OOTUNO

ấ

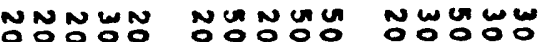
$z=2 x$

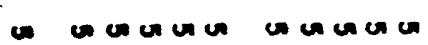

$z=z z z=z z z z$

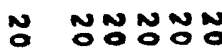
NONONO NONONN

$z=z z=$ $z x=2=$ unumer

zzzzz

unuenco

$z z z z z$

nonuon

$z \geq 2 x \geq$

onumu

$z z \geq z z$

nunum

$z z z \geq z$

NONONO

NONONO

NOOONO

$x \geq z=2$

$z x \geq z$
NOOON

$z=25$

NNW $z=x \in$

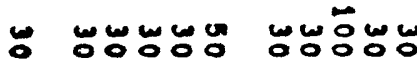

よ

- NONONO NUEONO
مَّم
س

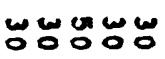

Now ow

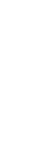

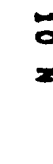

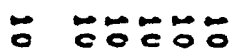

z I. $222 x$

우웅

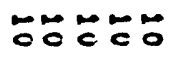

モே゚ேேே

ேㅜㅇㅠ

00000

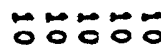

$z z z z$

$z 2 z 2 x$

$z \geq 2 z z$

$z z z z$

$z z z z=$

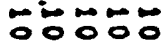

$z z z z=$

$\Xi$

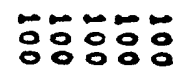

ละลััะั

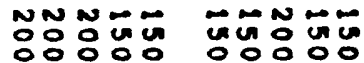

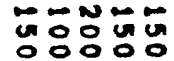

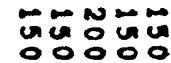

วัจำัตัด

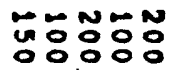




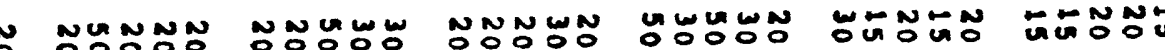

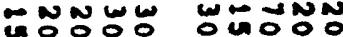

2

N OOOOOOOO OOOOOOOOO OOOOOOOOOOO

r $\quad z=z z$

C八: 22

ZZETE
NNNNN Oㅇㅇㅇㅇㅇㅇ

f $z 2 z 2$
OOOOOS

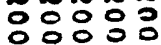

$z 25 z$

NNNNN NNNNN

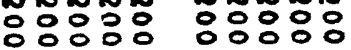
$z z z z z \quad z z z z z$

N $\begin{array}{lllll}0 & 1 & 1 & 1 & 1 \\ 0 & 0 & 0 & 0 \\ 0 & 0 & 0 & 0\end{array}$

سم

N م N N

wan

wnunw 영ㅇㅇㅇ

우 웅운둥요 웅웅 $z \quad 2 z \geq 2 z$ $z \geq z z z$

$\because \because \div \quad \because \therefore \circ$

든은웅은

-000ㄷ

둔운운

웅ㅇㅇㅇ

$c z \geq x z$

$z \geq z<x$

$5 \geq 2$

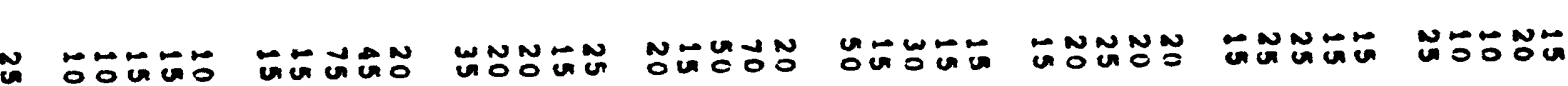

웅요요

$\geq z \geq \geq z$

3

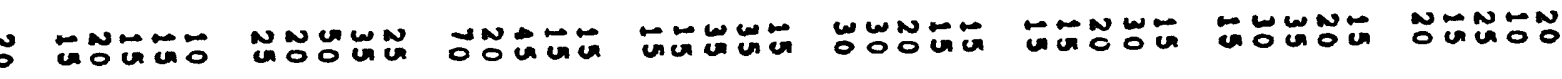

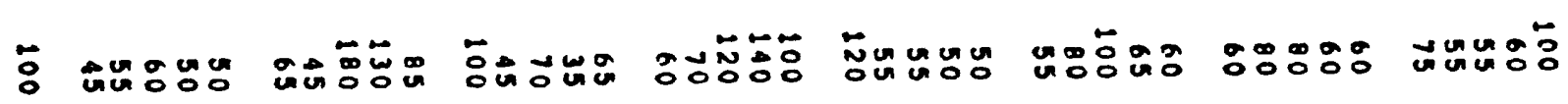

$\because$ 훙휴

$z z z z$

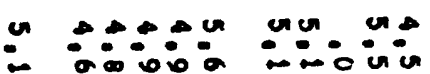

-

-

- ainiso aivio:

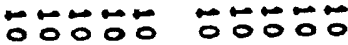

$z z \quad z$

$\therefore-\infty$

$\infty$

$\infty \pi \infty$

or $n=\infty$

wi:

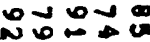

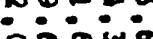

뚜웅

$z+z$

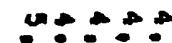

$00+\infty=0$

БОБО유 뉴융유

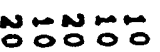

$z \mathbf{z}$

$r \ldots z z$

antua ususa una un

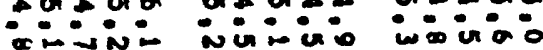

$\mathbf{x}$

$\underset{\infty}{\infty}$

con

வ

(2)

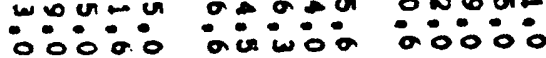


- 운요

aros a

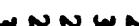

ט

un
W as a o

NNNNN

$\cos 2 \pi$

-

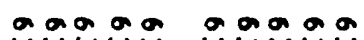
ט

مَ

कำ

a.0.0

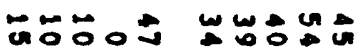

wo

ڤNNNNO

NNNNN

○०־U⿺
00900

UN口-

unn w

- م

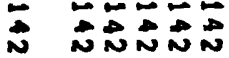

N NNNN

$\therefore$ SNDU

$\simeq$ N

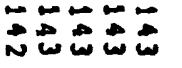

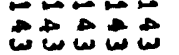
Un wO NO

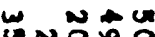

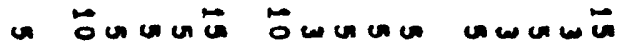

$\operatorname{sinnN}$

$\because 00000$

$n=0.000$ ०0:00
OOOO:0
NonnN n

- O0O0: 0 :00: ๔ N

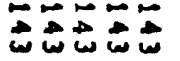

$N=N-N$

D

ผ
w
c

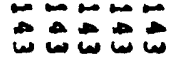
NN

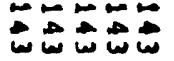
N

o wo:
1
$\omega$

$\omega \rightarrow n$ w

ת

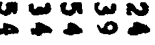

NNNNN

:0:0:
nNon

i0:0i0
05000000

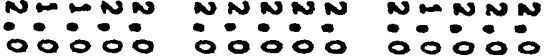

:00:0:0

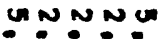

OO:O-
UNNNN

O:00:0
nN-nN jo.0.0 ibi.i boinn

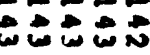

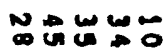

uñ

5
2
2
0
4
5

winis

ininis

ivinivis

inivis

inivivis

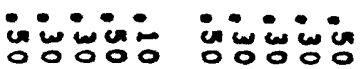

م ڤ̆

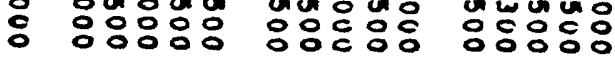

?
뗭융뭉ㅇㅇㅇ 응ㅇㅇㅇㅇㅇ
능 w눙응 응융유유 a

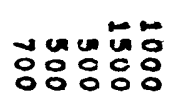

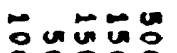
¿ㄷㅇㅇㅇㅇㅇㅇㅇ

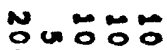

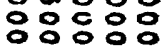

a in inin univis 00000 $z \quad 2 x \geq 2 x$ $2 z 2 z x$

22222

00000 univin

00000 in in in 00000 00000 00000 $z z \geq z z$

$z \geq z \geq 2$

$z x \geq z=$

$\geq \geq 2 \geq 2$

inisivi

:

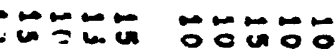

- 0000 5

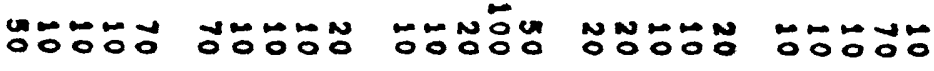
te

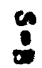

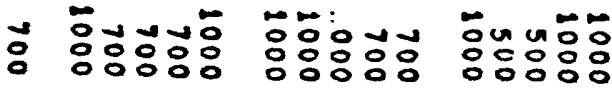

un u v

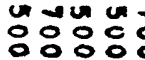




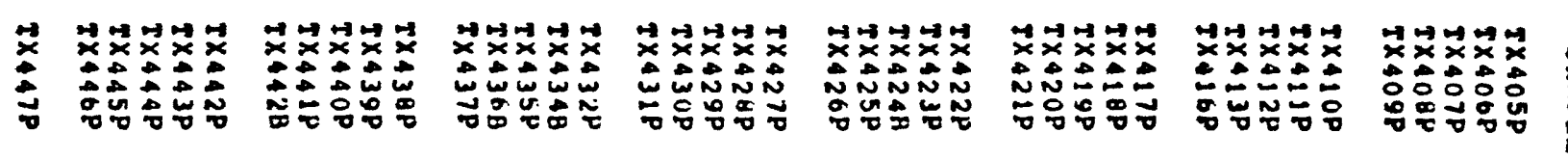

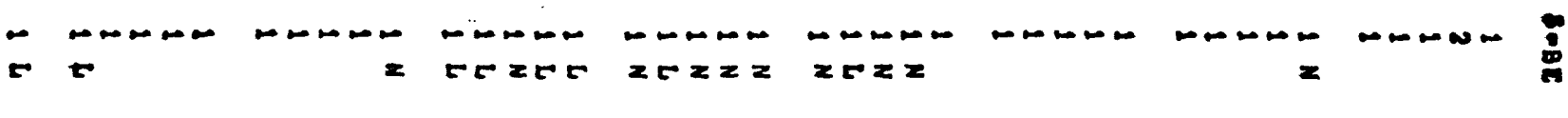

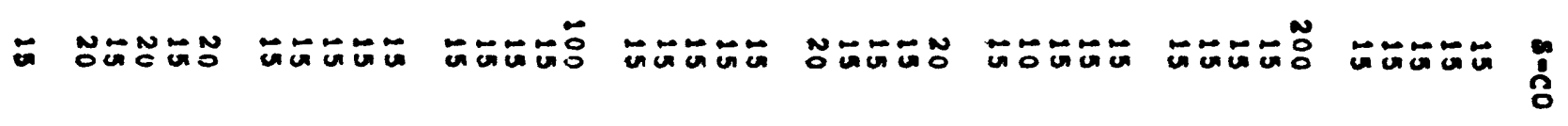

ฐ

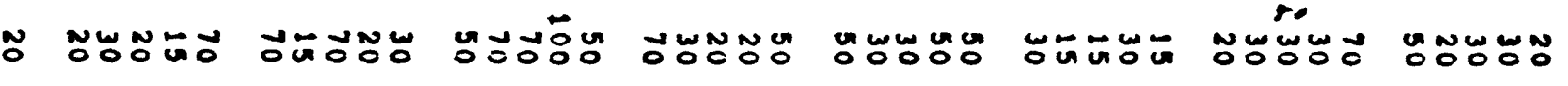

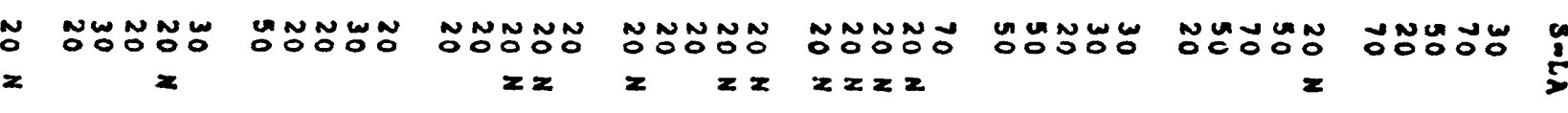

م

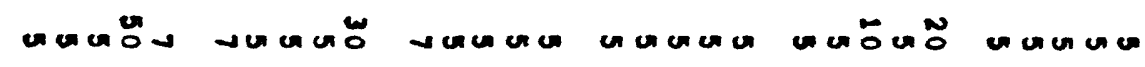

canca ca

$z z z z z z$

$z \geq z$

$z \geq z z$

$z z \geq z z$

$z=2$

$z \geq 2 \geq 2$

O NNONONO

NNONO

NONNO

ONONN

NONNN

NNNNO

NONONO

NONNO

$z \geq z \geq z$

$z z 2 z=$

$z \geq z \geq z$

$z z Z z Z$ ZZZZ

$z \geq z \geq z$

$z \geq z \geq z$

$z \geq z \geq z$

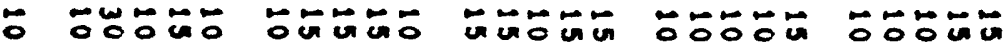

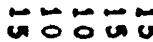

5

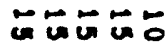

ேேேேேே

r

:

O WNONOW NGENO

WON N

$N \sim \sim 0 N$

Noñ

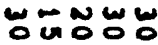

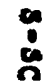

$\because$ 루유유

뚱후웅

두ㅇㅠㅜ웅

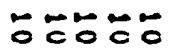

둥둥후

Сே0ே0

뚜ㅇㅠㅜㅇ

둥ㅇㅇ

$z \quad z z z z z$

$2 z 22 z$

$z=2 z 2$

22222 .

$z 2 Z z=22 z 2 z$

$z z \geq z z$

$z \geq z \geq z$

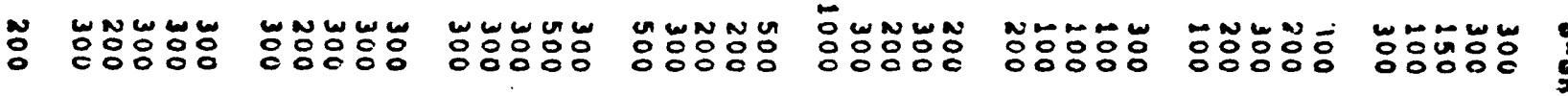

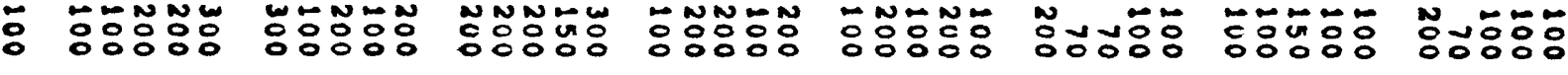


NOOOTO

WN UN

NU⿺辶一

MNN N N

6

N NONONO

응ㅇㅇㅇㅇㅇㅇ

$z \quad z=2=2$
nNNNN

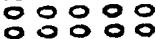

nNNNN ㅇㅇㅇㅇㅇㅇㅇㅇ

$22=22$ $z \geq z z$
NNNNN ㅇㅇㅇㅇㅇㅇㅇㅇ $z z z \geq z$
NNNNN 응ㅇㅇㅇㅇㅛ tr $x \geq 2$
ํํㅇํํㅇํำ

$z z z z$
$\begin{array}{llll}N & N & N & 0 \\ O & \circ & \circ & O\end{array}$

$z z z z$
NNNNONO OOOO $z z z z$

N N N N

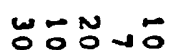

- NNNT

NNNW

- 온윤요

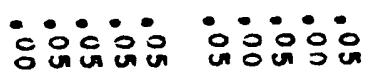
웅웁웅응 웅ㅇㅇ웅

$\div \div \div 0$

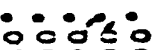

wzzz心

$\infty \geq x \geq 2$

$20 z z z$ $2 x=00$

둥ㅇㅇ $z=2 \geq 2 z$

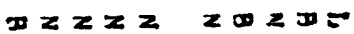

$z \geq z z z$

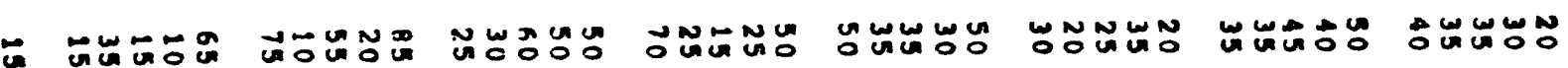
O OOOOOO OOOOOOOO

in

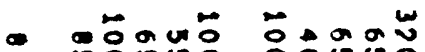

- 눙영영

ơ uno

$\ln \infty \geq \infty$

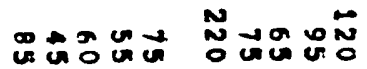

Uิ

눙ㅇㅇㅇ

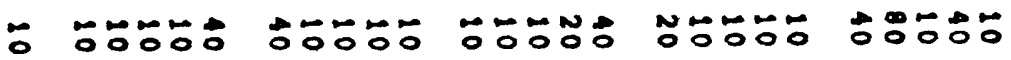

NOOOO

유융

$\frac{3}{1}$

$=$

$z$ z

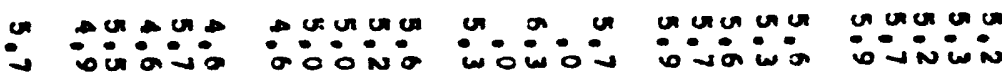

$0 \quad n \rightarrow u$

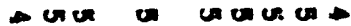
$\infty \quad \infty$

I

0

(1)

山ే

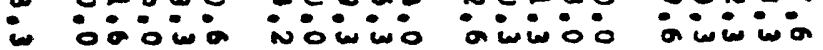

กั

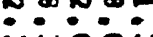

$\infty \subset ⿻ \infty$ :0ं0:

InNO

$\infty \infty \infty$ แ2 No: $\sigma$

$\infty$

ONNNN

owo

NMNNN $-N O N$

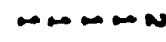

$\sim N \omega \sim N$

$\sim \boldsymbol{N} \boldsymbol{N} \mathbf{N}$

$$
\text { - }
$$$$
\text { - } 0
$$

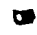

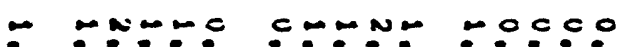

- Oㅜㅇi anc $\infty$

o o in in

- $\boldsymbol{0}$

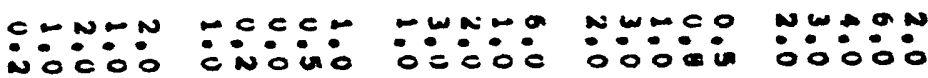

i $\rightarrow$ 


\section{- 90:00

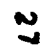 \\ is 090 is \\ 운 ○一⿻上 NNNNN

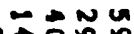

$a$

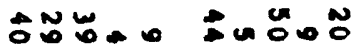

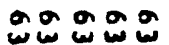

is 0 is NNNNN $\omega \omega \rightarrow N M$ ט

wn

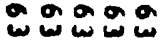

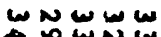

Uั

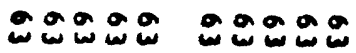

ט"

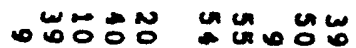

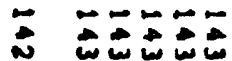

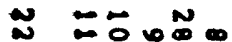 \\ - แ。ะ์}

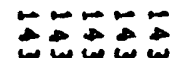

DNNNN

WNNN

UN NNㅡ음 $\rightarrow \infty \rightarrow+\infty$ NANAN

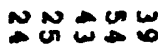
ज岗。

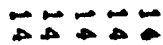
NNNEN

แă

won

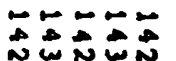

W

○ NuWo $\because \leftarrow \leftarrow \leftarrow$

Nㅗ

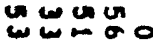

an

0๐O

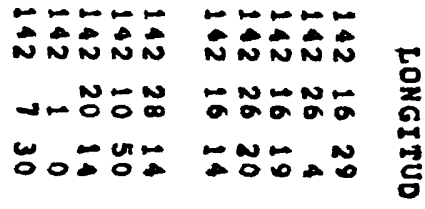

re

N wnom

NNNNN

NNNNN

NNNGN

NNNNN

NNNNG

$\operatorname{Dod}^{-00}$

- oivin

OOO:O :00:0

$000: 0$

00000000

$0: 00: 00000$ i juinuin uniö univin uninis wisinis in wivis iogon

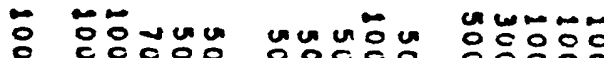

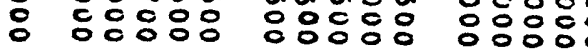

\section{nตㅇำ} ㄷㅇㅇㅇㅇㅇㅇㅇㅇ
눙두 웅응ㅇำ जũ̃o 응
ㅎㅇ웅ㅂ 응응ㅇㅇㅇ응

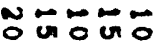
옹응ㅇㅇㅇ응

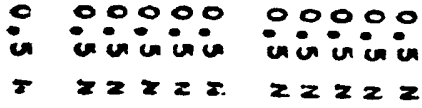

00000 ininivis $z \geq 2 z 2$
웅ㅇㅇㅇ

$z \geq \geq z=$
응웅

$z z z \geq z$ in in in

$z \geq z z 2$

\section{in in in in}

$z z=2$

00000
in in in in

$z \geq z z$
0
2

룽후웅 -

두웅

$\because \div \div$

뜽융 부ㅇㅠㅡ

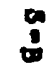

- ㅜㅜ눙 응영영영
5

ㅎํㅇํㅇㅇํㅇ

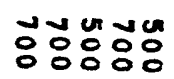

รัดัวัวัวัว 등영형영 충융형융융 

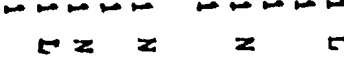

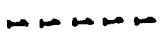

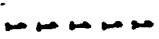

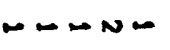

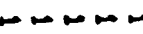

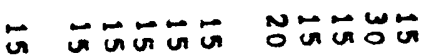

$\cos (\pi)$

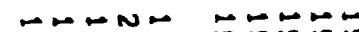

브능ㅇㅇ

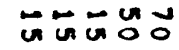

准范范

ô

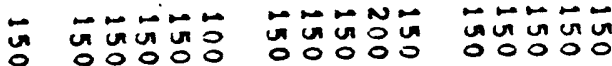

$\ddot{M} N=$

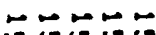

n

nOLN

药比比代

ถุ

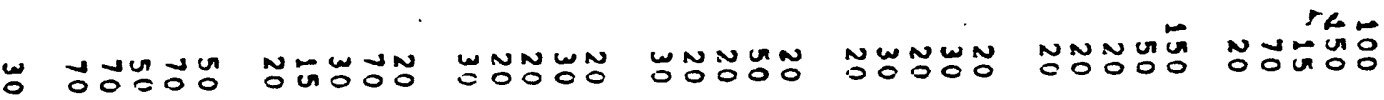

OOOO

Nono
जัOEับำ

ถุ

NONONO NNNOOOO NNONON

ํํ융ำ

NONNN

$z \geq z z$

$z z x$

W ำ

N ONOOON

$z \quad z z z z z$ un un u $z z z z$

$\omega N N N \omega$

$z z z$
NONONO

$z \geq z z z$
NONONO

$z z z z z$
NNONONO $z \geq z z 2$ unu un

$z z z$

NNN

$z z z z z$ unumu

$z z z z$

ONONONO $z z z z z$

- ONONONO ํํำ ำ 뜽ํㅇㅇํ 능ํㅇㅇํㅇ 뚜융ํㅇ 5

두유

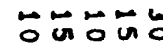

ํㅜㅇํำ

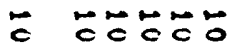

응융ㅇㅇ

묻유유 $z z z z z$
융루응

$z z z z z$
주융

$z z z z z$
त्वृत्व

$z z z z z$
뭉두융

$z z=z z$

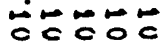

$z=22 z$ es

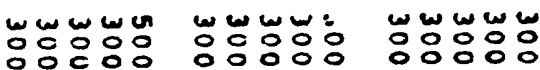

W NNNNN WNNNN

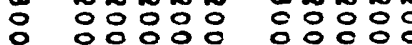

w้ ผ 응응응 ww w w 응ㅇㅇㅇㅇㅇ
ผ็ 응유유 $0000 \%$ 
O

ON

NONONO

Nㅛ용요

NONONON

z T $z: 2$

$z=2 z=$
NNNNN 응ํำ $z z=2 z$
NNNNN ㅇㅇㅇㅇㅇㅇㅇㅇㅇ $z z z=2$
NNNNN ㅇㅇㅇㅇㅇㅇㅇㅇ $z \geq z z$

NNNNN

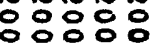
$z z=z=$

N NWwWW W-

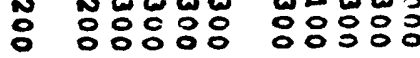

WWNONO

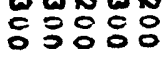

NNOW

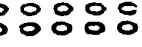

WNㅜㅇㅇㅛ 응ㅇㅇㅇㅇㅇㅇ WN. NONN
- co:0.

은운욘우

$z=2 z z z$

웅ㅇㅇ욷 $z \geq \geq z \geq$

운웅둔다 운운용 $z \geq z z z$

$z z z z=$

운운운요

$z z z<z$

은등ㅇㅇㅇㅇㅇ

र2 250

둔뭉ㅇㅇㅇ

$z=200$

응운웅

$x=2 z$

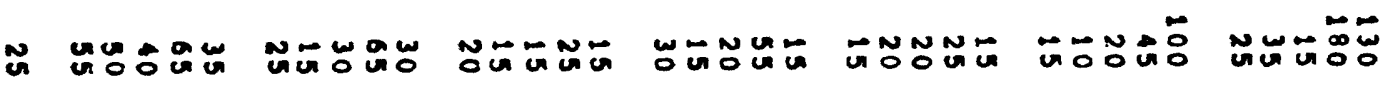

$\because \quad$ ONUNAO

$\because-\omega N N$

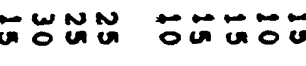

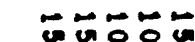

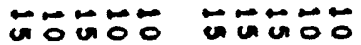

뚠훙우

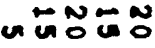

U NOテ

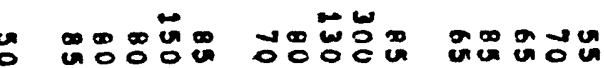
,

$\because$ 유융 OООООО 유유 $z z$ $z z z$

ONOڤัOО

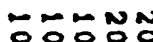

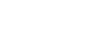

in o:o:io cinasin $\because 00 \div 0 \div$ C N0:in no un: $z x z$

OัOОNO

ㅜㅠㅇㅠ

$z$

manu un $+\infty \infty$

-

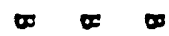

in:

$\because \because .0$

$\rightarrow 000$

. $\infty$

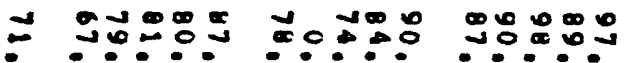

$\infty \times \infty$

$\omega$ U.

$\infty \times \infty \infty$

$\therefore:-0$.

in:

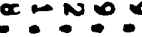

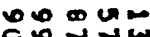

:00i०

$\infty$

- OnLnN nNane tanno

OWNWN

$\infty$

NNWNN

$\bullet$

- 뉴N

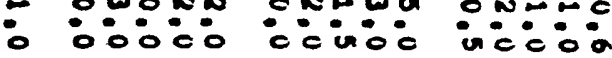

-

$\boldsymbol{\infty}$

ำ

-

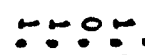

íni

$\approx 0 \forall N$

$\infty \infty \infty \infty$

$\infty \infty+\infty$ wisio

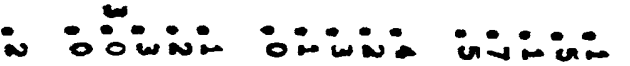
$-$

ivión ivivio $\because 0 ; 0 \circ$ + $\therefore: 3: 0$ - $\mathbf{0}$ unvivi

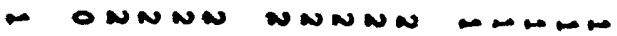
a. 
i 엠ำ

ט

N

w wöำ
का

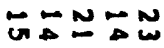

No UN

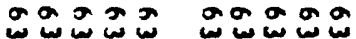

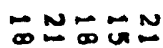

$u=0 \quad \tilde{0}$
UNaN

س a

न人

un
ט

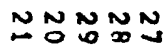

ט â

م

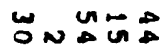

مa

ס

م

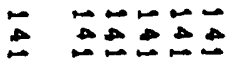 * $\quad$ wan un \\ u $u \rightarrow$ un}

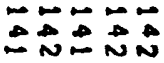
W

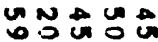

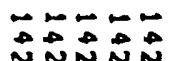
NNNNN $N-\omega N$ Aิ

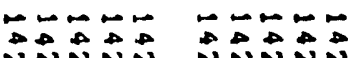
NNNNN

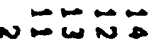

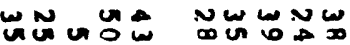

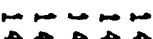
NASAA NN Wปコส UN

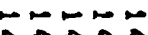
$\mathbb{N}: \mathbb{N}: \infty$ un $\ln 0$ W $\rightarrow \infty \rightarrow \infty$ ס

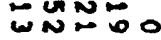
Uم

is inisi

NNNNN

NNNNN

NNNNE

NNNNN

NNNNN

NNNNN

NNNNN $\frac{a}{3}$

n nNNNN NNNNN

NNNNN

nNGTU unNann

nNNNN

NNNNÓO

NUNNN

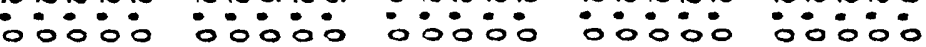

w

w w w

in inisis

in in in

iwivi

uninisin

in isis

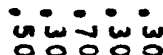

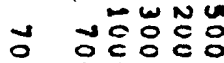

누ㅇㅠㅜㅇ 웅응응응

ज N 등엉ㅇㅇ응

OON N

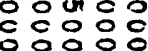

UNN N NO

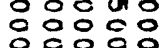
ฉ

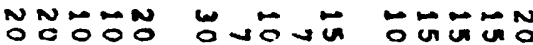

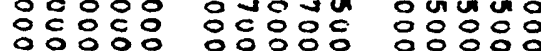

- 00000

in in in ivi

z $2222 z$
00000 in in in in $z 2 z 2 z$
00000 in in in in $z z z z$
00000 univin $z \geq z z z$
00000 irivinis $z=22$
00000 unin in un 22222
00000 in inivi $z z z z z$
00000 in in in $z z z z z$
N NNNNN TNNNN
NOOOOO wañ no no
Un w N N
NW:
: 
$\infty$

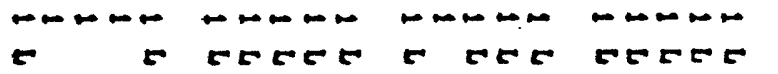

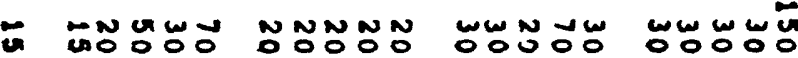

insungu

UMGOOOOO

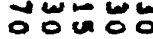

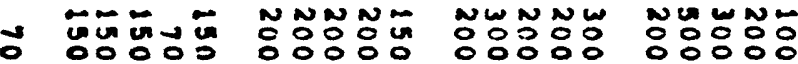

00000

응유

응영영

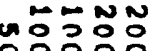

ज此NNN

능응응

जㄴ.

능응응

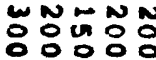

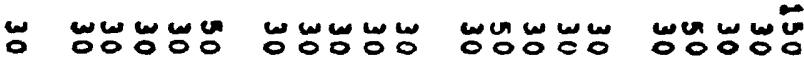

Sung wo:

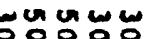

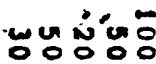

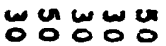

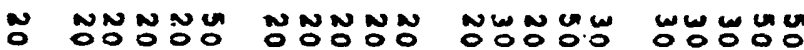

NNNNN №요유.

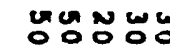

ณัญํํㅇำ

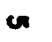

$z$

$z z z z$

enen en en

$z \geq z \geq z$

unucoso

$z z z z$

№№n

z $z z z z z$

ONONON

$z \geq x z 2$

ONNONONO

$z z z z$

NONNNONO

$z z z z z$ de u u e

$z z z z$

NNN N

zzzzz unemen

$z z z z z$

ONONONONO

$z z z z z$ on une

$z z z z z$

un un u

$z z z z z$

NNNONO

$z z z z$

NONONONO

$z z z z z$

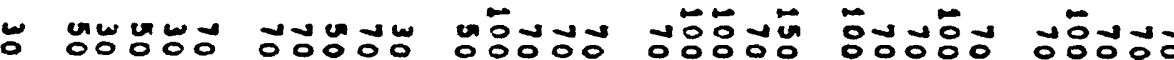

뭉ㅇㅇㅁㅜ

뭉ํㅇ뭉ㅇㅇ

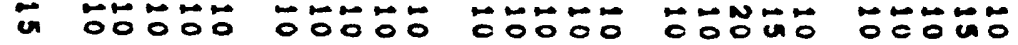
ET
ำㅇํㅇㅇำ tet
ํำำ

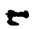

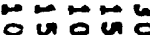
r

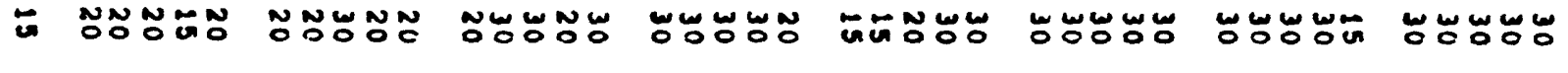

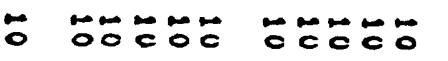

$z=2 z z=2 z 2 z$

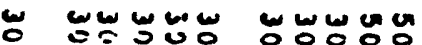

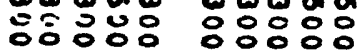

뭉두웅

$z z \geq z z$

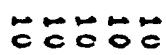

$z 2 z 2 x$

ล유유

$\geq \geq x \geq 2$

Сृㄷํㅇำ

둥ㅇㅇ을

등ㅇㅇㅇㅇㅇ

$z z z z z$

$z z z z$

$z z z z z$

م مN

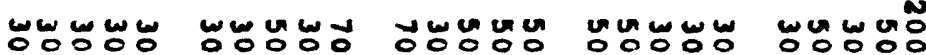

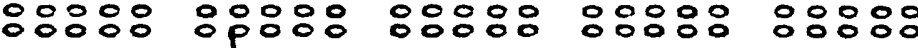

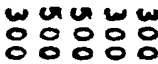




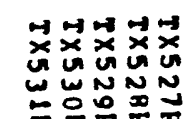

$\overrightarrow{x \rightarrow} \rightarrow \vec{x} \vec{x}$

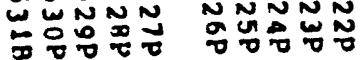

uñ $\vec{x} \vec{x} \vec{x} \vec{x} \vec{x}$

ưvưu

NNNO $\vec{x} \vec{x} \dot{x} \vec{x} \dot{x}$

ux ư ưu



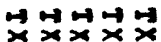

un ư जu

-

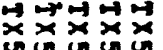

unu un un

웅으의

ơ

버배 $\rightarrow$

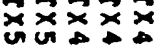

00000

ชำ

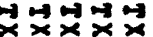

स्र:

更

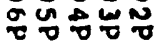

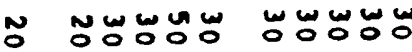

जั
00000 o 000 nu una

0000

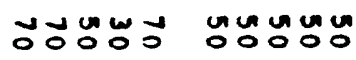

co
O OOOOOOO

$z \quad z z z F^{\circ}$
nNNNN 응ㅇㅇㅇㅇㅇ

$z z z z$
NNNNN

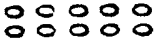

$z z z z$
NNNN $\therefore \circ$

$z z z$
NNNNN

응ㅇㅇㅇㅛ

5
NNNNN

ㅇㅇㅇㅇㅇㅇㅇㅇ

닺?

NNNNN $\therefore: \circ: \circ$

NNNNN

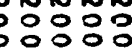

$25 z 2$

z zz

:

E

NNNN WNNNNN

N WNN

Num

مڤN

Now 응잉ㅇㅇㅇ

W

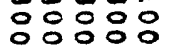

UWWN Oㅇㅇㅇㅇㅇ

סֶ.

in 용ㅇํ요

응응요

운윤운

요분웅

둥용웅

웅후

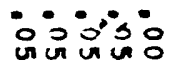

웅웅ㅇㅇㅇㅜ

$z \quad z z z z$

$z z z z z$

$z z z z z$

$z \quad z \geq \infty$

$\infty z 2 z$

$z<2<2$

$z z z z \infty$

$z \geq z \geq z$

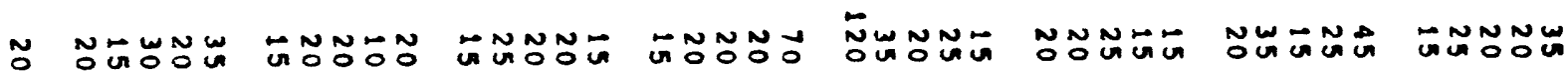

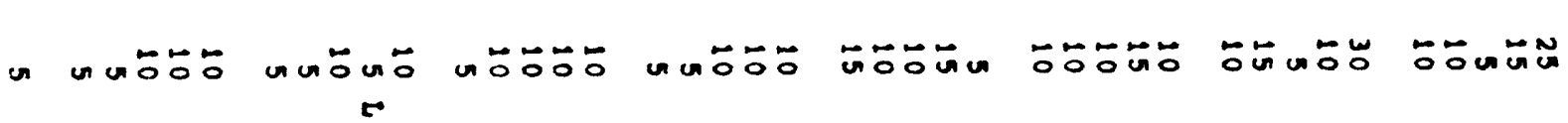

$\underset{2}{2}$

ü un

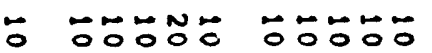

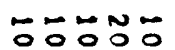

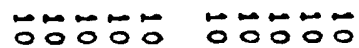

م0000

$z z z z z z$

$z \geq \varepsilon z$

FT 22

NOOOON

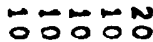

tr $\geq 2$

$z z \quad z$

$\frac{3}{3}$

- nimio

a un un un

$u \rightarrow \infty n \rightarrow$

ant unumuan

$\infty$ (1)

$\underset{x}{x}$ D

W $\div \div: \div \div 0^{\infty} \infty$

$\exists \mathbb{N}_{0 \rightarrow \infty}^{\infty}$

i⿺辶⿻

$\because-\infty \overrightarrow{0}$ in: $\stackrel{\infty}{\infty} \underset{0}{\infty} \infty \omega^{\infty}$

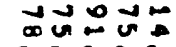
nicis:

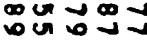

०ण 0 का

No⿱

ODNON NWNNN RNMRN

-NNNO

OONNN

NNRr

NNNNO

NNNNN

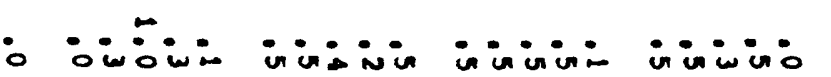

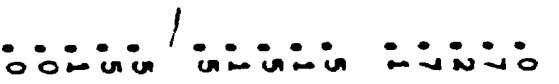
$\infty$ 


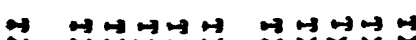

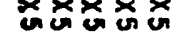

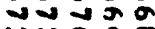
ช。ำ

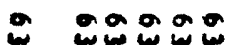

$\cos 20$

¿ UNNNN

NN $N N$

UnO
- UNOUO OOENE
NNN N N

- Wun

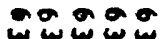

ing ois on

NONOF

ห.
운요요

$\therefore \approx N N$

Non $\begin{aligned} & 0 \\ & 0\end{aligned}$

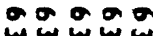

FNNN

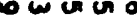

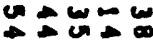

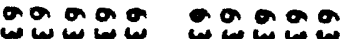

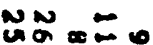

ONNNN

UNजEA ANOONG

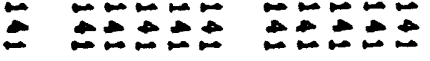 \\ -

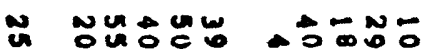 \\ 嘻ま NNָำ Nă}

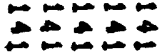

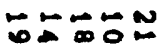

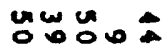

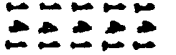
ow 0 o ט
五さきさ

- Oே㇒ in u

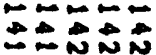

$\omega \widetilde{O} \omega$

wnwu

مأه

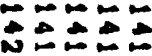

o î : in

م

$r$

i wiviw wiwiv wnivi

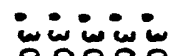

iw w

ivivi

iwimin

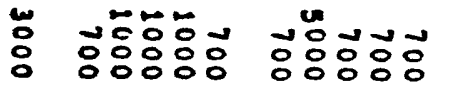

a 등ㅎㅇㅇㅇㅇㅇㅇㅇ 눙응ㄷㅇㅇㅇㅇ 넝응ㅇㅇㅇㅇㅇㅇ 응응ㅇㅇㅇㅇㅇㅇ

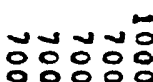

겅중ㅇㅇ융

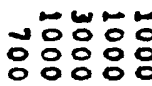

$\frac{0}{2}$

00000 un union

00000 ininivi

00000 inciví $z \geq z \geq z$

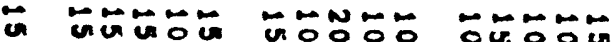

n N N N

$\operatorname{MNON}$

NNON

CNOONN RNOUONO

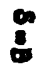

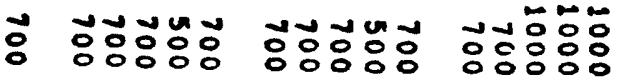

눙ํำ

ㅇํㅇํํㅇ 뭉ํํㅇํㅇ응

눈ㄷํㅇํㅇ 응유

ㅎํㅇำ 
- חRח

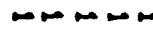

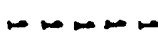

$\infty-\infty$

C TETET TETE

C. TEC

CECET

$\leftarrow r$

250

$\boldsymbol{c}$

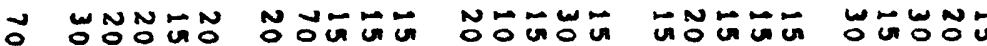

$\operatorname{OMMNG}$

ज次苗

जேㅇํㅇ

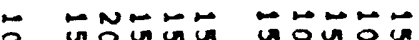

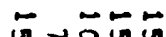

$\operatorname{MON}=0$

ONnNa

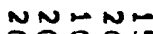

TNNNH

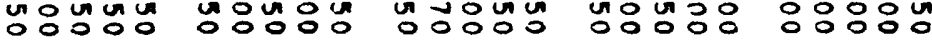

Oㅇㅇㅇㅛ م

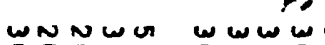

N NONONO ᄃ

N WNNN

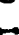

NONGNO

NON ONO 5
NONON

NONON

unumu

zzzzz

cuncuener

un unuon

unumurar

$z=25$

$z \geq 2 z z$

$z z z z z$

unumua

$z z z z$

unuence

$z z z z$

unuerur

$z z z z$

unumu $z \geq z z z$

NNNNN

NNNNN

NONON

NONNO

ONNNN

z z 2 z

$z \geq z z$

$z z z z z$

$z \geq z z z$

$z \geq z z$

NNNNN

$z 20 z=$

N N NON

t z z zz

NNNNN

$z z z z$

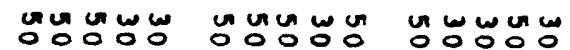

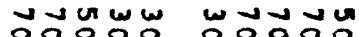

va w w w

unumu un

ununu un

$\sum_{i=1}^{\infty}$

-

- TEE

두용

in $0=0$

MNOOG

ேㅠㅇํㅇ

م0\%

Nㅜㅇㅠㅜ

CET

후유

$r$

:

NONO

n-NNN

NNNN-

NOWNN

NNNWN NNNNN

NONONO

:

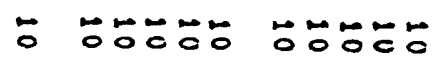

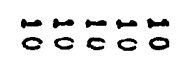

뚜ㅇㅠㅜ웅

둥ㄷㅇ

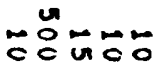

웅응

22222

$z z z z z$

$z z z z z$

$z$

$z z$

$z \geq z 2 z$

뭉유유

$z \geq z z z$

צ

年

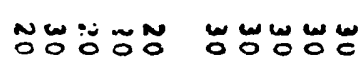

دو

แัน

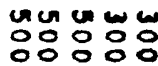



Nơ

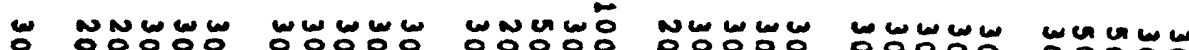

OOOOONO NONOWO

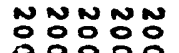

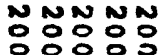

r I ZZ2Z

$z=z z$ $x \geq z=$

$z z z z$
NNONONO

영융요

$z z 2 x z$
NNNNN 응융유

$z=25$
NNNNN 응융융

$z=z z=$

NWWNN 응응웅 $x$

i 웅요요

inio:

$x=5 z=2$ unisis:

: 웅요

$z x z x z$

T) $2=2$

品品宫它

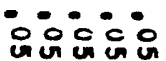

웅웅요

$52 \pi 2 z$

$x \quad 22 z$

$z z \geq z$

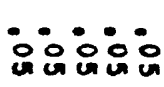

a NONNE NWNNN

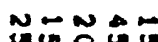

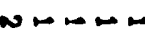

innNo

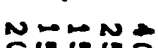

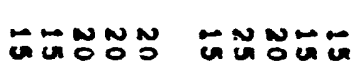

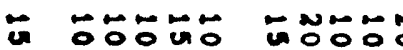

Non

$\because N=5$

ேேーேே

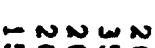

nand Oํㅠ음

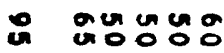

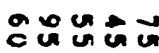

요용

anous

orentaun

00000

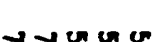

ํㅜㅇำ

ㅇ ㅇํㅇํํㅇ 뭉ํㅇ

ธேัОธัดั

두융ำ

등ํํ응

$0 \times 222$

$2 t<2=$

$2 x \geq z$

$z z z z$

$2 z 2 z$

ООООㅇํㅇ

뭉ㅁㅇㅇ 유융유

- cucruar an un un

cos un un

is uñas acisio

ingu

$\Delta u \rightarrow u s$

un unta

z $2 \geq 25$

$z z z \geq z \quad z L$

$\frac{2}{3}$

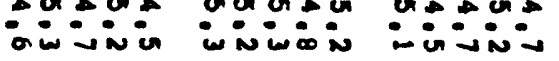

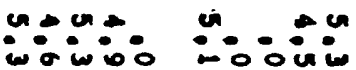

$\rightarrow$

$\underset{x}{0}$

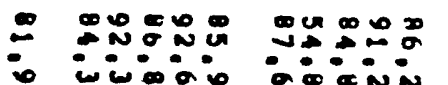

$\infty \infty \infty \infty$

$\infty \infty \infty \infty$

$\infty \infty 0 \times$

$\infty \infty \infty$

एะ

uniod

Un:

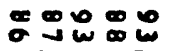

능요:

:

n nom

- MNNN

NNNNN

$\sim N N N$ N

NNNND

N NNNN

NMNNM RNNNN

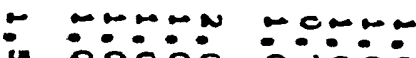

nNEN

-w-

$-0,00$

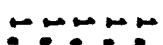

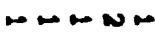

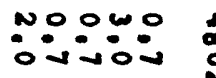

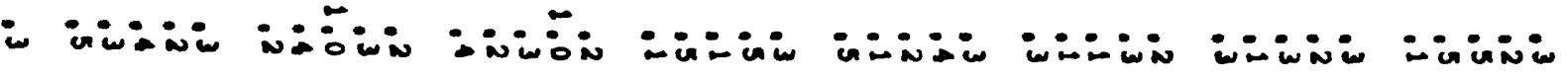




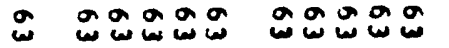

$N \rightarrow \infty u \rightarrow+200=$

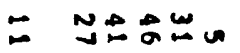

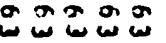

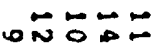
un ज०:

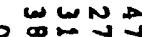

주요

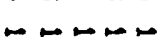

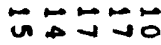

w w n
ำ

$=0$ O

$n \in N G M$

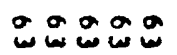

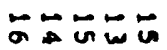

u un

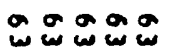

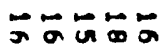

UNENO UNẼ UN $\rightarrow \infty-\infty$

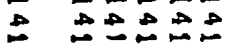

$-\infty-\infty$

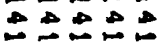

$\rightarrow \infty+\infty$

0 400

J 00000

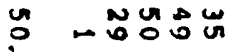

幽萬 and

$N \stackrel{N}{N} \sim N$

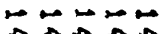

$\pm D \pm \pm E$

$\vec{U} \approx \vec{a} \rightarrow \infty$

$0 \lim _{0}^{\infty}-\frac{\pi}{4}$ $\rightarrow \infty \rightarrow \vec{a}$

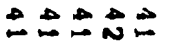

$\Delta \rightarrow \infty$ w

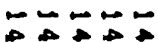

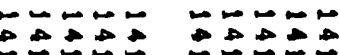

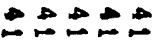

ara

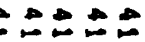

AีAW

W

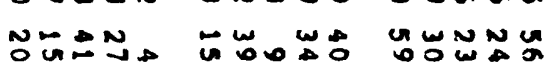

$r$

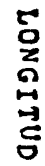

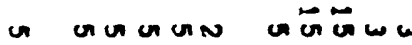

uncum

N N N U

unuma

on unu

un won

טw w w ט n

i 능융ㅇㅇㅇㅇㅇㅇㅇㅛ

ด

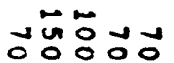
응ㅇㅇㅇㅇㅇㅇㅇㅇ

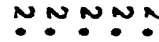

NNNNN

$: 0: 00$
Namn

N-NNN NNNMN

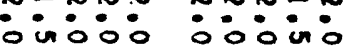

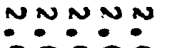

?

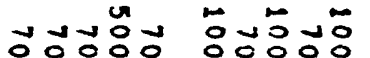

जㄴํㅇำ

궁료융

웅혀 ㅇㅇㅇ잉ㅇㅇㅇㅇ $\frac{0}{3}$
ฉ in iniuivin
$z \quad 2 z z 2 z$

00000 in univi $z 2 \geq 22$
00000 inivin

$z \geq 2 z$ in un uni in in univi

$z \geq z z$

$z z z z$
00000 in in in 00000 $z z z \geq z z z z z$
00000 in un in $z z z z 2$
:

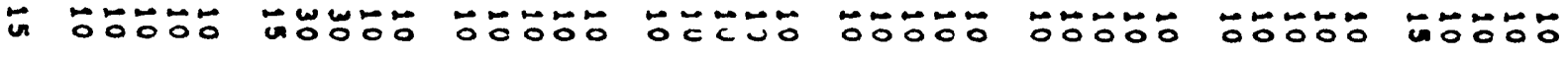


•

$\rightarrow \infty x^{2}$

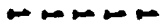

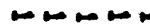

$\sim \infty m$

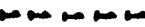

$\sim \infty \cdots$

- Cetcetecte

$\leftarrow$ CE

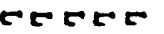

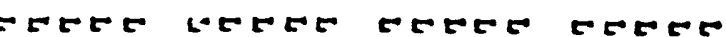

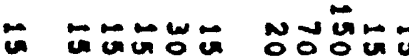

ถักกับั

ேேேンั

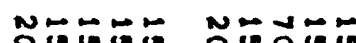

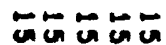

คำนีล

ถ்

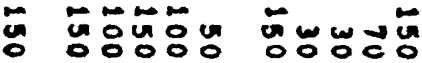

NOMOON

NGน十:

它落它品

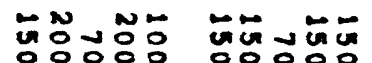

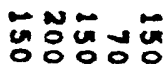

:

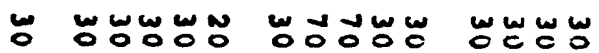

WWNW

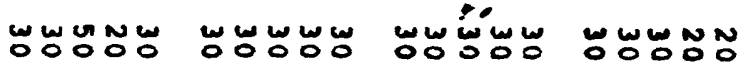

:

N NNNNN NNNNA NNNNN

nNNNN

nNNNN

nNNNN

ONNNN

مNOOOONO

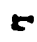

:

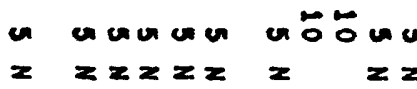

mun un

ununu

ancuan

anuai

onuma

oru un

:

NOONONO ONOCNO

NONONO

NONONO

ENNNN

$z \geq z \geq z$

$z x z z z \quad z x z z z$

$z=z z z \quad z Z z Z z$

$z \geq z z z$

$z \geq 2 z=$

$z \geq z \geq z$

NONNN

NNNNN

ONNNN

$x \geq 2 z 2$

$z z z z z \quad z z z z z$

$\sum_{0}^{\infty}$

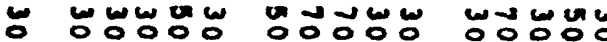

unw un

wus wor

nun

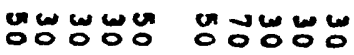

$\sum_{i=1}^{0}$

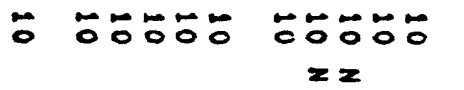

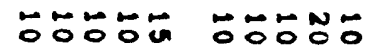

두ㅇㅠㅜㅇ

(2)

$r$ TE

ํํㅇํำ

무ㅇㅠㅜㅇ

Nㅜ융

5

r TE

$\underset{0}{0}$

O NONONG NUOOOOO ONONONO

NNGNN

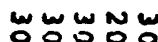

ONOWO

Wִ

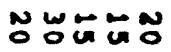

:

$\because$ 두유 유유

$z z z z z=2 z z$

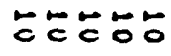

z z z z

둥두웅

두웅

$z \geq z=2$

$z z z z z$

둥두

무융유

$z z z z$

$z z z z z$

무두두

$z z z z$

0
0
2

ڤ

ww w

w w w w

w w w w w w

wWw

W.

융ㅇㅇㅇㅇㅇㅇ

$\because$

N O ONONE NWLNON NNNNN

NONNN

nNnTN

nN NNN

N-N N N N

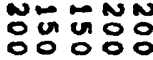

NON N

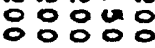

$\stackrel{8}{2}$ 


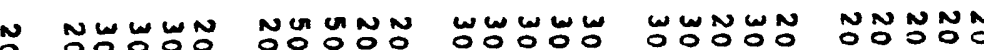

w w w w w w w w

م مس مس مس مس

W무요

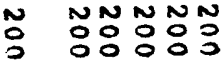

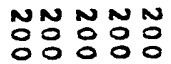

OOOOOOOO

NNONON

$z z z z$

$z=\quad z z$

$z z z z$

$z \geq z=\pi$

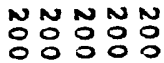

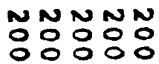

$Z Z Z F=$

$z z z z$

NNNNN

Oㅇㅇㅇㅇㅇㅇㅇㅛ

$z z z z z$

NNNNN

ㅇㅇㅇㅇㅇㅇ

$z z z z z$

$\underset{⿱ 亠 䒑}{\mathbf{N}}$

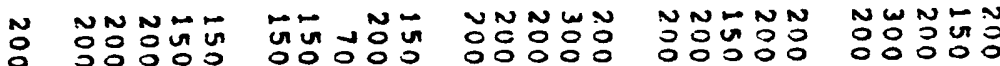

NNNNN

N

w

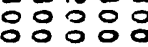

i 요요요

운응ㅇㅇㅇㅇㅇㅇ

엉ㅇㅇㅇㅜ

$Z r=25$

$\infty x \geq z$

z $\boldsymbol{C}^{\circ} \mathrm{z}$

운은웅으

(

엉ㅇㅇㅇㅇㅇㅡ

2 $匚 \geq \geq$
웅웡ㅇㅇ

$z z \geq z z$.

\section{过想品品}

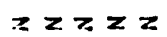

엉ㅇㅇㅇ
$z x z z z$

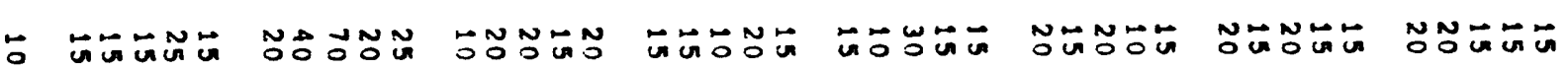

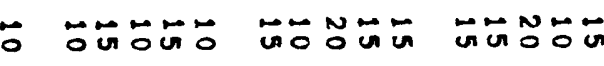
on

\section{noou}

in in $\operatorname{in}$ un un un
무요
MNDN

MCOO

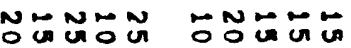

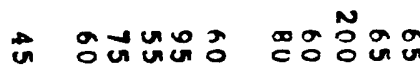

in $0 \rightarrow 0$
Un U⿺辶寸 in ungu in 0 O ouna ovicun ơ ữ ư ư ingun un

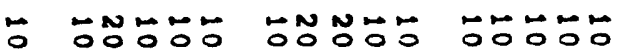

5

5

$z$

$z 2 Z 5$

무융

무융유

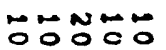

zEz $z \geq$

$z Z \quad z E^{\circ}$

$z z z z$

00000 두유유

Zt 2 Z

$z$ c $2 z$

$$
\text { : }
$$
$\infty \stackrel{\infty}{\infty} \infty$

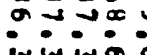

N NGNMN NOONN NMMMM $\infty$

¿ o. कां००

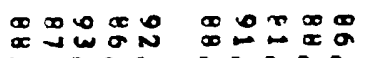

¿ OD:OUD

$-0:-$

:D:DO $\infty$

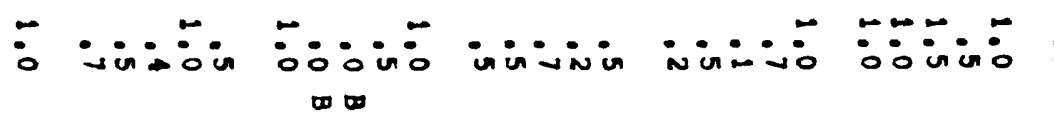

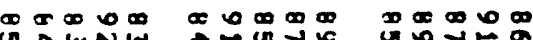


i 0 is

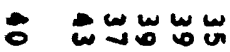

^ on 9090 N

Un
웅요

б un w -

-NNE-

- NN=0 ana0a

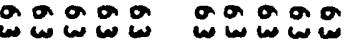

N WONO

№n
NDW

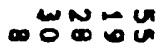
$\rightarrow-N a-$

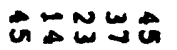

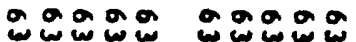

-

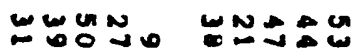

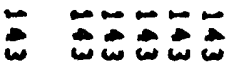 \\ $\because N \approx N N$ \\ ○}

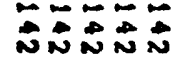

$\simeq \widetilde{O}=\mathbb{N}$

U요요

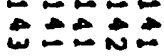

$\because$ Gun 0

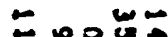

$\because ロ-\square$

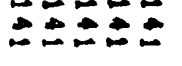

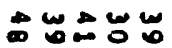

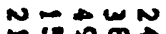

シミミミミ

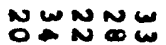

แกธัด

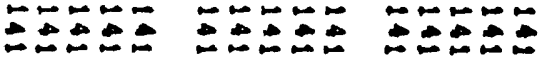

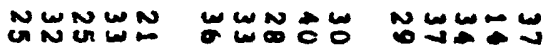

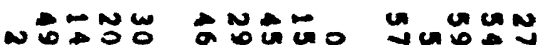

unwor

wnoruon

Druma

nosen on

in inivir inisi

iniviv

Uini:

inivin

inivin

证证

-

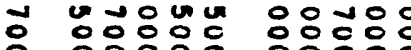
등ํㅇ영융유

눙ㅇㅇㅇㅇㅇㅇ

두눙 응응ㅇㅇㅇㅇㅇ

두웅ㅇㅇ 영영영융

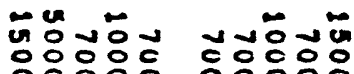

$\circ \Omega$

. 
מ ח ח ח ח ח

5 C TE⿱

ח-

cecter

-

$\operatorname{coc} 20$

nama

CETCE

and

CEETE

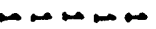

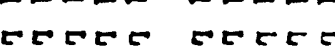

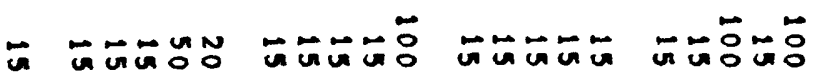

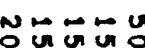

GUUG

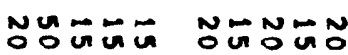

$\stackrel{\infty}{\vdots}$

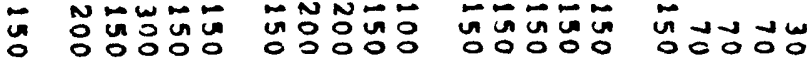

Nö un un

Non

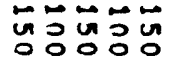

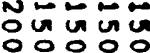

$\stackrel{8}{0}$

م

ט

w

wUN'Ńn

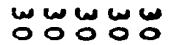

$\stackrel{n}{\grave{c}}$

23.

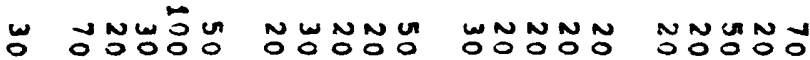

NNNNN

nNNNN

NNNNN

NNNNN

$\stackrel{2}{i}$

unu un un

unumer ununua

$$
\stackrel{2}{2}
$$

\section{NONONN}

$z z z z z z z z$
CNOCON

c $z 2 z 2$
NON N N N $z \geq z z$
NNNNO $z x z z$
$\begin{array}{llll}N & N & N \\ O & 0 & 0 & 0\end{array}$ O 0000

$z z z z$
NNNNN

OONON

$z z z z z$

NNNNN

$z z z z z$
N

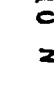

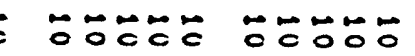

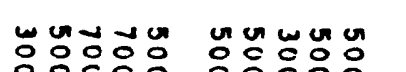

ேㅡ융ํㅇ $22 z 2 z$

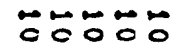
$z z z=2$
뭉두웅

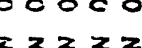

$\because$

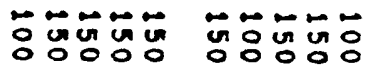

$\begin{array}{lllll}N & N & N & 0 & 0\end{array}$

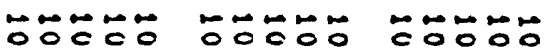
$z z z z \quad z z z z z \quad z z z z z$ 


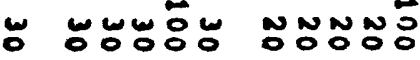

2000\%

\section{NNNNN}

$z x \geq z x$
Nㅡㅇํㅠ융

$z z \geq z z$
WNONON

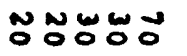

WNONO
NONONO
שั

ธักับัก

ONNNNN $\circ 0000$

$z z z z$

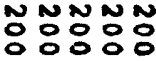

Z Z E 2

\section{NNNO용요}

$z z z z z$
NNNNNN

$z z z z$

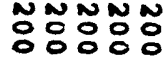

ET $2 \geq 2$
Nㅛㅇ용ㅇㅇㅇㅇ

$z z z z z$

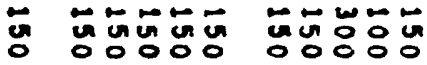

ลํํํํำ

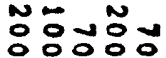

OOOOOO:O

กับnN

NNWNN

NNNNN

:ํ:ดำ

- 운욤운ㅇ

$z=z \quad z z$

운운웅웅

$z \geq \geq$
운운운웅

$z \geq z z z$
운운웅융

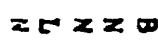

웅운웅요

$z \geq z \geq 2$
운둔웅요

$z=2 z=$

inisio

:io:$z x z z z \quad z z=$

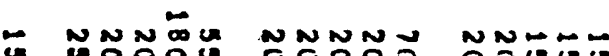

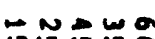

$\sim N N \sim N$

OUGNON

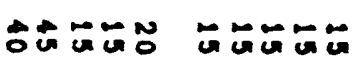

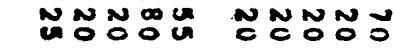

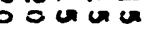

$\underset{0}{0}$

$\stackrel{n}{\mathbf{c}}$

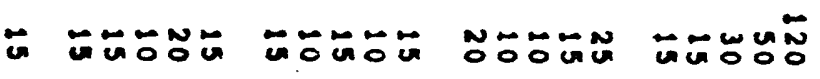

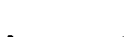

$\infty \ldots$

OMNOM

$\approx \approx 000$

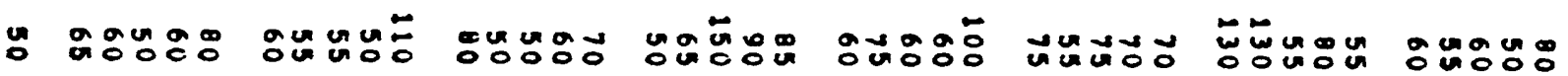
100000

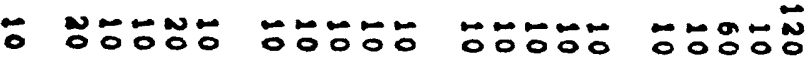

x

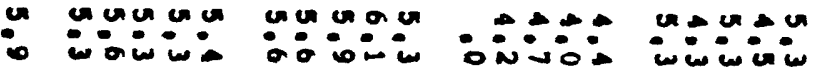
$\infty$

$\infty \quad \infty \infty \omega$

$+\infty \pm \infty$

- ivioi

$0 \infty \infty N$

$\div$ ก

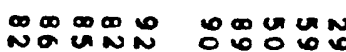
ㅁํㅇ O

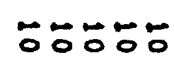

$\geq \geq E$

$-c \rightarrow c-$ - in:

$\infty \pi \infty \infty$ $\infty<0$ oivisio
후웅

रा 20

$u \rightarrow u+c$

:ㄴ:-

$\infty \infty \sim \infty \infty$

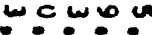

무요 $=-0:-0$

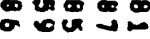
:0:

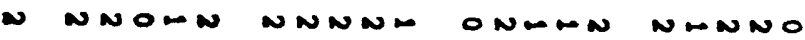
$\boldsymbol{\omega}$

NNNRN

Non

-nNNN

NMNNN

$\stackrel{\infty}{\infty}$

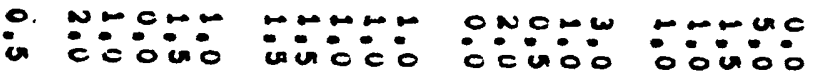
$\infty$ $\infty$

NnN-N

N-NWN

M

- on we

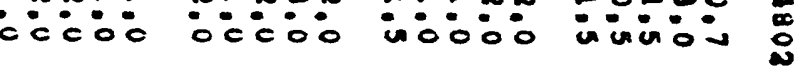

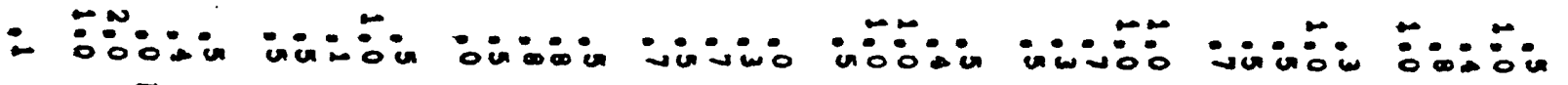
$-$

$\rightarrow \infty C^{\circ}$ 


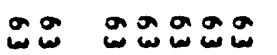

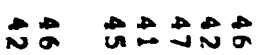

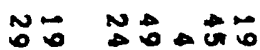

س

$\rightarrow$ un un nos $\rightarrow$

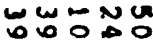

운

$\rightarrow \rightarrow \infty$

Uñ
운

\&

- $\begin{aligned} & 0 \neq 0 \\ & 0\end{aligned}$
NE

W

०ै $\overrightarrow{A \rightarrow A M}$

DW

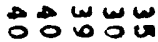

$\overrightarrow{A \rightarrow \infty}$

NNN

$\rightarrow$ W

م

$\because \square \div \div$

NANEA

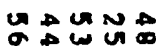

UN UN

un surat wañon sasen

oncuens

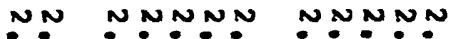

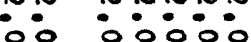

웅유

NNNN 0000

NNNWG 0000

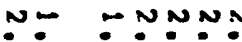

NNNNN

NN- - N

0

in in

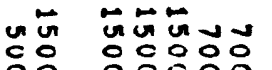

훈부웅

ㅇㅇㅇ응ํำ

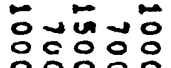

inivio

00000

$2222 z$

$z z z z$

$222 z$

$z z 2 z 2$

30 웅응 Et

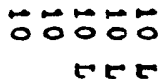

t5E
뭉우웅
C5

5

5 


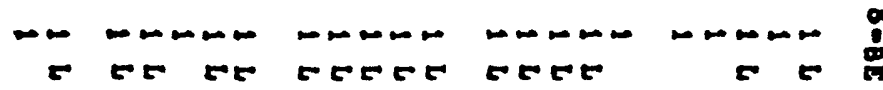

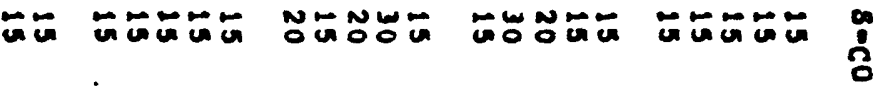

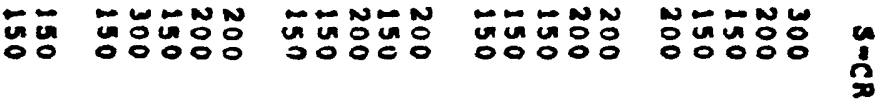

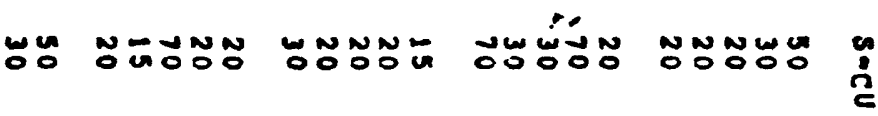

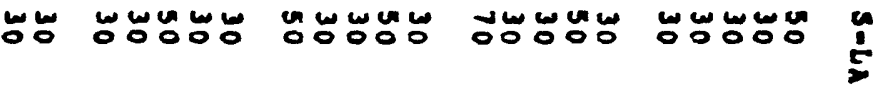

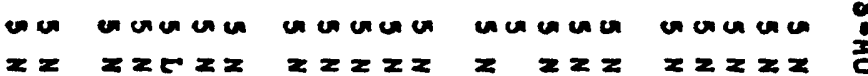

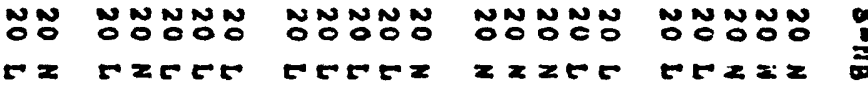

苗

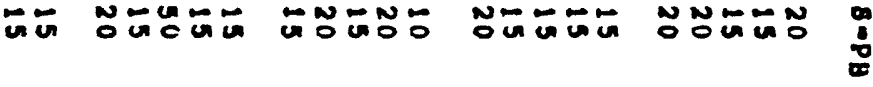

ONO O ONOOOOO $O N O N O N O$ ONONOOWN

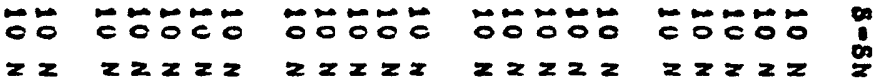

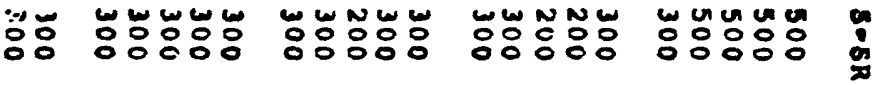

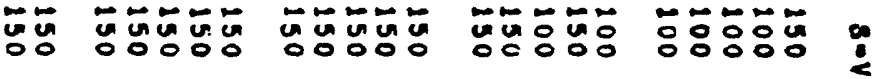


NNNNN

$z z \quad z z \geq z z$

$z z z z z$

다 $z \geq z$

ํำดั

\section{ing}

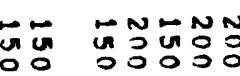

可证牙

舟N5

z z 20

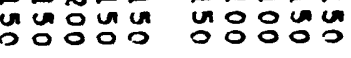

$n$
$\vdots$
0

品 㝝品品品

zE $\quad z \geq z z z$

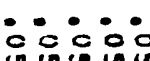

둥웅

$\because 0: 0$

$z=z z$

$z=z \geq z$

$\geq z \geq z z$

3
2
0

مूN

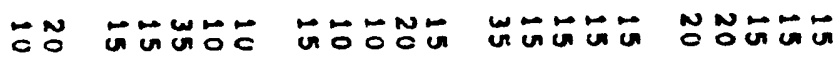

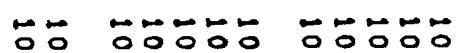

ZE $Z E Z Z Z$ ZEZZZ

ัํํํำ

뭉무유

3

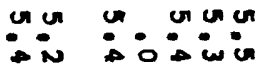

a

u un un r $z<$

$z z z z z$

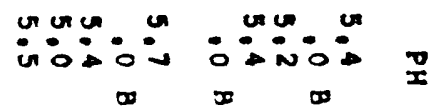

$\infty \infty \infty+\infty$ $\infty_{0}^{\infty} \div \mathbf{\infty}$

$\rightarrow x \infty$

a c w

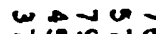

$\infty \mathbf{\infty}$

-

$\omega$

$N N N N$

$N N N N N$

n NON

NNMN

$\boldsymbol{x}$

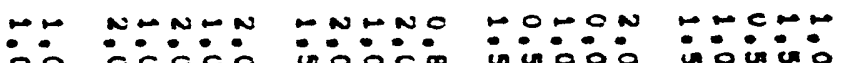

$\boldsymbol{\infty}$

:- in inivi : 
Rocks:

S-Au was looked for not found.

The following elements were not detected except in the samples listed:

$$
\begin{aligned}
& \text { S-Cd. .... TX 145RA (100 ppm) } \\
& \text { TX. 145PR (30 ppm) } \\
& \text { S-Sb..... TX 150RA (61000 ppm) } \\
& \text { TX 15ORB (100 ppm) }
\end{aligned}
$$




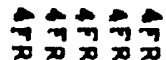

$2 \geq \pm 02$

a $00 \%$

曲

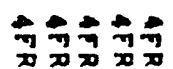
$0 \geq \geq 2 \geq$

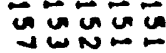

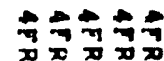
$12 \div 2$ 둥두

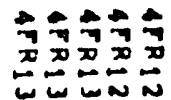

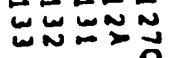

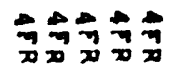

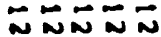

西似N
$-1=-2$

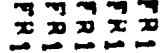
ニニ

$\approx$ जैป二

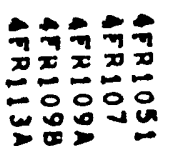

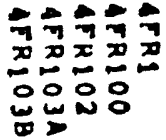

我
운

un un un

$\rightarrow \rightarrow \infty N$

운운

a 9000 טwa

눟ㅎㅇ

பㄴㅎㅇ

a o a a

-

00000 w w w (م)

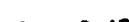

MNu $\rightarrow$ N

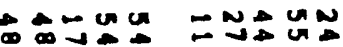

UnW

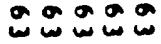

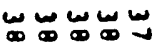

un $\infty \infty$

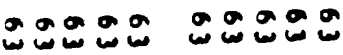

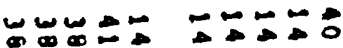

n: 0 0

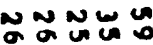

굴

$\infty-\infty$

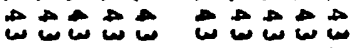

سָ س

A unun un

\&N NOEO

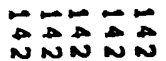

Wニ-

NN N

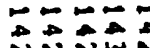

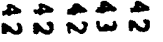

مَ

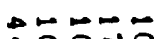

$\therefore=-\infty$ 就むむ

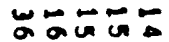
incon

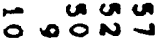

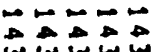

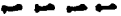

-

TOOOR

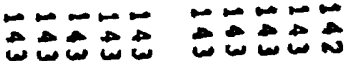

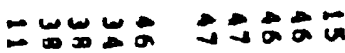

DAZ

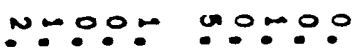

nusto

ounnw

:Nก: N

는

moOn-

w w w w w

Oonio uㅑㄴ

웅웅ㅇㅇㅇ

ㅇㅇㅇㅇㅇㅇㅇㅇ

ㅇㅇㅇㅇㅇㅇ

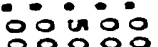

inio:

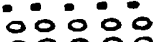

ca

-0000 WO-OOD WN WN

พ 20 N

OOOON

0000\%

00000

$00 \omega-5$

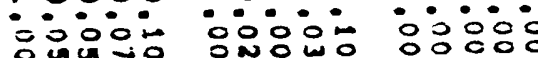

OOOOO

in:

00

inivio

जis:

$-0000$

No00

- NTM

TNNTN

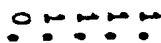

00005

$000=-$

OUก:-

웅ㅇㅇ웅

O이 00000

:00\%:

웅ㅇㅇㅇ

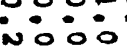

00000

te

$\infty$
2
2

5

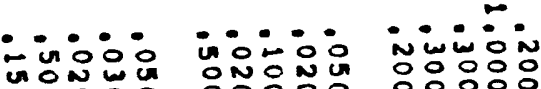

iw

inini

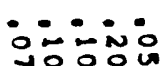

isi:i

Un

๑

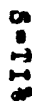




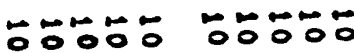
TCERE
5005: TEETE $\leftarrow \infty \div+\infty$ 0000 CEECE
ООㅠㅇ 05525

50

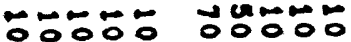
TEE $C$ TE

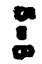

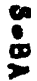

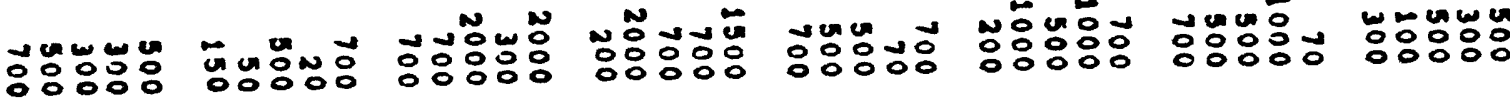
$z$

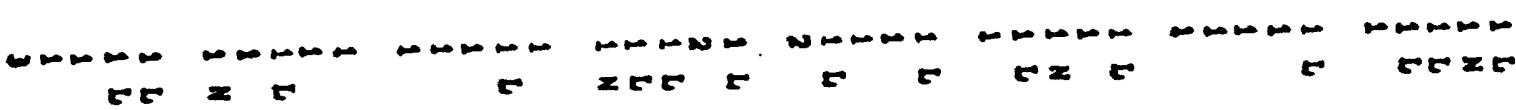

Нே0\%ั

$z z 2 z=$

두웅 $z=2 z z$

두웅 $z \geq z=$

$00 \div \div$

م००0\%

두융ㅇㅇㅇㅠ

$z z z x z \quad z x z z z \quad z z z z z$

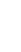

$\div$

nמo

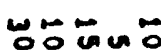

unuence

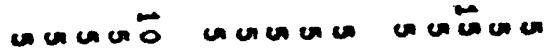
r $2 x$

z $\quad x$

$z \geq \geq 5$

$z Z 2 z$ L 25

$x \geq z z$

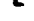

두우

$z \geq 2=$

مـ

$\cos ^{2}$

ON.

$z \quad z x$

잉

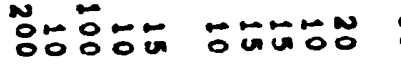

$z=$

$z z z z$

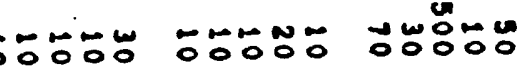

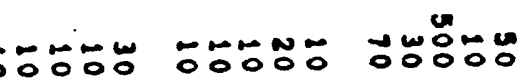

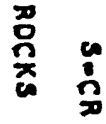
$z \geq z=$

unumo $z \geq z z$

OONNN $z \geq 2$

uncuna $z z z z z$

Nㅡㅇㅇㅛ CEEt

Oㅜㅇㅠ NONON TEETE
N wONO $z=$
NONO NOO $z=$ anuan andor:

exzer ze 2
NONONO cozz $z$ unumon

uneruen

anoug

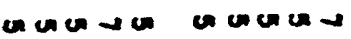
$z z z z z \quad z z z z z z z z z z$

TNONOONO NOOOOOON ONONONO NONONONO ZLZZ EETEE TEECZ TZZZE. ancen un CELE $\because 0$ in un a r ter

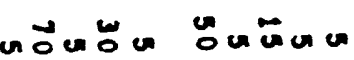

reraciance anumö CEcto unuma anỡ cte to

บㅜㅜㄴ

טم$00 \div 0$

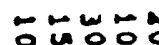

튼 능

مró

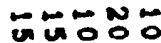

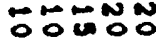
c 5 c 5 $5 \quad 5$ 20

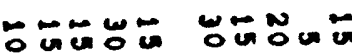

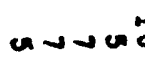
c $\geq 5$

c 


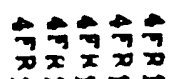 \\ क्ष Non 田

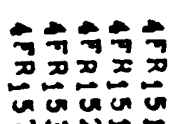 \\ जU U U \\ $\infty=$}

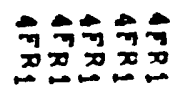

$\rightarrow \infty$

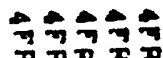

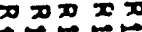

W

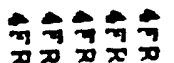

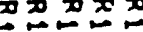

NNN然

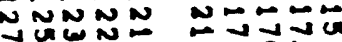

$\because \overrightarrow{0} \overrightarrow{0}=$

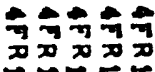

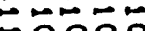

둥웅요

$\omega 000$

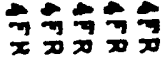

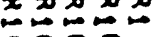

웅웅ㅇㅇ
무융 유유 $z \geq z z z$

$z z z z$

무유

두웅우

무유

$z z z z z \quad z z z z$

다지

두웅

두융ำ

22222

$z \geq z z x$

두웅

$z \geq x \geq 5$

:

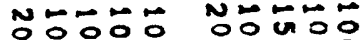

웅영ㅇㅇㅇ

$z z=z$

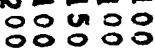

$z=x$

ผ ติ

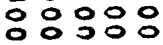

مس

응영응

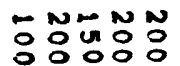

둥분

00000

$z$

뭉ㅇㅇㅇ 웅ㄴㅇㅇㅇㅇ

$z z z z$

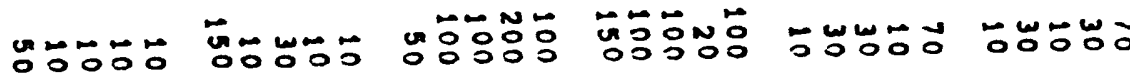

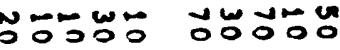
EC

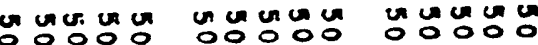

un unu un

ungun

$z z z z z$

$z \geq \geq z \geq$

$z z z z z$

uncuna

00000

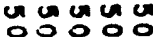

$z z z z z z z z z$

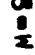

$z z z z z$

$z z z z z$

MNNON

जேㅇํㅇ 5

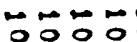

OMnOM

Nم CE E

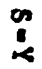

NNNNN NNNONO

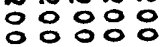
ㅇㅇㅇㅇㅇㅇㅇㅇ

nNNNN

응ㅇㅇㅇㅇㅇ

NNNNN 응영ㅇㅇㅇ

nNNNN

NNNNN

NNNNN

NNNNN 응응응 응응융 OOOOO 잉ㅇㅇㅇㅇㅇㅇ zzzzz

$z z z z$

$z z z z z$

z z z z z

z z z z

$z z z z z$

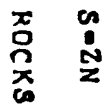

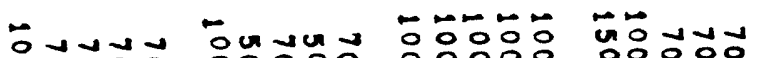

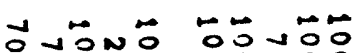

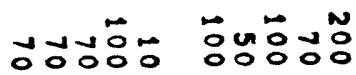

:

웅ㅇㅇㅇㅇㅇ

웅요

웅웅요

$\therefore \circ 000$

웅웅

웅우

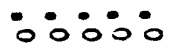

$\because \therefore \circ \circ$

$z 2 z z 2$

$z z z z z \quad z z z z z$

$z z z z$

$z z z z z$

$z z z z z$

$z \geq 2 \geq 0$

$z \geq \infty z z$

:

$\because 0 \div 00$

$\because \therefore \div \div$

:00:0

웅우

융유 NON W $12 z=20$ W

Ш

z

inisio

$8 \infty 60 \%$

$\div 0 \div 0 \div$

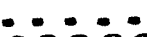

叫 2000

$000 \%$

ш

00000

00000

00000

00000

ம

00000

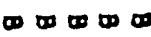

- ब必必

का एक 000

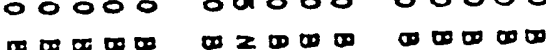

(1)

00000

00000

00000

00000

00000

ம 뚜

a $\infty \infty \infty$

ம0区

ద
00000
00000

0 0000
ए

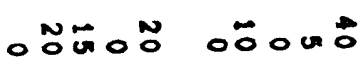

- 100

00000

ம0
00000

00000

由 2000
W Ũ 000

$\infty \infty \infty$
00000 $\infty \infty \infty \infty 6$
No 000 C जunoo $\infty$
No000 வாய
Uึ:000 $\infty \infty$ 


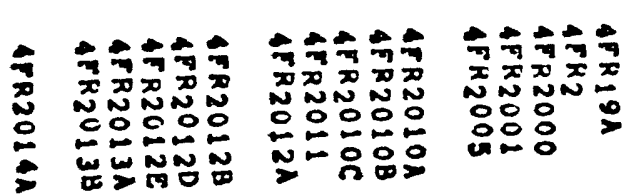

0.000

- w w

- 두눈

- wพ⿳宀口́⿴囗十
00000 ம 누수웅 encuca

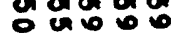

o.0.0

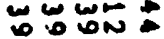
$\exists N$ ON

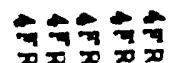
xख̃ $500 \div 0$ ww

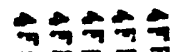
$x \geq 0 x$ $\infty-\infty \infty \infty$ $\infty$
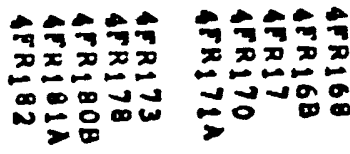

$x$ x जैज0 $\rightarrow \infty$ acosa

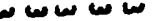
טun

ט Oேニ

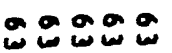
ancono (t) 우유
우웅 a was unun un un agunan

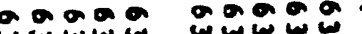

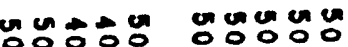
NNบN

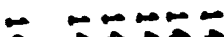

$\therefore \leq \mathbb{N}=5$

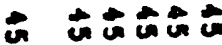
Nov日 $\because-\infty \cdots$ INANA 현현

$=-\infty=$ م مس مس can $u \rightarrow$ م $\because \div \div \div$ w\% NNNNA

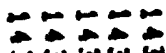

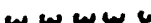
and 0 Na
ேㅡ는 ص

\section{N} Oa:
Eะ

w

wnu $\operatorname{con} 0$

$\infty \rightarrow n \rightarrow \infty$

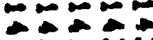

क

سِّ

ט

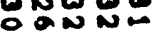

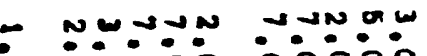

- 0000 :0:00.

:
- Ondar anock

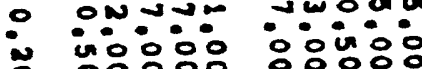

navan unoman

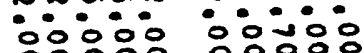

W

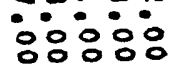
n.000 $\therefore \circ 00$ :
W:ONN 웅웅ㅇㅇㅇ
- 은 -

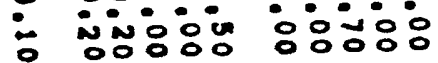

OnN:0

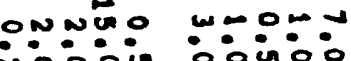
ㅇㅇㅇㅇㅛ
NNOOW

M:O:- : : NO:N

ODNO:

둥ㅎㅇ응 $\therefore \therefore$ i. in: OOOW:

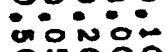

으유:웅ㅇㅇㅇ

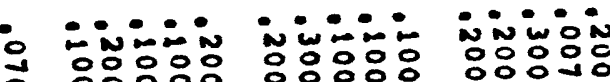

- inin ninin

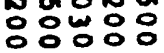
沙的泣 녕용응융
$0 \% \div:-\infty$ :6:0: :융
으응 둥웅웅
No000 웅웅웅은

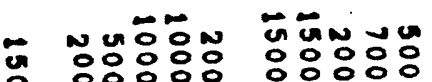

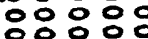

unven

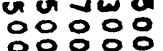
תัn un ũ 잉요요 응융유

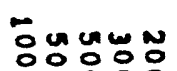
ㅇㅇㅇㅇㅇㅇㅇㅇ

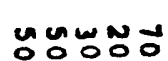
ㅇㅇㅇㅇㅇㅇㅇㅇ

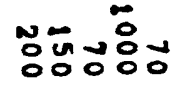

영영융영
- oo:00 o0000

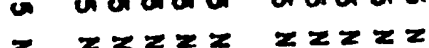

00000 inivivi $z z z z z$
00000 uniui $z \geq z \geq z$
0000000000 - ininar univina $z z z z z \quad z z z z z$
00000 univivi

$z=z z z$

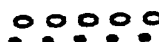
unuivi

$z z z z$ $\frac{1}{3}$

NNNNN NNNNN 응융영 영ㅇㅇㅇ영

$z z z z z \quad z z z z z$
NONONO 응응

$z z z z$
NNNNN 응응응응

$z z z z z$ 


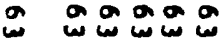

un unun un

N NNNNN

$\therefore \square-\infty N$

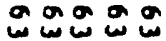

uncon on en

No 000
운

un uner un

w

エேニ a

unu un

$\omega \omega \omega \omega$

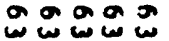

on on un a

o

क Noñ

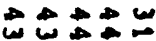

a
合合苛家

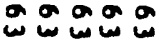

항ㅎㅇㅇㅠ

$\omega A$ क
NDWW

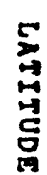

\section{ث

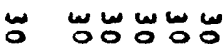 \\ $\sim \sim \sim \sim \mathcal{O}$}

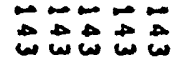

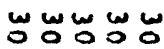

NNㅗㄴ

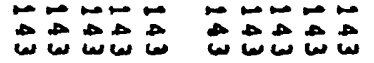

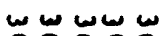

NUNONONO
مَ

NOTNN
ش志芯

لَ

N

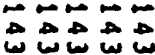

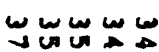

0
W

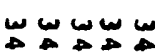

$\rightarrow \infty=0$

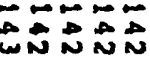

W

م

\section{$a \rightarrow-20$ \\ UNUNN \\ w Un- \\ Rancu \\ $\ln 2 \pi m$ \\ MUNNO \\ ONDNO \\ $\because 20$ un un

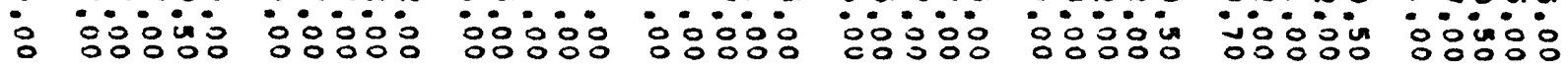

N NWwON

- -000

OODON NNWWL

-

00000

$0 \omega 0=-$

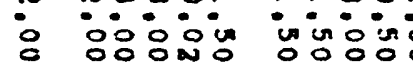

O융 wo in i:

$\because \because \therefore \circ$

åñ

Niñ

-

w"wo:

$\because 0000$

OONON

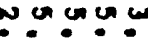

$0 \div \div 0$

00000

$\because 2000$

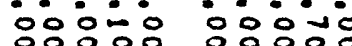

둥ㅇㅇ

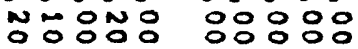

WOONO

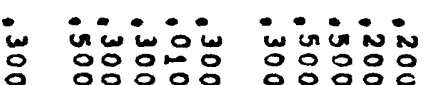

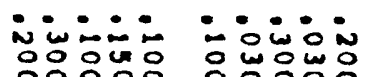

ininis

WNㅡㅇㅛ

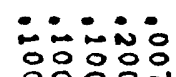

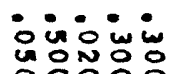

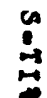

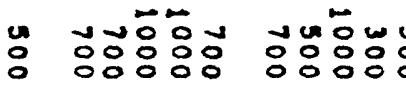

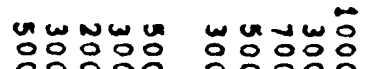

을 응응ㅇㅇㅇㅇㅇㅇ

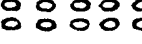

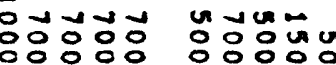

Nongug

nํำ

$\frac{n}{x}$

- 00000

in ininisin

$z \quad z z z z z$
00000 univinis 00000 inivin to $z \geq z z$ $z z z z z$
00000

uninis in

$z \geq z z$
00000 in in in i $z \geq z z$
00000 in in in is $z z z z z$
00000 in un inis in $z x z z$

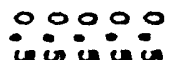
$z z z z z$
Nㅛ용ㅇㅇㅇ

$z=z z z$
N

$z z z z z \quad z z z z z$
NNNNNN

$z \geq 2 z=$
Nㅗ용용ㅇㅇ

$z z z z z$
Nㅡㅇㅇㅇㅇㅇㅇㅇㅇ

$z z z z z$

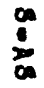




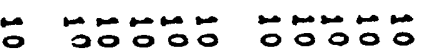

مि

$z z=2 z$

x. $2 x \geq 5$

$z \geq z 2 z$

ேㅜ유유

두웅

00000

c $z z z$ I $z z z z$

$z z \geq z$

מ以n

o

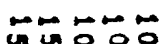
$z$

$z z z$

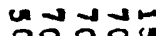

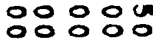

$\rightarrow$ NNNO 등ㅇㅇㅇㅇㅇㅇ

$z z$
둥ํำ 25 응영영영 영영명 $z z z z z$

舟

유ㅇㅠㅛ융

응슝무

Uूก

뭉무웅 5 C

영

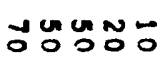

מש एా

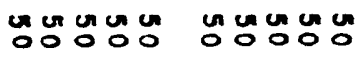

जưncuño

$z z z z z$

$z z \geq z z$

जัตํํㅇํㅇ

Uরu uñ

$z z z z z \quad z z z z z$

$z z z z z \quad z z z z z$

范

NบONO

Un UnN W

00000

NNOOOOO $z$

n NNNNN ㅇㅇㅇㅇㅇㅇㅇㅇㅇ NNNNN 영ㅇㅇㅇㅇㅇ <5ez

z. $z \geq z$

nN NNN 응ㅇㅇㅇㅇㅇㅇ

nNNNN 응응유

NNNNN 응응ㅇㅇㅇ응

$z z 2 z z$

$z z z z z$

$z z z z z$

NONNNO

$z \geq z z z$

NNNNN

응융응

Z r $2 \geq 2$

NNNNN

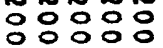
$z z z z$

N Un

\section{N}

nN n n

- Un M MNNNN

n

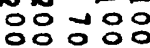

용ำ

MNONN uñôno

Hat

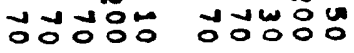

욘운운운

$z \quad z z z z z$

운운웅

응용ㅇㅇㅇㅛ

은웅영요

$z z z z z$

$z z z z z$

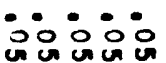

웅웅 은언용

$z \geq z z z$

$z \geq z z z$
웅ㅇㅇㅇㅇㅜ

$z \geq z z z$

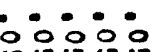

$z \geq z \geq z$

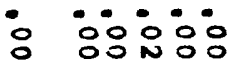

Ш Ш匚飞山
웅융ㅇㅇ ఉய
웅융요 × 2
응용ㅇㅇㅇ

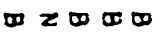

영응영응 $\infty \omega<\infty$

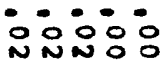

2000
두음 ㄷNㅇㅇㅇㅇㅇㅇ 由于四田
무웅 오ㅇㅛㅛ $\infty z z$
000000

-

00000 00 0000
0000000000 வ
00000 由 00000
00000 வ1000
00000 10000
00000 由田 由
: $\stackrel{0}{0}$
000000

ఉ ๘
00000 எயய四
00000 Ш
00000 1000
00000 - 000
00000 由
00000 由
00000

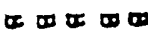




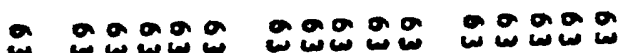

00000

00000

unu un un

$\operatorname{lng} 0$ orogor $\cos 2$ o $0 N \pi N$ uncou un or or or o ununa NNNNN

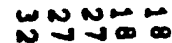

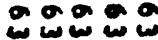
con un un NNNNN C cavan
운둔 avuca on an oviváa onga a 00 o مَّم

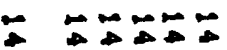 \\ - "⿻ \\ - unucó \\ N NNNNN}

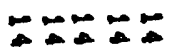

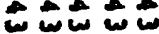
cucu un ס $\because \div \div=$

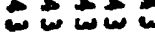
con ung

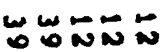

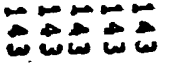

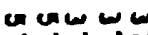

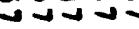

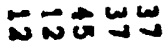

$\infty=\infty-\infty$ $\omega \approx \approx \omega \omega$ w w w w w w w w

(2) UN⿱N口Nบ

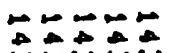
ט

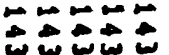

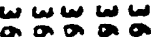
NNN WN每范 - OOD- MNNNN

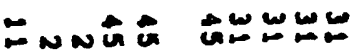

a 050 wㄷำ Uñun

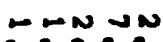
nUnON

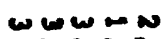

noon N우웅

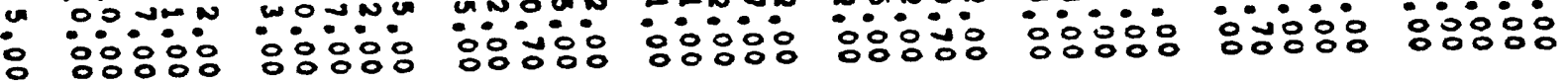
$\frac{6}{8}$

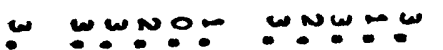

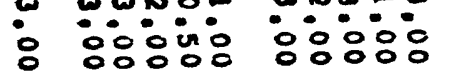

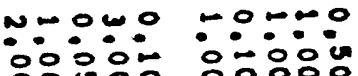

w0:0 우요 용융ㅇㅇ 000
$0: 00: 0$ NONOW :OON: $-\infty-\infty w$ 뭉웅

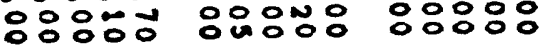

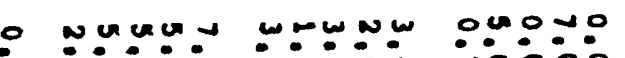

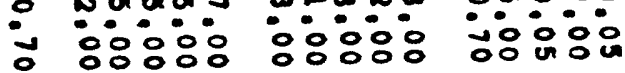
wom:0 영웅

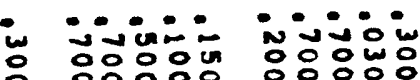
영영영 양녕영

in-0; 능우운 웅영잉응

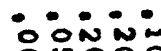

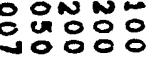

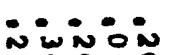
능ํำ 응응녕응 $\because: 000$

NONON

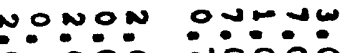
N:? 영영웅 응웅웅응

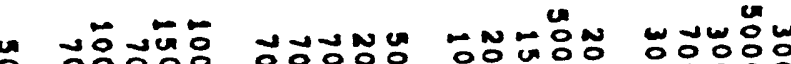

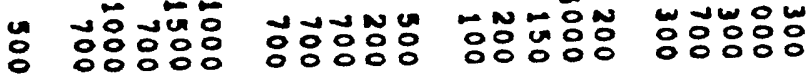
ค
W

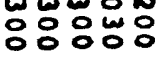
웅용 너옹응 00000 a

in univis

00000

$z z z z z$
- 00000 inivin in univin o iniviog $z \geq z \geq z$
0000잉 inuvin $z z z z z$
00ㅇ응 $z z z z$ inivin
응ㅇㅇ

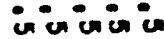
$z z z z$
00000 uviue $z z z z z$

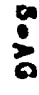

กับกับั

NNNNN 응ㅇㅇㅇ NONONON 응응

NNNNN 응응융
NNNNN

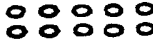

$z z z z z$
NNNNN 응잉ㅇㅇㅇㅇㅇ $z z z z=$
NNNNN

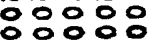

$z \geq z x z$ 
-

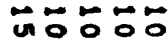

555
ேㅜ웅

5

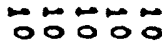
- एक्ट
두융 TELEE
जேㅜㅇㅇㅠ ए5
둥ㅇㅇ ОООООО EL TE TEEE

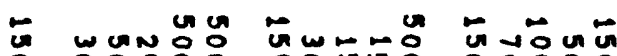

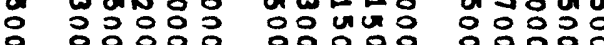

a

$\Omega$

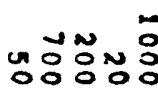
$z$

-

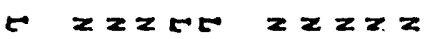

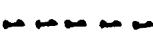

- $c \geq 2$

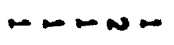

ח מ

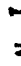

2

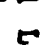

2

n w

융유융

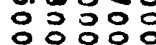

웅 응융유

$\div$ 0우웅 둥ํㅇ 두웅웅

$z \quad z z z z$

$z z z z z$

$z z z z x$

ேㅜ웅

ேㅜㅇㅠ

ேㅠㅇㅠ

$z z z z$

r $\geq 2 \geq 2$

$z z z z z$

후웅

두웅

$z \geq z z z$

$2 z 22 z$

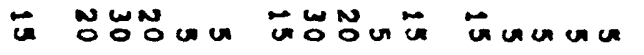

unutina

un un un un

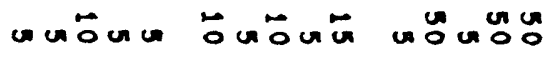

5

to ze zet

C. E

25

C $25 z$

: ैㅜㅇ융

Nonn

$\ddot{0} \div \overrightarrow{0}$

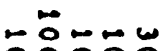

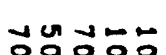

$z$

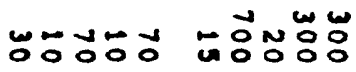

$\mathfrak{d}_{\substack{\infty \\ 0}}^{\infty}$

z z

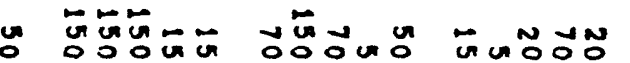
5

uö un un

r t TEEt

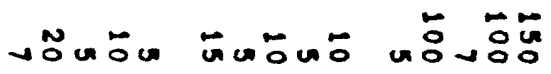

$\stackrel{a}{2}$

c 5 z z z

NNNNN

$z$

$z \geq \geq z z$

NONONO

$z x$

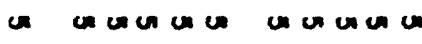

$z z z z z \quad z z z z z$

unu un

$z z z z z$

ununu

$z x z z z$
NNNNN

$z \geq z z$

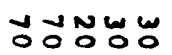

NNOUNOO WNNONO

$z x z$

$z 2 z z$ a

$:$

竞

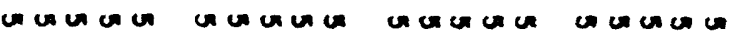

0
0
0
0

N ONONONO NONONONO ONONONO

NONON

NNONON

NONNON

NONNN

OOONN

$z \quad z \geq z \geq z$

$2 x \geq 2 x$

$z z z z z$

$2 \geq 250$

$z \geq 2 z z$

CLETE

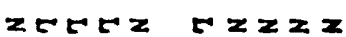

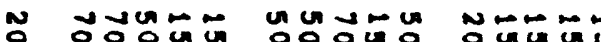

जैuvin$$
\text { c } 5
$$

uñü

r 5 ö̈ unu co

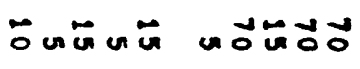

$r$ c

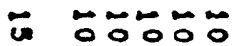
$5 \quad 5$

유융

50000 $+5 z$
00000 CELE
ज: :

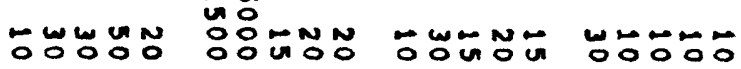

$z \geq z 5$

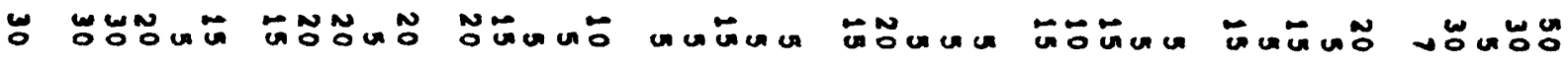
5 
-

z $z z z z$

$\div-1$

$20 \div 0$

-

$z=2 z=$

$=2$

$z z z \quad z$

-

e $-N N N$

N-wo

BOF

o $00 \% 0 \%$

$::: 8:$
00000
-50000
$2 x=2$

-

융후웅

$z=z z$
둥후웅 $z 2 z 2 x$
둥후웅

c $2 \geq z=$

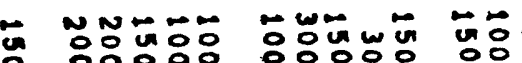

unumir

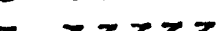

unumen

con unu un

000

$<\geq=2$

anugu

$z \geq z \geq z$

ựug

ำํํㅇํำ

$z \geq 2 x z$

$z z z z z$

ํํㅇํํำดั

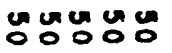

$z z z x z \quad x z z z z$

O유유

$\div 0 \div 00$

5

5

c

NONONO 응융응

NNNNN 응ㅇㅇㅇㅇㅇㅇ

$z \geq 2 z 2$

nO№n

$z \geq z \geq z$

$z z z z z$ $z=z z$

$\div \div \div$

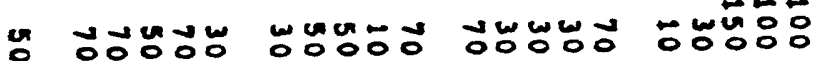
z

- 웅웅ㅇㅇㅇㅇㅇㅇ 운운운

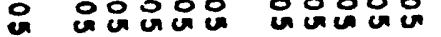

$z z z z z z \quad z z z z z$

$z z z z=$ iinio: $z z \geq z$ iㅜㅇ뭉요 $z z \geq z z$ sinciois: $z=z z$
웅ㅇㅇㅇㅇㅇㅇ

$z=z z z$

오웅우웅

$z \geq z \geq z$

영 웅유 $\infty .0000$

- 00000 10000 i:o:o

$$
\text { io } 0 \text { z } z
$$

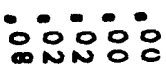
$\geq \geq 00$
웅융웅 -
웅영웅웅 $\infty 0000$
- 0.0 응ㅇㅇㅇㅇㅇㅇ - 0 का

웅융융요 由 2 E

:

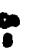
$\underset{d}{\infty}$ 


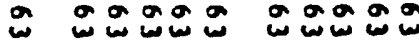

is

0

is a

a

acoso

$-\infty-\infty$

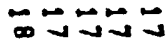

0

NWw口

ง v No

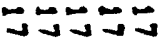

J־

00000

w

JoOJ

๘

NNNNN

NNNA:

00000

it $\rightarrow$ in

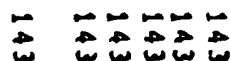

w

un anun un

$\because$ - $\underset{\omega \rightarrow \infty}{\rightarrow \infty}+\infty$

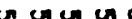

INN N O O

कू पूर्व

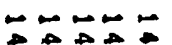

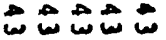

can un un

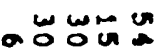

$\sum_{i=\infty} \rightarrow \infty \rightarrow$

cunum un

N $\underset{\infty}{\infty}+\infty$

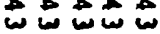
unumu

$\rightarrow N N N N$

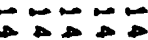
山屯 cun un a

NNNNN $\underset{\infty}{N N N}$

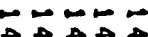
w $\operatorname{lum}_{0} u \in$ NNNUG $\therefore N$ N

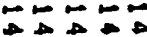

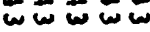
un un uno U. $\infty \infty \cdots$
-

-

$\overrightarrow{0} 00800$ 영ㅇㅇ 00000
M NOO N

n. 잉ㅇㅇㅇ

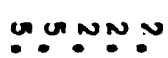
영ㅇㅇㅇ ăcuca

i0:0i 웅ㅇㅇㅇㅇ

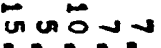
O 웅ㅇㅇㅇ $\div \div \div \div$

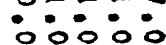
웅ㅇㅇㅇㅇㅛ

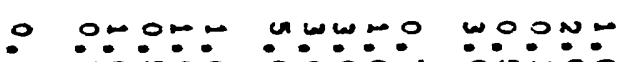

등ㅇㅇㅇㅇㅇㅇㅇㅇㅇㅇ 융영 nut o잉ㅇㅇㅇ w: 웅용 W:- N:-:ㅇㅇㅇㅇㅇㅇ
DOONN iniOOOO ON OROOO
wOMON

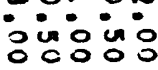

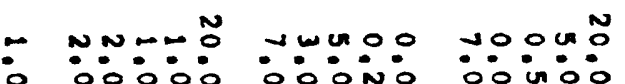

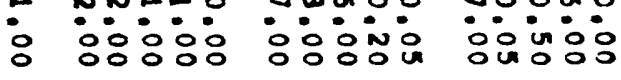
$\Omega$ w 1000

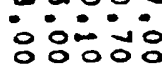

N.

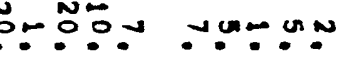
ㅇㅇㅇㅇㅇㅇㅇㅇㅇ un w w w $\therefore \therefore \circ$

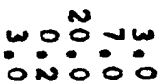
응용ㅇㅇㅇ

- Niñir jun un unimi n:Din

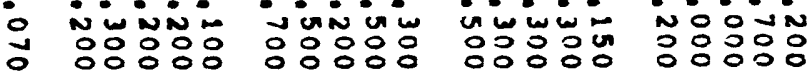

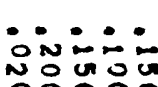

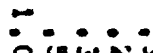

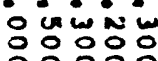
$\Omega$

$\Omega$

요용요

نே:-

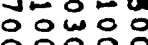
is

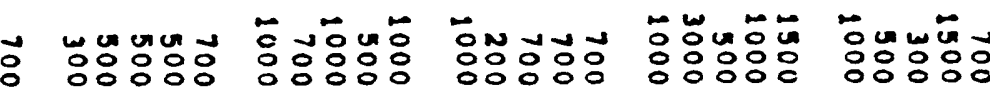

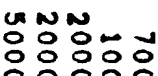

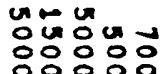

웅둥웅잉 a $\mathbf{0}$

$\because 0000000000$

in iniving in in in in

00000 in un un

$z \quad z z z z$ $x \geq z 2 z$ $z z z z$
00000 univiu $z x \geq z z$
00000 in unin 00000 $z \geq z z$
0000 00000
univin inivivi
$z z z z z y z z z$

:
N

$z$
NNNNN ㅇㅇㅇㅇㅇㅇㅇㅇ NNNNN OOOOOO $z z z z z \quad z z z z$
OOOON 응응ㅇㅇㅇㅇㅇㅇ $z z z z$
OONNN ㅇㅇㅇㅇㅇㅇㅇㅠ $z z z z \quad z z z z$ 


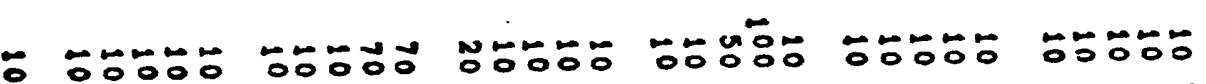

05050

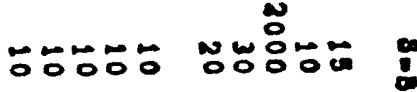

C TrEt ED

CE

5

5 TE

NET

5

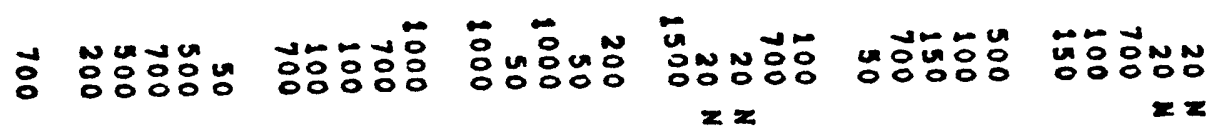

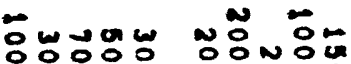

영융융

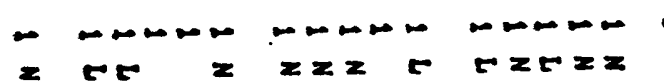

$\infty-\infty+\infty-\infty$

$\infty-\infty+\infty$

FEnz=

$\geq 2+2=\mathbb{E}$

$2 x \quad \mathrm{x}$

$\because \div 0 \div$

-

$\div 5 \%$

$\because \div \div \div$

$\because 50 \% 0 \div$

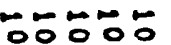

مே0\%ڤ

두ㅇㅠㅜㅇ

$z \geq 2 \geq 2$

$\varliminf_{\infty}^{\infty}$

$x=2 x \geq z$

$z \geq z \geq z$

$2 z 22 z$

22222

$z=2 z 2$

$z \geq z \geq z$

$\underset{0}{0}$

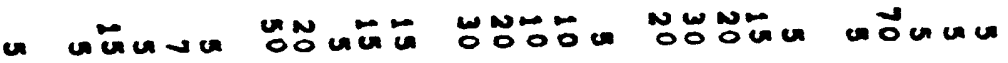

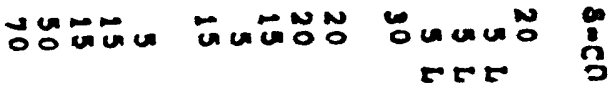
z

- $C$ C

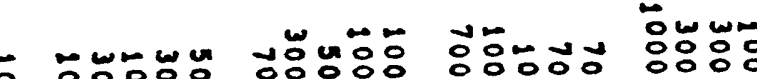

- $=0000$ 00000

W OOOOㅇ OOO유 5

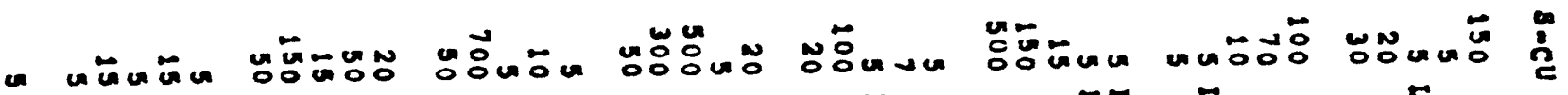
r $c$

$\leftarrow$

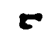

5

$\leftarrow 5$

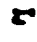

5

N NOOOOONON

NNNO응

W้ํํำ:

NMNN N

NNNNN

Nowno

NWNNN

NONNN $z z z$

$z=$

$z=\mathbf{z}=$

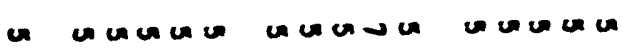

$z=2 \geq 2 x$

$z=z \geq z$
NONOOON

$z=z \quad r$
NONOOO ONONONO

CECEX $x \geq 2 z z$
NNNONO

Zr $2 \geq 1$ aruan

$z z z z z$ anuar

$z \geq \geq z z$ unuer

$z \geq z \geq z$

ONNONO

$x$ IE⿺

$\angle E \geq Z 2$

$z \geq z \geq z$

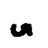

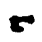

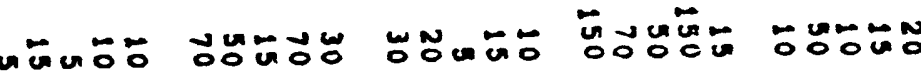

융ำ on $\tau$

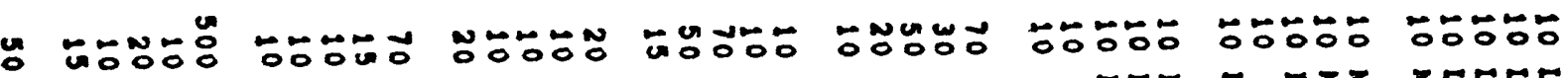
to

$z$

$\leftarrow 5$

एE

F trz zeter

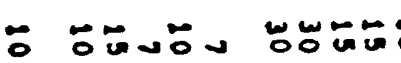

W№

Nowoñ uñuna

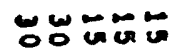

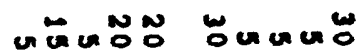
$z$

$z$

0
$\vdots$
0 


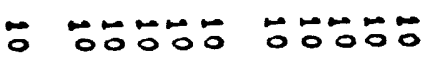

$z \quad z \geq x z$

$z \geq z z$
00000

$z \geq z=2$
무융

$z \geq z 2 z$
ํํㅇํํ웅

$z x z z$
둥응ㅇํ

$z z z z z$
Nㅇ융

ேㅠㅇㅠ

$z \quad z z z \quad z z z z z$

w w م ㅇㅇㅇㅇㅇㅇㅇㅇㅇ $z z$

ํㅜㅇํㅇ

룽ำ

تNم

n U

ผำํํㅇ z z

$z$

$=$

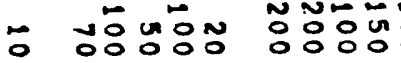

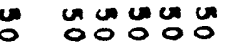

z $z z z z z$

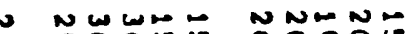

cun un un

$z z z z z$

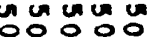

$z \geq z z z$ जuñon

$z=z z z$ जuñon

$z z z z$ avuno:

$z z z z z$

س م nunura

00000

$z z z z z$

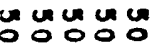

$z x z z z$

ONONN 5

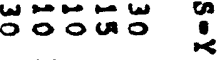
tr

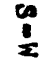

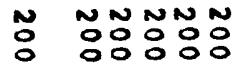

NNN NON

NNNNN

nNNGN

nNNNN

กNNNN NNNNN ㅇㅇㅇㅇㅇㅇㅇㅇㅇ $z=z$ zzzz

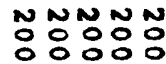
$z x \geq z z$ - $z$

궁ํㅇำ

ํํㅇํำ $\overrightarrow{0}$ 잉ㅇㅇ

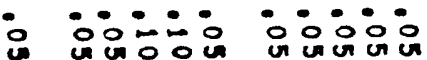
$z \quad z z$

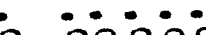

응용요웅

$\infty$ 용요
운웅웅ㅇ

$z z \geq z 2$
운웅웅요 $z z=2$ io io: $z \geq z z z$
웅ㅇㅇㅇㅇ

जư un

$z z 2 z 2$
웅ㅇㅇ웅

$z \geq \geq \geq z$

웅ㅇㅇㅇ

$z z z z z$

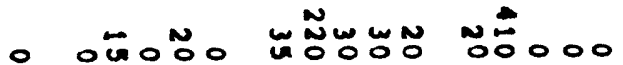
- 0 \%
용ㅇㅇㅇㅇㅇ $z \infty 00$
응ํํ유 (1)
웅ㅇㅇㅇㅇㅇㅇ 100
응융ㅇㅇ $z$ का

응ㅇㅇㅇㅇㅇ $z \geq \infty \times$

$\div 0 \div 00$

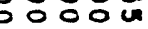
由ण口

- 0000000000

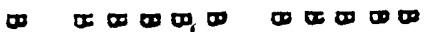

00000 யण口心
00000

00000

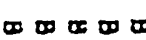

WN

WONONO or

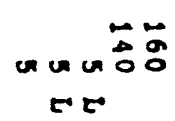

00000 뚜
00000 따
00000 - 0000

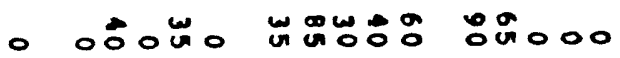
- 0 0

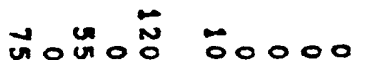

Uू. wo $\infty \infty$

Nั0\% - 0000 


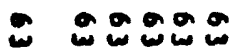

$\because \approx \approx \approx 00$ no
운 cuca un

$\rightarrow+\infty-$
운 on unoro -

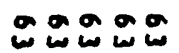
cúcucu

กN $\cos$
00009

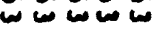
sing o o vinu

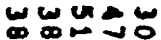

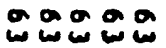
crucucor unun

운 0900 cun un NNNNO

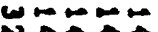
$\rightarrow+\infty \omega$

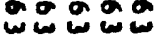
uncu ONNNN WN

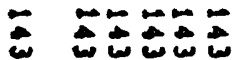 \\ o vo \\ م}

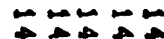

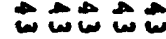
$\sim N N N N$ nNN un ONOOONOAN

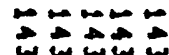
N N NNN -NNNN Q

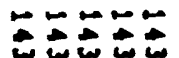
NNNNN NNNWN

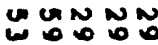

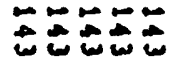
U UNN NN G W UN w एक
WE= NNN UaNáa

$\because \underset{\infty}{\infty}$

ca can a

ש مس مس س

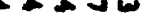

$\vec{\omega}=\vec{\omega} \vec{\omega}$ un a cua 車 J w $\begin{array}{lll}0 & 0 & 0 \\ 0 & 0 & 0\end{array}$

- unna ounnu

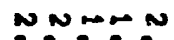

00000

눈

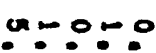

우웅

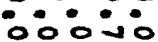

엉요

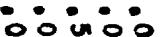

एே:

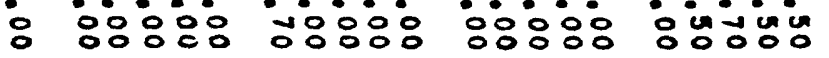

영영뭉

n

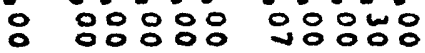

0000-

inivin: ỡ
-0000

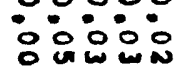

wa: $\therefore 0$ 웅잉잉 $\div 0000$ OOO
$0-0 \cdots 2$

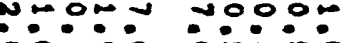

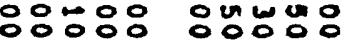

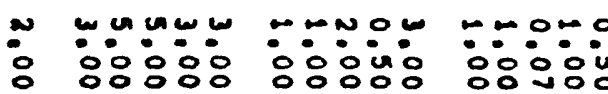

no 000

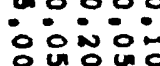

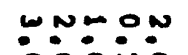

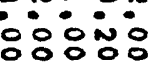

No0:0 ONN两每 w口un in:00 영영영 um oivio

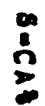
0000

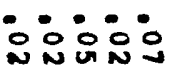
ON ONOA

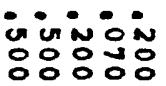

눙우응요

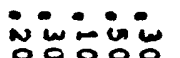
응용ㅇㅇ

UNiNin MNN N N N OOOO:

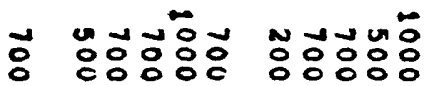

unumu ư
뭉유응ㅁㅇ

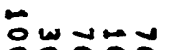
ㅇㅇㅇㅇㅇㅇㅇㅇ

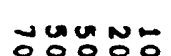
응ㅇㅇㅇㅇㅇㅇ $\rightarrow+60$

로으웅ㅇㅇㅇㅇㅇ
융주영 응ㅇㅇㅇㅇㅠ

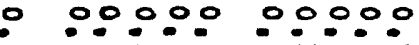
in ivivin $z \quad z z z z$
00000 inuviv $z x z z$ ú:00 o0:00 in inin in 웅응 $z z \quad z \quad z z z z$ $z \geq z \geq z$ $z z z z$ inivin $z \geq z \geq z$

:

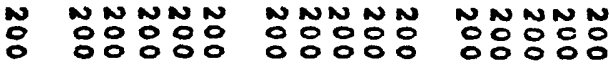

$z \quad x z z z z \quad z z z z$ $z z z z$

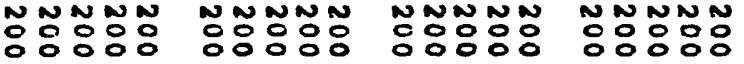

$z z z z z \quad z z z z \quad z z z z z \quad z z z z \quad z z z z$ 


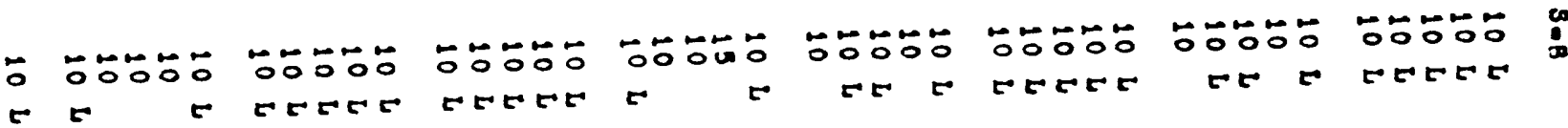

N

מחNN

Nルールー -

우융

$z \quad z 2 z 2 z$

ธேธㅇㅇำ

$x \geq z \geq$

$\because \div \div \div$

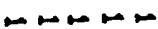

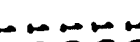

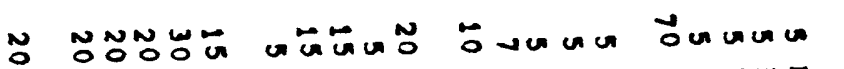

कैजu uno $z=$

$z z$ $r=$

$z \geq z$

Ni⿱

$\underset{\substack{n u \cos \\ z=}}{0}$

$x$

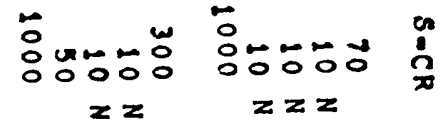

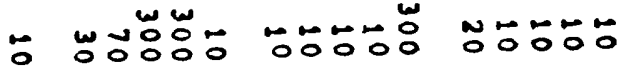

م.

응으융 $z z=z$ $\div: 0$

EZEF

\section{0} $z z z$
-50 $z z x z$

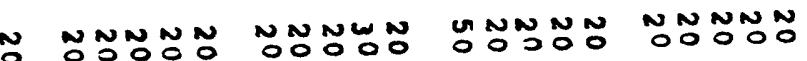
$z \quad z z z z$

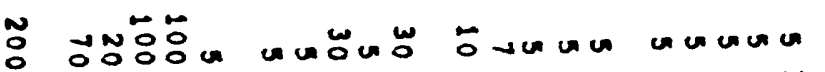
$r F t$

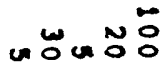
$r$ r Öunuon

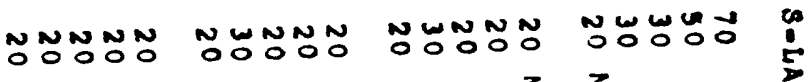
$z z$

$z z$

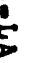

uncula

un unu

unuun

un un in

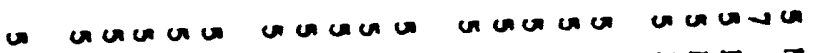
$z \geq z \geq z$

$z z z z z \quad z z z z$

$z z z z$

:

N NOOOOOO OOOOOOO

ONOOOOO NOOOOON

NNONONN

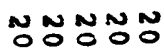

NNNOOOOO NNOOOOOOOOO

:

$z \quad z 2 z Z$

$z z 2 z 2$

FZZZZZZZZ

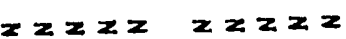

$Z Z Z Z Z Z Z Z Z$

No

Oununue

용

r.

cere

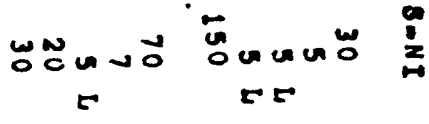

$\boldsymbol{c}$

TEC

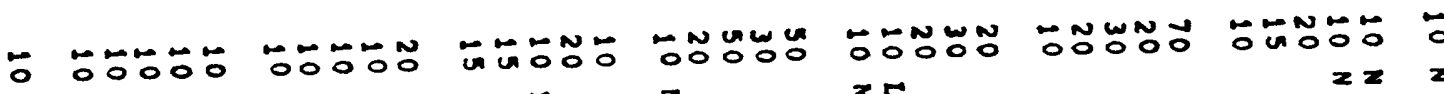

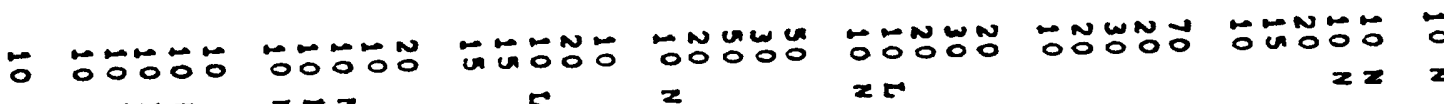

- OOOOOOO

r ICE 202

5

2

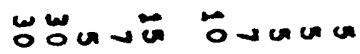

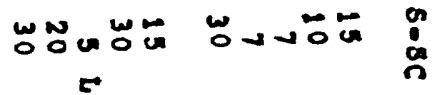




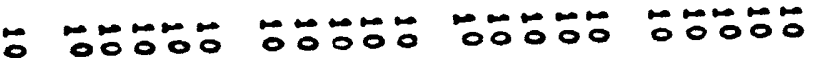

- $z=z z z$

$\geq \geq \geq=2$

$z x z x z$

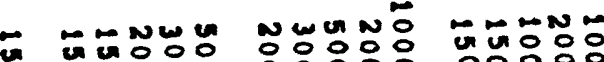

- $00 \%$ 유
둥ํ

$z x z 2 x \quad z z x z z$ $\because \div \div \div$

$x \geq z \geq z \geq z z_{2}=2$
두웅웅

$z z z z$
aN.

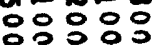
$=2$

$$
5
$$

둥후웅 trz $z$

Nㅜ융

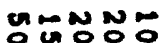<smiles></smiles>
능 웅융영

- caunu un - 00000

$z=x \geq z z$

unung

$z z z z z$

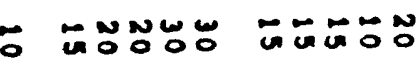

:

*

닝ㅎㅇㅇㅇㅇ

Oㅇㅇㅁㅇㅇㅁ
UUN UnN 年0
두유 CEET
융융유 으ㅇㅠㅜㅇㅠ c
2

두웅ㅇㅇㅇ

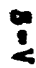

c

웅잉영

$z \geq z z z \quad z z z z z$

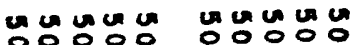

unnug nunua 00000

$z x z z z$

$z \geq z \geq z$

$z z z z z$

$z z z z z$

wan w

HUNE GNNNN

0
$\vdots$
$\vdots$
0
z
- 웅우웅

웅ㅇㅇㅇㅇㅇ 웅우 웅ㅇㅇㅇㅇ 웅웅

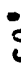

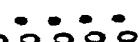

:

$z \quad z z z z$

$z z z z z$

$z \geq 2 \geq 5$

$z \geq z \geq z$

$z z z z z$

NNNNN

NNNNN 응ㅇㅇㅇㅇㅛ 00000

$z z E z \quad z z z z$

i

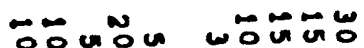
영영영영영붕응

:

영영웅

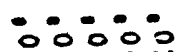

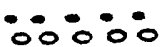

-

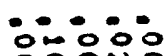

웅웅 $000 \mathrm{O}$ zo⿴囗十 w $=$

$z$

- $\infty 20$

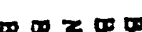

00000 由ण口z

$\pm \infty$

- $000 \% 0$

no 006

-0000 눙ํ․ o 0 u 00000 $0000 \%$ 2

-

- 10

(1)

100

00000 ம

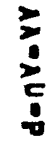

- 00000 -
00000 由ण西四
00000 00000
00000 10000
00000 $\infty 000$
00000 따 00
00000 -
00000 ம000
5000 由 wo o o: O

$$
\infty 000
$$

O0000 00000 - 0 -
00000 600 00
\& 0000 - 
a) a cas a

u unum un

u WN W N U U U U UNA onago

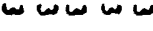
$00 \hat{0} \vec{\infty}$ $\rightarrow \leftrightarrow N$ U

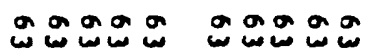

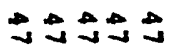

Uి

$\rightarrow-\infty$
ט.


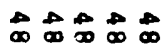

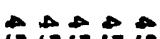

a con

D U Uun

ANNA

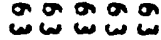

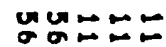

Buna.

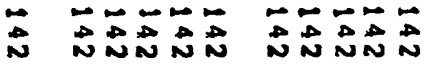

a ununuen

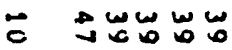

$\rightarrow \infty \rightarrow$

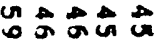

$\rightarrow \rightarrow \infty \rightarrow$ $\vec{W} \vec{N} \vec{N}$

U.

س

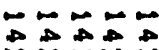
NNNN ט

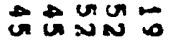

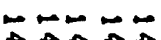
NNNNA

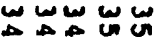

-NNN $\rightarrow \sim \infty$ NANA芯 W unu unu

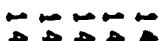
NANA心 ه U⿺辶一兀
Dم स屯心 - nun un का N
- OONnm omoun nonon

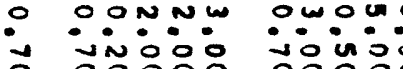

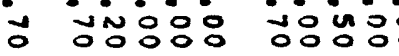

응용요 un vam 웅ㅇㅇㅇ응 wur un ioinin 응영잉 $\ln u n$ :0i்

م०००० 웅ํㅇำ

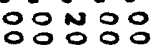

$\rightarrow \because n=0$ 응영영 $\therefore$ OOOD:O

ODONN

n.․․ㅇㅇㅇ

m mo:

ㅇㅇㅇ영영
Non:0 읭ㅇㅇㅇㅇ

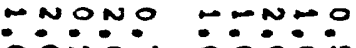

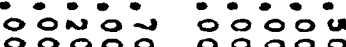
응영영
-NONn ㅇㅇㅇ웅 응 용응
NNONO ionin OOU용
- oouton zann unno:

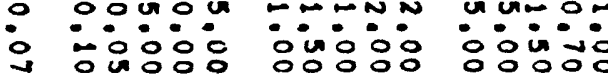

mONN:

엉응ㅇㅇㅇ
NUON-

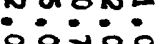
ㅇㅇㅇ뎡요
UNกั0!

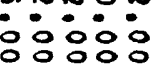

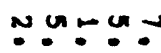
웅ㅇㅇㅇㅇㅇ
N M N N 융응

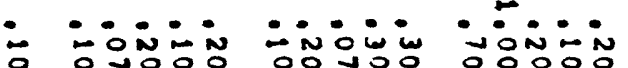

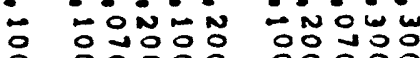
응ㅇㅇㅇㅇㅇㅇㅇㅇ ninin nininin

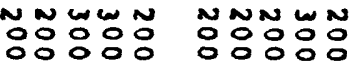

inis WO Ni⿱宀⿻三丨口
Win:

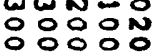

$\underset{0}{a}$

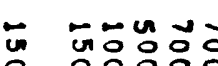
둥응응
응요유

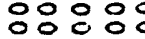

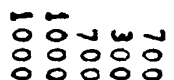

두용 ㅇㅇㅇㅇㅇㅇㅇㅇㅇㅇㅇㅇ

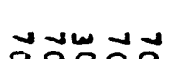
궁ㅁㅇㅇㅇㅇㅇㅇㅇㅇㅇ 궁응 둥융ㅇㅇㅇ 응뭉ㅇㅇㅇㅇㅇㅇㅇ
- 00000

in in uninis

$z \quad z z z \geq z$
00000 inivion $z \geq z \geq z$
웅요 univin 000?0 a vin in 00000 univin

00000 in in in in

00000 in in in $x \geq \geq \geq$
00000 inivini $z \geq z z=$
NNNNN

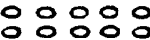

$z \quad z z$

NONNN ㅇㅇㅇㅇㅇㅇ응 


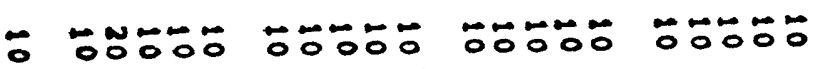

ㅜㅇㅇㅠ

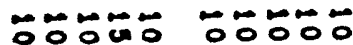

ㅜㅜ웅

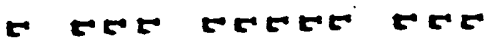

TEETE

tetCE

जE 5 C

EE 5

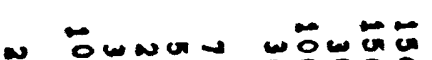

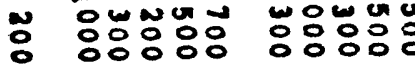

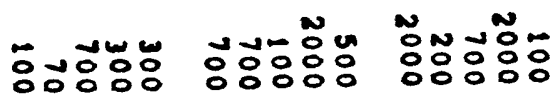

م:

जO융

ㅜㅜㄷㅜ

웅

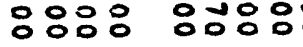

$=2$

잉ํㅇํㅇ

ㅇㅇㅇㅇㅇㅇㅇ

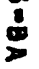

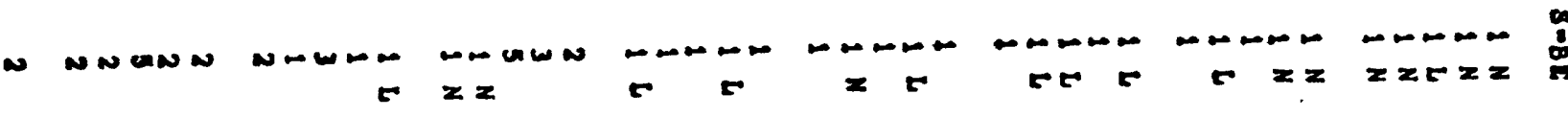

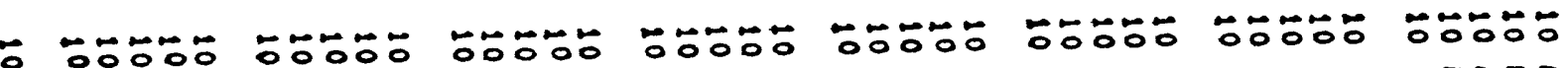

$z \geq z=2$

$z=2 x z$

$z \geq z 2 x$

$z z z z z \quad z z z z z \quad z z z z=$

$\underset{4}{6}$

$z \quad 2 x 22 x$

$2 z 5 \times 2$

$2=22$

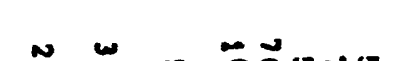

a com

cöuำ

sonus

r

든

\title{
- w w
}

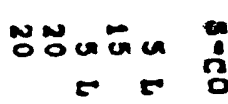

$z=2$ $=z$

$z$

2

$z$

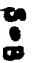

\section{.}

-

- ㅎํㅇํㅇ

$z z z$

웅우

$z=$

ํํ융

․ㅜㅇㅛ

$$
=z
$$

$00000=000$
5E $=1$

O 뭉유유

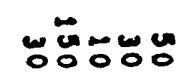

$=$

늉융유

$+2$

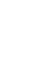

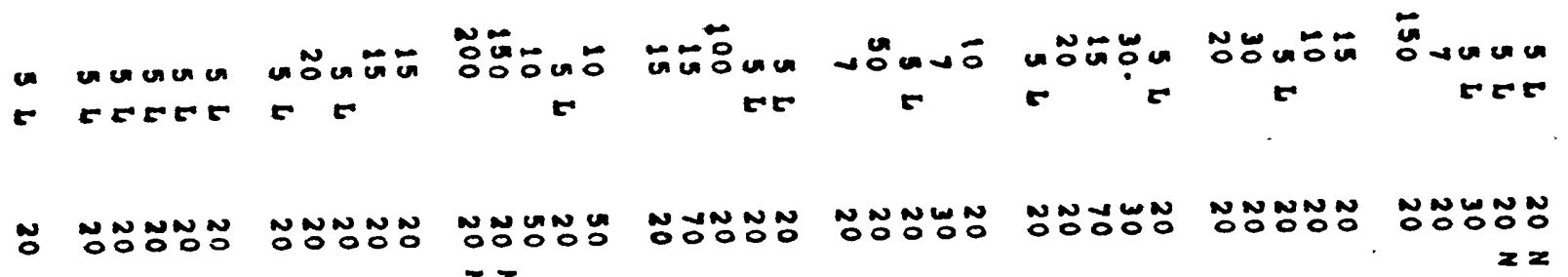
$x=$

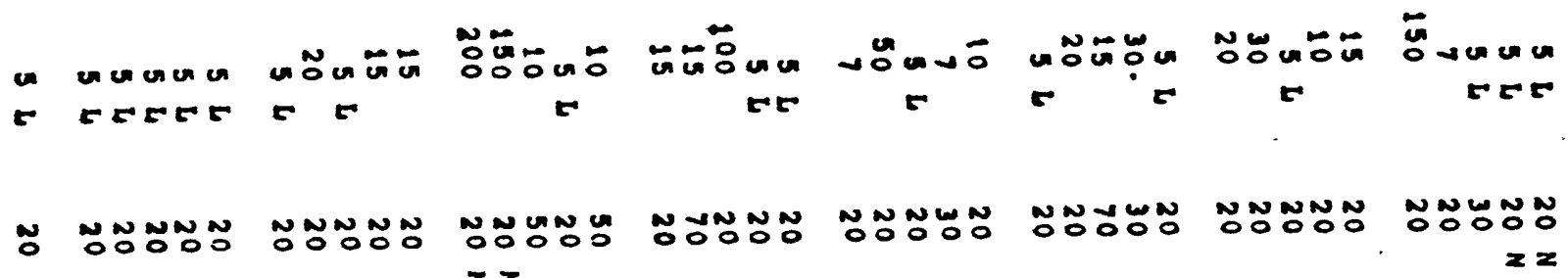
22

2

a cancuan

unciou unuca

$z z z z z$

$z z z z z$

$z \geq z \geq z$

unu un

anconos

$z \geq z z z$

$z z \geq z z$

$z z z z z \quad z z z z z \quad z z z z z$

:

O

Nㅜㅇㅇㅛ

NNNNO

NONONO

NONOON

NNNNO

$z z z z z$

$\geq x \geq 2 x$

$z \geq z \geq z$

NNNNN

NONON

$z z z z z$

$z z z z 2 \quad 2 z 2 z z$

a MUCOO

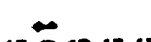

कैơuñ

용

- 5

$r 5$

5

ज०ैuñ

W.OOڤ

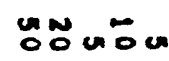

5

c

5

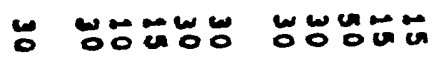

웅유

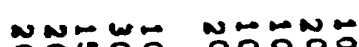

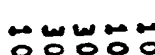

둥ํㅠㅇㅠ

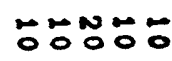

$r=$

r $x$

$z z z z$

$x$ re

:

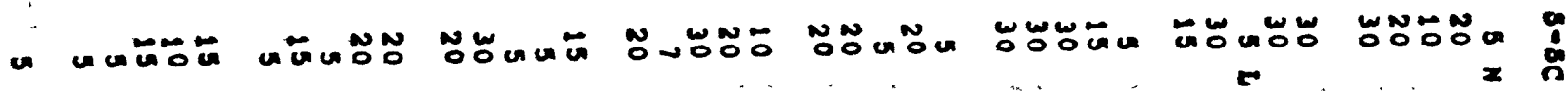




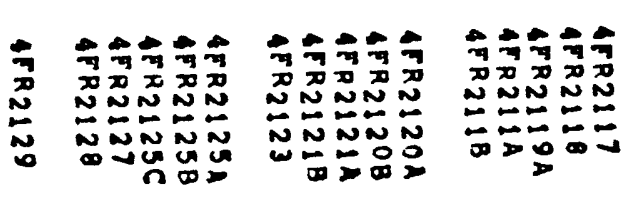

योग

NNNNN

$\angle \div \div \div=$

is

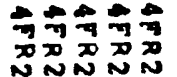

NNNN

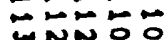

Nㅓㅇ요용

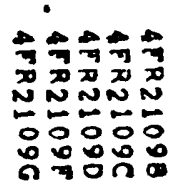

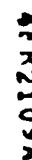

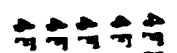

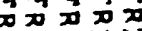

NNNNN

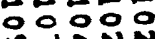

웅요언

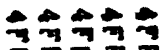

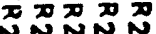

둥ㅎㅇ응

点
- Бேே00

0 00000

$x \quad x \geq x z$
00000

$z x=2 x$
후융우

$x<2 z$
두요 00000

zx $x \geq 2$
우웅

$x \geq 2 x x$

wㄷㅇㅇㅠ

후웅웅

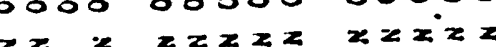

\section{\&}

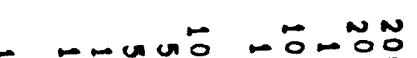

C 5

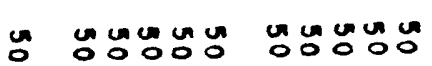

ํํㅇํํㅇํㅇ

$z z \geq z z$

$z z z z z$

$z z z z$

nugunou

$z \geq z z z$

unumu

00000

unungu o0000

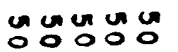

$z z z z z$

z $z \geq z 2$

जuñun

$z x \geq 2 z$

W NNONON OัO

NWNOT

nucan-

OOOOU

$\because \sim N O$

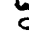

סת משי م000

n nnnnn nunnn

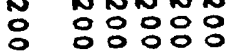

ㅇํㅇํำ

NNNNN

응ㅇㅇㅇㅇㅇㅇ

NNNNN

ㅇㅇㅇㅇㅇㅇㅇㅇ

NNNNN

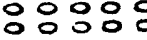

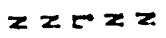

$z z z z$

ํㅡㅇํำ

$z z z z$

NNNNNN

z $z=2 z$

$2 \quad 22222$

ว

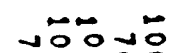

r.0.0

응ㅇㅇㅇㅇ

- W-N WER

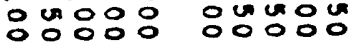

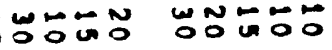

5

tr $x$

OOO 0 O

CE 2 Z

ํㅜㅇำ w으무

봉중ㅇㅇㅇㅇㅇㅇ

$z z$

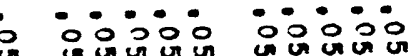

언은응

웅ㅇ

웅ㅇㅇ

00000

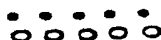
unvu 은은요 unuvin $z z z z z$

$z \geq z \geq z$

웅웅 r $2 \geq 2$

$z$ z $z 2$

$z \quad z z z z z$

CECEF $22 z 25$

$2 z 2 z$

$x \geq \geq z z$

ì

- 응ㅇㅇ 영유

웅우 NNOON W 10

$\infty$ வ

$\div 0000$ -OONO

$\circ \circ 000$ ம

$2 \infty \infty 20$

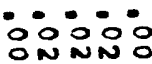

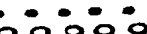

由 $222 \infty$

$\infty 2200$

웅웅웅

ம $\infty \mathbf{\infty}$
No

- $000 \mathrm{0} 000000$

-
00000 1000
00000

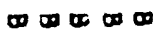

00000 由
$000 \% \overline{0}$ $\infty \infty$ w

0000 $\infty \infty \infty$
- 0000000000

-
00000

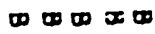

00000 ద $\infty \boldsymbol{\infty}$
00000 ॠण
00000

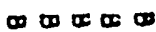

00000

10000

00000 ம $匚 000$
3

?

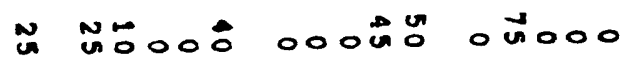
$\varpi$
No $0: 0$ $\infty \infty$
Uno 000 $\infty 0 \infty$

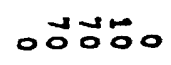
岗, 00 $\infty$
00000 - 00000 
- 00000

- avúu

20000 a un on a

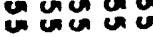

웅aㅇ

a. 0000 encuca ما

acooga ט 00000

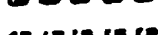

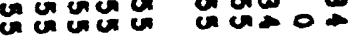
क uUnor NNNN

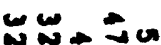
on on:

WW

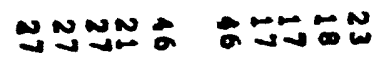

W N
\% un unu

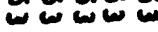
$\therefore=$

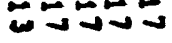

훙aㅇ w 000

م 중

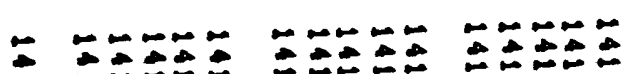

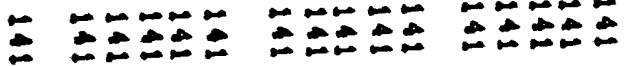

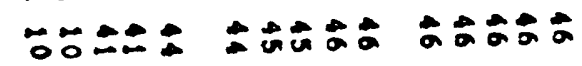

둥 wow us

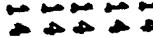

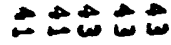
웅

Q

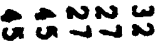

$\because ே ே ே \div$ $-\infty-\infty u$ a is a o

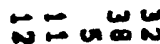

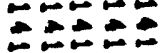
a uncuso un un w in

NM⿻

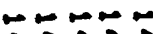

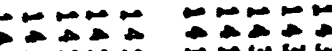
U⿺辶⿻二丨

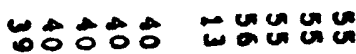

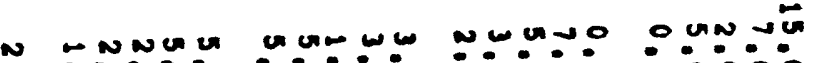

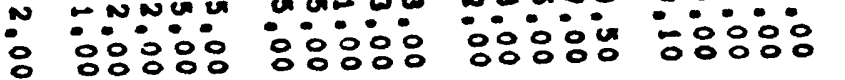

엔 눙유

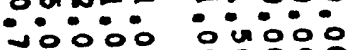
ㅅㅇㅇ영영 잉영영 $\div 000$ $\therefore \circ 0 \%$ 응유요

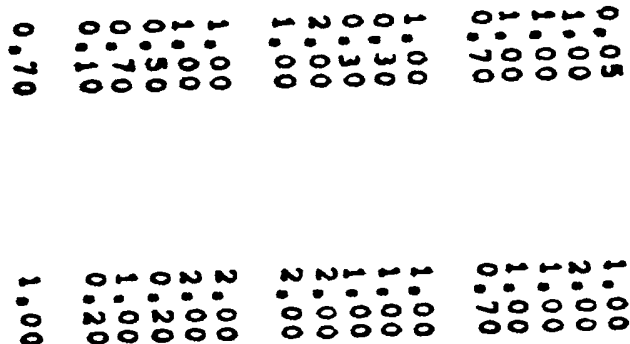

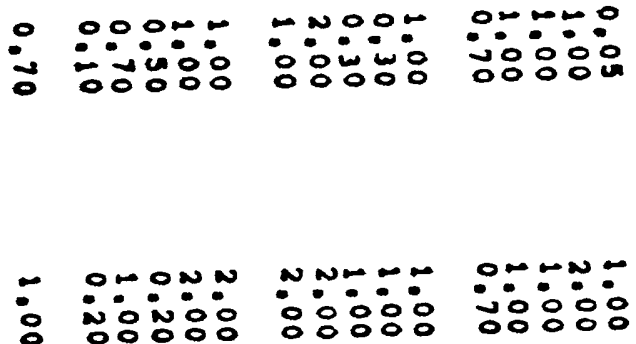

on $n$

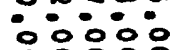
$a$
ONOOO OOONN

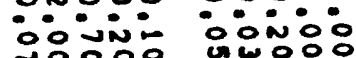

ON NOO iOONO O Oس س 0.00 $\cos ^{0}$ 영승용
O: 붕ㅇㅇㅇㅇ (t)
$0000=$ 은둥ㅇㅇㅇ 5

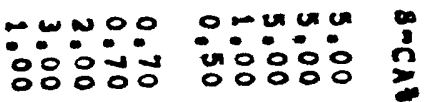

n onjin nimin 응ㅇㅇㅇㅇㅇ 응붕ㅇㅇㅇ

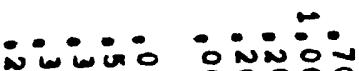
00000

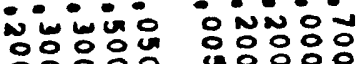

훙융용영

atan 뭉영응용

궁

중융ㅁㅇ

둥

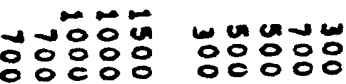
응ㅇㅇㅇ 证证 승융융 응용ㅇㅇㅇ W:눙:

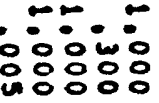

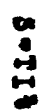
능ㅇㅇㅇㅇㅠ 두잉

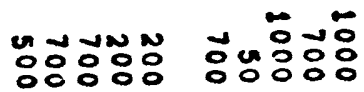

00000 uniun

- inivio iniviu

$z$ $z z \geq z$
00000 ivivin $z z z z=$

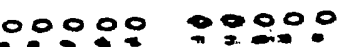
inince $\Rightarrow=0 i n$ $z z z z z y z z z$
0000000000 uninis uninu $z z z z z y z z z$ n nNnNn nNnNn \& 000000 $z z \geq z z$
NNNNN 응ㅇㅇㅇㅇㅇㅇ $z z z z=$

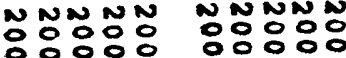
$z z z z z \quad z z z z$
NNNNN :ㅇํㅇำ

$z z z z z$
NNNNN ㅇㅇㅇㅇㅇㅇㅇㅇ 


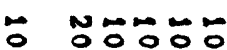

6
응ํㅇำ

che
ํํㅇํำ

CER 20
ㅁํㅇํํㅇํำ
ธัดัดัด

CER
№응ㅇㅇ

$c$ er
드응ㅇ

coter

Nㅇ응

W WMNON

ER TE
NUMnO

$z \quad 20$

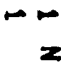

$z$

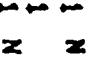

UNN N

UNMR

$z z=$
:

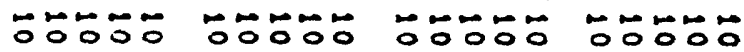
$z z z z z \quad z z x z z \quad z z z z z \quad z z z z$

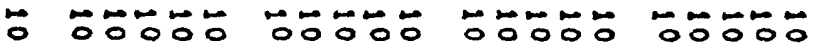

$z z z z z$
$20 \geq 2$

$x \geq 2 \geq$

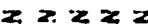

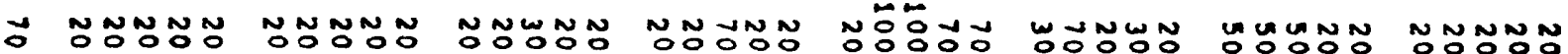
c

5

ए 5

$z \geq z$

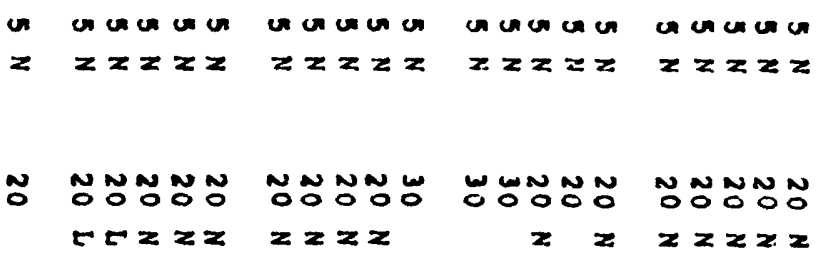

NONON

$z z z z z$
NONONO

$z \geq \geq z 2$
NONOOOOO ONONONO

$z=z z z \quad z z z z x$

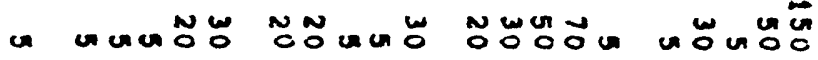
CE

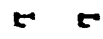

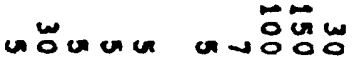

cte un unu un unomo $z z z z z \quad z z z z z$
ํํำำ
○О Nㅠ 5
ONOOO

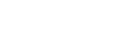

พم⿱宀0⿱一兀)

๐ㅜㅇㅠ

Cㄷz 2

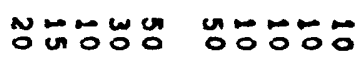
255
0

○ U 


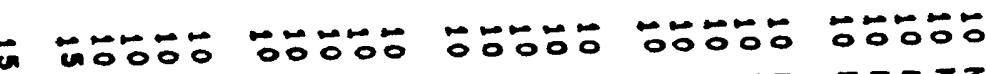

$=\geq 2$

லே0ே0\%

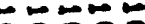

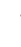

$z x z=x \geq z z x$

ㅜㅜ웅

$z=2 z z$

(2)

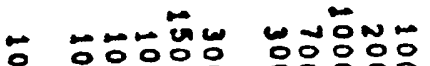

: $8: 8: 8:$

$=2$

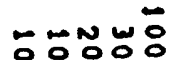
응융융 $z z x$

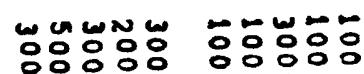
농무융유

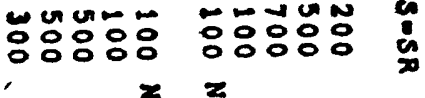

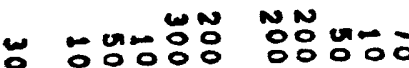
to

\& gungưon

$z=z z z z$

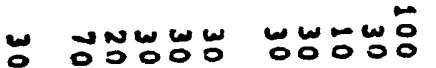
E

n nNNNN 응융용

NNNNN

융ㅇㅇㅇ응

$z=z z z z$

$z z z z$

$z z z z$

:

NNNC

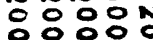

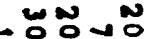
융유융 2

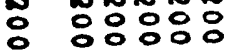

NNNNN
nNNNN nNNNN 응융영응 응유: $z z z z z$
nNNNN

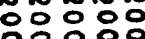
z t

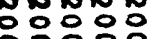
zx $x=$ 영영융응 $z z z z z$

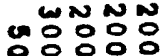

NEN

Nww

응ㅇㅇㅇ영

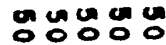

$x z z z z$

NOํํㅇํำ

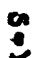

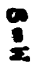
c $z z z z$

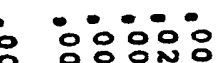

- :000:-

-
웅용ㅇㅇㅇ

웅웅용 Guvion स 522 $z \geq z z z$

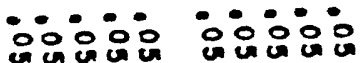
zEzzZ $z=2 z z$
웅웅요 ZCEI2
웅ㅇㅇ Uuunu $z z z z z$

$\therefore \circ 000$

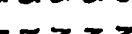

$\therefore 000$ 유용

$\therefore \therefore: \circ$

$\because \because 0$

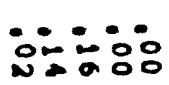

w0

OOOON

-

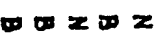

zo $02 z$
2
0.0000 송응유 20002 $\therefore: 00:$ OONDO - $\infty$ -
$000 \% 0$

- 000 Uñ 0000000000

-
60 000 $\infty \omega \infty \boldsymbol{0}$ -
00000 100000
00 ON口 -1006.
00000 - 0000
00 Not范 $\infty$ 


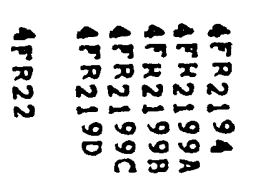

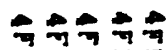

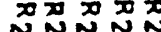

NNNㅗㄴ

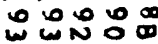
W

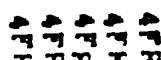

గ

舟

$\infty$
$\infty$
0
0

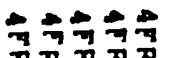

NNNNN

NNNNN

مि0

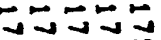

ज००न

>

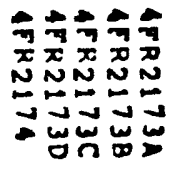

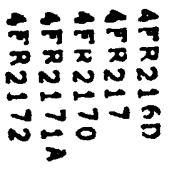

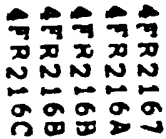

م

웅aㅇ

문

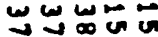

ה

$\leftarrow \infty-$

$\omega n=\omega N$

जos

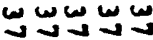

a unu un

눙

no000

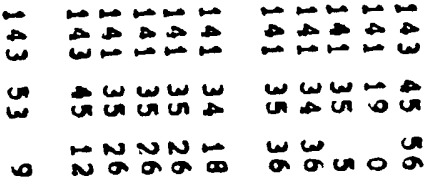

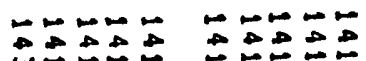

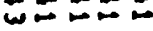

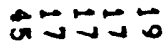

un

م

مَ

a

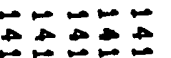

-

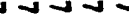

ONNNG $\underset{\infty}{\infty}-\infty$

セカD心

NNNNN

مَ $\stackrel{\infty}{\infty}+\infty$

$N \omega-\infty$

\# N Un

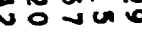

$\rightarrow \infty+\infty$ ш $\omega=$

$\rightarrow \infty \rightarrow \infty$

Nֶ๊

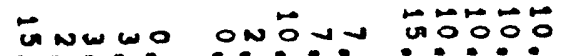

잉 ivino

능 un 잉요 u unwo now:

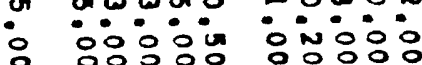

nuuno 웅윤ㄱ

\section{- ㄴ.}

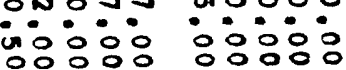
영영

w WNWWO

-

- ON wo

NO은

- NNNO

응으

Non:o

으

Nํํ유

용ㅇㅇㅇ

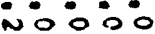

응ㅇㅇㅇㅇㅇㅇ

no

운영영ㅇㅇㅇㅇ

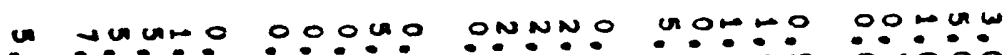

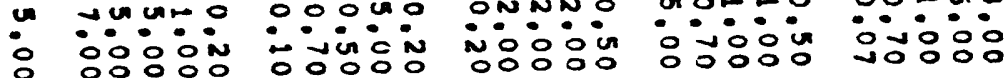

un un : o $\because 000$ Et
U으으

iㅇㅇㅇ

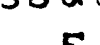

N

W.:-

$\circ \circ 80$ 웅

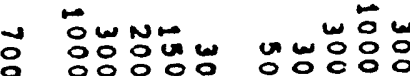
OOOO0:
N

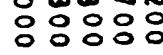
붕ㅇㅇㅇㅇㅇㅇㅇㅇㅇ 붕명ㅇㅇㅇㅇㅇ

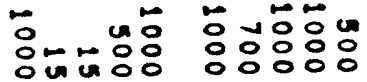

$\frac{0}{3}$ in inivin

$z \quad z z z z$
응으 in un un un

00000 in univin z $z \geq z$
00000 univin un 00000 ininiv

00000 inivivi $z z z z z$ $z z z z x \quad z z z z$
00000 ininin $z \geq z z z$
00:0? univin

$z z z z$
2
N NONONON NOOOOOOOOO

N

$z z z z z \quad z z z z$
nN NNN

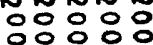

NNnNN nNNNN

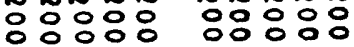
ㅈ z $z z z z z \quad z z z z$ $19 a^{2}$
NNNNN N 응ㅇㅇㅇㅇㅇㅇㅇㅇ $z z z z$
Nㅛ용요

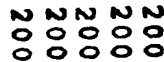

$z z x z z z z z z$ $\sum_{0}^{\infty}$ 


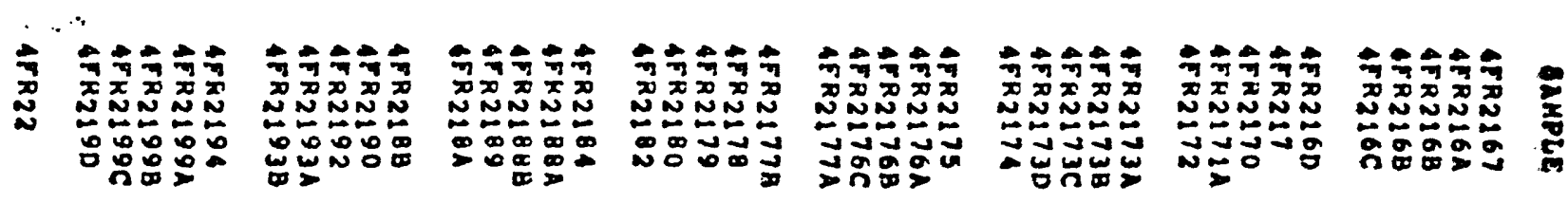

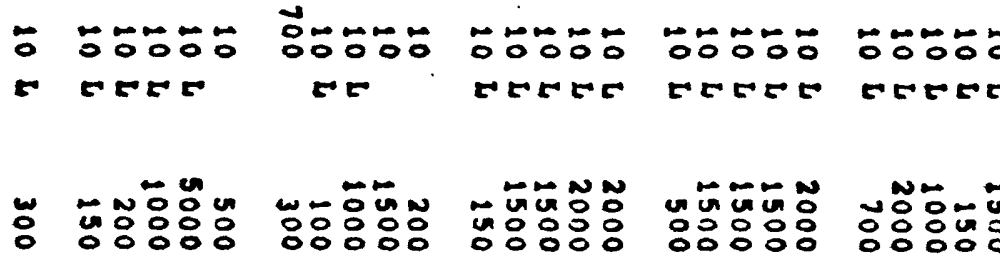

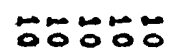

유워

TEET FE TEE

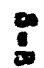

OOOOOOO

W

$x=2 x=$

ㅇㅇㅇㅇㅠ Nㅜㅇㅇㅠ:

$z z z z z$

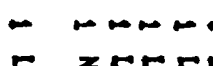

- WNA

ח N M

- n n

$\infty m \infty$

$\infty$

URn

$\underset{2}{0}$

$\because$ 두유

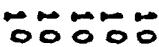

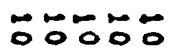

두웅

무융

무융유

ํํㅇํㅇ

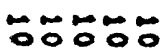

$z \quad z z z x z$

$z \geq 2 z 2$

$z z 2 z 2 \quad z 2 z 2 z$

$z 2222$

$z 2 z 2 z$

$z z z z z \quad z x z x z$

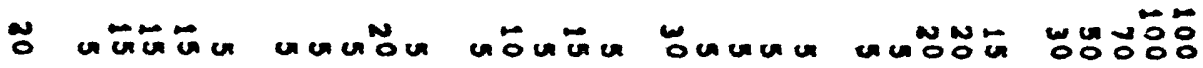
$z=z$

tr $2=5$

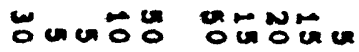
$z z$

unua

$\overrightarrow{0}$

으ㅇㅠㅜ

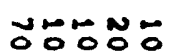

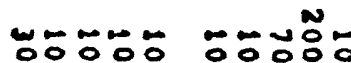
웅ㅇㅇㅇㅇㅇ $z=z$ $z z \geq x \quad x$

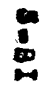

N N NON

NOWW $z z z z 2 z$

a cuncur

$z \quad z z z z$

onucuo

$z \geq z z z$

unumur

$z x \geq z x$

on unu

$z z z z z$

uncuuca

$z z=2 z$

acouna

$x \geq \geq 2 x$

unuaro

$z x \geq \geq z$

unaug

$x z z x z$

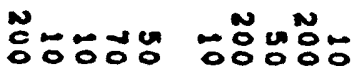
$z z$

응Nㅠㅇ N - $z \quad z x \geq z$
○ NNO융

$z$ ZTER
مNOONO

thex
NONONO

F z z z z
ONONON

ZIE $=2$
ONONON NNNNNO

$z z z z z \quad z z z z$
WNONONON NONONON

zzex $\quad z=2 z$
:

:

$:$

5

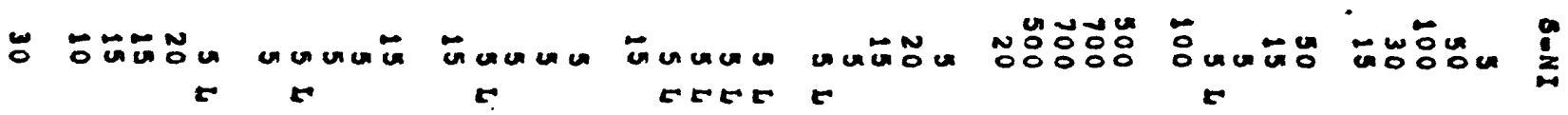

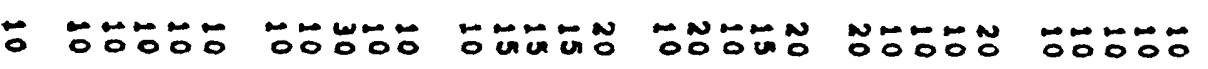

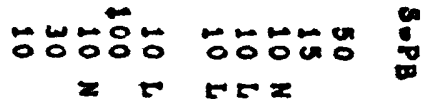

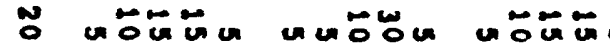
$5 t$

$t$

$z z z z$

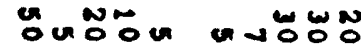

두요요

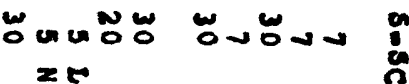




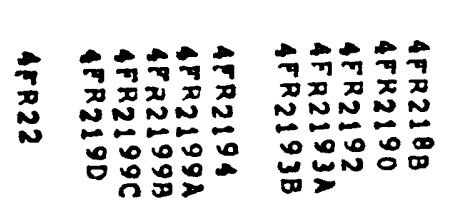

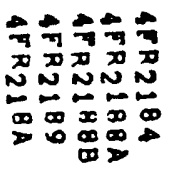

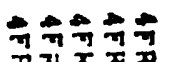

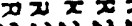

NNNNN

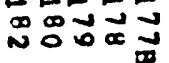

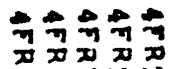

NNNNN

$\exists \overrightarrow{2} \vec{a}$

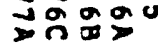

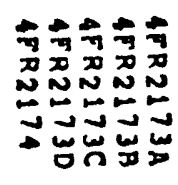

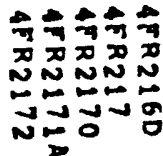

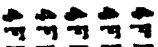

$\boldsymbol{D} \boldsymbol{D} \boldsymbol{D}$

NNNNN

뭉중

要

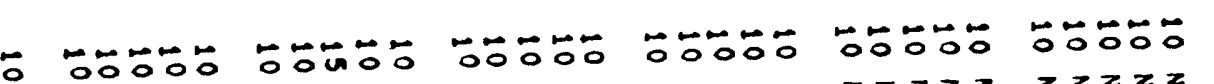

$z$ $z \geq z \geq 2$ $z \geq 2 z 2$

$z z z z z$ $z z z z z$

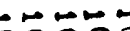

cos

०ே00\%

$z z \dot{z} z z$

$\underset{0}{0}$

뭉.

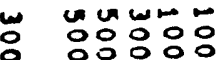

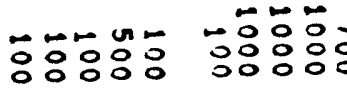

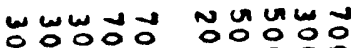

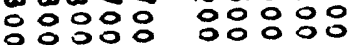
$z$ $\approx$

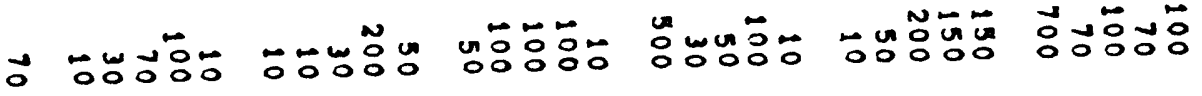

ununu

o

$z \quad z z z z z$

-

000000000000000 ununu un 00000

numun un

uñugun

$z z z z z$

wow

(1)

ỡ

0 unune 00000

$z z z z$ ununugur

z Z z z

NOOOO

c $z z$

NNNNN

잉ㅇㅇㅇㅇㅇㅇ

NNNNN NNNNNN 응ㅇㅇㅇ응

$z z z z z$

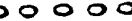

$z z z z$

$z z z z z$

$z z z z$ $z z z z$

$z z z z$

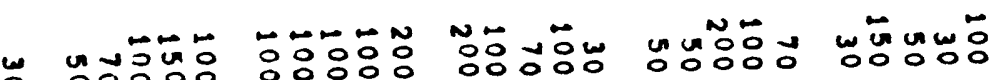

$\omega \div 0 \div 0$ $z z z x$
U.

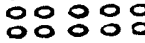

tr

NNWN2

영잉잉 5

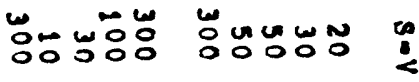
5

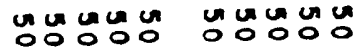
$z z z z z z z z z$

NOOOOON NOOOOO $\underset{1}{\infty}$ $z z$

5 ए

NNNNN ㅇㅇㅇㅇㅇㅇㅇㅇ

NNNONO $z z z z z \quad z z z z$

Nم

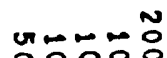

:

$z$

$z z z$

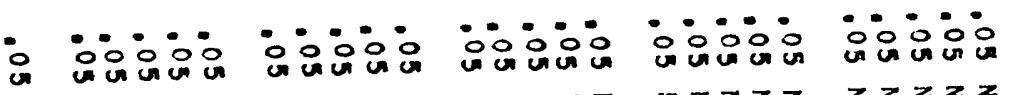
은운운운

\title{
$\therefore \circ \circ \circ$
} $z z z z z$

$z \geq z z z$

$\div 0 \div 0$

$z \quad z z z z z$

$z \geq 2 z 2$

$z z z z z \quad z z z z z$

$z \geq z \geq$

\section{(2)}

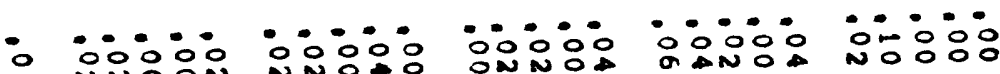

ㅇㅇㅇㅇㅇㅇㅇ

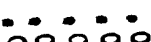

西西

काष्टz

읏ㅇㅇㅇㅇㅇㅇ

$z \infty \stackrel{x}{\Omega}$

W $z \infty z z z \infty \quad \infty \quad z \infty$

$\infty$

ग0

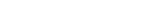

(1)

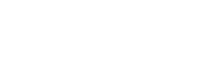

- no000 0000000000

a 50100

다

E0000 ம

00000

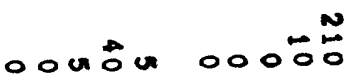

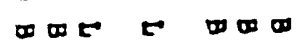

- 00000

-

00000

00000

மம
00000

世

00000

எᄄ山ய
00000

Ш ᄄᄄ口
00000

ம
00000 - 


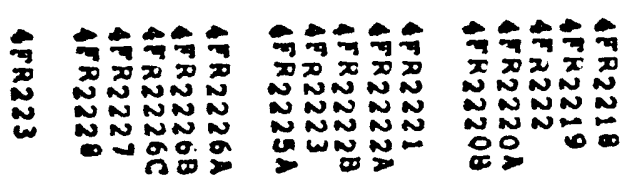

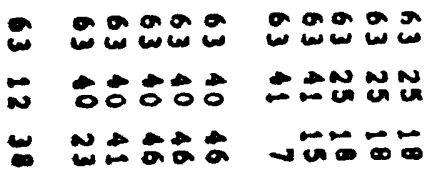

is NNDN Uuñu

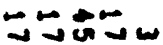
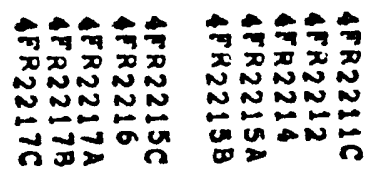

前落

x $x$ x

NNNNNN $\sim N$ ONO $\rightarrow=0 \frac{1}{2}$

낙하군

NNNNN NNNNN 응요 $\div \div \div \div \div$ NNN NNNNN ONOOOOO $\therefore \&$ W.

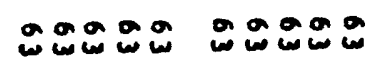
NNNNN $\rightarrow \rightarrow \infty$ N N NNN unuva

우요

WN

$\leftarrow \stackrel{\infty}{\infty}$

0.090.

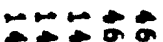

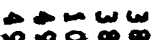

$\operatorname{lom}_{0 \rightarrow \infty N}$

NNoub

-

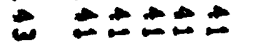

○

- $N \in \div 5=$

\section{玨却}

atur un

w U

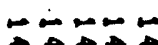
w正业 - óño -

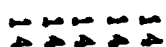

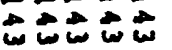

ano $\rightarrow \infty$

U U⿴囗十⺝

a.a.

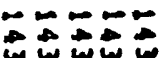
$\infty \infty+\infty$ it

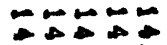

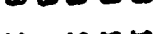
무웜 항ㅎㅇ 0 영여
푸

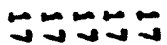

م

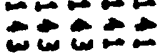

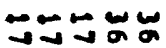

م

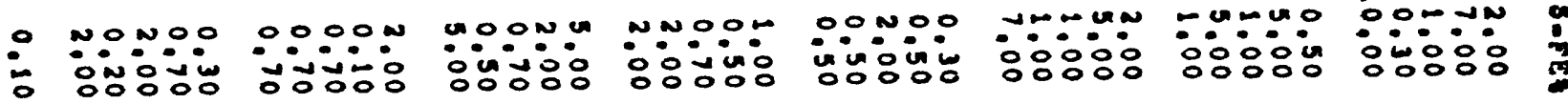

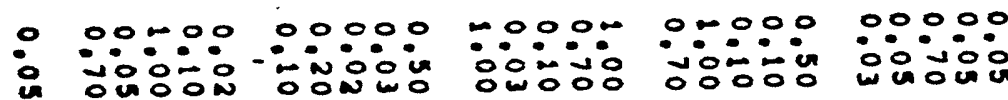

No으 ㄴivio

OMONO isioin -00 uno 응수 00000 00000 80000

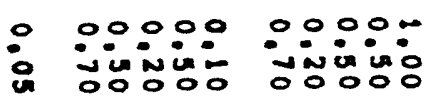

$\because 0: \div \div \div \div 0:$

aㄴㅇㅇㅇㅢ

unvo:

$00 \ldots$

i. i.

N-No

$00-1 \times 0$

$200 \div 0$ 응 훙유

o

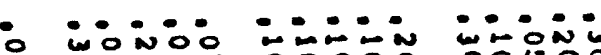

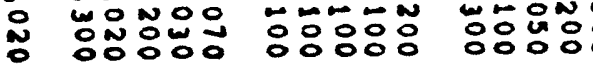

NND: 응응요
응ㅇㅇㅇ 닝영 w0:1 W언ㄷㅇㅛ
NWENO 녕영요

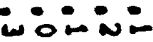

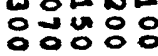

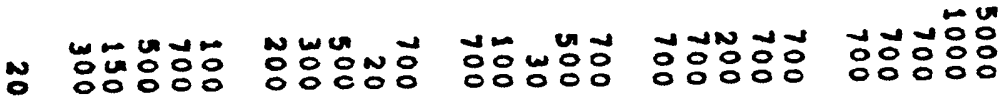

- 00000 00000

$z$

$z z z z$

$z z z z$
00000 00000 unicis iniviniu $z z z z z z z$
00000 iniviv $z z z z$
눈능ํㅇ 응응응융
누융충융 응응ㅇㅇㅇ
몽ㅁㅇ용 녕응

\section{NONONO 융ㅇㅇㅇ응 \\ NNNNN}

$z z \geq z z$
NNNNN 영영영 $z z z z z$
NNNNN

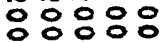

$z \geq 2 z 2$
NN NNN N 응ㅇㅇㅇㅇㅇㅇ $z z z z$ invivin $z \quad z z z$
0000잉 uviuín

$z z z z$
응ㅇㅇ úuivi $z z z=$ $\frac{6}{6}$

NNNNNN
NNNNN ลํํํำ $z z z z=$ NNNNN N $z z z=2$ 


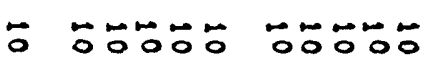

뚱ํㅇㅇํㅇ

مि000

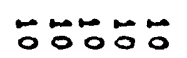

o.

ОООООО

눙ํํㅇ

$\leftarrow$

5

Ctet

$\infty$

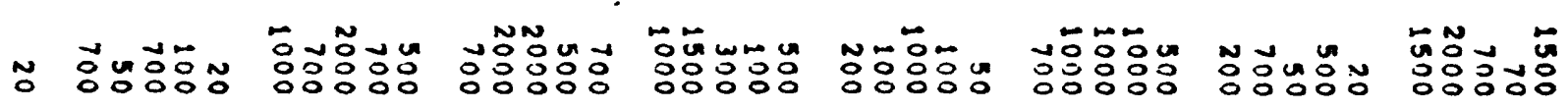

$z$

N

W N $\sim N$

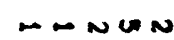

$\sim \omega \sim \omega N$

$\omega \sim N \leftarrow N$

-m-m

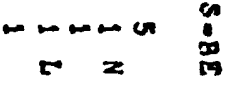

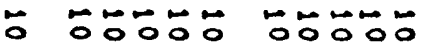

$z=z z$

$z \geq z 2 z$

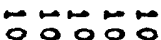

m00\%

$000 \% 0$

م0000

- $0 \div 00$

등ํㅇ

z zzz zzzzz zzzzz

co

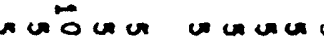

$z z z \quad z z z z$ un unu un

$2 \geq x$ wö unum

$$
z z z
$$

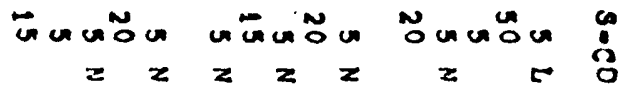

u

$z$ üundua unumuan c 5 t $2 z E$
5ั0ง

$\geq z$

z $\quad z z z$

सே등 Fz zZ ZzZzz

onnna nNNNN

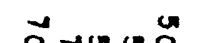

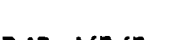
$r F \quad z=2 x$ o in wै wै .

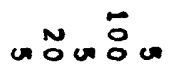

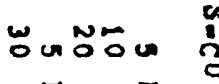

56

$r \quad z$

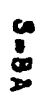




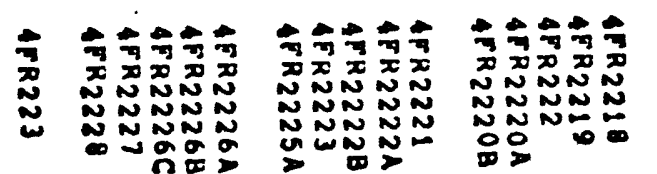

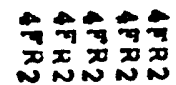
NNNNN

范荡
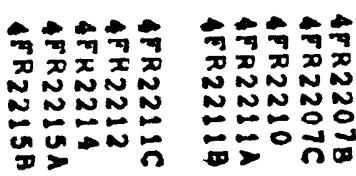

$\div$ -

- zzzzZ

둥후

\%о

$\div \div \div 0$

z. $z \geq z z$

$z x z z z$

$z=2$

$z x x z 2$

a 0

0000

$x z=2$

$\div \div \div$

00000

zz又iz
둥ㅎㅇ웅

$\div 50 \div 0$

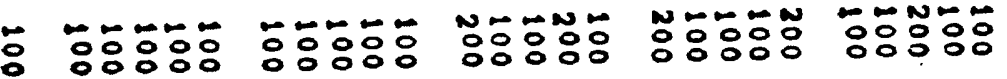

$=$

$z$
2 $z x z x$

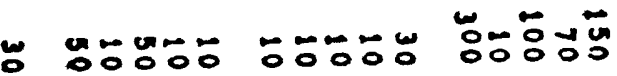
ए 5

\& gongon

unun un

unumu

z $z \geq z z z$

$z z z z$

$z z z x z$

눙ํํㅇํㅇ

(2)

ำํํㅇํำ

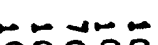
5 TE TE

응뜽ㅇㅇㅇ융

두우웅 웅혀융ㅇ

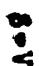
5

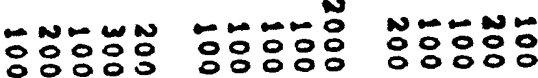
co $\div$

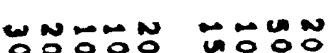
UOOOO

Nㅜㅇㅠ -

MNNNN

○ N ONONON 응ㅇㅇㅇㅇㅇㅇ

NNNNN 응ㅇㅇㅇㅇㅇㅇ

NNNNN 응ㅇㅇㅇㅇㅇ

Nํㅇํำ

NNNNN

ㅇㅇㅇㅇㅠ

NOOOO

$z z z z z \quad z z z z z$

$x \geq x z$

ㄱ. $x \geq z z$

NNNNN 잉ㅇㅇㅇㅇ $z z z z$

z $2 \geq \pi$ r

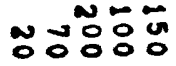

พับ

$-\infty, 0$

\% 유ㅇㅠㅜ

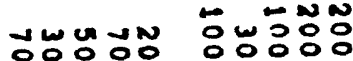

눙 00000
은욘용요 운운웅요 $x=z \geq z z$ $z=2 z 2$
00000 oúu $x \geq 2 z 2$

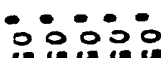
$\geq \geq \geq 2$
웅웅 윰은용 $2 \geq 202$
웅운운 $z z z z z$ iㅜ용

$z x z z 2$

$\therefore 0: 0$

zxezz

- 0000

용여

$=\quad 200$
엿ㅇㅇㅇ융ㅇㅇㅇ z 0020
영융ㅇㅇㅇ

:0000 $\infty=00$ $\therefore \therefore: 00$

$2 \infty=\infty$ $\because \therefore \therefore \circ$ 10000
$000 \div 0$

응응응

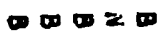

응영웅 $\omega=002$ a 00000

r 00 a o o no

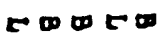

0500000000

no 00 to 00
00000 때 000
$000 \stackrel{0}{0}$ - 006

00000 由 200

00000

00000 Ш ㇒木
00000 - 1000

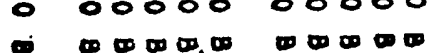
00000 வ
世
0
$\vdots$
$\vdots$

:

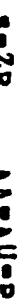

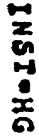

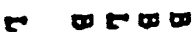

Noono

$\infty 0 \infty$
05000

-
- o ovo - 0000 coouno CDOEO
00000 10000
00000 由
$0000 \%$ w 200 
a $900 \%$

$\because \approx N \cong N$

$\therefore$ U. $-N N$ $\cos a$

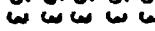
N

N
운

$\infty \infty \infty \infty$

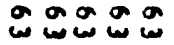

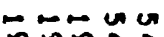

NNNNron un agara ט

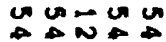

$N=w n$ $\cos \pi 0$ ט unu un un Uuजu $\cos 00$

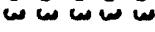

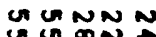

anaga ט

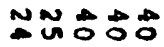
NNNGN

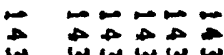

u vuver

م Nobि

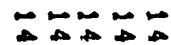
ज西心 Uजि

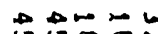

DE

$\rightarrow+\infty+\infty$

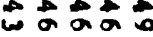

waraú $\rightarrow \infty \leftarrow \infty$

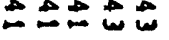

bun:

un w w

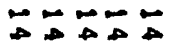
至点心

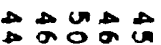

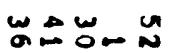

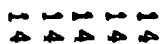

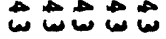

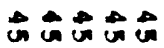

ลNNW $\because \underset{\square}{\square}$ แENลN UE UNA م0 WN $\rightarrow \vec{N} \rightarrow \rightarrow \infty$ w

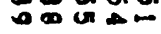
NָN
N 2 uñ

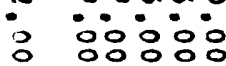

UnNNN

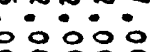

- -000

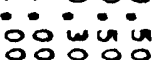

ㄴ -00ío

\section{M} n

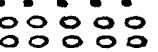

둥요

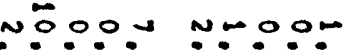

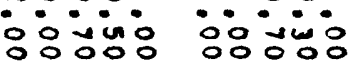

은 in:0:

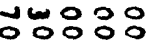

-
00000 모용
$0=000$ in 0 i
OnNOO OOOON ㅇㅇㅇㅇㅛ

OANOOOO in:

00000 inom niñon

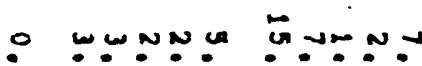

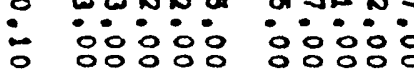

$-0000$

우웅웅

$0000 \mathrm{n}$ - 0.0. C5 55
ONNOO NOOOW FO:OH ino in: 00000

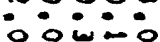
낭응

t०

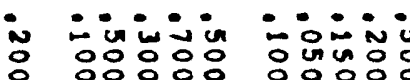

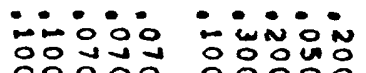

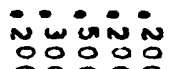

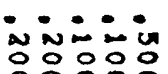

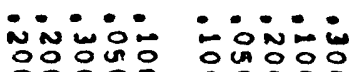
a

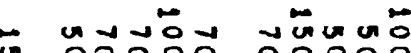

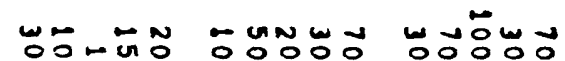
OOOOOO

MNNNO 응ㅇㅇㅇㅇㅇㅇ
당ㅎㅇㅇㅇㅇㅇ

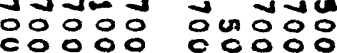

0 $\therefore \quad 00000$

in inivin

z $z z z$
00000 vininiv $z z z z z$
00000 जuivi $z \geq z \geq z$ inininin inivin inivinin $z z z z \quad z z \quad z z \quad z z z z$
0000000000

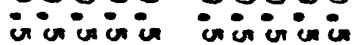
$z z z z z z z z$
: 


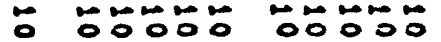

- 5 EET

ผ

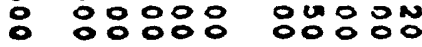
2
두웅

$\operatorname{ctc} 5$

Non 옹유유

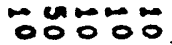
r TET ER
뚱ㄴํㅇํำ

5
둥후응 $\leftarrow$

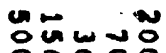

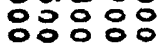
w눙ㅇㅇㅇㅇㅇㅇㅇㅇ
N WNOㅇㅇㅇㅇㅠ

2
O유유 CE 5

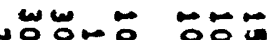

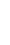

-

$\infty-\infty-\infty$

N N

$-\infty$

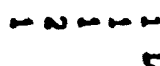

Wr Won

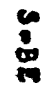

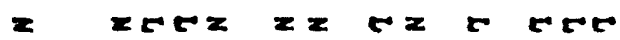

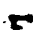

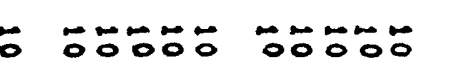

유융

ㅜㅇ유유

유유

두유

ํํ융

우웅

$z \quad 22222$

$x \geq 222$

$2 z 2 z 2$

$z z z z$

$z=2 z 2$

$2 z 22 z$

$z \geq z \geq z$

$z \geq 2 z 2$

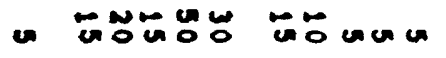

unuara unuara

wöóna unumo unaug unciana

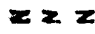

$z z z z z z$

$z \geq z$

$z z \quad z z$

흥 뭉둥ㅇㅇ

영웅ㅇㅇㅇ

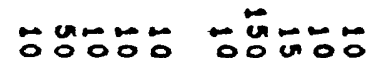

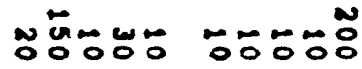

두웅

둥두유 $r z$ $z=z$

r $z z z z z$

$x=$ $z z z z=z$ L

- 붕ํㅇㅇㅠ

ounuar

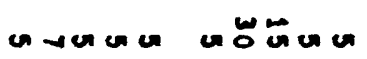

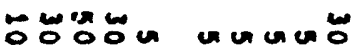

andue

uncun है

5

CE 5 CEe 5 te

$r$ CEE

FECET ER

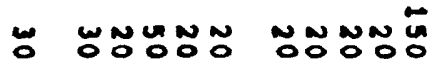
$z=2 \quad z$

นNNWN

\section{GNNNN}

ํํㅇํำ

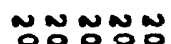

NNONOOO NONONO

$$
\text { Cr }
$$

r

u cuanua

anenca

unuan unuca

unuenur unumu

uncuoner

uncuen

$z \quad z z=2 z$

$z \geq z z z$

$z z z z z \quad z z z z z$

$z z z z z z z$

$z \geq z \geq z$

$z \geq z \geq z$

\section{ํ กOO융}

กํㅇํㅇํㅇ

$z \quad z z z z z$

$z z z z$

ONOOOOOOO N ONONOOONO

ONOOOON

مัOดัOก:

กัOOกัO

NOㅇㅇㅇㅇ

$z 2 z 2 z$

$z z z z z$

$z 252 z$

$z x z=$

$z z z z z$

$z \geq z z z$

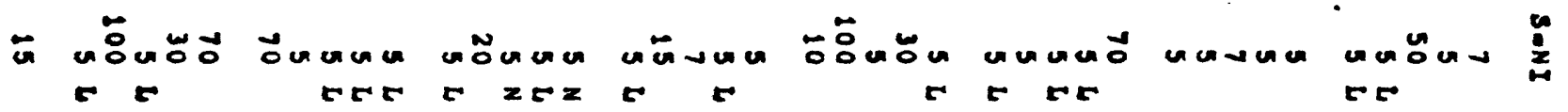

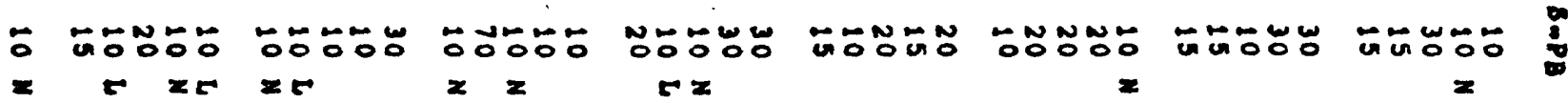

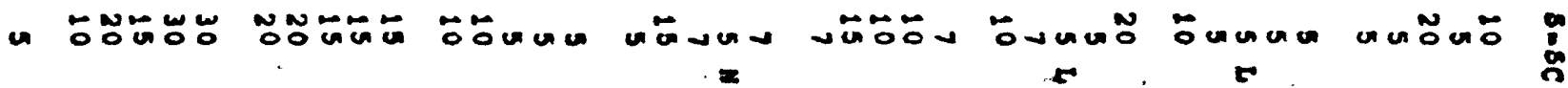




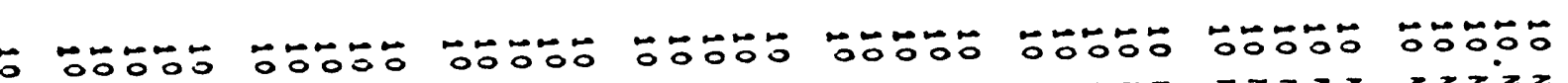

$z z z z z z z z z$

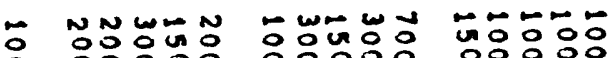

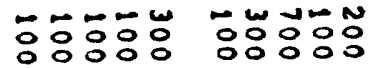
$z z z$

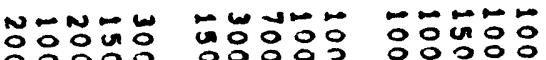

$z z$

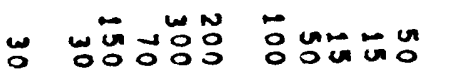

- ununung

$z \quad z z \geq z z$

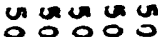

$z \geq z z z$

¿ WOLOOON OWONOO

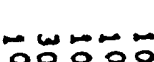

त्t
뭉뭉여 5

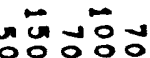

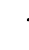

$\leftarrow$

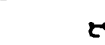

뭉ㅁㅇㅇㅇㅇ

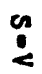

5

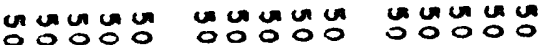

$z z z z z \quad z z z z z \quad z z z z z$

un un un in

00000

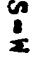

Nw-w

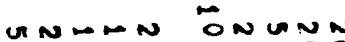

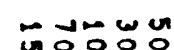

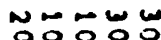

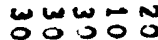
$\leftarrow$

ลํํํํํำ

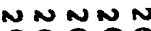

:อะ:ำ

nNNกN

:ํ:

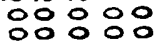

N NNNOOOO

OOOOOOOOOO

$z \geq z-2 z$

$z z z z z$

$z z z=z$

$z z z z 5 z$

$z z z z z$

Z $2 z z$

$z \geq z z z$

z $z \geq z z$

NNDON OONUN

ก M

ma m nn

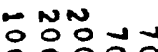

응융ㅇㅇ

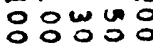

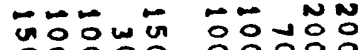

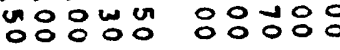

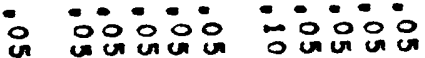

$z x z 20+$ $\therefore 0 \div 0$ on un un un $z \geq 2 z 2$
:0:0: जn जun

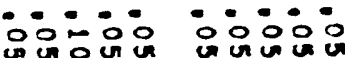

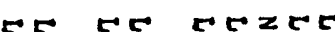

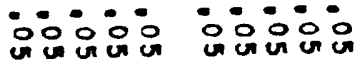
c $z z z z \quad z z z z z$

- in:-0: 00000

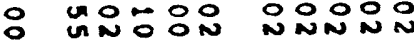
$\infty$ 2 m 2 $x \geq 2 \geq 2$ $\because 00: 0$ NNNN: $z \geq 2 z 0$ $\therefore \circ 0^{\circ}$ 옹유은 $z z z z z$ $\therefore 0.0$ NONNO

영영ㅇㅇㅇ Z

w 20 $\because 00 \div 0$

오유응 w $z=2$

00000 ก:ㅇㅇㅇ $2 \infty=\infty$
ล

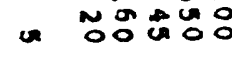

000000

-

00000

00000

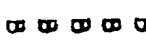

00000

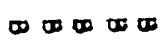

00000 क 0000
00000 -
00000 100000
00000 - 0000 


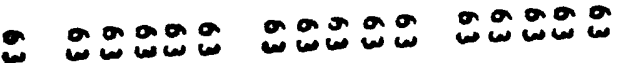

co

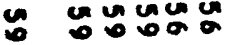
$\begin{array}{lllll}0 & 0 & 0 & 0 & 0 \\ \infty & \infty & 0 & 0 & 0\end{array}$

NOWNONO

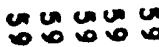

a un u $\rightarrow-\infty W N$ acos 0 WN $x \rightarrow \infty \infty$

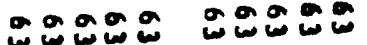
$\sim \infty N \omega$

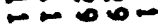
$\omega \infty \omega \infty$
N $\rightarrow \rightarrow \infty-1$
우우웅

e cou unvas

N-Nu

a. 0 an
두우요 둥후 $\omega \omega \omega \omega$ Nس 0 \%

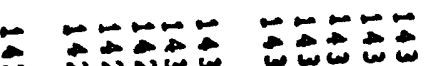
$\therefore \quad$ N

c) en un

a

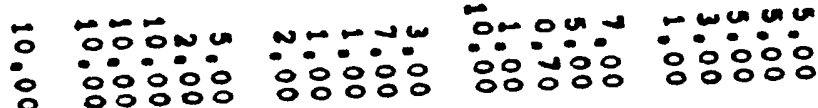

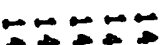

$\because \rightarrow-\infty$

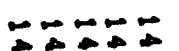

$\because \square=\square$

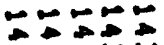

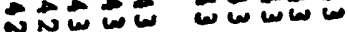

Aिcu

un unu

un un

־NNN

Iang

पsing

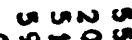

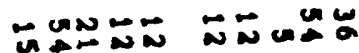

NNNOOO

ZNNN

U⿺辶一

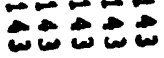
on unua

政

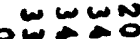

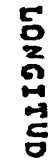

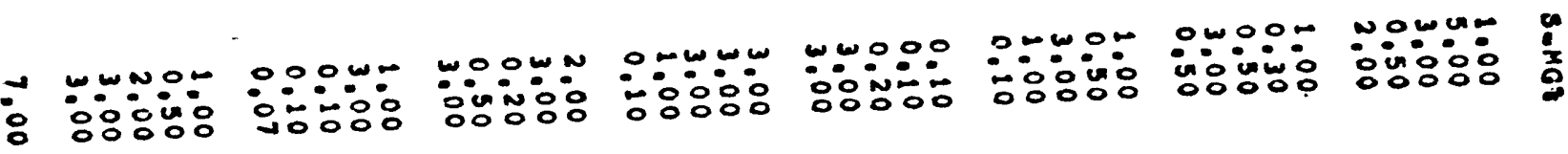

$\rightarrow \quad: \because 4: 50: 0: 5$

UOOON ORUNN

unooo

ornor

N

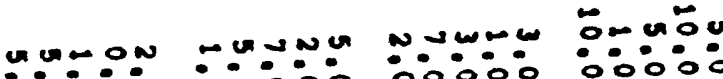

$\frac{0}{8}$

웅ㅇㅇ ini: 0 :0:0

요응

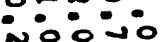

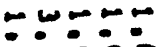

Lon unn

:ㅇ:웅

a

$\leftarrow$

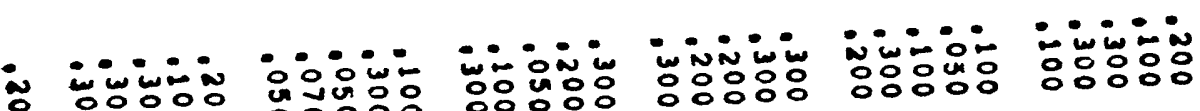

$\therefore$ inin

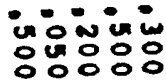

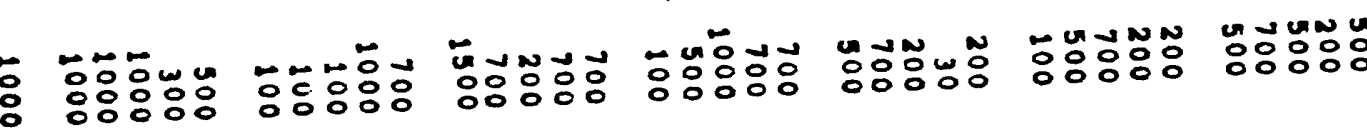

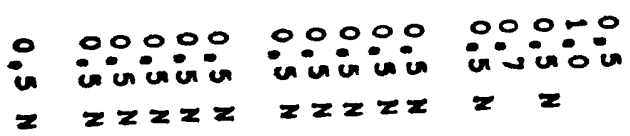

00000 uninivi $z \geq z z z$

00000 invin $z z z z$
00000
univivi
$z z z z$

웅으

$z z z z$
00000

univiv

$z z z z$
NNNNN NNNONO ㅇㅇㅇ융ㅇㅇㅇㅇㅇㅇㅇㅇ $z z z z z z z z z$
NNNNN :융 $z \geq 2 x=$
NNNNN 잉ㅇㅇㅇㅇㅇ $z=2 z$
NNNNN응 ำ०० $z=2 z$
NNN N N 웅ㅇㅇㅇ응 $z z z z z$
응ํㅠㅇํำ

$z=2 z=$
NNNNN 응유융 $z \geq z z z$ 


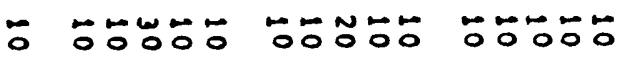

무융

둥ㄷㅇ

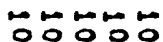

ํํㅇํㅇ

จำำ

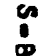
55

- terte t5

c erE

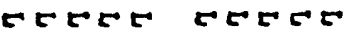

CETE

ผ

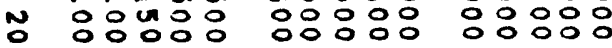

$z$

กัown un wiñ

영영영

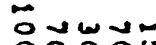
응영ํำ

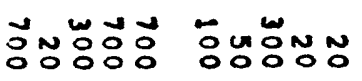

z

$2 z$

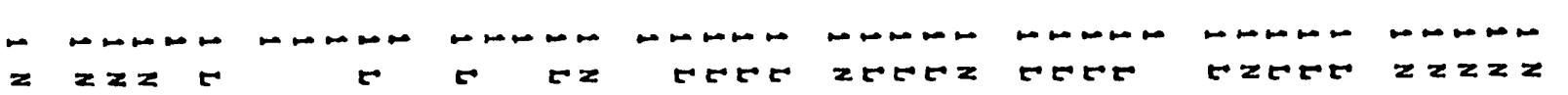

-

z $z z z z z$
등ํㅇㅇ

$z z x \geq z$
00500

z $22 z 2$
뭉유유

$z \geq 2 \geq z$
ㅁํㅇํㅇ

무융ㅇ

ํํำธ

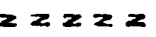

$z z z z z z z z z$

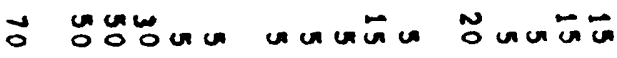
co

z $2 z$

CE unts

$\ddot{\circ}$

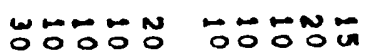
25
00000

$z \mathbf{z}$ พr viow wo

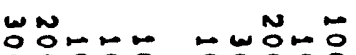

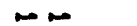

Noun än Noñ Criz z

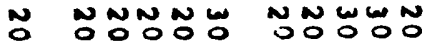

$z \quad z x \geq z$
OOOOOON

$z$
ัㅇㅇํํํํํ ํํㅇํำ

$2 z z z$

$\underset{\infty}{\infty}$

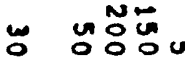
ctetre

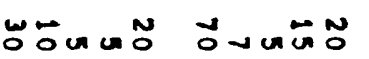
$c$ जin.

C5

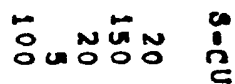
c 52
N N

$z=z z z z$
NNNNN $5+52=$
NNNNNO NNNNON NONONO

$z+r z z z z z z z \geq z+z z$
NNONONO

zEzZr
ONOOOOON NNONOOOOO CZLEF $2 \dot{2} z=2$ a

$z z z=$

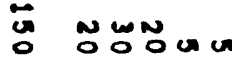
$+\infty$

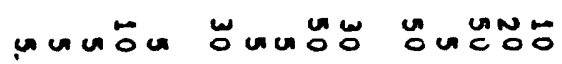

DET 5 TE

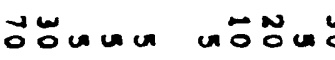
ret
พษธีชั

5

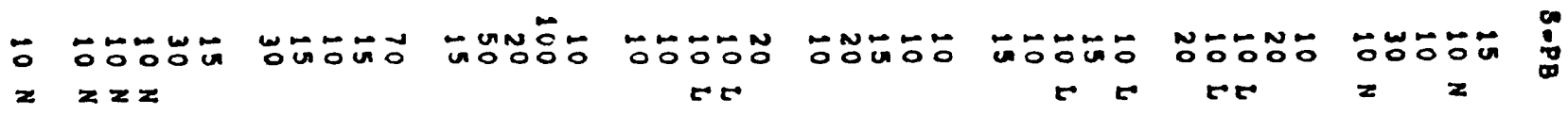

O 


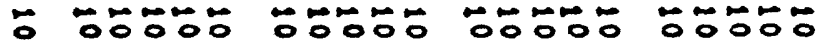

z $z=2 x z$

$2 x \geq 2 x$

Z2EZz

$z x z z x$

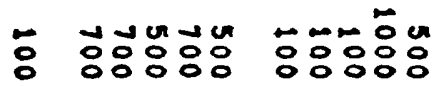

우운 웅영응

z

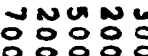
0000

NUํㅇㅇㅠ $z=2$

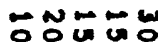

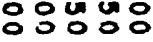
$z \geq 2 x$

웅

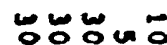

$-\infty \div$

웅두영

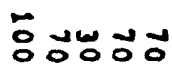

응후웅

두웅

봉뭉

G.

응붕응응

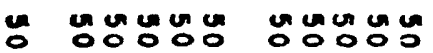

$x \quad \geq \geq z$

$z \geq z \geq z$

जuncon

uncun un ú

$x \geq x=2$

z $\geq x z 2$

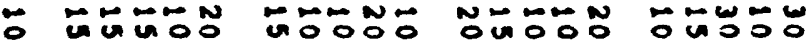

Niñ unung

$x \geq \geq x \geq$

unumua

$\geq \geq \geq \geq 2$

unuara

0080

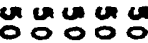

$z \geq z \geq x$

№ OOH $z$

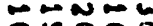

NTNNG

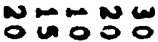

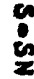

:

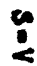

:

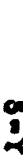

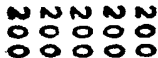

NNNNN Oㅇㅇㅇㅇㅇㅇ $z=2 z=$

$z z=2$

NNNNN 응응융 $z=2 z$ $z x \geq z=$ $z \geq z z z$

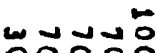

붕ํㅠ

\%N

anw wo

뭉녕ㅇㅇㅇ
웅ㅇㅇㅇㅇ 웅ㅇㅇ웅 웅용ㅇㅇ 은웅용요 जU जún

$z x \geq z z$ $z z z z$

$z \geq z \geq z$
웅요웅웅

$z \geq z \geq 2$
웅ㅇㅇㅇㅇㅇ

$z \geq 2 \geq 2$
웅웅ㅇㅇ웅

$x \geq 2 z=$

운웅웅웅

cox $x=$

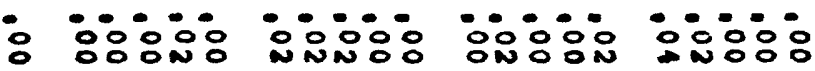

- $\infty \pi z=200$

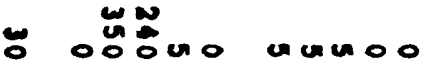
- CDEz⿻上丨

$0000 \%$ : 0000 -

$+\infty 0$

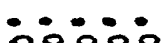
우융ㅇㅇ OONNO का $x \geq 0$

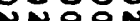
$z \geq \infty \pi x$
웅웅 (1)

$\infty+\infty 20$

웅요

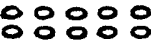
10000

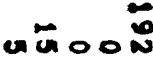
-

कण
00000 0050
- 0000000000

$\infty$
-

00000 10000
00000 000000
00000 1000
00000 由
00000 -

00000

0000

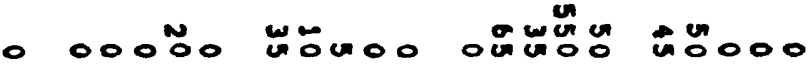

$+\infty \infty$ $\infty \boldsymbol{\infty}$
$000^{N} 0$ w
Un $\infty$ ○oயัoo $\infty$ -
- in 000 - 
arara

*

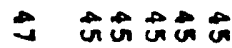

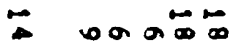

a) a o o م

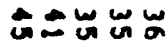
م an ono on cora

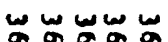

NNNOF下 w \& $00 \pm 5$

س oroga

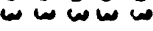
UNUNŨ NuU
ก) w ungung \$ arana

mosa iagas un $\ln _{0}$ on

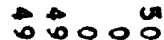

unca

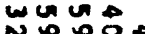

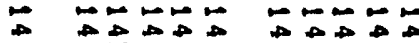

$\Rightarrow \quad \overrightarrow{N A N A} \mathbb{N} \mathbb{N} \mathbb{N}$

u unuma a

U ANNAB in w $\rightarrow \rightarrow \rightarrow \infty$

NNANA

N

$\rightarrow 0 \rightarrow$

a

N

$\rightarrow \vec{N} \rightarrow \vec{N}$

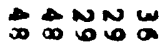

$\approx$ N
$\underset{N \rightarrow \infty}{\infty} \div$ NNNNN

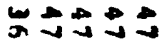
w w w w

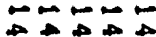
NNANA $\rightarrow \infty \rightarrow \infty u$ BNNN

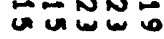

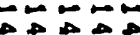
WNNNN $\infty u \cup u$
$\infty$
$\infty$

$\omega \infty \quad n$ NOSON

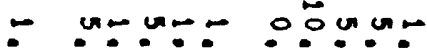

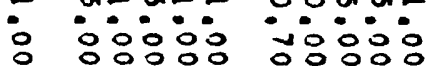

onoun ono:inioio iom:

ONUNM in:00i

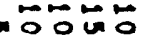

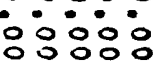

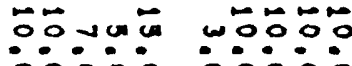
ㅇㅇㅇ영영ㅇㅇㅇㅇㅇㅇ in 응숭
10000

ONONN 이유유

- 0000

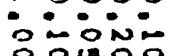

$00-\infty 0$ 이유

$\because 0: n N$

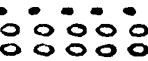

LU UnN N

NWW N N NOOOO OOUOO UNOOO
- nONOO

- Oñن́
ENNNO 웅ㅇㅇ웅
ODON N

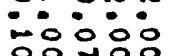

No000 융웅응
OONNN

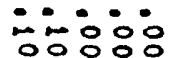

N

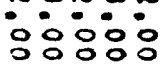

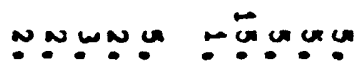
웅ㅇ

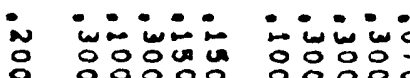
O००0

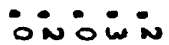

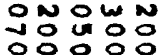

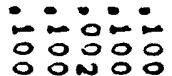

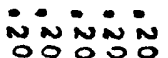

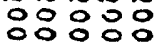

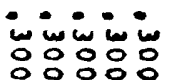

w

noinin

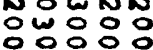

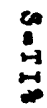

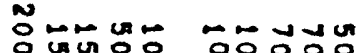

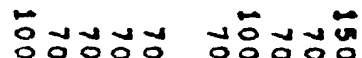

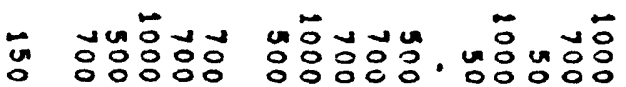

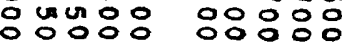

in in inivin 00000 in uncion $z z z z z$
00000 in uninis $z \geq z z$
00000 univion $z z z z$

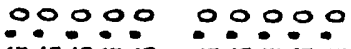
inivin $z \geq z z$

unuivin
00000 in in in $z z z z$ जo

이잉 inoini $z \quad z z=$

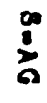




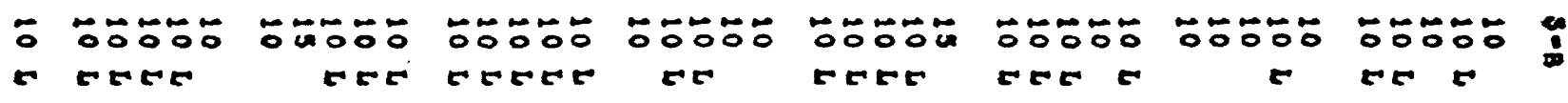

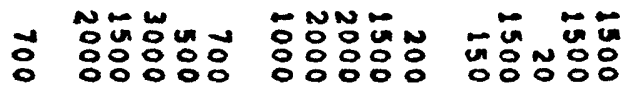
$z$

\section{w}

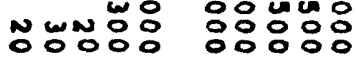
$=r$
N w w w w w w w 융융ㅇㅇㅇㅇㅇㅇ

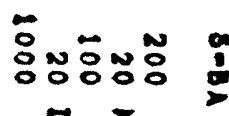

- $=$

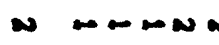
co

$\div \% 0 \% \%$

$z \quad z z 2 z 2$ $\omega m-\infty$

and

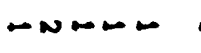

$\infty x$

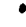

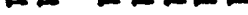
$r z z+x=2 x=$
른

0

ㅜㅜㅇㅠ

$z 2 z=2$

$z=z z$

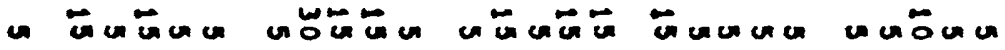

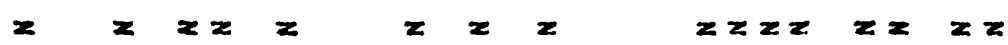

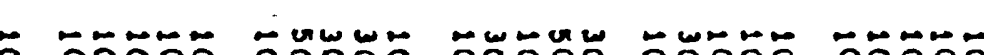
00000 00000 00000 00000 00000

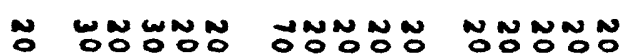

OONNO NNNNN $z \geq z$

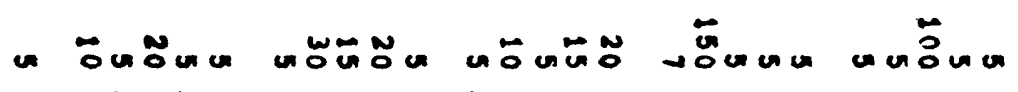

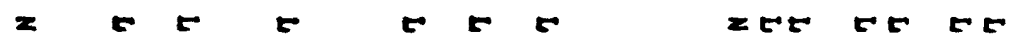

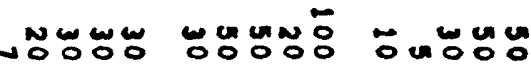
พㅜㅇㅁㅇ 붕용ㅇㅇㅇ

?

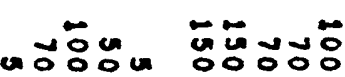

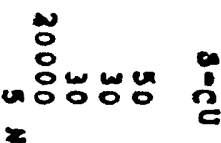

SNNNN

NNNNN

NONONO

$:$

- cucucanca

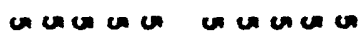

uncuua

unumu

unuma

uncouco

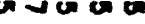

$z \quad z z z z z$

$z z z z$

$z z z z$

$z \geq z \geq z$

$z 2 z 2 z$

$z \geq z z 2$

$z \geq z \geq z$

$z=z z$

N NONONO

NONONO

NONNN

NNNNN

NNNNN

$z z z z r$

$z \geq 2 z 5$

$z z \geq z z$

$z \geq 2 z 2$

$z E \geq z z$

NONON

$z \geq z \geq z$

OONON

NONON $z \geq z z z$

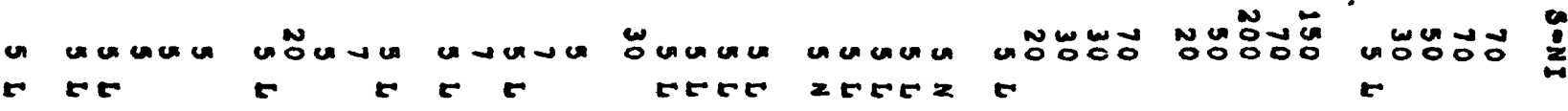

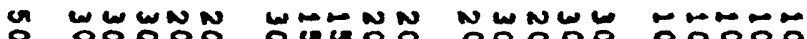
$=20$

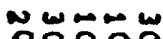

r

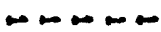
$z z z z$ $\underset{0}{0}$

$\div 0 \% 0 \%$ $z z z z z$
$00 \div 0$ $z z z$

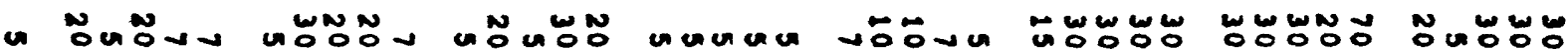
यEN 

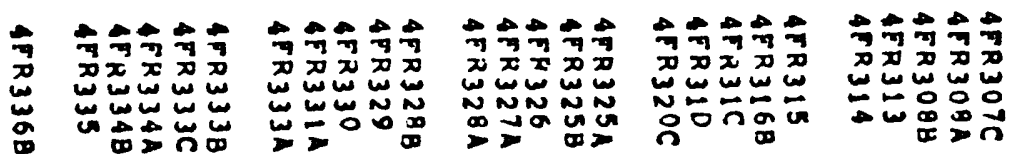

"

둥요 đே

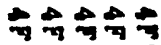

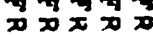

w w w

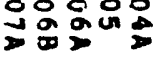

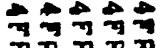

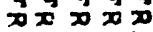

م

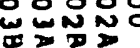

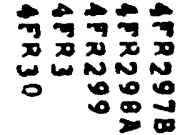

둥유

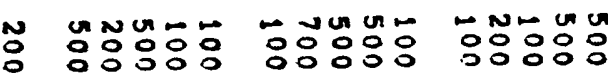

EE

z $z 2$

O OOOOOOO

$-\ddot{U}-\stackrel{0}{0} 0$

$\overrightarrow{0}$

$\leftarrow$

E

5 c

un ugunoun

nonuon un

uncurar un

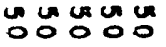

$z \quad z z z z$

$z z z z 2$

$z \geq z z z$

- NONONO NONONO WOLWN
드융응 ㅇㅇㅇㅇㅇㅇㅇㅇ $z z z z$
둥유 응융

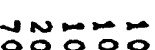
5
둥ㅇㅇㅇㅇㅇ

55
ேㅜ융

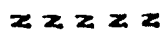

두웅

$z z \geq z$ $\div$

두을

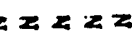

जㅡ웅

$z \quad z x z$ วัnunw

WNNWn-

w-unu un ำㅇำ

$$
\text { r. } z
$$

$z z z z$

mancun un

$z z z z z$

०0ㅇㅇㅇ

$z z \geq z z$

ñuguñ

$z \geq \geq \geq z$

ñ̂ㅇํㅇํㅇ

z

Nom 드 W

NO NOO

븡

NONOO n nNNNN

응ㅇㅇㅇㅇㅇㅇ

NNNNN 응응ㅇㅇㅇ

z r z z Z
NNNNN 영ㅇㅇㅇㅇㅇㅇ Z E Z ZZ
NNNNN 응ㅇㅇㅇㅇㅇㅇ re $z \geq z z$
NONN

$z z z z$
NNNNN OOOOOO zenter
Nㅡㅇ싱용ㅇㅇㅇ

TEE NONNNN Z Z T C

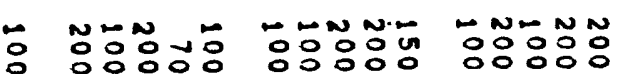
$000 \%$
W

No음

OUw Un

우융ำ $z$

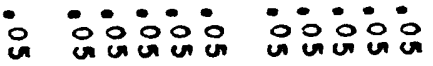

웅요요 $z x \geq z z$ $z \geq z z z$
웅응ㅇㅇㅇ जง जou

$r 2202$
연용ㅇㅇ

cr $2 \geq z z$
웅ㅎㅇ웅

$z z \geq z$ $\div \div 000$ un un un un $z \geq z z z$ ivio: 응분요 $z=z z$ a

is

영영요 영영요

$\infty$
20002
응응응

20200 $\div 0000$ N요욤 $z$ W 2

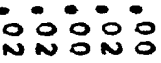

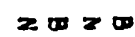

$\therefore \circ \div 00$ 응ㅇㅇㅇㅇㅇㅇ $\infty \infty \infty$

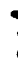

응ㅇㅇㅇ영 ம

웅ㅇㅇㅇㅇㅇㅇ 00000 $\infty \pi \infty$

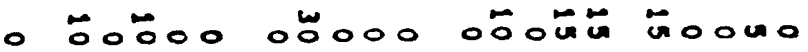

C
काषण

00000

ம

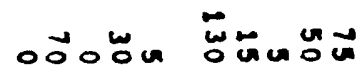
(1)
- 0000000000 - எ
00000 $\infty \infty \infty \omega$
00000

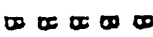

00000 மார
00000

00000
00000

1000
00000

1000

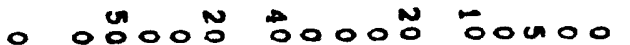

- 00 ய ö́⿴囗十 or

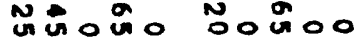

- $\infty$
00000

0000

00000

由 
- 운후 * coscó nugua

$00 a 90$

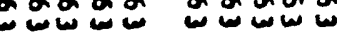
unco UUU二⿺辶一 ט - 10000

00000

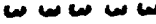
ه una-
ENNON

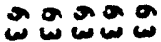
$\omega \omega \rightarrow-\infty$

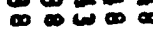
N OONN

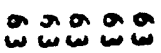

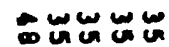

osan
- 20003

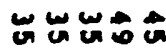

w
00000 - oc후웅 ㄴon wo

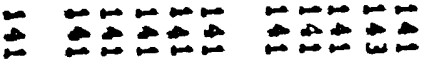 \\ $\therefore \approx \approx 00$ \\ N Na요 $\infty+0$ a unu - $\infty 000$}

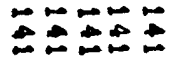

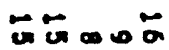
wㅜ․ oner

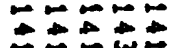
穴云合舟

జN

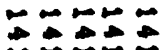

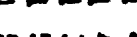

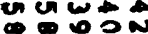

NNN些 $\because \underset{\infty}{\infty} \approx \infty$

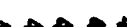

$\triangle \mathbb{N}=$

W. $00 \%$ $\rightarrow \infty$

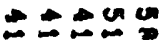
añ⿺ $\vec{\omega}+\infty \neq$ $\rightarrow \infty-\infty$

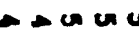
aลaแ

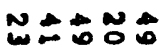

- แำง

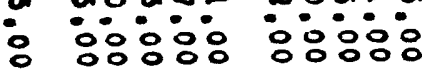

n n w n 웅ㅇㅇㅇㅇㅇㅇ
ำ ㄷㅇㅇㅇㅇㅇ 영영영 wom

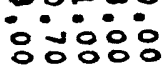

- unuo iojoi 응웅영 un 2 on o io:ini ㅇㅇㅇㅇ․ $\frac{1}{3}$

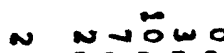
용요
근 :NOEO

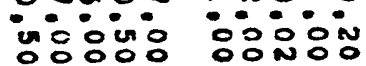
영영영
$00 \div 20$ wㅇㅇㅇㅇ

OOONN

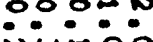

$000-N$

ON:mo 0.000 닝ㅇㅇㅇㅇㅇ OOOO

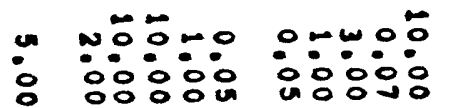

n w oro Nonm: OONU:

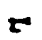

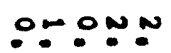
웅응ㅇㅇㅇ -

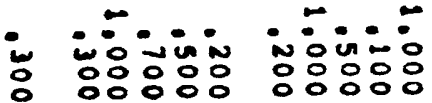
$\Omega$

a 추ㅇㅠㅛ N㤠前 $00 \%$

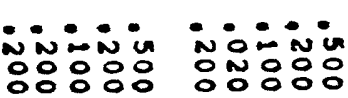

으누: 녕ㅇㅇㅇㅇㅇㅇ
븐ㅇㅇㅇㅇ ․ํㅇㅇㅇㅇㅇㅇㅇ 웅주응
무웅 ฉัลํํํำ 붕셩ㅎㅇㅇㅇㅇ 둥융융

롱ํㅇㅇㅇㅇㅁㅇ 응ㅇㅇㅇㅇㅇㅇ 양뭉용을

뜽융ㅇㅇㅇㅇㅇ

Nan웅ㅇㅇ웅 


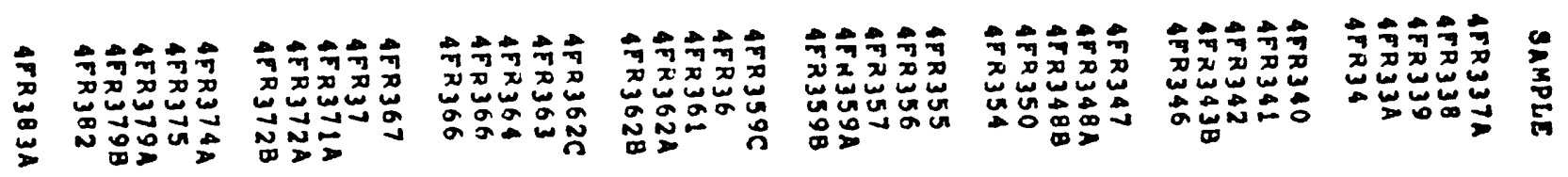

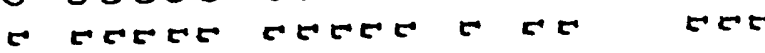

EF TEE

FIE TEETE

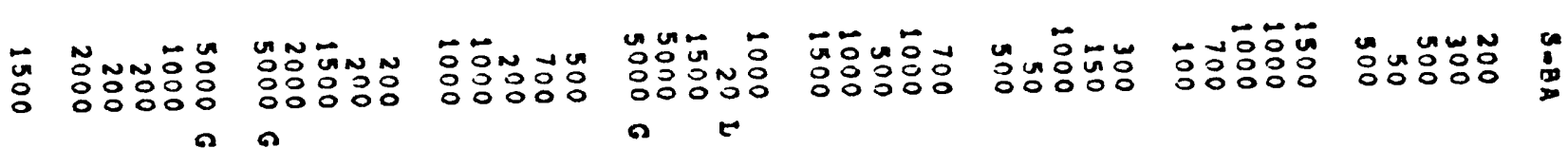
ค

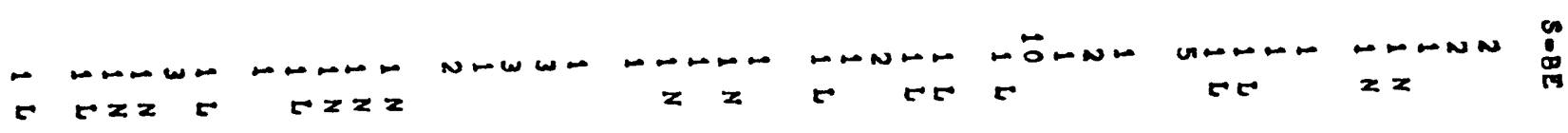

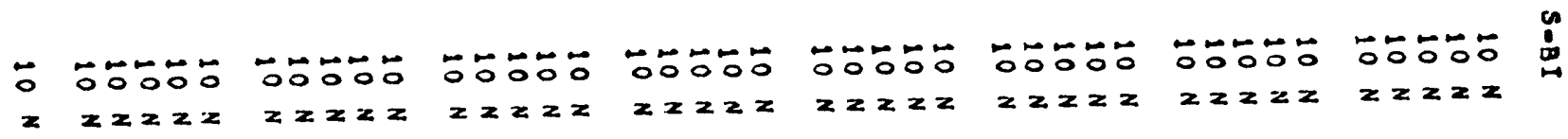

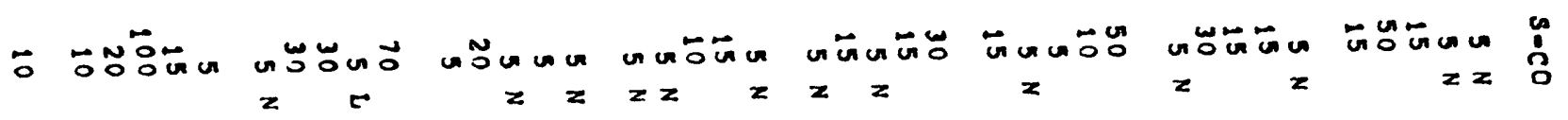

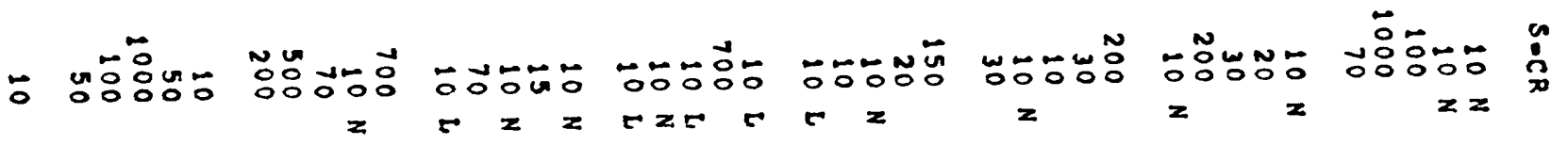

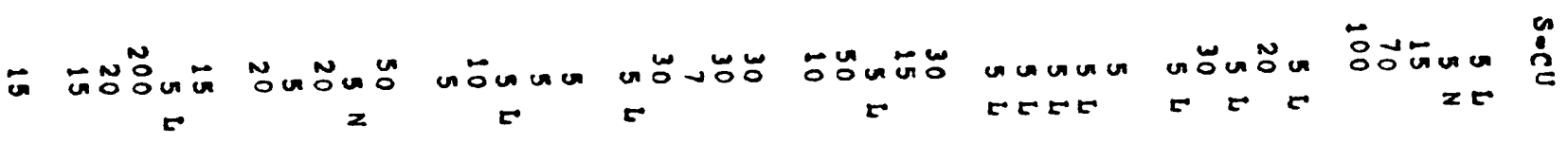

$z=2 z z=z=z z$

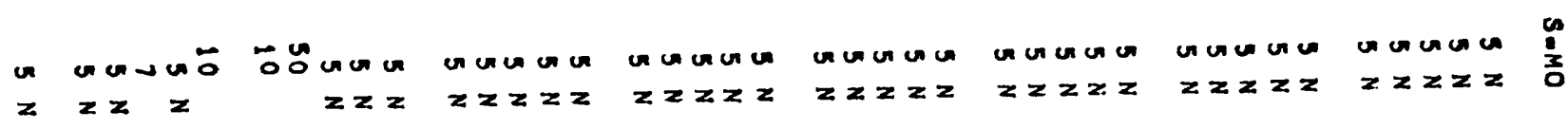

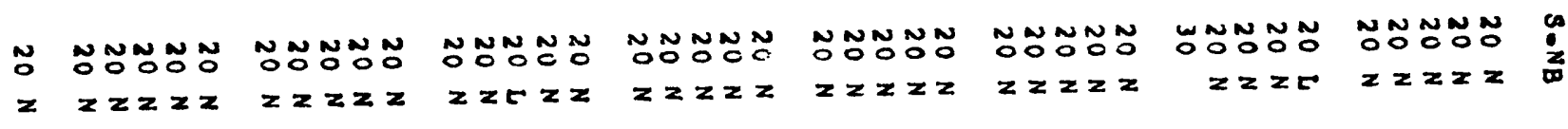

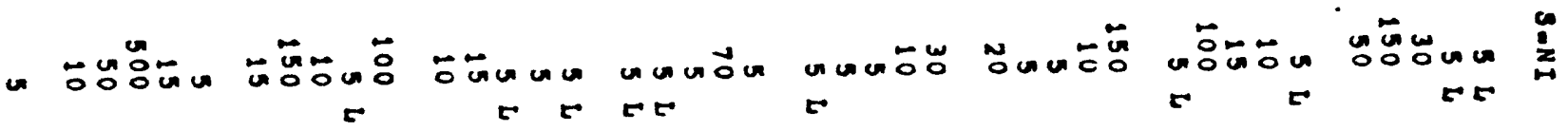

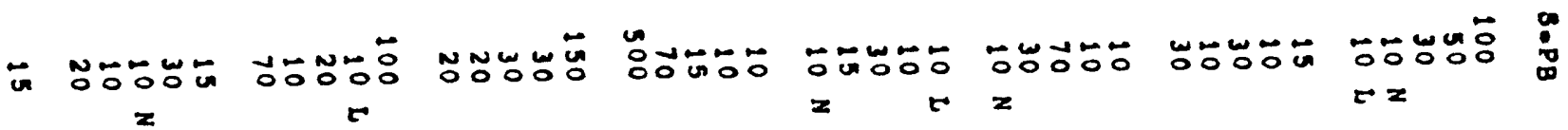

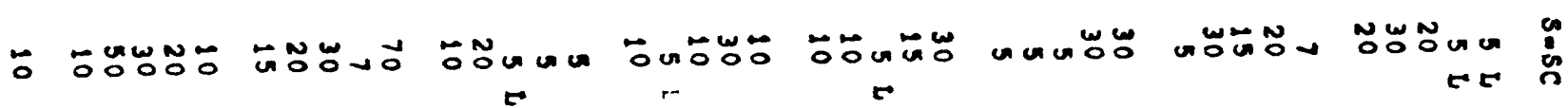
27 


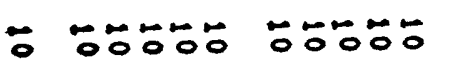

z $z z z z z$

亏ั ถัะัะั

ํํㅇํํㅇํㅇ

응응융

$z$

ํํㅇํㅇํำ

영융유

$z$

nữ

영융융

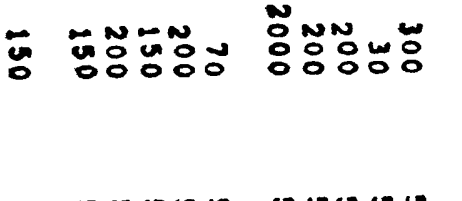

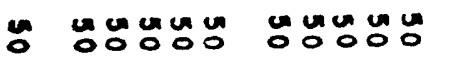

unumut un

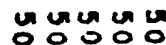

gunugus:

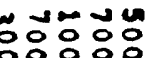

2

$z z=2 z$

$z z 2 z z$

unumun

00000

ancons

$z \geq z x z$

$z \geq z \geq z$

$z \geq z z z$

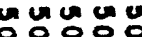

$z x z=2$

-NUTH

WNNDO

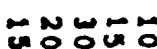

$\because \div \div \div$

O0000

$\div$

N $N N N N N N N N N$

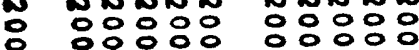

NNNNN

응영유융

NNNNN

OOOO:O

NNNNN

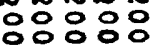

NNNNN

응유응

$z \geq \geq z=$

$z z z Z \quad z Z z Z z$

$z$ z $z \geq z$

อัดำำำ

$z z z z=$

กับnN :ะะ:

z $z=E z \quad z z z z z$

$\cos$

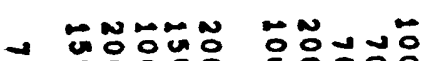
荲以ัم 둥ㅇㅇㅇㅇㅇㅇㅇㅠ

NN

N-

200 00010 융뮤: 뮹
:

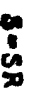
D

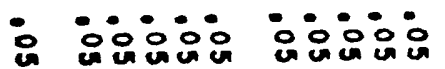

$z=2 \geq 2 z$

$z \geq 2 z z$

운은웅용

운은운윤요

둥웅

Z X E 2

c $x \geq z$

c $5 z 2$

웅ㅇㅇ웅

$\therefore \div \div 0$

$z=5$

$z z$

영윤운운 w2 252

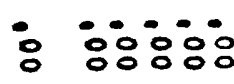

- 00000 $\therefore: 00: 0$ (10 0 $\because 0000$ 옹ㅇㅇㅇㅇㅇㅇㅛ 20002
은응ㅇㅇㅇㅇㅛ $z=\infty 12$

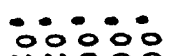
NNOOO 22000
웅영ㅇㅇㅇㅇㅇ 1000
두웅 NOOON 20002
두웅웅 요연 - $\quad z z$ co

co
- 0000000000

-
00000 - $\boldsymbol{\omega}=$
Muoo: nó000 - $\infty$
काष
$0000 \mathrm{a}$ Шण0ய
00000 10000
00000 10000
- 00000

-

00000 W 때
00000 - 0000
00000

$\infty \pi \times \infty$
00000 10000
00000 ம
00000 ம0000
00000

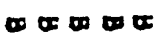

000000

- 0000 जuooo 厂0
Uno 000 U.
nU000 05000

-
00000 10000
:0000 -

$000=0$ மை) 


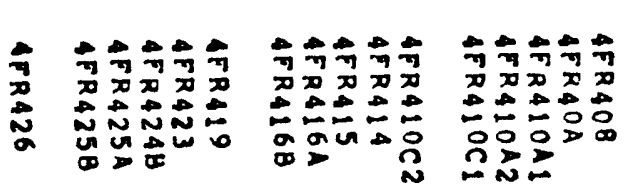

a na

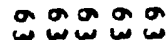

- $\overrightarrow{0} 0$ ○ी

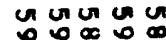

$\infty N_{N}^{N} \omega \underset{0}{0}$

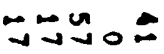

a 090

un un un

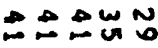

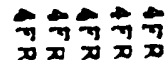

D可观

영ㅇㅇㅇㅇㅇ

व

N
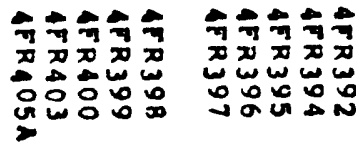

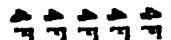

Dर $\omega_{0} \omega_{0}^{\infty} \omega_{\infty}^{\infty} \omega_{\infty}^{\infty}$

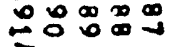

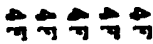

$\boldsymbol{x} \boldsymbol{x}$ م

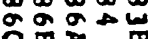

a cong

inaga araos

oc 0 a 00

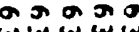

un un un

ט

w

con

$\infty \infty \infty \infty \infty$

$\infty \rightarrow \infty$ 舟

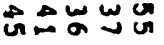

जu un un un

-

$\cos 20$

N

ט

$N \triangleq \infty \cong$

जUun

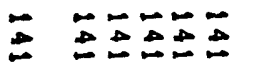 \\ $\rightarrow$ it \\ $\rightarrow N N E$ NO}

-

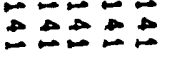

unaras

UN $-\rightarrow-\infty$

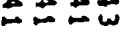

$\Delta \Delta \vec{k} \omega$

영지

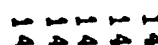

$\rightarrow D E \pm$

ט

cóá
$\ddot{\Delta} \ddot{N} \dot{\sim}$ UN

un

$0 \underset{\infty}{n} \omega \boldsymbol{0}$ $\underset{n \rightarrow \infty}{\rightarrow \infty}$

NNNND

NOE节

- NNAN $\operatorname{lam}_{\rightarrow \infty} \infty \rightarrow \infty$

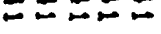
u vuvunan W

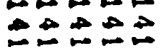

un un un a八刀

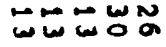

- - -00 으융

$\because \div 0:-20000$ 누웅

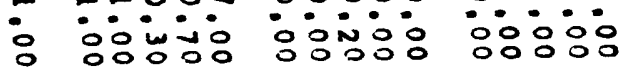

Noun un

in:oi:

눙용응

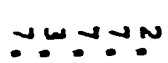

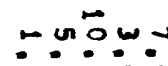

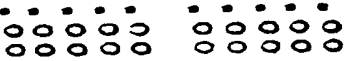

ton un un Danum $\therefore \circ: 000$

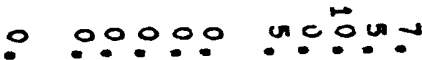

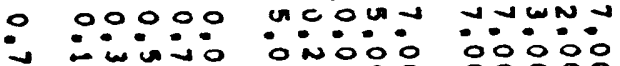
- 00003 w 0 un 웅:

- 00000

- 용ㅇㅇㅇ
Un un un w :0:0:0
응 w $\ln 4=$

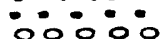
응ㅇㅇㅇ응
- 0 ON -i000 oOnOO
wNO

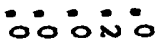
00000
O

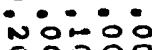

000 N ionio 5 un un -

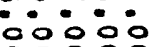
응응웅

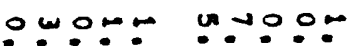
-000 :0

OWNNU

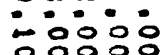

yoㅇㅇㅇ

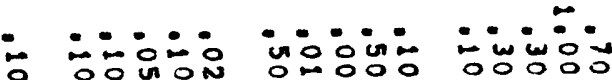

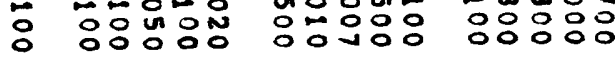

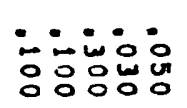
우ㅇㅝㅜ웡 눙요
نั

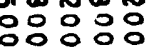

ivivi

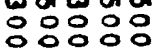

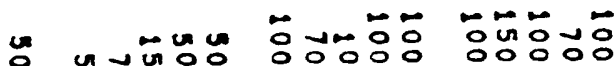

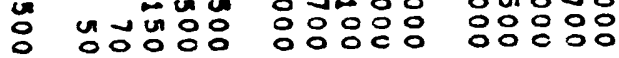

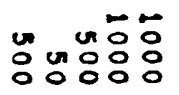

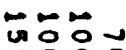

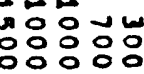

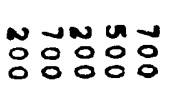

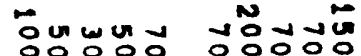
ㅇㅇㅇㅇㅇㅇㅇㅇㅇ 영영ㅇㅇㅇ in inuinum univiu

$z \quad z z z z$

$z z z z z$ ouninin unuvinu

$x \geq z z \quad z z z z$
00000 ivinis $z \geq z z z$
00000 uivivi $z \geq z z$
00000 inivini $z \geq z z$ uivini $z z z z=$ $\frac{6}{3}$

NNNNN NNNNN 


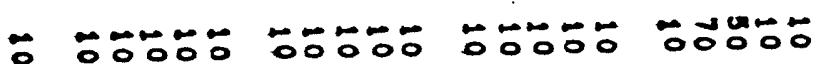

$\div 5005$

동ำ

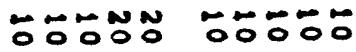

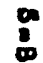

c cretr

EE $=55$

जसETE

- TEEt

CECE

TET

T5E

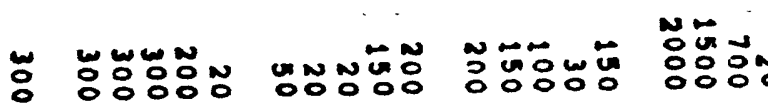
$=x z$

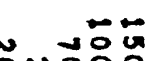
융유:

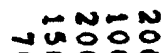
긍ㅇㅇㅇㅇㅠ 융유

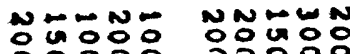
영융영 00000 : $z x \quad x$

n) NNUND $-\infty+\infty+\infty-\infty$

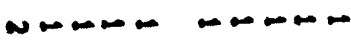

$\infty \sim \infty-\infty$

- מ מ -

$\omega \infty+\infty$

ct

$z=z x \geq E \quad+2 z 2 z$

$z=2 \geq 225$

$x=5$

$\underset{0}{0}$

- 00000 0000050000

무유

ேㅜㅇ

5005

5०००००

뭉유

:

$z=2222$

$z z z 2 \quad z 2 z 2 z$

$222 z=$

$z z z=2$

$\geq \geq \geq 2=$

$z x z z z \quad z z \geq z z$

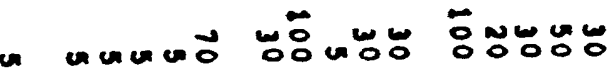

$z \quad z 2 z 2$

$\div$ ேํㅇㅇㅠ

$z \quad x \geq z z$

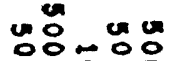 융유유} $a x$
눙응

$x=$

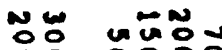

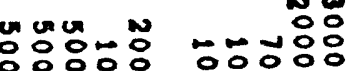

$x$

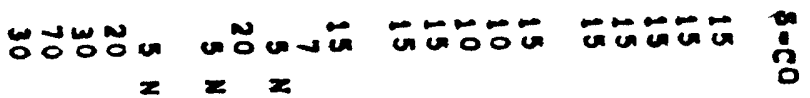

- unuñ

r trer

x

\% OOOOOOO ONOOOOO OOOOOOOO $z \quad z z z z z \quad z z z z z$

$\boldsymbol{4}$

z

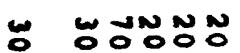
$z \geq z$ กํํㅇํำ กํㅇㅇํㅇำ $z \geq z=2$ ounuv $z z z z z$ $z z z z z$ unum a $z \geq z \geq z$
NNN WN $z=2$

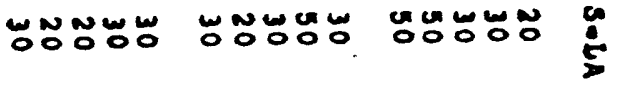

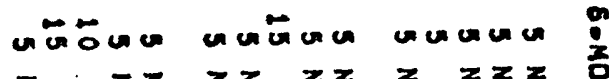
$x \geq 2 \geq$
№NONON Tr
NNONONO

$z z z z z$
등ํํㅇㅇㅇ $=r$

:

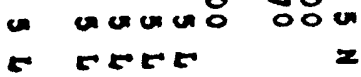

NNNNN

NNWNN

ONONON

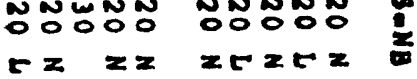

$\frac{2}{0}$ 


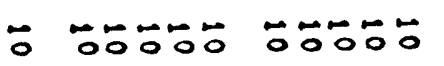

z $2 x 2 z$

100000

$5000-$

50500

БОО:О

(n)

$x<2 z 2$

$x<2 z x$

$z \geq 2 z 2$

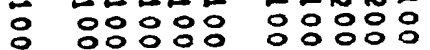

$z$ ZFELZ z

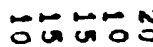

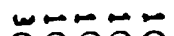

(2)

$z z$

n. Unu Un Un:

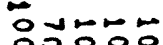

०००००००

in

$z$

00000

$z$

- OT-OOO

C TELE

능

$z z z z z$

un ununut

$z z z z z$

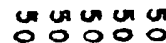

$z \geq 2 \geq 2$

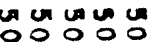

$z z \geq z=$

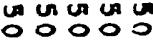

$z z z z z$

un un un wa

00000

$z z z z$

un unu un

wo nog

N w

w5 ww:

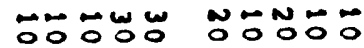

$z z z z$

WUOOONO W

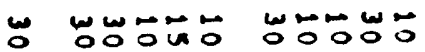
$z z z x z$

N

O

z $z z z z z$

$z z z z$

nNNNN

응영유

nNNNN

$\therefore \circ: \circ$

nNNNN

응응응

nNNNN

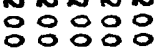

$z \geq z z z$

$z \geq z z z$

$z z z z$

$z z z z$

NNNNN

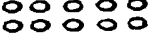

$z z z$ z $z$

NNNOOOO

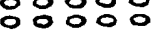

ex $x \geq$

$\stackrel{n}{2}$

ำ



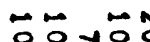

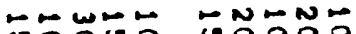

응 ำำ

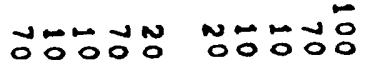
$z \mathbf{z}$

$z z$

00000

OOOOOO

noono

능응ㅇㅇㅇㅇㅇ

$z$ i 웅영ㅇㅇㅇ

$z \quad 22220$

$\therefore \circ: 0$.

응이요

웅ㅇㅇㅇㅇㅇㅇ

은웅ㅇㅇㅇ

$\therefore \circ \div 0$

은은응요

$\therefore 0 \div 00$ 응은은

연연은

$\therefore \circ-\circ$

$z \geq z \infty z$

$z z z z z=2 z z$

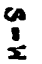

- ODio

옹용응

응옹응

D $z \geq z 00$

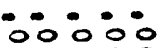
응ㅇㅇㅇㅇㅇ 山ய
웅ㅇㅇㅇ $\infty \infty$
웅ㅇㅇㅇ - $\therefore 0000$ NONNN $z \cos$

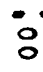
D $\therefore \circ$ 20

$\infty$

00000 00000 வ000

ए

مّ人

c
வ
- 0
00000 ம
00000 데 000
00000 ம யய
00000

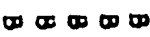

00000

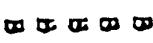

00000

Шயர口心
- जEv00 00000 -
由
00000

வ
W:000 04000 ம
웅유

$\infty$
O $\infty$
Noc 00000 $\infty$ 


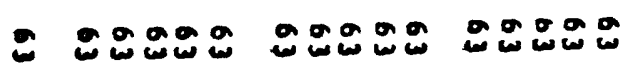

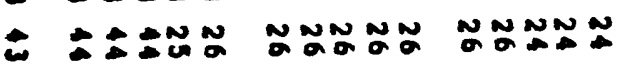

a arom 0 w

$\infty \infty \infty$

- $\infty \dot{0} \boldsymbol{0}$ a o o o

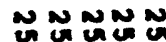

$\infty \rightarrow \infty \rightarrow$
\%

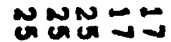

$\infty \bullet \bullet-\infty$

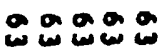

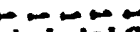

(1)
연요

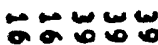

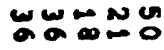

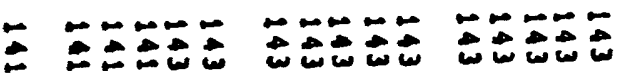

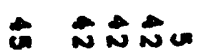 \\ $\omega=N N=0$ \\ WNATO 00000

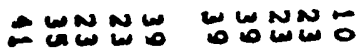

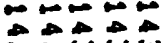

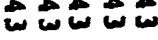

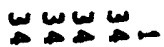
un un un wn

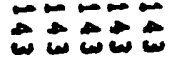

$-0000$

UัOல $\because ே \div \div \div$

N N

$0000 \%$

aunat

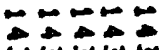

NNNNN

N

และละด

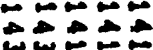

Nㅓㄴㄷㅡ:

us un un

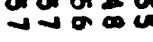

- w-

$\div$ :

nno:

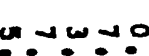

พั0 No $\therefore \therefore: 0 \%$ :웅

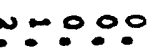
:0:

onoun

ธun- 0

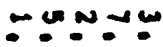

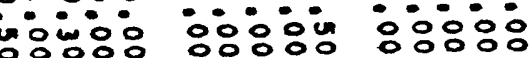

- 0w000 0000-

$\therefore$ ion:o: DOOUNO WNNNO in in: $: 0 \%$ :

$00: 00$ o은요

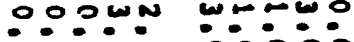

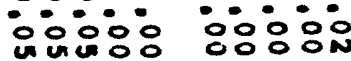

OnOB: 영ㅇㅇㅇ

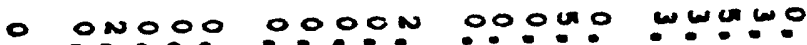
-
00000 niñó
0 OONO in:nํํㅇํㅇำ

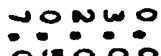
응영융
OnOO$\because \because \div i:$

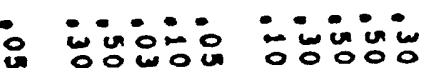

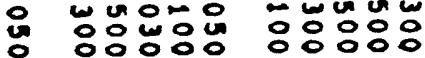
inin: $\therefore \circ: \circ$ $\because: \div:-0$

$\because \therefore: \div 0$ 응응 io:ius

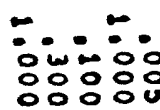
$\Omega$

מั

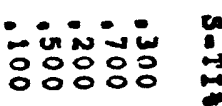

- 0000

in inivis

$z \quad z z z z z$
응요 univi

$z z x$
0000:

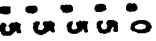

$z z z z$
00000 inivivis $z z z z$
00000 iviuía $z z z z z$
$00=00$ univiur $z z \quad z z$
00000 00000 $z z z=2 z z z z$ univion inuiviu

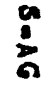

nNnNN nNnNa

กNํ유: NNNNNO :0:0:웅

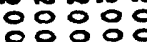
$z \geq z z=$ $\because: O: O$

$z z z z \quad z z 2 z z$
NNNNON

$x \geq z \geq 2$
NNNNN 응ำ $z \geq z z z$
NNNNNN ํํำ $z z z z z$ 
-

C 5 Er

مOOOO

5

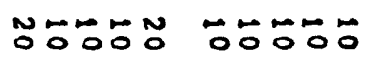
C5
ET E
웅중 terte
두ㅇㅠㅜ웅 ceter

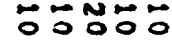

जE TE

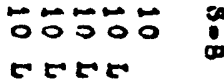

누ㅇㅠㅜㅇㅠ

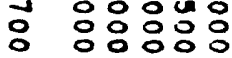

$4 \sqrt{4}$ 휘웡ㅇㅇㅇㅇㅇ

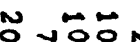
응ㅇㅇㅇㅇㅇ z
ज $\ddot{O}$ UัOO

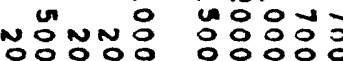

뭉
웅영ㅇㅇ 0000

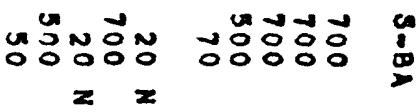

$\vec{G}-N-N$

$z$

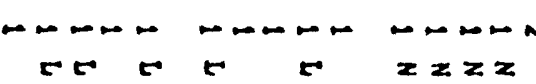

D-nNN

NNNNN

-

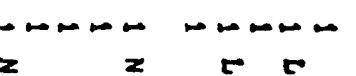

$\div$ 유우

무유

두ㅇㅠㅜㅇ

두웅

०००000

무ㅇㅠㅜ웅

두유

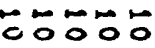

$z 22 z 2 x$

22222

$x \geq 2 x 2$

$z 2 z 2 z$

$z 2 z 2 z$

$2 z 2 z 2$

$z \geq x \geq 2$

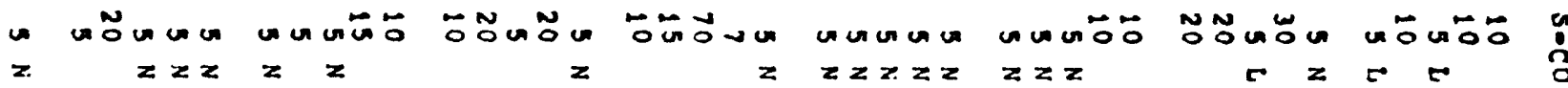

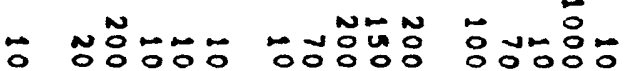

z $\quad z \geq z$ $z=z \geq z$

두웅
무융ㅇㅇ $z \geq z=$

ํํㅇํํㅇํํㅇำ 지 $x \geq z$

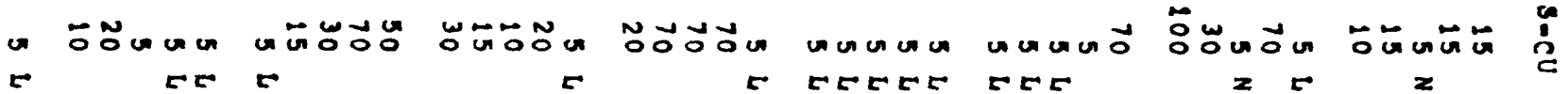

응 ONO유 N z $z z z z z$

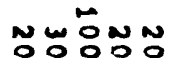

2

nOMNOTO

$$
z
$$

Nㅡㅇㅁㅇㅇㅇㅛ Nㅜ융ㅇ $z \quad z z z$ a unumun

$z \quad z \geq z \geq$

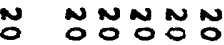

$z$ C5 25 unumer

$z z \geq z$

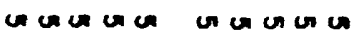

$z z z z \quad z z z z z$ un un un un

$z \geq z \geq z$ uncuu

$z z z z$

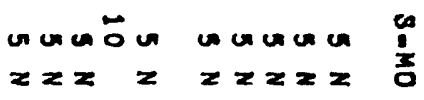

NNOOOOO NOOOONONO

$z z z=2 z E$

:
NNONON tyzzz
NNNONO NONONON

ZEFZ ZZZZZ CTCEE
NNNONO
ONONON

CEC $2=2$

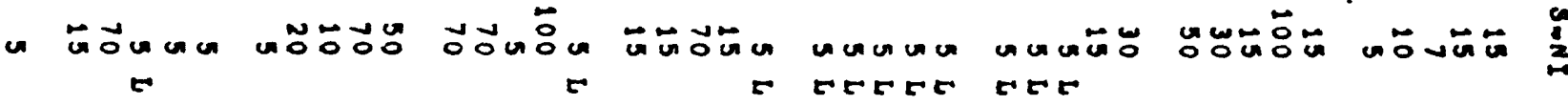




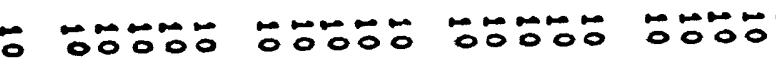

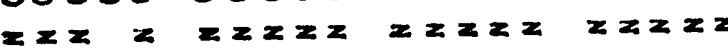

०000\% 05005

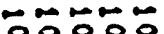

ธธธธธ

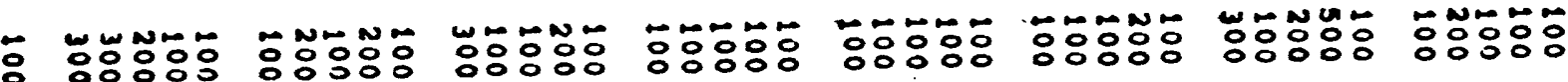

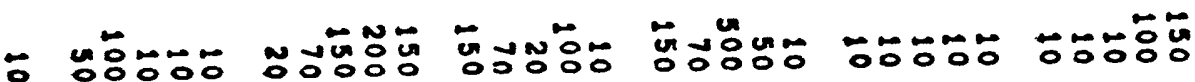

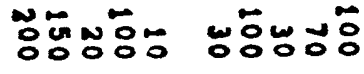

uncuncts

ununus un

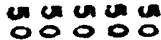

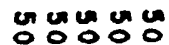

$z \quad z z z z z$

$z z z z$

$z \geq z \geq z$

$z \geq z \geq z$

$2 \geq 222$

$z \geq z \geq z$

Uñongung:

$z x z z$

uncusuch

$z \geq z=2$

W 2

n nNNNN nNNNN nNnNn

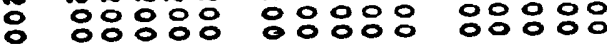

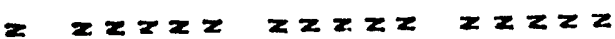

กัดกลกั

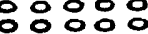

$z z \geq z$

NNNNN

영듕융

NNNNN

응응응응

$\geq z \geq z$

$z=z z z$

NNNNN

응응응응

$z \geq 20$

NNNNN

$z \geq z z z$

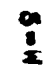
웅응유

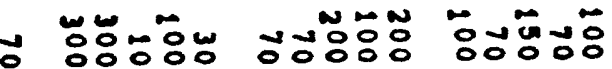

nonton

$\sim \circ$

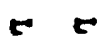

-0

응무유

$\operatorname{raㅇㅠ~}$

둥유:-

O000:

$\underset{⿱ 亠 䒑}{*}$

$=$

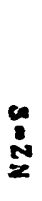

:

용용요

$\because 000$

$\because 0000$

웅ㅇㅇㅇㅇㅇ

웅요웅

웅웅웅웅

웅웅우

웅웅요

$z=2 z z$

$z z z z z$

$z z z z z$

$x z z z z$
영영

- $\omega * 2$
웅유 $\infty \infty$
둥유

\section{-}

$\therefore 0: 0$

웅응

$\because \because 0$

- 6
OOONN

- $0=2$
융유 $z=0$ $\therefore \therefore 00$

$\therefore \therefore \therefore: 0$ 10

-
- 00000

- DOUt5 o ounto

$\infty \infty$

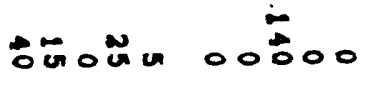

$$
\text { - } \infty \boldsymbol{\infty}
$$

000un 由ा由 unconot F

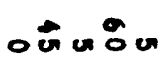
1.
ज. 0000 -1000
- 00000

- 0000
00000 Шய凶ய
00000

10000
00000 ம
00000 10000
00000 1000
00000 -
00000 $\infty \boldsymbol{\infty} \times \infty$

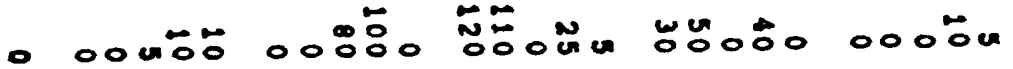

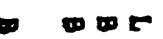

$\mathbf{0} \mathbf{0}$

- 5

(1)

-

nOㅇㅇㅇㅛ

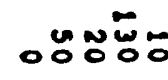
-

00000 $\infty 000$ 


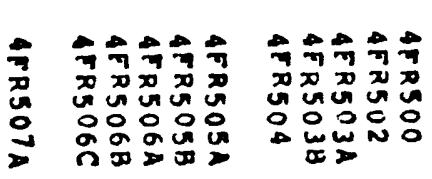

a) on on on

ono on

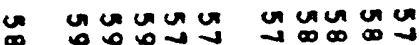

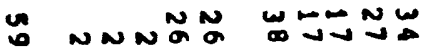

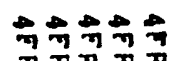

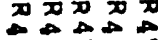

$\because 0 \% 0$ :

क्षे
合节命击节

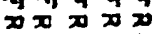

$\rightarrow+0 \overrightarrow{0}$

م。요

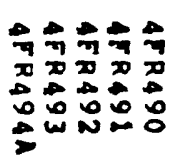

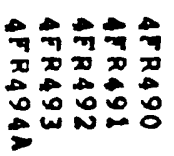

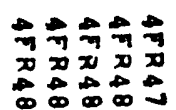
i 잉 or a o a d a un unu unun un مo u un un

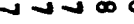
UUNN $\rightarrow \rightarrow N N$ טa un un un

$N= \pm$ un

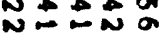

arogo

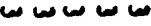
Nun No Dutan a a oa 0 w NNNNN - Nun un DN ONN ocosos

$N N=0$ uñ NONa

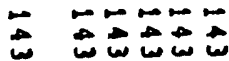

u nu unu un

- vuvióo

- a a

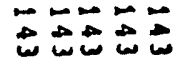
un un un

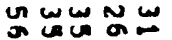

$\because \underset{-\infty}{\triangle A}$ ש u U Unu

นดำ $\because \approx-\infty$ 政 ט unu un

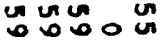

$-\infty-\infty$

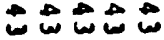

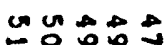
هـ ه $\because \underset{\infty}{-\infty}$ แ૯屯心

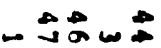
苛以感

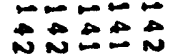

$\vec{N} \leq \overrightarrow{0}$

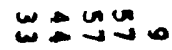

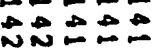

$\rightarrow \underset{N}{n} \rightarrow \vec{a}$

$\stackrel{\sim}{\infty} \rightarrow \infty$ m

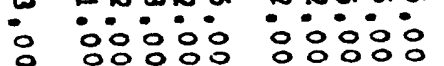

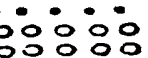

un un w w 웅ㅇㅇㅇㅇㅇ

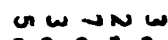

i.

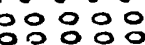

TWNOM :OOUi: 잉영용

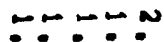
잉ㅇ 응응응응
$00: 50$

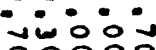

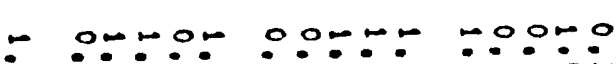

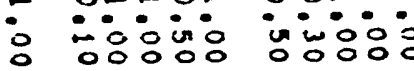

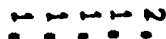
․․․ Nㅡㅇㅇㅇㅇㅇㅇ n- N-N

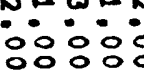

w- 00 웅요 00000 $\rightarrow \infty 00 \%$

00000 OON in wO OOO
- OnOOW FONNN WOONH

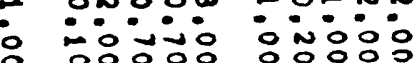
... ONONOO
NNTNN

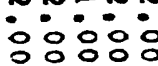

NOWN:000

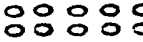

WNNOO

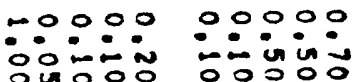

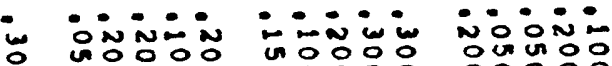

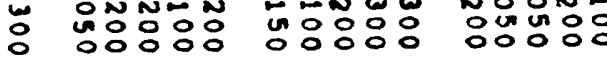

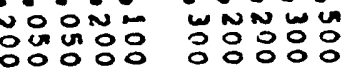

is ivis 잉잉ㅇㅇㅇ

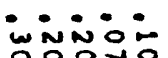
영응
웅우웅 ớño
우웅웅 능ㅇㅇㅇㅇㅇ u

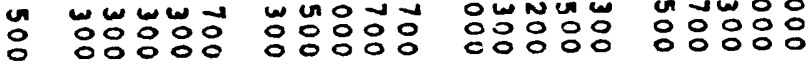
궁ㅇㅇㅇㅇㅇㅇㅇㅇ 뮹ㅇㅇㅇㅇㅇㅇㅇㅇ

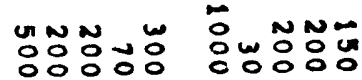

00000 univin $z z z z=$
00000 vivinin $z x z z$
00000 in uninin ininivin on un $z z z z z$
00000
00000 inivin $z z z z z$
0000 in in un $z z z z$

\footnotetext{
:
}

$z z z \geq z$
NNNNN NNNNN NNNONO OOOOOOOO $z z: \because z$

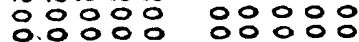

$z z z z \quad x z z z z$
NNNNN 응ㅇㅇㅇㅇㅇㅇㅇ

$z z z z z$
NNNNN ㅇㅇㅇㅇㅇㅇㅇㅇ $z \geq z z z$

ํㅡㅇํㅇㅇํㅇ

$z z z z$ 
$\because$ 뜌유

r TETER

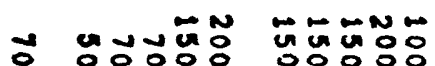

융 영영융 영영융
두ㅇㅠㅜ웅

WEECE

유융ㅇ

TETER

जu 응ㅇㅇㅇㅇㅇㅇㅇㅇ
50ㅜ웅

WEETE
5웅웅

CEETE
두웅

enter
둥후

WETE

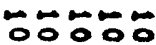

CEETE

6

\section{जษ}

\section{जu}

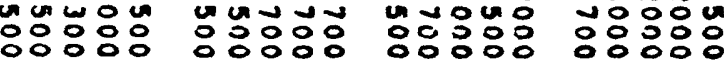

जuजि

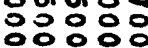

- nethen

- M N

$\infty \infty \infty+\infty$

$\infty \infty \infty+\infty$

$\leftrightarrow \infty+\infty$

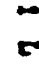

L

wam

c

$\because$ 무융ㅇ

두웅

두웅유

두ㅇㅜㅜ웅

두유유

$z=2 x \geq 2$

$z z z z z z z z z$

$z \geq z z=$

$z=2 z 2$

두우웅

$x \geq z 2 z$

a cricanum

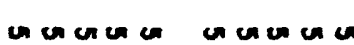

$u x+a$

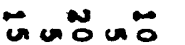

ouna <e 2 $c \geq c$

$z z \geq z$

$c$

$x \geq$

-

$z=25 E$ $\div 000 \%$

F $2 x \geq 2$
둥후웅

$\mapsto \%$ $x \geq x z$

$z z$
둥유유

- 2 z
영영응

$z \geq z$ a uneno

C Canec

บ

$z=$

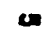

anumo EEt unum zertzes unumas

ctec uñón जuanó

CE $=$

CE
무ㅇㅠㅜ웅

ํㅡ웅ㅇ

$z z \geq z z$

$z \geq x \geq z$

unaug ancuar

$z \geq z=2$
ㅜㅜ융유

$z x \geq z z$
훙후웅

$z x \geq z=$ ancana

unour

$5 \times 20=$

:

3

$\stackrel{0}{0}$

:

CECEL

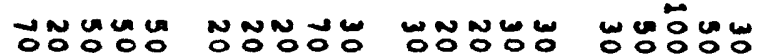

2 .

unumar $z \geq z z z$ uncuna $z z z z$ $\operatorname{lngun}$ 자 $2 \geq z$ unuun $z z z z z$ un un un $x z z \geq z$ unumu $z z z z z$ num $z z z z$
NNNNON CETC:
NONNO NONONO ZZELZZZZZZ
NONN N $z \geq E \geq z$
NONONO

$z z z z z$
NNONONO NONONON GECTE TETCE
:

웅 웅 ancina cauna

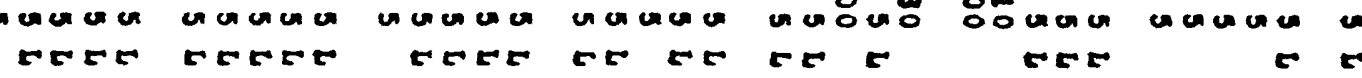

O WU⿺辶⿻ WO WONONO

TNE

- NNN

חNNNL

wawn ungwa

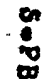

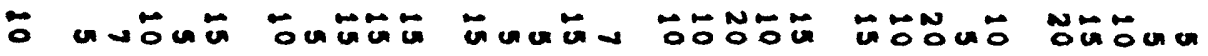
5

tr

ouncä sarara 5

CED 
$\because$ 유융 유유

두웅

Бேоั0ั0

두융우

$z z z z z$

$z z z z z$

z $z z x z$

z z $2 \geq 2$

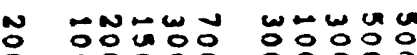

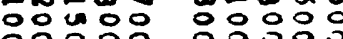
$z$

NWN N

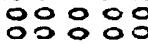

w

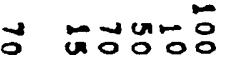

Nㅜㅇㄴㅇㅇㅇㅇ

ํํำำ

둥흐웅

등ㅇㅇㅇㅇㅇㅇ

웅웅응

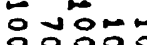
5

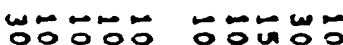

뚱ํํ응

$000 \div \%$

$z=$

00000

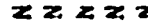

un w w w

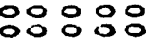

NN=5

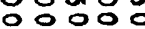

누응

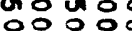

5

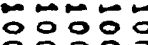

$z$

\& 0

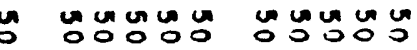

unumu

ununur

ununum

Mañono

un

nongug

$z z x z z \quad z<z z z$

$z \geq z z z$

$z z z z z$

Z2 2 Z 2

$z z z z z$

- ETEOOE WOTNW

NNNWN

NNNW

NNWNN

WEN N

WNजE

뚜유유 n nnNnN nNnNn nNnNn

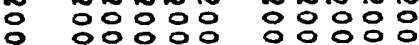

$z \quad z z z z \quad z z z z$

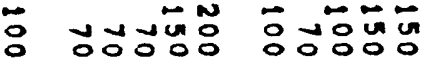

응웅ㅇㅇㅇ

$z z \geq z$
nNNNN 응응융ㅇㅇㅇㅇㅠ

$z z z z$
nNNNN 응ㅇㅇㅇㅇㅠ

$z z z=$
Noñ

잉ㅇㅇㅇㅇㅇㅇ

$z \geq z z$
NOOOON 응응응

$\geq \geq z \geq z$

NNNNN 응ㅇㅇㅇ응 $z \geq z z z$

$0+000$

두요 00000 00000

- 웅응요 $x \geq 2 x \geq$ onicio io

$z \geq z z z$ 品 in on $z \geq z z$

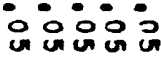

$z \geq z z$ inionio

$z z z z z$
웅웅웅요

का $x \geq z \geq$
웅영요

$z x \geq z 2$
언엉요

$z \geq x_{2} z$

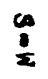

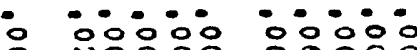
웅우

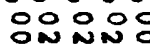
응웅웅응 영응영웅 응영요

w 2220 山ய
由 $\boldsymbol{0} \mathbf{z}$
웅ㅇㅇ

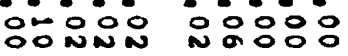

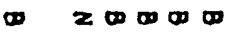

( 由ा 由

- $z z z$

$=\infty 00$
- noo noo

由 5010010 o noo

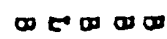

o on uno 更
00000 由
0000000000 ம oncuna wetre un o o o c $\geq 000$

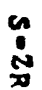

- 00000

வ
00000 ம0心
00000

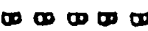

00000

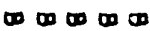

00000

$\infty 0 \infty \Phi$
00000 10000
00000 100000
00000 1000
- 0000000000

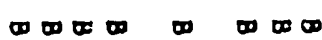

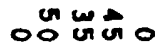

$\infty$
00000

00000 - 0000
$0000 \%$

由
ON N

$\infty$
N5000 100 


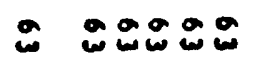

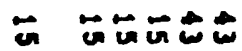

accos

onaga

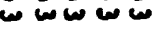

a o a o 0

$\rightarrow-\infty \sim$

N N N

NNNNN

-

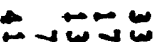

w

w

W conoro ט

NNNNN

W
운웅우

NN N N N N N

NDNN

$0 \omega_{0}=\omega$

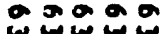
NNNNN שט

(1) w

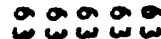

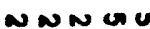
W $\because \approx N 0$

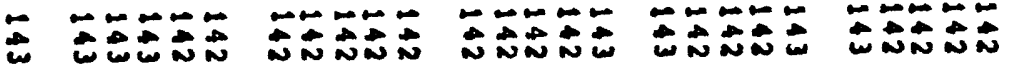 \\ แ艹

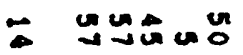

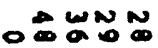 \\ $\because \ln _{0} \in \mathbb{0}$ \\ or ua \\ - $\infty \infty$ \\ N \\ NONN

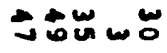
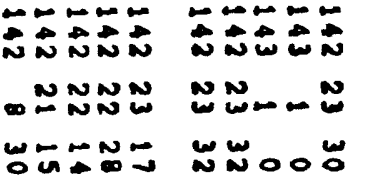

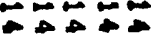

NNNGE

N NN U⿴囗十

مأم

ง 뜨우

: $8: 80:$
ANMON

io in: 잉ㅇㅇㅇㅇㅇ
- unw

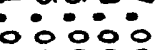
영융ㅇㅇ
nONN U $\therefore 0: 0$

NOONW inio: 영 눙영
Un w00 ㅇㅇ인 영유인
- w n

영영웅
$0 \div 0 \div$ 닝충ㅇㅇ
- n-mun monoo

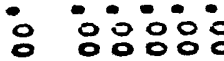

ㄴ W $\because 0000$

$\because 00-1$

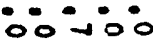

1000: 웅ํㅇ 00000 웅언ㄱㅇㅇㅇ

ONOOO OUMOO w-0응 uvio 응요요

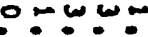

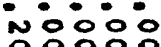

00000

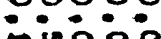
눙웡
- ar-2u 20 noo

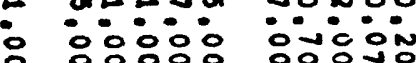
0 NwN-

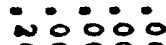
$\because 0 m N N$ 운우잉 NOOHN

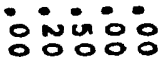

UNEOO 웅영웅 ONNNE

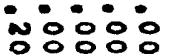
우응 응응 ㄴ 0000

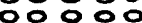

ज证定

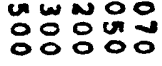

:

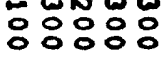

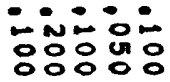

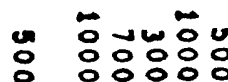

의.

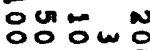
승요융용 응 잉ㅇㅇㅇㅇㅇㅇ

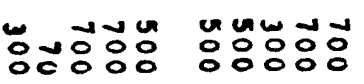

जิํํ유 00080

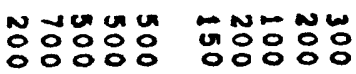

0

$\therefore \quad 0000$
in unin
$z \quad z z z z$

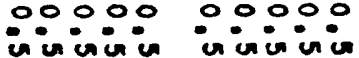

$z z z z z$ $z z z z$
000 00

$z z z z$
00:00 unuivin

z2ZZZ
00000

ininivi

$z z z z z$
00000 ivivivin

$z \geq z \geq z$
00000 in in in

$z z z z$

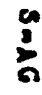

nNNNN NNNNN :O:O:

NNNNN ㅇㅇㅇㅇㅇㅇㅇㅇ

$z z z z=$

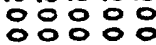

$z=2 z 2$
NNNNN 


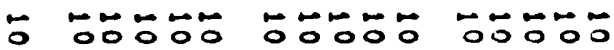

\%ั0

जேㅇํ유

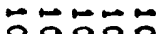

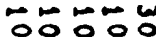

붕ํํ유

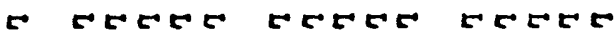

CEC

C5E

$r$ C

C.5

re

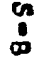

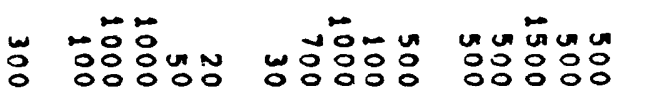

$\rightarrow$ N N N

M N N N N

00000

이의요잉

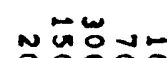

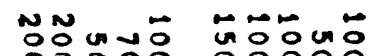
z

n nam
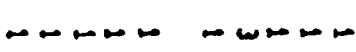

- W N N

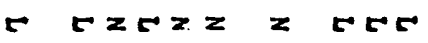

TECE

e

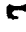

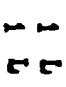

ேㅠㅇㅠ

ஸே0ல0ั0

$z x z z z$

$z \geq z z 2$

$z \quad z z z z$

$z \geq z z$

$z \geq z z 2$

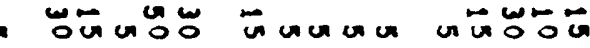

to

CE 5

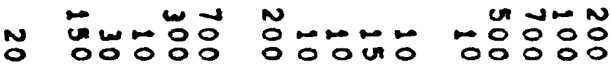

$$
z
$$

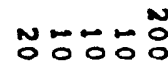
$z z=$
ํㅜㅇㅠㅜ웅 $z \geq z=$
No

0000 c $z 2 z$
๐ㅡㅇ요유 22

ேㅠ뭉 z zzzz

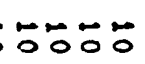
$25 \geq 22$

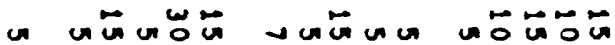
c $z$ un un un CEE
Onumor 225 oñunu zEC

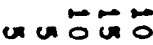

c

union un ECt5zz $\underset{⿱ ㇒}{\mathbb{m}}$

(4)
O OKONOO N

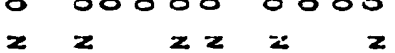

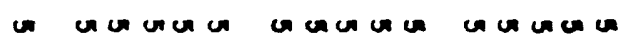

$z \quad z z z z \quad z z z z \quad z z z z z$

N ONONON NONONON NONONON

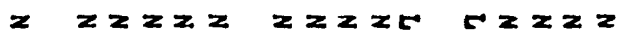

unuma

$z \geq z z z$ unuman

$z \geq z z z$ unu un

$z z z z z$

uenou

$z z z z z$

unuun

$z z z z z$

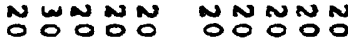
$z \quad z z$
ZEZ2Z
NNNN O

$z z z$
NNONONO NONONO

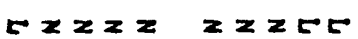

$\underset{x}{x}$

我

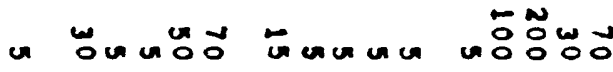
$c$

$$
\text { reter }
$$

unuugo oैunua CEE
CERE
\% retec
ज ज

tre wonum

C TEE

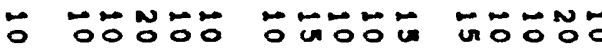
$x$ CE 5

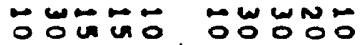
5
ONOEON NONOOO NֵO

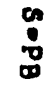

- WOE to

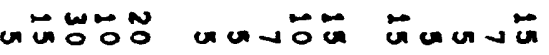
$c$

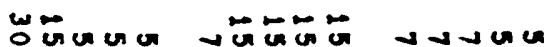


.
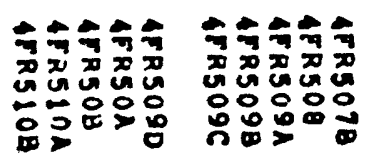

$-\quad \div-\div \div \div-0 \div 0$

- 00000

$z=z z z$

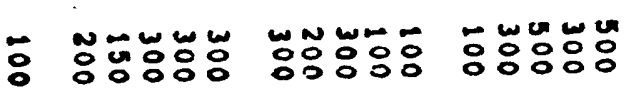

$0 \div 0 \div 0$

$z=z=$
ㄷํㅇ유웅

$z=2 z=$
ேㅜ웅

$z=z z$
Бேㅜ웅

$z \geq z z z$

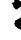

50505

$z x \geq z 2$

뭉ㅇㅇㅇ

co

$z=x^{\circ} z$

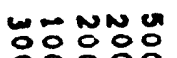

$$
z
$$

ํํํํํㅇํำ

$z=$ $z=$

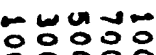
2.5

둥ㅎㅇ응

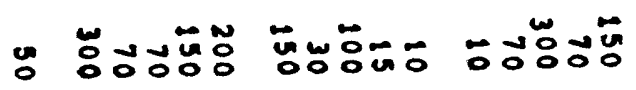

- cununa

z

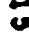
0000

cuncus na

Uncuñú

$z z z z$

NNWN $z z z z z$

$z z \geq z z$

ํํㅇ둥융 5

응둥응

noñon

$z \geq z z z$

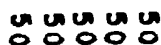

00008

$z z z=z$

anugug

$x=2$

unaun 00000

$z z z z z$

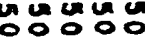

$z z z z=$

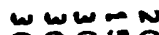
GOWON

$M N N=6$

UNO

UENONO

$z$
응 융융

$z=z z z$

NONNON 응융융

$z \geq z=2$

nNNNN

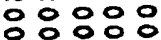

NNNNN

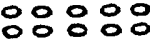

NONONO

$z z z z z$ $z z z z \pi$

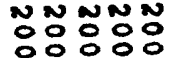

$z z z z$
NONONO $z z z z z$

NNNNN 영ㅇㅇㅇ응 $z z z z z$ $n$
$\vdots$
2

: 무융유

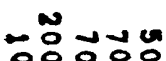
궁ㄱㅇ 겅ㅇㅇ

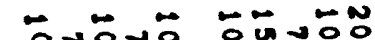
붕늉ㅇㅇ 눙융유 :

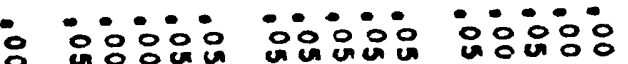

웅ㅇㅇㅇ

웅웅

운운운웅

웅:웅웅

우웅우 $z z z z z \quad z=00 z$ $z x \geq 2 x$

$z \geq 2=0$

w $z \geq z z$

영융웅유 $\infty \geq \infty x$ $\circ \div 000$ OnNaC $\infty \geq z \geq \infty$ $\div 0 \div 00$ 웅용ㅇㅇ

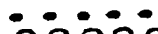
온옹요 W $12 z=2002$ $\therefore 000$ NNN요 $z \geq z \geq 2$
1000000000000000

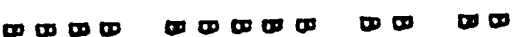

00000 00000
00000 00000
00000 10 0000
00000 - 0000 o0 oun W
- 00000

- 1000.0
00000 ம
00000 1000
0000000000

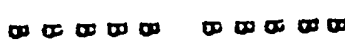

00000 का
00000

00000 以四比四四四
- 0 Uు 000 - 100
00000 - 0000
00000

10000

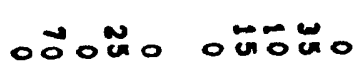

$\infty \quad 00$
Nov $\infty$

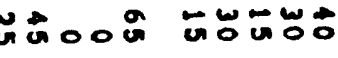
- 
as a a o

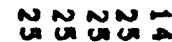
ט.

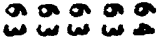
比率。 a voun

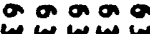

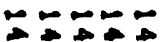
N NNN N 00000 00000 山' W世NO 두융요

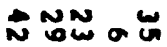

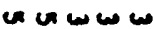

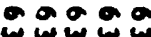

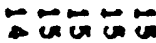
wNN

\begin{abstract}
00000
00000

00000

-
\end{abstract}

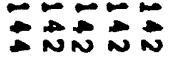
UNー مَ

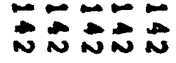
مN טم

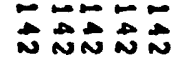

OेO

wั

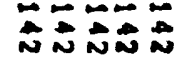

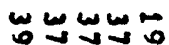
แ
ลேニニニล $\because=-\infty$

un $\rightarrow 00 \%$

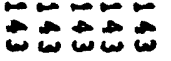
ن

w w w a a

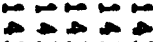
的的 훙후 $=0$ nôn Un nO Unnm - NnNnm

- - monn unuUn

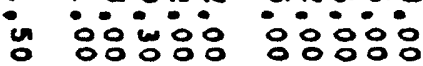

N w w 웅ㅇㅇㅇㅇㅇ
UnN WN 영ㅇㅇㅇ
ONNOO

ए人山 w

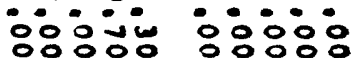

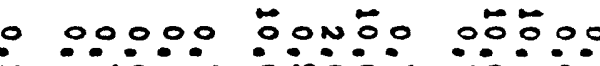

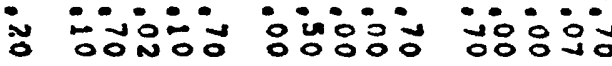

a

ก $\because 00=0$ is is: in 00:00 n?:-70 엉ㅇㅇㅇ 엥ㅇㅇㅇㅇ

W0:00 www:

- ONOEN a 20
TnN? 웅ㅇㅇㅇ응

to $\because \because \div \div$

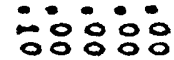

M-mNo w0000 엉융ㅇㅇ हم
- Onion moini inimin

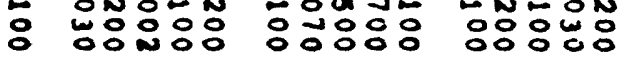

웅:-i. 40906 0000

0000 겅ㅇㅇㅇㅇㅇㅇ

inNNN

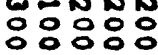

$\because$ in: 잉ㅇㅇㅇ응

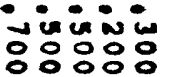

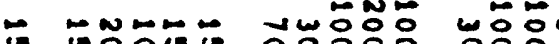

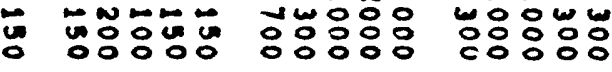
드융유 누ㅇㅝㅠㅇㅇㅠ

눙웅 웅영

mN" nom 영웅영웅 
두유유 TE

뚜유유

두융

우융웅 NE C C T TETE

뚜융유 सETE

ํㅜㅇ웅 C5te

뚱ํำ

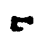

\$

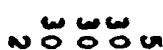

Non

Un 응응응

응ㅇㅇㅇㅇㅇㅇ w

응ㅇㅇㅇㅇㅇㅇ

능ㅇㅇ

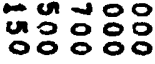
둥융웅
후유 to ze

$\because$ 우융

z $z x z z z$
무융

$z z z z z$
무웅

$z \geq z \geq z$
๐ㅜㅇㅠ

$z x z z z$
ㅜㅜ유유

$z z z z z$
ஜேர்ே0

$x \geq 2 z$
ேேேேே0

$x z z z z$

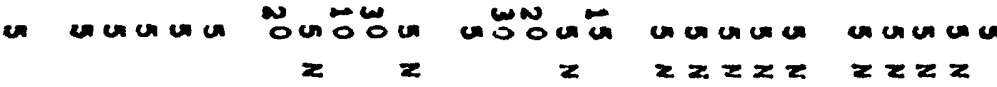

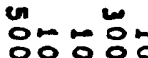

$\div \div \div \div \div \div$

O유유

$\geq \geq z$

$z z z x$ $z$
두유ำ $z \geq z \geq$ $\div \div 500$ $z z=25$
No

on $\rightarrow 00$

○ $z z z x$
으유유 TEE
유ㅇㅠㅜㅇㅠ $z \geq x$

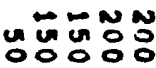

- cuncua

r rer مua ơ

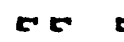

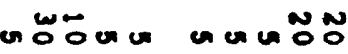

cet

cunan

te

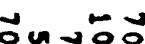

두웅우

$z x z z$

กำ 5

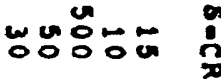

Eunuu

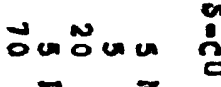

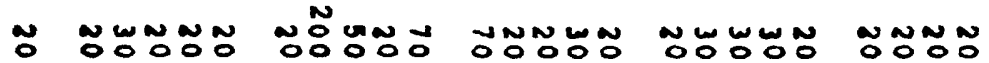

$z z z z x$

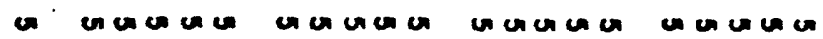

$z \geq z \geq z$

$2 \geq 2 z=$

$x \geq z z$

$z z z z=$ cucuucu

$2 \geq 2 z 2$ anum

zx z z z anuma

$z z z z$ $\operatorname{anang}$
$z z=2$

NONONON TETE $\because 00$ NONO

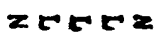

NONONON

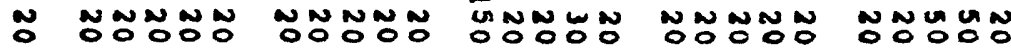

$z z z z z=2 z$

$x=$

(n)

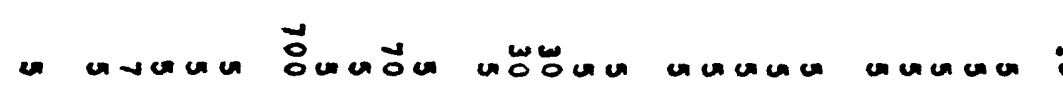

or una

$$
\text { ret }
$$

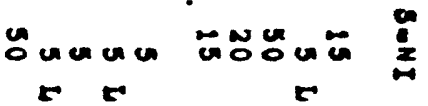

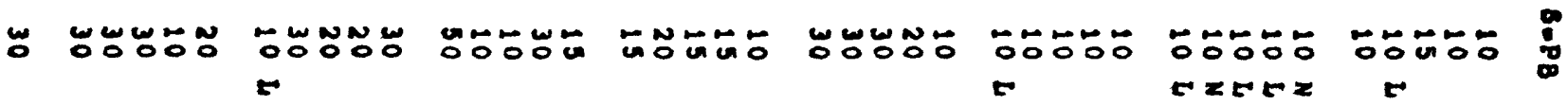

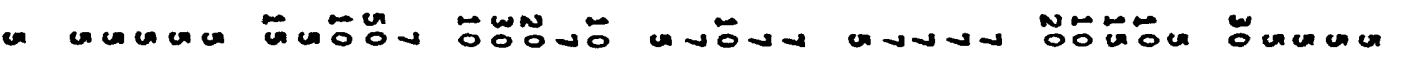


$\because$ 유웅

둥휴

05005

뜽후웅

두웅웅

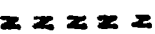

$z x \geq z$

$z z z=$

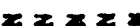

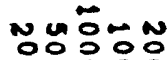

-

NพUUN

Nㅜ융유

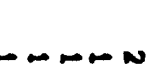

z $z$

- 00000

눙ㅎㅇ

5
5
융ㅁㅇㅇ 융ㅇㅇ

:ะ::

둥웅영영

두웅

$z \geq z=2$
뭉유웅

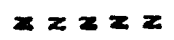

후융

$z \geq z=2$
- cuncon

$=x \geq 2 \geq$

- Nم$x=$

응 응융요

NONNN N

NONONO

unum

$z \geq z z z$

ungun

$z \geq z \geq$

sucos

$z \geq z z z$

जoñog

$x z \geq z$

जuํํㅇㅇㅇ

$z<2 z 2$

$\because$ NN W

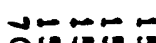
ouvun NŐ:

GN

NNER o ono

wo

$=$

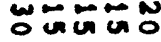

NNNNN NNNNN

O:O:융

ㅇํㅇํำ

NNNNN

ㅇㅇㅇㅇㅇㅇㅇㅇ

NNNNN

ㅇㅇㅇㅇㅇㅇㅇㅇ

NNNNN

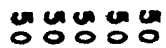

$z z z z z$

-

웅영영

$z$

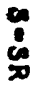

$z \quad z z z z$

$z z z$

$z \geq z \geq 2$

$z=2 z z$

$z \geq 2 z=$

$z \geq z z z$

$z z z z z$

뮹요

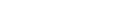

○

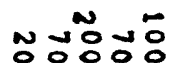

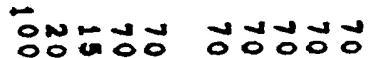

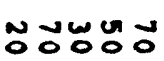

눙응유유

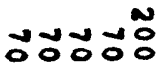

$x$

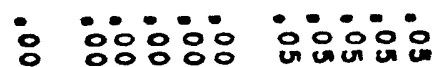

1.0000

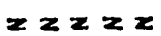

웅우 은은요요 웅웅ㅇㅇ $z \geq z z z$
운운웅응

$z \geq z z$
운운운웅 $z 2 z=2$
은은웅웅 $z z 2 z=$

응유:

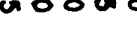
20020 : $\frac{1}{2}$

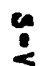

: 웅ㅇㅇㅇ

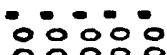

$0-0$ ㅇㅇㅇ웅 - 000

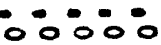

- 60000

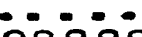
응웅웅 $\infty \infty 000$

00000 응ㅇㅇㅇ잉 10000
운응유 $\geq z$

웅웅 웅요 - 000

00000

00000 -

00000 1000
00000 00
00000 100000
00000 10000
[00000
00000

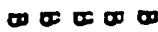

00000

10000
0000000000

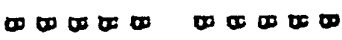

00000 - $\infty 00$
00000 ம
00000 ம
00000

0000000000

-
10010
ம0100
10000 wัo num cre 
000000

000000

- 00000

-

\section{$\begin{array}{cccc}-\cdots & \cdots\end{array}$}

0000000000

00000

0000
00000
00000

00000

क
00000 00000 00000 வ
00000 00000 00000 00000 దుய
00000

00000

00000 1000
00000

00000

00000 ద
5
in
है
00000

00000

00000

00000
0000000000

0000000000

100000000
0000000000
0000000000 0000 00000 0000000000 00000
- ․․․․ 눙ㅇㅇㅇㅇㅇㅇ
Nuㄴ. 웅잉 OUUno muntu 웅 웅연 영영영

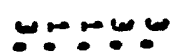
영영영 영영영

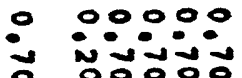
웅웅

orm: is: $\div 0 \div 00$ : ज: 0 in. NONOO OOOF= in:-0 0itio

$00-00$

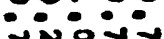
숭유

$\omega \leftarrow+\infty$ num vo 웅으

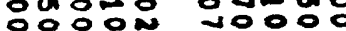

- 00000

- 웅ㅇㅇ웅 CECE
00000 눙숭웅 OONE

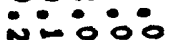
w은 등ㅇㅇㅇㅇㅇ
00000 IONNO c 5
00060 능웅 $0000 \%$

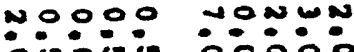
영웡: 영영영 \%궁

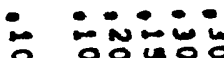
느웅 oUnin innio. 응요융

웅ㅎㅇ 엉영영

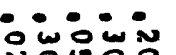

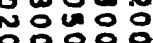

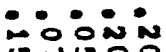
붕영ㅇㅇㅇ

NN는

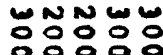

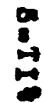

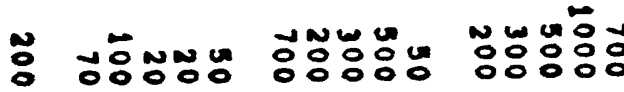
00000 뭉요용

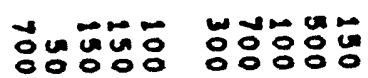

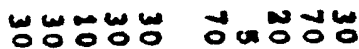

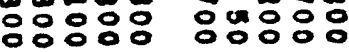

- 0000000000

i inivis inivin

$z \quad z z z z=z z z z$
00000 in in $z z z z=$
OOONO univio $z z z=2$ inuiv

$z=2 z$
00000 inivis $z=2 z$
00000 inisiv

$x \geq x=2$ ion:

$z x \geq z$

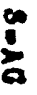

NNNNN 응ㅇㅇㅇㅇㅛ

NNNNN

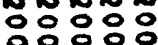

NNN NONO NONONO 응영ㅇㅇㅇ 융영영 $x \geq 2 z=$ 


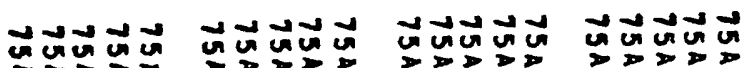
ing

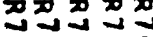
$\rightarrow \overrightarrow{a \rightarrow \infty}+\vec{a}$ $\rightarrow+\infty=\infty$ 入观政

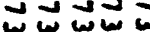

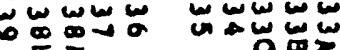

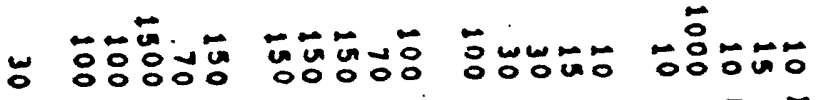
$=$

-

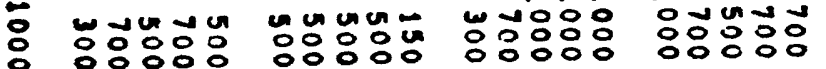

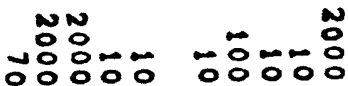

0000000000

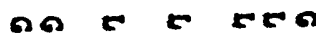

Nan

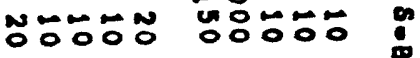

a

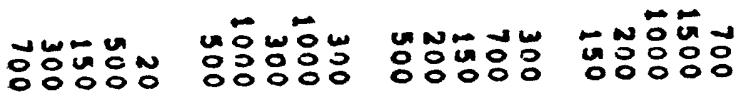

מח מח מח

NחR

WーW

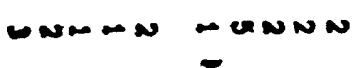

:

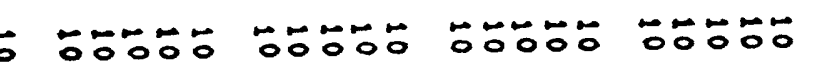

뚜융

두ㅇㅠㅜ웅

두웅

$\div 0 \div 0$

$\sum_{0}^{\infty}$

$z \quad z \geq z z z$

$z x \geq z z$

$z z z z z \quad z z z z z$

$2 z 2 \geq 2$

$z 2 z=c$

$z z z=$

$z=2=$

a $z$

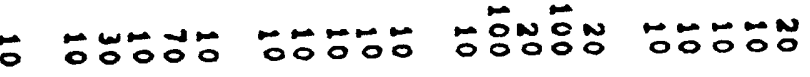
$z=$ $z$

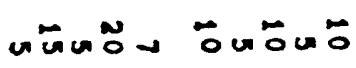

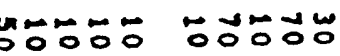
$x \geq x \geq x$

s.s.

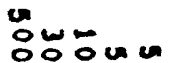

a

WONGO Ẽuna 5

$c t$ r

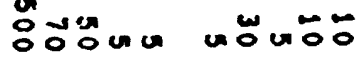
tr 25
NWNN

$z=z z$

MNN NONO

$z z z$

NNNNN

$z z z z$

NNNN

O NONONO NONON

$z \geq z z$

$z z z z 2$

$z \quad z \geq z$

unum unuara

$x z z z \quad z z z z z$

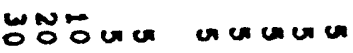
$z z \quad z z z z z$

$z \quad 2 x \geq 2 x$

NNNN

NNNNN

느요요

NUNON N N

00000

$z 2 x$

$z z z z$

$z \geq z \geq z$

NONN N N

$z \geq z z z$

NNNNN

$z \geq z z z$

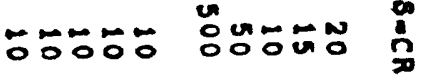
$=z$

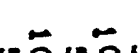

눙

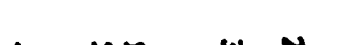

NONONON NONOONO

$z z z z \quad z z$

unuma unoua : $z z z z z z z \quad z z$ 
$\because$ 후유 우융

$\div \div 00$

뚜응

두ㅇㅜㅜ

두웅

두웅

z $=2 \mathrm{C}$

$x z z=E \quad z Z z z$

$z z=2$

$z$

$=2$

$x \geq z=$

$z=2 z 2$

$z=z z$

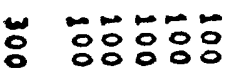

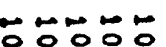

๑๐: $z z z x z$
둥뭉뭉 영ㅇㅇㅇ $2 z$

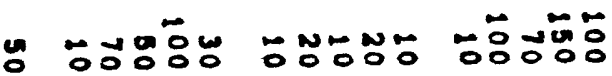

$z=z z z$

$\rightarrow$

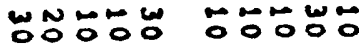

$z$

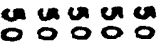

$z z z z z$

ஸே0000 $z=$

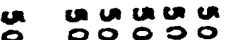

00000 10000

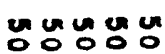

$z x z z x$ 05000

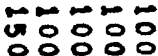
$z \geq z z$
둥융융유

$z z z=$
ลัลำำ

$x z$
OND。

유ㅇㅠㅠ유유유 $\infty$

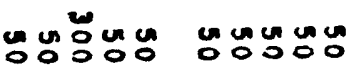

$z=$

$z \geq z \geq z$

ununu 00000

$z \geq z z=$

พ5000

ODON= $z=z Z z$
Nㅠㅇㅠ

x
00000

2

"

mucu $z=2 z=$

Nㅡㅇㅠ:

NNNNN 응응융응

NONNNO

NNNNN

NNNNN

NNNNN 응ㅇㅇㅇㅇㅇ

$z z z z z$

$\underset{⿱ 亠 乂}{2}$

$z \quad z z z z$

$z x z z z$

$z \geq \geq \geq 2$

$z \geq z z z$

$z z z z$

zZzZz

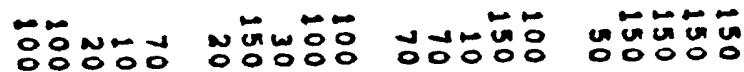
$z$ z
웅융웅 웅ㅇㅇ 웅융융 훙ㅇㅇㅇㅇㅇ 由 $\therefore \circ \div 0: 0$ 四 ㅇㅇㅇㅇㅇㅇ

웅ㅇㅇㅇ 四
웅ㅇㅇㅇㅇㅇㅇㅇㅜ 1000

웅ㅇㅇㅇㅇㅇㅇ

$\circ 0000$

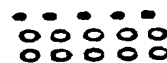
ம0

\section{0}

-
00000 山四四四
00000 10000
00000 ய00
00000 10 0060
00000 - 0000
00000.00000 வ1000
00000

由ण

00000

00000 ம
-0000000000
-0000000000000000

- 00000

00000

-
00000 10
00000 -
00900 00000
00000 100000
00000

100000
00000 - 0000 


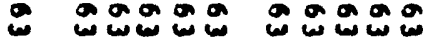

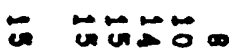

N NN=0 a

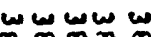

0
00

$\omega \infty 000$

W.

0000

(1)
00000

000000

00000

00000

00000

00000

00000

00000

0000000000

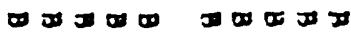

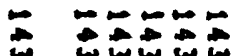

-

$\therefore= \pm \pm \pi a$

- ๑๑๐ั

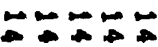

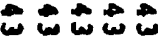
COUNN $\because \leftarrow 0$ un $\because-\infty=$ ace

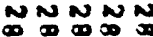
un unu a $\because \infty$

N000 00000000000000000000

NLON0000000000000000000000 notor $\infty \pi$

00000 00000 00000

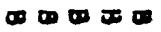

$0 \approx \infty \infty$

गण

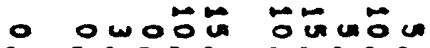
is 용ㅇㅇㅇ 웅우

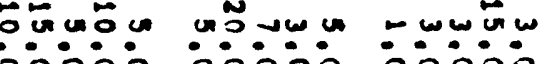
$\therefore 0000$ ㅇㅇㅇㅇㅇㅇ

man

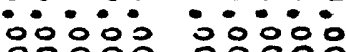

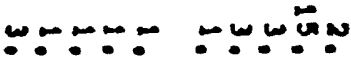

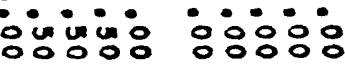

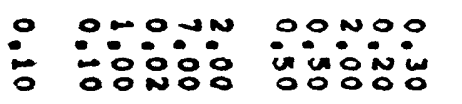

00000

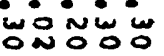

$=$ io:00 mo0:0 0mo00 n이의 영응응 O0, noñin

OOOOO

00000 누요 Nㅜㅇ두웅
- O-0ำ

은 영영응

-
Nut u

응영용

FE
유:0 웅ㅇㅇㅇㅡ.

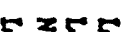

Nㅜㅇ

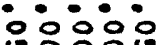

$\leftarrow$

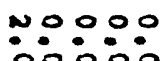

옹언요요

TEET
Nㅜㅇㅇㅡ

웅: úu un

r tzz
유유 은은응요

z $z \geq z z$

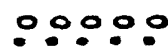
웅웅 जดง

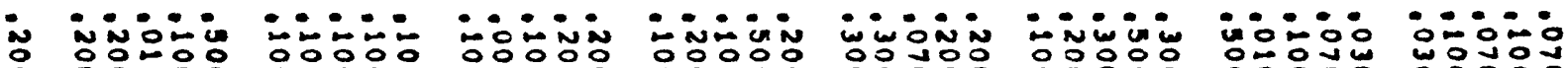
$\frac{6}{2}$

-

- 눙ㅇㅇㅇㅇㅇㅇ

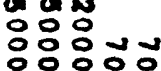

$\ln 200$

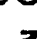

Un:

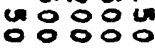

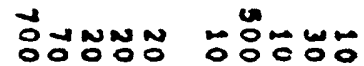
드유 in 0000

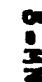

- i0:00

$z x z=0$
눙ㅇㅇ 인iv $z=0$
00000 ivivin X tox $x$
00000 inivin 00000 inivi $z x z x=$ $z \geq x \geq z$
00000 uivivín $z \geq z z z$
응ㅇㅇ in in is $z \geq z=2$
00000 inivivi $z=x \geq z$
N. NNGี

ㅇㅇㅇㅇㅇㅇㅇㅇ

$x z z=$
NONNN ㅇㅇㅇㅇㅇㅇㅇㅛ $z=2 z$
NONNN

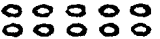

$z z z x=$
NNNNN NNNNN 응영융유 $x=2 x \frac{x}{3} 55^{z x}=$
NNNNN ㅇㅇㅇㅇㅇㅇㅇㅇㅇ $z z z z=$
NONNNN ㅇㅇㅇㅇㅇㅇㅇㅇ

$z x z=2$ 슝융융응 $z=z z=$ 


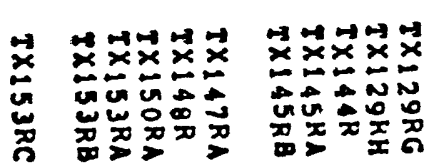

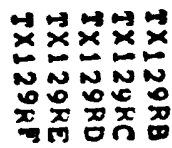

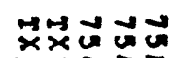

$\infty=\infty>0$

엄

ॠ u u
부부분

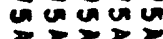

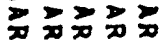

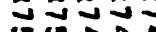

जU जि:

융ํํ

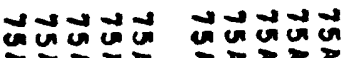

$\sum_{x \rightarrow \infty}>$

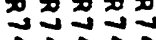

$\lim _{\substack{1 \\ 0} \rightarrow \infty}$

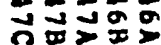

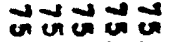

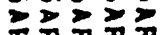

ग刃⿻

inti:

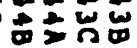

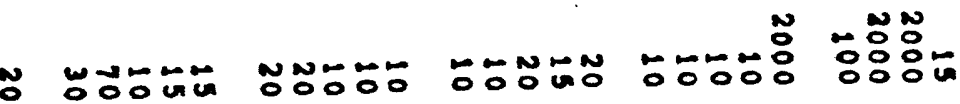

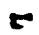

$c$

r
TE

as

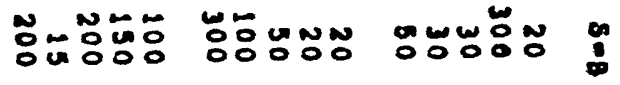
诺

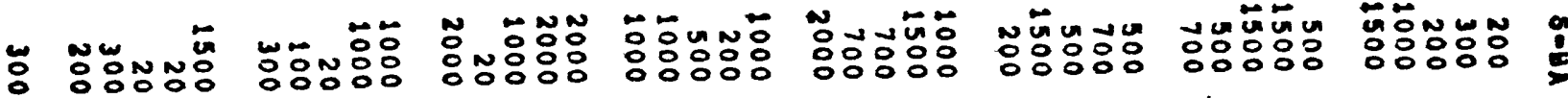
$z=2 \geq$

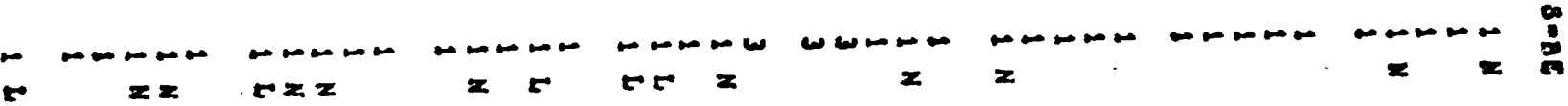

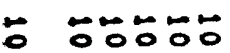

z $z \geq 2 z z$
5005

$z=$

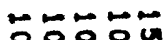

$z \quad z z$
․ํำำ $z x \geq z z$
뭉ํㅇㅇㅇㅇ $z \geq z z z$
๐ㅜㅇㅇㅠ

$z z z z z$

ํํㅇํํ응

ํํㅇํㅇ

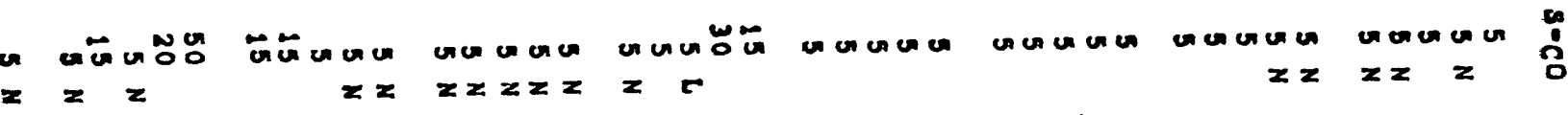

$\ddot{\circ}$

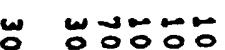
$z=2$

$z z z z z$

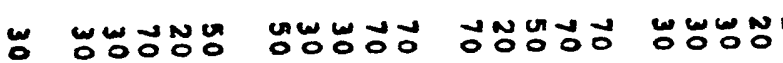
$x$
$=$

$z z$
UNNNNN
Nㅡ융유

ज.

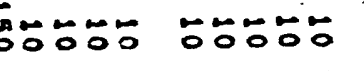

$z=z Z z=2$
2

N ONOOON

$z \quad z z z z z$

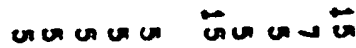
$z z x z$ $z z$

unenen

$z z z z z$

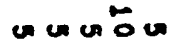
$z=2 z$ un un u

$z z z z z$ un unen

$z z z x z$ noumun
$z \quad z z z$
NNNNNO

00000

$z x z z z$
NNNNล OOOON

NNNNN OCOO०

NNNNN

NNONON

NNNNN

$z z=z Z z Z z Z z=$

$z \geq z \geq z$

NNล์ำ

OOOOOO $z=$

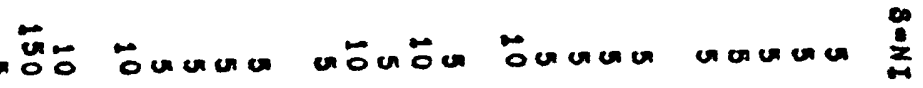

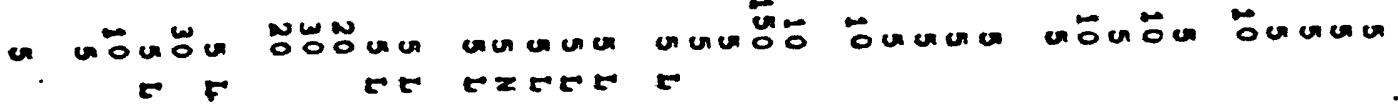

-

$5 x$

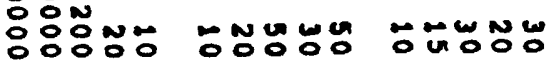

ㄸํㅇํํ유

2
눙융응

$\because \div 0 \div \div$

웅ํㅜ웅

- $z=z z$

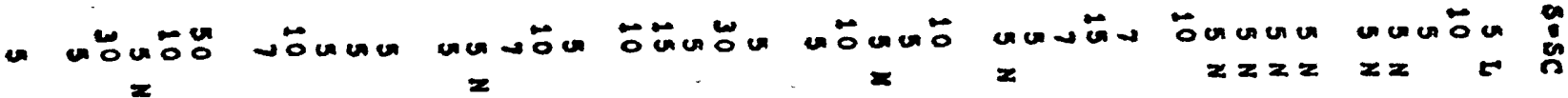
2.76 

un us

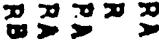

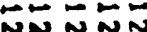

잉
$N=刀 x 又$

ஜx

D 0 U
버ำปี

xхज़

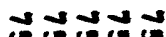

$>0$

वर

$\overrightarrow{0} \overrightarrow{1}=$

-0ㅇㅇㅇㅇㅇ

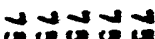
$>>>$ 점지 네는 - 541

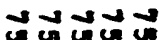

$>\geq \geq>$

ग》2

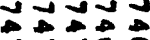

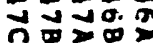

$>>2$

ग्]

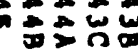

ธีงีயี $x \times x x$

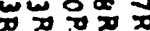

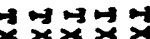

$\vec{x} \not \vec{x} \rightarrow \vec{x} \not \vec{x}$

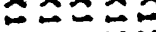
$\rightarrow-1 N$

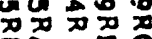

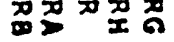

$\vec{x} \vec{x} \vec{x} \vec{x} \dot{x}$

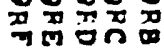

\section{乐}

-

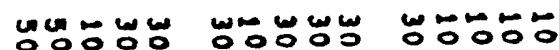

$x$

$z$

ㅜㅜ웅

무웅

ேㅜㅇㅜ

웅유유

$z x \geq x$

$=5 E$

$z \geq z z z$

C $x z x=$

$z x \geq x$

ڤ둥

سํㅇㅇํㅇ

믕

ธ므유요

고음

unn

두응

ேㅗㅇㅜ ดัดั 응 ๑о:อั

영용요

$z \geq z z$

$z \quad z z z$

$z z z z z \quad z z z z z$

$z=$ $z z=$

$z=2$

N

두융ㅇㅇ

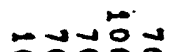

ONum.
잉

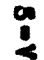

กับ

- Munư

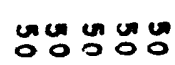

nun une

vunun un

unene

un un en

wusur

uncuncu

$z z z z z$

$z z z z z$

$z z z z z$

$z z z z z$

$z z z z$

$z z z z z$

$z z z z z$

$z z z z z$

- Бே000 2

$N N-N-$

-NN N

- NNNN

$\rightarrow \omega M-\infty N N$ $z=$

Non $z$

ேㅠㅇㅛ $z 2$

20

$\begin{array}{llll}N & N N N & N & N \\ 0 & 0 & 0 & 0\end{array}$

$z \quad z=2 z$

$\rightarrow \infty-\infty$

인

00080

$\begin{array}{llll}N N & N & N \\ 0 & 0 & 0 & 0 \\ 0 & 0 & 0 & 0\end{array}$

กำกับก

NNNNN

$a z z z z z z z$

$z=z z z$

ㅇํㅇำ

NNNNN

00008

NNNNN

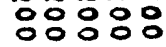

NNNNN

$z \geq z z=$

$z z z z z$

$z \geq z z$

$\underset{2}{\infty}$

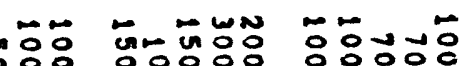

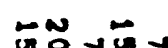

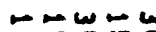

- NN

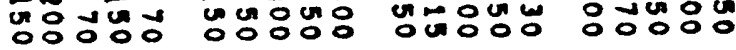

응ㅇㅇㅇㅇㅇ

눙

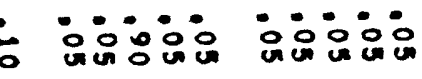
은은으 Zा 252 $z=z z-z z=$

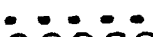

$\div 0000$
웅웅ㅇㅇㅇ 영ㅇㅇㅇㅇㅇㅇ - 000
웅웅ㅇㅇㅇ

0000
웅ㅇㅇㅇㅇㅇㅇ 00000 00000

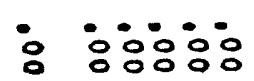

$\therefore \therefore: 0:$ का का एक

$\therefore \therefore \therefore$ कण 00

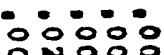
四

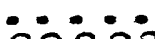
읃ㅇㅇㅇㅇㅇㅇ 웅ㅇㅇㅇㅇㅇㅇ

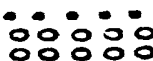
ఉ 000

$\infty+\infty \infty$

웅웅ㅇㅇㅇ 1000

눙용.

뚠ํㅇㅇㅇ $z=$

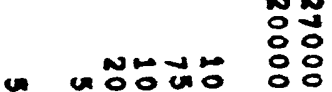

$=\infty$

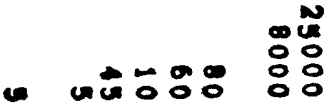

-

- 두융웅

:0ะ

ํํำ แล

10000

0000000000

00000

00000 5

$\infty \mathbf{0}$

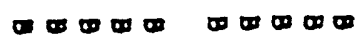

- 0 खण

- 000 $z=$

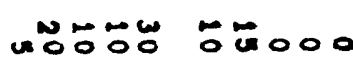

00000

00000

0000000000 - 000000

0000

0000

ए

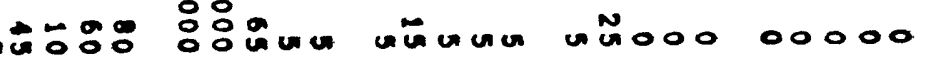

00000

0000000000 $\frac{2}{2}$

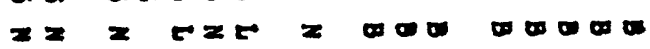
10000 - 

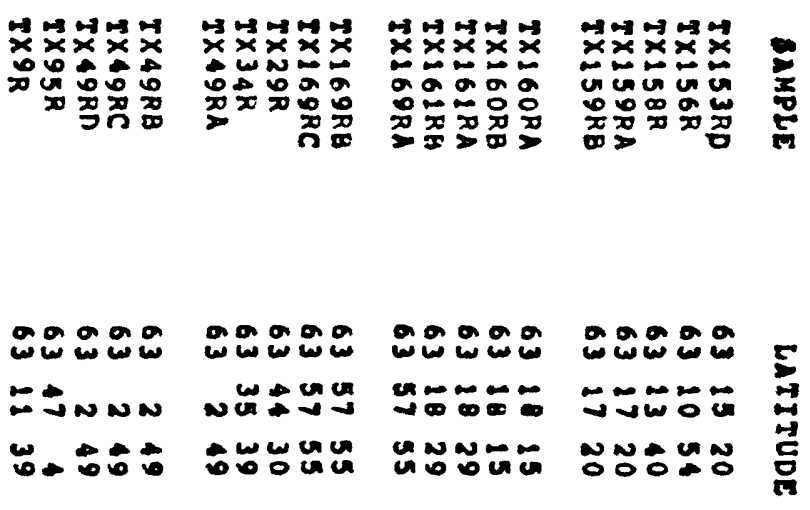

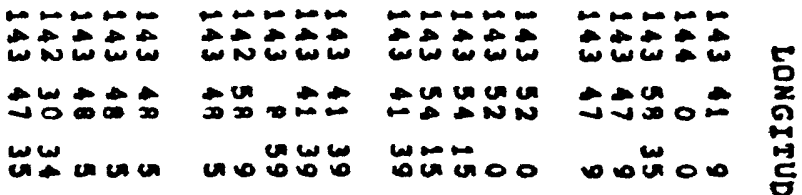

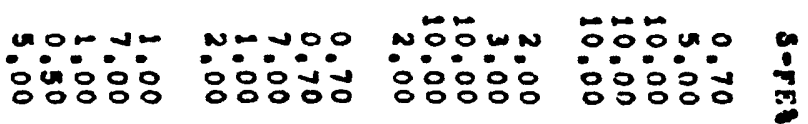

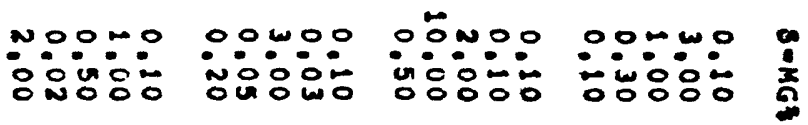

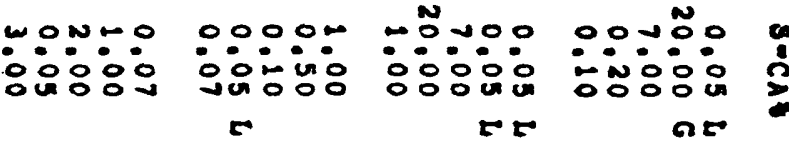

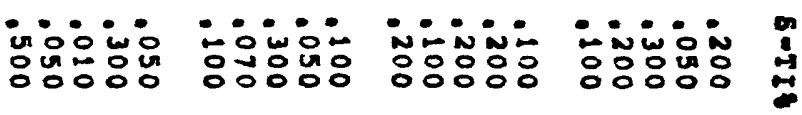

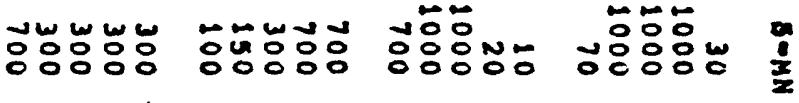

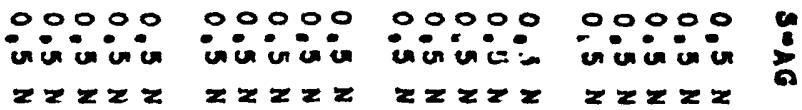

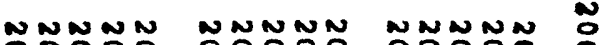

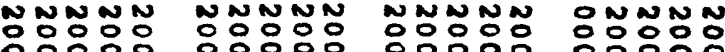

$z z z z z, z z z z z \quad z z z z z \quad z z z z$ 
W00

무웅웅

후융

두웅웅

스ㅇㅠㅠ

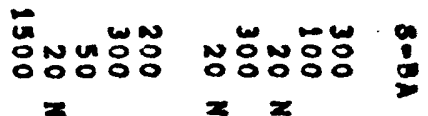

W

MNMN

-

$\geq E \geq z \geq \geq 0$

$$
\underset{8}{0}
$$

뚜웅

두융웅

둥후웅

$z z z z z$

Zx $2 x=$

$z=2 z$

뭉응ㅇㅇ

zx $x \geq 2$

unu ưo

unounon
$z z$

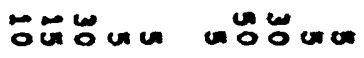

c

$z$

:

둥후

뚱유웅

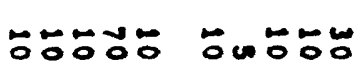

8

25

$z=2$

$z=25$

$x$

ㄸํㅇํㅇㅇ

E

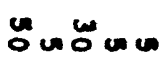

z 5

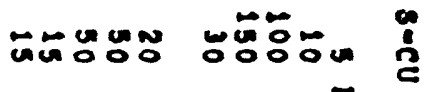

NNONONO

NNNNN

NNOOOOW NWONOO

NONOOOO

$z=2 x$

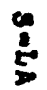

un unin

onum

nencua

$z \geq x z$

$z z z z=$

$z z z z x \quad z z z z x$

$\frac{1}{0}$

Nㅜㅇํㅠㅇㅛ

NONONO

NNNON

단

$2522=$

$z=2 z$

ลNㅇㅇㅇㅇ

$z=2 z$

na웅

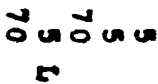

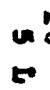

$t$

5

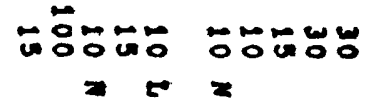

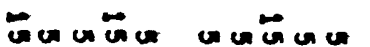

$r=5$

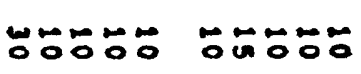

$x=$

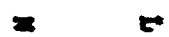


ํํํำ

ัํㅇํํำ

$\div 5 \div 0 \div$

ํํㅇํำ

$z=2 z 2$

Z

$x \geq x \geq z$

$z x z z z$

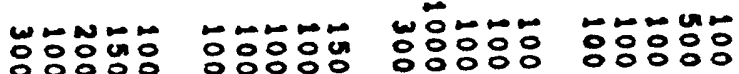

$z z z z$

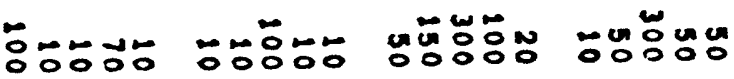

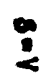

\begin{tabular}{|c|c|c|c|}
\hline 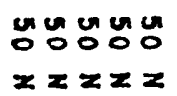 & 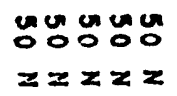 & 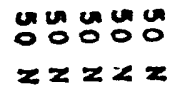 & $\begin{array}{l}000 \\
x \geq z z=\end{array}$ \\
\hline 0 & مّة & GE & 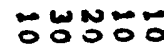 \\
\hline
\end{tabular}

NNNNN

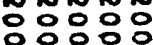

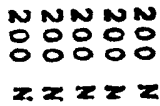

NNNNN :ํ:ดั:

NNNNN

유융유

$z z z z$

$z x \geq z=$

$z x z z$

Na n N

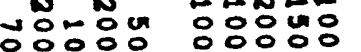

ํํㅇํํำ

\section{$\because \cdots$}

$\ldots . .$.

$\therefore \therefore$

טu

$z \geq E x$

둥윤용

$z \geq z z z$

$z z z z z$

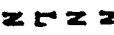

$\therefore 00$ in

$\therefore \therefore 0: 0$

$\therefore \therefore \div 0$

$\because \cdots$

$\infty$

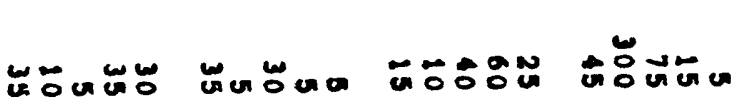

